\title{
Higher Spin Currents in Wolf Space: Part III
}

\author{
Changhyun Ahn \\ Department of Physics, Kyungpook National University, Taegu 702-701, Korea \\ ahn@knu.ac.kr
}

\begin{abstract}
The large $\mathcal{N}=4$ linear superconformal algebra (generated by four spin- $\frac{1}{2}$ currents, seven spin-1 currents, four spin- $\frac{3}{2}$ currents and one spin- 2 current) found by Sevrin, Troost and Van Proeyen (and other groups) was realized in the $\mathcal{N}=4$ superconformal coset $\frac{S U(5)}{S U(3)}$ theory previously. The lowest 16 higher spin currents of spins $\left(1, \frac{3}{2}, \frac{3}{2}, 2\right),\left(\frac{3}{2}, 2,2, \frac{5}{2}\right),\left(\frac{3}{2}, 2,2, \frac{5}{2}\right)$ and $\left(2, \frac{5}{2}, \frac{5}{2}, 3\right)$ are obtained by starting with the operator product expansions (OPEs) between the four spin- $\frac{3}{2}$ currents from the above large $\mathcal{N}=4$ linear superconformal algebra and the lowest higher spin-1 current which is the same as the one in the Wolf space coset $\frac{S U(5)}{S U(3) \times S U(2) \times U(1)}$ theory. These OPEs determine the four higher spin- $\frac{3}{2}$ currents and the next six higher spin2 currents are obtained from the OPEs between the above four spin- $\frac{3}{2}$ currents associated with the $\mathcal{N}=4$ supersymmetry and these four higher spin- $\frac{3}{2}$ currents. The four higher spin- $\frac{5}{2}$ currents can be determined by calculating the OPEs between the above four spin- $\frac{3}{2}$ currents and the higher spin-2 currents. Similarly, the higher spin-3 current is obtained from the OPEs between the four spin- $\frac{3}{2}$ currents and the higher spin- $\frac{5}{2}$ currents. The explicit relations between the above 16 higher spin currents and the corresponding 16 higher spin currents which were found in the extension of large $\mathcal{N}=4$ nonlinear superconformal algebra previously are given. By examining the OPEs between the 16 currents from the large $\mathcal{N}=4$ linear superconformal algebra and the 16 higher spin currents, the match with the findings of Beccaria, Candu and Gaberdiel is also given. The next 16 higher spin currents of spins $\left(2, \frac{5}{2}, \frac{5}{2}, 3\right),\left(\frac{5}{2}, 3,3, \frac{7}{2}\right),\left(\frac{5}{2}, 3,3, \frac{7}{2}\right)$ and $\left(3, \frac{7}{2}, \frac{7}{2}, 4\right)$ occur from the OPEs between the above lowest 16 higher spin currents.
\end{abstract}




\section{Introduction}

It has been studied in [1] that the better understanding for the string theory in the tensionless limit can be described from the Vasiliev higher spin theory. The string theory is described by the type IIB string theory on $A d S_{3} \times \mathbf{S}^{3} \times \mathbf{T}^{4}$. The dual conformal field theory (CFT) is believed to be on the moduli space of the free symmetric orbifold. This CFT contains $4(N+1)$ free fermions and bosons where $(N+1)$ is the product of $D 1$ brane and $D 5$ brane charges. On the other hand, the large $\mathcal{N}=4$ coset theory in two dimensional CFT, which is dual to the Vasiliev higher spin theory on $A d S_{3}$, has a factor $S O(4 N+4)$ in the numerator of the coset. This describes $(4 N+4)$ real free fermions. In the large level limit of the large $\mathcal{N}=4$ coset theory, the coset factor $\frac{S U(N+2)}{S U(N)}$ leads to the $4(N+1)=\left[(N+2)^{2}-1\right]-\left[N^{2}-1\right]$ free bosons. Then the number of free bosons and fermions is the same both in the symmetric orbifold theory and in the continuous orbifold theory (described in [1]). After precise analysis of the embedding of the permutation group $S_{N+1}$ into the $U(N)$, they have concluded that the $U(N)$ singlet sector of the large $\mathcal{N}=4$ coset theory in the large level limit is a subsector of the $S_{N+1}$ singlet sector of the symmetric orbifold theory. In other words, the perturbative Vasiliev theory is a subsector of the tensionless string theory. See also the recent work in [2] for relevant discussions.

In the large level limit, the parameter $\gamma$ of large $\mathcal{N}=4$ linear superconformal algebra [3] becomes zero. Then the large $\mathcal{N}=4$ linear superconformal algebra contracts to the small $\mathcal{N}=4$ linear superconformal algebra (generated by spin-2 stress tensor, four spin- $\frac{3}{2}$ supersymmetry currents and three spin-1 currents associated with the affine $S U(2)$ algebra) with four free bosons and fermions. In the type IIB string theory on $A d S_{3} \times \mathbf{S}^{3} \times \mathbf{S}^{3} \times \mathbf{S}^{1}$, the above parameter $\gamma$ is described in terms of the radius of $A d S_{3}$ space and the radius of one of the three spheres. There exists a relation between the radius of $A d S_{3}$ and the radii of two three spheres. As $\gamma \rightarrow 0$, one of the two three spheres becomes flat and decompactifies to $R^{3}$ (and to three torus $\mathbf{T}^{3}$ ) and then one realizes the string theory on $A d S_{3} \times \mathbf{S}^{3} \times \mathbf{T}^{4}$ mentioned before.

What happens for finite level before taking the large level limit? One should understand the dual CFT corresponding to the above type IIB string theory on $A d S_{3} \times \mathbf{S}^{3} \times \mathbf{S}^{3} \times \mathbf{S}^{1}$. In addition to the large $\mathcal{N}=4$ linear superconformal algebra, the large $\mathcal{N}=4$ coset theory has an extended chiral algebra which contains the higher spin currents initiated in [4]. Therefore, one immediate thing to do is to obtain the lowest higher spin currents for generic $N$. Once the higher spin currents are obtained for generic $N$, then it is straightforward to calculate the three point functions with two scalars in CFT. Before describing the higher spin currents 
for general $N$, one fixes $N=3$ in this paper because one can see most of the structures of the extended large $\mathcal{N}=4$ linear superconformal algebra. One can vary $N$ and obtain the higher spin currents for several $N$ values in near future. One expects that one can determine the structure constants for general $N$ from the above low $N$ values results. The present work will give some hints for the construction of the three point functions with two scalars for low higher spins of $s$ with arbitrary $N$ and level $k$. Eventually, the general spin $s$ dependence of the three point functions corresponding to the three point function in the $A d S_{3}$ bulk theory will be obtained.

For the 16 currents in the large $\mathcal{N}=4$ linear superconformal algebra, the explicit results for $N=3$ in terms of the coset fields were present in Part I [5]. The general $N$ dependence can be found from the earlier work in [6, 7].

How does one determine the 16 lowest higher spin currents in terms of coset fields? For the lowest higher spin-1 current in the linear version, one takes the one in the nonlinear version in Part I [5] 1. In other words, the starting point is based on this higher spin-1 current in the linear (or nonlinear) version. As described in the abstract, the next four higher spin$\frac{3}{2}$ currents can be obtained from the OPEs between the four spin- $\frac{3}{2}$ currents which are the supersymmetry generators of the large $\mathcal{N}=4$ linear superconformal algebra and this higher spin-1 current. Each higher spin- $\frac{3}{2}$ current has the same $U(1)$ charge (which will be discussed in next section) as the corresponding above spin- $\frac{3}{2}$ current because the $U(1)$ charge of the higher spin-1 current is zero. Two of them belong to the same $\mathcal{N}=2$ multiplet with the higher spin-1 current and other two belong to two other $\mathcal{N}=2$ multiplets respectively. Then the next thing is to obtain the next six higher spin-2 currents. Since there are four spin- $\frac{3}{2}$ currents and four higher spin- $\frac{3}{2}$ currents, there exist 16 possible OPEs between them. Among them, the only 12 OPEs are nontrivial ones. Then the above six higher spin-2 currents appear twice in these OPEs. One of them completes the last component of the above first $\mathcal{N}=2$ multiplet containing the higher spin-1 current. Two of them belong to the above second $\mathcal{N}=2$ multiplet as the second and third components. Two of them belong to the third $\mathcal{N}=2$ multiplet (second component and third component). The last one belongs to the new fourth $\mathcal{N}=2$ multiplet as a first component.

For the next four higher spin- $\frac{5}{2}$ currents one continues to calculate similar OPEs. Since there are four spin- $\frac{3}{2}$ currents and six higher spin- 2 currents, there exist 24 possible OPEs

\footnotetext{
${ }^{1}$ The fields in the linear version are defined as the ones in an extension of large $\mathcal{N}=4$ 'linear' superconformal algebra (generated by spin- 2 current, four spin- $\frac{3}{2}$ currents, seven spin- 1 currents and four spin- $\frac{1}{2}$ currents) where the OPEs between the spin- $\frac{3}{2}$ currents are linear while the fields in the nonlinear version are defined as in an extension of large $\mathcal{N}=4$ 'nonlinear' superconformal algebra (generated by spin-2 current, four spin- $\frac{3}{2}$ currents and six spin-1 currents) where the OPEs between the spin- $\frac{3}{2}$ currents are nonlinear. The OPEs are nonlinear in both linear and nonlinear versions.
} 
between them. Among them, the only 12 OPEs are nontrivial ones. Then the above four higher spin- $\frac{5}{2}$ currents appear three times in these OPEs. One of the four higher spin- $\frac{5}{2}$ currents plays the role of the last component of the above second $\mathcal{N}=2$ multiplet while one of them is the last component of the third $\mathcal{N}=2$ multiplet. Two of them consist of the second and third components of the fourth $\mathcal{N}=2$ multiplet. For the higher spin-3 current, there are four spin- $\frac{3}{2}$ currents and four higher spin- $\frac{5}{2}$ currents and so there are 16 OPEs between them. The nontrivial OPEs are given by four and the higher spin-3 current appears four times in these OPEs. This higher spin-3 current will be the last component of the fourth $\mathcal{N}=2$ multiplet. One expects that the above OPEs between the spin- $\frac{3}{2}$ currents and the higher spin currents of $s=1, \frac{3}{2}, 2$ and $\frac{5}{2}$ will be generalized to the general $N$ case without any difficulty because the structure constants will have very simple functions of the level $k$ and several $N$ examples will fix them completely.

In [4], the large $\mathcal{N}=4$ holography is proposed and some of the currents and higher spin currents are constructed using the oscillator formalism in the context of asymptotic symmetry algebra in $A d S_{3}$ bulk theory. One expects that the extension of large $\mathcal{N}=4$ linear superconformal algebra should match with the corresponding higher spin algebra defined in the $A d S_{3}$ bulk theory. Therefore, it is necessary to calculate the OPEs between the 16 lowest higher spin currents, as a first step, to obtain the corresponding algebra in dual CFT associated with the above asymptotic symmetry algebra in $A d S_{3}$. One should calculate $16 \times 16=256$ OPEs but the reversed OPE between any two higher spin currents can be obtained from the original OPE between them. Therefore, one should calculate $\sum_{n=16}^{1} n=\frac{1}{2} \times 16 \times(16+1)=136$ OPEs which are nontrivial in the sense that there are singular terms. For the corresponding OPEs in the nonlinear version, the number of OPEs is less than this number because there exist some trivial OPEs. The OPEs are written in terms of coset fields because the higher spin currents are given in terms of those coset fields.

Therefore, in order to rewrite them in terms of known 16 currents and 16 higher spin currents (and their derivatives), one should introduce the possible candidates with arbitrary coefficients in the right hand side of the OPEs. Since there is $U(1)$ charge constraint in the given OPE, the number of arbitrary coefficients is reduced. If one cannot write any OPE in terms of the known 16 currents and 16 higher spin currents (and their derivatives), then one should expect that there should exist a new primary field for given pole in the OPE. Since the extended algebra contains infinite number of higher spin currents, one expects that there should be new primary currents in the 136 OPEs and actually the next 16 higher spin currents occur. So it is better to obtain those 16 next lowest higher spin currents instead of calculating the above 136 OPEs. From the experience in the construction of 16 lowest higher 
spin currents one can apply the prescriptions used in there to the 16 next lowest higher spin currents.

Now then one can calculate the 136 OPEs explicitly and each pole of each OPE is written in terms of multiple products of various coset fields. One puts each pole of the OPE to be equal to be multiple product of known 16 currents, 16 higher spin currents and the next 16 higher spin currents (and their derivatives) satisfying the $U(1)$ charge constraint. Compared to the corresponding OPEs in the nonlinear version of Part II [8], the present 136 OPEs do not have any multiplet products between the 16 currents of large $\mathcal{N}=4$ linear superconformal algebra and the 16 lowest higher spin currents (and their derivatives) except a few OPEs. Furthermore, if one goes to different basis found in [9] where it is not clear how the coset fields arise explicitly, those nonlinear terms disappear. One of the reasons why the current issue on the construction of 136 OPEs is interested in is that if one sees the corresponding OPEs in the nonlinear version from Part II, it is hard to extract the simple structure behind these OPEs because there are too many nonlinear terms between the 16 currents and the 16 lowest higher spin currents in the OPEs. In the linear version of present paper, the right hand side of the OPEs consists of linear terms having either 16 currents, 16 lowest higher spin currents, or 16 next lowest higher spin currents (and their derivatives) and nonlinear terms having 16 currents (and their derivatives) from the large $\mathcal{N}=4$ linear superconformal algebra up to the poles of the OPEs considered in this paper. This indicates that if one goes to $\mathcal{N}=4$ superspace description, then the above 136 OPEs can fit in one single OPE in $\mathcal{N}=4$ superspace and the right hand side of this OPE contains linear terms as well as nonlinear terms consisting of multiple product of $\mathcal{N}=4$ stress tensor (and their derivatives) up to the poles considered in this paper.

In this paper, the analysis done in Part I [5] and Part II [8] for the higher spin currents is extended to the large $\mathcal{N}=4$ linear superconformal algebra. The extra two $\mathcal{N}=4$ multiplets (16 lowest higher spin currents and the next 16 higher spin currents) are obtained. In particular, the complete OPEs between the first $\mathcal{N}=4$ multiplet in component approach are described.

In section 2, the review of large $\mathcal{N}=4$ linear superconformal algebra is described.

In section 3, the 16 lowest higher spin currents are constructed. Let us describe the notations one uses in this paper. In order to emphasize the extra higher spin currents, one uses a boldface current. In other words, the boldface currents are the higher spin currents. Furthermore, in order to characterize the higher spin currents in the nonlinear version, the word of 'non' is added in the subscript of the boldface higher spin currents. The boldface higher spin currents without 'non' are the higher spin currents in the linear version. For 
example, the 16 lowest higher spin currents have the following spins 2

$$
\begin{array}{rll}
\left(1, \frac{3}{2}, \frac{3}{2}, 2\right): & \left(\mathbf{T}_{\text {non }}^{(\mathbf{1})}, \mathbf{T}_{+, \text {non }}^{\left(\frac{3}{2}\right)}, \mathbf{T}_{-, \text {non }}^{\left(\frac{3}{2}\right)}, \mathbf{T}_{\text {non }}^{(\mathbf{2})}\right) \leftrightarrow\left(\mathbf{T}^{(\mathbf{1})}, \mathbf{T}_{+}^{\left(\frac{3}{2}\right)}, \mathbf{T}_{-}^{\left(\frac{3}{2}\right)}, \mathbf{T}^{(\mathbf{2})}\right), \\
\left(\frac{3}{2}, 2,2, \frac{5}{2}\right): & \left(\mathbf{U}_{\text {non }}^{\left(\frac{3}{2}\right)}, \mathbf{U}_{+, \text {non }}^{(\mathbf{2})}, \mathbf{U}_{-, \text {non }}^{(\mathbf{2})}, \mathbf{U}_{\text {non }}^{\left(\frac{5}{2}\right)}\right) \leftrightarrow\left(\mathbf{U}^{\left(\frac{3}{2}\right)}, \mathbf{U}_{+}^{(2)}, \mathbf{U}_{-}^{(\mathbf{2})}, \mathbf{U}^{\left(\frac{5}{2}\right)}\right), \\
\left(\frac{3}{2}, 2,2, \frac{5}{2}\right): & \left(\mathbf{V}_{\text {non }}^{\left(\frac{3}{2}\right)}, \mathbf{V}_{+, \text {non }}^{(\mathbf{2})}, \mathbf{V}_{-, \text {non }}^{(\mathbf{2})}, \mathbf{V}_{\text {non }}^{\left(\frac{5}{2}\right)} \leftrightarrow\left(\mathbf{V}^{\left(\frac{3}{2}\right)}, \mathbf{V}_{+}^{(\mathbf{2})}, \mathbf{V}_{-}^{(2)}, \mathbf{V}^{\left(\frac{5}{2}\right)}\right),\right. \\
\left(2, \frac{5}{2}, \frac{5}{2}, 3\right): & \left(\mathbf{W}_{\text {non }}^{(2)}, \mathbf{W}_{+, \text {non }}^{\left(\frac{5}{2}\right)}, \mathbf{W}_{-, \text {non }}^{\left(\frac{5}{2}\right)}, \mathbf{W}_{\text {non }}^{(3)}\right) \leftrightarrow\left(\mathbf{W}^{(2)}, \mathbf{W}_{+}^{\left(\frac{5}{2}\right)}, \mathbf{W}_{-}^{\left(\frac{5}{2}\right)}, \mathbf{W}^{(3)}\right) .
\end{array}
$$

These are primary fields under each stress tensor in the nonlinear or linear version. In section 2 , the $U(1)$ charges of these higher spin currents will be described. The precise relations between the higher spin currents in the nonlinear and linear versions (1.1) are described.

In section 4, the OPEs between the 16 currents from the large $\mathcal{N}=4$ linear superconformal algebra and the the 16 lowest higher spin currents found in section 3 are given. The comparison with the field contents in different basis [9] is given.

In section 5, the 16 next lowest higher spin currents are constructed. One can specify these 16 higher spin currents according to their spins and $\mathcal{N}=2$ multiplets as follows:

$$
\begin{array}{lll}
\left(2, \frac{5}{2}, \frac{5}{2}, 3\right): & \left(\mathbf{P}_{\text {non }}^{(2)}, \mathbf{P}_{+, \text {non }}^{\left(\frac{5}{2}\right)}, \mathbf{P}_{-, \text {non }}^{\left(\frac{5}{2}\right)}, \mathbf{P}_{\text {non }}^{(3)}\right) \leftrightarrow\left(\mathbf{P}^{(2)}, \mathbf{P}_{+}^{\left(\frac{5}{2}\right)}, \mathbf{P}_{-}^{\left(\frac{5}{2}\right)}, \mathbf{P}^{(3)}\right), \\
\left(\frac{5}{2}, 3,3, \frac{7}{2}\right): & \left(\mathbf{Q}_{\text {non }}^{\left(\frac{5}{2}\right)}, \mathbf{Q}_{+, \text {non }}^{(3)}, \mathbf{Q}_{-, \text {non }}^{(3)}, \mathbf{Q}_{\text {non }}^{\left(\frac{7}{2}\right)}\right) \leftrightarrow\left(\mathbf{Q}^{\left(\frac{5}{2}\right)}, \mathbf{Q}_{+}^{(3)}, \mathbf{Q}_{-}^{(3)}, \mathbf{Q}^{\left(\frac{7}{2}\right)}\right), \\
\left(\frac{5}{2}, 3,3, \frac{7}{2}\right): & \left(\mathbf{R}_{\text {non }}^{\left(\frac{5}{2}\right)}, \mathbf{R}_{+, \text {non }}^{(3)}, \mathbf{R}_{-, \text {non }}^{(3)}, \mathbf{R}_{\text {non }}^{\left(\frac{7}{2}\right)}\right) \leftrightarrow\left(\mathbf{R}^{\left(\frac{5}{2}\right)}, \mathbf{R}_{+}^{(3)}, \mathbf{R}_{-}^{(3)}, \mathbf{R}^{\left(\frac{7}{2}\right)}\right), \\
\left(3, \frac{7}{2}, \frac{7}{2}, 4\right): & \left(\mathbf{S}_{\text {non }}^{(3)}, \mathbf{S}_{+, \text {non }}^{\left(\frac{7}{2}\right)}, \mathbf{S}_{-, \text {non }}^{\left(\frac{7}{2}\right)}, \mathbf{S}_{\text {non }}^{(4)}\right) \leftrightarrow\left(\mathbf{S}^{(3)}, \mathbf{S}_{+}^{\left(\frac{7}{2}\right)}, \mathbf{S}_{-}^{\left(\frac{7}{2}\right)}, \mathbf{S}^{(4)}\right) .
\end{array}
$$

In section 6 , the OPEs between the 16 currents from the large $\mathcal{N}=4$ linear superconformal algebra and the 16 next lowest higher spin currents found in section 5 are described.

In section 7, the OPEs between the 16 lowest higher spin currents found in section 3 and themselves are given.

In section 8, the summary of this paper is given and the future directions are also described.

In Appendices $A-F$, some details appearing in previous sections are presented.

The mathematica package by Thielemans [10] is used.

\footnotetext{
${ }^{2}$ In Part I [5], the higher spin currents in the nonlinear version were written without 'non' and there were no boldfaces. In part II [8], all the higher spin currents were presented without 'non' and the boldface higher spin currents were the second $\mathcal{N}=4$ multiplet (next 16 lowest higher spin currents).
} 


\section{The large $\mathcal{N}=4$ linear superconformal algebra in the coset: review}

The large $\mathcal{N}=4$ linear superconformal algebra is generated by spin-2 stress tensor $T(z)$, four spin- $\frac{3}{2}$ supersymmetry generators $G_{a}(z)$, seven spin-1 currents $A_{i}(z), B_{i}(z)$ and $U(z)$ and four spin- $\frac{1}{2}$ currents $F_{a}(z)$. Six spin-1 currents are the generators of two $S U(2)$ affine algebras where the levels are denoted by $\left(k^{+}, k^{-}\right)$and one spin- 1 current is the $U(1)$ current. The central charge appearing in the OPE between the spin- 2 current and itself is given by $c=\frac{6 k^{+} k^{-}}{\left(k^{+}+k^{-}\right)}$. The parameter $\gamma=\frac{k^{-}}{\left(k^{+}+k^{-}\right)}$appears in the large $\mathcal{N}=4$ linear superconformal algebra. One presents the large $\mathcal{N}=4$ linear superconformal algebra in Appendix $A$.

The 16 currents can be rearranged into the following four $\mathcal{N}=2$ multiplets as follows:

$$
\begin{array}{lll}
\left(0, \frac{1}{2}, \frac{1}{2}, 1\right): & \left(\int U d z, F_{21}, F_{12}, A_{3} \text { and } B_{3}\right), \\
\left(\frac{1}{2}, 1,1, \frac{3}{2}\right): & \left(F_{11}, B_{-}, A_{+}, G_{11} \text { and } \partial F_{11}\right), \\
\left(\frac{1}{2}, 1,1, \frac{3}{2}\right): & \left(F_{22}, A_{-}, B_{+}, G_{22} \text { and } \partial F_{22}\right), \\
\left(1, \frac{3}{2}, \frac{3}{2}, 2\right): & \left(A_{3} \text { and } B_{3}, G_{21}, G_{12}, T\right) .
\end{array}
$$

The last multiplet contains the currents in the usual $\mathcal{N}=2$ superconformal algebra. The $U(1)$ charges of (2.1) have the same behavior of those of (1.1) and $A_{ \pm}(z) \equiv A_{1}(z) \pm i A_{2}(z)$ and $B_{ \pm}(z) \equiv B_{1}(z) \pm i B_{2}(z)$.

By factoring out the spin- 1 current and four spin- $\frac{1}{2}$ currents from the above large $\mathcal{N}=4$ linear superconformal algebra, the large $\mathcal{N}=4$ nonlinear superconformal algebra (where the OPEs between the spin- $\frac{3}{2}$ currents are nonlinear) is obtained. For example, the spin- $\frac{3}{2}$ currents of large $\mathcal{N}=4$ nonlinear superconformal algebra are written in terms of the fields in the linear version as follows:

$$
\begin{aligned}
\hat{G}_{11}(z) & =\left[G_{11}+\frac{2}{(5+k)} U F_{11}-\frac{4}{(5+k)^{2}} F_{12} F_{21} F_{11}+\frac{2 i}{(5+k)} F_{11} A_{3}\right. \\
& \left.+\frac{2 i}{(5+k)} F_{21} A_{+}+\frac{2 i}{(5+k)} F_{12} B_{-}+\frac{2 i}{(5+k)} F_{11} B_{3}\right](z), \\
\hat{G}_{12}(z) & =\left[G_{12}+\frac{2}{(5+k)} U F_{12}-\frac{4}{(5+k)^{2}} F_{12} F_{11} F_{22}+\frac{2 i}{(5+k)} F_{12} A_{3}\right. \\
& \left.-\frac{2 i}{(5+k)} F_{22} A_{+}+\frac{2 i}{(5+k)} F_{11} B_{+}-\frac{2 i}{(5+k)} F_{12} B_{3}\right](z), \\
\hat{G}_{21}(z) & =\left[G_{21}+\frac{2}{(5+k)} U F_{21}-\frac{4}{(5+k)^{2}} F_{21} F_{11} F_{22}-\frac{2 i}{(5+k)} F_{21} A_{3}\right.
\end{aligned}
$$




$$
\begin{aligned}
& \left.+\frac{2 i}{(5+k)} F_{11} A_{-}-\frac{2 i}{(5+k)} F_{22} B_{-}+\frac{2 i}{(5+k)} F_{21} B_{3}\right](z), \\
\hat{G}_{22}(z) & =\left[G_{22}+\frac{2}{(5+k)} U F_{22}-\frac{4}{(5+k)^{2}} F_{21} F_{12} F_{22}-\frac{2 i}{(5+k)} F_{22} A_{3}\right. \\
& \left.-\frac{2 i}{(5+k)} F_{12} A_{-}-\frac{2 i}{(5+k)} F_{21} B_{+}-\frac{2 i}{(5+k)} F_{22} B_{3}\right](z) .
\end{aligned}
$$

One can also write down the spin- $\frac{3}{2}$ currents in the linear version in terms of the fields in the nonlinear version using (2.2) and the equations (3.6) and (3.10) of Part I (by writing down the spin-1 currents in the linear version in terms of the spin-1 currents in the nonlinear version plus spin- $\frac{1}{2}$ current terms) 3 .

One has the following explicit relations between the spin- $\frac{3}{2}$ currents with $S O(4)$ indices and those with $S U(2) \times S U(2)$ indices

$$
\begin{array}{rlrl}
G^{0}(z) & =-\frac{i}{\sqrt{2}}\left(G_{12}-G_{21}\right)(z), & G^{1}(z)=\frac{1}{\sqrt{2}}\left(G_{11}+G_{22}\right)(z), \\
G^{2}(z)=\frac{i}{\sqrt{2}}\left(G_{11}-G_{22}\right)(z), & G^{3}(z)=-\frac{1}{\sqrt{2}}\left(G_{12}+G_{21}\right)(z) .
\end{array}
$$

Similarly, one has the following relations for the spin- $\frac{1}{2}$ currents

$$
\begin{array}{ll}
F^{0}(z)=-\frac{i}{\sqrt{2}}\left(F_{12}-F_{21}\right)(z), & F^{1}(z)=\frac{1}{\sqrt{2}}\left(F_{11}+F_{22}\right)(z), \\
F^{2}(z)=\frac{i}{\sqrt{2}}\left(F_{11}-F_{22}\right)(z), & F^{3}(z)=-\frac{1}{\sqrt{2}}\left(F_{12}+F_{21}\right)(z) .
\end{array}
$$

These relations (2.3) and (2.4) will be used later to describe the OPEs in Appendix $D$.

The $U(1)$ charge can be obtained from the coefficient of the first order pole in the OPE between the $U(1)$ current $\left(-2 i \gamma A_{3}-2 i(1-\gamma) B_{3}\right)(z)$ and each primary field $\phi(w)$. This $U(1)$ current appears in the half of the second order pole of $G_{21}(z) G_{12}(w)$ in Appendix $A$. One describes the whole $U(1)$ charges for 16 currents from the large $\mathcal{N}=4$ linear superconformal

\footnotetext{
${ }^{3}$ The remaining seven currents in the nonlinear version have the following relations with those in the linear version:

$$
\begin{aligned}
& \hat{T}(z)=T(z)+\frac{1}{(5+k)}\left(U U+\partial F^{a} F_{a}\right)(z), \\
& \hat{A}_{i}(z)=A_{i}(z)-\frac{1}{(5+k)} \alpha_{a b}^{+i} F^{a} F^{b}(z), \\
& \hat{B}_{i}(z)=B_{i}(z)-\frac{1}{(5+k)} \alpha_{a b}^{-i} F^{a} F^{b}(z) .
\end{aligned}
$$

Also using these relations, one can express the fields in the linear version in terms of those in the nonlinear version. The fields in the nonlinear version have trivial OPEs between the spin- 1 current $U(w)$ and the four spin- $\frac{1}{2}$ currents $F_{a}(w)$.
} 
algebra and two 16 higher spin currents in Table 1. One can construct any composite fields with definite $U(1)$ charge based on this Table 1 . Although the 48 currents have different $U(1)$ charges compared to the ones in the nonlinear version, one realizes that their behaviors appearing in all the OPEs in this paper are the same. In other words, one can consider the extra composite fields due to the four spin- $\frac{1}{2}$ currents and one spin- 1 current in addition to the Tables (with the presence of the corresponding composite fields in the linear version) in Part I.

\begin{tabular}{|c|c|}
\hline$U(1)$ charge & 16 currents and 32 higher spin currents \\
\hline$\frac{(2+2 k)}{(5+k)}$ & $\begin{array}{lll}B_{-} ; & \mathbf{U}_{+}^{(2)} ; & \mathbf{Q}_{+}^{(3)}\end{array}$ \\
\hline 1 & $\begin{array}{llllll}G_{21}, & F_{21} ; & \mathbf{T}_{+}^{\left(\frac{3}{2}\right)}, & \mathbf{W}_{+}^{\left(\frac{5}{2}\right)} ; & \mathbf{P}_{+}^{\left(\frac{5}{2}\right)}, & \mathbf{S}_{+}^{\left(\frac{7}{2}\right)}\end{array}$ \\
\hline$\frac{(-3+k)}{(5+k)}$ & 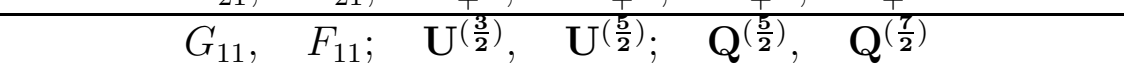 \\
\hline$\frac{8}{(5+k)}$ & $A_{-} ; \quad \mathbf{V}_{+}^{(2)} ; \quad \mathbf{R}_{+}^{(3)}$ \\
\hline 0 & $A_{3}, B_{3}, U, T ; \mathbf{T}^{(1)}, \mathbf{T}^{(2)}, \mathbf{W}^{(2)}, \mathbf{W}^{(3)} ; \mathbf{P}^{(2)}, \mathbf{P}^{(3)}, \mathbf{S}^{(3)}, \mathbf{S}^{(4)}$ \\
\hline$-\frac{8}{(5+k)}$ & $A_{+} ; \quad \mathbf{U}_{-}^{(2)} ; \quad \mathbf{Q}_{-}^{(3)}$ \\
\hline$-\frac{(-3+k)}{(5+k)}$ & $\begin{array}{llllll}G_{22}, & F_{22} ; & \mathbf{V}^{\left(\frac{3}{2}\right)}, & \mathbf{V}^{\left(\frac{5}{2}\right)} ; & \mathbf{R}^{\left(\frac{5}{2}\right)}, & \mathbf{R}^{\left(\frac{7}{2}\right)} \\
\end{array}$ \\
\hline-1 & $\begin{array}{llllll}G_{12}, & F_{12} ; & \mathbf{T}_{-}^{\left(\frac{3}{2}\right)}, & \mathbf{W}_{-}^{\left(\frac{5}{2}\right)} ; & \mathbf{P}_{-}^{\left(\frac{5}{2}\right)}, & \mathbf{S}_{-}^{\left(\frac{7}{2}\right)}\end{array}$ \\
\hline$-\frac{(2+2 k)}{(5+k)}$ & $B_{+} ; \quad \mathbf{V}_{-}^{(2)} ; \quad \mathbf{R}_{-}^{(3)}$ \\
\hline
\end{tabular}

Table 1: The $U(1)$ charges for the 16 currents from the large $\mathcal{N}=4$ linear superconformal algebra and two 16 higher spin currents. The first 18 upper currents with positive $(k>3)$ $U(1)$ charges have their conjugated currents with each opposite (negative) $U(1)$ charge.

\section{The higher spin currents where the lowest spin is 1 in the coset}

Let us construct the 16 lowest higher spin currents.

One has the two results given in (4.9) and (4.13) of [5] where the two higher spin- $\frac{3}{2}$ currents in the nonlinear version are generated. Then one considers the spin- $\frac{3}{2}$ currents $G_{21}(z)$ and $G_{12}(z)$ in the large $\mathcal{N}=4$ linear superconformal algebra and they act on the lowest spin-1 current $\mathbf{T}^{(1)}(w)$. From the explicit expressions in terms of WZW currents living in $S U(5)$, (2.21) corresponding to $G_{21}(z),(2.24)$ corresponding to $G_{12}(z)$ and (4.7) corresponding to $\mathbf{T}^{(\mathbf{1})}(w)$ of [5], the OPEs between them can be obtained and it turns out that

$$
\left(\begin{array}{c}
G_{12} \\
G_{21}
\end{array}\right)(z) \mathbf{T}^{(\mathbf{1})}(w)=\frac{1}{(z-w)}\left[\mp\left(\begin{array}{c}
G_{12} \\
G_{21}
\end{array}\right)+2 \mathbf{T}_{\mp}^{\left(\frac{3}{2}\right)}\right](w)+\cdots .
$$


This is the same as its nonlinear version and each higher spin- $\frac{3}{2}$ current in (3.1) contains 31 terms consisting of multiple product of spin- $\frac{1}{2}$ current $Q^{a}(w)$ and the spin-1 current $V^{b}(w)$ (the indices $a, b$ run as $a, b=1,2, \cdots, 24)$ introduced in [11. One expects that as one generalizes the spin- $\frac{3}{2}$ currents and higher spin- 1 current for $N=3$ to satisfy for general $N$, the OPEs for general $N$ have the same form as (3.1) with the corresponding higher spin- $\frac{3}{2}$ currents for general $N$. There will be no $(N, k)$ dependence in the right hand side of the OPE.

What about other higher spin- $\frac{3}{2}$ currents? Once again (4.20) and (4.34) of [5] describe the corresponding higher spin currents in the nonlinear version. Starting from (2.11) of [5] associated with $G_{11}(z),(2.12)$ of [5] associated with $G_{22}(z)$ and (4.7) of [5] corresponding to $\mathbf{T}^{(\mathbf{1})}(w)$, the following OPEs can be obtained

$$
\left(\begin{array}{l}
G_{11} \\
G_{22}
\end{array}\right)(z) \mathbf{T}^{(\mathbf{1})}(w)=\frac{1}{(z-w)}\left[ \pm\left(\begin{array}{c}
G_{11} \\
G_{22}
\end{array}\right)+2\left(\begin{array}{c}
\mathbf{U}^{\left(\frac{3}{2}\right)} \\
\mathbf{V}^{\left(\frac{3}{2}\right)}
\end{array}\right)\right](w)+\cdots .
$$

These OPES are exactly the same forms as the ones in the nonlinear version. Moreover, each higher spin- $\frac{3}{2}$ current contains 26 terms written in terms of the above $Q^{a}(w)$ and $V^{b}(w)$ (and their derivatives). The relative coefficients in the right hand side of (3.2) are numerical constants and one expects that the OPEs of (3.2) for general $N$ remain unchanged discussed as before.

Let us move on the next higher spin currents. The equation (4.16) in [5] determines the higher spin-2 current. Now one considers the similar OPEs in the linear version. The spin- $\frac{3}{2}$ currents of large $\mathcal{N}=4$ linear superconformal algebra are given in [5] as before and the higher spin- $\frac{3}{2}$ currents are determined via (3.1). From these explicit expressions, it turns out that

$$
\begin{aligned}
\left(\begin{array}{c}
G_{12} \\
G_{21}
\end{array}\right)(z) \mathbf{T}_{ \pm}^{\left(\frac{3}{2}\right)}(w) & =\mp \frac{1}{(z-w)^{3}} \frac{8(1+k)}{(5+k)} \\
& +\frac{1}{(z-w)^{2}}\left[-\frac{8 i}{(5+k)} A_{3}-\frac{2 i(1+k)}{(5+k)} B_{3}+\mathbf{T}^{(\mathbf{1})}\right](w) \\
& +\frac{1}{(z-w)}\left[\frac{1}{2} \partial(\text { pole- } 2) \mp \mathbf{T}^{(\mathbf{2})} \mp T\right](w)+\cdots
\end{aligned}
$$

Recall that the equations (2.16), (2.33) and (2.39) of Part I provide the currents $A_{3}(w)$, $B_{3}(w)$ and $T(w)$ living in the large $\mathcal{N}=4$ linear superconformal algebra respectively. In the first order pole of (3.3), the first term denotes the derivative of second order pole of (3.3). Compared to the corresponding OPEs in the nonlinear version, the field contents are the same but the structure constants appearing in the right hand side are different. The higher spin-2 current in (3.3) contains 64 terms written in terms of $Q^{a}(w)$ and $V^{b}(w)$ (and their derivatives). One expects that the corresponding OPEs for general $N$ have $(N, k)$ dependence in three places of (3.3) explicitly. 
The footnotes 48 and 49 of [5] determine other two higher spin-2 currents and the corresponding OPEs from the higher spin- $-\frac{3}{2}$ currents obtained in (3.2) can be summarized as

$$
\begin{aligned}
\left(\begin{array}{l}
G_{12} \\
G_{21}
\end{array}\right)(z)\left(\begin{array}{c}
\mathbf{U}^{\left(\frac{3}{2}\right)} \\
\mathbf{V}^{\left(\frac{3}{2}\right)}
\end{array}\right)(w) & =\frac{1}{(z-w)^{2}} \frac{8 i}{(5+k)} A_{ \pm}(w) \\
& +\frac{1}{(z-w)}\left[\frac{1}{2} \partial(\text { pole- } 2)+\left(\begin{array}{c}
\mathbf{U}_{-}^{(\mathbf{2})} \\
\mathbf{V}_{+}^{(\mathbf{2})}
\end{array}\right)\right](w)+\cdots
\end{aligned}
$$

Recall that the $A_{ \pm}(w)$ appearing in the right hand side are obtained from the equation (2.13) of [5]. The second order pole of (3.4) has different structure constant compared to the ones in the nonlinear version and depends on $(N, k)$ dependence for general $N$. Each higher spin-2 current contains 28 terms written in terms of $Q^{a}(w)$ and $V^{b}(w)$ (and their derivatives).

Similarly the footnotes 47 and 50 of [5] determine other two higher spin-2 currents and the corresponding OPEs from the higher spin- $\frac{3}{2}$ currents obtained in (3.2) can be described as

$$
\begin{aligned}
\left(\begin{array}{l}
G_{12} \\
G_{21}
\end{array}\right)(z)\left(\begin{array}{c}
\mathbf{V}^{\left(\frac{3}{2}\right)} \\
\mathbf{U}^{\left(\frac{3}{2}\right)}
\end{array}\right)(w) & =-\frac{1}{(z-w)^{2}} \frac{2 i(1+k)}{(5+k)} B_{ \pm}(w) \\
& +\frac{1}{(z-w)}\left[\frac{1}{2} \partial(\text { pole- } 2)+\left(\begin{array}{c}
\mathbf{V}_{-}^{(\mathbf{2})} \\
\mathbf{U}_{+}^{(\mathbf{2})}
\end{array}\right)\right](w)+\cdots
\end{aligned}
$$

Recall that the $B_{ \pm}(w)$ appearing in the right hand side are obtained from the equation (2.31) of [5]. Each higher spin-2 current in (3.5) contains 42 terms written in terms of $Q^{a}(w)$ and $V^{b}(w)$ (and their derivatives). The second order pole of (3.5) has different structure constant compared to the ones in the nonlinear version and depends on $(N, k)$ dependence for general $N$ as before.

The equation (4.48) of [5] determines the remaining higher spin-2 current. From the higher spin- $\frac{3}{2}$ currents determined in (3.2), one has the following OPEs

$$
\begin{aligned}
\left(\begin{array}{l}
G_{11} \\
G_{22}
\end{array}\right)(z)\left(\begin{array}{c}
\mathbf{V}^{\left(\frac{3}{2}\right)} \\
\mathbf{U}^{\left(\frac{3}{2}\right)}
\end{array}\right)(w) & = \pm \frac{1}{(z-w)^{3}} \frac{8(1+k)}{(5+k)} \\
& +\frac{1}{(z-w)^{2}}\left[\frac{8 i}{(5+k)} A_{3}-\frac{2 i(1+k)}{(5+k)} B_{3}+\mathbf{T}^{(\mathbf{1})}\right](w) \\
& +\frac{1}{(z-w)}\left[\frac{1}{2} \partial(\text { pole- }-2) \pm \mathbf{W}^{(\mathbf{2})} \pm T\right](w)+\cdots
\end{aligned}
$$

Compared to the OPEs in the nonlinear version, the fields contents in (3.6) are the same form but the structure constants have different forms. Note that the footnote 13 of [11] describes the primary current $\hat{W}^{(2)}(w)$ while the higher spin-2 current $W^{(2)}(w)$ is a quasi primary field. 
The higher spin-2 current in (3.6) contains 76 terms written in terms of $Q^{a}(w)$ and $V^{b}(w)$ (and their derivatives).

The equation (4.31) of [5] determines the higher spin- $\frac{5}{2}$ current. From the explicit expressions appearing in the OPEs in (3.4), one can construct the following OPEs

$$
\begin{aligned}
\left(\begin{array}{l}
G_{12} \\
G_{21}
\end{array}\right)(z)\left(\begin{array}{c}
\mathbf{V}_{+}^{(\mathbf{2})} \\
\mathbf{U}_{-}^{(2)}
\end{array}\right)(w) & =\frac{1}{(z-w)^{2}} \frac{2(3+k)}{(5+k)}\left[\mp\left(\begin{array}{c}
G_{22} \\
G_{11}
\end{array}\right)+2\left(\begin{array}{c}
\mathbf{V}^{\left(\frac{3}{2}\right)} \\
\mathbf{U}^{\left(\frac{3}{2}\right)}
\end{array}\right)\right](w) \\
& +\frac{1}{(z-w)}\left[\frac{1}{3} \partial(\text { pole- } 2) \mp\left(\begin{array}{c}
\mathbf{V}^{\left(\frac{5}{2}\right)} \\
\mathbf{U}^{\left(\frac{5}{2}\right)}
\end{array}\right)\right](w)+\cdots .
\end{aligned}
$$

Compared to the corresponding OPEs in the nonlinear version, the field contents are the same but the structure constant in the second order pole behaves differently. Furthermore, the second order pole contains the overall $k$ dependent factor. Note that the corresponding nonlinear version does not have this kind of behavior. There is no common factor in the second order pole in (4.31) of [5]. We will see this feature in next section. Definitely this structure constant depends on $(N, k)$ explicitly for general $N$. The higher spin- $\frac{5}{2}$ currents in (3.7) contain 283 and 279 terms written in terms of $Q^{a}(w)$ and $V^{b}(w)$ (and their derivatives).

Again the equations (4.52) and (4.55) of Part I determine the remaining higher spin- $\frac{5}{2}$ currents. With the help of (3.6), one can calculate the following OPEs

$$
\begin{aligned}
\left(\begin{array}{l}
G_{12} \\
G_{21}
\end{array}\right)(z) \mathbf{W}^{(2)}(w) & =\frac{1}{(z-w)^{2}} \frac{(-3+k)}{2(5+k)}\left[\left(\begin{array}{c}
G_{12} \\
G_{21}
\end{array}\right) \mp 2 \mathbf{T}_{\mp}^{\left(\frac{3}{2}\right)}\right](w) \\
& +\frac{1}{(z-w)}\left[\frac{1}{3} \partial(\text { pole- } 2)+\mathbf{W}_{\mp}^{\left(\frac{5}{2}\right)}\right](w)+\cdots .
\end{aligned}
$$

The higher spin- $\frac{5}{2}$ currents in (3.8) contain 279 and 287 terms written in terms of $Q^{a}(w)$ and $V^{b}(w)$ (and their derivatives). One cannot compare the above OPEs and the equation (4.52) of [5] directly because the higher spin-2 current in the nonlinear version is a quasi primary current. As before, the second order pole contains the overall $k$ dependent factor. Recall that the $(k-3)$ factor can be written in terms of $\left(k^{+}-k^{-}\right)=(k+1)-(N+1)=(k-N)$ for general $N$ [11]. Therefore, for $k^{+}=k^{-}$, there is no second order pole in (3.8). The combination of spin- $\frac{3}{2}$ currents and higher spin- $\frac{3}{2}$ currents will appear in next section.

Let us describe the final higher spin-3 current. The nonlinear version appears in the equation (4.59) of [5]. With the help of (3.8), one can construct the following OPEs

$$
\begin{aligned}
\left(\begin{array}{c}
G_{12} \\
G_{21}
\end{array}\right)(z) \mathbf{W}_{ \pm}^{\left(\frac{5}{2}\right)}(w) & =\mp \frac{1}{(z-w)^{3}} \frac{8(-3+\mathbf{k})}{3(5+k)} \mathbf{T}^{(\mathbf{1})}(w) \\
& +\frac{1}{(z-w)^{2}}\left[\frac{4(-3+k)}{3(5+k)} \mathbf{T}^{(\mathbf{2})}+4 \mathbf{W}^{(\mathbf{2})}\right](w)
\end{aligned}
$$




$$
\begin{aligned}
& +\frac{1}{(z-w)}\left[\frac{1}{4} \partial(\text { pole- } 2) \mp \mathbf{W}^{(\mathbf{3})}\right. \\
& \left.\mp \frac{4(-\mathbf{3}+\mathbf{k})}{(17+13 k)}\left(T \mathbf{T}^{(\mathbf{1})}-\frac{1}{2} \partial^{2} \mathbf{T}^{(\mathbf{1})}\right)\right](w)+\cdots .
\end{aligned}
$$

The field contents are different compared to the nonlinear version but the right hand side is simpler than the one in the nonlinear version. There are no nonlinear terms in the second order pole of (3.9) . Furthermore, one regards the first order pole except the derivative term as a quasi primary field because the last two terms is a quasi primary field. We will describe this issue further in next section. The higher spin-3 current in (3.9) contain 1130 terms written in terms of $Q^{a}(w)$ and $V^{b}(w)$ (and their derivatives). As explained before, the $(k-3)$ factor, emphasized by a boldface, appears in the higher spin- 1 current dependent terms in the third order and the first order poles. In particular, for $k^{+}=k^{-}$, there is no nonlinear term in the OPE of (3.9) and the coefficient of $\mathbf{T}^{(2)}(w)$ vanishes.

In Appendix $B$, the precise relations of the higher spin currents in the nonlinear version and those in the linear version are presented.

\section{The OPEs between the 16 currents in the large $\mathcal{N}=$ 4 linear superconformal algebra and the 16 lowest higher spin currents}

In order to compare the results in [9], one should calculate the OPEs between the 16 currents in the large $\mathcal{N}=4$ linear superconformal algebra and the 16 higher spin currents found in previous section. The complete OPEs between them are presented in Appendix $C$. In Appendix $D$, the fourth equation in Appendix D.1 is the definition of their higher spin- $\frac{3}{2}$ currents for given higher spin- 1 current and the four spin- $\frac{3}{2}$ currents. The four spin- $\frac{3}{2}$ currents have explicit relations in (2.3). From the results of Part I [5] (or (3.2) and (3.1)), one has the following OPEs

$$
\begin{aligned}
& \left(\begin{array}{l}
G_{11} \\
G_{22}
\end{array}\right)(z) \mathbf{T}^{(\mathbf{1})}(w)=\frac{1}{(z-w)}\left[ \pm\left(\begin{array}{c}
G_{11} \\
G_{22}
\end{array}\right)+2\left(\begin{array}{c}
\mathbf{U}^{\left(\frac{3}{2}\right)} \\
\mathbf{V}^{\left(\frac{3}{2}\right)}
\end{array}\right)\right](w)+\cdots, \\
& \left(\begin{array}{l}
G_{12} \\
G_{21}
\end{array}\right)(z) \mathbf{T}^{(\mathbf{1})}(w)=\frac{1}{(z-w)}\left[\mp\left(\begin{array}{l}
G_{12} \\
G_{21}
\end{array}\right)+2 \mathbf{T}_{\mp}^{\left(\frac{3}{2}\right)}\right](w)+\cdots .
\end{aligned}
$$

With the identification of $V_{0}^{(1)}(w)=\mathbf{T}^{(\mathbf{1})}(w)$, , one can make the linear combinations from (4.1) with the help of (2.3). By reading off the first order poles in (4.1) and in the fourth

\footnotetext{
${ }^{4}$ Strictly speaking, the exact relation is given by $V_{0}^{(1)}(w)= \pm i \mathbf{T}^{(\mathbf{1})}(w)$.
} 
equation of Appendix D.1, one obtains the higher spin- $\frac{3}{2}$ currents in [9] in terms of the spin- $\frac{3}{2}$ currents and the higher spin- $\frac{3}{2}$ currents as follows:

$$
\begin{aligned}
V_{\frac{1}{2}}^{(1), 0}(z) & =-\frac{i}{\sqrt{2}}\left(-G_{12}-G_{21}-2 \mathbf{T}_{+}^{\left(\frac{3}{2}\right)}+2 \mathbf{T}_{-}^{\left(\frac{3}{2}\right)}\right)(z), \\
V_{\frac{1}{2}}^{(1), 1}(z) & =\frac{1}{\sqrt{2}}\left(G_{11}-G_{22}+2 \mathbf{U}^{\left(\frac{3}{2}\right)}+2 \mathbf{V}^{\left(\frac{3}{2}\right)}\right)(z), \\
V_{\frac{1}{2}}^{(1), 2}(z) & =\frac{i}{\sqrt{2}}\left(G_{11}+G_{22}+2 \mathbf{U}^{\left(\frac{3}{2}\right)}-2 \mathbf{V}^{\left(\frac{3}{2}\right)}\right)(z), \\
V_{\frac{1}{2}}^{(1), 3}(z) & =-\frac{1}{\sqrt{2}}\left(-G_{12}+G_{21}+2 \mathbf{T}_{+}^{\left(\frac{3}{2}\right)}+2 \mathbf{T}_{-}^{\left(\frac{3}{2}\right)}\right)(z) .
\end{aligned}
$$

Let us identify other higher spin currents. The eighth equation of Appendix D.1 implies that one should calculate the OPEs between the spin- $\frac{3}{2}$ currents and the higher spin- $\frac{3}{2}$ currents in (4.2). When the indices $(a, b)$ of the eighth equation of Appendix D.1 become $(a, b)=$ $(0,1)$, then the right hand side of the first order pole is given by $-\frac{1}{2}\left(V_{1}^{(1), 1}-V_{1}^{(1),-1}\right)(w)$. Furthermore the indices are given by $(a, b)=(2,3)$, then the first order pole is given by $\frac{1}{2}\left(V_{1}^{(1), 1}+V_{1}^{(1),-1}\right)(w)$. On the other hand, one can express the relevant OPEs from the relations (4.2) and the defining OPEs between the spin- $\frac{3}{2}$ currents in the large $\mathcal{N}=4$ linear superconformal algebra. For the former, one has the relevant OPEs given by (3.4) and (3.5) . For the latter, one has the following OPEs

$$
\begin{aligned}
\left(\begin{array}{c}
G_{11} \\
G_{22}
\end{array}\right)(z) \mathbf{T}_{ \pm}^{\left(\frac{\mathbf{3}}{2}\right)}(w) & =\frac{1}{(z-w)^{2}} \frac{2 i(1+k)}{(5+k)} B_{\mp}(w) \\
& +\frac{1}{(z-w)}\left[\frac{1}{2} \partial(\text { pole- } 2)-\left(\begin{array}{c}
\mathbf{U}_{+}^{(\mathbf{2})} \\
\mathbf{V}_{-}^{(2)}
\end{array}\right)\right](w)+\cdots \\
\left(\begin{array}{c}
G_{11} \\
G_{22}
\end{array}\right)(z) \mathbf{T}_{\mp}^{\left(\frac{\mathbf{3}}{2}\right)}(w) & =\frac{1}{(z-w)^{2}} \frac{8 i}{(5+k)} A_{ \pm}(w) \\
& +\frac{1}{(z-w)}\left[\frac{1}{2} \partial(\text { pole- } 2)-\left(\begin{array}{c}
\mathbf{U}_{-}^{(\mathbf{2})} \\
\mathbf{V}_{+}^{(\mathbf{2})}
\end{array}\right)\right](w)+\cdots
\end{aligned}
$$

These are obtained from the relation (3.1) and the result on the spin- $\frac{3}{2}$ currents from Part I. Eventually, one can write down $V_{1}^{(1), \pm 1}(z)$ as a linear combinations of $\mathbf{U}_{ \pm}^{(2)}(z)$ and $\mathbf{V}_{ \pm}^{(2)}(z)$ which will be shown later.

Similarly, when the indices $(a, b)$ of the eighth equation of Appendix D.1 become $(a, b)=$ $(0,2)$, then the right hand side of the first order pole is given by $-\frac{1}{2}\left(V_{1}^{(1), 2}-V_{1}^{(1),-2}\right)(w)$. For the indices $(a, b)=(1,3)$, then the first order pole is given by $-\frac{1}{2}\left(V_{1}^{(1), 2}+V_{1}^{(1),-2}\right)(w)$. One can write down $V_{1}^{(1), \pm 2}(z)$ as a linear combinations of $\mathbf{U}_{ \pm}^{(\mathbf{2})}(z)$ and $\mathbf{V}_{ \pm}^{(\mathbf{2})}(z)$ as analyzed before (4.3). 
Finally, when the indices $(a, b)$ of the eighth equation of Appendix D.1 become $(a, b)=$ $(0,3)$, then the right hand side of the first order pole is given by $-\frac{1}{2}\left(V_{1}^{(1), 3}-V_{1}^{(1),-3}\right)(w)$. For the indices $(a, b)=(1,2)$, then the first order pole is given by $\frac{1}{2}\left(V_{1}^{(1), 3}+V_{1}^{(1),-3}\right)(w)$. For the former, the relevant OPEs are described in (3.3). For the latter, one has the OPEs in (3.6). One can write down $V_{1}^{(1), \pm 3}(z)$ as a linear combinations of $\mathbf{T}^{(\mathbf{2})}(z)$ and $\mathbf{W}^{(2)}(z)$. Therefore, the explicit higher spin-2 currents have the following relations

$$
\begin{aligned}
& V_{1}^{(1), \pm 1}(z)=2 i\left(\mathbf{U}_{\mp}^{(\mathbf{2})}-\mathbf{V}_{ \pm}^{(\mathbf{2})}\right)(z), \\
& V_{1}^{(1), \pm 2}(z)=-2\left(\mathbf{U}_{\mp}^{(\mathbf{2})}+\mathbf{V}_{ \pm}^{(\mathbf{2})}\right)(z), \\
& V_{1}^{(1), \pm 3}(z)= \pm 2 i\left(\mathbf{T}^{(\mathbf{2})} \mp \mathbf{W}^{(\mathbf{2})}\right)(z) .
\end{aligned}
$$

Let us move to the next higher spin- $-\frac{5}{2}$ current. When the indices $(a, i)$ of the thirteenth equation of Appendix D.1 become $(a, i)=(3,3)$, then the nonderivative term in the right hand side of the first order pole is given by $-\frac{1}{2} V_{\frac{3}{2}}^{(1), 0}(w)$. Then the relevant OPEs are given by (3.8) and furthermore one can calculate the following OPEs

$$
\begin{aligned}
\left(\begin{array}{l}
G_{12} \\
G_{21}
\end{array}\right)(z) \mathbf{T}^{(2)}(w) & =\frac{1}{(z-w)^{2}} \frac{3}{2}\left[-\left(\begin{array}{l}
G_{12} \\
G_{21}
\end{array}\right) \pm 2 \mathbf{T}_{\mp}^{\left(\frac{3}{2}\right)}\right](w) \\
& +\frac{1}{(z-w)} \frac{1}{3} \partial(\operatorname{pole}-2)(w)+\cdots .
\end{aligned}
$$

One realizes that one can write down $V_{\frac{3}{2}}^{(1), 0}(z)$ as a linear combinations of $\mathbf{W}_{ \pm}^{\left(\frac{5}{2}\right)}(z)$.

When the indices $(a, i)$ of the thirteenth equation of Appendix D.1 become $(a, i)=(0,3)$, then the nonderivative term in the right hand side of the first order pole is given by $\frac{1}{2} V_{\frac{3}{2}}^{(1), 3}(w)$. In this case also, one can write down $V_{\frac{3}{2}}^{(1), 3}(z)$ as a linear combinations of $\mathbf{W}_{ \pm}^{\left(\frac{5}{2}\right)}(z)$ as analyzed before in (4.5).

When the indices $(a, i)$ of the thirteenth equation of Appendix D.1 become $(a, i)=$ $(0,2)$, then the nonderivative term in the right hand side of the first order pole is given by $\frac{1}{2} V_{\frac{3}{2}}^{(1), 2}(w)$. In this case, the corresponding OPEs are given by (3.7). Similarly, for the indices $(a, i)=(0,1)$, the nonderivative term in the right hand side of the first order pole is given by $\frac{1}{2} V_{\frac{3}{2}}^{(1), 1}(w)$. Then the remaining higher spin- $\frac{5}{2}$ currents can be written in terms of the linear combinations of $\mathbf{U}^{\left(\frac{5}{2}\right)}(z)$ and $\mathbf{V}^{\left(\frac{5}{2}\right)}(z)$. Therefore one obtains the following simple relations

$$
\begin{aligned}
& V_{\frac{3}{2}}^{(1), 0}(z)=-2 i \sqrt{2}\left(\mathbf{W}_{+}^{\left(\frac{5}{2}\right)}+\mathbf{W}_{-}^{\left(\frac{5}{2}\right)}\right)(z), \quad V_{\frac{3}{2}}^{(1), 1}(z)=-2 \sqrt{2}\left(\mathbf{U}^{\left(\frac{5}{2}\right)}-\mathbf{V}^{\left(\frac{5}{2}\right)}\right)(z), \\
& V_{\frac{3}{2}}^{(1), 2}(z)=-2 i \sqrt{2}\left(\mathbf{U}^{\left(\frac{5}{2}\right)}+\mathbf{V}^{\left(\frac{5}{2}\right)}\right)(z), \quad V_{\frac{3}{2}}^{(1), 3}(z)=2 \sqrt{2}\left(\mathbf{W}_{+}^{\left(\frac{5}{2}\right)}-\mathbf{W}_{-}^{\left(\frac{5}{2}\right)}\right)(z) .
\end{aligned}
$$


Let us consider the final higher spin-3 current. According to the OPE between the spin- $\frac{3}{2}$ currents and the higher spin- $\frac{5}{2}$ currents in Appendix $D$, the first order pole with the indices $(a, b)=(0,0)$ provides the higher spin- 3 current $V_{2}^{(1)}(z)$ which is a quasi primary field. One has the OPEs (3.9). Then by examining the first order pole, one arrives at the final relation

$$
V_{2}^{(1)}(z)=4\left[\mathbf{W}^{(\mathbf{3})}+\frac{4(-\mathbf{3}+\mathbf{k})}{(17+13 k)}\left(T \mathbf{T}^{(\mathbf{1})}-\frac{1}{2} \partial^{2} \mathbf{T}^{(\mathbf{1})}\right)\right](z) .
$$

When $k=3$, this quasi primary field becomes a primary one. One can check that using the right hand side of (4.7) instead of using $\mathbf{W}^{(3)}(w)$ itself allows us to remove all the nonlinear terms appearing in Appendix $C$. Some of nonlinear terms in Appendix $F$ will disappear if one uses $V_{2}^{(1)}(w)$ rather than $\mathbf{W}^{(3)}(w)$. It would be interesting to see their precise relation in the $\mathcal{N}=4$ superspace.

\section{The higher spin currents where the lowest spin is 2 in the coset}

In order to the complete OPEs between the lowest higher spin currents, one should find out the next higher spin currents (1.2) as in Part II 8].

The higher spin-1 current has the same form as the one given in Part I [5] in the nonlinear version (B.1). From the explicit results in (3.7), one can calculate the following OPEs

$$
\begin{aligned}
\mathbf{T}^{(\mathbf{1})}(z)\left(\begin{array}{c}
\mathbf{U}^{\left(\frac{5}{2}\right)} \\
\mathbf{V}^{\left(\frac{5}{2}\right)}
\end{array}\right)(w) & =\mp \frac{1}{(z-w)^{3}} \frac{12 k}{(5+k)^{2}}\left(\begin{array}{c}
F_{11} \\
F_{22}
\end{array}\right)(w) \\
& +\frac{1}{(z-w)^{2}}\left[-\partial(\text { pole- } 3)+\frac{4 i(6+k)}{3(5+k)^{2}}\left(\begin{array}{c}
F_{11} \\
F_{22}
\end{array}\right) B_{3}\right. \\
& \mp \frac{4(-3+k)}{3(5+k)}\left(\begin{array}{c}
G_{11} \\
G_{22}
\end{array}\right) \mp \frac{8(-3+k)}{3(5+k)^{2}} U\left(\begin{array}{c}
F_{11} \\
F_{22}
\end{array}\right) \\
& +\frac{4(-3+k)}{3(5+k)^{3}} F_{12} F_{21}\left(\begin{array}{c}
F_{11} \\
F_{22}
\end{array}\right)-\frac{4 i(3+2 k)}{3(5+k)^{2}}\left(\begin{array}{c}
F_{11} \\
F_{22}
\end{array}\right) A_{3} \\
& \left.-\frac{4 i(3+2 k)}{3(5+k)^{2}}\left(\begin{array}{c}
F_{21} \\
F_{12}
\end{array}\right) A_{ \pm}+\frac{4 i(6+k)}{3(5+k)^{2}}\left(\begin{array}{c}
F_{12} \\
F_{21}
\end{array}\right) B_{\mp}\right](w) \\
& +\frac{1}{(z-w)}\left[ \pm\left(\begin{array}{c}
\mathbf{U}^{\left(\frac{5}{2}\right)} \\
\mathbf{V}^{\left(\frac{5}{2}\right)}
\end{array}\right)+\left(\begin{array}{c}
\mathbf{Q}^{\left(\frac{5}{2}\right)} \\
\mathbf{R}^{\left(\frac{5}{2}\right)}
\end{array}\right)(w)\right](w)+\cdots .
\end{aligned}
$$

Compared to the corresponding OPEs in the nonlinear version, the second order pole does not contain the higher spin- $\frac{3}{2}$ currents. Of course, the extra terms due to the spin- $\frac{1}{2}$ currents and the spin-1 current in the linear version of this paper occur in the above OPEs. In the 
basis of (4.6), the higher spin-1 current transforms the higher spin- $\frac{5}{2}$ current $V_{\frac{3}{2}}^{(1), 1}(w)$ into the higher spin- $\frac{5}{2}$ current $V_{\frac{3}{2}}^{(1), 2}(w)$ and vice versa.

Similarly, from the explicit expression in (3.8) one can calculate the following OPEs

$$
\begin{aligned}
\mathbf{T}^{(\mathbf{1})}(z) \mathbf{W}_{ \pm}^{\left(\frac{5}{2}\right)}(w) & =\mp \frac{1}{(z-w)^{3}} \frac{12 k}{(5+k)^{2}}\left(\begin{array}{c}
F_{21} \\
F_{12}
\end{array}\right)(w) \\
& +\frac{1}{(z-w)^{2}}\left[-\partial\left(\text { pole-3) }+\frac{4 i(6+k)}{3(5+k)^{2}}\left(\begin{array}{c}
F_{21} \\
F_{12}
\end{array}\right) B_{3}\right.\right. \\
& \mp \frac{4(-3+k)}{3(5+k)}\left(\begin{array}{c}
G_{21} \\
G_{12}
\end{array}\right) \mp \frac{8(-3+k)}{3(5+k)^{2}} U\left(\begin{array}{c}
F_{21} \\
F_{12}
\end{array}\right) \\
& -\frac{4(-3+k)}{3(5+k)^{3}}\left(\begin{array}{c}
F_{21} \\
F_{12}
\end{array}\right) F_{11} F_{22}+\frac{4 i(3+2 k)}{3(5+k)^{2}}\left(\begin{array}{c}
F_{21} \\
F_{12}
\end{array}\right) A_{3} \\
& \left.-\frac{4 i(3+2 k)}{3(5+k)^{2}}\left(\begin{array}{c}
F_{11} \\
F_{22}
\end{array}\right) A_{\mp}-\frac{4 i(6+k)}{3(5+k)^{2}}\left(\begin{array}{c}
F_{22} \\
F_{11}
\end{array}\right) B_{\mp}\right](w) \\
& +\frac{1}{(z-w)}\left[\begin{array}{l}
\left(\frac{5}{2}\right) \\
\pm
\end{array} \mathbf{P}_{ \pm}^{\left(\frac{5}{2}\right)}\right](w)+\cdots
\end{aligned}
$$

In this case also, the higher spin- $\frac{3}{2}$ currents are not present in the second order pole. In the basis of (4.6), the higher spin-1 current transforms the higher spin- $\frac{5}{2}$ current $V_{\frac{3}{2}}^{(1), 0}(w)$ into the higher spin- $\frac{5}{2}$ current $V_{\frac{3}{2}}^{(1), 3}(w)$ and vice versa.

In order to obtain the next higher spin currents, one should calculate the spin- $\frac{3}{2}$ currents and the higher spin- $\frac{5}{2}$ currents obtained before. Let us take one of the spin- $\frac{3}{2}$ currents in the large $\mathcal{N}=4$ linear superconformal algebra and the higher spin- $\frac{5}{2}$ current from (5.2). It turns out that

$$
\begin{aligned}
& G_{21}(z) \mathbf{P}_{-}^{\left(\frac{5}{2}\right)}(w)=\frac{1}{(z-w)^{3}}\left[\frac{8(-\mathbf{3}+\mathbf{k})}{3(5+k)} \mathbf{T}^{(\mathbf{1})}-\frac{4 i(18+5 k)}{(5+k)^{2}} A_{3}+\frac{4 i k(21+4 k)}{3(5+k)^{2}} B_{3}\right. \\
& \left.-\frac{8\left(27+9 k+2 k^{2}\right)}{3(5+k)^{3}} F_{11} F_{22}+\frac{8(-3+k)(9+2 k)}{3(5+k)^{3}} F_{12} F_{21}-\frac{12 k}{(5+k)^{2}} U\right](w) \\
& +\frac{1}{(z-w)^{2}} \mathbf{P}^{(\mathbf{2})}(w)+\frac{1}{(z-w)}\left[\frac{1}{4} \partial \mathbf{P}^{(\mathbf{2})}+\frac{4(-\mathbf{3}+\mathbf{k})}{(17+13 k)}\left(T \mathbf{T}^{(\mathbf{1})}-\frac{1}{2} \partial^{2} \mathbf{T}^{(\mathbf{1})}\right)+\mathbf{P}^{(\mathbf{3})}\right. \\
& -\frac{6 i(18+5 k)}{(5+k)(17+13 k)}\left(T A_{3}-\frac{1}{2} \partial^{2} A_{3}\right)+\frac{2 i k(21+4 k)}{(5+k)(17+13 k)}\left(T B_{3}-\frac{1}{2} \partial^{2} B_{3}\right) \\
& -\frac{18 k}{(5+k)(17+13 k)}\left(T U-\frac{1}{2} \partial^{2} U\right)-\frac{4\left(27+9 k+2 k^{2}\right)}{(5+k)^{2}(17+13 k)}\left(T F_{11} F_{22}-\frac{1}{2} \partial^{2}\left(F_{11} F_{22}\right)\right) \\
& \left.+\frac{4(-3+k)(9+2 k)}{(5+k)^{2}(17+13 k)}\left(T F_{12} F_{21}-\frac{1}{2} \partial^{2}\left(F_{12} F_{21}\right)\right)\right](w)+\cdots .
\end{aligned}
$$

Note the appearance of the factor $(k-3)$ in the higher spin-1 current. The corresponding terms in the nonlinear version appear in the above OPEs also. The last three extra expressions 
in the first order pole are quasi primary fields. Note that the higher spin- 2 current $\mathbf{P}^{(2)}(w)$ is not equal to the one in the basis of [9]. The explicit relation will be described in next section.

Now one considers the following OPEs with the explicit results in (5.1)

$$
\begin{aligned}
& \left(\begin{array}{c}
G_{21} \\
G_{12}
\end{array}\right)(z)\left(\begin{array}{c}
\mathbf{Q}^{\left(\frac{5}{2}\right)} \\
\mathbf{R}^{\left(\frac{5}{2}\right)}
\end{array}\right)(w)=\frac{1}{(z-w)^{3}}\left[\frac{4 i k(21+4 k)}{3(5+k)^{2}} B_{\mp} \mp \frac{32 k(3+k)}{3(5+k)^{3}}\left(\begin{array}{c}
F_{11} F_{21} \\
F_{22} F_{12}
\end{array}\right)\right](w) \\
& +\frac{1}{(z-w)^{2}}\left[\frac{16(3+k)}{3(5+k)}\left(\begin{array}{c}
\mathbf{U}_{+}^{(2)} \\
\mathbf{V}_{-}^{(\mathbf{2}}
\end{array}\right) \mp \frac{4(-3+k)}{3(5+k)^{2}} A_{3} B_{\mp}+\frac{4 i(-3+k)}{3(5+k)^{3}} A_{3}\left(\begin{array}{c}
F_{11} F_{21} \\
F_{22} F_{12}
\end{array}\right)\right. \\
& -\frac{4 i(33+13 k)}{3(5+k)^{3}} B_{3}\left(\begin{array}{c}
F_{11} F_{21} \\
F_{22} F_{12}
\end{array}\right) \pm \frac{8 i(3+k)}{(5+k)^{3}} B_{\mp} F_{11} F_{22} \mp \frac{4 i(15+7 k)}{3(5+k)^{3}} B_{\mp} F_{12} F_{21} \\
& \mp \frac{2(21+5 k)}{3(5+k)^{2}}\left(\begin{array}{c}
F_{11} G_{21} \\
F_{22} G_{12}
\end{array}\right) \mp \frac{4(9+5 k)}{(5+k)^{3}}\left(\begin{array}{l}
\partial F_{11} F_{21} \\
\partial F_{22} F_{12}
\end{array}\right) \mp \frac{4(39+11 k)}{3(5+k)^{3}}\left(\begin{array}{c}
F_{11} \partial F_{21} \\
F_{22} \partial F_{12}
\end{array}\right) \\
& \left. \pm \frac{4(6+k)}{3(5+k)^{2}}\left(\begin{array}{c}
F_{21} G_{11} \\
F_{12} G_{22}
\end{array}\right)+\frac{4 i k}{(5+k)^{2}} U B_{\mp} \mp \frac{4(33+13 k)}{3(5+k)^{3}} U\left(\begin{array}{c}
F_{11} F_{21} \\
F_{22} F_{12}
\end{array}\right)\right](w) \\
& +\frac{1}{(z-w)}\left[\frac{1}{4} \partial(\text { pole- } 2)+\left(\begin{array}{l}
\mathbf{Q}_{+}^{(3)} \\
\mathbf{R}_{-}^{(3)}
\end{array}\right)+\frac{2 i k(21+4 k)}{(5+k)(17+13 k)}\left(T B_{\mp}-\frac{1}{2} \partial^{2} B_{\mp}\right)\right. \\
& \left.\mp \frac{16 k(3+k)}{(5+k)^{2}(17+13 k)}\left(\begin{array}{l}
T F_{11} F_{21}-\frac{1}{2} \partial^{2}\left(F_{11} F_{21}\right) \\
T F_{22} F_{12}-\frac{1}{2} \partial^{2}\left(F_{22} F_{12}\right)
\end{array}\right)\right](w)+\cdots
\end{aligned}
$$

Compared to the corresponding OPEs in the nonlinear version, the nonlinear term between the higher spin current and the currents from the large $\mathcal{N}=4$ linear superconformal algebra does not appear in the OPE. The last expression containing the spin- $\frac{1}{2}$ currents in the first order pole is a quasi primary field.

Now one can consider the following OPEs where the spin- $\frac{3}{2}$ currents are interchanged in the left hand side of (5.4)

$$
\begin{aligned}
& \left(\begin{array}{c}
G_{12} \\
G_{21}
\end{array}\right)(z)\left(\begin{array}{c}
\mathbf{Q}^{\left(\frac{5}{2}\right)} \\
\mathbf{R}^{\left(\frac{5}{2}\right)}
\end{array}\right)(w)=\frac{1}{(z-w)^{3}}\left[\frac{4 i(18+5 k)}{(5+k)^{2}} A_{ \pm} \pm \frac{16(9+k)}{(5+k)^{3}}\left(\begin{array}{c}
F_{11} F_{12} \\
F_{22} F_{21}
\end{array}\right)\right](w) \\
& +\frac{1}{(z-w)^{2}}\left[-\frac{8(9+k)}{3(5+k)}\left(\begin{array}{c}
\mathbf{U}_{-}^{(2)} \\
\mathbf{V}_{+}^{(2)}
\end{array}\right) \mp \frac{4(-3+k)}{3(5+k)^{2}} A_{ \pm} B_{3}-\frac{4 i(57+5 k)}{3(5+k)^{3}} A_{3}\left(\begin{array}{c}
F_{11} F_{12} \\
F_{22} F_{21}
\end{array}\right)\right. \\
& -\frac{4 i(-3+k)}{3(5+k)^{3}} B_{3}\left(\begin{array}{c}
F_{11} F_{12} \\
F_{22} F_{21}
\end{array}\right) \pm \frac{4 i(9+k)}{(5+k)^{3}} A_{ \pm} F_{11} F_{22} \pm \frac{8 i(15+k)}{3(5+k)^{3}} A_{ \pm} F_{12} F_{21} \\
& \mp \frac{8(6+k)}{3(5+k)^{2}}\left(\begin{array}{c}
F_{11} G_{12} \\
F_{22} G_{21}
\end{array}\right) \pm \frac{4(21+k)}{(5+k)^{3}}\left(\begin{array}{l}
\partial F_{11} F_{12} \\
\partial F_{22} F_{21}
\end{array}\right) \pm \frac{4(51+7 k)}{3(5+k)^{3}}\left(\begin{array}{c}
F_{11} \partial F_{12} \\
F_{22} \partial F_{21}
\end{array}\right) \\
& \left. \pm \frac{2(15+k)}{3(5+k)^{2}}\left(\begin{array}{c}
F_{12} G_{11} \\
F_{21} G_{22}
\end{array}\right)-\frac{12 i}{(5+k)^{2}} U A_{ \pm} \mp \frac{4(57+5 k)}{3(5+k)^{3}} U\left(\begin{array}{c}
F_{11} F_{12} \\
F_{22} F_{21}
\end{array}\right)\right](w) \\
& +\frac{1}{(z-w)}\left[\frac{1}{4} \partial(\text { pole- } 2)+\left(\begin{array}{c}
\mathbf{Q}_{-}^{(\mathbf{3})} \\
\mathbf{R}_{+}^{(3)}
\end{array}\right)+\frac{6 i(18+5 k)}{(5+k)(17+13 k)}\left(T A_{ \pm}-\frac{1}{2} \partial^{2} A_{ \pm}\right)\right.
\end{aligned}
$$




$$
\left.\pm \frac{24(9+k)}{(5+k)^{2}(17+13 k)}\left(\begin{array}{l}
T F_{11} F_{12}-\frac{1}{2} \partial^{2}\left(F_{11} F_{12}\right) \\
T F_{22} F_{21}-\frac{1}{2} \partial^{2}\left(F_{22} F_{21}\right)
\end{array}\right)\right](w)+\cdots
$$

As in previous OPEs, the nonlinear term in the corresponding OPEs in the nonlinear version does not appear in the second order pole.

For the last unknown higher spin-3 current, one considers the following OPE

$$
\begin{aligned}
& G_{22}(z) \mathbf{Q}^{\left(\frac{5}{2}\right)}(w)=\frac{1}{(z-w)^{3}}\left[\frac{8(-3+\mathbf{k})}{3(5+k)} \mathbf{T}^{(\mathbf{1})}+\frac{4 i(18+5 k)}{(5+k)^{2}} A_{3}+\frac{4 i k(21+4 k)}{3(5+k)^{2}} B_{3}\right. \\
& \left.+\frac{12 k}{(5+k)^{2}} U-\frac{8(-3+k)(9+2 k)}{3(5+k)^{3}} F_{11} F_{22}+\frac{8\left(27+9 k+2 k^{2}\right)}{3(5+k)^{3}} F_{12} F_{21}\right](w) \\
& +\frac{1}{(z-w)^{2}}\left[-\mathbf{P}^{(\mathbf{2})}-\frac{8(9+k)}{3(5+k)} \mathbf{T}^{(\mathbf{2})}+\frac{8(9+k)}{3(5+k)} \mathbf{W}^{(\mathbf{2})}-\frac{8(-3+k)}{3(5+k)^{2}} A_{3} B_{3}\right. \\
& +\frac{4 i(-3+k)}{3(5+k)^{3}} A_{3} F_{11} F_{22}-\frac{4 i(-3+k)}{3(5+k)^{3}} A_{3} F_{12} F_{21}-\frac{4 i(-3+k)}{3(5+k)^{3}} B_{3} F_{11} F_{22} \\
& -\frac{4 i(-3+k)}{3(5+k)^{3}} B_{3} F_{12} F_{21}-\frac{4 i(33+13 k)}{3(5+k)^{3}} B_{-} F_{12} F_{22}+\frac{4 i(33+13 k)}{3(5+k)^{3}} B_{+} F_{11} F_{21} \\
& -\frac{2(21+5 k)}{3(5+k)^{2}} F_{11} G_{22}-\frac{4(9+5 k)}{(5+k)^{3}} \partial F_{11} F_{22}-\frac{4(39+11 k)}{3(5+k)^{3}} F_{11} \partial F_{22} \\
& +\frac{2(21+5 k)}{3(5+k)^{2}} F_{12} G_{21}+\frac{4(9+5 k)}{(5+k)^{3}} \partial F_{12} F_{21}+\frac{4(39+11 k)}{3(5+k)^{3}} F_{12} \partial F_{21}-\frac{4(6+k)}{3(5+k)^{2}} F_{21} G_{12} \\
& \left.+\frac{4(6+k)}{3(5+k)^{2}} F_{22} G_{11}+\frac{8 i k}{(5+k)^{2}} U B_{3}-\frac{4(33+13 k)}{3(5+k)^{3}} U F_{11} F_{22}+\frac{4(33+13 k)}{3(5+k)^{3}} U F_{12} F_{21}\right](w) \\
& +\frac{1}{(z-w)}\left[\frac{1}{4} \partial\left\{G_{22} \mathbf{Q}^{\left(\frac{\mathbf{5}}{2}\right)}\right\}_{-2}+\frac{4(-\mathbf{3}+\mathbf{k})}{(17+13 k)}\left(T \mathbf{T}^{(\mathbf{1})}-\frac{1}{2} \partial^{2} \mathbf{T}^{(\mathbf{1})}\right)+\mathbf{S}^{(\mathbf{3})}\right. \\
& +\frac{18 k}{(5+k)(17+13 k)}\left(T U-\frac{1}{2} \partial^{2} U\right)+\frac{6 i(18+5 k)}{(5+k)(17+13 k)}\left(T A_{3}-\frac{1}{2} \partial^{2} A_{3}\right) \\
& +\frac{2 i k(21+4 k)}{(5+k)(17+13 k)}\left(T B_{3}-\frac{1}{2} \partial^{2} B_{3}\right)-\frac{4(-3+k)(9+2 k)}{(5+k)^{2}(17+13 k)}\left(T F_{11} F_{22}-\frac{1}{2} \partial^{2}\left(F_{11} F_{22}\right)\right) \\
& \left.+\frac{4\left(27+9 k+2 k^{2}\right)}{(5+k)^{2}(17+13 k)}\left(T F_{12} F_{21}-\frac{1}{2} \partial^{2}\left(F_{12} F_{21}\right)\right)\right](w)+\cdots .
\end{aligned}
$$

In the first order pole, one sees the nonlinear term between the higher spin-1 current and the spin-2 stress tensor from the large $\mathcal{N}=4$ linear superconformal algebra. Note that in Appendix $C$, the OPE between $G_{22}(z)$ and $\mathbf{U}^{\left(\frac{5}{2}\right)}(w)$ contains this kind of nonlinear term in the first order pole. Therefore simply adding the higher spin- $\frac{5}{2}$ current $\mathbf{U}^{\left(\frac{5}{2}\right)}(w)$ to the left hand side of the OPE (5.6) will eliminate the above nonlinear term because they have opposite signs.

So far, the four higher spin- $\frac{5}{2}$ currents and the six higher spin-3 currents are obtained. The remaining four higher spin- $\frac{7}{2}$ currents and one single higher spin- 4 current will be present 
in Appendix E. Contrary to the previous section, the exact relations between the higher spin currents in (1.2) and those in [9] are complicated because the precise relation between the lowest higher spin- 2 currents is rather involved and this will further give rise to the complexity as the spin increases.

\section{The OPEs between the 16 currents in the large $\mathcal{N}=4$ linear superconformal algebra and the next 16 lowest higher spin currents}

Because the 16 currents of large $\mathcal{N}=4$ linear superconformal algebra and the 16 next higher spin currents are known in terms of the coset fields, it is straightforward to obtain the OPEs between them. For example, the previous OPEs, (5.3), (5.4), (5.5), and (5.6) are some of those OPEs. The right hand side of these OPEs contain the nonlinear terms. On the other hands, the description of [9] implies that there exist linear OPEs between the 16 currents of large $\mathcal{N}=4$ linear superconformal algebra and the 16 next higher spin currents.

Recall that the lowest higher spin-2 current [9] living in the next 16 higher spin currents has the same form in the linear and nonlinear version. Then it is easy to obtain their higher spin-2 current in terms of the higher spin-2 currents (and some composite fields) of this paper by requiring that the higher spin- 2 current should satisfy the first three equations of Appendix D.1. It turns out that one obtains

$$
\begin{aligned}
V_{0}^{(2)}(z) & =\left[\mathbf{P}^{(\mathbf{2})}-4 \mathbf{W}^{(\mathbf{2})}-\frac{4(k-3)}{3(k+5)} \mathbf{T}^{(\mathbf{2})}+\frac{\left(41 k^{2}+400 k+783\right)}{5(k+5)^{2}} \mathbf{T}^{(\mathbf{1})} \mathbf{T}^{(\mathbf{1})}\right. \\
& -\frac{4(k+3)(5 k+34)}{3(k+5)^{2}} T-\frac{4(5 k+24)}{3(k+5)^{2}} A_{3} A_{3}-\frac{4 i(5 k+24)}{3(k+5)^{2}} \partial A_{3} \\
& +\frac{8 i(k+15)}{3(k+5)^{3}} A_{3} F_{11} F_{22}+\frac{4 i(k+9)}{(k+5)^{3}} A_{3} F_{12} F_{21}+\frac{4 i(5 k+57)}{3(k+5)^{3}} A_{-} F_{11} F_{12} \\
& -\frac{4(5 k+24)}{3(k+5)^{2}} A_{+} A_{-}-\frac{4\left(9 k^{2}+70 k+102\right)}{15(k+5)^{2}} B_{3} B_{3}-\frac{4 i\left(9 k^{2}+70 k+102\right)}{15(k+5)^{2}} \partial B_{3} \\
& -\frac{8 i\left(9 k^{2}+80 k+177\right)}{15(k+5)^{3}} B_{3} F_{11} F_{22}+\frac{4 i\left(6 k^{2}+55 k+113\right)}{5(k+5)^{3}} B_{3} F_{12} F_{21} \\
& -\frac{8 i\left(9 k^{2}+65 k+132\right)}{15(k+5)^{3}} B_{-} F_{12} F_{22}-\frac{4\left(9 k^{2}+70 k+102\right)}{15(k+5)^{2}} B_{+} B_{-} \\
& -\frac{4 i\left(6 k^{2}+65 k+143\right)}{5(k+5)^{3}}+\frac{10(k+3)}{3(k+5)^{2}} F_{11} G_{22}-\frac{8\left(9 k^{2}+80 k+127\right)}{5(k+5)^{4}} F_{11} F_{12} F_{21} F_{22} \\
& -\frac{2\left(77 k^{2}+630 k+1401\right)}{15(k+5)^{3}} \partial F_{11} F_{22}+\frac{2\left(77 k^{2}+810 k+2301\right)}{15(k+5)^{3}} F_{11} \partial F_{22}
\end{aligned}
$$




$$
\begin{aligned}
& -\frac{4}{(k+5)^{2}} F_{12} G_{21}-\frac{2\left(77 k^{2}+780 k+1671\right)}{15(k+5)^{3}} \partial F_{12} F_{21}+\frac{14\left(11 k^{2}+100 k+273\right)}{15(k+5)^{3}} F_{12} \partial F_{21} \\
& +\frac{4}{(k+5)} F_{21} G_{12}+\frac{4(2 k+9)}{3(k+5)^{2}} F_{22} G_{11}-\frac{12 i}{(k+5)^{2}} U A_{3}-\frac{4 i k}{(k+5)^{2}} U B_{3} \\
& \left.-\frac{4(k+3)(5 k+34)}{3(k+5)^{3}} U U+\frac{16(k-3)}{3(k+5)^{3}} U F_{11} F_{22}-\frac{12}{(k+5)^{2}} U F_{12} F_{21}\right](z) .
\end{aligned}
$$

Therefore, one can check that the OPEs between the 16 currents of large $\mathcal{N}=4$ linear superconformal algebra and the higher spin-2 current $\mathbf{P}^{(\mathbf{2})}(w)$ can be obtained from the coset fields and they will exactly produce the OPEs between the 16 currents and $V_{0}^{(2)}(w)+\cdots(=$ $\left.\mathbf{P}^{(\mathbf{2})}(w)\right)$ in (6.1).

The four higher spin- $\frac{5}{2}$ currents [9] can be written in terms of cost fields because the higher spin-2 current is given in (6.1) and also further can be written in terms of other higher spin currents as in Appendix $E$. Then the OPEs between the 16 currents in the large $\mathcal{N}=4$ linear superconformal algebra and the four higher spin- $\frac{5}{2}$ currents, $\mathbf{P}_{ \pm}^{\left(\frac{5}{2}\right)}(w), \mathbf{Q}^{\frac{5}{2}}(w)$ and $\mathbf{R}^{\left(\frac{5}{2}\right)}(w)$ can be obtained from the results in [9] and Appendices $A$ and $C$. On the other hands, they can be also obtained from the explicit expressions for the coset fields. In this case, the nontrivial thing is to identify the OPEs in terms of the known 16 currents and higher spin currents (and their derivatives). Again the eighth equation of Appendix D.1 allows us to obtain the six higher spin-3 currents, $V_{1}^{(2),+i}(w)$ and $V_{1}^{(2),-i}(w)$ from the previous higher spin- $\frac{5}{2}$ currents, $V_{\frac{1}{2}}^{(2), a}(w)$ as done in section 4. Then the corresponding OPEs between the above 16 currents and the six higher spin-3 currents will be obtained by following previous procedure. In this way, one can obtain the OPEs between the above 16 currents and the single higher spin- 4 current which is the last component of the 16 next lowest higher spin currents.

\section{The OPEs between the higher spin currents where the lowest spin is 1}

The full OPEs for these are given in Appendix F. When the OPEs for low spins are calculated, one follows what has been done in the nonlinear version. In other words, because the 16 currents and the 16 higher spin currents are known in terms of coset fields explicitly, after calculating the OPE, the right hand side of the OPE is written in terms of the coset fields. Then one should rewrite them in terms of the above known 16 currents and 16 higher spin currents by assuming the possible composite fields with definite $U(1)$ charges introduced in section 2 together with arbitrary coefficient functions which depend on $k$. All the results in Appendix $F$ are done by using this procedure explicitly. 
On the other hands, one can use the results of Part I and Part II in the nonlinear version because the above 16 currents and 16 higher spin currents in the linear version have the corresponding currents in the nonlinear version. For example, let us consider the OPE $\mathbf{T}^{(\mathbf{1})}(z) \mathbf{T}_{ \pm}^{\left(\frac{3}{2}\right)}(w)$. The higher spin-1 current has the same form in the linear and nonlinear versions. The higher spin- $-\frac{3}{2}$ current is given in the last expression in $(\underline{B .2})$ where $\mathbf{T}_{ \pm}^{\left(\frac{3}{2}\right)}(w)$ can be written in terms of $\mathbf{T}_{ \pm, \text {non }}^{\left(\frac{3}{2}\right)}(w)$ plus other six terms which depend on the spin- $\frac{1}{2}$ current, the spin- 1 current, the other spin-1 currents $\hat{A}_{i}(w)$ and $\hat{B}_{i}(w)$ living in the nonlinear version. Now one should calculate the OPE between $\mathbf{T}^{(\mathbf{1})}(z)$ and $\mathbf{T}_{ \pm, \text {non }}^{\left(\frac{3}{2}\right)}(w)$. However, this was done in Part II already but the right hand side is written in terms of the field $\mathbf{T}_{ \pm \text {,non }}^{\left(\frac{3}{2}\right)}(w)$ in the nonlinear version. Therefore, one should rewrite this in the linear version using the first expression in (B.2) where all the expressions are given in terms of the fields in the linear version. Now one should calculate the OPE between the $\mathbf{T}^{(\mathbf{1})}(z)$ and other six terms mentioned above. By the definition of the higher spin- 1 current, the four spin- $\frac{1}{2}$ currents and the spin- 1 current do not have any singular terms in the OPE with the higher spin-1 current. Furthermore, the higher spin-1 current does not have any singular terms with the above spin-1 currents, $\hat{A}_{i}(w)$ and $\hat{B}_{i}(w)$. Then there is no contribution from the OPE between the $\mathbf{T}^{(\mathbf{1})}(z)$ and other six terms. Therefore, the OPE $\mathbf{T}^{(\mathbf{1})}(z) \mathbf{T}_{ \pm}^{\left(\frac{3}{2}\right)}(w)$ has the first order pole given in $\mathbf{T}_{ \pm, \mathbf{n}}^{\left(\frac{3}{2}\right)}(w)$ and this should be written in terms of the fields in the linear version using (B.2). Then the explicit result is given in Appendix $F$. Some of the structures (the composite fields of spin- $\frac{7}{2}$, spin-4, spin- $\frac{9}{2}$ or spin-5) in the OPEs of Appendix $F$ are not presented but they can be obtained using this procedure.

There are three cases in Appendix F. The first case is that the right hand side of the OPEs do not have the higher spin currents but they have only the multiple product (including the derivatives) of the 16 currents of large $\mathcal{N}=4$ linear superconformal algebra. The second case is that the right hand side of the OPEs contains the higher spin currents in (1.1) or the higher spin currents (1.2) as well as the composite fields from the 16 currents. The third case is that the right hand side of OPEs possess the higher spin currents in (1.1) and in (1.2) as well as the multiple products in the above 16 currents.

One realizes that any OPE between any higher spin current and itself does not contain the higher spin currents in the right hand side. The only exception is the higher spin-3 current. Moreover, the OPEs between $\mathbf{U}^{\frac{3}{2}}(z)\left[\mathbf{V}^{\frac{3}{2}}(z)\right]$ and any other component fields in the second [third] $\mathcal{N}=2$ multiplet do not contain any higher spin currents in the right hand side. Some the OPEs containing $\mathbf{T}^{(\mathbf{1})}(z)$ or $\mathbf{T}_{ \pm}^{\frac{3}{2}}(z)$ do not have the higher spin currents in the right hand side from Appendix $F$. One sees that the OPEs between the second components of $\mathcal{N}=2$ second, third or fourth multiplets do not have the higher spin currents in the right hand side. 
Similarly, the OPEs between the third components of these $\mathcal{N}=2$ multiplets contain only the 16 currents in the large $\mathcal{N}=4$ linear superconformal algebra. Finally, the OPEs between the higher spin-1 current and the higher spin currents of integer spin do not contain the higher spin currents in the right hand side.

If one use the OPEs $V_{\frac{1}{2}}^{(1), 0}(z) V_{1}^{(1), \pm 3}(w)$ and $V_{\frac{1}{2}}^{(1), 3}(z) V_{1}^{(1), \pm 3}(w)$ in different basis, then the right hand side do not contain the higher spin currents $\mathbf{T}_{ \pm}^{\left(\frac{\mathbf{3}}{2}\right)}(w)$ in the OPEs of $\mathbf{T}_{ \pm}^{\left(\frac{3}{2}\right)}(z) \mathbf{T}^{(\mathbf{2})}(w)$. Similarly, the OPEs $V_{\frac{1}{2}}^{(1), 1}(z) V_{1}^{(1), \pm 3}(w)$ and $V_{\frac{1}{2}}^{(1), 2}(z) V_{1}^{(1), \pm 3}(w)$ remove the higher spin currents $\mathbf{U}^{\left(\frac{3}{2}\right)}(w)$ and $\mathbf{V}^{\left(\frac{3}{2}\right)}(w)$ which appear in the OPEs $\mathbf{U}^{\left(\frac{3}{2}\right)}(z) \mathbf{W}^{(\mathbf{2})}(w)$ and $\mathbf{V}^{\left(\frac{3}{2}\right)}(z) \mathbf{W}^{(\mathbf{2})}(w)$. Some of OPEs contain the higher spin currents $\mathbf{Q}_{ \pm}^{(\mathbf{3})}(w)$ or $\mathbf{R}_{ \pm}^{(\mathbf{3})}(w)$ in the right hand side where there are no other types of higher spin currents. For example, the OPE between $\mathbf{T}^{(\mathbf{2})}(z)$ and $\mathbf{U}_{+}^{(2)}(w)$ has only the higher spin current $\mathbf{Q}_{+}^{(\mathbf{3})}(w)$ in the first order pole. Most of the OPEs have both the lowest higher spin currents and the next lowest higher spin currents as well as the 16 currents in the large $\mathcal{N}=4$ linear superconformal algebra in the right hand side of the OPEs in general.

\section{Conclusions and outlook}

The main result of this paper is the fact that the OPEs between the 16 lowest higher spin currents and itself (presented in Appendix $F$ ) do not have any nonlinear terms between the 16 currents from the large $\mathcal{N}=4$ linear superconformal algebra and the 16 lowest higher spin currents except few cases. For the nonlinear version, this kind of nonlinear terms occurs in the corresponding various OPEs. In particular, for the OPEs where the left hand side contains the higher spin-3 current $\mathbf{W}^{(\mathbf{3})}(w)$ there exist the nonlinear terms containing the higher spin1 current $\mathbf{T}^{(\mathbf{1})}(w)$. One can easily see that these nonlinear terms disappear once one uses the higher spin-3 current (quasi primary field) defined in (4.7). Note also that the OPE $V_{2}^{(1)}(z) V_{2}^{(1)}(w)$ corresponding to the $\mathrm{OPE} \mathbf{W}^{(\mathbf{3})}(z) \mathbf{W}^{(\mathbf{3})}(w)$ does not contain the nonlinear term $\mathbf{T}^{(\mathbf{1})} \mathbf{T}^{(\mathbf{1})}(w)$ in the fourth order pole because the contribution from the extra terms in (4.7) cancel exactly.

Among the OPEs between the higher spin- $\frac{3}{2}$ currents and the higher spin- $\frac{5}{2}$ currents, the different kind of nonlinear terms occur: $T \mathbf{T}^{(\mathbf{1})}(w)$. Specifically, there are four OPEs, $\mathbf{T}_{ \pm}^{\left(\frac{3}{2}\right)}(z) \mathbf{W}_{\mp}^{\left(\frac{5}{2}\right)}(w), \mathbf{U}^{\left(\frac{3}{2}\right)}(z) \mathbf{V}^{\left(\frac{5}{2}\right)}(w)$ and $\mathbf{V}^{\left(\frac{3}{2}\right)}(z) \mathbf{U}^{\frac{5}{2}}(w)$ from Appendix $F$. Also these nonlinear terms can be removed by using the higher spin- $\frac{3}{2}$ currents (4.2) and the higher spin- $\frac{5}{2}$ currents (4.6) which are primary fields. In other words, the first order poles of the following OPEs do not contain the above nonlinear term: $V_{\frac{1}{2}}^{(1), 0}(z) V_{\frac{3}{2}}^{(1), 0}(w), V_{\frac{1}{2}}^{(1), 0}(z) V_{\frac{3}{2}}^{(1), 3}(w)$, $V_{\frac{1}{2}}^{(1), 3}(z) V_{\frac{3}{2}}^{(1), 0}(w), V_{\frac{1}{2}}^{(1), 3}(z) V_{\frac{3}{2}}^{(1), 3}(w), V_{\frac{1}{2}}^{(1), 1}(z) V_{\frac{3}{2}}^{(1), 1}(w), V_{\frac{1}{2}}^{(1), 1}(z) V_{\frac{3}{2}}^{(1), 2}(w), V_{\frac{1}{2}}^{(1), 2}(z) V_{\frac{3}{2}}^{(1), 1}(w)$, 
and $V_{\frac{1}{2}}^{(1), 2}(z) V_{\frac{3}{2}}^{(1), 2}(w)$. So far, the nonlinearity in the OPEs disappears if one uses the higher spin-3 current in the quasi-primary basis. It would be interesting to obtain the precise relation between the nonlinearity and the property of quasi-primary field (The OPE between the spin-2 stress tensor and the quasi primary field has the vanishing third order pole and nontrivial higher order poles).

The small $\mathcal{N}=4$ linear superconformal algebra can be obtained by rescaling the four spin$\frac{1}{2}$ currents, one spin-1 current and three spin-1 currents with level $k^{+}$and taking $k^{+} \rightarrow \infty$ limit. For example, in the OPE between $\mathbf{T}^{(\mathbf{1})}(z)$ and $\mathbf{T}_{ \pm}^{\left(\frac{3}{2}\right)}(w)$ given in Appendix $F$, the third, the sixth and the seventh terms in the right hand side are vanishing while the first, the second, the fourth and the fifth terms survive in this limit. One can analyze all the other OPEs presented in Appendix $F$ and obtains 136 OPEs in an extension of small $\mathcal{N}=4$ linear superconformal algebra. It would be interesting to see how this plays the role in [1].

- The higher spin currents for general $N$

In this paper, the $N$ in the coset $\frac{S U(N+2)}{S U(N)}$ is fixed as $N=3$. The next question one can ask is how one can obtain the 16 higher spin currents for generic $N$. There are several ways to obtain the higher spin currents for general $N$. One of them is to use the higher spin currents for generic $N$ in the nonlinear version. From the experience of this paper, there should exist the exact relations between the higher spin currents in the nonlinear and linear versions for generic $N$. Their relations for $N=3$ are presented in Appendix $B$. All the $k$ dependent coefficients can be generalized to the ones with $N$ dependence explicitly. Or one can start with the four spin- $\frac{3}{2}$ currents of large $\mathcal{N}=4$ linear superconformal algebra and the higher spin-1 current fro general $N$. They have explicit their coset field realizations. Then it is straightforward to calculate the various OPEs and to extract the higher spin currents by hand.

- The $\mathcal{N}=4$ superspace description

For the 16 currents of large $\mathcal{N}=4$ linear superconformal algebra, one can write down them in one single $\mathcal{N}=4$ superfield (of superspin 0) and the various OPEs in Appendix $A$ can be written as a single OPE in $\mathcal{N}=4$ superspace. According to the observation of [9], the other single $\mathcal{N}=4$ superfield (of superspin 1) representing the lowest 16 higher spin currents transform as linearly under the above $\mathcal{N}=4$ superfield. In other words, the Appendix $D$ can be written in terms of the OPE between the $\mathcal{N}=4$ supercurrent with superspin 0 and the $\mathcal{N}=4$ supercurrent with superspin 1 and the right hand side of this OPE depends on the $\mathcal{N}=4$ supercurrent with superspin 1 linearly. It would be an open problem to rewrite Appendix $F$ in terms of one single $\mathcal{N}=4$ super OPE. The nontrivial thing is to express the nonlinear terms between the above $\mathcal{N}=4$ super current of superspin zero which will be 
complicated. The linear terms from the $\mathcal{N}=4$ higher spin currents of superspin 1 or 2 will be rather simple form. Furthermore, in $\mathcal{N}=2$ superspace, because the $\mathcal{N}=4$ multiplet corresponds to four independent $\mathcal{N}=2$ multiplets as in (1.1), one has the ten $\mathcal{N}=2$ super OPEs. It would be also interesting to write down the above single $\mathcal{N}=4$ super OPE in terms of ten $\mathcal{N}=2$ super OPEs.

- The higher spin currents in the linear version in the orthogonal Wolf space

In [12], the higher spin currents construction was given for the different type of $\mathcal{N}=4$ super coset theory. It would be interesting to describe the linear version of the higher spin currents.

\section{Acknowledgments}

We would like to thank H. Kim and M.H. Kim for discussions. This work was supported by the Mid-career Researcher Program through the National Research Foundation of Korea (NRF) grant funded by the Korean government (MEST) (No. 2012-045385/2013056327/2014-051185). CA acknowledges warm hospitality from the School of Liberal Arts (and Institute of Convergence Fundamental Studies), Seoul National University of Science and Technology. 


\section{Appendix A The large $\mathcal{N}=4$ linear superconformal al- gebra}

The large $\mathcal{N}=4$ linear superconformal algebra (generated by spin-2 current $T(z)$, four spin- $\frac{3}{2}$ currents $G_{a}(z)$, three spin- 1 currents $A^{+i}(z)$, three spin- 1 currents $A^{-i}(z)$, spin- 1 current $U(z)$ and four spin- $\frac{1}{2}$ currents $\left.\Gamma_{a}(z)\right)$ can be summarized by [3]

$$
\begin{aligned}
& T(z) T(w)=\frac{1}{(z-w)^{4}} \frac{c}{2}+\frac{1}{(z-w)^{2}} 2 T(w)+\frac{1}{(z-w)} \partial T(w)+\cdots, \\
& T(z) \phi(w)=\frac{1}{(z-w)^{2}} h_{\phi} \phi(w)+\frac{1}{(z-w)} \partial \phi(w)+\cdots, \\
& G_{a}(z) G_{b}(w)=\frac{1}{(z-w)^{3}} \frac{4 c}{3} \delta_{a b}-\frac{1}{(z-w)^{2}} 16\left[\gamma \alpha_{a b}^{+i} A_{i}^{+}+(1-\gamma) \alpha_{a b}^{-i} A_{i}^{-}\right](w) \\
& +\frac{1}{(z-w)}\left[\frac{1}{2} \partial(\text { pole- } 2)+4 \delta_{a b} T\right](w)+\cdots, \\
& A^{+i}(z) G_{a}(w)=-\frac{1}{(z-w)^{2}} 2(1-\gamma) \alpha_{a}^{+i b} \Gamma_{b}(w)+\frac{1}{(z-w)} \alpha_{a}^{+i b} G_{b}(w)+\cdots, \\
& A^{-i}(z) G_{a}(w)=\frac{1}{(z-w)^{2}} 2 \gamma \alpha_{a}^{-i b} \Gamma_{b}(w)+\frac{1}{(z-w)} \alpha_{a}^{-i b} G_{b}(w)+\cdots, \\
& A^{+i}(z) A^{+j}(w)=-\frac{1}{(z-w)^{2}} \frac{1}{2} k^{+} \delta^{i j}+\frac{1}{(z-w)} \epsilon^{i j k} A_{k}^{+}(w)+\cdots, \\
& A^{-i}(z) A^{-j}(w)=-\frac{1}{(z-w)^{2}} \frac{1}{2} k^{-} \delta^{i j}+\frac{1}{(z-w)} \epsilon^{i j k} A_{k}^{-}(w)+\cdots, \\
& \Gamma_{a}(z) G_{b}(w)=\frac{1}{(z-w)} 2\left[2\left(\alpha_{a b}^{+i} A_{i}^{+}-\alpha_{a b}^{-i} A_{i}^{-}\right)+\delta_{a b} U\right](w)+\cdots, \\
& A^{+i}(z) \Gamma_{a}(w)=\frac{1}{(z-w)} \alpha_{a}^{+i b} \Gamma_{b}(w)+\cdots, \\
& A^{-i}(z) \Gamma_{a}(w)=\frac{1}{(z-w)} \alpha_{a}^{-i b} \Gamma_{b}(w)+\cdots, \\
& U(z) G_{a}(w)=\frac{1}{(z-w)^{2}} \Gamma_{a}(w)+\cdots, \\
& \Gamma_{a}(z) \Gamma_{b}(w)=-\frac{1}{(z-w)} \frac{c}{6 \gamma(1-\gamma)} \delta_{a b}+\cdots, \\
& U(z) U(w)=-\frac{1}{(z-w)^{2}} \frac{c}{12 \gamma(1-\gamma)}+\cdots .
\end{aligned}
$$

Here the conformal dimension is as follows: $h_{G_{a}}=\frac{3}{2}, h_{A^{ \pm i}}=1, h_{U}=1$ and $h_{\Gamma_{a}}=\frac{1}{2}$. The numerical values for the quantities in the right hand side are given in [3]. The indices $a, b$ are bispinor notations. One has the following letters where $A^{+i}(z) \equiv A_{i}(z), A^{-i}(z) \equiv B_{i}(z)$ and $\Gamma_{a}(z) \equiv F_{a}(z)$. The coset field realization representing this algebra for $N=3$ is already given in Part I [5]. 


\section{Appendix B The 16 lowest higher spin currents in the nonlinear version in terms of those in linear version}

In this Appendix, the explicit relations between the 16 lowest higher spin currents in the nonlinear version and the linear version are given. For the lowest higher spin-1 current, the nonlinear version in [5] and the linear version of this paper are the same each other

$$
\mathbf{T}_{\text {non }}^{(1)}(z)=\mathbf{T}^{(1)}(z) .
$$

In (3.1), the higher spin- $\frac{3}{2}$ currents are determined. The corresponding currents in the nonlinear version can be obtained from the equations (4.9) and (4.13) of [5]. The spin- $\frac{3}{2}$ currents of large $\mathcal{N}=4$ superconformal algebra are related to each other in equation (3.13) of [5]. Then one can substitute the right hand side of (3.13) of Part I into the equations (4.9) and (4.13) of Part I. The nontrivial OPEs come from only the spin- $\frac{3}{2}$ currents because there are no singular terms between $\left(U, F_{a}, \hat{A}_{i}, \hat{B}_{i}\right)(z)$ and $\mathbf{T}^{(\mathbf{1})}(w)$. This implies that the left hand sides of the OPEs in the nonlinear version and the linear version are the same and one has the following relations

$$
\mp\left(\begin{array}{c}
\hat{G}_{12} \\
\hat{G}_{21}
\end{array}\right)(z)+2 \mathbf{T}_{\mp, \mathbf{n o n}}^{\left(\frac{3}{2}\right)}(z)=\mp\left(\begin{array}{c}
G_{12} \\
G_{21}
\end{array}\right)(z)+2 \mathbf{T}_{\mp}^{\left(\frac{3}{2}\right)}(z) .
$$

Or the higher spin- $\frac{3}{2}$ currents can be written as

$$
\mathbf{T}_{\mp, \text { non }}^{\left(\frac{3}{2}\right)}(z)=\mathbf{T}_{\mp}^{\left(\frac{3}{2}\right)}(z) \pm \frac{1}{2}\left(\begin{array}{c}
\hat{G}_{12} \\
\hat{G}_{21}
\end{array}\right)(z) \mp \frac{1}{2}\left(\begin{array}{c}
G_{12} \\
G_{21}
\end{array}\right)(z) .
$$

Therefore, the extra terms between the higher spin- $\frac{3}{2}$ currents in the nonlinear and linear versions are exactly those from the spin- $\frac{3}{2}$ currents. We arrive at the following results

$$
\begin{aligned}
\mathbf{T}_{ \pm, \text {non }}^{\left(\frac{3}{2}\right)}(z) & =\left[\mathbf{T}_{ \pm}^{\left(\frac{3}{2}\right)} \mp \frac{1}{(5+k)} U\left(\begin{array}{c}
F_{21} \\
F_{12}
\end{array}\right)+\frac{4}{(5+k)^{2}}\left(\begin{array}{c}
F_{21} \\
F_{12}
\end{array}\right) F_{11} F_{22}+\frac{i}{(5+k)}\left(\begin{array}{c}
F_{21} \\
F_{12}
\end{array}\right) A_{3}\right. \\
& \left.-\frac{i}{(5+k)}\left(\begin{array}{c}
F_{11} \\
F_{22}
\end{array}\right) A_{\mp}+\frac{i}{(5+k)}\left(\begin{array}{c}
F_{22} \\
F_{11}
\end{array}\right) B_{\mp}-\frac{i}{(5+k)}\left(\begin{array}{c}
F_{21} \\
F_{12}
\end{array}\right) B_{3}\right](z) \\
& =\left[\mathbf{T}_{ \pm}^{\left(\frac{3}{2}\right)} \mp \frac{1}{(5+k)} U\left(\begin{array}{c}
F_{21} \\
F_{12}
\end{array}\right)-\frac{2}{(5+k)^{2}}\left(\begin{array}{c}
F_{21} \\
F_{12}
\end{array}\right) F_{11} F_{22}+\frac{i}{(5+k)}\left(\begin{array}{c}
F_{21} \\
F_{12}
\end{array}\right) \hat{A}_{3}\right. \\
& \left.-\frac{i}{(5+k)}\left(\begin{array}{c}
F_{11} \\
F_{22}
\end{array}\right) \hat{A}_{\mp}+\frac{i}{(5+k)}\left(\begin{array}{c}
F_{22} \\
F_{11}
\end{array}\right) \hat{B}_{\mp}-\frac{i}{(5+k)}\left(\begin{array}{c}
F_{21} \\
F_{12}
\end{array}\right) \hat{B}_{3}\right](z) . \quad(\mathrm{B} .2)
\end{aligned}
$$

In the first expression of (B.2), the extra term in the right hand side is written in terms of the fields in the linear version and in the second expression of (B.2), the extra term in the 
right hand side is given by the fields in the nonlinear version using the equations (3.5), (3.6) and (3.10) of [5].

Similarly, one has the following relations from the equation (3.2) together with (4.20) and (4.34) of [5]

$$
\left(\begin{array}{l}
\mathbf{U}_{\text {non }}^{\left(\frac{3}{2}\right)} \\
\mathbf{V}_{\text {non }}^{\left(\frac{3}{2}\right)}
\end{array}\right)(z)=\left(\begin{array}{l}
\mathbf{U}^{\left(\frac{3}{2}\right)} \\
\mathbf{V}^{\left(\frac{3}{2}\right)}
\end{array}\right)(z) \pm \frac{1}{2}\left(\begin{array}{c}
\hat{G}_{11} \\
\hat{G}_{22}
\end{array}\right)(z) \mp \frac{1}{2}\left(\begin{array}{c}
G_{11} \\
G_{22}
\end{array}\right)(z) .
$$

In other words, one has, as done before,

$$
\begin{aligned}
\left(\begin{array}{c}
\mathbf{U}_{\mathbf{n}}^{\left(\frac{3}{2}\right)} \\
\mathbf{V}_{\mathbf{n o n}}^{\left(\frac{3}{2}\right)}
\end{array}\right)(z) & =\left[\left(\begin{array}{c}
\mathbf{U}^{\left(\frac{3}{2}\right)} \\
\mathbf{V}^{\left(\frac{3}{2}\right)}
\end{array}\right) \mp \frac{1}{(5+k)} U\left(\begin{array}{c}
F_{11} \\
F_{22}
\end{array}\right)-\frac{4}{(5+k)^{2}}\left(\begin{array}{c}
F_{11} \\
F_{22}
\end{array}\right) F_{12} F_{21}-\frac{i}{(5+k)}\left(\begin{array}{c}
F_{11} \\
F_{22}
\end{array}\right) A_{3}\right. \\
& \left.-\frac{i}{(5+k)}\left(\begin{array}{c}
F_{21} \\
F_{12}
\end{array}\right) A_{ \pm}-\frac{i}{(5+k)}\left(\begin{array}{c}
F_{12} \\
F_{21}
\end{array}\right) B_{\mp}-\frac{i}{(5+k)}\left(\begin{array}{c}
F_{11} \\
F_{22}
\end{array}\right) B_{3}\right](z) \\
& =\left[\left(\begin{array}{c}
\mathbf{U}^{\left(\frac{3}{2}\right)} \\
\mathbf{V}^{\left(\frac{3}{2}\right)}
\end{array}\right) \mp \frac{1}{(5+k)} U\left(\begin{array}{c}
F_{11} \\
F_{22}
\end{array}\right)+\frac{2}{(5+k)^{2}}\left(\begin{array}{c}
F_{11} \\
F_{22}
\end{array}\right) F_{12} F_{21}-\frac{i}{(5+k)}\left(\begin{array}{c}
F_{11} \\
F_{22}
\end{array}\right) \hat{A}_{3}\right. \\
& \left.-\frac{i}{(5+k)}\left(\begin{array}{c}
F_{21} \\
F_{12}
\end{array}\right) \hat{A}_{\mp}-\frac{i}{(5+k)}\left(\begin{array}{c}
F_{12} \\
F_{21}
\end{array}\right) \hat{B}_{\mp}-\frac{i}{(5+k)}\left(\begin{array}{c}
F_{11} \\
F_{22}
\end{array}\right) \hat{B}_{3}\right](z) .
\end{aligned}
$$

One can use the second relation of (B.4) to express the higher spin- $\frac{3}{2}$ currents in the linear version in terms of the fields in the nonlinear version.

For the higher spin-2 currents, one has the equation (3.5) and the corresponding footnotes 47 and 50 of [5]. Now one can substitute the spin- $\frac{3}{2}$ currents in the nonlinear version using (3.13) of [5] and the relation (B.3) into those equations. Then one can identify the following relations as follows:

$$
\begin{aligned}
\left(\begin{array}{c}
\mathbf{U}_{+, \text {non }}^{(2)} \\
\mathbf{V}_{-, \text {non }}^{(2)}
\end{array}\right)(z) & =\left[\left(\begin{array}{c}
\mathbf{U}_{+}^{(2)} \\
\mathbf{V}_{-}^{(\mathbf{2})}
\end{array}\right)+\frac{2}{(5+k)}\left(\begin{array}{c}
F_{11} \\
F_{22}
\end{array}\right) \mathbf{T}_{ \pm}^{\left(\frac{3}{2}\right)}-\frac{2}{(5+k)}\left(\begin{array}{l}
F_{21} \mathbf{U}^{\left(\frac{3}{2}\right)} \\
F_{12} \mathbf{V}^{\left(\frac{3}{2}\right)}
\end{array}\right)\right. \\
& \mp \frac{2}{(5+k)} A_{3} B_{\mp}-\frac{4 i}{(5+k)^{2}} A_{3}\left(\begin{array}{c}
F_{11} F_{21} \\
F_{22} F_{12}
\end{array}\right) \pm \frac{2 i}{(5+k)^{2}} B_{\mp} F_{11} F_{22} \\
& \pm \frac{2 i}{(5+k)^{2}} B_{\mp} F_{12} F_{21} \pm \frac{1}{(5+k)}\left(\begin{array}{c}
F_{11} G_{21} \\
F_{22} G_{12}
\end{array}\right) \\
& \left. \pm \frac{2}{(5+k)^{2}}\left(\begin{array}{l}
\partial F_{11} F_{21} \\
\partial F_{22} F_{12}
\end{array}\right) \mp \frac{2}{(5+k)^{2}}\left(\begin{array}{c}
F_{11} \partial F_{21} \\
F_{22} \partial F_{12}
\end{array}\right) \mp \frac{1}{(5+k)}\left(\begin{array}{c}
F_{21} G_{11} \\
F_{12} G_{22}
\end{array}\right)\right](z) \\
& =\left[\left(\begin{array}{c}
\mathbf{U}_{+}^{(2)} \\
\mathbf{V}_{-}^{(2)}
\end{array}\right)+\frac{2}{(5+k)}\left(\begin{array}{c}
F_{11} \\
F_{22}
\end{array}\right) \mathbf{T}_{ \pm, \text {non }}^{\left(\frac{3}{2}\right)}-\frac{2}{(5+k)}\left(\begin{array}{l}
F_{21} \mathbf{U}_{\text {non }}^{\left(\frac{3}{2}\right)} \\
F_{12} \mathbf{V}_{\text {non }}^{\left(\frac{3}{2}\right)}
\end{array}\right)\right. \\
& \left.\mp \frac{2}{(5+k)} \hat{A}_{3} \hat{B}_{\mp} \pm \frac{1}{(5+k)}\left(\begin{array}{c}
F_{11} \hat{G}_{21} \\
F_{22} \hat{G}_{12}
\end{array}\right) \mp \frac{1}{(5+k)}\left(\begin{array}{c}
F_{21} \hat{G}_{11} \\
F_{12} \hat{G}_{22}
\end{array}\right)\right](z) .
\end{aligned}
$$


In the second equation of (B.5), the extra terms are written in terms of the fields in the nonlinear version. Note the appearance of the nonlinear terms between the higher spin currents and the spin- $\frac{1}{2}$ currents.

Similarly, from the equation (3.4) and the footnotes 48 and 49 of [5], one can obtain the following relations

$$
\begin{aligned}
\left(\begin{array}{c}
\mathbf{U}_{-, \text {non }}^{(2)} \\
\mathbf{V}_{+, \text {non }}^{(2)}
\end{array}\right)(z) & =\left[\left(\begin{array}{c}
\mathbf{U}_{-}^{(2)} \\
\mathbf{V}_{+}^{(2)}
\end{array}\right)-\frac{2}{(5+k)}\left(\begin{array}{l}
F_{11} \\
F_{22}
\end{array}\right) \mathbf{T}_{ \pm}^{\left(\frac{3}{2}\right)}+\frac{2}{(5+k)}\left(\begin{array}{l}
F_{12} \mathbf{U}^{\left(\frac{3}{2}\right)} \\
F_{21} \mathbf{V}^{\left(\frac{3}{2}\right)}
\end{array}\right)\right. \\
& \mp \frac{2}{(5+k)} A_{ \pm} B_{3} \mp \frac{2 i}{(5+k)^{2}} A_{ \pm} F_{11} F_{22}+\frac{2 i}{(5+k)^{2}} A_{ \pm} F_{12} F_{21} \\
& +\frac{4 i}{(5+k)^{2}} B_{3}\left(\begin{array}{c}
F_{11} F_{21} \\
F_{22} F_{12}
\end{array}\right) \pm \frac{1}{(5+k)}\left(\begin{array}{c}
F_{11} G_{12} \\
F_{22} G_{21}
\end{array}\right) \\
& \left. \pm \frac{2}{(5+k)^{2}}\left(\begin{array}{l}
\partial F_{11} F_{12} \\
\partial F_{22} F_{21}
\end{array}\right) \mp \frac{2}{(5+k)^{2}}\left(\begin{array}{c}
F_{11} \partial F_{12} \\
F_{22} \partial F_{21}
\end{array}\right) \pm \frac{1}{(5+k)}\left(\begin{array}{l}
F_{12} G_{11} \\
F_{21} G_{22}
\end{array}\right)\right](z) \\
& =\left[\left(\begin{array}{c}
\mathbf{U}_{-}^{(2)} \\
\mathbf{V}_{+}^{(2)}
\end{array}\right)-\frac{2}{(5+k)}\left(\begin{array}{l}
F_{11} \\
F_{22}
\end{array}\right) \mathbf{T}_{\mp, \text { non }}^{\left(\frac{3}{2}\right)}+\frac{2}{(5+k)}\left(\begin{array}{c}
F_{12} \mathbf{U}_{\text {non }}^{\left(\frac{3}{2}\right)} \\
F_{21} \mathbf{V}_{\text {non }}^{\left(\frac{3}{2}\right)}
\end{array}\right)\right. \\
& \left.\mp \frac{2}{(5+k)} \hat{A}_{ \pm} \hat{B}_{3} \pm \frac{1}{(5+k)}\left(\begin{array}{l}
F_{11} \hat{G}_{12} \\
F_{22} \hat{G}_{21}
\end{array}\right) \pm \frac{1}{(5+k)}\left(\begin{array}{c}
F_{12} \hat{G}_{11} \\
F_{21} \hat{G}_{22}
\end{array}\right)\right](z) .
\end{aligned}
$$

In this case, the second equation of (B.6) contains the extra terms written in terms of the fields in the nonlinear version. As before, one sees the appearance of the nonlinear terms between the higher spin currents and the spin- $\frac{1}{2}$ currents.

The equation (4.16) of [5] and the equation (3.3) determine the explicit relation between the higher spin-2 current in the nonlinear version and in the linear version

$$
\begin{aligned}
\mathbf{T}_{\text {non }}^{(\mathbf{2})}(z) & =\left[\mathbf{T}^{(\mathbf{2})}-\frac{2}{(5+k)} F_{11} \mathbf{V}^{\left(\frac{3}{2}\right)}+\frac{2}{(5+k)} F_{22} \mathbf{U}^{\left(\frac{3}{2}\right)}+\frac{(1+k)(21+k)}{(5+k)^{2}(3+7 k)} \partial F_{12} F_{21}\right. \\
& +\frac{(3+k)}{(3+7 k)} T+\frac{1}{(5+k)} A_{3} A_{3}-\frac{2}{(5+k)} A_{3} B_{3}+\frac{i}{(5+k)} \partial A_{3}-\frac{4 i}{(5+k)^{2}} A_{3} F_{11} F_{22} \\
& -\frac{2 i}{(5+k)^{2}} A_{-} F_{11} F_{12}+\frac{1}{(5+k)} A_{+} A_{-}-\frac{2 i}{(5+k)^{2}} A_{+} F_{21} F_{22}+\frac{1}{(5+k)} B_{3} B_{3}+\frac{i}{(5+k)} \partial B_{3} \\
& +\frac{2 i}{(5+k)^{2}} B_{-} F_{12} F_{22}+\frac{1}{(5+k)} B_{+} B_{-}+\frac{2 i}{(5+k)^{2}} B_{+} F_{11} F_{21}+\frac{1}{(5+k)} F_{22} G_{11} \\
& +\frac{1}{(5+k)} F_{11} G_{22}+\frac{\left(27+36 k+k^{2}\right)}{(5+k)^{2}(3+7 k)} \partial F_{11} F_{22}+\frac{4 i}{(5+k)^{2}} B_{3} F_{11} F_{22} \\
& \left.-\frac{\left(27+36 k+k^{2}\right)}{(5+k)^{2}(3+7 k)} F_{11} \partial F_{22}-\frac{(1+k)(21+k)}{(5+k)^{2}(3+7 k)} F_{12} \partial F_{21}+\frac{(3+k)}{(5+k)(3+7 k)} U U\right](z) \\
& =\left[\mathbf{T}^{(\mathbf{2})}-\frac{2}{(5+k)} F_{11} \mathbf{V}_{\text {non }}^{\left(\frac{3}{2}\right)}+\frac{2}{(5+k)} F_{22} \mathbf{U}_{\text {non }}^{\left(\frac{3}{2}\right)}+\frac{i}{(5+k)} \partial \hat{B}_{3}+\frac{1}{(5+k)} \hat{B}_{+} \hat{B}_{-}\right.
\end{aligned}
$$




$$
\begin{aligned}
& +\frac{(3+k)}{(3+7 k)} \hat{T}+\frac{1}{(5+k)} \hat{A}_{3} \hat{A}_{3}-\frac{2}{(5+k)} \hat{A}_{3} \hat{B}_{3}+\frac{i}{(5+k)} \partial \hat{A}_{3} \\
& \left.+\frac{1}{(5+k)} \hat{A}_{+} \hat{A}_{-}+\frac{1}{(5+k)} \hat{B}_{3} \hat{B}_{3}+\frac{1}{(5+k)} F_{11} \hat{G}_{22}+\frac{1}{(5+k)} F_{22} \hat{G}_{11}\right](z) .
\end{aligned}
$$

As explained before, one can express the higher spin-2 current in the linear version in terms of the fields in the nonlinear version using the second equation of (B.7). The nonlinear terms between the higher spin currents and the spin- $\frac{1}{2}$ currents occur in the right hand side.

Let us continue to consider the last higher spin-2 current. With the help of (3.6) and the equation (4.48) of [5], one can express the following relation between the higher spin-2 current in the nonlinear version and in the linear version

$$
\begin{aligned}
\mathbf{W}_{\text {non }}^{(2)}(z) & =\left[\mathbf{W}^{(\mathbf{2})}+\frac{2}{(5+k)} F_{12} \mathbf{T}_{+}^{\left(\frac{3}{2}\right)}-\frac{2}{(5+k)} F_{21} \mathbf{T}_{-}^{\left(\frac{3}{2}\right)}+\frac{1}{(5+k)} B_{3} B_{3}+\frac{i}{(5+k)} \partial B_{3}\right. \\
& +T+\frac{1}{(5+k)} A_{3} A_{3}+\frac{2}{(5+k)} A_{3} B_{3}+\frac{i}{(5+k)} \partial A_{3}-\frac{4 i}{(5+k)^{2}} A_{3} F_{12} F_{21} \\
& -\frac{2 i}{(5+k)^{2}} A_{-} F_{11} F_{12}+\frac{1}{(5+k)} A_{+} A_{-}-\frac{2 i}{(5+k)^{2}} A_{+} F_{21} F_{22} \\
& +\frac{2 i}{(5+k)^{2}} B_{-} F_{12} F_{22}+\frac{1}{(5+k)} B_{+} B_{-}+\frac{2 i}{(5+k)^{2}} B_{+} F_{11} F_{21}+\frac{1}{(5+k)} F_{12} G_{21} \\
& +\frac{(7+k)}{(5+k)^{2}} \partial F_{11} F_{22}-\frac{4 i}{(5+k)^{2}} B_{3} F_{12} F_{21}-\frac{(7+k)}{(5+k)^{2}} F_{11} \partial F_{22}+\frac{(9+k)}{(5+k)^{2}} \partial F_{12} F_{21} \\
& \left.+\frac{1}{(5+k)} F_{21} G_{12}-\frac{(9+k)}{(5+k)^{2}} F_{12} \partial F_{21}+\frac{1}{(5+k)} U U\right](z) \\
& =\left[\mathbf{W}(\mathbf{2})+\frac{2}{(5+k)} F_{12} \mathbf{T}_{+, \text {non }}^{\left(\frac{3}{2}\right)}-\frac{2}{(5+k)} F_{21} \mathbf{T}_{-, \text {non }}^{\left(\frac{3}{2}\right)}+\frac{1}{(5+k)} F_{21} \hat{G}_{12}\right. \\
& +\hat{T}+\frac{1}{(5+k)} \hat{A}_{3} \hat{A}_{3}+\frac{2}{(5+k)} \hat{A}_{3} \hat{B}_{3}+\frac{i}{(5+k)} \partial \hat{A}_{3}+\frac{1}{(5+k)} F_{12} \hat{G}_{21} \\
& \left.+\frac{1}{(5+k)} \hat{A}_{+} \hat{A}_{-}+\frac{1}{(5+k)} \hat{B}_{3} \hat{B}_{3}+\frac{i}{(5+k)} \partial \hat{B}_{3}+\frac{1}{(5+k)} \hat{B}_{+} \hat{B}_{-}\right](z) .
\end{aligned}
$$

Furthermore, the second equation of (B.8) allows us to write down the higher spin-2 current in the linear version in terms of the fields in the nonlinear version. One observes the appearance of the nonlinear terms between the higher spin currents and the spin- $\frac{1}{2}$ currents in the right hand side.

From the relation in (3.7) and the explicit result for the corresponding higher spin currents in Part I, the following relation holds

$$
\mathbf{U}_{\text {non }}^{\left(\frac{5}{2}\right)}(z)=\left[\mathbf{U}^{\left(\frac{5}{2}\right)}-\frac{2 i}{(5+k)} A_{3} \mathbf{U}^{\left(\frac{3}{2}\right)}+\frac{2 i}{(5+k)} B_{3} \mathbf{U}^{\left(\frac{3}{2}\right)}+\frac{2 i}{(5+k)} B_{-} \mathbf{T}_{-}^{\left(\frac{3}{2}\right)}+\frac{4}{(5+k)^{2}} F_{11} F_{12} \mathbf{T}_{+}^{\left(\frac{3}{2}\right)}\right.
$$




$$
\begin{aligned}
& +\frac{2}{(5+k)} F_{11} \mathbf{W}^{(\mathbf{2})}-\frac{8}{(5+k)^{2}} F_{11} F_{21} \mathbf{T}_{-}^{\left(\frac{3}{2}\right)}-\frac{4}{(5+k)^{2}} F_{11} F_{22} \mathbf{U}^{\left(\frac{3}{2}\right)}-\frac{2}{(5+k)} \partial F_{11} \mathbf{T}^{(\mathbf{1})} \\
& +\frac{1}{(5+k)} F_{11} \partial \mathbf{T}^{(\mathbf{1})}-\frac{2}{(5+k)} F_{12} \mathbf{U}_{+}^{(\mathbf{2})}+\frac{4}{(5+k)^{2}} F_{12} F_{21} \mathbf{U}^{\left(\frac{3}{2}\right)}+\frac{2}{(5+k)} U \mathbf{U}^{\left(\frac{3}{2}\right)} \\
& -\frac{2}{(5+k)} F_{21} \mathbf{U}_{-}^{(2)}+\frac{4}{3(5+k)} \partial \mathbf{U}^{\left(\frac{3}{2}\right)}+\frac{4}{(5+k)^{2}} F_{11} F_{21} G_{12} \\
& \text { - } \frac{i}{(5+k)} A_{3} G_{11}+\frac{4}{(5+k)^{2}} A_{3} B_{3} F_{11}+\frac{4}{(5+k)^{2}} B_{3} B_{3} F_{11}-\frac{8 i}{3(5+k)^{2}} \partial B_{3} F_{11} \\
& \text { - } \frac{8 i}{3(5+k)^{2}} \partial A_{3} F_{11}-\frac{4 i}{3(5+k)^{2}} A_{3} \partial F_{11}(z)-\frac{8 i}{(5+k)^{3}} A_{3} F_{11} F_{12} F_{21}+\frac{i}{(5+k)} A_{+} G_{21} \\
& +\frac{2}{(5+k)^{2}} A_{+} A_{3} F_{21}-\frac{2}{(5+k)^{2}} A_{+} A_{-} F_{11}+\frac{2}{(5+k)^{2}} A_{+} B_{3} F_{21}+\frac{2}{(5+k)^{2}} A_{+} B_{-} F_{22} \\
& +\frac{4 i}{(5+k)^{3}} A_{+} F_{11} F_{21} F_{22}-\frac{4 i}{3(5+k)^{2}} \partial A_{+} F_{21}-\frac{4 i}{3(5+k)^{2}} A_{+} \partial F_{21}-\frac{i}{(5+k)} B_{3} G_{11} \\
& \text { - } \frac{4 i}{3(5+k)^{2}} B_{3} \partial F_{11}-\frac{16 i}{(5+k)^{3}} B_{3} F_{11} F_{12} F_{21}-\frac{i}{(5+k)} B_{-} G_{12}+\frac{4}{(5+k)^{2}} B_{-} B_{3} F_{12} \\
& -\frac{8 i}{(5+k)^{3}} B_{-} F_{11} F_{12} F_{22}+\frac{8 i}{3(5+k)^{2}} \partial B_{-} F_{12}-\frac{4 i}{3(5+k)^{2}} B_{-} \partial F_{12}+\frac{4}{(5+k)^{2}} F_{11} F_{12} G_{21} \\
& -\frac{4}{3(5+k)^{3}} \partial F_{11} F_{12} F_{21}+\frac{8}{3(5+k)^{3}} F_{11} \partial F_{12} F_{21}-\frac{4}{3(5+k)^{3}} F_{11} F_{12} \partial F_{21} \\
& +\frac{1}{(5+k)} U G_{11}+\frac{2 i}{(5+k)^{2}} U A_{+} F_{21}-\frac{4 i}{(5+k)^{2}} U B_{3} F_{11} \\
& \text { - } \left.\frac{4}{3(5+k)^{2}} U \partial F_{11}-\frac{4}{3(5+k)^{2}} F_{11} G_{22} F_{11}-\frac{4}{(5+k)^{3}} F_{11} \partial F_{11} F_{22}\right](z) \\
& =\left[\mathbf{U}^{\left(\frac{5}{2}\right)}-\frac{2 i}{(5+k)} \hat{A}_{3} \mathbf{U}_{\text {non }}^{\left(\frac{3}{2}\right)}+\frac{2 i}{(5+k)} \hat{B}_{3} \mathbf{U}_{\text {non }}^{\left(\frac{3}{2}\right)}+\frac{2 i}{(5+k)} \hat{B}_{-} \mathbf{T}_{-, \text {non }}^{\left(\frac{3}{2}\right)}-\frac{2}{(5+k)} \partial F_{11} \mathbf{T}^{(\mathbf{1})}\right. \\
& +\frac{2}{(5+k)} F_{11} \mathbf{W}_{\text {non }}^{(2)}-\frac{4}{(5+k)^{2}} F_{11} F_{12} \mathbf{T}_{+, \text {non }}^{\left(\frac{3}{2}\right)}+\frac{4}{(5+k)^{2}} F_{11} F_{21} \mathbf{T}_{-, \text {non }}^{\left(\frac{3}{2}\right)}+\frac{2}{(5+k)} U \mathbf{U}_{\text {non }}^{\left(\frac{3}{2}\right)} \\
& +\frac{1}{(5+k)} F_{11} \partial \mathbf{T}^{(\mathbf{1})}-\frac{2}{(5+k)} F_{12} \mathbf{U}_{+, \text {non }}^{(2)}-\frac{4}{(5+k)^{2}} F_{12} F_{21} \mathbf{U}_{\text {non }}^{\left(\frac{3}{2}\right)}+\frac{4}{3(5+k)} \partial \mathbf{U}_{\text {non }}^{\left(\frac{3}{2}\right)} \\
& -\frac{2}{(5+k)} F_{21} \mathbf{U}_{-, \text {non }}^{(2)}-\frac{i}{(5+k)} \hat{B}_{3} \hat{G}_{11}-\frac{2}{(5+k)^{2}} \hat{B}_{3} \hat{B}_{3} F_{11}-\frac{2 i}{(5+k)^{2}} \partial \hat{B}_{3} F_{11} \\
& -\frac{i}{(5+k)} \hat{A}_{3} \hat{G}_{11}-\frac{2}{(5+k)^{2}} \hat{A}_{3} \hat{A}_{3} F_{11}-\frac{4}{(5+k)^{2}} \hat{A}_{3} \hat{B}_{3} F_{11}-\frac{4}{(5+k)^{2}} \hat{A}_{3} \hat{B}_{-} F_{12} \\
& -\frac{2 i}{(5+k)^{2}} \partial \hat{A}_{3} F_{11}+\frac{i}{(5+k)} \hat{A}_{+} \hat{G}_{21}-\frac{2}{(5+k)^{2}} \hat{A}_{+} \hat{A}_{-} F_{11}-\frac{4}{(5+k)^{2}} \hat{A}_{+} \hat{B}_{3} F_{21} \\
& -\frac{2}{(5+k)^{2}} \hat{B}_{+} \hat{B}_{-} F_{11}-\frac{i}{(5+k)} \hat{B}_{-} \hat{G}_{12}-\frac{2}{(5+k)^{2}} F_{11} F_{12} \hat{G}_{21}-\frac{2}{(5+k)} \hat{T} F_{11} \\
& \left.-\frac{2}{(5+k)^{2}} F_{11} F_{21} \hat{G}_{12}+\frac{4}{(5+k)^{2}} F_{12} F_{21} \hat{G}_{11}+\frac{1}{(5+k)} U \hat{G}_{11}\right](z),
\end{aligned}
$$




$$
\begin{aligned}
& \mathbf{V}_{\text {non }}^{\left(\frac{5}{2}\right)}(z)=\left[\mathbf{V}^{\left(\frac{5}{2}\right)}+\frac{2 i}{(5+k)} A_{3} \mathbf{V}^{\left(\frac{3}{2}\right)}+\frac{2 i}{(5+k)} A_{-} \mathbf{T}_{-}^{\left(\frac{3}{2}\right)}-\frac{2 i}{(5+k)} B_{3} \mathbf{V}^{\left(\frac{3}{2}\right)}+\frac{2}{(5+k)} F_{22} \mathbf{W}^{(2)}\right. \\
& -\frac{8}{(5+k)^{2}} F_{22} F_{21} \mathbf{T}_{-}^{\left(\frac{3}{2}\right)}-\frac{2}{(5+k)} U \mathbf{V}^{\left(\frac{3}{2}\right)}+\frac{4}{(5+k)^{2}} F_{22} F_{12} \mathbf{T}_{+}^{\left(\frac{3}{2}\right)}-\frac{4}{(5+k)^{2}} F_{22} F_{11} \mathbf{V}^{\left(\frac{3}{2}\right)} \\
& +\frac{2}{(5+k)} \partial F_{22} \mathbf{T}^{(\mathbf{1})}-\frac{1}{(5+k)} F_{22} \partial \mathbf{T}^{(\mathbf{1})}+\frac{2}{(5+k)} F_{21} \mathbf{V}_{-}^{(\mathbf{2})}+\frac{4}{3(5+k)} \partial \mathbf{V}^{\left(\frac{\mathbf{3}}{\mathbf{2}}\right)} \\
& -\frac{4}{(5+k)^{2}} F_{21} F_{12} \mathbf{V}^{\left(\frac{3}{2}\right)}+\frac{2}{(5+k)} F_{12} \mathbf{V}_{+}^{(2)} \\
& +\frac{i}{(5+k)} A_{3} G_{22}+\frac{4}{(5+k)^{2}} A_{3} A_{3} F_{22}+\frac{16 i}{(5+k)^{3}} A_{3} F_{22} F_{21} F_{12}-\frac{i}{(5+k)} A_{-} G_{12} \\
& +\frac{4}{(5+k)^{2}} A_{3} B_{3} F_{22}+\frac{2}{(5+k)^{2}} A_{3} B_{+} F_{21}-\frac{4 i}{3(5+k)^{2}} A_{3} \partial F_{22}+\frac{4}{(5+k)^{2}} A_{-} A_{3} F_{12} \\
& +\frac{2}{(5+k)^{2}} A_{-} B_{+} F_{11}-\frac{8 i}{(5+k)^{3}} A_{-} F_{22} F_{12} F_{11}+\frac{8 i}{3(5+k)^{2}} \partial A_{-} F_{12}-\frac{4 i}{3(5+k)^{2}} A_{-} \partial F_{12} \\
& +\frac{4 i}{(5+k)^{2}} \partial B_{3} F_{22}-\frac{4 i}{3(5+k)^{2}} B_{3} \partial F_{22}-\frac{2}{(5+k)^{2}} B_{-} B_{+} F_{22}+\frac{4}{(5+k)^{2}} F_{22} F_{21} G_{12} \\
& +\frac{8 i}{(5+k)^{3}} B_{3} F_{22} F_{21} F_{12}-\frac{i}{(5+k)} B_{+} G_{21}+\frac{2}{(5+k)^{2}} B_{+} B_{3} F_{21}+\frac{i}{(5+k)} B_{3} G_{22} \\
& +\frac{4 i}{(5+k)^{3}} B_{+} F_{22} F_{21} F_{11}-\frac{4 i}{3(5+k)^{2}} \partial B_{+} F_{21}-\frac{4 i}{3(5+k)^{2}} B_{+} \partial F_{21}+\frac{4}{3(5+k)^{2}} U \partial F_{22} \\
& +\frac{4}{3(5+k)^{3}} \partial F_{22} F_{21} F_{12}+\frac{4}{3(5+k)^{3}} F_{22} \partial F_{21} F_{12}-\frac{8}{3(5+k)^{3}} F_{22} F_{21} \partial F_{12} \\
& +\frac{1}{(5+k)} U G_{22}+\frac{4 i}{(5+k)^{2}} U A_{3} F_{22}-\frac{2 i}{(5+k)^{2}} U B_{+} F_{21}+\frac{8}{3(5+k)^{2}} \partial U F_{22} \\
& \left.-\frac{4}{3(5+k)^{2}} F_{22} G_{11} F_{22}-\frac{4}{(5+k)^{3}} F_{22} \partial F_{22} F_{11}\right](z) \\
& =\left[\mathbf{V}^{\left(\frac{\mathbf{5}}{\mathbf{2}}\right)}+\frac{2 i}{(5+k)} \hat{A}_{3} \mathbf{V}_{\text {non }}^{\left(\frac{\mathbf{3}}{2}\right)}+\frac{2 i}{(5+k)} \hat{A}_{-} \mathbf{T}_{-, \text {non }}^{\left(\frac{\mathbf{3}}{2}\right)}-\frac{2 i}{(5+k)} \hat{B}_{3} \mathbf{V}_{\text {non }}^{\left(\frac{\mathbf{3}}{2}\right)}+\frac{4}{3(5+k)} \partial \mathbf{V}_{\text {non }}^{\left(\frac{3}{2}\right)}\right. \\
& +\frac{2}{(5+k)} F_{22} \mathbf{W}_{\text {non }}^{(\mathbf{2})}+\frac{4}{(5+k)^{2}} F_{22} F_{21} \mathbf{T}_{-, \text {non }}^{\left(\frac{3}{2}\right)}-\frac{2}{(5+k)} U \mathbf{V}_{\text {non }}^{\left(\frac{3}{2}\right)}+\frac{2}{(5+k)} F_{12} \mathbf{V}_{+, \text {non }}^{(\mathbf{2})} \\
& -\frac{4}{(5+k)^{2}} F_{22} F_{12} \mathbf{T}_{+, \text {non }}^{\left(\frac{\mathbf{3}}{2}\right)}+\frac{2}{(5+k)} \partial F_{22} \mathbf{T}^{(\mathbf{1})}-\frac{1}{(5+k)} F_{22} \partial \mathbf{T}^{(\mathbf{1})}+\frac{2}{(5+k)} F_{21} \mathbf{V}_{-, \text {non }}^{(\mathbf{2})} \\
& +\frac{4}{(5+k)^{2}} F_{21} F_{12} \mathbf{V}_{\text {non }}^{\left(\frac{3}{2}\right)}-\frac{2}{(5+k)^{2}} \hat{B}_{3} \hat{B}_{3} F_{22}+\frac{2 i}{(5+k)^{2}} \partial \hat{B}_{3} F_{22}-\frac{i}{(5+k)} \hat{B}_{+} \hat{G}_{21} \\
& +\frac{i}{(5+k)} \hat{A}_{3} \hat{G}_{22}-\frac{2}{(5+k)^{2}} \hat{A}_{3} \hat{A}_{3} F_{22}-\frac{4}{(5+k)^{2}} \hat{A}_{3} \hat{B}_{3} F_{22}-\frac{2}{(5+k)^{2}} F_{22} F_{12} \hat{G}_{21} \\
& -\frac{4}{(5+k)^{2}} \hat{A}_{3} \hat{B}_{+} F_{21}+\frac{2 i}{(5+k)^{2}} \partial \hat{A}_{3} F_{22}-\frac{i}{(5+k)} \hat{A}_{-} \hat{G}_{12}-\frac{2}{(5+k)^{2}} F_{22} F_{21} \hat{G}_{12} \\
& -\frac{2}{(5+k)^{2}} \hat{A}_{-} \hat{A}_{+} F_{22}-\frac{4}{(5+k)^{3}} \hat{A}_{-} \hat{B}_{3} F_{12}+\frac{i}{(5+k)} \hat{B}_{3} \hat{G}_{22}-\frac{2}{(5+k)^{2}} \hat{B}_{-} \hat{B}_{+} F_{22}
\end{aligned}
$$




$$
\left.-\frac{2}{(5+k)^{2}} F_{21} F_{12} \hat{G}_{22}-\frac{2}{(5+k)} \hat{T} F_{22}+\frac{1}{(5+k)} U \hat{G}_{22}\right](z) .
$$

As before, the extra terms except the higher spin currents in the linear version are written in terms of composite fields living in the nonlinear version. Note the appearance of the nonlinear terms (containing the higher spin currents) between the higher spin currents and the spin- $\frac{1}{2}$ currents (the spin-1 currents).

For the remaining higher spin- $\frac{5}{2}$ currents, one has the following relations which obtained from the relation (3.8) and some results from Part I

$$
\begin{aligned}
& \mathbf{W}_{+, \text {non }}^{\left(\frac{5}{2}\right)}(z)=\left[\mathbf{W}_{+}^{\left(\frac{5}{2}\right)}-\frac{2 i}{(5+k)} A_{3} \mathbf{T}_{+}^{\left(\frac{3}{2}\right)}+\frac{2 i}{(5+k)} B_{3} \mathbf{T}_{+}^{\left(\frac{3}{2}\right)}+\frac{2}{(5+k)} F_{11} \mathbf{V}_{+}^{(\mathbf{2})}+\frac{2}{(5+k)} U \mathbf{T}_{+}^{\left(\frac{3}{2}\right)}\right. \\
& +\frac{4}{(5+k)^{2}} F_{11} F_{21} \mathbf{V}^{\left(\frac{3}{2}\right)}-\frac{8}{(5+k)^{2}} F_{11} F_{22} \mathbf{T}_{+}^{\left(\frac{3}{2}\right)}+\frac{2}{(5+k)} F_{21} \mathbf{T}^{(\mathbf{2})}+\frac{2}{(5+k)} F_{22} \mathbf{U}_{+}^{(\mathbf{2})} \\
& +\frac{4}{(5+k)^{2}} F_{21} F_{22} \mathbf{U}^{\left(\frac{3}{2}\right)}-\frac{2}{(5+k)} \partial F_{21} \mathbf{T}^{(\mathbf{1})}+\frac{1}{(5+k)} F_{21} \partial \mathbf{T}^{(\mathbf{1})} \\
& -\frac{3 i}{(5+k)} A_{3} G_{21}-\frac{4}{(5+k)^{2}} A_{3} A_{3} F_{21}+\frac{2}{(5+k)^{2}} B_{+} B_{-} F_{21}-\frac{2}{(5+k)^{2}} A_{3} B_{-} F_{22} \\
& -\frac{8 i}{(5+k)^{3}} A_{3} F_{11} F_{21} F_{22}-\frac{4 i}{3(5+k)^{2}} \partial A_{3} F_{21}+\frac{8 i}{3(5+k)^{2}} A_{3} \partial F_{21}+\frac{i}{(5+k)} A_{-} G_{11} \\
& +\frac{2}{(5+k)^{2}} A_{-} A_{3} F_{11}+\frac{2}{(5+k)^{2}} A_{-} B_{3} F_{11}+\frac{4 i}{3(5+k)^{2}} \partial A_{-} F_{11}-\frac{8 i}{3(5+k)^{2}} A_{-} \partial F_{11} \\
& -\frac{4 i}{(5+k)^{3}} A_{-} F_{11} F_{12} F_{21}-\frac{2}{(5+k)^{2}} A_{+} A_{-} F_{21}-\frac{i}{(5+k)} B_{3} G_{21}+\frac{4}{(5+k)^{2}} B_{3} B_{3} F_{21} \\
& -\frac{8 i}{(5+k)^{3}} B_{3} F_{11} F_{21} F_{22}+\frac{8 i}{3(5+k)^{2}} B_{-} \partial F_{22}+\frac{4 i}{3(5+k)^{2}} \partial B_{3} F_{21}-\frac{8 i}{3(5+k)^{2}} B_{3} \partial F_{21} \\
& +\frac{i}{(5+k)} B_{-} G_{22}-\frac{2}{(5+k)^{2}} B_{-} B_{3} F_{22}+\frac{4 i}{(5+k)^{3}} B_{-} F_{12} F_{21} F_{22}-\frac{4 i}{3(5+k)^{2}} \partial B_{-} F_{22} \\
& -\frac{4}{(5+k)^{2}} F_{11} F_{21} G_{22}+\frac{4}{3(5+k)^{3}} \partial F_{11} F_{21} F_{22}-\frac{4 i}{(5+k)^{2}} U A_{3} F_{21}+\frac{2 i}{(5+k)^{2}} U B_{-} F_{22} \\
& -\frac{8}{3(5+k)^{3}} F_{11} \partial F_{21} F_{22}+\frac{4}{3(5+k)^{3}} F_{11} F_{21} \partial F_{22}-\frac{4}{(5+k)^{2}} F_{11} F_{22} G_{21} \\
& -\frac{4}{(5+k)^{2}} F_{12} F_{21} G_{21}+\frac{4}{(5+k)^{2}} F_{21} F_{22} G_{11}-\frac{4}{3(5+k)} \partial G_{21}-\frac{4 i}{(5+k)^{2}} U B_{3} F_{21} \\
& \left.+\frac{2 i}{(5+k)^{2}} U A_{-} F_{11}-\frac{8}{3(5+k)^{2}} \partial U F_{21}-\frac{8}{3(5+k)^{2}} U \partial F_{21}+\frac{1}{(5+k)} U G_{21}\right](z) \\
& =\left[\mathbf{W}_{+}^{\left(\frac{5}{2}\right)}-\frac{2 i}{(5+k)} \hat{A}_{3} \mathbf{T}_{+, \text {non }}^{\left(\frac{3}{2}\right)}+\frac{2 i}{(5+k)} \hat{B}_{3} \mathbf{T}_{+, \text {non }}^{\left(\frac{3}{2}\right)}+\frac{2}{(5+k)} F_{11} \mathbf{V}_{+, \text {non }}^{(\mathbf{2})}\right. \\
& +\frac{1}{(5+k)} F_{21} \partial \mathbf{T}^{(\mathbf{1})}-\frac{4}{(5+k)^{2}} F_{11} F_{21} \mathbf{V}_{\text {non }}^{\left(\frac{3}{2}\right)}+\frac{4}{(5+k)^{2}} F_{11} F_{22} \mathbf{T}_{+, \text {non }}^{\left(\frac{3}{2}\right)}
\end{aligned}
$$




$$
\begin{aligned}
& +\frac{2}{(5+k)} F_{21} \mathbf{T}_{\text {non }}^{(\mathbf{2})}-\frac{2}{(5+k)} \partial F_{21} \mathbf{T}^{(\mathbf{1})}-\frac{4}{(5+k)^{2}} F_{21} F_{22} \mathbf{U}_{\mathbf{n o n}}^{\left(\frac{3}{2}\right)} \\
& +\frac{2}{(5+k)} F_{22} \mathbf{U}_{+, \text {non }}^{(2)}+\frac{2}{(5+k)} U \mathbf{T}_{+, \text {non }}^{\left(\frac{3}{2}\right)}+\frac{2}{(5+k)^{2}} F_{11} F_{21} \hat{G}_{22} \\
& -\frac{3 i}{(5+k)} \hat{A}_{3} \hat{G}_{21}-\frac{2}{(5+k)^{2}} \hat{A}_{3} \hat{A}_{3} F_{21}+\frac{4}{(5+k)^{2}} \hat{A}_{3} \hat{B}_{3} F_{21}+\frac{4}{(5+k)^{2}} \hat{A}_{3} \hat{B}_{-} F_{22} \\
& +\frac{i}{(5+k)} \hat{A}_{-} \hat{G}_{11}-\frac{4}{(5+k)^{2}} \hat{A}_{-} \hat{B}_{3} F_{11}-\frac{2}{(5+k)^{2}} \hat{A}_{+} \hat{A}_{-} F_{21}-\frac{i}{(5+k)} \hat{B}_{3} \hat{G}_{21} \\
& -\frac{2 i}{(5+k)^{2}} \partial \hat{A}_{3} F_{21}-\frac{2 i}{(5+k)^{2}} \partial \hat{B}_{3} F_{21}+\frac{i}{(5+k)} \hat{B}_{-} \hat{G}_{22}-\frac{2}{(5+k)^{2}} \hat{B}_{+} \hat{B}_{-} F_{21} \\
& +\frac{2}{(5+k)^{2}} F_{11} F_{22} \hat{G}_{21}-\frac{2}{(5+k)^{2}} F_{21} F_{22} \hat{G}_{11}-\frac{4}{3(5+k)} \partial \hat{G}_{21}-\frac{2}{(5+k)^{2}} \hat{B}_{3} \hat{B}_{3} F_{21} \\
& \left.+\frac{1}{(5+k)} U \hat{G}_{21}-\frac{2(k+3)}{(5+k)(7 k+3)} \hat{T} F_{21}\right](z) \\
& \mathbf{W}_{-, \text {non }}^{\left(\frac{5}{2}\right)}(z)=\left[\mathbf{W}_{-}^{\left(\frac{5}{2}\right)}-\frac{2 i}{(5+k)} A_{3} \mathbf{T}_{-}^{\left(\frac{3}{2}\right)}+\frac{2 i}{(5+k)} B_{3} \mathbf{T}_{-}^{\left(\frac{3}{2}\right)}-\frac{2}{(5+k)} F_{11} \mathbf{V}_{-}^{(\mathbf{2})}-\frac{2}{(5+k)} U \mathbf{T}_{-}^{\left(\frac{3}{2}\right)}\right. \\
& +\frac{4}{(5+k)^{2}} F_{11} F_{12} \mathbf{V}^{\left(\frac{3}{2}\right)}-\frac{8}{(5+k)^{2}} F_{11} F_{22} \mathbf{T}_{-}^{\left(\frac{3}{2}\right)}+\frac{2}{(5+k)} F_{12} \mathbf{T}^{(\mathbf{2})}-\frac{2}{(5+k)} F_{22} \mathbf{U}_{-}^{(\mathbf{2})} \\
& +\frac{4}{(5+k)^{2}} F_{12} F_{22} \mathbf{U}^{\left(\frac{3}{2}\right)}+\frac{2}{(5+k)} \partial F_{12} \mathbf{T}^{(\mathbf{1})}-\frac{1}{(5+k)} F_{12} \partial \mathbf{T}^{(\mathbf{1})} \\
& +\frac{3 i}{(5+k)} A_{3} G_{12}-\frac{4}{(5+k)^{2}} A_{3} A_{3} F_{12}-\frac{8 i}{(5+k)^{3}} B_{3} F_{11} F_{12} F_{22}-\frac{2}{(5+k)^{2}} A_{3} B_{+} F_{11} \\
& -\frac{8 i}{(5+k)^{3}} A_{3} F_{11} F_{12} F_{22}-\frac{8 i}{3(5+k)^{2}} \partial A_{3} F_{12}-\frac{8 i}{3(5+k)^{2}} A_{3} \partial F_{12}-\frac{i}{(5+k)} A_{+} G_{22} \\
& +\frac{2}{(5+k)^{2}} A_{+} A_{3} F_{22}-\frac{2}{(5+k)^{2}} A_{+} A_{-} F_{12}+\frac{2}{(5+k)^{2}} A_{+} B_{3} F_{22}-\frac{4 i}{(5+k)^{3}} A_{+} F_{12} F_{21} F_{22} \\
& -\frac{4 i}{3(5+k)^{2}} \partial A_{+} F_{22}+\frac{8 i}{3(5+k)^{2}} A_{+} \partial F_{22}+\frac{i}{(5+k)} B_{3} G_{12}+\frac{8 i}{3(5+k)^{2}} \partial B_{3} F_{12} \\
& +\frac{8 i}{3(5+k)^{2}} B_{3} \partial F_{12}-\frac{i}{(5+k)} B_{+} G_{11}-\frac{2}{(5+k)^{2}} B_{+} B_{3} F_{11}+\frac{2}{(5+k)^{2}} B_{+} B_{-} F_{12} \\
& +\frac{4 i}{3(5+k)^{2}} \partial B_{+} F_{11}-\frac{8 i}{3(5+k)^{2}} B_{+} \partial F_{11}+\frac{4 i}{(5+k)^{3}} B_{+} F_{11} F_{12} F_{21}+\frac{4}{(5+k)^{2}} B_{3} B_{3} F_{12} \\
& -\frac{4}{(5+k)^{2}} F_{11} F_{12} G_{22}-\frac{4}{3(5+k)^{3}} \partial F_{11} F_{12} F_{22}+\frac{4 i}{(5+k)^{2}} U A_{3} F_{12}-\frac{2 i}{(5+k)^{2}} U B_{+} F_{11} \\
& +\frac{8}{3(5+k)^{3}} F_{11} \partial F_{12} F_{22}-\frac{4}{3(5+k)^{3}} F_{11} F_{12} \partial F_{22}+\frac{4}{(5+k)^{2}} F_{11} F_{22} G_{12} \\
& +\frac{4}{(5+k)^{2}} F_{12} F_{21} G_{12}+\frac{4}{(5+k)^{2}} F_{12} F_{22} G_{11}-\frac{4}{3(5+k)} \partial G_{12}+\frac{4 i}{(5+k)^{2}} U B_{3} F_{12} \\
& \left.-\frac{2 i}{(5+k)^{2}} U A_{+} F_{22}-\frac{8}{3(5+k)^{2}} \partial U F_{12}-\frac{8}{3(5+k)^{2}} U \partial F_{12}+\frac{1}{(5+k)} U G_{12}\right](z)
\end{aligned}
$$




$$
\begin{aligned}
& =\left[\mathbf{W}_{-}^{\left(\frac{5}{2}\right)}-\frac{2 i}{(5+k)} A_{3} \mathbf{T}_{-, \text {non }}^{\left(\frac{3}{2}\right)}+\frac{2 i}{(5+k)} B_{3} \mathbf{T}_{-, \text {non }}^{\left(\frac{3}{2}\right)}-\frac{2}{(5+k)} F_{11} \mathbf{V}_{-, \text {,non }}^{(\mathbf{2})}\right. \\
& -\frac{4}{(5+k)^{2}} F_{11} F_{12} \mathbf{V}_{\mathbf{n o n}}^{\left(\frac{3}{2}\right)}+\frac{4}{(5+k)^{2}} F_{11} F_{22} \mathbf{T}_{-, \text {,non }}^{\left(\frac{3}{2}\right)}+\frac{2}{(5+k)} F_{12} \mathbf{T}_{\mathbf{n o n}}^{(\mathbf{2})} \\
& +\frac{2}{(5+k)} \partial F_{12} \mathbf{T}^{(\mathbf{1})}-\frac{4}{(5+k)^{2}} F_{12} F_{22} \mathbf{U}_{\mathbf{n o n}}^{\left(\frac{3}{2}\right)}-\frac{2}{(5+k)} F_{22} \mathbf{U}_{-, \text {non }}^{(\mathbf{2})} \\
& -\frac{2}{(5+k)} U \mathbf{T}_{-, \text {non }}^{\left(\frac{3}{2}\right)}-\frac{1}{(5+k)} F_{12} \partial \mathbf{T}^{(\mathbf{1})}+\frac{2}{(5+k)^{2}} F_{11} F_{12} \hat{G}_{22} \\
& +\frac{3 i}{(5+k)} \hat{A}_{3} \hat{G}_{12}-\frac{2}{(5+k)^{2}} \hat{A}_{3} \hat{A}_{3} F_{12}+\frac{2}{(5+k)^{2}} \hat{A}_{3} \hat{B}_{3} F_{12}-\frac{2 i}{(5+k)^{2}} \partial \hat{A}_{3} F_{12} \\
& -\frac{i}{(5+k)} \hat{A}_{+} \hat{G}_{22}-\frac{2}{(5+k)^{2}} \hat{A}_{+} \hat{A}_{-} F_{12}-\frac{2}{(5+k)^{2}} \hat{A}_{+} \hat{B}_{3} F_{22}+\frac{4}{(5+k)^{2}} \hat{A}_{3} \hat{B}_{+} F_{11} \\
& +\frac{i}{(5+k)} \hat{B}_{3} \hat{G}_{12}+\frac{4 i}{(5+k)^{3}} \hat{B}_{3} F_{11} F_{12} F_{22}-\frac{2 i}{(5+k)^{2}} \partial \hat{B}_{3} F_{12}-\frac{i}{(5+k)} \hat{B}_{+} \hat{G}_{11} \\
& -\frac{2}{(5+k)^{2}} \hat{B}_{+} \hat{B}_{3} F_{11}-\frac{2}{(5+k)^{2}} \hat{B}_{+} \hat{B}_{-} F_{12}+\frac{2 i}{(5+k)^{2}} \partial \hat{B}_{+} F_{11} \\
& -\frac{2}{(5+k)^{2}} F_{11} F_{22} \hat{G}_{12}-\frac{2}{(5+k)^{2}} F_{12} F_{22} \hat{G}_{11}-\frac{4}{3(5+k)} \partial \hat{G}_{12} \\
& \left.+\frac{1}{(5+k)} U \hat{G}_{12}-\frac{2(k+3)}{(k+5)(7 k+3)} \hat{T} F_{12}+\frac{2 i}{(5+k)^{2}} U \hat{B}_{3} F_{12}\right](z) .
\end{aligned}
$$

The nonlinear terms between the higher spin currents and the spin- $\frac{1}{2}$ currents (and the spin1 currents) are present. Due to these nonlinear terms, some of the nonlinear terms in the nonlinear version will disappear in the context of Appendix $F$.

Similarly, the final higher spin-3 current, from (3.9) and the corresponding equation in Part I has the following relation

$$
\begin{aligned}
\mathbf{W}_{\text {non }}^{(\mathbf{3})}(z) & =\left[\mathbf{W}^{(\mathbf{3})}-\frac{4 i}{(5+k)} A_{3} \mathbf{T}^{(\mathbf{2})}+\frac{8 i}{(5+k)^{2}} A_{3} F_{11} \mathbf{V}^{\left(\frac{3}{2}\right)}-\frac{8 i}{(5+k)^{2}} A_{3} F_{22} \mathbf{U}^{\left(\frac{3}{2}\right)}\right. \\
& -\frac{i}{(5+k)} \partial A_{3} \mathbf{T}^{(\mathbf{1})}+\frac{i}{(5+k)} A_{3} \partial \mathbf{T}^{(\mathbf{1})}-\frac{2 i}{(5+k)} A_{-} \mathbf{U}_{-}^{(\mathbf{2})}+\frac{4 i}{(5+k)^{2}} A_{-} F_{11} \mathbf{T}_{-}^{\left(\frac{3}{2}\right)} \\
& -\frac{4 i}{(5+k)^{2}} A_{-} F_{12} \mathbf{U}^{\left(\frac{3}{2}\right)}+\frac{4 i}{(5+k)} B_{3} \mathbf{T}^{(\mathbf{2})}-\frac{8 i}{(5+k)^{2}} B_{3} F_{11} \mathbf{V}^{\left(\frac{3}{2}\right)}+\frac{8 i}{(5+k)^{2}} B_{3} F_{22} \mathbf{U}^{\left(\frac{3}{2}\right)} \\
& +\frac{i}{(5+k)} \partial B_{3} \mathbf{T}^{(\mathbf{1})}-\frac{i}{(5+k)} B_{3} \partial \mathbf{T}^{(\mathbf{1})}+\frac{2 i}{(5+k)} B_{-} \mathbf{V}_{-}^{(\mathbf{2})}+\frac{4 i}{(5+k)^{2}} B_{-} F_{22} \mathbf{T}_{-}^{\left(\frac{3}{2}\right)} \\
& -\frac{4 i}{(5+k)^{2}} B_{-} F_{12} \mathbf{V}^{\left(\frac{3}{2}\right)}-\frac{8}{(5+k)^{3}} F_{11} F_{12} F_{21} \mathbf{V}^{\left(\frac{3}{2}\right)}-\frac{4}{(5+k)^{2}} F_{11} F_{21} \mathbf{V}_{-}^{(\mathbf{2})} \\
& -\frac{8}{(5+k)^{2}} F_{11} F_{22} \mathbf{T}^{(\mathbf{2})}-\frac{2\left(-41+31 k+4 k^{2}\right)}{(5+k)^{2}(19+23 k)} \partial F_{11} F_{22} \mathbf{T}^{(\mathbf{1})}-\frac{2}{(5+k)} U \partial \mathbf{T}^{(\mathbf{1})} \\
& +\frac{2\left(-79-15 k+4 k^{2}\right)}{(5+k)^{2}(19+23 k)} F_{11} \partial F_{22} \mathbf{T}^{(\mathbf{1})}+\frac{8(-3+k)}{(5+k)(19+23 k)} F_{12} \partial F_{21} \mathbf{T}^{(\mathbf{1})}
\end{aligned}
$$




$$
\begin{aligned}
& +\frac{2}{(5+k)^{2}} F_{11} F_{22} \partial \mathbf{T}^{(\mathbf{1})}-\frac{6}{(5+k)} \partial F_{11} \mathbf{V}^{\left(\frac{3}{2}\right)}+\frac{2}{(5+k)} F_{11} \partial \mathbf{V}^{\left(\frac{3}{2}\right)}+\frac{2}{(5+k)} \partial U \mathbf{T}^{(\mathbf{1})} \\
& -\frac{8}{(5+k)^{3}} F_{12} F_{21} F_{22} \mathbf{U}^{\left(\frac{3}{2}\right)}-\frac{8(-3+k)}{(5+k)(19+23 k)} \partial F_{12} F_{21} \mathbf{T}^{(\mathbf{1})}-\frac{2(14+3 k)}{(5+k)^{2}} \partial F_{12} \mathbf{T}_{+}^{\left(\frac{3}{2}\right)} \\
& +\frac{2(6+k)}{(5+k)^{2}} F_{12} \partial \mathbf{T}_{+}^{\left(\frac{3}{2}\right)}-\frac{4}{(5+k)^{2}} F_{21} F_{22} \mathbf{U}_{-}^{(2)}-\frac{12(-3+k)(5+k)}{(17+13 k)(19+23 k)} T \mathbf{T}^{(\mathbf{1})} \\
& -\frac{2(16+3 k)}{(5+k)^{2}} \partial F_{21} \mathbf{T}_{-}^{\left(\frac{3}{2}\right)}+\frac{2(4+k)}{(5+k)^{2}} F_{21} \partial \mathbf{T}_{-}^{\left(\frac{3}{2}\right)}-\frac{6}{(5+k)} \partial F_{22} \mathbf{U}^{\left(\frac{3}{2}\right)} \\
& +\frac{2}{(5+k)} F_{22} \partial \mathbf{U}^{\left(\frac{3}{2}\right)}+\frac{1}{(5+k)} \partial \mathbf{W}^{(\mathbf{2})}+\frac{6(-3+k)(5+k)}{(17+13 k)(19+23 k)} \partial^{2} \mathbf{T}^{(\mathbf{1})} \\
& -\frac{8(-3+k)}{(5+k)(19+23 k)} U U \mathbf{T}^{(\mathbf{1})} \\
& +\frac{4 i}{(5+k)^{2}} A_{3} A_{3} A_{3}-\frac{4 i}{(5+k)^{2}} A_{3} A_{3} B_{3}-\frac{10 i}{(5+k)^{3}} \partial A_{-} F_{11} F_{12}+\frac{6 i}{(5+k)^{3}} A_{-} \partial F_{11} F_{12} \\
& -\frac{4}{(5+k)^{2}} \partial A_{3} A_{3}+\frac{16}{(5+k)^{3}} A_{3} A_{3} F_{11} F_{22}-\frac{4 i}{(5+k)^{2}} A_{3} B_{3} B_{3}-\frac{2(k-3)}{(5+k)^{2}} \partial A_{3} B_{3} \\
& +\frac{2(-3+k)}{(5+k)^{2}} A_{3} \partial B_{3}-\frac{8}{(5+k)^{3}} A_{3} B_{-} F_{12} F_{22}-\frac{8}{(5+k)^{3}} A_{3} B_{+} F_{11} F_{21} \\
& -\frac{\left(-3-85 k+10 k^{2}\right)}{(5+k)^{2}(19+23 k)} i \partial^{2} A_{3}-\frac{4 i}{(5+k)^{2}} A_{3} F_{11} G_{22}+\frac{16 i}{(5+k)^{4}} A_{3} F_{11} F_{12} F_{21} F_{22} \\
& +\frac{24 i}{(5+k)^{3}} \partial A_{3} F_{11} F_{22}-\frac{4 i}{(5+k)^{2}} A_{3} F_{12} G_{21}-\frac{2 i\left(-225-172 k+13 k^{2}\right)}{(5+k)^{3}(19+23 k)} A_{3} \partial F_{12} F_{21} \\
& +\frac{2 i\left(263+256 k+33 k^{2}\right)}{(5+k)^{3}(19+23 k)} A_{3} \partial F_{11} F_{22}+\frac{2 i\left(-377-356 k+13 k^{2}\right)}{(5+k)^{3}(19+23 k)} A_{3} F_{11} \partial F_{22} \\
& -\frac{6 i\left(37+24 k+11 k^{2}\right)}{(5+k)^{3}(19+23 k)} A_{3} F_{12} \partial F_{21}-\frac{4 i}{(5+k)^{2}} A_{3} F_{21} G_{12}+\frac{8}{(5+k)^{3}} A_{-} B_{3} F_{11} F_{12} \\
& +\frac{2 i}{(5+k)^{2}} A_{-} F_{11} G_{12}-\frac{4 i}{(5+k)^{2}} A_{3} F_{22} G_{11}-\frac{2 i}{(5+k)^{3}} A_{-} F_{11} \partial F_{12} \\
& -\frac{2 i}{(5+k)^{2}} A_{-} F_{12} G_{11}+\frac{4}{(5+k)^{3}} A_{+} A_{-} F_{12} F_{21}-\frac{1}{(5+k)^{2}} \partial A_{+} A_{-}+\frac{1}{(5+k)^{2}} A_{+} \partial A_{-} \\
& +\frac{12}{(5+k)^{3}} A_{+} A_{-} F_{11} F_{22}+\frac{4 i}{(5+k)^{2}} B_{3} F_{11} G_{22}-\frac{16 i}{(5+k)^{4}} B_{3} F_{11} F_{12} F_{21} F_{22} \\
& +\frac{10 i}{(5+k)^{3}} \partial A_{+} F_{21} F_{22}+\frac{2 i}{(5+k)^{3}} A_{+} \partial F_{21} F_{22}-\frac{6 i}{(5+k)^{3}} A_{+} F_{21} \partial F_{22}+\frac{4 i}{(5+k)^{2}} A_{+} A_{-} A_{3} \\
& +\frac{4 i}{(5+k)^{2}} A_{+} F_{22} G_{21}+\frac{4 i}{(5+k)^{2}} B_{3} B_{3} B_{3}+\frac{8}{(5+k)^{3}} A_{+} B_{3} F_{21} F_{22}+\frac{4 i}{(5+k)^{2}} B_{3} F_{21} G_{12} \\
& -\frac{4}{(5+k)^{2}} \partial B_{3} B_{3}-\frac{16}{(5+k)^{3}} B_{3} B_{3} F_{11} F_{22}+\frac{i\left(-456+29 k+97 k^{2}\right)}{3(5+k)^{2}(19+23 k)} \partial^{2} B_{3} \\
& +\frac{2 i\left(323+248 k+29 k^{2}\right)}{(5+k)^{3}(19+23 k)} B_{3} \partial F_{11} F_{22}+\frac{2 i\left(-437-348 k+17 k^{2}\right)}{(5+k)^{3}(19+23 k)} B_{3} F_{11} \partial F_{22}
\end{aligned}
$$




$$
\begin{aligned}
& +\frac{4 i}{(5+k)^{2}} B_{3} F_{12} G_{21}+\frac{8 i}{(5+k)^{3}} \partial B_{3} F_{12} F_{21}+\frac{4 i}{(5+k)^{2}} B_{3} F_{22} G_{11}-\frac{2 i k}{(5+k)^{3}} B_{-} F_{12} \partial F_{22} \\
& +\frac{2 i\left(-285-164 k+17 k^{2}\right)}{(5+k)^{3}(19+23 k)} B_{3} F_{12} \partial F_{21}+\frac{2 i\left(171+64 k+29 k^{2}\right)}{(5+k)^{3}(19+23 k)} B_{3} \partial F_{12} F_{21} \\
& +\frac{2 i}{(5+k)^{2}} B_{-} F_{12} G_{22}+\frac{2 i(2+k)}{(5+k)^{3}} \partial B_{-} F_{12} F_{22}-\frac{2 i(-4+k)}{(5+k)^{3}} B_{-} \partial F_{12} F_{22} \\
& -\frac{6 i}{(5+k)^{2}} B_{-} F_{22} G_{12}+\frac{4 i}{(5+k)^{2}} B_{+} B_{-} B_{3}+\frac{4 i}{(5+k)^{2}} B_{3} F_{22} G_{11}-\frac{(-2+k)}{(5+k)^{2}} \partial B_{+} B_{-} \\
& +\frac{(-2+k)}{(5+k)^{2}} B_{+} \partial B_{-}-\frac{12}{(5+k)^{3}} B_{+} B_{-} F_{11} F_{22}+\frac{(-3+k)(-71+5 k)}{3(5+k)^{3}(19+23 k)} F_{11} \partial^{2} F_{22} \\
& +\frac{4}{(5+k)^{3}} B_{+} B_{-} F_{12} F_{21}-\frac{4 i}{(5+k)^{2}} B_{+} F_{11} G_{21}-\frac{2 i(2+k)}{(5+k)^{3}} \partial B_{+} F_{11} F_{21} \\
& +\frac{2 i k}{(5+k)^{3}} B_{+} \partial F_{11} F_{21}+\frac{2 i(-4+k)}{(5+k)^{3}} B_{+} F_{11} \partial F_{21}+\frac{(6+k)}{(5+k)^{2}} F_{12} \partial G_{21} \\
& +\frac{24\left(33+26 k+9 k^{2}\right)}{(5+k)^{4}(19+23 k)} \partial F_{11} F_{12} F_{21} F_{22}+\frac{4}{(5+k)^{3}} F_{11} F_{12} F_{21} G_{22}-\frac{4}{(5+k)^{3}} F_{12} F_{21} F_{22} G_{11} \\
& -\frac{16(-3+k)(7+11 k)}{(5+k)^{4}(19+23 k)} F_{11} \partial F_{12} F_{21} F_{22}-\frac{8(-3+k)}{(5+k)^{3}(19+23 k)} F_{11} F_{12} \partial F_{21} F_{22} \\
& -\frac{32\left(39+32 k+k^{2}\right)}{(5+k)^{4}(19+23 k)} F_{11} F_{12} F_{21} \partial F_{22}+\frac{16}{(5+k)^{3}} F_{11} F_{12} F_{22} G_{21}+\frac{16}{(5+k)^{3}} F_{11} F_{21} F_{22} G_{12} \\
& +\frac{(-3+k)(-71+5 k)}{3(5+k)^{3}(19+23 k)} \partial^{2} F_{11} F_{22}-\frac{16(-3+k)}{3(5+k)^{3}} \partial F_{11} \partial F_{22}-\frac{(11+3 k)}{(5+k)^{2}} \partial F_{22} G_{11} \\
& +\frac{(11+3 k)}{(5+k)^{2}} \partial F_{11} G_{22}-\frac{(1+k)}{(5+k)^{2}} F_{11} \partial G_{22}-\frac{(14+3 k)}{(5+k)^{2}} \partial F_{12} G_{21}+\frac{8 i}{(5+k)^{3}} U A_{-} F_{11} F_{12} \\
& -\frac{\left(1611+874 k+95 k^{2}\right)}{3(5+k)^{3}(19+23 k)} \partial^{2} F_{12} F_{21}+\frac{8(3+2 k)}{3(5+k)^{3}} \partial F_{12} \partial F_{21}-\frac{4 i}{(5+k)^{2}} U \partial A_{3} \\
& -\frac{\left(1611+874 k+95 k^{2}\right)}{3(5+k)^{3}(19+23 k)} F_{12} \partial^{2} F_{21}+\frac{(16+3 k)}{(5+k)^{2}} \partial F_{21} G_{12}-\frac{(4+k)}{(5+k)^{2}} F_{21} \partial G_{12} \\
& +\frac{(1+k)}{(5+k)^{2}} F_{22} \partial G_{11}-\frac{2}{(5+k)} G_{12} G_{21}+\frac{4 i(13+5 k)}{(5+k)(19+23 k)} T A_{3} \\
& +\frac{4 i(19+3 k)}{(5+k)(19+23 k)} T B_{3}+\frac{2}{(5+k)} \partial T+\frac{8(-3+k)}{(5+k)^{3}(19+23 k)} U U F_{11} F_{22} \\
& +\frac{8(-3+k)}{(5+k)^{2}(19+23 k)} T F_{11} F_{22}+\frac{32(4+k)}{(5+k)^{2}(19+23 k)} T F_{12} F_{21}+\frac{4 i}{(5+k)^{2}} \partial U A_{3} \\
& -\frac{8 i}{(5+k)^{3}} U A_{+} F_{21} F_{22}-\frac{4 i}{(5+k)^{2}} \partial U B_{3}+\frac{4 i}{(5+k)^{2}} U \partial B_{3}+\frac{8 i}{(5+k)^{3}} U B_{-} F_{12} F_{22} \\
& -\frac{8 i}{(5+k)^{3}} U B_{+} F_{11} F_{21}-\frac{4}{(5+k)^{2}} U F_{12} G_{21}+\frac{4}{(5+k)^{2}} U F_{21} G_{12}+\frac{(-1+k)}{(5+k)^{3}} F_{21} G_{12} F_{21} F_{12}
\end{aligned}
$$




$$
\begin{aligned}
& +\frac{4 i(13+5 k)}{(5+k)^{2}(19+23 k)} U U A_{3}+\frac{4 i(19+3 k)}{(5+k)^{2}(19+23 k)} U U B_{3}-\frac{8(3+19 k)}{(5+k)^{3}(19+23 k)} U U F_{12} F_{21} \\
& \left.-\frac{(-5+k)}{(5+k)^{3}} F_{22} G_{11} F_{22} F_{11}-\frac{(11+k)}{(5+k)^{3}} F_{11} G_{22} F_{11} F_{22}+\frac{(-1+k)}{(5+k)^{3}} F_{12} G_{21} F_{12} F_{21}\right](z) \\
& =\left[\mathbf{W}^{(\mathbf{3})}-\frac{4 i}{(5+k)} A_{3} \mathbf{T}_{\mathbf{n o n}}^{(\mathbf{2})}-\frac{i}{(5+k)} \partial A_{3} \mathbf{T}^{(\mathbf{1})}+\frac{i}{(5+k)} A_{3} \partial \mathbf{T}^{(\mathbf{1})}+\frac{2}{(5+k)} F_{11} \partial \mathbf{V}_{\text {non }}^{\left(\frac{3}{2}\right)}\right. \\
& -\frac{2 i}{(5+k)} A_{-} \mathbf{U}_{-, \text {non }}^{(\mathbf{2})}+\frac{4 i}{(5+k)} B_{3} \mathbf{T}_{\mathbf{n o n}}^{(\mathbf{2})}+\frac{i}{(5+k)} \partial B_{3} \mathbf{T}^{(\mathbf{1})}-\frac{6}{(5+k)} \partial F_{11} \mathbf{V}_{\mathbf{n o n}}^{\left(\frac{\mathbf{3}}{2}\right)} \\
& -\frac{i}{(5+k)} B_{3} \partial \mathbf{T}^{(\mathbf{1})}+\frac{2 i}{(5+k)} B_{-} \mathbf{V}_{-, \text {non }}^{(\mathbf{2})}-\frac{4(k-3)}{(5+k)(17+13 k)} \partial F_{11} F_{22} \mathbf{T}^{(\mathbf{1})} \\
& +\frac{4(k-3)}{(5+k)(17+13 k)} F_{11} \partial F_{22} \mathbf{T}^{(\mathbf{1})}+\frac{4(-3+k)}{(5+k)(17+13 k)} F_{12} \partial F_{21} \mathbf{T}^{(\mathbf{1})}+\frac{2}{(5+k)} F_{12} \partial \mathbf{T}_{+, \text {non }}^{\left(\frac{\mathbf{3}}{2}\right)} \\
& -\frac{4(-3+k)}{(5+k)(17+13 k)} \partial F_{12} F_{21} \mathbf{T}^{(\mathbf{1})}-\frac{4(-3+k)}{(5+k)(17+13 k)} U U \mathbf{T}^{(\mathbf{1})}-\frac{6}{(5+k)} \partial F_{12} \mathbf{T}_{+, \text {non }}^{\left(\frac{\mathbf{3}}{2}\right)} \\
& -\frac{6}{(5+k)} \partial F_{21} \mathbf{T}_{-, \text {non }}^{\left(\frac{3}{2}\right)}+\frac{2}{(5+k)} F_{21} \partial \mathbf{T}_{-, \text {non }}^{\left(\frac{3}{2}\right)}-\frac{6}{(5+k)} \partial F_{22} \mathbf{U}_{\text {non }}^{\left(\frac{3}{2}\right)} \\
& +\frac{2}{(5+k)} F_{22} \partial \mathbf{U}_{\text {non }}^{\left(\frac{3}{2}\right)}+\frac{1}{(5+k)} \partial \mathbf{W}_{\text {non }}^{(\mathbf{2})}+\frac{6(-3+k)(5+k)}{(17+13 k)(19+23 k)} \partial^{2} \mathbf{T}^{(\mathbf{1})} \\
& -\frac{12(-3+k)(5+k)}{(17+13 k)(19+23 k)} \hat{T} \mathbf{T}^{(\mathbf{1})}+\frac{2}{(5+k)} \partial U \mathbf{T}^{(\mathbf{1})}-\frac{2}{(5+k)} U \partial \mathbf{T}^{(\mathbf{1})} \\
& +\frac{8 i}{(5+k)^{2}} \hat{A}_{3} \hat{A}_{3} \hat{A}_{3}-\frac{16 i}{(5+k)^{2}} \hat{A}_{3} \hat{A}_{3} \hat{B}_{3}-\frac{6}{(5+k)^{2}} \partial \hat{A}_{3} \hat{A}_{3}+\frac{8 i}{(5+k)^{2}} \hat{A}_{3} \hat{B}_{3} \hat{B}_{3} \\
& -\frac{2(k-6)}{(5+k)^{2}} \partial \hat{A}_{3} \hat{B}_{3}+\frac{2(-3+k)}{(5+k)^{2}} \hat{A}_{3} \partial \hat{B}_{3}-\frac{2 i\left(58 k^{3}+k^{2}+460 k+309\right)}{(k+5)^{2}(7 k+3)(23 k+19)} \partial^{2} \hat{A}_{3} \\
& -\frac{8 i}{(5+k)^{2}} \hat{A}_{+} \hat{A}_{-} \hat{B}_{3}+\frac{8 i}{(5+k)^{2}} \hat{A}_{+} \hat{A}_{-} \hat{A}_{3}+\frac{6}{(5+k)^{2}} \partial \hat{A}_{+} \hat{A}_{-}-\frac{3}{(5+k)} \partial F_{22} \hat{G}_{11} \\
& -\frac{4}{(5+k)^{2}} \hat{A}_{+} \partial \hat{A}_{-}-\frac{(-1+k)}{(5+k)^{2}} \partial \hat{B}_{+} \hat{B}_{-}+\frac{(1+k)}{(5+k)^{2}} \hat{B}_{+} \partial \hat{B}_{-}-\frac{1}{(5+k)} F_{21} \partial \hat{G}_{12} \\
& +\frac{2}{(5+k)^{2}} \partial \hat{B}_{3} \hat{B}_{3}+\frac{i\left(817 k^{3}+1229 k^{2}-729 k+171\right)}{3(k+5)^{2}(7 k+3)(23 k+19)} \partial^{2} \hat{B}_{3}+\frac{3}{(5+k)} \partial F_{21} \hat{G}_{12} \\
& +\frac{3}{(5+k)} \partial F_{11} \hat{G}_{22}-\frac{(1+k)}{(5+k)} F_{11} \partial \hat{G}_{22}-\frac{3}{(5+k)} \partial F_{12} \hat{G}_{21}+\frac{1}{(5+k)} F_{12} \partial \hat{G}_{21} \\
& +\frac{1}{(5+k)} F_{22} \partial \hat{G}_{11}-\frac{2}{(5+k)} \hat{G}_{12} \hat{G}_{21}+\frac{8 i\left(29 k^{2}+97 k+48\right)}{(k+5)(7 k+3)(23 k+19)} \hat{T} \hat{A}_{3} \\
& \left.-\frac{8 i(k-27) k}{(k+5)(7 k+3)(23 k+19)} \hat{T} \hat{B}_{3}+\frac{1}{(5+k)} \partial \hat{T}\right](z) \text {. }
\end{aligned}
$$

Now the higher spin currents in the linear version can be written in terms of those in the nonlinear version and this implies that using the previous results in Part I and Part II one can write 136 OPEs in the linear version (although one should rewrite all the fields written 
in the nonlinear version in the basis of linear version and make the mathematica file which defines the results of Part I and Part II inside Thielemans package).

Due to these nonlinear terms between the boldface higher spin currents and the currents from the large $\mathcal{N}=4$ linear superconformal algebra, some of the nonlinear terms in the nonlinear version will disappear in the context of Appendix $F$.

\section{Appendix C The complete OPEs between the 16 currents of large $\mathcal{N}=4$ linear superconformal algebra and the 16 lowest higher spin currents}

In this Appendix, one describes the complete OPEs between the 16 currents of large $\mathcal{N}=4$ linear superconformal algebra and the 16 lowest higher spin currents. Except the few cases, these are linear.

\section{Appendix C.1 The OPEs between the spin- $\frac{1}{2}$ currents and the 16 lowest higher spin currents}

One can perform the various OPEs between the four spin- $\frac{1}{2}$ currents found in Part I and 16 higher spin currents obtained in section 3 as follows:

$$
\begin{aligned}
& \left(\begin{array}{c}
F_{11} \\
F_{22}
\end{array}\right)(z) \mathbf{T}_{ \pm}^{\left(\frac{3}{2}\right)}(w)=\frac{1}{(z-w)} \frac{i}{2} B_{\mp}(w)+\cdots, \\
& \left(\begin{array}{l}
F_{11} \\
F_{22}
\end{array}\right)(z) \mathbf{T}_{\mp}^{\left(\frac{3}{2}\right)}(w)=-\frac{1}{(z-w)} \frac{i}{2} A_{ \pm}(w)+\cdots, \\
& \left(\begin{array}{c}
F_{11} \\
F_{22}
\end{array}\right)(z) \mathbf{T}^{(2)}(w)=\frac{1}{(z-w)}\left[\frac{1}{2}\left(\begin{array}{c}
G_{11} \\
G_{22}
\end{array}\right) \pm\left(\begin{array}{c}
\mathbf{U}^{\left(\frac{3}{2}\right)} \\
\mathbf{V}^{\left(\frac{3}{2}\right)}
\end{array}\right)\right](w)+\cdots, \\
& \left(\begin{array}{l}
F_{11} \\
F_{22}
\end{array}\right)(z)\left(\begin{array}{l}
\mathbf{V}^{\left(\frac{3}{2}\right)} \\
\mathbf{U}^{\left(\frac{3}{2}\right)}
\end{array}\right)(w)=\frac{1}{(z-w)} \frac{1}{2}\left[-i A_{3}-i B_{3} \pm U\right](w)+\cdots, \\
& \left(\begin{array}{c}
F_{11} \\
F_{22}
\end{array}\right)(z)\left(\begin{array}{c}
\mathbf{V}_{+}^{(2)} \\
\mathbf{U}_{-}^{(2)}
\end{array}\right)(w)=-\frac{1}{(z-w)}\left[ \pm \frac{1}{2}\left(\begin{array}{c}
G_{21} \\
G_{12}
\end{array}\right)+\mathbf{T}_{ \pm}^{\left(\frac{3}{2}\right)}\right](w)+\cdots, \\
& \left(\begin{array}{c}
F_{11} \\
F_{22}
\end{array}\right)(z)\left(\begin{array}{c}
\mathbf{V}_{-}^{(2)} \\
\mathbf{U}_{+}^{(2)}
\end{array}\right)(w)=\frac{1}{(z-w)}\left[\mp \frac{1}{2}\left(\begin{array}{c}
G_{12} \\
G_{21}
\end{array}\right)+\mathbf{T}_{\mp}^{\left(\frac{3}{2}\right)}\right](w)+\cdots, \\
& \left(\begin{array}{l}
F_{11} \\
F_{22}
\end{array}\right)(z)\left(\begin{array}{c}
\mathbf{V}^{\left(\frac{5}{2}\right)} \\
\mathbf{U}^{\left(\frac{5}{2}\right)}
\end{array}\right)(w)= \pm \frac{1}{(z-w)^{2}} \mathbf{T}^{(\mathbf{1})}(w)+\frac{1}{(z-w)}\left[\mp \frac{1}{2} \partial \mathbf{T}^{(\mathbf{1})}+\mathbf{W}^{(\mathbf{2})}\right](w)+\cdots, \\
& \left(\begin{array}{c}
F_{11} \\
F_{22}
\end{array}\right)(z) \mathbf{W}_{ \pm}^{\left(\frac{5}{2}\right)}(w)= \pm \frac{1}{(z-w)}\left(\begin{array}{c}
\mathbf{U}_{+}^{(2)} \\
\mathbf{V}_{-}^{(2)}
\end{array}\right)(w)+\cdots,
\end{aligned}
$$




$$
\begin{aligned}
& \left(\begin{array}{c}
F_{11} \\
F_{22}
\end{array}\right)(z) \mathbf{W}_{\mp}^{\left(\frac{5}{2}\right)}(w)=\mp \frac{1}{(z-w)}\left(\begin{array}{c}
\mathbf{U}_{-}^{(2)} \\
\mathbf{V}_{+}^{(2)}
\end{array}\right)(w)+\cdots \\
& \left(\begin{array}{l}
F_{11} \\
F_{22}
\end{array}\right)(z) \mathbf{W}^{(\mathbf{3})}(w)=\frac{1}{(z-w)^{2}}\left[\mp \frac{3}{2}\left(\begin{array}{c}
G_{11} \\
G_{22}
\end{array}\right)-3\left(\begin{array}{c}
\mathbf{U}^{\left(\frac{3}{2}\right)} \\
\mathbf{V}^{\left(\frac{3}{2}\right)}
\end{array}\right)-\frac{2(-\mathbf{3}+\mathbf{k})}{(17+13 k)} \mathbf{T}^{(\mathbf{1})}\left(\begin{array}{c}
F_{11} \\
F_{22}
\end{array}\right)\right](w) \\
& +\frac{1}{(z-w)}\left[-\frac{1}{3} \partial(\text { pole- } 2)+\frac{4(-\mathbf{3}+\mathbf{k})}{3(17+13 k)} \partial\left(\begin{array}{c}
F_{11} \\
F_{22}
\end{array}\right) \mathbf{T}^{(\mathbf{1})}\right. \\
& \left.-\frac{2(-\mathbf{3}+\mathbf{k})}{3(17+13 k)}\left(\begin{array}{c}
F_{11} \\
F_{22}
\end{array}\right) \partial \mathbf{T}^{(\mathbf{1})}\right](w)+\cdots \\
& \left(\begin{array}{l}
F_{12} \\
F_{21}
\end{array}\right)(z) \mathbf{T}_{ \pm}^{\left(\frac{3}{2}\right)}(w)=\frac{1}{(z-w)} \frac{1}{2}\left[i A_{3}-i B_{3} \mp U\right](w)+\cdots, \\
& \left(\begin{array}{l}
F_{12} \\
F_{21}
\end{array}\right)(z)\left(\begin{array}{c}
\mathbf{U}^{\left(\frac{3}{2}\right)} \\
\mathbf{V}^{\left(\frac{3}{2}\right)}
\end{array}\right)(w)=-\frac{1}{(z-w)} \frac{i}{2} A_{ \pm}(w)+\cdots, \\
& \left(\begin{array}{c}
F_{12} \\
F_{21}
\end{array}\right)(z)\left(\begin{array}{c}
\mathbf{U}_{+}^{(2)} \\
\mathbf{V}_{-}^{(\mathbf{2})}
\end{array}\right)(w)=-\frac{1}{(z-w)}\left[ \pm \frac{1}{2}\left(\begin{array}{c}
G_{11} \\
G_{22}
\end{array}\right)+\left(\begin{array}{c}
\mathbf{U}^{\left(\frac{3}{2}\right)} \\
\mathbf{V}^{\left(\frac{3}{2}\right)}
\end{array}\right)\right](w)+\cdots, \\
& \left(\begin{array}{l}
F_{12} \\
F_{21}
\end{array}\right)(z)\left(\begin{array}{c}
\mathbf{U}^{\left(\frac{5}{2}\right)} \\
\mathbf{V}^{\left(\frac{5}{2}\right)}
\end{array}\right)(w)=\mp \frac{1}{(z-w)}\left(\begin{array}{c}
\mathbf{U}_{-}^{(\mathbf{2})} \\
\mathbf{V}_{+}^{(\mathbf{2})}
\end{array}\right)(w)+\cdots, \\
& \left(\begin{array}{c}
F_{12} \\
F_{21}
\end{array}\right)(z)\left(\begin{array}{c}
\mathbf{V}^{\left(\frac{3}{2}\right)} \\
\mathbf{U}^{\left(\frac{3}{2}\right)}
\end{array}\right)(w)=-\frac{1}{(z-w)} \frac{i}{2} B_{ \pm}(w)+\cdots, \\
& \left(\begin{array}{l}
F_{12} \\
F_{21}
\end{array}\right)(z)\left(\begin{array}{c}
\mathbf{V}_{+}^{(\mathbf{2})} \\
\mathbf{U}_{-}^{(\mathbf{2})}
\end{array}\right)(w)=-\frac{1}{(z-w)}\left[ \pm \frac{1}{2}\left(\begin{array}{c}
G_{22} \\
G_{11}
\end{array}\right)-\left(\begin{array}{c}
\mathbf{V}^{\left(\frac{3}{2}\right)} \\
\mathbf{U}^{\left(\frac{3}{2}\right)}
\end{array}\right)\right](w)+\cdots, \\
& \left(\begin{array}{c}
F_{12} \\
F_{21}
\end{array}\right)(z)\left(\begin{array}{c}
\mathbf{V}^{\left(\frac{5}{2}\right)} \\
\mathbf{U}^{\left(\frac{5}{2}\right)}
\end{array}\right)(w)= \pm \frac{1}{(z-w)}\left(\begin{array}{c}
\mathbf{V}_{-}^{(2)} \\
\mathbf{U}_{+}^{(2)}
\end{array}\right)(w)+\cdots \\
& \left(\begin{array}{c}
F_{12} \\
F_{21}
\end{array}\right)(z) \mathbf{W}^{(2)}(w)=\frac{1}{(z-w)}\left[\frac{1}{2}\left(\begin{array}{c}
G_{12} \\
G_{21}
\end{array}\right) \mp \mathbf{T}_{\mp}^{\left(\frac{3}{2}\right)}\right](w)+\cdots, \\
& \left(\begin{array}{c}
F_{12} \\
F_{21}
\end{array}\right)(z) \mathbf{W}_{ \pm}^{\left(\frac{5}{2}\right)}(w)=\mp \frac{1}{(z-w)^{2}} \mathbf{T}^{(\mathbf{1})}(w)+\frac{1}{(z-w)}\left[ \pm \frac{1}{2} \partial \mathbf{T}^{(\mathbf{1})}+\mathbf{T}^{(2)}\right](w)+\cdots \\
& \left(\begin{array}{c}
F_{12} \\
F_{21}
\end{array}\right)(z) \mathbf{W}^{(\mathbf{3})}(w)=\frac{1}{(z-w)^{2}}\left[ \pm \frac{3}{2}\left(\begin{array}{c}
G_{12} \\
G_{21}
\end{array}\right)-3 \mathbf{T}_{\mp}^{\left(\frac{3}{2}\right)}-\frac{2(-\mathbf{3}+\mathbf{k})}{(17+13 k)} \mathbf{T}^{(\mathbf{1})}\left(\begin{array}{c}
F_{12} \\
F_{21}
\end{array}\right)\right](w) \\
& +\frac{1}{(z-w)}\left[-\frac{1}{3} \partial(\text { pole- } 2)+\frac{4(-\mathbf{3}+\mathbf{k})}{3(17+13 k)} \partial\left(\begin{array}{c}
F_{12} \\
F_{21}
\end{array}\right) \mathbf{T}^{(\mathbf{1})}\right. \\
& \left.-\frac{2(-\mathbf{3}+\mathbf{k})}{3(17+13 k)}\left(\begin{array}{c}
F_{12} \\
F_{21}
\end{array}\right) \partial \mathbf{T}^{(\mathbf{1})}\right](w)+\cdots .
\end{aligned}
$$

It is easy to see that the OPEs containing the higher spin-3 current (which is a primary field) do have the nonlinear terms in the right hand side. Again, by considering the quasi primary field containing the higher spin-1 current (that is (4.7)) one can remove these nonlinear terms with the relations in (2.4). For the corresponding OPEs in the nonlinear version, there are 
trivial OPEs because the OPEs between the higher spin currents and the spin- $\frac{1}{2}$ currents are regular by construction. One also checks that the above OPEs are equivalent to the corresponding OPEs in [9] via the field identifications in section 4.

\section{Appendix C.2 The OPEs between the spin-1 current and the 16 lowest higher spin currents}

Let us perform the various OPEs between the spin-1 current found in Part I and 16 higher spin currents obtained in section 3 as follows:

$$
\begin{aligned}
U(z) \mathbf{T}_{ \pm}^{\left(\frac{3}{2}\right)}(w) & =\mp \frac{1}{(z-w)^{2}} \frac{1}{2}\left(\begin{array}{c}
F_{21} \\
F_{12}
\end{array}\right)(w)+\cdots, \\
U(z)\left(\begin{array}{c}
\mathbf{U}^{\left(\frac{3}{2}\right)} \\
\mathbf{V}^{\left(\frac{3}{2}\right)}
\end{array}\right)(w) & =\mp \frac{1}{(z-w)^{2}} \frac{1}{2}\left(\begin{array}{c}
F_{11} \\
F_{22}
\end{array}\right)(w)+\cdots, \\
U(z)\left(\begin{array}{c}
\mathbf{U}^{\left(\frac{5}{2}\right)} \\
\mathbf{V}^{\left(\frac{5}{2}\right)}
\end{array}\right)(w) & =\frac{1}{(z-w)^{2}}\left[\frac{1}{2}\left(\begin{array}{c}
G_{11} \\
G_{22}
\end{array}\right) \pm\left(\begin{array}{c}
\mathbf{U}^{\left(\frac{3}{2}\right)} \\
\mathbf{V}^{\left(\frac{3}{2}\right)}
\end{array}\right)\right](w)+\cdots, \\
U(z)\left(\begin{array}{c}
\mathbf{W}_{+}^{\left(\frac{5}{2}\right)} \\
\mathbf{W}_{-}^{\left(\frac{5}{2}\right)}
\end{array}\right)(w) & =\frac{1}{(z-w)^{2}}\left[\frac{1}{2}\left(\begin{array}{c}
G_{21} \\
G_{12}
\end{array}\right) \pm \mathbf{T}_{ \pm}^{\left(\frac{3}{2}\right)}\right](w)+\cdots, \\
U(z) \mathbf{W}^{(3)}(w) & =\frac{1}{(z-w)^{3}} 2 \mathbf{T}^{(\mathbf{1})}(w) \\
& -\frac{1}{(z-w)^{2}}\left[\frac{1}{2} \partial\left(\text { pole-3) }+\frac{4(-\mathbf{3}+\mathbf{k})}{(17+13 k)} U \mathbf{T}^{(\mathbf{1})}\right](w)+\cdots\right.
\end{aligned}
$$

It is easy to see that the last OPE containing the higher spin-3 current has the nonlinear terms in the right hand side. This will disappear by using the quasi primary field in (4.7). For the corresponding OPEs in the nonlinear version, there are trivial OPEs because the OPEs between the higher spin currents and the spin-1 current are regular by construction. One also checks that the above OPEs are equivalent to the corresponding OPEs in [9] (or in Appendix $D$ ) via the field identifications in section 4.

\section{Appendix C.3 The OPEs between the spin-1 currents and the 16 lowest higher spin currents}

The OPEs between the three spin-1 currents found in Part I and the 16 higher spin currents obtained in section 3 are

$$
A_{ \pm}(z) \mathbf{T}_{ \pm}^{\left(\frac{3}{2}\right)}(w)=-\frac{1}{(z-w)^{2}} \frac{i(1+k)}{(5+k)}\left(\begin{array}{c}
F_{11} \\
F_{22}
\end{array}\right)(w) \mp \frac{1}{(z-w)} i\left(\begin{array}{c}
\mathbf{U}^{\left(\frac{3}{2}\right)} \\
\mathbf{V}^{\left(\frac{3}{2}\right)}
\end{array}\right)(w)+\cdots
$$




$$
\begin{aligned}
& A_{ \pm}(z) \mathbf{T}^{(\mathbf{2})}(w)=\frac{1}{(z-w)} i\left(\begin{array}{c}
\mathbf{U}_{-}^{(2)} \\
\mathbf{V}_{+}^{(2)}
\end{array}\right)(w)+\cdots, \\
& A_{ \pm}(z)\left(\begin{array}{c}
\mathbf{V}^{\left(\frac{3}{2}\right)} \\
\mathbf{U}^{\left(\frac{3}{2}\right)}
\end{array}\right)(w)=-\frac{1}{(z-w)^{2}} \frac{i(1+k)}{(5+k)}\left(\begin{array}{c}
F_{12} \\
F_{21}
\end{array}\right)(w) \pm \frac{1}{(z-w)} i \mathbf{T}_{\mp}^{\left(\frac{3}{2}\right)}(w)+\cdots \\
& A_{ \pm}(z)\left(\begin{array}{c}
\mathbf{V}_{+}^{(\mathbf{2})} \\
\mathbf{U}_{-}^{(\mathbf{2})}
\end{array}\right)(w)=\mp \frac{1}{(z-w)^{2}} i \mathbf{T}^{(\mathbf{1})}(w)+\frac{1}{(z-w)} i\left[\mathbf{T}^{(\mathbf{2})}-\mathbf{W}^{(\mathbf{2})}\right](w)+\cdots \\
& A_{ \pm}(z)\left(\begin{array}{c}
\mathbf{V}^{\left(\frac{5}{2}\right)} \\
\mathbf{U}^{\left(\frac{5}{2}\right)}
\end{array}\right)(w)=\frac{1}{(z-w)^{2}} \frac{i(21+5 k)}{3(5+k)}\left[2 \mathbf{T}_{\mp}^{\left(\frac{3}{2}\right)} \mp\left(\begin{array}{c}
G_{12} \\
G_{21}
\end{array}\right)\right](w) \\
& \pm \frac{1}{(z-w)} i \mathbf{W}_{\mp}^{\left(\frac{5}{2}\right)}(w)+\cdots \\
& A_{ \pm}(z) \mathbf{W}^{(2)}(w)=-\frac{1}{(z-w)} i\left(\begin{array}{c}
\mathbf{U}_{-}^{(2)} \\
\mathbf{V}_{+}^{(2)}
\end{array}\right)(w)+\cdots \\
& A_{ \pm}(z) \mathbf{W}_{ \pm}^{\left(\frac{5}{2}\right)}(w)=\frac{1}{(z-w)^{2}} \frac{i(21+5 k)}{3(5+k)}\left[2\left(\begin{array}{c}
\mathbf{U}^{\left(\frac{3}{2}\right)} \\
\mathbf{V}^{\left(\frac{3}{2}\right)}
\end{array}\right) \pm\left(\begin{array}{c}
G_{11} \\
G_{22}
\end{array}\right)\right](w) \\
& \mp \frac{1}{(z-w)} i\left(\begin{array}{c}
\mathbf{U}^{\left(\frac{5}{2}\right)} \\
\mathbf{V}^{\left(\frac{5}{2}\right)}
\end{array}\right)(w)+\cdots \\
& A_{ \pm}(z) \mathbf{W}^{(\mathbf{3})}(w)=\frac{1}{(z-w)^{2}}\left[\mp 4 i\left(\begin{array}{c}
\mathbf{U}_{-}^{(\mathbf{2})} \\
\mathbf{V}_{+}^{(\mathbf{2})}
\end{array}\right)-\frac{4(-\mathbf{3}+\mathbf{k})}{(17+13 k)} A_{ \pm} \mathbf{T}^{(\mathbf{1})}\right](w)+\cdots \\
& A_{3}(z) \mathbf{T}_{ \pm}^{\left(\frac{3}{2}\right)}(w)=\frac{1}{(z-w)^{2}} \frac{i(1+k)}{2(5+k)}\left(\begin{array}{c}
F_{21} \\
F_{12}
\end{array}\right)(w) \pm \frac{1}{(z-w)} \frac{i}{2} \mathbf{T}_{ \pm}^{\left(\frac{3}{2}\right)}(w)+\cdots, \\
& A_{3}(z)\left(\begin{array}{c}
\mathbf{T}^{(\mathbf{2})} \\
\mathbf{W}^{(2)}
\end{array}\right)(w)= \pm \frac{1}{(z-w)^{2}} \frac{i}{2} \mathbf{T}^{(\mathbf{1})}(w)+\cdots, \\
& A_{3}(z)\left(\begin{array}{c}
\mathbf{U}^{\left(\frac{3}{2}\right)} \\
\mathbf{V}^{\left(\frac{3}{2}\right)}
\end{array}\right)(w)=-\frac{1}{(z-w)^{2}} \frac{i(1+k)}{2(5+k)}\left(\begin{array}{c}
F_{11} \\
F_{22}
\end{array}\right)(w) \mp \frac{1}{(z-w)} \frac{i}{2}\left(\begin{array}{c}
\mathbf{U}^{\left(\frac{3}{2}\right)} \\
\mathbf{V}^{\left(\frac{3}{2}\right)}
\end{array}\right)(w)+\cdots \\
& A_{3}(z)\left(\begin{array}{c}
\mathbf{U}_{-}^{(\mathbf{2})} \\
\mathbf{V}_{+}^{(2)}
\end{array}\right)(w)=\mp \frac{1}{(z-w)} i\left(\begin{array}{c}
\mathbf{U}_{-}^{(2)} \\
\mathbf{V}_{+}^{(2)}
\end{array}\right)(w)+\cdots \\
& A_{3}(z)\left(\begin{array}{c}
\mathbf{U}^{\left(\frac{5}{2}\right)} \\
\mathbf{V}^{\left(\frac{5}{2}\right)}
\end{array}\right)(w)=\frac{1}{(z-w)^{2}} \frac{i(21+5 k)}{6(5+k)}\left[2\left(\begin{array}{c}
\mathbf{U}^{\left(\frac{3}{2}\right)} \\
\mathbf{V}^{\left(\frac{3}{2}\right)}
\end{array}\right) \pm\left(\begin{array}{c}
G_{11} \\
G_{22}
\end{array}\right)\right](w) \\
& \mp \frac{1}{(z-w)} \frac{i}{2}\left(\begin{array}{c}
\mathbf{U}^{\left(\frac{5}{2}\right)} \\
\mathbf{V}^{\left(\frac{5}{2}\right)}
\end{array}\right)(w)+\cdots, \\
& A_{3}(z) \mathbf{W}_{ \pm}^{\left(\frac{5}{2}\right)}(w)=-\frac{1}{(z-w)^{2}} \frac{i(21+5 k)}{6(5+k)}\left[2 \mathbf{T}_{ \pm}^{\left(\frac{3}{2}\right)} \pm\left(\begin{array}{c}
G_{21} \\
G_{12}
\end{array}\right)\right](w) \pm \frac{1}{(z-w)} \frac{i}{2} \mathbf{W}_{ \pm}^{\left(\frac{5}{2}\right)}(w)+\cdots, \\
& A_{3}(z) \mathbf{W}^{(\mathbf{3})}(w)=\frac{1}{(z-w)^{2}}\left[-2 i \mathbf{T}^{(\mathbf{2})}+2 i \mathbf{W}^{(\mathbf{2})}-\frac{4(-\mathbf{3}+\mathbf{k})}{(17+13 k)} A_{3} \mathbf{T}^{(\mathbf{1})}\right](w)+\cdots .
\end{aligned}
$$

It is easy to see that the OPEs containing the higher spin-3 current do have the nonlinear terms in the right hand side. This will be eliminated if one uses (4.7). One can also compare 
the expressions in the nonlinear version in Part I and the above expressions. Some of the OPEs in Part I were present but the corresponding OPEs in this paper do not appear. The nonlinear terms in Part I disappear in the above OPEs. It is easy to see that the above OPEs are equivalent to the corresponding OPEs in [9] via the field identifications in section 4 .

\section{Appendix C.4 The OPEs between the spin-1 currents and the 16 lowest higher spin currents}

The OPEs between the other three spin-1 currents found in Part I and the 16 higher spin currents obtained in section 3 are

$$
\begin{aligned}
& B_{ \pm}(z) \mathbf{T}_{ \pm}^{\left(\frac{3}{2}\right)}(w)=\frac{1}{(z-w)^{2}} \frac{4 i}{(5+k)}\left(\begin{array}{c}
F_{22} \\
F_{11}
\end{array}\right)(w)+\frac{1}{(z-w)} i\left(\begin{array}{c}
-\mathbf{V}^{\left(\frac{3}{2}\right)}+G_{22} \\
\mathbf{U}^{\left(\frac{3}{2}\right)}+G_{11}
\end{array}\right)(w)+\cdots, \\
& B_{ \pm}(z) \mathbf{T}^{(\mathbf{2})}(w)=\frac{1}{(z-w)} i\left(\begin{array}{c}
\mathbf{V}_{-}^{(2)} \\
\mathbf{U}_{+}^{(2)}
\end{array}\right)(w)+\cdots, \\
& B_{ \pm}(z)\left(\begin{array}{c}
\mathbf{U}^{\left(\frac{3}{2}\right)} \\
\mathbf{V}^{\left(\frac{3}{2}\right)}
\end{array}\right)(w)=-\frac{1}{(z-w)^{2}} \frac{4 i}{(5+k)}\left(\begin{array}{c}
F_{12} \\
F_{21}
\end{array}\right)(w) \\
& +\frac{1}{(z-w)} i\left[ \pm \mathbf{T}_{\mp}^{\left(\frac{3}{2}\right)}-\left(\begin{array}{c}
G_{12} \\
G_{21}
\end{array}\right)\right](w)+\cdots, \\
& B_{ \pm}(z)\left(\begin{array}{c}
\mathbf{U}_{+}^{(2)} \\
\mathbf{V}_{-}^{(\mathbf{2})}
\end{array}\right)(w)=\mp \frac{1}{(z-w)^{2}} i \mathbf{T}^{(\mathbf{1})}(w)+\frac{1}{(z-w)} i\left[\mathbf{T}^{(\mathbf{2})}+\mathbf{W}^{(\mathbf{2})}\right](w)+\cdots, \\
& B_{ \pm}(z)\left(\begin{array}{c}
\mathbf{U}^{\left(\frac{5}{2}\right)} \\
\mathbf{V}^{\left(\frac{5}{2}\right)}
\end{array}\right)(w)=\frac{1}{(z-w)^{2}} \frac{4 i(6+k)}{3(5+k)}\left[2 \mathbf{T}_{\mp}^{\left(\frac{3}{2}\right)} \mp\left(\begin{array}{c}
G_{12} \\
G_{21}
\end{array}\right)\right](w) \mp \frac{1}{(z-w)} i \mathbf{W}_{\mp}^{\left(\frac{5}{2}\right)}(w)+\cdots, \\
& B_{ \pm}(z) \mathbf{W}^{(\mathbf{2})}(w)=\frac{1}{(z-w)} i\left(\begin{array}{c}
\mathbf{V}_{-}^{(\mathbf{2})} \\
\mathbf{U}_{+}^{(\mathbf{2})}
\end{array}\right)(w)+\cdots, \\
& B_{ \pm}(z) \mathbf{W}_{ \pm}^{\left(\frac{5}{2}\right)}(w)=\frac{1}{(z-w)^{2}} \frac{4 i(6+k)}{3(5+k)}\left[-2\left(\begin{array}{c}
\mathbf{V}^{\left(\frac{3}{2}\right)} \\
\mathbf{U}^{\left(\frac{3}{2}\right)}
\end{array}\right) \pm\left(\begin{array}{c}
G_{22} \\
G_{11}
\end{array}\right)\right](w) \\
& \pm \frac{1}{(z-w)} i\left(\begin{array}{c}
\mathbf{V}^{\left(\frac{5}{2}\right)} \\
\mathbf{U}^{\left(\frac{5}{2}\right)}
\end{array}\right)(w)+\cdots \\
& B_{ \pm}(z) \mathbf{W}^{(\mathbf{3})}(w)=\frac{1}{(z-w)^{2}}\left[ \pm 4 i\left(\begin{array}{c}
\mathbf{V}_{-}^{(\mathbf{2})} \\
\mathbf{U}_{+}^{(\mathbf{2})}
\end{array}\right)-\frac{4(-\mathbf{3}+\mathbf{k})}{(17+13 k)} B_{ \pm} \mathbf{T}^{(\mathbf{1})}\right](w)+\cdots, \\
& B_{3}(z) \mathbf{T}_{ \pm}^{\left(\frac{3}{2}\right)}(w)=-\frac{1}{(z-w)^{2}} \frac{2 i}{(5+k)}\left(\begin{array}{c}
F_{21} \\
F_{12}
\end{array}\right)(w) \pm \frac{1}{(z-w)} \frac{i}{2} \mathbf{T}_{ \pm}^{\left(\frac{3}{2}\right)}(w)+\cdots, \\
& B_{3}(z)\left(\begin{array}{c}
\mathbf{T}^{(\mathbf{2})} \\
\mathbf{W}^{(\mathbf{2})}
\end{array}\right)(w)=\frac{1}{(z-w)^{2}} \frac{i}{2} \mathbf{T}^{(\mathbf{1})}(w)+\cdots, \\
& B_{3}(z)\left(\begin{array}{c}
\mathbf{U}^{\left(\frac{3}{2}\right)} \\
\mathbf{V}^{\left(\frac{3}{2}\right)}
\end{array}\right)(w)=-\frac{1}{(z-w)^{2}} \frac{2 i}{(5+k)}\left(\begin{array}{c}
F_{11} \\
F_{22}
\end{array}\right)(w) \pm \frac{1}{(z-w)} \frac{i}{2}\left(\begin{array}{c}
\mathbf{U}^{\left(\frac{3}{2}\right)} \\
\mathbf{V}^{\left(\frac{3}{2}\right)}
\end{array}\right)(w)+\cdots,
\end{aligned}
$$




$$
\begin{aligned}
B_{3}(z)\left(\begin{array}{c}
\mathbf{U}_{+}^{(2)} \\
\mathbf{V}_{-}^{(\mathbf{2})}
\end{array}\right)(w) & = \pm \frac{1}{(z-w)} i\left(\begin{array}{c}
\mathbf{U}_{+}^{(\mathbf{2})} \\
\mathbf{V}_{-}^{(\mathbf{2})}
\end{array}\right)(w)+\cdots \\
B_{3}(z)\left(\begin{array}{c}
\mathbf{U}^{\left(\frac{5}{2}\right)} \\
\mathbf{V}^{\left(\frac{5}{2}\right)}
\end{array}\right)(w) & =\frac{1}{(z-w)^{2}} \frac{2 i(6+k)}{3(5+k)}\left[2\left(\begin{array}{c}
\mathbf{U}^{\left(\frac{3}{2}\right)} \\
\mathbf{V}^{\left(\frac{\mathbf{3}}{2}\right)}
\end{array}\right) \pm\left(\begin{array}{c}
G_{11} \\
G_{22}
\end{array}\right)\right](w) \\
& \pm \frac{1}{(z-w)} \frac{i}{2}\left(\begin{array}{c}
\mathbf{U}^{\left(\frac{5}{2}\right)} \\
\mathbf{V}^{\left(\frac{5}{2}\right)}
\end{array}\right)(w)+\cdots \\
B_{3}(z) \mathbf{W}_{ \pm}^{\left(\frac{5}{2}\right)}(w) & =\frac{1}{(z-w)^{2}} \frac{2 i(6+k)}{3(5+k)}\left[2 \mathbf{T}_{ \pm}^{\left(\frac{3}{2}\right)} \pm\left(\begin{array}{c}
G_{21} \\
G_{12}
\end{array}\right)\right](w) \pm \frac{1}{(z-w)} \frac{i}{2} \mathbf{W}_{ \pm}^{\left(\frac{5}{2}\right)}(w)+\cdots \\
B_{3}(z) \mathbf{W}^{(3)}(w) & =\frac{1}{(z-w)^{2}}\left[2 i \mathbf{T}^{(\mathbf{2})}+2 i \mathbf{W}^{(\mathbf{2})}-\frac{4(-\mathbf{3}+\mathbf{k})}{(17+13 k)} B_{3} \mathbf{T}^{(\mathbf{1})}\right](w)+\cdots
\end{aligned}
$$

It is easy to see that the OPEs containing the higher spin-3 current do have the nonlinear terms in the right hand side. This can be removed if one uses (4.7). One can compare the expressions in the nonlinear version in Part I and the above expressions. Some of the OPEs in Part I were present but the corresponding OPEs in this paper do not appear. The nonlinear terms in Part I disappear in the above OPEs. Due to the symmetry in the structure constants one can present the above OPEs by combining separate OPEs together. One also checks that the above OPEs are equivalent to the corresponding OPEs in [9] (or in Appendix D) via the field identifications in section 4 .

\section{Appendix C.5 The OPEs between the spin- $\frac{3}{2}$ currents and the 16 lowest higher spin currents}

The OPEs between the four spin- $\frac{3}{2}$ currents found in Part I and the 16 higher spin currents obtained in section 3 are

$$
\begin{aligned}
\left(\begin{array}{l}
G_{11} \\
G_{22}
\end{array}\right)(z) \mathbf{T}^{(\mathbf{1})}(w) & =\frac{1}{(z-w)}\left[ \pm\left(\begin{array}{c}
G_{11} \\
G_{22}
\end{array}\right)+2\left(\begin{array}{c}
\mathbf{U}^{\left(\frac{3}{2}\right)} \\
\left.\mathbf{V}^{\left(\frac{3}{2}\right.}\right)
\end{array}\right)\right](w)+\cdots, \\
\left(\begin{array}{c}
G_{11} \\
G_{22}
\end{array}\right)(z) \mathbf{T}_{ \pm}^{\left(\frac{3}{2}\right)}(w) & =\frac{1}{(z-w)^{2}} \frac{2 i(1+k)}{(5+k)} B_{\mp}(w) \\
& +\frac{1}{(z-w)}\left[\frac{1}{2} \partial(\text { pole- } 2)-\left(\begin{array}{c}
\mathbf{U}_{+}^{(\mathbf{2})} \\
\mathbf{V}_{-}^{(\mathbf{2})}
\end{array}\right)\right](w)+\cdots, \\
\left(\begin{array}{c}
G_{11} \\
G_{22}
\end{array}\right)(z) \mathbf{T}_{\mp}^{\left(\frac{3}{2}\right)}(w) & =\frac{1}{(z-w)^{2}} \frac{8 i}{(5+k)} A_{ \pm}(w) \\
& +\frac{1}{(z-w)}\left[\frac{1}{2} \partial(\text { pole- } 2)-\left(\begin{array}{c}
\mathbf{U}_{-}^{(\mathbf{2})} \\
\mathbf{V}_{+}^{(\mathbf{2})}
\end{array}\right)\right](w)+\cdots, \\
\left(\begin{array}{c}
G_{11} \\
G_{22}
\end{array}\right)(z) \mathbf{T}^{(\mathbf{2})}(w) & =\frac{1}{(z-w)^{2}} \frac{(-3+k)}{2(5+k)}\left[\left(\begin{array}{c}
G_{11} \\
G_{22}
\end{array}\right) \pm 2\left(\begin{array}{c}
\mathbf{U}^{\left(\frac{3}{2}\right)} \\
\mathbf{V}^{\left(\frac{3}{2}\right)}
\end{array}\right)\right](w)
\end{aligned}
$$




$$
\begin{aligned}
& +\frac{1}{(z-w)}\left[\frac{1}{3} \partial(\text { pole- } 2)+\left(\begin{array}{c}
\mathbf{U}^{\left(\frac{5}{2}\right)} \\
\mathbf{V}^{\left(\frac{5}{2}\right)}
\end{array}\right)\right](w)+\cdots, \\
& \left(\begin{array}{l}
G_{11} \\
G_{22}
\end{array}\right)(z)\left(\begin{array}{l}
\mathbf{V}^{\left(\frac{3}{2}\right)} \\
\mathbf{U}^{\left(\frac{3}{2}\right)}
\end{array}\right)(w)= \pm \frac{1}{(z-w)^{3}} \frac{8(1+k)}{(5+k)} \\
& +\frac{1}{(z-w)^{2}}\left[\frac{8 i}{(5+k)} A_{3}-\frac{2 i(1+k)}{(5+k)} B_{3}+\mathbf{T}^{(\mathbf{1})}\right](w) \\
& +\frac{1}{(z-w)}\left[\frac{1}{2} \partial(\text { pole- } 2) \pm \mathbf{W}^{(2)} \pm T\right](w)+\cdots, \\
& \left(\begin{array}{l}
G_{11} \\
G_{22}
\end{array}\right)(z)\left(\begin{array}{c}
\mathbf{V}_{+}^{(2)} \\
\mathbf{U}_{-}^{(2)}
\end{array}\right)(w)=-\frac{1}{(z-w)^{2}} \frac{2(3+k)}{(5+k)}\left[ \pm\left(\begin{array}{c}
G_{21} \\
G_{12}
\end{array}\right)+2 \mathbf{T}_{ \pm}^{\left(\frac{3}{2}\right)}\right](w) \\
& +\frac{1}{(z-w)}\left[\frac{1}{3} \partial(\text { pole- } 2) \mp \mathbf{W}_{ \pm}^{\left(\frac{5}{2}\right)}\right](w)+\cdots \\
& \left(\begin{array}{l}
G_{11} \\
G_{22}
\end{array}\right)(z)\left(\begin{array}{c}
\mathbf{V}_{-}^{(2)} \\
\mathbf{U}_{+}^{(2)}
\end{array}\right)(w)=\frac{1}{(z-w)^{2}} \frac{(9+k)}{(5+k)}\left[ \pm\left(\begin{array}{c}
G_{12} \\
G_{21}
\end{array}\right)-2 \mathbf{T}_{\mp}^{\left(\frac{3}{2}\right)}\right](w) \\
& +\frac{1}{(z-w)}\left[\frac{1}{3} \partial(\text { pole- } 2) \mp \mathbf{W}_{\mp}^{\left(\frac{5}{2}\right)}\right](w)+\cdots, \\
& \left(\begin{array}{l}
G_{11} \\
G_{22}
\end{array}\right)(z)\left(\begin{array}{l}
\mathbf{V}^{\left(\frac{5}{2}\right)} \\
\mathbf{U}^{\left(\frac{5}{2}\right)}
\end{array}\right)(w)= \pm \frac{1}{(z-w)^{3}} \frac{8(-3+\mathbf{k})}{3(5+k)} \mathbf{T}^{(\mathbf{1})}(w) \\
& +\frac{1}{(z-w)^{2}}\left[4 \mathbf{T}^{(2)}+\frac{4(-3+k)}{3(5+k)} \mathbf{W}^{(\mathbf{2})}\right](w) \\
& +\frac{1}{(z-w)}\left[\frac{1}{4} \partial(\text { pole- } 2) \pm \mathbf{W}^{(3)}\right. \\
& \left. \pm \frac{4(-\mathbf{3}+\mathbf{k})}{(17+13 k)}\left(T \mathbf{T}^{(\mathbf{1})}-\frac{1}{2} \partial^{2} \mathbf{T}^{(\mathbf{1})}\right)\right](w)+\cdots, \\
& \left(\begin{array}{l}
G_{11} \\
G_{22}
\end{array}\right)(z) \mathbf{W}^{(2)}(w)=-\frac{1}{(z-w)^{2}} \frac{3}{2}\left[\left(\begin{array}{c}
G_{11} \\
G_{22}
\end{array}\right) \pm 2\left(\begin{array}{c}
\mathbf{U}^{\left(\frac{3}{2}\right)} \\
\mathbf{V}^{\left(\frac{3}{2}\right)}
\end{array}\right)\right](w) \\
& +\frac{1}{(z-w)} \frac{1}{3} \partial(\text { pole- } 2)(w)+\cdots, \\
& \left(\begin{array}{l}
G_{11} \\
G_{22}
\end{array}\right)(z) \mathbf{W}_{ \pm}^{\left(\frac{5}{2}\right)}(w)= \pm \frac{1}{(z-w)^{2}} \frac{16(3+k)}{3(5+k)}\left(\begin{array}{c}
\mathbf{U}_{+}^{(2)} \\
\mathbf{V}_{-}^{(2)}
\end{array}\right)(w) \\
& +\frac{1}{(z-w)} \frac{1}{4} \partial(\text { pole- } 2)(w)+\cdots \\
& \left(\begin{array}{l}
G_{11} \\
G_{22}
\end{array}\right)(z) \mathbf{W}_{\mp}^{\left(\frac{5}{2}\right)}(w)= \pm \frac{1}{(z-w)^{2}} \frac{8(9+k)}{3(5+k)}\left(\begin{array}{c}
\mathbf{U}_{-}^{(\mathbf{2})} \\
\mathbf{V}_{+}^{(\mathbf{2})}
\end{array}\right)(w)+\frac{1}{(z-w)} \frac{1}{4} \partial(\text { pole- } 2)(w)+\cdots, \\
& \left(\begin{array}{l}
G_{11} \\
G_{22}
\end{array}\right)(z) \mathbf{W}^{(\mathbf{3})}(w)=-\frac{1}{(z-w)^{3}} \frac{2(-3+k)(113+61 k)}{3(5+k)(17+13 k)}\left[ \pm\left(\begin{array}{c}
G_{11} \\
G_{22}
\end{array}\right)+2\left(\begin{array}{l}
\mathbf{U}^{\left(\frac{3}{2}\right)} \\
\mathbf{V}^{\left(\frac{3}{2}\right)}
\end{array}\right)\right](w)
\end{aligned}
$$




$$
\begin{aligned}
& +\frac{1}{(z-w)^{2}}\left[\mp 5\left(\begin{array}{l}
\mathbf{U}^{\left(\frac{5}{2}\right)} \\
\mathbf{V}^{\left(\frac{5}{2}\right)}
\end{array}\right) \pm \frac{4(-3+k)}{(17+13 k)} \partial\left(\begin{array}{l}
G_{11} \\
G_{22}
\end{array}\right)\right. \\
& \left.+\frac{8(-3+k)}{(17+13 k)} \partial\left(\begin{array}{c}
\mathbf{U}^{\left(\frac{3}{2}\right)} \\
\mathbf{V}^{\left(\frac{3}{2}\right)}
\end{array}\right)-\frac{6(-3+\mathbf{k})}{(17+13 k)}\left(\begin{array}{l}
G_{11} \\
G_{22}
\end{array}\right) \mathbf{T}^{(\mathbf{1})}\right](w) \\
& +\frac{1}{(z-w)}\left[\frac{1}{5} \partial(\text { pole- } 2) \pm \frac{6(-3+k)}{5(17+13 k)} \partial^{2}\left(\begin{array}{l}
G_{11} \\
G_{22}
\end{array}\right)\right. \\
& -\frac{4(-\mathbf{3}+\mathbf{k})}{5(17+13 k)} \partial\left(\begin{array}{c}
G_{11} \\
G_{22}
\end{array}\right) \mathbf{T}^{(\mathbf{1})}+\frac{12(-3+k)}{5(17+13 k)} \partial^{2}\left(\begin{array}{c}
\mathbf{U}^{\left(\frac{3}{2}\right)} \\
\mathbf{V}^{\left(\frac{3}{2}\right)}
\end{array}\right) \\
& \mp \frac{4(-3+k)}{(17+13 k)} T\left(\begin{array}{c}
G_{11} \\
G_{22}
\end{array}\right)-\frac{8(-3+\mathbf{k})}{(17+13 k)} T\left(\begin{array}{c}
\mathbf{U}^{\left(\frac{3}{2}\right)} \\
\mathbf{V}^{\left(\frac{3}{2}\right)}
\end{array}\right) \\
& \left.+\frac{6(-\mathbf{3}+\mathbf{k})}{5(17+13 k)}\left(\begin{array}{c}
G_{11} \\
G_{22}
\end{array}\right) \partial \mathbf{T}^{(\mathbf{1})}\right](w)+\cdots, \\
& \left(\begin{array}{l}
G_{12} \\
G_{21}
\end{array}\right)(z) \mathbf{T}^{(\mathbf{1})}(w)=\frac{1}{(z-w)}\left[\mp\left(\begin{array}{l}
G_{12} \\
G_{21}
\end{array}\right)+2 \mathbf{T}_{\mp}^{\left(\frac{3}{2}\right)}\right](w)+\cdots, \\
& \left(\begin{array}{l}
G_{12} \\
G_{21}
\end{array}\right)(z) \mathbf{T}_{ \pm}^{\left(\frac{3}{2}\right)}(w)=\mp \frac{1}{(z-w)^{3}} \frac{8(1+k)}{(5+k)} \\
& +\frac{1}{(z-w)^{2}}\left[-\frac{8 i}{(5+k)} A_{3}-\frac{2 i(1+k)}{(5+k)} B_{3}+\mathbf{T}^{(\mathbf{1})}\right](w) \\
& +\frac{1}{(z-w)}\left[\frac{1}{2} \partial(\text { pole- } 2) \mp \mathbf{T}^{(2)} \mp T\right](w)+\cdots, \\
& \left(\begin{array}{l}
G_{12} \\
G_{21}
\end{array}\right)(z) \mathbf{T}^{(2)}(w)=\frac{1}{(z-w)^{2}} \frac{3}{2}\left[-\left(\begin{array}{c}
G_{12} \\
G_{21}
\end{array}\right) \pm 2 \mathbf{T}_{\mp}^{\left(\frac{3}{2}\right)}\right](w)+\frac{1}{(z-w)} \frac{1}{3} \partial(\text { pole- } 2)(w)+\cdots, \\
& \left(\begin{array}{l}
G_{12} \\
G_{21}
\end{array}\right)(z)\left(\begin{array}{l}
\mathbf{U}^{\left(\frac{3}{2}\right)} \\
\mathbf{V}^{\left(\frac{3}{2}\right)}
\end{array}\right)(w)=\frac{1}{(z-w)^{2}} \frac{8 i}{(5+k)} A_{ \pm}(w) \\
& +\frac{1}{(z-w)}\left[\frac{1}{2} \partial(\text { pole- } 2)+\left(\begin{array}{c}
\mathbf{U}_{-}^{(2)} \\
\mathbf{V}_{+}^{(2)}
\end{array}\right)\right](w)+\cdots, \\
& \left(\begin{array}{l}
G_{12} \\
G_{21}
\end{array}\right)(z)\left(\begin{array}{c}
\mathbf{U}_{+}^{(2)} \\
\mathbf{V}_{-}^{(\mathbf{2})}
\end{array}\right)(w)=\frac{1}{(z-w)^{2}} \frac{(9+k)}{(5+k)}\left[ \pm\left(\begin{array}{c}
G_{11} \\
G_{22}
\end{array}\right)+2\left(\begin{array}{l}
\mathbf{U}^{\left(\frac{3}{2}\right)} \\
\mathbf{V}^{\left(\frac{3}{2}\right)}
\end{array}\right)\right](w) \\
& +\frac{1}{(z-w)}\left[\frac{1}{3} \partial(\text { pole- } 2) \mp\left(\begin{array}{c}
\mathbf{U}^{\left(\frac{5}{2}\right)} \\
\mathbf{V}^{\left(\frac{5}{2}\right)}
\end{array}\right)\right](w)+\cdots, \\
& \left(\begin{array}{l}
G_{12} \\
G_{21}
\end{array}\right)(z)\left(\begin{array}{c}
\mathbf{U}^{\left(\frac{5}{2}\right)} \\
\mathbf{V}^{\left(\frac{5}{2}\right)}
\end{array}\right)(w)= \pm \frac{1}{(z-w)^{2}} \frac{8(9+k)}{3(5+k)}\left(\begin{array}{c}
\mathbf{U}_{-}^{(\mathbf{2})} \\
\mathbf{V}_{+}^{(2)}
\end{array}\right)(w)+\frac{1}{(z-w)} \frac{1}{4} \partial(\text { pole- } 2)(w)+\cdots, \\
& \left(\begin{array}{l}
G_{12} \\
G_{21}
\end{array}\right)(z)\left(\begin{array}{l}
\mathbf{V}^{\left(\frac{3}{2}\right)} \\
\mathbf{U}^{\left(\frac{3}{2}\right)}
\end{array}\right)(w)=-\frac{1}{(z-w)^{2}} \frac{2 i(1+k)}{(5+k)} B_{ \pm}(w) \\
& +\frac{1}{(z-w)}\left[\frac{1}{2} \partial(\text { pole- } 2)+\left(\begin{array}{c}
\mathbf{V}_{-}^{(\mathbf{2})} \\
\mathbf{U}_{+}^{(2)}
\end{array}\right)\right](w)+\cdots,
\end{aligned}
$$




$$
\begin{aligned}
& \left(\begin{array}{l}
G_{12} \\
G_{21}
\end{array}\right)(z)\left(\begin{array}{c}
\mathbf{V}_{+}^{(\mathbf{2})} \\
\mathbf{U}_{-}^{(2)}
\end{array}\right)(w)=\frac{1}{(z-w)^{2}} \frac{2(3+k)}{(5+k)}\left[\mp\left(\begin{array}{c}
G_{22} \\
G_{11}
\end{array}\right)+2\left(\begin{array}{c}
\mathbf{V}^{\left(\frac{3}{2}\right)} \\
\mathbf{U}^{\left(\frac{3}{2}\right)}
\end{array}\right)\right](w) \\
& +\frac{1}{(z-w)}\left[\frac{1}{3} \partial(\text { pole- } 2) \mp\left(\begin{array}{c}
\mathbf{V}^{\left(\frac{5}{2}\right)} \\
\mathbf{U}^{\left(\frac{5}{2}\right)}
\end{array}\right)\right](w)+\cdots, \\
& \left(\begin{array}{l}
G_{12} \\
G_{21}
\end{array}\right)(z)\left(\begin{array}{c}
\mathbf{V}^{\left(\frac{5}{2}\right)} \\
\mathbf{U}^{\left(\frac{5}{2}\right)}
\end{array}\right)(w)= \pm \frac{1}{(z-w)^{2}} \frac{16(3+k)}{3(5+k)}\left(\begin{array}{c}
\mathbf{V}_{-}^{(\mathbf{2})} \\
\mathbf{U}_{+}^{(2)}
\end{array}\right)(w) \\
& +\frac{1}{(z-w)} \frac{1}{4} \partial(\text { pole- } 2)(w)+\cdots, \\
& \left(\begin{array}{l}
G_{12} \\
G_{21}
\end{array}\right)(z) \mathbf{W}^{(2)}(w)=\frac{1}{(z-w)^{2}} \frac{(-3+k)}{2(5+k)}\left[\left(\begin{array}{c}
G_{12} \\
G_{21}
\end{array}\right) \mp 2 \mathbf{T}_{\mp}^{\left(\frac{3}{2}\right)}\right](w) \\
& +\frac{1}{(z-w)}\left[\frac{1}{3} \partial(\text { pole- } 2)+\mathbf{W}_{\mp}^{\left(\frac{5}{2}\right)}\right](w)+\cdots, \\
& \left(\begin{array}{l}
G_{12} \\
G_{21}
\end{array}\right)(z) \mathbf{W}_{ \pm}^{\left(\frac{5}{2}\right)}(w)=\mp \frac{1}{(z-w)^{3}} \frac{8(-\mathbf{3}+\mathbf{k})}{3(5+k)} \mathbf{T}^{(\mathbf{1})}(w) \\
& +\frac{1}{(z-w)^{2}}\left[\frac{4(-3+k)}{3(5+k)} \mathbf{T}^{(2)}+4 \mathbf{W}^{(\mathbf{2})}\right](w) \\
& +\frac{1}{(z-w)}\left[\frac{1}{4} \partial(\text { pole- } 2) \mp \mathbf{W}^{(3)}\right. \\
& \left.\mp \frac{4(-\mathbf{3}+\mathbf{k})}{(17+13 k)}\left(T \mathbf{T}^{(\mathbf{1})}-\frac{1}{2} \partial^{2} \mathbf{T}^{(\mathbf{1})}\right)\right](w)+\cdots, \\
& \left(\begin{array}{l}
G_{12} \\
G_{21}
\end{array}\right)(z) \mathbf{W}^{(\mathbf{3})}(w)=\frac{1}{(z-w)^{3}} \frac{2(-3+k)(113+61 k)}{3(5+k)(17+13 k)}\left[ \pm\left(\begin{array}{c}
G_{12} \\
G_{21}
\end{array}\right)-2 \mathbf{T}_{\mp}^{\left(\frac{3}{2}\right)}\right](w) \\
& +\frac{1}{(z-w)^{2}}\left[ \pm 5 \mathbf{W}_{\mp}^{\left(\frac{5}{2}\right)} \mp \frac{4(-3+k)}{(17+13 k)} \partial\left(\begin{array}{c}
G_{12} \\
G_{21}
\end{array}\right)\right. \\
& \left.+\frac{8(-3+k)}{(17+13 k)} \partial \mathbf{T}_{\mp}^{\left(\frac{3}{2}\right)}-\frac{6(-\mathbf{3}+\mathbf{k})}{(17+13 k)}\left(\begin{array}{c}
G_{12} \\
G_{21}
\end{array}\right) \mathbf{T}^{(\mathbf{1})}\right](w) \\
& +\frac{1}{(z-w)}\left[\frac{1}{5} \partial(\text { pole- } 2) \mp \frac{6(-3+k)}{5(17+13 k)} \partial^{2}\left(\begin{array}{c}
G_{12} \\
G_{21}
\end{array}\right)\right. \\
& -\frac{4(-\mathbf{3}+\mathbf{k})}{5(17+13 k)} \partial\left(\begin{array}{c}
G_{12} \\
G_{21}
\end{array}\right) \mathbf{T}^{(\mathbf{1})}+\frac{12(-3+k)}{5(17+13 k)} \partial^{2} \mathbf{T}_{\mp}^{\left(\frac{\mathbf{3}}{2}\right)} \\
& \pm \frac{4(-3+k)}{(17+13 k)} T\left(\begin{array}{c}
G_{12} \\
G_{21}
\end{array}\right)-\frac{8(-3+k)}{(17+13 k)} T \mathbf{T}_{\mp}^{\left(\frac{3}{2}\right)} \\
& \left.+\frac{6(-\mathbf{3}+\mathbf{k})}{5(17+13 k)}\left(\begin{array}{c}
G_{12} \\
G_{21}
\end{array}\right) \partial \mathbf{T}^{(\mathbf{1})}\right](w)+\cdots \text {. }
\end{aligned}
$$

It is easy to see that the OPEs containing the higher spin- $\frac{5}{2}$ or spin-3 current do have the nonlinear terms in the right hand side. These nonlinear terms will disappear in the different basis in next Appendix. In doing this, one should realize that there exist nontrivial OPEs 
(4.1) in section 4. In the right hand side of those OPEs, the higher spin- $\frac{3}{2}$ currents occur and this will contribute to the corresponding terms in the first order pole above. In this case, the symmetry of the structure constants allows us to write the OPEs together and this is new feature compared to the expressions in Part I. The nonlinear terms in Part I do not appear in the above OPEs. One also checks that the above OPEs are equivalent to the corresponding OPEs in [9] (or in Appendix D) via the field identifications in section 4. In next Appendix, the above OPEs can be written in different basis where the nonlinear terms will disappear.

\section{Appendix D Other basis where the OPEs are linear}

In order to compare the fields in [9] with the ones in this paper, their defining relations are given.

\section{Appendix D.1 The OPEs in the linear version}

Let us present the description of [9] as follows:

$$
\begin{aligned}
& U(z) V_{0}^{(s)}(w)=+\cdots, \\
& Q^{a}(z) V_{0}^{(s)}(w)=+\cdots, \\
& A^{ \pm, i}(z) V_{0}^{(s)}(w)=+\cdots, \\
& G^{a}(z) V_{0}^{(s)}(w)=\frac{1}{(z-w)} V_{\frac{1}{2}}^{(s), a}(w)+\cdots, \\
& U(z) V_{\frac{1}{2}}^{(s), a}(w)=+\cdots, \\
& Q^{a}(z) V_{\frac{1}{2}}^{(s), b}(w)=+\cdots, \\
& A^{ \pm, i}(z) V_{\frac{1}{2}}^{(s), a}(w)=\frac{1}{(z-w)} \alpha_{a b}^{ \pm, i} V_{\frac{1}{2}}^{(s), b}(w)+\cdots, \\
& G^{a}(z) V_{\frac{1}{2}}^{(s), b}(w)=\frac{1}{(z-w)^{2}} 2 s \delta^{a b} V_{0}^{(s)}(w) \\
& +\frac{1}{(z-w)}\left[\alpha_{a b}^{+, i} V_{1}^{(s),+, i}+\alpha_{a b}^{-, i} V_{1}^{(s),-, i}+\delta^{a b} \partial V_{0}^{(s)}\right](w)+\cdots, \\
& U(z) V_{1}^{(s), \pm i}(w)=+\cdots, \\
& Q^{a}(z) V_{1}^{(s), \pm, i}(w)= \pm \frac{1}{(z-w)} 2 \alpha_{a b}^{ \pm, i} V_{\frac{1}{2}}^{(s), b}(w)+\cdots \\
& A^{ \pm, i}(z) V_{1}^{(s), \pm, j}(w)=\frac{1}{(z-w)^{2}} 2 s \delta^{i j} V_{0}^{(s)}(w)+\frac{1}{(z-w)} \epsilon^{i j k} V_{1}^{(s), \pm, k}(w)+\cdots, \\
& A^{ \pm, i}(z) V_{1}^{(s), \mp, j}(w)=+\cdots, \\
& G^{a}(z) V_{1}^{(s), \pm, i}(w)=\frac{1}{(z-w)^{2}} 4\left(s+\gamma_{\mp}\right) \alpha_{a b}^{ \pm, i} V_{\frac{1}{2}}^{(s), b}(w)
\end{aligned}
$$




$$
\begin{aligned}
& +\frac{1}{(z-w)}\left[\frac{1}{(2 s+1)} \partial(\text { pole- } 2) \mp \alpha_{a b}^{ \pm, i} V_{\frac{3}{2}}^{(s), b}\right](w)+\cdots, \\
& U(z) V_{\frac{3}{2}}^{(s), a}(w)=-\frac{1}{(z-w)^{2}} 2 V_{\frac{1}{2}}^{(s), a}(w)+\cdots, \\
& Q^{a}(z) V_{\frac{3}{2}}^{(s), b}(w)=\frac{1}{(z-w)^{2}} 4 s \delta^{a b} V_{0}^{(s)}(w) \\
& +\frac{1}{(z-w)} 2\left[\alpha_{a b}^{+, i} V_{1}^{(s),+, i}+\alpha_{a b}^{-, i} V_{1}^{(s),-, i}-\delta^{a b} \partial V_{0}^{(s)}\right](w)+\cdots, \\
& A^{ \pm, i}(z) V_{\frac{3}{2}}^{(s), a}(w)= \pm \frac{1}{(z-w)^{2}}\left[\frac{8 s(s+1)+4 \gamma_{\mp}}{(2 s+1)}\right] \alpha_{a b}^{ \pm, i} V_{\frac{1}{2}}^{(s), b}(w) \\
& +\frac{1}{(z-w)} \alpha_{a b}^{ \pm, i} V_{\frac{3}{2}}^{(s), b}(w)+\cdots, \\
& G^{a}(z) V_{\frac{3}{2}}^{(s), b}(w)=-\frac{1}{(z-w)^{3}}\left[\frac{16 s(s+1)(2 \gamma-1)}{(2 s+1)}\right] \delta^{a b} V_{0}^{(s)}(w) \\
& -\frac{1}{(z-w)^{2}} \frac{8(s+1)}{(2 s+1)}\left[\left(s+\gamma_{+}\right) \alpha_{a b}^{+, i} V_{1}^{(s),+, i}-\left(s+\gamma_{-}\right) \alpha_{a b}^{-, i} V_{1}^{(s),-, i}\right](w) \\
& +\frac{1}{(z-w)}\left[\frac{1}{2(s+1)} \partial(\text { pole- } 2)+\delta^{a b} V_{2}^{(s)}\right](w)+\cdots, \\
& U(z) V_{2}^{(s)}(w)=\frac{1}{(z-w)^{3}} 8 s V_{0}^{(s)}(w)-\frac{1}{(z-w)^{2}} 4 \partial V_{0}^{(s)}(w)+\cdots, \\
& Q^{a}(z) V_{2}^{(s)}(w)=-\frac{1}{(z-w)^{2}} 2(2 s+1) V_{\frac{1}{2}}^{(s), a}(w)+\frac{1}{(z-w)} 2 \partial V_{\frac{1}{2}}^{(s), a}(w)+\cdots, \\
& A^{ \pm, i}(z) V_{2}^{(s)}(w)= \pm \frac{1}{(z-w)^{2}} 2(s+1) V_{1}^{(s), \pm, i}(w)+\cdots, \\
& G^{a}(z) V_{2}^{(s)}(w)=\frac{1}{(z-w)^{3}}\left[\frac{16 s(s+1)(2 \gamma-1)}{(2 s+1)}\right] V_{\frac{1}{2}}^{(s), a}(w) \\
& +\frac{1}{(z-w)^{2}}(2 s+3) V_{\frac{3}{2}}^{(s), a}(w)+\frac{1}{(z-w)} \partial V_{\frac{3}{2}}^{(s), a}(w)+\cdots, \\
& T(z) V_{2}^{(s)}(w)=-\frac{1}{(z-w)^{4}}\left[\frac{24 s(s+1)(2 \gamma-1)}{(2 s+1)}\right] V_{0}^{(s)}(w) \\
& +\frac{1}{(z-w)^{2}}(s+2) V_{2}^{(s)}(w)+\frac{1}{(z-w)} \partial V_{2}^{(s)}(w)+\cdots, \\
& T(z) V_{0}^{(s)}(w)=\frac{1}{(z-w)^{2}} s V_{0}^{(s)}(w)+\frac{1}{(z-w)} \partial V_{0}^{(s)}(w)+\cdots, \\
& T(z) V_{\frac{1}{2}}^{(s), a}(w)=\frac{1}{(z-w)^{2}}\left(s+\frac{1}{2}\right) V_{\frac{1}{2}}^{(s), a}(w)+\frac{1}{(z-w)} \partial V_{\frac{1}{2}}^{(s), a}(w)+\cdots, \\
& T(z) V_{1}^{(s), \pm, i}(w)=\frac{1}{(z-w)^{2}}(s+1) V_{1}^{(s), \pm, i}(w)+\frac{1}{(z-w)} \partial V_{1}^{(s), \pm, i}(w)+\cdots, \\
& T(z) V_{\frac{3}{2}}^{(s), a}(w)=\frac{1}{(z-w)^{2}}\left(s+\frac{3}{2}\right) V_{\frac{3}{2}}^{(s), a}(w)+\frac{1}{(z-w)} \partial V_{\frac{3}{2}}^{(s), a}(w)+\cdots .
\end{aligned}
$$


Here the spin- $\frac{1}{2}$ currents $Q^{a}(z)$ play the role of previous one $\Gamma^{a}(z)$ in Appendix $A$. In the present notation, this is equivalent to the spin- $\frac{1}{2}$ current $F_{a}(z)$. One has $\gamma_{+} \equiv \gamma$ and $\gamma_{-} \equiv 1-\gamma$. Some of the typos appearing in [9] are corrected. In particular, note that the first order pole of the OPE $G^{a}(z) V_{\frac{3}{2}}^{(s), b}(w)$ should possess the $\delta^{a b} V_{2}^{(s)}$ term in the right hand side. These OPEs hold for any spin $s=1,2, \cdots$. The higher spin current $V_{2}^{(s)}(w)$ does not contain the third order pole in the OPE with $T(z)$ and has nontrivial fourth order pole. The spin- $\frac{3}{2}$ currents which are the supersymmetry generators determine all the higher spin currents via the OPEs containing $G_{a}(z)$ in the above OPEs.

\section{Appendix D.2 The higher spin currents in the nonlinear version}

One has the explicit relations between the higher spin currents in the nonlinear version [9] and the corresponding higher spin currents in this paper. For the higher spin-2 currents, one has

$$
\begin{aligned}
& \widetilde{V}_{1}^{(1),+1}(z)=\left[2 i\left(\mathbf{U}_{-, \text {non }}^{(\mathbf{2})}-\mathbf{V}_{+, \text {non }}^{(\mathbf{2})}\right)+\frac{8 i}{(5+k)} \hat{A}_{1} \hat{B}_{3}\right](z), \\
& \widetilde{V}_{1}^{(1),+2}(z)=\left[-2\left(\mathbf{U}_{-, \text {non }}^{(\mathbf{2})}+\mathbf{V}_{+, \text {non }}^{(\mathbf{2})}\right)-\frac{8 i}{(5+k)} \hat{A}_{2} \hat{B}_{3}\right](z), \\
& \widetilde{V}_{1}^{(1),+3}(z)=\left[2 i\left(\mathbf{T}_{\text {non }}^{(\mathbf{2})}-\mathbf{W}_{\text {non }}^{(\mathbf{2})}\right)+\frac{8 i}{(5+k)} \hat{A}_{3} \hat{B}_{3}+\frac{12 i k}{(3+7 k)} \hat{T}\right](z), \\
& \widetilde{V}_{1}^{(1),-1}(z)=\left[2 i\left(\mathbf{U}_{+, \text {non }}^{(\mathbf{2})}-\mathbf{V}_{-, \text {non }}^{(\mathbf{2})}\right)+\frac{8 i}{(5+k)} \hat{A}_{3} \hat{B}_{1}\right](z), \\
& \widetilde{V}_{1}^{(1),-2}(z)=\left[-2\left(\mathbf{U}_{+, \text {non }}^{(\mathbf{2})}+\mathbf{V}_{-, \text {non }}^{(\mathbf{2})}\right)+\frac{8 i}{(5+k)} \hat{A}_{3} \hat{B}_{2}\right](z), \\
& \widetilde{V}_{1}^{(1),+3}(z)=\left[-2 i\left(\mathbf{T}_{\text {non }}^{(\mathbf{2})}+\mathbf{W}_{\text {non }}^{(\mathbf{2})}\right)+\frac{4 i}{(5+k)}\left(\hat{A}_{i} \hat{A}_{i}+\hat{B}_{i} \hat{B}_{i}\right)+\frac{4 i(3+4 k)}{(3+7 k)} \hat{T}\right](z) .
\end{aligned}
$$

From the relations (4.23)-(4.25) of [9], one can rewrite the higher spin currents in the nonlinear version in terms of those in the linear version. Then using the explicit relations (4.2), (4.6) and (4.7), one can rewrite them in terms of the higher spin currents in the linear version. Finally, using the explicit relations appearing in Appendix $B$, one arrives at the final results in (D.1). This was also realized in [11].

One can analyze the other higher spin currents. The following relations can be obtained

$$
\begin{aligned}
\widetilde{V}_{\frac{3}{2}}^{(1), 0}(z) & =-2 i \sqrt{2}\left(\mathbf{W}_{+, \text {non }}^{\left(\frac{5}{2}\right)}+\mathbf{W}_{-, \text {non }}^{\left(\frac{5}{2}\right)}\right)(z)-\frac{4 \sqrt{2}}{(5+k)}\left[\hat{A}_{-} \hat{G}_{11}+\hat{A}_{-} \mathbf{U}_{\text {non }}^{\left(\frac{3}{2}\right)}-2 \hat{A}_{3} \hat{G}_{21}-2 \hat{A}_{3} \mathbf{T}_{+, \text {non }}^{\left(\frac{3}{2}\right)}\right. \\
& \left.+\hat{B}_{-} \hat{G}_{22}-\hat{B}_{-} \mathbf{V}_{\text {non }}^{\left(\frac{3}{2}\right)}+2 \hat{B}_{3} \mathbf{T}_{+, \text {non }}^{\left(\frac{3}{2}\right)}+\frac{2 i}{3} \partial \hat{G}_{21}\right](z)+\frac{4 \sqrt{2}}{(5+k)}\left[\hat{A}_{+} \hat{G}_{22}-\hat{A}_{+} \mathbf{V}_{\text {non }}^{\left(\frac{3}{2}\right)}\right.
\end{aligned}
$$




$$
\begin{aligned}
& \left.-2 \hat{A}_{3} \hat{G}_{12}+2 \hat{A}_{3} \mathbf{T}_{-, \text {non }}^{\left(\frac{\mathbf{3}}{\mathbf{2}}\right)}+\hat{B}_{+} \hat{G}_{11}+\hat{B}_{+} \mathbf{U}_{\mathbf{n o n}}^{\left(\frac{\mathbf{3}}{2}\right)}-2 \hat{B}_{3} \mathbf{T}_{-, \text {non }}^{\left(\frac{\mathbf{3}}{2}\right)}-\frac{2 i}{3} \partial \hat{G}_{12}\right](z), \\
& \widetilde{V}_{\frac{3}{2}}^{(1), 1}(z)=-2 \sqrt{2}\left(\mathbf{U}_{\text {non }}^{\left(\frac{5}{2}\right)}-\mathbf{V}_{\text {non }}^{\left(\frac{5}{2}\right)}\right)(z) \\
& +\frac{4 i \sqrt{2}}{(5+k)}\left[\hat{A}_{+} \hat{G}_{21}+\hat{A}_{+} \mathbf{T}_{+, \text {non }}^{\left(\frac{3}{2}\right)}-\hat{B}_{-} \hat{G}_{12}+2 \hat{B}_{-} \mathbf{T}_{-, \text {non }}^{\left(\frac{3}{2}\right)}+2 \hat{B}_{3} \mathbf{U}_{\mathbf{n o n}}^{\left(\frac{3}{2}\right)}-\frac{2 i}{3} \partial \mathbf{U}_{\text {non }}^{\left(\frac{3}{2}\right)}\right](z) \\
& +\frac{4 i \sqrt{2}}{(5+k)}\left[\hat{A}_{-} \hat{G}_{12}-2 \hat{A}_{-} \mathbf{T}_{-, \text {non }}^{\left(\frac{3}{2}\right)}-2 \hat{A}_{3} \mathbf{V}_{\text {non }}^{\left(\frac{3}{2}\right)}-\hat{B}_{+} \mathbf{T}_{+, \text {non }}^{\left(\frac{3}{2}\right)}+\frac{2 i}{3} \partial \mathbf{V}_{\text {non }}^{\left(\frac{3}{2}\right)}\right](z), \\
& \widetilde{V}_{\frac{3}{2}}^{(1), 2}(z)=-2 i \sqrt{2}\left(\mathbf{U}_{\text {non }}^{\left(\frac{5}{2}\right)}+\mathbf{V}_{\text {non }}^{\left(\frac{5}{2}\right)}\right)(z) \\
& -\frac{4 \sqrt{2}}{(5+k)}\left[\hat{A}_{+} \hat{G}_{21}+\hat{A}_{+} \mathbf{T}_{+, \text {non }}^{\left(\frac{3}{2}\right)}-\hat{B}_{-} \hat{G}_{12}+2 \hat{B}_{-} \mathbf{T}_{-, \text {non }}^{\left(\frac{3}{2}\right)}+2 \hat{B}_{3} \mathbf{U}_{\text {non }}^{\left(\frac{3}{2}\right)}-\frac{2 i}{3} \partial \mathbf{U}_{\text {non }}^{\left(\frac{3}{2}\right)}\right](z) \\
& +\frac{4 \sqrt{2}}{(5+k)}\left[\hat{A}_{-} \hat{G}_{12}-2 \hat{A}_{-} \mathbf{T}_{-, \text {non }}^{\left(\frac{3}{2}\right)}-2 \hat{A}_{3} \mathbf{V}_{\text {non }}^{\left(\frac{3}{2}\right)}-\hat{B}_{+} \mathbf{T}_{+, \text {non }}^{\left(\frac{3}{2}\right)}+\frac{2 i}{3} \partial \mathbf{V}_{\text {non }}^{\left(\frac{3}{2}\right)}\right](z), \\
& \widetilde{V}_{\frac{3}{2}}^{(1), 3}(z)=2 \sqrt{2}\left(\mathbf{W}_{+, \text {non }}^{\left(\frac{5}{2}\right)}-\mathbf{W}_{-, \text {non }}^{\left(\frac{5}{2}\right)}\right)(z)-\frac{4 i \sqrt{2}}{(5+k)}\left[\hat{A}_{-} \hat{G}_{11}+\hat{A}_{-} \mathbf{U}_{\text {non }}^{\left(\frac{3}{2}\right)}-2 \hat{A}_{3} \hat{G}_{21}-2 \hat{A}_{3} \mathbf{T}_{+, \text {non }}^{\left(\frac{3}{2}\right)}\right. \\
& \left.+\hat{B}_{-} \hat{G}_{22}-\hat{B}_{-} \mathbf{V}_{\text {non }}^{\left(\frac{3}{2}\right)}+2 \hat{B}_{3} \mathbf{T}_{+, \text {non }}^{\left(\frac{3}{2}\right)}+\frac{2 i}{3} \partial \hat{G}_{21}\right](z)-\frac{4 i \sqrt{2}}{(5+k)}\left[\hat{A}_{+} \hat{G}_{22}-\hat{A}_{+} \mathbf{V}_{\text {non }}^{\left(\frac{3}{2}\right)}\right. \\
& \left.-2 \hat{A}_{3} \hat{G}_{12}+2 \hat{A}_{3} \mathbf{T}_{-, \text {non }}^{\left(\frac{\mathbf{3}}{\mathbf{2}}\right)}+\hat{B}_{+} \hat{G}_{11}+\hat{B}_{+} \mathbf{U}_{\mathbf{n o n}}^{\left(\frac{3}{2}\right)}-2 \hat{B}_{3} \mathbf{T}_{-, \text {non }}^{\left(\frac{\mathbf{3}}{2}\right)}-\frac{2 i}{3} \partial \hat{G}_{12}\right](z) .
\end{aligned}
$$

These observations are also appeared in [1].

Now the final higher spin-3 current has the following relation

$$
\begin{aligned}
\widetilde{V}_{2}^{(1)}(z) & =4\left[\mathbf{W}_{\mathbf{n o n}}^{(\mathbf{3})}+\frac{8(-3+k)}{(19+23 k)} \mathbf{T}^{(\mathbf{1})} \hat{T}+\frac{2 i}{(5+k)} \hat{A}_{-} \mathbf{U}_{-, \mathbf{n o n}}^{(\mathbf{2})}+\frac{4 i}{(5+k)} \hat{A}_{3} \mathbf{T}_{\mathbf{n o n}}^{(\mathbf{2})}\right. \\
& -\frac{2 i}{(5+k)} \hat{B}_{-} \mathbf{V}_{-, \mathbf{n o n}}^{(\mathbf{2})}-\frac{4 i}{(5+k)} \hat{B}_{3} \mathbf{T}_{\mathbf{n o n}}^{(\mathbf{2})}-\frac{1}{(5+k)} \partial \mathbf{W}_{\mathbf{n o n}}^{(\mathbf{2})}+\frac{1}{2(5+k)} \hat{B}_{+} \mathbf{T}^{(\mathbf{1})} \hat{B}_{-} \\
& -\frac{i}{(5+k)} \partial \mathbf{T}^{(\mathbf{1})} \hat{A}_{3}+\frac{i}{(5+k)} \partial \mathbf{T}^{(\mathbf{1})} \hat{B}_{3}+\frac{i}{(5+k)} \mathbf{T}^{(\mathbf{1})} \partial \hat{A}_{3}-\frac{1}{2(5+k)} \hat{B}_{-} \mathbf{T}^{(\mathbf{1})} \hat{B}_{+} \\
& -\frac{2}{(5+k)^{2}} \hat{A}_{+} \partial \hat{A}_{-}-\frac{8 i\left(48+97 k+29 k^{2}\right)}{(5+k)(3+7 k)(19+23 k)} \hat{A}_{3} \hat{T}_{+} \frac{2}{(5+k)^{2}} \hat{A}_{3} \partial \hat{A}_{3} \\
& -\frac{(2+k)}{(5+k)^{2}} \hat{B}_{+} \partial \hat{B}_{-}+\frac{k}{(5+k)^{2}} \hat{B}_{-} \partial \hat{B}_{+}-\frac{i}{(5+k)^{2}} \hat{B}_{3} \hat{B}_{+} \hat{B}_{-} \\
& +\frac{8 i k(-27+k)}{(5+k)(3+7 k)(19+23 k)} \hat{B}_{3} \hat{T}_{+}+\frac{2(-6+k)}{(5+k)^{2}} \hat{B}_{3} \partial \hat{A}_{3} \\
& -\frac{1}{(5+k)} \partial \hat{T}_{-} \frac{6 i}{(5+k)^{2}} \partial^{2} \hat{A}_{3}-\frac{i(3+8 k)}{3(5+k)^{2}} \partial^{2} \hat{B}_{3}+\frac{2}{(5+k)} \hat{G}_{12} \hat{G}_{21} \\
& -\frac{2 i}{(5+k)^{2}} \hat{A}_{-} \hat{A}_{+} \hat{A}_{3}-\frac{6 i}{(5+k)^{2}} \hat{A}_{3} \hat{A}_{+} \hat{A}-\frac{8 i}{(5+k)^{2}} \hat{A}_{3} \hat{A}_{3} \hat{A}_{3}
\end{aligned}
$$




$$
\begin{aligned}
& -\frac{i(-4+k)}{(5+k)^{2}} \hat{B}_{+} \hat{A}_{3} \hat{B}_{-}+\frac{i(-4+k)}{(5+k)^{2}} \hat{B}_{-} \hat{A}_{3} \hat{B}_{+}+\frac{i}{(5+k)^{2}} \hat{B}_{-} \hat{B}_{+} \hat{B}_{3} \\
& \left.+\frac{8 i}{(5+k)^{2}} \hat{B}_{3} \hat{A}_{+} \hat{A}_{-}+\frac{16 i}{(5+k)^{2}} \hat{B}_{3} \hat{A}_{3} \hat{A}_{3}-\frac{8 i}{(5+k)^{2}} \hat{B}_{3} \hat{A}_{3} \hat{B}_{3}\right](z) .
\end{aligned}
$$

It would be interesting to see the OPEs between the higher spin currents in the basis above and how they behave differently compared to the previous results in Part I and Part II.

\section{Appendix E Some details on the construction of the 16 second lowest higher spin currents}

In section 5 , the higher spin currents of spin- $\frac{5}{2}$ and spin-3 are obtained explicitly. In this Appendix, the remaining higher spin currents are obtained. Due to the presence of four spin$\frac{1}{2}$ currents and the spin-1 current, the structures of the OPEs are rather complicated. It is rather nontrivial to extract the correct primary field from the given singular term because there exist many quasi primary fields.

\section{Appendix E.1 The remaining higher spin currents in the next 16 higher spin currents}

Let us consider the OPE between the spin- $\frac{3}{2}$ current and the higher spin-3 current found in (5.5)

$$
\begin{aligned}
& G_{21}(z) \mathbf{Q}_{-}^{(3)}(w)=-\frac{1}{(z-4)^{4}} \frac{6 k\left(303+334 k+47 k^{2}\right)}{(5+k)^{3}(17+13 k)} F_{11}(w) \\
& +\frac{1}{(z-w)^{3}}\left[-\frac{4\left(21+137 k+52 k^{2}\right)}{(5+k)^{2}(17+13 k)} U F_{11}-\frac{12}{(5+k)^{2}} F_{12} F_{21} F_{11}\right. \\
& -\frac{16 i\left(114+122 k+81 k^{2}+13 k^{3}\right)}{(5+k)^{3}(17+13 k)} F_{11} A_{3}-\frac{4 i\left(21+21 k+4 k^{2}\right)}{(5+k)^{3}} F_{21} A_{+} \\
& -\frac{4 i\left(21+86 k+13 k^{2}\right)}{(5+k)^{2}(17+13 k)} F_{12} B_{-}+\frac{4 i\left(-105-247 k+73 k^{2}+39 k^{3}\right)}{(5+k)^{3}(17+13 k)} F_{11} B_{3} \\
& \text { - } \left.\partial(\text { pole- } 4)-\frac{(2+k)\left(105+637 k+104 k^{2}\right)}{(5+k)^{2}(17+13 k)} G_{11}\right](w) \\
& +\frac{1}{(z-w)^{2}}\left[\frac{2(11+3 k)}{(5+k)} \mathbf{Q}^{\left(\frac{5}{2}\right)}+\frac{2(11+3 k)}{(5+k)} \mathbf{U}^{\left(\frac{5}{2}\right)}-\frac{i(3+5 k)}{2(5+k)^{2}} A_{3} G_{11}\right. \\
& -\frac{3}{(5+k)^{2}} A_{3} A_{3} F_{11}+\frac{20(3+k)}{(5+k)^{3}} A_{3} B_{3} F_{11}+\frac{(39+11 k)}{(5+k)^{3}} A_{3} B_{-} F_{12}
\end{aligned}
$$




$$
\begin{aligned}
& +\frac{2 i\left(-795-397 k-21 k^{2}+13 k^{3}\right)}{(5+k)^{3}(17+13 k)} \partial A_{3} F_{11}-\frac{(45+17 k)}{(5+k)^{3}} B_{3} B_{3} F_{11} \\
& -\frac{2 i\left(-651+322 k+29 k^{2}\right)}{3(5+k)^{3}(17+13 k)} A_{3} \partial F_{11}-\frac{i\left(2079+1244 k+181 k^{2}\right)}{2(5+k)^{2}(17+13 k)} A_{+} G_{21} \\
& -\frac{2(3+k)}{(5+k)^{3}} A_{+} A_{3} F_{21}-\frac{(9+k)}{(5+k)^{3}} A_{+} A_{-} F_{11}+\frac{18(3+k)}{(5+k)^{3}} A_{+} B_{3} F_{21} \\
& -\frac{3(11+3 k)}{(5+k)^{3}} A_{+} B_{-} F_{22}-\frac{4 i(15+4 k)}{3(5+k)^{3}} \partial A_{+} F_{21}-\frac{4 i(15+4 k)}{3(5+k)^{3}} A_{+} \partial F_{21} \\
& +\frac{3 i(3+k)}{2(5+k)^{2}} B_{3} G_{11}-\frac{2\left(-1671-271 k+223 k^{2}+39 k^{3}\right)}{3(5+k)^{3}(17+13 k)} \partial U F_{11} \\
& +\frac{4 i\left(618+1537 k+566 k^{2}+39 k^{3}\right)}{3(5+k)^{3}(17+13 k)} B_{3} \partial F_{11}-\frac{6 i}{(5+k)^{2}} B_{-} G_{12} \\
& -\frac{3(7+3 k)}{(5+k)^{3}} B_{-} B_{3} F_{12}+\frac{i\left(1518+715 k+308 k^{2}+39 k^{3}\right)}{3(5+k)^{3}(17+13 k)} \partial B_{-} F_{12} \\
& -\frac{i\left(741-784 k+265 k^{2}+78 k^{3}\right)}{3(5+k)^{3}(17+13 k)} B_{-} \partial F_{12}-\frac{8(3+k)}{(5+k)^{3}} B_{+} B_{-} F_{11} \\
& +\frac{\left(2367+2215 k+521 k^{2}+33 k^{3}\right)}{(5+k)^{3}(17+13 k)} \partial^{2} F_{11}-\frac{(9+k)(163+23 k)}{(5+k)^{3}(17+13 k)} F_{11} F_{12} G_{21} \\
& +\frac{2\left(-3+22 k+9 k^{2}\right)}{3(5+k)^{4}} \partial F_{11} F_{12} F_{21}-\frac{2\left(381+194 k+21 k^{2}\right)}{3(5+k)^{4}} F_{11} \partial F_{12} F_{21} \\
& +\frac{8\left(96+43 k+3 k^{2}\right)}{3(5+k)^{4}} F_{11} F_{12} \partial F_{21}-\frac{8(3+k)}{(5+k)^{3}} F_{11} F_{21} G_{12} \\
& -\frac{(15+7 k)}{(5+k)^{3}} F_{11} F_{22} G_{11}+\frac{10(3+k)}{(5+k)^{3}} F_{12} F_{21} G_{11}-\frac{i(39+11 k)}{(5+k)^{3}} U B_{-} F_{12} \\
& +\frac{\left(1977+1234 k+233 k^{2}\right)}{6(5+k)^{2}(17+13 k)} \partial G_{11}-\frac{4\left(153+88 k+11 k^{2}\right)}{(5+k)^{2}(17+13 k)} T F_{11} \\
& -\frac{(27+11 k)}{2(5+k)^{2}} U G_{11}-\frac{16 i(3+k)}{(5+k)^{3}} U A_{3} F_{11}-\frac{18 i(3+k)}{(5+k)^{3}} U A_{+} F_{21} \\
& -\frac{2 i(9+k)}{(5+k)^{3}} U B_{3} F_{11}+\frac{\left(-1365+116 k+391 k^{2}+78 k^{3}\right)}{3(5+k)^{3}(17+13 k)} F_{11} G_{22} F_{11} \\
& +\frac{8\left(-303+71 k+82 k^{2}\right)}{3(5+k)^{3}(17+13 k)} U \partial F_{11}-\frac{(63+19 k)}{(5+k)^{3}} U U F_{11} \\
& \left.-\frac{2\left(105+42 k+k^{2}\right)}{(5+k)^{4}} F_{11} \partial F_{11} F_{22}\right](w) \\
& +\frac{1}{(z-w)}\left[\frac{1}{5} \partial\left\{G_{21} \mathbf{Q}_{-}^{(\mathbf{3})}\right\}_{-2}+\mathbf{Q}^{\left(\frac{7}{2}\right)}\right. \\
& -\frac{16\left(21+137 k+52 k^{2}\right)}{(5+k)(17+13 k)(67+39 k)}\left(T U F_{11}-\frac{3}{8} \partial^{2}\left(U F_{11}\right)\right)
\end{aligned}
$$




$$
\begin{aligned}
& -\frac{48}{(5+k)(67+39 k)}\left(T F_{12} F_{21} F_{11}-\frac{3}{8} \partial^{2}\left(F_{12} F_{21} F_{11}\right)\right) \\
& -\frac{64 i\left(114+122 k+81 k^{2}+13 k^{3}\right)}{(5+k)^{2}(17+13 k)(67+39 k)}\left(T F_{11} A_{3}-\frac{3}{8} \partial^{2}\left(F_{11} A_{3}\right)\right) \\
& -\frac{16 i\left(21+21 k+4 k^{2}\right)}{(5+k)^{2}(67+39 k)}\left(T F_{21} A_{+}-\frac{3}{8} \partial^{2}\left(F_{21} A_{+}\right)\right) \\
& -\frac{16 i\left(21+86 k+13 k^{2}\right)}{(5+k)(17+13 k)(67+39 k)}\left(T F_{12} B_{-}-\frac{3}{8} \partial^{2}\left(F_{12} B_{-}\right)\right) \\
& +\frac{16 i\left(-105-247 k+73 k^{2}+39 k^{3}\right)}{(5+k)^{2}(17+13 k)(67+39 k)}\left(T F_{11} B_{3}-\frac{3}{8} \partial^{2}\left(F_{11} B_{3}\right)\right) \\
& +\frac{192 k\left(303+334 k+47 k^{2}\right)}{5(5+k)^{2}(17+13 k)(43+47 k)}\left(T \partial F_{11}-\frac{1}{4} \partial T F_{11}-\frac{1}{3} \partial^{3} F_{11}\right) \\
& \left.-\frac{4(2+k)\left(105+637 k+104 k^{2}\right)}{(5+k)(17+13 k)(67+39 k)}\left(T G_{11}-\frac{3}{8} \partial^{2} G_{11}\right)\right](w)+\cdots
\end{aligned}
$$

In the third order pole there is no higher spin current. It is clear that there are no higher spin currents in the context of footnote 5 which will appear in Appendix $F$. It is easy to check that the various terms in the first order pole are quasi primary fields. Note that the second term from the last expression has different relative coefficients $-\frac{1}{4}$ and $-\frac{1}{3}$ rather than $-\frac{3}{8}$. There are no nonlinear terms between the higher spin currents in the above.

Let us consider the OPE between the spin- $\frac{3}{2}$ current and the higher spin-3 current found in (5.4)

$$
\begin{aligned}
G_{21}(z) \mathbf{R}_{-}^{(3)}(w) & =\frac{1}{(z-4)^{4}} \frac{6 k\left(237+314 k+61 k^{2}\right)}{(5+k)^{3}(17+13 k)} F_{22}(w) \\
& +\frac{1}{(z-w)^{3}}\left[-\partial(\text { pole- } 4)-\frac{8\left(1683+1713 k+391 k^{2}+17 k^{3}\right)}{3(5+k)^{3}(17+13 k)} U F_{22}\right. \\
& -\frac{12}{(5+k)^{2}} F_{21} F_{12} F_{22}+\frac{4 i\left(-1683-1200 k+197 k^{2}+34 k^{3}\right)}{3(5+k)^{3}(17+13 k)} F_{22} A_{3} \\
& +\frac{4 i\left(1071+1212 k+431 k^{2}+34 k^{3}\right)}{3(5+k)^{3}(17+13 k)} F_{12} A- \\
& +\frac{4 i\left(66+15 k+k^{2}\right)}{(5+k)^{3}} F_{21} B_{+}+\frac{4 i\left(3366+3405 k+556 k^{2}+5 k^{3}\right)}{3(5+k)^{3}(17+13 k)} F_{22} B_{3} \\
& \left.-\frac{\left(6732+6753 k+1717 k^{2}+68 k^{3}\right)}{3(5+k)^{2}(17+13 k)} G_{22}\right](w) \\
& +\frac{1}{(z-w)^{2}}\left[\frac{4(7+k)}{(5+k)} \mathbf{R}^{\left(\frac{5}{2}\right)}-\frac{4(7+k)}{(5+k)} \mathbf{V}^{\left(\frac{5}{2}\right)}+\frac{9 i}{(5+k)^{2}} A_{3} G_{22}\right. \\
& +\frac{(75+7 k)}{(5+k)^{3}} A_{3} A_{3} F_{22}-\frac{10(9+k)}{(5+k)^{3}} A_{3} B_{3} F_{22}-\frac{9(9+k)}{(5+k)^{3}} A_{3} B_{+} F_{21}
\end{aligned}
$$




$$
\begin{aligned}
& -\frac{i(3+k)\left(765+842 k+61 k^{2}\right)}{3(5+k)^{3}(17+13 k)} \partial A_{3} F_{22}+\frac{3}{(5+k)^{2}} B_{3} B_{3} F_{22} \\
& +\frac{2 i\left(-6171-4954 k-263 k^{2}+24 k^{3}\right)}{3(5+k)^{3}(17+13 k)} A_{3} \partial F_{22}-\frac{3 i(1+k)}{2(5+k)^{2}} A_{-} G_{12} \\
& +\frac{3(13+k)}{(5+k)^{3}} A_{-} A_{3} F_{12}+\frac{4(9+k)}{(5+k)^{3}} A_{-} A_{+} F_{22}-\frac{(51+7 k)}{(5+k)^{3}} A_{-} B_{3} F_{12} \\
& +\frac{6(7+k)}{(5+k)^{3}} A_{-} B_{+} F_{11}-\frac{i\left(255+878 k+439 k^{2}+24 k^{3}\right)}{3(5+k)^{3}(17+13 k)} \partial A_{-} F_{12} \\
& +\frac{i\left(-1785-458 k+527 k^{2}+48 k^{3}\right)}{3(5+k)^{3}(17+13 k)} A_{-} \partial F_{12}+\frac{i(-12+k)}{(5+k)^{2}} B_{3} G_{22} \\
& -\frac{2 i\left(-2244-1067 k+430 k^{2}+37 k^{3}\right)}{3(5+k)^{3}(17+13 k)} B_{3} \partial F_{22}+\frac{(9+k)}{(5+k)^{3}} B_{+} B_{3} F_{21} \\
& -\frac{i\left(561+1144 k+311 k^{2}+24 k^{3}\right)}{2(5+k)^{2}(17+13 k)} B_{+} G_{21}+\frac{i(1+k)(21+2 k)}{3(5+k)^{3}} \partial B_{+} F_{21} \\
& -\frac{i\left(-183+13 k+4 k^{2}\right)}{3(5+k)^{3}} B_{+} \partial F_{21}+\frac{2(3+k)}{(5+k)^{3}} B_{-} B_{+} F_{22} \\
& -\frac{\left(21573+14907 k+2023 k^{2}+113 k^{3}\right)}{6(5+k)^{3}(17+13 k)} \partial^{2} F_{22}-\frac{4(9+k)}{(5+k)^{3}} F_{22} F_{21} G_{12} \\
& -\frac{2\left(-147-2 k+k^{2}\right)}{3(5+k)^{4}} \partial F_{22} F_{21} F_{12}+\frac{4\left(177+88 k+7 k^{2}\right)}{3(5+k)^{4}} F_{22} \partial F_{21} F_{12} \\
& -\frac{2\left(501+178 k+13 k^{2}\right)}{3(5+k)^{4}} F_{22} F_{21} \partial F_{12}-\frac{2(15+k)}{(5+k)^{3}} F_{22} F_{11} G_{22} \\
& -\frac{2(3+k)(-1+3 k)(17+4 k)}{(5+k)^{3}(17+13 k)} F_{22} F_{12} G_{21}-\frac{5(9+k)}{(5+k)^{3}} F_{21} F_{12} G_{22} \\
& +\frac{\left(867+1310 k+259 k^{2}+24 k^{3}\right)}{6(5+k)^{2}(17+13 k)} \partial G_{22}+\frac{\left(663+338 k+43 k^{2}\right)}{(5+k)^{2}(17+13 k)} T F_{22} \\
& +\frac{2(12+k)}{(5+k)^{2}} U G_{22}-\frac{4 i(3+k)}{(5+k)^{3}} U A_{3} F_{22}-\frac{i(51+7 k)}{(5+k)^{3}} U A_{-} F_{12} \\
& -\frac{8 i(9+k)}{(5+k)^{3}} U B_{3} F_{22}-\frac{9 i(9+k)}{(5+k)^{3}} U B_{+} F_{21}-\frac{\left(-21+52 k+k^{2}\right)}{3(5+k)^{3}} \partial U F_{22} \\
& -\frac{2\left(-663-538 k-107 k^{2}+24 k^{3}\right)}{3(5+k)^{3}(17+13 k)} U \partial F_{22}+\frac{(87+11 k)}{(5+k)^{3}} U U F_{22} \\
& +\frac{\left(510+1345 k+664 k^{2}+37 k^{3}\right)}{3(5+k)^{3}(17+13 k)} F_{22} G_{11} F_{22} \\
& \left.+\frac{8\left(15+12 k+k^{2}\right)}{(5+k)^{4}} F_{22} \partial F_{22} F_{11}\right](w) \\
& +\frac{1}{(z-w)}\left[\frac{1}{5} \partial\left\{G_{21} \mathbf{R}_{-}^{(\mathbf{3})}\right\}_{-2}+\mathbf{R}^{\left(\frac{7}{2}\right)}\right.
\end{aligned}
$$




$$
\begin{aligned}
& -\frac{32\left(1683+1713 k+391 k^{2}+17 k^{3}\right)}{3(5+k)^{2}(17+13 k)(67+39 k)}\left(T U F_{22}-\frac{3}{8} \partial^{2}\left(U F_{22}\right)\right) \\
& -\frac{48}{(5+k)(67+39 k)}\left(T F_{21} F_{12} F_{22}-\frac{3}{8} \partial^{2}\left(F_{21} F_{12} F_{22}\right)\right) \\
& +\frac{16 i\left(-1683-1200 k+197 k^{2}+34 k^{3}\right)}{3(5+k)^{2}(17+13 k)(67+39 k)}\left(T F_{22} A_{3}-\frac{3}{8} \partial^{2}\left(F_{22} A_{3}\right)\right) \\
& +\frac{16 i\left(1071+1212 k+431 k^{2}+34 k^{3}\right)}{3(5+k)^{2}(17+13 k)(67+39 k)}\left(T F_{12} A_{-}-\frac{3}{8} \partial^{2}\left(F_{12} A_{-}\right)\right) \\
& +\frac{16 i\left(66+15 k+k^{2}\right)}{(5+k)^{2}(67+39 k)}\left(T F_{21} B_{+}-\frac{3}{8} \partial^{2}\left(F_{21} B_{+}\right)\right) \\
& +\frac{16 i\left(3366+3405 k+556 k^{2}+5 k^{3}\right)}{3(5+k)^{2}(17+13 k)(67+39 k)}\left(T F_{22} B_{3}-\frac{3}{8} \partial^{2}\left(F_{22} B_{3}\right)\right) \\
& -\frac{192 k\left(237+314 k+61 k^{2}\right)}{5(5+k)^{2}(17+13 k)(43+47 k)}\left(T \partial F_{22}-\frac{1}{4} \partial T F_{22}-\frac{1}{3} \partial^{3} F_{22}\right) \\
& \left.-\frac{4\left(6732+6753 k+1717 k^{2}+68 k^{3}\right)}{3(5+k)(17+13 k)(67+39 k)}\left(T G_{22}-\frac{3}{8} \partial^{2} G_{22}\right)\right](w) \\
& +\quad \cdots
\end{aligned}
$$

There is no boldface higher spin current in the third order pole. As before, various nonlinear terms in Part II do not appear in this OPE. In this case also, various quasi primary fields appear in the first order pole. Furthermore, there are no nonlinear terms between the higher spin currents in the above.

Let us consider the OPE between the spin- $\frac{3}{2}$ current and the higher spin-3 current found in $(5.6)$

$$
\begin{aligned}
G_{21}(z) \mathbf{S}^{(3)}(w) & =\frac{1}{(z-4)^{4}} \frac{12 k\left(189+182 k+25 k^{2}\right)}{(5+k)^{3}(17+13 k)} F_{21}(w) \\
& +\frac{1}{(z-w)^{3}}\left[-\partial(\text { pole- } 4)-\frac{4(-3+k)(113+61 k)}{3(5+k)(17+13 k)} \mathbf{T}_{+}^{\left(\frac{3}{2}\right)}\right. \\
& +\frac{2\left(3987+5676 k+1895 k^{2}+190 k^{3}\right)}{3(5+k)^{3}(17+13 k)} U F_{21} \\
& +\frac{8(-15+k)}{(5+k)^{3}} F_{21} F_{11} F_{22}-\frac{2 i\left(3375+4596 k+1427 k^{2}+190 k^{3}\right)}{3(5+k)^{3}(17+13 k)} F_{21} A_{3} \\
& +\frac{2 i\left(3969+3744 k+1325 k^{2}+190 k^{3}\right)}{3(5+k)^{3}(17+13 k)} F_{11} A_{-} \\
& +\frac{2 i\left(-3987-4788 k-751 k^{2}+34 k^{3}\right)}{3(5+k)^{3}(17+13 k)} F_{22} B_{-} \\
& -\frac{2 i\left(-3987-3432 k+433 k^{2}+278 k^{3}\right)}{3(5+k)^{3}(17+13 k)} F_{21} B_{3}
\end{aligned}
$$




$$
\begin{aligned}
& \left.+\frac{\left(7377+6955 k+1578 k^{2}+68 k^{3}\right)}{3(5+k)^{2}(17+13 k)} G_{21}\right](w) \\
& +\frac{1}{(z-w)^{2}}\left[-\frac{2\left(4239+5758 k+1735 k^{2}+40 k^{3}\right)}{(5+k)^{3}(17+13 k)} F_{21} \mathbf{T}_{-}^{\left(\frac{3}{2}\right)} F_{21}\right. \\
& -\frac{\left(489-142 k-223 k^{2}+8 k^{3}\right)}{4(5+k)(17+13 k)}\left(F_{21} \mathbf{P}^{(\mathbf{2})}-\mathbf{P}^{(\mathbf{2})} F_{21}\right) \\
& -\frac{(-3+k)}{(5+k)} \mathbf{P}_{+}^{\left(\frac{5}{2}\right)}-\frac{2(11+3 k)}{(5+k)} \mathbf{W}_{+}^{\left(\frac{5}{2}\right)} \\
& -\frac{6(-\mathbf{3}+\mathbf{k})}{(17+13 k)} G_{21} \mathbf{T}^{(\mathbf{1})}+\frac{\left(369-126 k-215 k^{2}+8 k^{3}\right)}{(5+k)(17+13 k)} \partial \mathbf{T}_{+}^{\left(\frac{\mathbf{3}}{2}\right)} \\
& +\frac{2 i\left(14883+8577 k-847 k^{2}-341 k^{3}+24 k^{4}\right)}{3(5+k)^{4}(17+13 k)} F_{21}\left(\left(F_{11} B_{+}\right) F_{21}\right) \\
& +\frac{8 i\left(77+30 k+k^{2}\right)}{(5+k)^{4}} F_{21}\left(\left(F_{12} B_{3}\right) F_{21}\right)-\frac{10(3+k)}{(5+k)^{3}} B_{-} B_{3} F_{22} \\
& -\frac{2(1+k)}{(5+k)^{3}} A_{3} A_{3} F_{21}+\frac{2(29+9 k)}{(5+k)^{3}} A_{3} B_{3} F_{21}-\frac{4(7+2 k)}{(5+k)^{3}} A_{3} B_{-} F_{22} \\
& +\frac{2 i\left(119+53 k+2 k^{2}\right)}{(5+k)^{3}} \partial A_{3} F_{21}-\frac{3 i\left(389+237 k+28 k^{2}\right)}{(5+k)^{2}(17+13 k)} A_{3} G_{21} \\
& -\frac{4 i(-17+5 k)}{(5+k)^{3}} A_{3} \partial F_{21}+\frac{i(9+2 k)}{(5+k)^{2}} A_{-} G_{11}-\frac{2 i(3+k)}{(5+k)^{2}} B_{-} G_{22} \\
& +\frac{2(3+k)}{(5+k)^{3}} A_{-} A_{3} F_{11}+\frac{4}{(5+k)^{3}} A_{+} A_{-} F_{21}-\frac{4(16+5 k)}{(5+k)^{3}} A_{-} B_{3} F_{11} \\
& -\frac{2(17+5 k)}{(5+k)^{3}} A_{-} B_{-} F_{12}-\frac{4 i\left(141-422 k-262 k^{2}+9 k^{3}\right)}{(5+k)^{3}(17+13 k)} \partial A_{-} F_{11} \\
& -\frac{i\left(3397+254 k-879 k^{2}+24 k^{3}\right)}{(5+k)^{3}(17+13 k)} A_{-} \partial F_{11}-\frac{4(5+2 k)}{(5+k)^{3}} F_{11} F_{21} G_{22} \\
& -\frac{3 i\left(51+161 k+54 k^{2}+4 k^{3}\right)}{(5+k)^{2}(17+13 k)} B_{3} G_{21}+\frac{8(5+2 k)}{(5+k)^{3}} B_{3} B_{3} F_{21} \\
& -\frac{8 i(14+k)}{(5+k)^{3}} B_{3} \partial F_{21}+\frac{4\left(157+76 k+7 k^{2}\right)}{(5+k)^{4}} F_{11} F_{21} \partial F_{22} \\
& +\frac{i\left(1971+1491 k-535 k^{2}-527 k^{3}+24 k^{4}\right)}{3(5+k)^{3}(17+13 k)} \partial B_{-} F_{22} \\
& +\frac{i\left(1122+903 k-2020 k^{2}-881 k^{3}+24 k^{4}\right)}{3(5+k)^{3}(17+13 k)} B_{-} \partial F_{22} \\
& +\frac{2(5+3 k)}{(5+k)^{3}} B_{+} B_{-} F_{21}+\frac{\left(-317+458 k+195 k^{2}+12 k^{3}\right)}{(5+k)^{3}(17+13 k)} F_{11} F_{22} G_{21} \\
& -\frac{2\left(8253+855 k-2977 k^{2}-419 k^{3}+24 k^{4}\right)}{3(5+k)^{4}(17+13 k)} F_{11} \partial F_{21} F_{22}
\end{aligned}
$$




$$
\begin{aligned}
& -\frac{3\left(389+286 k+77 k^{2}+4 k^{3}\right)}{(5+k)^{3}(17+13 k)} F_{12} F_{21} G_{21}-\frac{2(7+k)}{(5+k)^{3}} F_{21} F_{22} G_{11} \\
& -\frac{\left(-5007+1968 k+1205 k^{2}-770 k^{3}+24 k^{4}\right)}{3(5+k)^{3}(17+13 k)} \partial^{2} F_{21} \\
& +\frac{\left(882-543 k-1060 k^{2}-187 k^{3}+8 k^{4}\right)}{2(5+k)^{2}(17+13 k)} \partial G_{21}-\frac{20 i(3+k)}{(5+k)^{3}} U A_{3} F_{21} \\
& +\frac{2(17+2 k)(11+7 k)}{(5+k)^{2}(17+13 k)} T F_{21}+\frac{2\left(153+109 k+32 k^{2}\right)}{(5+k)^{2}(17+13 k)} U G_{21} \\
& +\frac{18 i(3+k)}{(5+k)^{3}} U A_{-} F_{11}+\frac{2 i(9+k)}{(5+k)^{3}} U B_{3} F_{21}-\frac{12 i(4+k)}{(5+k)^{3}} U B_{-} F_{22} \\
& \left.+\frac{4\left(819+1417 k+493 k^{2}+7 k^{3}\right)}{(5+k)^{3}(17+13 k)} \partial U F_{21}+\frac{2(29+9 k)}{(5+k)^{3}} U U F_{21}\right](w) \\
& +\frac{1}{(z-w)}\left[\frac{1}{5} \partial\left\{G_{21} \mathbf{S}^{(\mathbf{3})}\right\}_{-2}\right. \\
& -\frac{16(-3+k)(113+61 k)}{3(17+13 k)(67+39 k)}\left(T \mathbf{T}_{+}^{\left(\frac{3}{2}\right)}-\frac{3}{8} \partial^{2} \mathbf{T}_{+}^{\left(\frac{3}{2}\right)}\right)+\mathbf{S}_{+}^{\left(\frac{7}{2}\right)} \\
& +\frac{8\left(3987+5676 k+1895 k^{2}+190 k^{3}\right)}{3(5+k)^{2}(17+13 k)(67+39 k)}\left(T U F_{21}-\frac{3}{8} \partial^{2}\left(U F_{21}\right)\right) \\
& +\frac{32(-15+k)}{(5+k)^{2}(67+39 k)}\left(T F_{21} F_{11} F_{22}-\frac{3}{8} \partial^{2}\left(F_{21} F_{11} F_{22}\right)\right) \\
& -\frac{8 i\left(3375+4596 k+1427 k^{2}+190 k^{3}\right)}{3(5+k)^{2}(17+13 k)(67+39 k)}\left(T F_{21} A_{3}-\frac{3}{8} \partial^{2}\left(F_{21} A_{3}\right)\right) \\
& +\frac{8 i\left(3969+3744 k+1325 k^{2}+190 k^{3}\right)}{3(5+k)^{2}(17+13 k)(67+39 k)}\left(T F_{11} A_{-}-\frac{3}{8} \partial^{2}\left(F_{11} A_{-}\right)\right) \\
& +\frac{8 i\left(-3987-4788 k-751 k^{2}+34 k^{3}\right)}{3(5+k)^{2}(17+13 k)(67+39 k)}\left(T F_{22} B_{-}-\frac{3}{8} \partial^{2}\left(F_{22} B_{-}\right)\right) \\
& -\frac{8 i\left(-3987-3432 k+433 k^{2}+278 k^{3}\right)}{3(5+k)^{2}(17+13 k)(67+39 k)}\left(T F_{21} B_{3}-\frac{3}{8} \partial^{2}\left(F_{21} B_{3}\right)\right) \\
& -\frac{384 k\left(189+182 k+25 k^{2}\right)}{5(5+k)^{2}(17+13 k)(43+47 k)}\left(T \partial F_{21}-\frac{1}{4} \partial T F_{21}-\frac{1}{3} \partial^{3} F_{21}\right) \\
& \left.+\frac{4\left(7377+6955 k+1578 k^{2}+68 k^{3}\right)}{3(5+k)(17+13 k)(67+39 k)}\left(T G_{21}-\frac{3}{8} \partial^{2} G_{21}\right)\right](w) \\
& +\ldots \text {. }
\end{aligned}
$$

One can reexpress the first term in the second order pole by moving the higher spin current to the right hand side and will be written in term of known other terms. Note that one can rewrite the two terms containing the higher spin-2 current $\mathbf{P}^{(\mathbf{2})}(w)$ in terms of the known other currents and then there will be no $\mathbf{P}^{(\mathbf{2})}(w)$ dependence eventually. From the previous experience, one can consider the nonlinear term between the $G_{21}$ and the higher spin-1 current 
and this will be removed by redefining the spin-3 current with $T \mathbf{T}^{(\mathbf{1})}(w)$ into the left hand side. In the first order pole, one sees many quasi primary fields. One can also consider the OPE between the $G_{21}(z)$ and the quasi primary field appearing in (4.7). Then from the results in Appendix $C$, the terms having $\mathbf{T}_{+}^{\left(\frac{3}{2}\right)}(w)$ in the second term of the first order pole can be seen from this OPE. Then, those nonlinear terms can be removed in the redefined higher spin-3 current by adding the quasi primary field to the left hand side of above OPE.

Similarly one has

$$
\begin{aligned}
& G_{12}(z) \mathbf{S}^{(3)}(w)=\frac{1}{(z-4)^{4}} \frac{48 k\left(39+43 k+8 k^{2}\right)}{(5+k)^{3}(17+13 k)} F_{12}(w) \\
& +\frac{1}{(z-w)^{3}}\left[-\partial(\text { pole- } 4)-\frac{4(-3+k)(113+61 k)}{3(5+k)(17+13 k)} \mathbf{T}_{-}^{\left(\frac{3}{2}\right)}\right. \\
& -\frac{2\left(3375+5412 k+2051 k^{2}+190 k^{3}\right)}{3(5+k)^{3}(17+13 k)} U F_{12} \\
& -\frac{32 k}{(5+k)^{3}} F_{12} F_{11} F_{22}-\frac{2 i\left(-5193-2364 k+1115 k^{2}+190 k^{3}\right)}{3(5+k)^{3}(17+13 k)} F_{12} A_{3} \\
& +\frac{2 i\left(909+1608 k+1481 k^{2}+190 k^{3}\right)}{3(5+k)^{3}(17+13 k)} F_{22} A_{+} \\
& \text {- } \frac{2 i\left(3375+4728 k+1267 k^{2}+122 k^{3}\right)}{3(5+k)^{3}(17+13 k)} F_{11} B_{+} \\
& +\frac{2 i\left(3375+4596 k+1427 k^{2}+190 k^{3}\right)}{3(5+k)^{3}(17+13 k)} F_{12} B_{3} \\
& \left.-\frac{\left(6765+6691 k+1734 k^{2}+68 k^{3}\right)}{3(5+k)^{2}(17+13 k)} G_{12}\right](w) \\
& +\frac{1}{(z-w)^{2}}\left[-\frac{\left(-523+65 k+235 k^{2}+31 k^{3}\right)}{8(5+k)^{2}(17+13 k)}\left(F_{12} \mathbf{P}^{(\mathbf{2})}-\mathbf{P}^{(\mathbf{2})} F_{12}\right)\right. \\
& -\frac{(-3+k)}{(5+k)} \mathbf{P}_{-}^{\left(\frac{5}{2}\right)}+\frac{2(11+3 k)}{(5+k)} \mathbf{W}_{-}^{\left(\frac{5}{2}\right)} \\
& -\frac{6(-\mathbf{3}+\mathbf{k})}{(17+13 k)} G_{12} \mathbf{T}^{(\mathbf{1})}-\frac{\left(677+145 k+123 k^{2}+15 k^{3}\right)}{2(5+k)^{2}(17+13 k)} \partial \mathbf{T}_{-}^{\left(\frac{3}{2}\right)} \\
& +\frac{2(35+3 k)}{(5+k)^{3}} A_{3} A_{3} F_{12}+\frac{2(41+5 k)}{(5+k)^{3}} A_{3} B_{3} F_{12}-\frac{(91+11 k)}{(5+k)^{3}} A_{3} B_{+} F_{11} \\
& +\frac{i\left(77+24 k+3 k^{2}\right)}{(5+k)^{3}} \partial A_{3} F_{12}-\frac{3 i\left(693+392 k+43 k^{2}\right)}{2(5+k)^{2}(17+13 k)} A_{3} G_{12} \\
& +\frac{i\left(4143+4325 k+421 k^{2}+15 k^{3}\right)}{2(5+k)^{3}(17+13 k)} A_{3} \partial F_{12}-\frac{i(9+k)}{(5+k)^{2}} A_{+} G_{22} \\
& -\frac{5(9+k)}{(5+k)^{3}} A_{+} A_{3} F_{22}+\frac{(25+k)}{(5+k)^{3}} A_{+} A_{-} F_{12}-\frac{(37+5 k)}{(5+k)^{3}} A_{+} B_{3} F_{22}
\end{aligned}
$$




$$
\begin{aligned}
& -\frac{2(23+3 k)}{(5+k)^{3}} A_{+} B_{+} F_{21}-\frac{i\left(263+1269 k+469 k^{2}+39 k^{3}\right)}{2(5+k)^{3}(17+13 k)} \partial A_{+} F_{22} \\
& -\frac{i\left(-1347-1703 k-181 k^{2}+15 k^{3}\right)}{2(5+k)^{3}(17+13 k)} A_{+} \partial F_{22} \\
& -\frac{3 i\left(187+404 k+121 k^{2}+8 k^{3}\right)}{2(5+k)^{2}(17+13 k)} B_{3} G_{12}-\frac{8}{(5+k)^{3}} B_{3} B_{3} F_{12} \\
& -\frac{i\left(-2861-759 k+833 k^{2}+171 k^{3}\right)}{2(5+k)^{3}(17+13 k)} B_{3} \partial F_{12}+\frac{i(12+k)}{(5+k)^{2}} B_{+} G_{11} \\
& +\frac{(9+k)}{(5+k)^{3}} B_{+} B_{3} F_{11}-\frac{i\left(2091+3441 k+1361 k^{2}+139 k^{3}\right)}{6(5+k)^{3}(17+13 k)} \partial B_{+} F_{11} \\
& +\frac{i\left(-11337-2229 k+3745 k^{2}+557 k^{3}\right)}{6(5+k)^{3}(17+13 k)} B_{+} \partial F_{11}+\frac{(1+k)}{(5+k)^{3}} B_{+} B_{-} F_{12} \\
& +\frac{(35+3 k)}{(5+k)^{3}} F_{11} F_{12} G_{22}+\frac{4\left(6165+6006 k+1313 k^{2}+64 k^{3}\right)}{3(5+k)^{4}(17+13 k)} F_{11} F_{12} \partial F_{22} \\
& -\frac{2\left(5979+9435 k+3629 k^{2}+301 k^{3}\right)}{3(5+k)^{4}(17+13 k)} \partial F_{11} F_{12} F_{22}+\frac{9 i(9+k)}{(5+k)^{3}} U B_{+} F_{11} \\
& -\frac{4\left(822+1581 k+556 k^{2}+53 k^{3}\right)}{3(5+k)^{4}(17+13 k)} F_{11} \partial F_{12} F_{22}-\frac{10 i(9+k)}{(5+k)^{3}} U B_{3} F_{12} \\
& +\frac{\left(-1507-690 k+13 k^{2}+12 k^{3}\right)}{(5+k)^{3}(17+13 k)} F_{11} F_{22} G_{12}-\frac{3 i(19+3 k)}{(5+k)^{3}} U A_{+} F_{22} \\
& -\frac{3\left(491+330 k+51 k^{2}+4 k^{3}\right)}{(5+k)^{3}(17+13 k)} F_{12} F_{21} G_{12}+\frac{(11+3 k)}{(5+k)^{3}} F_{12} F_{22} G_{11} \\
& +\frac{\left(-14013-9129 k-587 k^{2}+65 k^{3}\right)}{6(5+k)^{3}(17+13 k)} \partial^{2} F_{12} \\
& +\frac{\left(551+529 k+261 k^{2}+27 k^{3}\right)}{2(5+k)^{2}(17+13 k)} \partial G_{12}+\frac{4 i(3+k)}{(5+k)^{3}} U A_{3} F_{12} \\
& +\frac{\left(425+268 k+27 k^{2}\right)}{(5+k)^{2}(17+13 k)} T F_{12}+\frac{\left(969+590 k+37 k^{2}\right)}{2(5+k)^{2}(17+13 k)} U G_{12} \\
& +\frac{\left(-277+877 k+369 k^{2}+15 k^{3}\right)}{2(5+k)^{3}(17+13 k)} U \partial F_{12}+\frac{2(41+5 k)}{(5+k)^{3}} U U F_{12} \\
& \left.+\frac{\left(25+12 k+3 k^{2}\right)}{2(5+k)^{3}} F_{12} G_{21} F_{12}+\frac{4\left(47+34 k+3 k^{2}\right)}{(5+k)^{4}} F_{12} \partial F_{12} F_{21}\right](w) \\
& +\frac{1}{(z-w)}\left[\frac{1}{5} \partial\left\{G_{12} \mathbf{S}^{(3)}\right\}_{-2}\right. \\
& -\frac{16(-3+k)(113+61 k)}{3(17+13 k)(67+39 k)}\left(T \mathbf{T}_{-}^{\left(\frac{3}{2}\right)}-\frac{3}{8} \partial^{2} \mathbf{T}_{-}^{\left(\frac{\mathbf{3}}{2}\right)}\right)+\mathbf{S}_{-}^{\left(\frac{7}{2}\right)} \\
& -\frac{8\left(3375+5412 k+2051 k^{2}+190 k^{3}\right)}{3(5+k)^{2}(17+13 k)(67+39 k)}\left(T U F_{12}-\frac{3}{8} \partial^{2}\left(U F_{12}\right)\right)
\end{aligned}
$$




$$
\begin{aligned}
& -\frac{128 k}{(5+k)^{2}(67+39 k)}\left(T F_{12} F_{11} F_{22}-\frac{3}{8} \partial^{2}\left(F_{12} F_{11} F_{22}\right)\right) \\
& -\frac{8 i\left(-5193-2364 k+1115 k^{2}+190 k^{3}\right)}{3(5+k)^{2}(17+13 k)(67+39 k)}\left(T F_{12} A_{3}-\frac{3}{8} \partial^{2}\left(F_{12} A_{3}\right)\right) \\
& +\frac{8 i\left(909+1608 k+1481 k^{2}+190 k^{3}\right)}{3(5+k)^{2}(17+13 k)(67+39 k)}\left(T F_{22} A_{+}-\frac{3}{8} \partial^{2}\left(F_{22} A_{+}\right)\right) \\
& -\frac{8 i\left(3375+4728 k+1267 k^{2}+122 k^{3}\right)}{3(5+k)^{2}(17+13 k)(67+39 k)}\left(T F_{11} B_{+}-\frac{3}{8} \partial^{2}\left(F_{11} B_{+}\right)\right) \\
& +\frac{8 i\left(3375+4596 k+1427 k^{2}+190 k^{3}\right)}{3(5+k)^{2}(17+13 k)(67+39 k)}\left(T F_{12} B_{3}-\frac{3}{8} \partial^{2}\left(F_{12} B_{3}\right)\right) \\
& -\frac{1536 k\left(39+43 k+8 k^{2}\right)}{5(5+k)^{2}(17+13 k)(43+47 k)}\left(T \partial F_{12}-\frac{1}{4} \partial T F_{12}-\frac{1}{3} \partial^{3} F_{12}\right) \\
& \left.-\frac{4\left(6765+6691 k+1734 k^{2}+68 k^{3}\right)}{3(5+k)(17+13 k)(67+39 k)}\left(T G_{12}-\frac{3}{8} \partial^{2} G_{12}\right)\right](w) \\
& +\quad \ldots .
\end{aligned}
$$

Note that one can rewrite the two terms containing the higher spin-2 current $\mathbf{P}^{(\mathbf{2})}(w)$ in terms of the known other currents and then there will be no $\mathbf{P}^{(2)}(w)$ dependence eventually. The nonlinear term between the $G_{12}$ and the higher spin-1 current will be removed by redefining the spin-3 current with $T \mathbf{T}^{(\mathbf{1})}(w)$ into the left hand side. The terms having $\mathbf{T}_{-}^{\left(\frac{\mathbf{3}}{2}\right)}(w)$ in the second term of the first order pole can be removed in the redefined higher spin-3 current by adding the quasi primary field in (4.7) to the left hand side of above OPE.

Let us consider the OPE between the spin- $\frac{3}{2}$ current and the higher spin- $\frac{7}{2}$ current found in previous OPE

$$
\begin{aligned}
G_{21}(z) \mathbf{S}_{-}^{\left(\frac{7}{2}\right)}(w) & =\frac{1}{(z-w)^{4}}\left[-\frac{16(\mathbf{k}-\mathbf{3})(23 k+19)(61 k+113)}{15(k+5)(13 k+17)(39 k+67)} \mathbf{T}^{(\mathbf{1})}\right. \\
& -\frac{8 i}{5(k+5)^{3}(13 k+17)(39 k+67)(47 k+43)} \\
& \times\left(812455 k^{5}+6422062 k^{4}+16948458 k^{3}+20543408 k^{2}+11175575 k\right. \\
& +1957962) A_{3}+\frac{8 i k}{15(k+5)^{3}(13 k+17)(39 k+67)(47 k+43)} \\
& \times\left(410780 k^{5}+10154773 k^{4}+64740756 k^{3}+155849878 k^{2}\right. \\
& +159556664 k+58487229) B_{3} \\
& -\frac{24 k\left(6277 k^{3}+34435 k^{2}+48679 k+19689\right)}{5(k+5)^{3}(13 k+17)(47 k+43)} U \\
& -\frac{16}{15(k+5)^{4}(13 k+17)(39 k+67)}\left(4370 k^{5}+94701 k^{4}+517032 k^{3}\right. \\
& \left.+914974 k^{2}+592158 k+68301\right) F_{11} F_{22}
\end{aligned}
$$




$$
\begin{aligned}
& +\frac{16}{15(k+5)^{4}(13 k+17)(39 k+67)}\left(4370 k^{5}+63819 k^{4}+274248 k^{3}\right. \\
& \left.\left.+520234 k^{2}+269646 k-68301\right) F_{12} F_{21}\right](w) \\
& +\frac{1}{(z-w)^{3}}\left[\frac{12(k-3)}{5(k+5)} \mathbf{P}^{(\mathbf{2})}-\frac{48(k-3)}{5(k+5)} \mathbf{W}^{(\mathbf{2})}\right. \\
& \text { - } \frac{16(k-3)\left(1543 k^{3}+225 k^{2}-10179 k-11101\right)}{15(k+5)^{2}(13 k+17)(39 k+67)} \mathbf{T}^{(\mathbf{2})} \\
& +\frac{128 i(\mathbf{k}-\mathbf{3})}{5(k+5)(13 k+17)} A_{3} \mathbf{T}^{(\mathbf{1})}+\frac{32 i(\mathbf{k}-\mathbf{3})(k+1)}{5(k+5)(13 k+17)} B_{3} \mathbf{T}^{(\mathbf{1})} \\
& +\frac{8(\mathbf{k}-\mathbf{3})(23 k+19)(61 k+113)}{15(k+5)(13 k+17)(39 k+67)} \partial \mathbf{T}^{(\mathbf{1})} \\
& -\frac{16\left(1613 k^{4}+18563 k^{3}+106625 k^{2}+284877 k+223074\right)}{15(k+5)^{2}(13 k+17)(39 k+67)} T \\
& -\frac{16\left(6218 k^{4}+72179 k^{3}+335333 k^{2}+710217 k+525069\right)}{15(k+5)^{3}(13 k+17)(39 k+67)} A_{3} A_{3} \\
& -\frac{16\left(7922 k^{4}+90179 k^{3}+180017 k^{2}-14607 k-169263\right)}{15(k+5)^{3}(13 k+17)(39 k+67)} A_{3} B_{3} \\
& +\frac{4 i}{15(k+5)^{3}(13 k+17)(39 k+67)(47 k+43)} \\
& \times\left(1268381 k^{5}+5934390 k^{4}-9953250 k^{3}-74286536 k^{2}-106361763 k\right.
\end{aligned}
$$

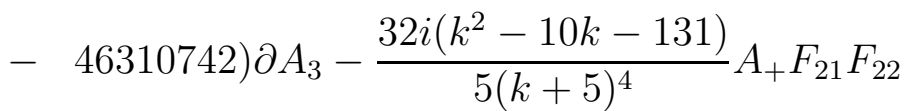

$$
\begin{aligned}
& \text { - } \frac{64 i\left(637 k^{4}-1209 k^{3}-34981 k^{2}-100635 k-79596\right)}{5(k+5)^{4}(13 k+17)(39 k+67)} A_{3} F_{11} F_{22} \\
& +\frac{48 i\left(27 k^{3}+261 k^{2}+937 k+1023\right)}{5(k+5)^{4}(13 k+17)} A_{3} F_{12} F_{21} \\
& +\frac{16 i\left(125 k^{3}+2287 k^{2}+5575 k+4757\right)}{5(k+5)^{4}(39 k+67)} A_{-} F_{11} F_{12} \\
& -\frac{16\left(6218 k^{4}+65225 k^{3}+263723 k^{2}+481683 k+303399\right)}{15(k+5)^{3}(13 k+17)(39 k+67)} A_{+} A_{-} \\
& -\frac{32\left(234 k^{5}-701 k^{4}+6760 k^{3}+88144 k^{2}+185490 k+111537\right)}{15(k+5)^{3}(13 k+17)(39 k+67)} B_{3} B_{3} \\
& -\frac{4 i}{15(k+5)^{3}(13 k+17)(39 k+67)(47 k+43)} \\
& \times\left(410780 k^{6}+10444857 k^{5}+73693068 k^{4}\right. \\
& \left.+211858366 k^{3}+283781640 k^{2}+173849361 k+38368728\right) \partial B_{3} \\
& -\frac{32 i\left(78 k^{5}-243 k^{4}+6026 k^{3}+65908 k^{2}+161880 k+113967\right)}{5(k+5)^{4}(13 k+17)(39 k+67)} B_{3} F_{11} F_{22}
\end{aligned}
$$




$$
\begin{aligned}
& +\frac{16 i\left(4 k^{4}+107 k^{3}+813 k^{2}+2649 k+2259\right)}{5(k+5)^{4}(13 k+17)} B_{3} F_{12} F_{21} \\
& +\frac{32 i\left(191 k^{3}+423 k^{2}-1527 k-3551\right)}{5(k+5)^{4}(39 k+67)} B_{-} F_{12} F_{22} \\
& -\frac{16\left(1543 k^{4}+46207 k^{3}+255643 k^{2}+426885 k+223074\right)}{15(k+5)^{3}(13 k+17)(39 k+67)} B_{+} B_{-} \\
& -\frac{16 i\left(11 k^{2}+78 k+227\right)}{5(k+5)^{4}} B_{+} F_{11} F_{21} \\
& +\frac{8\left(16687 k^{4}+122554 k^{3}+344764 k^{2}+530214 k+322677\right)}{15(k+5)^{3}(13 k+17)(39 k+67)} F_{11} G_{22} \\
& -\frac{8}{15(k+5)^{4}(13 k+17)(39 k+67)}\left(8066 k^{5}+12481 k^{4}\right. \\
& \left.+374680 k^{3}+3383510 k^{2}+7061982 k+4345953\right) \partial F_{11} F_{22} \\
& +\frac{8}{15(k+5)^{4}(13 k+17)(39 k+67)}\left(16806 k^{5}+280975 k^{4}\right. \\
& \left.+2000920 k^{3}+6245866 k^{2}+8394762 k+4004175\right) F_{11} \partial F_{22} \\
& -\frac{4}{5(k+5)^{3}(13 k+17)(39 k+67)(47 k+43)}\left(212589 k^{5}+2511712 k^{4}\right. \\
& \left.+6213530 k^{3}+971776 k^{2}-8108847 k-4883080\right) F_{12} G_{21} \\
& -\frac{8}{5(k+5)^{4}(13 k+17)(39 k+67)}\left(5602 k^{5}+84477 k^{4}\right. \\
& \left.+531736 k^{3}+1929966 k^{2}+3036574 k+1647789\right) \partial F_{12} F_{21} \\
& +\frac{8}{15(k+5)^{4}(13 k+17)(39 k+67)}\left(8066 k^{5}+78369 k^{4}\right. \\
& \left.+751944 k^{3}+3118438 k^{2}+5205102 k+2947761\right) F_{12} \partial F_{21} \\
& +\frac{8\left(165 k^{3}+1186 k^{2}+1351 k+34\right)}{5(k+5)^{3}(13 k+17)} F_{21} G_{12} \\
& -\frac{16\left(1387 k^{4}+35356 k^{3}+179710 k^{2}+272436 k+127143\right)}{15(k+5)^{3}(13 k+17)(39 k+67)} F_{22} G_{11} \\
& +\frac{48 i\left(13 k^{3}+4187 k^{2}+2431 k-4879\right)}{5(k+5)^{3}(13 k+17)(39 k+67)} U A_{3} \\
& +\frac{32 i k\left(1443 k^{3}+20601 k^{2}+53657 k+38083\right)}{5(k+5)^{3}(13 k+17)(39 k+67)} U B_{3} \\
& +\frac{12 k\left(6277 k^{3}+34435 k^{2}+48679 k+19689\right)}{5(k+5)^{3}(13 k+17)(47 k+43)} \partial U \\
& -\frac{32\left(3109 k^{4}+39658 k^{3}+154630 k^{2}+225810 k+111537\right)}{15(k+5)^{3}(13 k+17)(39 k+67)} U U \\
& +\frac{64\left(76 k^{3}+373 k^{2}+2126 k+2613\right)}{5(k+5)^{4}(39 k+67)} U F_{11} F_{22}
\end{aligned}
$$




$$
\begin{aligned}
& \left.-\frac{16(13 k-7)}{5(k+5)^{3}} U F_{12} F_{21}\right](w) \\
& +\frac{1}{(z-w)^{2}}\left[\frac{6(k-3)}{5(k+5)} \mathbf{P}^{(\mathbf{3})}+6 \mathbf{S}^{(\mathbf{3})}-\frac{12(3 k+11)}{5(k+5)} \mathbf{W}^{(\mathbf{3})}\right. \\
& +\frac{96 i(\mathbf{k}-\mathbf{3})}{5(k+5)(13 k+17)} \partial A_{3} \mathbf{T}^{(\mathbf{1})}-\frac{96 i(\mathbf{k}-\mathbf{3})}{5(k+5)(13 k+17)} A_{3} \partial \mathbf{T}^{(\mathbf{1})} \\
& +\frac{24 i(\mathbf{k}-\mathbf{3})(k+1)}{5(k+5)(13 k+17)} \partial B_{3} \mathbf{T}^{(\mathbf{1})}-\frac{24 i(\mathbf{k}-\mathbf{3})(k+1)}{5(k+5)(13 k+17)} B_{3} \partial \mathbf{T}^{(\mathbf{1})} \\
& -\frac{12(k-3)}{5(13 k+17)} G_{12} \mathbf{T}_{+}^{\left(\frac{3}{2}\right)}+\frac{20(k-3)(k+5)}{(13 k+17)(39 k+67)} G_{21} \mathbf{T}_{-}^{\left(\frac{3}{2}\right)} \\
& -\frac{4(k-3)(71 k+163)}{5(13 k+17)(39 k+67)} \partial \mathbf{T}^{(\mathbf{2})}+\frac{8(\mathbf{k}-\mathbf{3})(23 k+19)}{5(13 k+17)(39 k+67)} \partial^{2} \mathbf{T}^{(\mathbf{1})} \\
& -\frac{32(\mathbf{k}-\mathbf{3})(23 k+19)}{15(13 k+17)(39 k+67)} T \mathbf{T}^{(\mathbf{1})}-\frac{4 i(11 k+79)}{5(k+5)^{3}} A_{3} F_{11} G_{22} \\
& +\frac{16 i}{5(k+5)^{2}} A_{3} A_{3} A_{3}-\frac{96 i(k+3)}{5(k+5)^{3}} A_{3} A_{3} B_{3}-\frac{16}{5(k+5)^{2}} \partial A_{3} A_{3} \\
& -\frac{4\left(1638 k^{4}-20469 k^{3}-190079 k^{2}-392311 k-251283\right)}{5(k+5)^{3}(13 k+17)(39 k+67)} \partial A_{3} B_{3} \\
& +\frac{4\left(1638 k^{4}-8301 k^{3}-116759 k^{2}-254527 k-169275\right)}{5(k+5)^{3}(13 k+17)(39 k+67)} A_{3} \partial B_{3} \\
& +\frac{2 i}{5(k+5)^{3}(13 k+17)(39 k+67)(47 k+43)}\left(221563 k^{5}+2925184 k^{4}\right. \\
& \left.+12258818 k^{3}+11607476 k^{2}-12780037 k-14213564\right) \partial^{2} A_{3} \\
& +\frac{2 i\left(1287 k^{4}+29908 k^{3}+207762 k^{2}+192324 k-118657\right)}{5(k+5)^{4}(13 k+17)(39 k+67)} A_{3} \partial F_{11} F_{22} \\
& +\frac{2 i\left(23595 k^{4}+304260 k^{3}+1360330 k^{2}+2098996 k+952003\right)}{5(k+5)^{4}(13 k+17)(39 k+67)} A_{3} F_{11} \partial F_{22} \\
& -\frac{2 i\left(3070 k^{4}+47971 k^{3}+191033 k^{2}+178769 k-963\right)}{5(k+5)^{3}(13 k+17)(39 k+67)} A_{3} F_{12} G_{21} \\
& +\frac{8 i\left(115 k^{3}-485 k^{2}-4995 k-4619\right)}{5(k+5)^{4}(13 k+17)} \partial A_{3} F_{12} F_{21}-\frac{96 i(k+3)}{5(k+5)^{3}} A_{+} A_{-} B_{3} \\
& +\frac{2 i\left(307 k^{3}+5933 k^{2}+24657 k+19479\right)}{5(k+5)^{4}(13 k+17)} A_{3} \partial F_{12} F_{21}+\frac{16 i}{5(k+5)^{2}} A_{+} A_{-} A_{3} \\
& -\frac{2 i\left(473 k^{3}+391 k^{2}-10701 k-10299\right)}{5(k+5)^{4}(13 k+17)} A_{3} F_{12} \partial F_{21}+\frac{4 i(31 k+131)}{5(k+5)^{3}} B_{3} F_{11} G_{22} \\
& \text { - } \frac{2 i\left(6 k^{3}-151 k^{2}-680 k-411\right)}{5(k+5)^{3}(13 k+17)} A_{3} F_{21} G_{12}+\frac{4 i(7 k+59)}{5(k+5)^{3}} A_{3} F_{22} G_{11} \\
& +\frac{2 i\left(6 k^{3}-221 k^{2}-1780 k-2241\right)}{5(k+5)^{3}(13 k+17)} A_{-} F_{11} G_{12}+\frac{4 i(11 k+59)}{5(k+5)^{3}} A_{-} F_{12} G_{11}
\end{aligned}
$$




$$
\begin{aligned}
& -\frac{8 i\left(99 k^{3}+2695 k^{2}+14461 k+16281\right)}{5(k+5)^{4}(39 k+67)} \partial A_{-} F_{11} F_{12}-\frac{16(5 k+13)}{5(k+5)^{3}} \partial B_{3} B_{3} \\
& -\frac{16 i\left(87 k^{3}-216 k^{2}-4617 k-6298\right)}{5(k+5)^{4}(39 k+67)} A_{-} \partial F_{11} F_{12}+\frac{16 i(5 k+13)}{5(k+5)^{3}} B_{3} B_{3} B_{3} \\
& +\frac{16 i\left(186 k^{3}+2479 k^{2}+9844 k+9983\right)}{5(k+5)^{4}(39 k+67)} A_{-} F_{11} \partial F_{12}+\frac{4 i(11 k+59)}{5(k+5)^{3}} B_{-} F_{12} G_{22} \\
& -\frac{4\left(826 k^{3}-1759 k^{2}-19756 k-20675\right)}{5(k+5)^{2}(13 k+17)(39 k+67)} \partial A_{+} A_{-}+\frac{4 i(13 k-7)}{5(k+5)^{3}} B_{3} F_{22} G_{11} \\
& +\frac{4\left(826 k^{3}-1759 k^{2}-19756 k-20675\right)}{5(k+5)^{2}(13 k+17)(39 k+67)} A_{+} \partial A_{-}+\frac{16 i(5 k+13)}{5(k+5)^{3}} B_{+} B_{-} B_{3} \\
& \text { - } \frac{4 i(9 k+49)}{5(k+5)^{3}} A_{+} F_{21} G_{22}-\frac{16 i\left(k^{2}+30 k+129\right)}{5(k+5)^{4}} \partial A_{+} F_{21} F_{22} \\
& \text { - } \frac{32 i\left(k^{2}-k-32\right)}{5(k+5)^{4}} A_{+} \partial F_{21} F_{22}+\frac{32 i\left(2 k^{2}+29 k+97\right)}{5(k+5)^{4}} A_{+} F_{21} \partial F_{22} \\
& +\frac{2 i\left(3070 k^{4}+44065 k^{3}+181611 k^{2}+186907 k+32787\right)}{5(k+5)^{3}(13 k+17)(39 k+67)} A_{+} F_{22} G_{21} \\
& +\frac{2}{5(k+5)^{3}(13 k+17)(39 k+67)(47 k+43)} i\left(23312 k^{6}+533811 k^{5}\right. \\
& \left.+2300220 k^{4}-431454 k^{3}-11134868 k^{2}-11008445 k-2079136\right) \partial^{2} B_{3} \\
& \text { - } \frac{12 i}{5(k+5)^{4}(13 k+17)(39 k+67)}\left(156 k^{5}+1295 k^{4}\right. \\
& \text { - } \left.3100 k^{3}-64246 k^{2}-105576 k-24321\right) \partial B_{3} F_{11} F_{22} \\
& +\frac{2 i}{5(k+5)^{4}(13 k+17)(39 k+67)}\left(936 k^{5}+11085 k^{4}\right. \\
& \left.+232412 k^{3}+1112022 k^{2}+1962372 k+1161445\right) B_{3} \partial F_{11} F_{22} \\
& +\frac{2 i}{5(k+5)^{4}(13 k+17)(39 k+67)}\left(936 k^{5}+29337 k^{4}\right. \\
& \left.+64556 k^{3}-314882 k^{2}-937964 k-619951\right) B_{3} F_{11} \partial F_{22} \\
& +\frac{2 i\left(3070 k^{4}+47035 k^{3}+227457 k^{2}+492889 k+345429\right)}{5(k+5)^{3}(13 k+17)(39 k+67)} B_{3} F_{12} G_{21} \\
& +\frac{4 i\left(12 k^{4}+87 k^{3}+2237 k^{2}+11541 k+11299\right)}{5(k+5)^{4}(13 k+17)} \partial B_{3} F_{12} F_{21} \\
& -\frac{2 i\left(24 k^{4}+785 k^{3}+2959 k^{2}+8187 k+7077\right)}{5(k+5)^{4}(13 k+17)} B_{3} \partial F_{12} F_{21} \\
& -\frac{2 i\left(24 k^{4}+317 k^{3}+7651 k^{2}+33375 k+30945\right)}{5(k+5)^{4}(13 k+17)} B_{3} F_{12} \partial F_{21} \\
& +\frac{2 i\left(6 k^{3}+889 k^{2}+3800 k+3669\right)}{5(k+5)^{3}(13 k+17)} B_{3} F_{21} G_{12}
\end{aligned}
$$




$$
\begin{aligned}
& -\frac{2 i\left(267 k^{3}+4789 k^{2}+22521 k+30351\right)}{5(k+5)^{4}(39 k+67)} \partial B_{-} F_{12} F_{22} \\
& +\frac{2 i\left(345 k^{3}+4455 k^{2}+17427 k+22981\right)}{5(k+5)^{4}(39 k+67)} B_{-} \partial F_{12} F_{22} \\
& +\frac{2 i\left(189 k^{3}+5123 k^{2}+27615 k+37721\right)}{5(k+5)^{4}(39 k+67)} B_{-} F_{12} \partial F_{22} \\
& +\frac{2 i\left(6 k^{3}-221 k^{2}-1780 k-2241\right)}{5(k+5)^{3}(13 k+17)} B_{-} F_{22} G_{12} \\
& -\frac{4\left(1475 k^{4}+9959 k^{3}+31887 k^{2}+78277 k+64666\right)}{5(k+5)^{3}(13 k+17)(39 k+67)} \partial B_{+} B_{-} \\
& +\frac{4\left(1475 k^{4}+9959 k^{3}+31887 k^{2}+78277 k+64666\right)}{5(k+5)^{3}(13 k+17)(39 k+67)} B_{+} \partial B_{-} \\
& -\frac{2 i\left(50 k^{4}-985 k^{3}+19149 k^{2}+172733 k+181413\right)}{5(k+5)^{3}(13 k+17)(39 k+67)} B_{+} F_{11} G_{21} \\
& -\frac{2 i\left(39 k^{2}+382 k+903\right)}{5(k+5)^{4}} \partial B_{+} F_{11} F_{21}+\frac{2 i\left(37 k^{2}+410 k+1093\right)}{5(k+5)^{4}} B_{+} \partial F_{11} F_{21} \\
& +\frac{2 i\left(41 k^{2}+354 k+713\right)}{5(k+5)^{4}} B_{+} F_{11} \partial F_{21}+\frac{192 k}{(k+5)^{2}(39 k+67)} F_{11} F_{12} F_{22} G_{21} \\
& +\frac{2}{5(k+5)^{4}(13 k+17)(39 k+67)}\left(1156 k^{5}+57927 k^{4}\right. \\
& \left.+\quad 484204 k^{3}+1404610 k^{2}+1377864 k+100911\right) \partial^{2} F_{11} F_{22} \\
& -\frac{4}{5(k+5)^{4}(13 k+17)(39 k+67)}\left(496 k^{5}+68487 k^{4}\right. \\
& \left.+589748 k^{3}+1717458 k^{2}+2192244 k+1275327\right) \partial F_{11} \partial F_{22} \\
& +\frac{2}{5(k+5)^{4}(13 k+17)(39 k+67)}\left(1156 k^{5}+57927 k^{4}\right. \\
& \left.+\quad 484204 k^{3}+1404610 k^{2}+1377864 k+100911\right) F_{11} \partial^{2} F_{22} \\
& +\frac{\left(7321 k^{4}-33698 k^{3}-523364 k^{2}-1342878 k-943605\right)}{5(k+5)^{3}(13 k+17)(39 k+67)} \partial F_{11} G_{22} \\
& -\frac{\left(2251 k^{4}-18618 k^{3}-244984 k^{2}-676278 k-499395\right)}{5(k+5)^{3}(13 k+17)(39 k+67)} F_{11} \partial G_{22} \\
& -\frac{2}{5(k+5)^{4}(13 k+17)(39 k+67)}\left(1156 k^{5}+46107 k^{4}\right. \\
& \left.+428708 k^{3}+1545554 k^{2}+2544784 k+1682763\right) \partial^{2} F_{12} F_{21} \\
& +\frac{4}{5(k+5)^{4}(13 k+17)(39 k+67)}\left(496 k^{5}+52191 k^{4}\right. \\
& \left.+509484 k^{3}+1617994 k^{2}+2018524 k+686031\right) \partial F_{12} \partial F_{21} \\
& -\frac{2}{5(k+5)^{4}(13 k+17)(39 k+67)}\left(1156 k^{5}+46107 k^{4}\right.
\end{aligned}
$$




$$
\begin{aligned}
& \left.+\quad 428708 k^{3}+1545554 k^{2}+2544784 k+1682763\right) F_{12} \partial^{2} F_{21} \\
& -\frac{1}{5(k+5)^{3}(13 k+17)(39 k+67)(47 k+43)}\left(946375 k^{5}+11001867 k^{4}\right. \\
& \left.+54265174 k^{3}+149710406 k^{2}+178493139 k+70730399\right) \partial F_{12} G_{21} \\
& +\frac{1}{5(k+5)^{3}(13 k+17)(39 k+67)(47 k+43)}\left(410085 k^{5}+4466445 k^{4}\right. \\
& \left.+17966834 k^{3}+36726202 k^{2}+35009881 k+11610473\right) F_{12} \partial G_{21} \\
& +\frac{2\left(63 k^{3}+40 k^{2}-2381 k-2726\right)}{5(k+5)^{3}(13 k+17)} \partial F_{21} G_{12}-\frac{4 i(9 k+49)}{5(k+5)^{3}} B_{+} F_{21} G_{11} \\
& -\frac{2\left(25 k^{3}+22 k^{2}-927 k-1292\right)}{5(k+5)^{3}(13 k+17)} F_{21} \partial G_{12} \\
& -\frac{2\left(6187 k^{4}+57202 k^{3}+194476 k^{2}+325326 k+195561\right)}{5(k+5)^{3}(13 k+17)(39 k+67)} \partial F_{22} G_{11} \\
& +\frac{2\left(1117 k^{4}+8400 k^{3}+33170 k^{2}+102888 k+86217\right)}{5(k+5)^{3}(13 k+17)(39 k+67)} F_{22} \partial G_{11} \\
& +\frac{18(k+3)}{5(k+5)^{2}} G_{11} G_{22}-\frac{2\left(1744 k^{3}+23367 k^{2}+59474 k+42267\right)}{5(k+5)(13 k+17)(39 k+67)} G_{12} G_{21} \\
& +\frac{4 i\left(11369 k^{4}+728349 k^{3}+5556781 k^{2}+11678039 k+6652702\right)}{5(k+5)^{2}(13 k+17)(39 k+67)(47 k+43)} T A_{3} \\
& +\frac{4 i}{15(k+5)^{2}(13 k+17)(39 k+67)(47 k+43)}\left(85352 k^{5}+1785237 k^{4}\right. \\
& \left.+11949591 k^{3}+28863259 k^{2}+26292429 k+7640412\right) T B_{3} \\
& +\frac{4\left(801 k^{4}+13102 k^{3}+73621 k^{2}+150124 k+102516\right)}{5(k+5)^{2}(13 k+17)(39 k+67)} \partial T \\
& -\frac{4\left(5059 k^{3}+44554 k^{2}+120379 k+78948\right)}{5(k+5)^{2}(13 k+17)(47 k+43)} T U-\frac{16(k-15)}{5(k+5)^{3}} U A_{3} B_{3} \\
& -\frac{16\left(454 k^{4}+11473 k^{3}+59023 k^{2}+62739 k-39153\right)}{15(k+5)^{3}(13 k+17)(39 k+67)} T F_{11} F_{22} \\
& +\frac{32\left(227 k^{3}+3820 k^{2}+12093 k+14508\right)}{15(k+5)^{2}(13 k+17)(39 k+67)} T F_{12} F_{21}-\frac{16(5 k+27)}{5(k+5)^{3}} U A_{3} A_{3} \\
& +\frac{16 i\left(413 k^{4}+4082 k^{3}+9344 k^{2}+8238 k+4419\right)}{5(k+5)^{3}(13 k+17)(39 k+67)} \partial U A_{3} \\
& -\frac{16 i\left(413 k^{4}+6617 k^{3}+30703 k^{2}+55351 k+35172\right)}{5(k+5)^{3}(13 k+17)(39 k+67)} U \partial A_{3} \\
& +\frac{8 i\left(929 k^{4}-5539 k^{3}-66895 k^{2}-171009 k-123894\right)}{5(k+5)^{3}(13 k+17)(39 k+67)} \partial U B_{3} \\
& -\frac{8 i\left(929 k^{4}-469 k^{3}-24177 k^{2}-76783 k-62388\right)}{5(k+5)^{3}(13 k+17)(39 k+67)} U \partial B_{3}
\end{aligned}
$$




$$
\begin{aligned}
& -\frac{16(5 k+27)}{5(k+5)^{3}} U B_{+} B_{-}+\frac{2\left(5059 k^{3}+44554 k^{2}+120379 k+78948\right)}{5(k+5)^{2}(13 k+17)(47 k+43)} \partial^{2} U \\
& -\frac{2\left(903 k^{3}+28265 k^{2}+140557 k+158187\right)}{5(k+5)^{4}(39 k+67)} U \partial F_{11} F_{22}-\frac{16(5 k+27)}{5(k+5)^{3}} U A_{+} A_{-} \\
& +\frac{2\left(2997 k^{3}+34907 k^{2}+141319 k+159393\right)}{5(k+5)^{4}(39 k+67)} U F_{11} \partial F_{22}-\frac{16(5 k+27)}{5(k+5)^{3}} U B_{3} B_{3} \\
& -\frac{2\left(3070 k^{4}+55303 k^{3}+291205 k^{2}+593469 k+386433\right)}{5(k+5)^{3}(13 k+17)(39 k+67)} U F_{12} G_{21} \\
& -\frac{2\left(83 k^{2}+710 k+1859\right)}{5(k+5)^{4}} U \partial F_{12} F_{21}+\frac{2\left(25 k^{2}+658 k+2281\right)}{5(k+5)^{4}} U F_{12} \partial F_{21} \\
& +\frac{6\left(2 k^{3}+123 k^{2}+520 k+543\right)}{5(k+5)^{3}(13 k+17)} U F_{21} G_{12}+\frac{4(11 k+31)}{5(k+5)^{3}} U F_{11} G_{22} \\
& -\frac{4(11 k+31)}{5(k+5)^{3}} U F_{22} G_{11}+\frac{16 i}{5(k+5)^{2}} U U A_{3}+\frac{16 i(5 k+13)}{5(k+5)^{3}} U U B_{3} \\
& -\frac{16(5 k+27)}{5(k+5)^{3}} U U U+\frac{4\left(167 k^{3}+1175 k^{2}+3401 k+1593\right)}{5(k+5)^{4}(13 k+17)} F_{22} G_{11} F_{22} F_{11} \\
& -\frac{2\left(585 k^{4}-53056 k^{3}-361318 k^{2}-565672 k-203211\right)}{5(k+5)^{4}(13 k+17)(39 k+67)} F_{11} G_{22} F_{11} F_{22} \\
& \left.+\frac{2\left(29 k^{2}+26 k-211\right)}{5(k+5)^{4}} F_{12} G_{21} F_{12} F_{21}\right](w) \\
& +\frac{1}{(z-w)}\left[\frac{1}{6} \partial\left\{G_{21} \mathbf{S}_{-}^{\left(\frac{7}{2}\right)}\right\}_{-2}+\frac{9(k-3)}{(41 k+85)}\left(T \mathbf{P}^{(\mathbf{2})}-\frac{3}{10} \partial^{2} \mathbf{P}^{(\mathbf{2})}\right)\right. \\
& -\frac{36(k-3)}{(41 k+85)}\left(T \mathbf{W}^{(\mathbf{2})}-\frac{3}{10} \partial^{2} \mathbf{W}^{(\mathbf{2})}\right) \\
& -\frac{4(k-3)\left(1543 k^{3}+225 k^{2}-10179 k-11101\right)}{(k+5)(13 k+17)(39 k+67)(41 k+85)}\left(T \mathbf{T}^{(\mathbf{2})}-\frac{3}{10} \partial^{2} \mathbf{T}^{(\mathbf{2})}\right) \\
& +\frac{96 i(\mathbf{k}-\mathbf{3})}{(13 k+17)(41 k+85)}\left(T A_{3} \mathbf{T}^{(\mathbf{1})}-\frac{3}{10} \partial^{2}\left(A_{3} \mathbf{T}^{(\mathbf{1})}\right)\right) \\
& +\frac{24 i(\mathbf{k}-\mathbf{3})(k+1)}{(13 k+17)(41 k+85)}\left(T B_{3} \mathbf{T}^{(\mathbf{1})}-\frac{3}{10} \partial^{2}\left(B_{3} \mathbf{T}^{(\mathbf{1})}\right)\right) \\
& +\frac{16(\mathbf{k}-\mathbf{3})(61 k+113)}{9(13 k+17)(39 k+67)}\left(T \partial \mathbf{T}^{(\mathbf{1})}-\frac{1}{2} \partial T \mathbf{T}^{(\mathbf{1})}-\frac{1}{4} \partial^{3} \mathbf{T}^{(\mathbf{1})}\right)+\mathbf{S}^{(\mathbf{4})} \\
& -\frac{2(5 k+13)^{-1}(7 k+11)^{-1}(37+17 k)^{-1}(73+53 k)^{-1}(71 k+115)^{-1}}{(k+5)(13 k+17)(23 k+19)(29 k+97)(39 k+67)(41 k+85)} \\
& \times\left(18582783268720 k^{11}+468783625312793 k^{10}+5611734564211938 k^{9}\right. \\
& +\quad 41116614702193245 k^{8}+200379415234559352 k^{7} \\
& +671296144948119522 k^{6}+1562566286025217548 k^{5} \\
& +\quad 2514238282804743954 k^{4}+2733706958184300744 k^{3}
\end{aligned}
$$




$$
\begin{aligned}
& +1910595867995994437 k^{2}+771960599593492834 k \\
& +136491146486617425)\left(T T-\frac{3}{10} \partial^{2} T\right) \\
& \text { - } \frac{4\left(6218 k^{4}+72179 k^{3}+335333 k^{2}+710217 k+525069\right)}{(k+5)^{2}(13 k+17)(39 k+67)(41 k+85)} \\
& \times \quad\left(T A_{3} A_{3}-\frac{3}{10} \partial^{2}\left(A_{3} A_{3}\right)\right) \\
& -\frac{4\left(7922 k^{4}+90179 k^{3}+180017 k^{2}-14607 k-169263\right)}{(k+5)^{2}(13 k+17)(39 k+67)(41 k+85)} \\
& \times\left(T A_{3} B_{3}-\frac{3}{10} \partial^{2}\left(A_{3} B_{3}\right)\right) \\
& -\frac{4\left(6218 k^{4}+65225 k^{3}+263723 k^{2}+481683 k+303399\right)}{5(k+5)^{2}(7 k+11)(13 k+17)(39 k+67)} \\
& \times\left(T A_{+} A_{-}-\frac{1}{2} \partial^{2} A_{+} A_{-}-\frac{1}{2} A_{+} \partial^{2} A_{-}-\frac{i}{12} \partial^{3} A_{3}+\frac{i}{2} \partial T A_{3}\right) \\
& -\frac{8\left(234 k^{5}-701 k^{4}+6760 k^{3}+88144 k^{2}+185490 k+111537\right)}{(k+5)^{2}(13 k+17)(39 k+67)(41 k+85)} \\
& \times\left(T B_{3} B_{3}-\frac{3}{10} \partial^{2}\left(B_{3} B_{3}\right)\right) \\
& -\frac{4\left(1543 k^{4}+46207 k^{3}+255643 k^{2}+426885 k+223074\right)}{5(k+5)^{2}(7 k+11)(13 k+17)(39 k+67)} \\
& \times\left(T B_{+} B_{-}-\frac{1}{2} \partial^{2} B_{+} B_{-}-\frac{1}{2} B_{+} \partial^{2} B_{-}-\frac{i}{12} \partial^{3} B_{3}+\frac{i}{2} \partial T B_{3}\right) \\
& -\frac{48 i\left(637 k^{4}-1209 k^{3}-34981 k^{2}-100635 k-79596\right)}{(k+5)^{3}(13 k+17)(39 k+67)(41 k+85)} \\
& \times\left(T A_{3} F_{11} F_{22}-\frac{3}{10} \partial^{2}\left(A_{3} F_{11} F_{22}\right)\right) \\
& +\frac{36 i\left(27 k^{3}+261 k^{2}+937 k+1023\right)}{(k+5)^{3}(13 k+17)(41 k+85)}\left(T A_{3} F_{12} F_{21}-\frac{3}{10} \partial^{2}\left(A_{3} F_{12} F_{21}\right)\right) \\
& +\frac{24 i\left(125 k^{3}+2287 k^{2}+5575 k+4757\right)}{5(k+5)^{3}(17 k+37)(39 k+67)} \\
& \times\left(T A_{-} F_{11} F_{12}-\frac{1}{2} \partial^{2} A_{-} F_{11} F_{12}-3 A_{-} \partial F_{11} \partial F_{12}\right. \\
& \text { - } \frac{i}{4} \partial T F_{11} F_{22}+\frac{3 i}{4} \partial^{2} F_{11} \partial F_{22}-\frac{i}{4} \partial T F_{12} F_{21}+\frac{3 i}{4} \partial^{2} F_{12} \partial F_{21} \\
& \text { - } \left.\frac{i}{12} \partial^{3} F_{11} F_{22}-\frac{i}{12} \partial^{3} F_{12} F_{21}\right) \\
& -\frac{48 i\left(k^{2}-10 k-131\right)}{5(k+5)^{3}(17 k+37)}\left(T A_{+} F_{21} F_{22}-\frac{1}{2} \partial^{2} A_{+} F_{21} F_{22}-3 A_{+} \partial F_{21} \partial F_{22}\right. \\
& +\frac{i}{4} \partial T F_{11} F_{22}+\frac{i}{12} F_{11} \partial^{3} F_{22}+\frac{i}{4} \partial T F_{12} F_{21}+\frac{i}{12} F_{12} \partial^{3} F_{21}
\end{aligned}
$$




$$
\begin{aligned}
& \left.-\frac{3 i}{4} \partial F_{11} \partial^{2} F_{22}-\frac{3 i}{4} \partial F_{12} \partial^{2} F_{21}\right) \\
& -\frac{24 i\left(78 k^{5}-243 k^{4}+6026 k^{3}+65908 k^{2}+161880 k+113967\right)}{(k+5)^{3}(13 k+17)(39 k+67)(41 k+85)} \\
& \times\left(T B_{3} F_{11} F_{22}-\frac{3}{10} \partial^{2}\left(B_{3} F_{11} F_{22}\right)\right) \\
& +\frac{12 i\left(4 k^{4}+107 k^{3}+813 k^{2}+2649 k+2259\right)}{(k+5)^{3}(13 k+17)(41 k+85)} \\
& \times\left(T B_{3} F_{12} F_{21}-\frac{3}{10} \partial^{2}\left(B_{3} F_{12} F_{21}\right)\right) \\
& +\frac{192 i\left(191 k^{3}+423 k^{2}-1527 k-3551\right)}{5(k+5)^{3}(39 k+67)(53 k+73)} \\
& \times\left(T B_{-} F_{12} F_{22}-\frac{3}{4} \partial^{2} B_{-} F_{12} F_{22}-\frac{3}{4} B_{-} F_{12} \partial^{2} F_{22}\right. \\
& +\frac{3}{2} \partial B_{-} F_{12} \partial F_{22}-3 B_{-} \partial F_{12} \partial F_{22}-\frac{i}{4} \partial T F_{11} F_{22}+\frac{i}{4} \partial T F_{12} F_{21} \\
& \left.+\frac{3 i}{4} \partial F_{11} \partial^{2} F_{22}-\frac{3 i}{4} \partial F_{12} \partial^{2} F_{21}\right) \\
& -\frac{6 i\left(11 k^{2}+78 k+227\right)}{5(k+5)^{3}(5 k+13)}\left(T B_{+} F_{11} F_{21}-\frac{3}{2} \partial B_{+} \partial F_{11} F_{21}\right. \\
& \text { - } \left.\frac{3}{2} \partial B_{+} F_{11} \partial F_{21}+\frac{i}{4} \partial T F_{11} F_{22}-\frac{i}{4} \partial T F_{12} F_{21}\right) \\
& +\frac{48\left(76 k^{3}+373 k^{2}+2126 k+2613\right)}{(k+5)^{3}(39 k+67)(41 k+85)}\left(T U F_{11} F_{22}-\frac{3}{10} \partial^{2}\left(U F_{11} F_{22}\right)\right) \\
& \text { - } \frac{12(13 k-7)}{(k+5)^{2}(41 k+85)}\left(T U F_{12} F_{21}-\frac{3}{10} \partial^{2}\left(U F_{12} F_{21}\right)\right) \\
& +\frac{2\left(16687 k^{4}+122554 k^{3}+344764 k^{2}+530214 k+322677\right)}{(k+5)^{2}(13 k+17)(39 k+67)(41 k+85)} \\
& \times\left(T F_{11} G_{22}-\frac{3}{10} \partial^{2}\left(F_{11} G_{22}\right)\right) \\
& -\frac{3}{(k+5)^{2}(13 k+17)(39 k+67)(41 k+85)(47 k+43)} \\
& \times\left(212589 k^{5}+2511712 k^{4}+6213530 k^{3}+971776 k^{2}\right. \\
& \text { - 8108847k-4883080) }\left(T F_{12} G_{21}-\frac{3}{10} \partial^{2}\left(F_{12} G_{21}\right)\right) \\
& +\frac{6\left(165 k^{3}+1186 k^{2}+1351 k+34\right)}{(k+5)^{2}(13 k+17)(41 k+85)}\left(T F_{21} G_{12}-\frac{3}{10} \partial^{2}\left(F_{21} G_{12}\right)\right) \\
& -\frac{4\left(1387 k^{4}+35356 k^{3}+179710 k^{2}+272436 k+127143\right)}{(k+5)^{2}(13 k+17)(39 k+67)(41 k+85)} \\
& \times\left(T F_{22} G_{11}-\frac{3}{10} \partial^{2}\left(F_{22} G_{11}\right)\right)
\end{aligned}
$$




$$
\begin{aligned}
& +\frac{36 i\left(13 k^{3}+4187 k^{2}+2431 k-4879\right)}{(k+5)^{2}(13 k+17)(39 k+67)(41 k+85)}\left(T U A_{3}-\frac{3}{10} \partial^{2}\left(U A_{3}\right)\right) \\
& +\frac{24 i k\left(1443 k^{3}+20601 k^{2}+53657 k+38083\right)}{(k+5)^{2}(13 k+17)(39 k+67)(41 k+85)}\left(T U B_{3}-\frac{3}{10} \partial^{2}\left(U B_{3}\right)\right) \\
& -\frac{8\left(3109 k^{4}+39658 k^{3}+154630 k^{2}+225810 k+111537\right)}{(k+5)^{2}(13 k+17)(39 k+67)(41 k+85)} \\
& \times \quad\left(T U U-\frac{3}{10} \partial^{2}(U U)\right) \\
& +\frac{4 i}{15(k+5)^{2}(7 k+11)(13 k+17)(23 k+19)(39 k+67)(47 k+43)} \\
& \times\left(36706876 k^{6}+292282887 k^{5}+654064523 k^{4}+91424438 k^{3}\right. \\
& \text { - } \left.1307825118 k^{2}-1527225197 k-528255129\right) \\
& \times \quad\left(T \partial A_{3}-\frac{1}{2} \partial T A_{3}-\frac{1}{4} \partial^{3} A_{3}\right) \\
& \text { - } \frac{4 i}{45(k+5)^{2}(7 k+11)(13 k+17)(23 k+19)(39 k+67)(47 k+43)} \\
& \times\left(28754600 k^{7}+771031757 k^{6}+6124561187 k^{5}+21312125212 k^{4}\right. \\
& \left.+37135458430 k^{3}+32925947125 k^{2}+13350908007 k+1640263122\right) \\
& \times\left(T \partial B_{3}-\frac{1}{2} \partial T B_{3}-\frac{1}{4} \partial^{3} B_{3}\right) \\
& +\frac{8 k\left(6277 k^{3}+34435 k^{2}+48679 k+19689\right)}{(k+5)^{2}(13 k+17)(23 k+19)(47 k+43)}\left(T \partial U-\frac{1}{2} \partial T U-\frac{1}{4} \partial^{3} U\right) \\
& (39 k+67)^{-1}(53 k+73)^{-1} \\
& -\overline{90(k+5)^{3}(5 k+13)(13 k+17)(17 k+37)(23 k+19)(29 k+97)} \\
& \times \quad\left(34502966080 k^{9}-393778019461 k^{8}-6294110274792 k^{7}\right. \\
& \text { - } 13713341570588 k^{6}+98506625033288 k^{5}+629802093225522 k^{4} \\
& +\quad 1546655751602536 k^{3}+1967790292232708 k^{2} \\
& +1287742927169400 k+343785031306731) \\
& \times\left(T \partial F_{11} F_{22}-\partial T F_{11} F_{22}+3 T F_{11} \partial F_{22}-3 \partial^{2} F_{11} \partial F_{22}-F_{11} \partial^{3} F_{22}\right) \\
& -\frac{(39 k+67)^{-1}(53 k+73)^{-1}}{90(k+5)^{3}(5 k+13)(13 k+17)(17 k+37)(23 k+19)(29 k+97)} \\
& \times \quad\left(151049118080 k^{9}+3422145311317 k^{8}+34193189819496 k^{7}\right. \\
& +202349378272988 k^{6}+763718572719544 k^{5}+1854666569799342 k^{4} \\
& +2846500244497688 k^{3}+2637697241887420 k^{2} \\
& +1332346363292616 k+278192359770597) \\
& \times\left(T \partial F_{12} F_{21}-\partial T F_{12} F_{21}+3 T F_{12} \partial F_{21}-3 \partial^{2} F_{12} \partial F_{21}-F_{12} \partial^{3} F_{21}\right)
\end{aligned}
$$




$$
\begin{aligned}
& +\frac{8(39 k+67)^{-1}(53 k+73)^{-1}}{45(k+5)^{3}(5 k+13)(13 k+17)(17 k+37)(23 k+19)(29 k+97)} \\
& \times \quad\left(20044114520 k^{9}+328144939453 k^{8}+3596066948700 k^{7}\right. \\
& +\quad 26963376212984 k^{6}+123301840570060 k^{5}+339182244047274 k^{4} \\
& +\quad 563131131917300 k^{3}+550433364392848 k^{2} \\
& +\quad 289951406263308 k+62921457327249) \\
& \times \quad\left(T F_{11} \partial F_{22}-\frac{1}{4} \partial T F_{11} F_{22}-\frac{3}{4} \partial F_{11} \partial^{2} F_{22}-\frac{1}{4} F_{11} \partial^{3} F_{22}\right) \\
& +\frac{8(39 k+67)^{-1}(53 k+73)^{-1}}{45(k+5)^{3}(5 k+13)(13 k+17)(17 k+37)(23 k+19)(29 k+97)} \\
& \times \quad\left(26343906520 k^{9}+540983572121 k^{8}+5334907265412 k^{7}\right. \\
& +\quad 32669384753056 k^{6}+128858905140884 k^{5}+325963171531530 k^{4} \\
& +\quad 520830224450668 k^{3}+505789721434088 k^{2} \\
& +\quad 271388045277732 k+61391736767061) \\
& \left.\times\left(T F_{12} \partial F_{21}-\frac{1}{4} \partial T F_{12} F_{21}-\frac{3}{4} \partial F_{12} \partial^{2} F_{21}-\frac{1}{4} F_{12} \partial^{3} F_{21}\right)\right](w)+\cdots
\end{aligned}
$$

In the fourth and fifth terms of the third order pole one sees the nonlinear terms. One can add the $G_{12} A_{3} \mathbf{T}^{(\mathbf{1})}(w)$ or $G_{12} B_{3} \mathbf{T}^{(\mathbf{1})}(w)$ into the left hand side. Then the nonzero contribution $A_{3} \mathbf{T}^{(\mathbf{1})}(w)$ or $B_{3} \mathbf{T}^{(\mathbf{1})}(w)$ will appear in the third order pole in the right hand side because one has the central term between $G_{21}(z)$ and $G_{12}(w)$. In the second order pole, the nonlinear terms containing $\mathbf{T}^{(\mathbf{1})}(w)$ can appear from the $\partial G_{12} \mathbf{T}^{(\mathbf{1})}(w)$ in the left hand side. The nonlinear terms containing $\partial \mathbf{T}^{(\mathbf{1})}(w)$ can appear from the $G_{12} \partial \mathbf{T}^{(\mathbf{1})}(w)$ in the left hand side as before. According to the result of (4.1), if one introduces $G_{12} \partial \mathbf{T}^{(\mathbf{1})}(w)$ in the left hand side, the nonlinear term $G_{12} \mathbf{T}_{+}^{\left(\frac{3}{2}\right)}(w)$ can appear in the second order pole of the right hand side. Similarly, if one introduces $\partial \mathbf{T}^{(1)} \mathbf{T}_{-}^{\left(\frac{3}{2}\right)}(w)$ in the left hand side, the nonlinear term $G_{21} \mathbf{T}_{-}^{\left(\frac{3}{2}\right)}(w)$ can appear in the second order pole of the right hand side. Among the various quasi primary fields appearing in the first order pole, the quasi primary field with relative coefficients $-\frac{1}{2}$ and $-\frac{1}{4}$ is special because it is not $-\frac{3}{10}$ as other cases. There are various quasi primary fields which behave very differently and contain many terms.

\section{Appendix E.2 The higher spin currents in the nonlinear version in terms of those in the linear version}

As done in Appendix $B$, one has the following relations

$\mathbf{P}_{+, \text {non }}^{\left(\frac{5}{2}\right)}(z)=\left[\mathbf{P}_{+}^{\left(\frac{5}{2}\right)}+\frac{6 i}{(5+k)} A_{3} \mathbf{T}_{+}^{\left(\frac{3}{2}\right)}+\frac{2 i}{(5+k)} B_{3} \mathbf{T}_{+}^{\left(\frac{3}{2}\right)}-\frac{2}{(5+k)} F_{11} \mathbf{V}_{+}^{(2)}+\frac{8}{(5+k)^{2}} F_{12} F_{21} \mathbf{T}_{+}^{\left(\frac{3}{2}\right)}\right.$ 


$$
\begin{aligned}
& +\frac{8}{(5+k)^{2}} F_{11} F_{22} \mathbf{T}_{+}^{\left(\frac{3}{2}\right)}-\frac{2}{(5+k)} F_{21} \mathbf{T}^{(\mathbf{2})}-\frac{8}{(5+k)^{2}} F_{21} F_{22} \mathbf{U}^{\left(\frac{\mathbf{3}}{\mathbf{2}}\right)}+\frac{2}{(5+k)} \partial F_{21} \mathbf{T}^{(\mathbf{1})} \\
& -\frac{1}{(5+k)} F_{21} \partial \mathbf{T}^{(\mathbf{1})}-\frac{2}{(5+k)} F_{22} \mathbf{U}_{+}^{(\mathbf{2})}-\frac{2}{(5+k)} U \mathbf{T}_{+}^{\left(\frac{\mathbf{3}}{2}\right)}-\frac{2 i}{(k+5)} A_{-} \mathbf{U}^{\left(\frac{\mathbf{3}}{\mathbf{2}}\right)} \\
& -\frac{2 i}{(k+5)} B_{-} \mathbf{V}^{\left(\frac{3}{2}\right)}+\frac{2}{3(k+5)} F_{21} \mathbf{P}^{(\mathbf{2})}-\frac{2}{3(k+5)} \mathbf{P}^{(\mathbf{2})} F_{21} \\
& +\frac{6 i}{(5+k)} A_{3} G_{21}+\frac{6}{(5+k)^{2}} A_{3} A_{3} F_{21}+\frac{4}{(5+k)^{2}} A_{3} B_{-} F_{22}+\frac{16 i}{(5+k)^{3}} A_{3} F_{11} F_{21} F_{22} \\
& +\frac{4 i(k+3)}{3(5+k)^{3}} \partial A_{3} F_{21}-\frac{8 i(k+6)}{3(5+k)^{3}} A_{3} \partial F_{21}-\frac{2 i}{(5+k)} A_{-} G_{11}-\frac{4}{(5+k)^{2}} A_{-} A_{3} F_{11} \\
& -\frac{4}{(5+k)^{2}} A_{3} B_{3} F_{21}-\frac{2 i(5 k+9)}{3(5+k)^{3}} \partial A_{-} F_{11}+\frac{8 i(k+9)}{3(5+k)^{3}} A_{-} \partial F_{11}+\frac{8 i}{(5+k)^{3}} A_{-} F_{11} F_{12} F_{21} \\
& +\frac{2}{(5+k)^{2}} A_{+} A_{-} F_{21}+\frac{2 i}{(5+k)} B_{3} G_{21}-\frac{2}{(5+k)^{2}} B_{3} B_{3} F_{21}-\frac{4 i(k+3)}{3(5+k)^{3}} \partial B_{3} F_{21} \\
& +\frac{8 i(k+6)}{3(5+k)^{2}} B_{3} \partial F_{21}-\frac{2 i(5 k+9)}{3(5+k)^{3}} \partial B_{-} F_{22}-\frac{16 i(k+3)}{3(5+k)^{3}} B_{-} \partial F_{22}-\frac{2}{(5+k)^{2}} B_{+} B_{-} F_{21} \\
& +\frac{4}{(5+k)^{2}} F_{11} F_{21} G_{22}+\frac{8}{(5+k)^{3}} \partial F_{11} F_{21} F_{22}+\frac{8}{(5+k)^{3}} F_{11} \partial F_{21} F_{22}+\frac{4}{(5+k)^{2}} F_{11} F_{22} G_{21} \\
& +\frac{8}{(5+k)^{2}} F_{12} F_{21} G_{21}+\frac{8(k-3)}{3(k+5)^{3}} \partial^{2} F_{21}-\frac{4}{(5+k)^{2}} F_{21} F_{22} G_{11}+\frac{4(k+6)}{3(5+k)^{2}} \partial G_{21} \\
& -\frac{2}{(5+k)} U G_{21}+\frac{8 i}{(5+k)^{2}} U A_{3} F_{21}-\frac{4 i}{(5+k)^{2}} U A_{-} F_{11}-\frac{2}{(5+k)^{2}} U U F_{21} \\
& \left.-\frac{8}{(5+k)^{3}} F_{21} \partial F_{21} F_{12}+\frac{8(k+6)}{3(5+k)^{3}} \partial U F_{21}+\frac{8(k+6)}{3(5+k)^{3}} U \partial F_{21}\right](z), \\
& \mathbf{P}_{-, \text {non }}^{\left(\frac{\mathbf{5}}{2}\right)}(z)=\left[\mathbf{P}_{-}^{\left(\frac{5}{2}\right)}-\frac{6 i}{(5+k)} A_{3} \mathbf{T}_{-}^{\left(\frac{3}{2}\right)}-\frac{2 i}{(5+k)} B_{3} \mathbf{T}_{-}^{\left(\frac{3}{2}\right)}-\frac{2}{(5+k)} F_{11} \mathbf{V}_{-}^{(2)}-\frac{8}{(k+5)^{2}} F_{12} F_{21} \mathbf{T}_{-}^{\left(\frac{3}{2}\right)}\right. \\
& +\frac{8}{(5+k)^{2}} F_{11} F_{12} \mathbf{V}^{\left(\frac{\mathbf{3}}{2}\right)}-\frac{8}{(5+k)^{2}} F_{11} F_{22} \mathbf{T}_{-}^{\left(\frac{\mathbf{3}}{2}\right)}+\frac{2}{(5+k)} F_{12} \mathbf{T}^{(\mathbf{2})}+\frac{2 i}{(k+5)} B_{+} \mathbf{U}^{\left(\frac{3}{2}\right)} \\
& +\frac{2}{(5+k)} \partial F_{12} \mathbf{T}^{(\mathbf{1})}-\frac{1}{(5+k)} F_{12} \partial \mathbf{T}^{(\mathbf{1})}+\frac{2 i}{(k+5)} A_{+} \mathbf{V}^{\left(\frac{\mathbf{3}}{2}\right)}+\frac{8}{3(k+5)} \partial \mathbf{T}_{-}^{\left(\frac{\mathbf{3}}{2}\right)} \\
& -\frac{2}{(5+k)} F_{22} \mathbf{U}_{-}^{(\mathbf{2})}-\frac{2}{(5+k)} U \mathbf{T}_{-}^{\left(\frac{3}{2}\right)} \\
& +\frac{6 i}{(5+k)} A_{3} G_{12}-\frac{6}{(5+k)^{2}} A_{3} A_{3} F_{12}+\frac{4}{(k+5)^{2}} A_{3} B_{3} F_{12}-\frac{4}{(5+k)^{2}} A_{3} B_{+} F_{11} \\
& +\frac{16 i}{(5+k)^{3}} A_{3} F_{11} F_{12} F_{22}-\frac{8 i}{3(5+k)^{2}} A_{3} \partial F_{12}-\frac{2 i}{(5+k)} A_{+} G_{22}+\frac{4}{(5+k)^{2}} A_{+} A_{3} F_{22} \\
& -\frac{2}{(5+k)^{2}} A_{+} A_{-} F_{12}-\frac{8 i}{(5+k)^{3}} A_{+} F_{12} F_{21} F_{22}-\frac{10 i}{3(5+k)^{2}} \partial A_{+} F_{22}+\frac{8 i}{3(5+k)^{2}} A_{+} \partial F_{22} \\
& +\frac{2 i}{(5+k)} B_{3} G_{12}+\frac{2}{(5+k)^{2}} B_{3} B_{3} F_{12}+\frac{8 i}{3(5+k)^{2}} B_{3} \partial F_{12}+\frac{2}{(5+k)^{2}} B_{+} B_{-} F_{12}
\end{aligned}
$$




$$
\begin{aligned}
& -\frac{2 i}{3(5+k)^{2}} \partial B_{+} F_{11}-\frac{8 i}{3(5+k)^{2}} B_{+} \partial F_{11}-\frac{4}{(5+k)^{2}} F_{11} F_{12} G_{22}-\frac{16}{3(5+k)^{3}} \partial F_{11} F_{12} F_{22} \\
& +\frac{8}{3(5+k)^{3}} F_{11} \partial F_{12} F_{22}+\frac{8}{3(5+k)^{3}} F_{11} F_{12} \partial F_{22}+\frac{4}{(5+k)^{2}} F_{11} F_{22} G_{12} \\
& +\frac{8}{(5+k)^{2}} F_{12} F_{21} G_{12}+\frac{4}{(5+k)^{2}} F_{12} F_{22} G_{11}-\frac{8}{3(5+k)} \partial G_{12}+\frac{2}{(5+k)} U G_{12} \\
& +\frac{8 i}{(5+k)^{2}} U A_{3} F_{12}+\frac{16 i}{(k+5)^{3}} F_{12} B_{3} F_{12} F_{21}-\frac{4 i}{(5+k)^{2}} U A_{+} F_{22}+\frac{2}{(5+k)^{2}} U U F_{12} \\
& \left.+\frac{8}{3(5+k)^{2}} F_{12} G_{21} F_{12}-\frac{16}{3(5+k)^{2}} \partial U F_{12}-\frac{8}{3(5+k)^{2}} U \partial F_{12}\right](z), \\
& \mathbf{Q}_{\text {non }}^{\left(\frac{5}{2}\right)}(z)=\left[\mathbf{Q}^{\left(\frac{5}{2}\right)}+\frac{2 i}{(5+k)} A_{3} \mathbf{U}^{\left(\frac{3}{2}\right)}+\frac{2 i}{(5+k)} B_{3} \mathbf{U}^{\left(\frac{3}{2}\right)}-\frac{2 i}{(5+k)} B_{-} \mathbf{T}_{-}^{\left(\frac{3}{2}\right)}-\frac{2}{(5+k)} U \mathbf{U}^{\left(\frac{3}{2}\right)}\right. \\
& -\frac{2}{(5+k)} F_{11} \mathbf{W}^{(\mathbf{2})}-\frac{8}{(5+k)^{2}} F_{11} F_{12} \mathbf{T}_{+}^{\left(\frac{3}{2}\right)}-\frac{2 i}{(5+k)} A_{+} \mathbf{T}_{+}^{\left(\frac{3}{2}\right)}-\frac{1}{(5+k)} F_{11} \partial \mathbf{T}^{(\mathbf{1})} \\
& +\frac{8}{(5+k)^{2}} F_{11} F_{21} \mathbf{T}_{-}^{\left(\frac{3}{2}\right)}+\frac{2}{(5+k)} \partial F_{11} \mathbf{T}^{(\mathbf{1})}+\frac{2}{(5+k)} F_{12} \mathbf{U}_{+}^{(2)}+\frac{2}{(5+k)} F_{21} \mathbf{U}_{-}^{(2)} \\
& +\frac{2 i}{(5+k)} A_{3} G_{11}-\frac{4}{(5+k)^{2}} A_{3} B_{3} F_{11}-\frac{2}{(k+5)^{2}} A_{3} A_{3} F_{11}-\frac{4 i}{(5+k)^{2}} \partial A_{3} F_{11} \\
& +\frac{16 i}{(5+k)^{3}} A_{3} F_{11} F_{12} F_{21}+\frac{2 i}{(5+k)} B_{3} G_{11}-\frac{2 i}{(5+k)} A_{+} G_{21}-\frac{4}{(5+k)^{2}} A_{+} A_{3} F_{21} \\
& +\frac{2}{(5+k)^{2}} A_{+} A_{-} F_{11}-\frac{4}{(5+k)^{2}} A_{+} B_{-} F_{22}-\frac{8 i}{(5+k)^{3}} A_{+} F_{11} F_{21} F_{22}+\frac{2 i}{(5+k)^{2}} \partial A_{+} F_{21} \\
& -\frac{2}{(5+k)^{2}} B_{3} B_{3} F_{11}+\frac{16 i}{(5+k)^{3}} B_{3} F_{11} F_{12} F_{21}-\frac{2}{(k+5)^{2}} U U F_{11}-\frac{4}{(5+k)^{2}} B_{-} B_{3} F_{12} \\
& +\frac{8 i}{(5+k)^{3}} B_{-} F_{11} F_{12} F_{22}-\frac{2 i}{(5+k)^{2}} \partial B_{-} F_{12}+\frac{2}{(5+k)^{2}} B_{+} B_{-} F_{11}-\frac{4}{(5+k)^{2}} F_{11} F_{12} G_{21} \\
& -\frac{8}{(5+k)^{3}} F_{11} \partial F_{12} F_{21}+\frac{8}{(5+k)^{3}} F_{11} F_{12} \partial F_{21}-\frac{4}{(5+k)^{2}} F_{11} F_{21} G_{12}-\frac{2}{(5+k)} U G_{11} \\
& -\frac{4 i}{(5+k)^{2}} U A_{+} F_{21}-\frac{4 i}{(5+k)^{2}} U B_{-} F_{12}+\frac{4}{(5+k)^{2}} \partial U F_{11}-\frac{4}{(5+k)^{2}} F_{11} G_{22} F_{11} \\
& \left.+\frac{4}{(5+k)^{2}} F_{12} F_{21} G_{11}\right](z) \text {, } \\
& \mathbf{R}_{\text {non }}^{\left(\frac{5}{2}\right)}(z)=\left[\mathbf{R}^{\left(\frac{5}{2}\right)}-\frac{2 i}{(5+k)} A_{3} \mathbf{V}^{\left(\frac{3}{2}\right)}+\frac{2 i}{(5+k)} A_{-} \mathbf{T}_{-}^{\left(\frac{3}{2}\right)}-\frac{2 i}{(5+k)} B_{3} \mathbf{V}^{\left(\frac{3}{2}\right)}+\frac{2}{(5+k)} F_{21} \mathbf{V}_{-}^{(2)}\right. \\
& +\frac{2}{(5+k)} F_{22} \mathbf{W}^{(\mathbf{2})}-\frac{8}{(5+k)^{2}} F_{22} F_{21} \mathbf{T}_{-}^{\left(\frac{3}{2}\right)}-\frac{2}{(5+k)} U \mathbf{V}^{\left(\frac{3}{2}\right)}+\frac{2}{(5+k)} F_{12} \mathbf{V}_{+}^{(2)} \\
& +\frac{8}{(5+k)^{2}} F_{22} F_{12} \mathbf{T}_{+}^{\left(\frac{3}{2}\right)}+\frac{2 i}{(k+5)} B_{+} \mathbf{T}_{+}^{\left(\frac{3}{2}\right)}+\frac{2}{(5+k)} \partial F_{22} \mathbf{T}^{(\mathbf{1})}-\frac{1}{(5+k)} F_{22} \partial \mathbf{T}^{(\mathbf{1})} \\
& +\frac{2 i}{(5+k)} A_{3} G_{22}+\frac{2}{(5+k)^{2}} A_{3} A_{3} F_{22}+\frac{4}{(5+k)^{2}} A_{3} B_{3} F_{22}+\frac{16 i}{(5+k)^{3}} A_{3} F_{22} F_{21} F_{12}
\end{aligned}
$$




$$
\begin{aligned}
& -\frac{2 i}{(5+k)} A_{-} G_{12}-\frac{4 i}{3(5+k)^{2}} \partial A_{3} F_{22}+\frac{4}{(5+k)^{2}} A_{-} A_{3} F_{12}+\frac{4}{(5+k)^{2}} A_{-} B_{+} F_{11} \\
& -\frac{8 i}{(5+k)^{3}} A_{-} F_{22} F_{12} F_{11}+\frac{2 i}{(5+k)^{2}} \partial A_{-} F_{12}-\frac{2}{(5+k)^{2}} A_{-} A_{+} F_{22}+\frac{2 i}{(5+k)} B_{3} G_{22} \\
& +\frac{2}{(5+k)^{2}} B_{3} B_{3} F_{22}+\frac{16 i}{(5+k)^{3}} B_{3} F_{22} F_{21} F_{12}+\frac{4}{(5+k)^{2}} B_{+} B_{3} F_{21} \\
& +\frac{8 i}{(5+k)^{3}} B_{+} F_{22} F_{21} F_{11}-\frac{2 i}{(5+k)^{2}} \partial B_{+} F_{21}-\frac{2}{(5+k)^{2}} B_{-} B_{+} F_{22}+\frac{4}{(5+k)^{2}} F_{22} F_{21} G_{12} \\
& +\frac{8}{(5+k)^{3}} F_{22} \partial F_{21} F_{12}-\frac{8}{(5+k)^{3}} F_{22} F_{21} \partial F_{12}+\frac{2}{(5+k)} U G_{22}-\frac{4 i}{(5+k)^{2}} U A_{-} F_{12} \\
& +\frac{4}{(5+k)^{2}} F_{22} F_{12} G_{21}-\frac{4 i}{(5+k)^{2}} U B_{+} F_{21}-\frac{4}{(5+k)^{2}} \partial U F_{22}-\frac{4}{(5+k)^{2}} F_{21} F_{12} G_{22} \\
& \left.+\frac{4}{(5+k)^{2}} F_{22} G_{11} F_{22}+\frac{2}{(5+k)^{2}} U U F_{22}\right](z) .
\end{aligned}
$$

One can also write down the above expressions in terms of the fields in the nonlinear version.

What about other higher spin current? For example, let us look at the equation (2.7) of Part II. One would like to determine the explicit relation between $\mathbf{Q}_{+, \text {non }}^{(\mathbf{3})}(z)$ and its linear version $\mathbf{Q}_{+}^{(3)}(z)$. The upper OPE in (2.7) of Part II is given by the OPE between $\hat{G}_{21}(z)$ and $\mathbf{Q}_{\mathbf{n o n}}^{\left(\frac{5}{2}\right)}(w)$. Then one can express each field in terms of its linear version plus other terms which are defined also in the linear version. Then one can express the equation (2.7) of Part II in terms of the OPE between $G_{21}(z)$ and $\mathbf{Q}^{\left(\frac{5}{2}\right)}(w)$ and other terms which can be calculated from the previous results. The former is given by (5.4) where one can see the first order pole containing $\mathbf{Q}_{+}^{(\mathbf{3})}(w)$. The latter can be calculated explicitly. For example, the next term in $\hat{G}_{21}(z)$ of (2.2) is given by $U F_{21}(z)$ and the next term in $\mathbf{Q}_{\mathbf{n o n}}^{\left(\frac{5}{2}\right)}(w)$ of above OPE (E.1) is given by $A_{3} \mathbf{U}^{\left(\frac{3}{2}\right)}(w)$. Then it is straightforward to calculate the OPE between $U F_{21}(z)$ and $A_{3} \mathbf{U}^{\left(\frac{3}{2}\right)}(w)$ and read off the first order pole in the linear version. Eventually one can equate the first order pole of (2.7) of Part II (where the other fields except $\mathbf{Q}_{+, \text {non }}^{(\mathbf{3})}(w)$ should be written in the linear version) to the first order pole of (5.4) plus first order pole from various OPEs from other contributions as explained above.

\section{Appendix E.3 The higher spin currents in different basis}

As done in (4.2), one can calculate the OPEs between the spin- $\frac{3}{2}$ currents and the higher spin-2 current in (6.1), use the property of the fourth equation of Appendix D.1 with $s=2$ and obtain the following results

$$
V_{\frac{1}{2}}^{(2), 0}(z)=\left[-2 i \sqrt{2} \mathbf{P}_{+}^{\left(\frac{5}{2}\right)}-2 i \sqrt{2} \mathbf{W}_{+}^{\left(\frac{5}{2}\right)}+\frac{i \sqrt{2}\left(41 k^{2}+400 k+783\right)}{5(k+5)^{2}} G_{21} \mathbf{T}^{(\mathbf{1})}\right.
$$




$$
\begin{aligned}
& +\frac{2 i \sqrt{2}\left(41 k^{2}+400 k+783\right)}{5(k+5)^{2}} \mathbf{T}^{(\mathbf{1})} \mathbf{T}_{+}^{\left(\frac{3}{2}\right)}-\frac{i\left(41 k^{2}+400 k+783\right)}{5 \sqrt{2}(k+5)^{2}}\left(F_{21} \mathbf{P}^{(\mathbf{2})}-\mathbf{P}^{(\mathbf{2})} F_{21}\right) \\
& -\frac{4 \sqrt{2}}{(k+5)^{2}} A_{3} G_{21}+\frac{8 i \sqrt{2}}{(k+5)^{3}} A_{3} A_{3} F_{21}+\frac{4 i \sqrt{2}\left(3 k^{2}+30 k+59\right)}{5(k+5)^{3}} A_{3} B_{3} F_{21} \\
& -\frac{4 i \sqrt{2}\left(3 k^{2}+30 k+59\right)}{5(k+5)^{3}} A_{3} B_{-} F_{22}+\frac{4 \sqrt{2}\left(9 k^{2}+80 k+127\right)}{5(k+5)^{4}} A_{3} F_{11} F_{21} F_{22} \\
& +\frac{2 \sqrt{2}\left(50 k^{3}+617 k^{2}+2150 k+2151\right)}{15(k+5)^{4}} \partial A_{3} F_{21}+\frac{4 \sqrt{2}}{(k+5)^{2}} A_{-} G_{11} \\
& +\frac{2 \sqrt{2}\left(50 k^{3}+617 k^{2}+2270 k+2751\right)}{15(k+5)^{4}} A_{3} \partial F_{21}-\frac{4 i \sqrt{2}\left(3 k^{2}+30 k+59\right)}{5(k+5)^{3}} A_{-} B_{3} F_{11} \\
& -\frac{4 i \sqrt{2}\left(3 k^{2}+30 k+59\right)}{5(k+5)^{3}} A_{-} B_{-} F_{12}-\frac{4 \sqrt{2}\left(25 k^{3}+124 k^{2}-695 k-2298\right)}{15(k+5)^{4}} \partial A_{-} F_{11} \\
& -\frac{4 \sqrt{2}\left(25 k^{3}+124 k^{2}-665 k-2148\right)}{15(k+5)^{4}} A_{-} \partial F_{11}-\frac{6 \sqrt{2}\left(k^{2}+10 k+23\right)}{5(k+5)^{2}} B_{3} G_{21} \\
& -\frac{4 \sqrt{2}\left(9 k^{2}+80 k+127\right)}{5(k+5)^{4}} A_{-} F_{11} F_{12} F_{21}+\frac{8 i \sqrt{2}}{(k+5)^{3}} A_{+} A_{-} F_{21} \\
& -\frac{12 i \sqrt{2}\left(k^{2}+10 k+23\right)}{5(k+5)^{3}} B_{3} B_{3} F_{21}-\frac{4 \sqrt{2}\left(9 k^{2}+80 k+127\right)}{5(k+5)^{4}} B_{3} F_{11} F_{21} F_{22} \\
& -\frac{2 \sqrt{2}(k+3)\left(14 k^{2}+35 k-463\right)}{15(k+5)^{4}} \partial B_{3} F_{21}-\frac{2 \sqrt{2}\left(50 k^{3}+617 k^{2}+2270 k+2751\right)}{15(k+5)^{4}} B_{3} \partial F_{21} \\
& +\frac{6 \sqrt{2}\left(k^{2}+10 k+23\right)}{5(k+5)^{2}} B_{-} G_{22}-\frac{4 \sqrt{2}\left(9 k^{2}+80 k+127\right)}{5(k+5)^{4}} B_{-} F_{12} F_{21} F_{22} \\
& -\frac{2 \sqrt{2}\left(91 k^{3}+853 k^{2}+1393 k-681\right)}{15(k+5)^{4}} \partial B_{-} F_{22} \\
& -\frac{2 \sqrt{2}\left(73 k^{3}+583 k^{2}+79 k-2751\right)}{15(k+5)^{4}} B_{-} \partial F_{22}-\frac{12 i \sqrt{2}\left(k^{2}+10 k+23\right)}{5(k+5)^{3}} B_{+} B_{-} F_{21} \\
& +\frac{4 i \sqrt{2}\left(3 k^{2}+25 k+44\right)}{5(k+5)^{3}} F_{11} F_{21} G_{22}-\frac{4 i \sqrt{2}\left(23 k^{2}+280 k+729\right)}{15(k+5)^{4}} \partial F_{11} F_{21} F_{22} \\
& -\frac{4 i \sqrt{2}\left(41 k^{2}+400 k+783\right)}{15(k+5)^{4}} F_{11} \partial F_{21} F_{22}-\frac{4 i \sqrt{2}\left(59 k^{2}+520 k+837\right)}{15(k+5)^{4}} F_{11} F_{21} \partial F_{22} \\
& -\frac{2 i \sqrt{2}\left(3 k^{2}+20 k+9\right)}{5(k+5)^{3}} F_{11} F_{22} G_{21}+\frac{2 i \sqrt{2}\left(3 k^{2}+30 k+79\right)}{5(k+5)^{3}} F_{12} F_{21} G_{21} \\
& -\frac{2 i \sqrt{2}(k-3)\left(41 k^{2}+400 k+783\right)}{5(k+5)^{4}} \partial^{2} F_{21}-\frac{4 i \sqrt{2}(k+7)}{(k+5)^{3}} F_{21} F_{22} G_{11} \\
& +\frac{i \sqrt{2}\left(50 k^{3}+617 k^{2}+2270 k+2751\right)}{15(k+5)^{3}} \partial G_{21}+\frac{2 i \sqrt{2}}{k+5} U G_{21}+\frac{4 \sqrt{2}(k+3)}{(k+5)^{3}} U A_{3} F_{21}
\end{aligned}
$$




$$
\begin{aligned}
& -\frac{4 \sqrt{2}(k+3)}{(k+5)^{3}} U A_{-} F_{11}-\frac{4 \sqrt{2}\left(3 k^{2}+35 k+94\right)}{5(k+5)^{3}} U B_{3} F_{21} \\
& +\frac{4 \sqrt{2}\left(3 k^{2}+35 k+94\right)}{5(k+5)^{3}} U B_{-} F_{22}+\frac{4 i \sqrt{2}\left(9 k^{2}+80 k+127\right)}{5(k+5)^{4}} U F_{11} F_{21} F_{22} \\
& +\frac{2 i \sqrt{2}\left(50 k^{3}+617 k^{2}+2270 k+2751\right)}{15(k+5)^{4}} \partial U F_{21} \\
& +\frac{2 i \sqrt{2}\left(50 k^{3}+617 k^{2}+2270 k+2751\right)}{15(k+5)^{4}} U \partial F_{21} \\
& \left.+\frac{4 i \sqrt{2}}{(k+5)^{2}} U U F_{21}-\frac{8 i \sqrt{2}\left(3 k^{2}+20 k+9\right)}{5(k+5)^{4}} F_{21} \partial F_{21} F_{12}\right](z) \\
& +\left[-2 i \sqrt{2} \mathbf{P}_{-}^{\left(\frac{5}{2}\right)}+2 i \sqrt{2} \mathbf{W}_{-}^{\left(\frac{5}{2}\right)}-\frac{i \sqrt{2}(k+3)(5 k+34)}{3(k+5)^{2}}\left(F_{12} \mathbf{P}^{(\mathbf{2})}-\mathbf{P}^{(\mathbf{2})} F_{12}\right)\right. \\
& +\frac{i \sqrt{2}\left(41 k^{2}+400 k+783\right)}{5(k+5)^{2}} G_{12} \mathbf{T}^{(\mathbf{1})}-\frac{2 i \sqrt{2}\left(173 k^{2}+1690 k+3369\right)}{15(k+5)^{2}} \partial \mathbf{T}_{-}^{\left(\frac{3}{2}\right)} \\
& -\frac{2 i \sqrt{2}\left(41 k^{2}+400 k+783\right)}{5(k+5)^{2}} \mathbf{T}^{(\mathbf{1})} \mathbf{T}_{-}^{\left(\frac{3}{2}\right)} \\
& -\frac{4 \sqrt{2}}{(k+5)^{2}} A_{3} G_{12}-\frac{8 i \sqrt{2}}{(k+5)^{3}} A_{3} A_{3} F_{12}-\frac{4 i \sqrt{2}\left(3 k^{2}+30 k+59\right)}{5(k+5)^{3}} A_{3} B_{3} F_{12} \\
& +\frac{4 i \sqrt{2}\left(3 k^{2}+30 k+59\right)}{5(k+5)^{3}} A_{3} B_{+} F_{11}-\frac{4 \sqrt{2}\left(9 k^{2}+80 k+127\right)}{5(k+5)^{4}} A_{3} F_{11} F_{12} F_{22} \\
& +\frac{4 \sqrt{2}}{(k+5)^{2}} A_{+} G_{22}+\frac{4 i \sqrt{2}\left(3 k^{2}+30 k+59\right)}{5(k+5)^{3}} A_{+} B_{3} F_{22}-\frac{8 i \sqrt{2}}{(k+5)^{3}} A_{+} A_{-} F_{12} \\
& +\frac{4 i \sqrt{2}\left(3 k^{2}+30 k+59\right)}{5(k+5)^{3}} A_{+} B_{+} F_{21}+\frac{4 \sqrt{2}\left(9 k^{2}+80 k+127\right)}{5(k+5)^{4}} A_{+} F_{12} F_{21} F_{22} \\
& +\frac{8 \sqrt{2}}{(k+5)^{3}} \partial A_{+} F_{22}-\frac{6 \sqrt{2}\left(k^{2}+10 k+23\right)}{5(k+5)^{2}} B_{3} G_{12}+\frac{12 i \sqrt{2}\left(k^{2}+10 k+23\right)}{5(k+5)^{3}} B_{3} B_{3} F_{12} \\
& +\frac{4 \sqrt{2}\left(9 k^{2}+80 k+127\right)}{5(k+5)^{4}} B_{3} F_{11} F_{12} F_{22}+\frac{6 \sqrt{2}\left(k^{2}+10 k+23\right)}{5(k+5)^{2}} B_{+} G_{11} \\
& +5(k+5)^{3} \\
& +
\end{aligned}
$$




$$
\begin{aligned}
& +\frac{2 i \sqrt{2}\left(3 k^{2}+30 k+79\right)}{5(k+5)^{3}} F_{12} F_{21} G_{12}+\frac{4 i \sqrt{2}\left(3 k^{2}+25 k+44\right)}{5(k+5)^{3}} F_{12} F_{22} G_{11} \\
& +\frac{i \sqrt{2}\left(173 k^{2}+1690 k+3369\right)}{15(k+5)^{2}} \partial G_{12}-\frac{2 i \sqrt{2}}{k+5} U G_{12}+\frac{4 \sqrt{2}(k+3)}{(k+5)^{3}} U A_{3} F_{12} \\
& -\frac{4 \sqrt{2}(k+3)}{(k+5)^{3}} U A_{+} F_{22}-\frac{4 \sqrt{2}\left(3 k^{2}+35 k+94\right)}{5(k+5)^{3}} U B_{3} F_{12} \\
& +\frac{4 \sqrt{2}\left(3 k^{2}+35 k+94\right)}{5(k+5)^{3}} U B_{+} F_{11}+\frac{4 i \sqrt{2}\left(9 k^{2}+80 k+127\right)}{5(k+5)^{4}} U F_{11} F_{12} F_{22} \\
& \left.-\frac{4 i \sqrt{2}}{(k+5)^{2}} U U F_{12}+\frac{8 i \sqrt{2}\left(3 k^{2}+20 k+9\right)}{5(k+5)^{4}} F_{12} \partial F_{12} F_{21}\right](z) \text {, } \\
& V_{\frac{1}{2}}^{(2), 1}=\left[-2 \sqrt{2} \mathbf{Q}^{\left(\frac{5}{2}\right)}-2 \sqrt{2} \mathbf{U}^{\left(\frac{5}{2}\right)}+\frac{\sqrt{2}\left(41 k^{2}+400 k+783\right)}{5(k+5)^{2}} G_{11} \mathbf{T}^{(\mathbf{1})}\right. \\
& -\frac{2 \sqrt{2}\left(41 k^{2}+400 k+783\right)}{5(k+5)^{2}} \partial \mathbf{U}^{\left(\frac{3}{2}\right)}+\frac{2 \sqrt{2}\left(41 k^{2}+400 k+783\right)}{5(k+5)^{2}} \mathbf{T}^{(\mathbf{1})} \mathbf{U}^{\left(\frac{3}{2}\right)} \\
& -\frac{4 i \sqrt{2}}{(k+5)^{2}} A_{3} G_{11}-\frac{4 \sqrt{2}\left(3 k^{2}+30 k+59\right)}{5(k+5)^{3}} A_{3} B_{3} F_{11}+\frac{8 \sqrt{2}}{(k+5)^{3}} A_{3} A_{3} F_{11} \\
& +\frac{4 i \sqrt{2}(k+3)(5 k+34)}{3(k+5)^{3}} \partial A_{3} F_{11}+\frac{4 i \sqrt{2}(k+3)(5 k+34)}{3(k+5)^{3}} A_{3} \partial F_{11} \\
& -\frac{4 \sqrt{2}\left(3 k^{2}+30 k+59\right)}{5(k+5)^{3}} A_{3} B_{-} F_{12}+\frac{4 i \sqrt{2}\left(9 k^{2}+80 k+127\right)}{5(k+5)^{4}} A_{3} F_{11} F_{12} F_{21} \\
& -\frac{4 i \sqrt{2}}{(k+5)^{2}} A_{+} G_{21}-\frac{4 \sqrt{2}\left(3 k^{2}+30 k+59\right)}{5(k+5)^{3}} A_{+} B_{3} F_{21}+\frac{8 \sqrt{2}}{(k+5)^{3}} A_{+} A_{-} F_{11} \\
& +\frac{4 \sqrt{2}\left(3 k^{2}+30 k+59\right)}{5(k+5)^{3}} A_{+} B_{-} F_{22}+\frac{4 i \sqrt{2}\left(9 k^{2}+80 k+127\right)}{5(k+5)^{4}} A_{+} F_{11} F_{21} F_{22} \\
& +\frac{4 i \sqrt{2}\left(5 k^{2}+49 k+96\right)}{3(k+5)^{3}} \partial A_{+} F_{21}+\frac{4 i \sqrt{2}(k+3)(5 k+34)}{3(k+5)^{3}} A_{+} \partial F_{21} \\
& +\frac{6 i \sqrt{2}\left(k^{2}+10 k+23\right)}{5(k+5)^{2}} B_{3} G_{11}-\frac{12 \sqrt{2}\left(k^{2}+10 k+23\right)}{5(k+5)^{3}} B_{3} B_{3} F_{11} \\
& +\frac{4 i \sqrt{2}\left(7 k^{2}+65 k+96\right)}{15(k+5)^{3}} \partial B_{3} F_{11}+\frac{4 i \sqrt{2}(k+3)(5 k+34)}{3(k+5)^{3}} B_{3} \partial F_{11} \\
& +\frac{4 i \sqrt{2}\left(9 k^{2}+80 k+127\right)}{5(k+5)^{4}} B_{3} F_{11} F_{12} F_{21}+\frac{6 i \sqrt{2}\left(k^{2}+10 k+23\right)}{5(k+5)^{2}} B_{-} G_{12} \\
& -\frac{4 i \sqrt{2}\left(9 k^{2}+80 k+127\right)}{5(k+5)^{4}} B_{-} F_{11} F_{12} F_{22}+\frac{4 i \sqrt{2}\left(16 k^{2}+155 k+303\right)}{15(k+5)^{3}} \partial B_{-} F_{12} \\
& +\frac{4 i \sqrt{2}(k+3)(5 k+34)}{3(k+5)^{3}} B_{-} \partial F_{12}-\frac{12 \sqrt{2}\left(k^{2}+10 k+23\right)}{5(k+5)^{3}} B_{+} B_{-} F_{11}
\end{aligned}
$$




$$
\begin{aligned}
& -\frac{4 \sqrt{2}(k+7)}{(k+5)^{3}} F_{11} F_{12} G_{21}+\frac{8 \sqrt{2}\left(41 k^{2}+400 k+783\right)}{15(k+5)^{4}} \partial F_{11} F_{12} F_{21} \\
& -\frac{32 \sqrt{2}\left(8 k^{2}+85 k+189\right)}{15(k+5)^{4}} F_{11} \partial F_{12} F_{21}+\frac{16 \sqrt{2}\left(5 k^{2}+46 k+81\right)}{3(k+5)^{4}} F_{11} F_{12} \partial F_{21} \\
& -\frac{4 \sqrt{2}\left(3 k^{2}+25 k+44\right)}{5(k+5)^{3}} F_{11} F_{21} G_{12}-\frac{2 \sqrt{2}\left(3 k^{2}+30 k+79\right)}{5(k+5)^{3}} F_{11} F_{22} G_{11} \\
& +\frac{2 \sqrt{2}\left(3 k^{2}+20 k+9\right)}{5(k+5)^{3}} F_{12} F_{21} G_{11}-\frac{\sqrt{2}\left(73 k^{2}+710 k+1329\right)}{15(k+5)^{2}} \partial G_{11} \\
& +\frac{2 \sqrt{2}}{k+5} U G_{11}+\frac{4 i \sqrt{2}(k+3)}{(k+5)^{3}} U A_{3} F_{11}+\frac{4 i \sqrt{2}(k+3)}{(k+5)^{3}} U A_{+} F_{21} \\
& +\frac{4 i \sqrt{2}\left(3 k^{2}+35 k+94\right)}{5(k+5)^{3}} U B_{3} F_{11}+\frac{4 i \sqrt{2}\left(3 k^{2}+35 k+94\right)}{5(k+5)^{3}} U B_{-} F_{12} \\
& +\frac{4 \sqrt{2}(k+3)(5 k+34)}{3(k+5)^{3}} \partial U F_{11}+\frac{4 \sqrt{2}(k+3)(5 k+34)}{3(k+5)^{3}} U \partial F_{11}+\frac{4 \sqrt{2}}{(k+5)^{2}} U U F_{11} \\
& \left.+\frac{4 \sqrt{2}\left(9 k^{2}+80 k+127\right)}{5(k+5)^{4}} U F_{11} F_{12} F_{21}-\frac{8 \sqrt{2}\left(3 k^{2}+20 k+9\right)}{5(k+5)^{4}} F_{11} \partial F_{11} F_{22}\right](z) \\
& +\left[2 \sqrt{2} \mathbf{R}^{\left(\frac{5}{2}\right)}-2 \sqrt{2} \mathbf{V}^{\left(\frac{5}{2}\right)}+\frac{4 \sqrt{2}\left(5 k^{2}+46 k+81\right)}{(k+5)(7 k+3)}\left(F_{22} \mathbf{P}^{(\mathbf{2})}-\mathbf{P}^{(\mathbf{2})} F_{22}\right)\right. \\
& -\frac{\sqrt{2}\left(41 k^{2}+400 k+783\right)}{5(k+5)^{2}} G_{22} \mathbf{T}^{(1)}+\frac{2 \sqrt{2}\left(1061 k^{3}+10009 k^{2}+17763 k-2673\right)}{15(k+5)^{2}(7 k+3)} \partial \mathbf{V}^{\left(\frac{3}{2}\right)} \\
& +\frac{2 \sqrt{2}\left(41 k^{2}+400 k+783\right)}{5(k+5)^{2}} \mathbf{T}^{(\mathbf{1})} \mathbf{V}^{\left(\frac{3}{2}\right)} \\
& +\frac{4 i \sqrt{2}}{(k+5)^{2}} A_{3} G_{22}+\frac{8 \sqrt{2}}{(k+5)^{3}} A_{3} A_{3} F_{22}-\frac{4 \sqrt{2}\left(3 k^{2}+30 k+59\right)}{5(k+5)^{3}} A_{3} B_{3} F_{22} \\
& -\frac{4 \sqrt{2}\left(3 k^{2}+30 k+59\right)}{5(k+5)^{3}} A_{3} B_{+} F_{21}+\frac{8 i \sqrt{2}\left(29 k^{3}+91 k^{2}-1143 k-2637\right)}{5(k+5)^{3}(7 k+3)} \partial A_{3} F_{22} \\
& +\frac{4 i \sqrt{2}\left(5 k^{3}+24 k^{2}-109 k-264\right)}{(k+5)^{3}(7 k+3)} A_{3} \partial F_{22}-\frac{4 i \sqrt{2}\left(9 k^{2}+80 k+127\right)}{5(k+5)^{4}} A_{3} F_{22} F_{21} F_{12} \\
& +\frac{4 i \sqrt{2}}{(k+5)^{2}} A_{-} G_{12}+\frac{8 \sqrt{2}}{(k+5)^{3}} A_{-} A_{+} F_{22}-\frac{4 \sqrt{2}\left(3 k^{2}+30 k+59\right)}{5(k+5)^{3}} A_{-} B_{3} F_{12} \\
& +\frac{4 \sqrt{2}\left(3 k^{2}+30 k+59\right)}{5(k+5)^{3}} A_{-} B_{+} F_{11}-\frac{4 i \sqrt{2}\left(9 k^{2}+80 k+127\right)}{5(k+5)^{4}} A_{-} F_{22} F_{12} F_{11} \\
& +\frac{4 i \sqrt{2}\left(5 k^{3}+24 k^{2}-95 k-258\right)}{(k+5)^{3}(7 k+3)} \partial A_{-} F_{12}+\frac{4 i \sqrt{2}\left(5 k^{3}+24 k^{2}-109 k-264\right)}{(k+5)^{3}(7 k+3)} A_{-} \partial F_{12} \\
& -\frac{6 i \sqrt{2}\left(k^{2}+10 k+23\right)}{5(k+5)^{2}} B_{3} G_{22}-\frac{12 \sqrt{2}\left(k^{2}+10 k+23\right)}{5(k+5)^{3}} B_{3} B_{3} F_{22}
\end{aligned}
$$




$$
\begin{aligned}
& -\frac{4 i \sqrt{2}\left(15 k^{3}+100 k^{2}-119 k-708\right)}{(k+5)^{3}(7 k+3)} B_{3} \partial F_{22} \\
& -\frac{4 i \sqrt{2}\left(9 k^{2}+80 k+127\right)}{5(k+5)^{4}} B_{3} F_{22} F_{21} F_{12}-\frac{6 i \sqrt{2}\left(k^{2}+10 k+23\right)}{5(k+5)^{2}} B_{+} G_{21} \\
& +\frac{4 i \sqrt{2}\left(9 k^{2}+80 k+127\right)}{5(k+5)^{4}} B_{+} F_{22} F_{21} F_{11}+\frac{4 i \sqrt{2}\left(46 k^{3}+639 k^{2}+2788 k+3747\right)}{5(k+5)^{3}(7 k+3)} \partial B_{+} F_{21} \\
& +\frac{4 i \sqrt{2}(k+3)\left(5 k^{2}+69 k+236\right)}{(k+5)^{3}(7 k+3)} B_{+} \partial F_{21}-\frac{12 \sqrt{2}\left(k^{2}+10 k+23\right)}{5(k+5)^{3}} B_{-} B_{+} F_{22} \\
& +\frac{8 \sqrt{2}(k-9)\left(5 k^{2}+46 k+81\right)}{(k+5)^{3}(7 k+3)} \partial^{2} F_{22}-\frac{4 \sqrt{2}(k+7)}{(k+5)^{3}} F_{22} F_{21} G_{12} \\
& -\frac{8 \sqrt{2}\left(3 k^{2}+20 k+9\right)}{5(k+5)^{4}} \partial F_{22} F_{21} F_{12}-\frac{16 \sqrt{2}\left(3 k^{2}+20 k+9\right)}{5(k+5)^{4}} F_{22} \partial F_{21} F_{12} \\
& -\frac{4 \sqrt{2}\left(3 k^{2}+25 k+44\right)}{5(k+5)^{3}} F_{22} F_{12} G_{21}-\frac{2 \sqrt{2}\left(3 k^{2}+30 k+79\right)}{5(k+5)^{3}} F_{22} F_{11} G_{22} \\
& +\frac{2 \sqrt{2}\left(3 k^{2}+20 k+9\right)}{5(k+5)^{3}} F_{21} F_{12} G_{22}-\frac{\sqrt{2}\left(1211 k^{3}+12529 k^{2}+31053 k+18567\right)}{15(k+5)^{2}(7 k+3)} \partial G_{22} \\
& +\frac{2 \sqrt{2}}{k+5} U G_{22}-\frac{4 i \sqrt{2}(k+3)}{(k+5)^{3}} U A_{3} F_{22}-\frac{4 i \sqrt{2}(k+3)}{(k+5)^{3}} U A_{-} F_{12} \\
& -\frac{4 i \sqrt{2}\left(3 k^{2}+35 k+94\right)}{5(k+5)^{3}} U B_{3} F_{22}-\frac{4 i \sqrt{2}\left(3 k^{2}+35 k+94\right)}{5(k+5)^{3}} U B_{+} F_{21} \\
& +\frac{8 \sqrt{2}\left(4 k^{3}-179 k^{2}-1978 k-3747\right)}{5(k+5)^{3}(7 k+3)} \partial U F_{22}-\frac{4 \sqrt{2}(k+3)\left(5 k^{2}+69 k+236\right)}{(k+5)^{3}(7 k+3)} U \partial F_{22} \\
& +\frac{4 \sqrt{2}\left(9 k^{2}+80 k+127\right)}{5(k+5)^{4}} U F_{22} F_{21} F_{12}-\frac{4 \sqrt{2}\left(33 k^{3}+62 k^{2}-1741 k-3954\right)}{5(k+5)^{3}(7 k+3)} F_{22} G_{11} F_{22} \\
& \left.-\frac{8 \sqrt{2}\left(3 k^{2}+20 k+9\right)}{5(k+5)^{4}} F_{22} \partial F_{22} F_{11}+\frac{4 \sqrt{2}}{(k+5)^{2}} U U F_{22}\right](z), \\
& V_{\frac{1}{2}}^{(2), 2}=\left[-2 i \sqrt{2} \mathbf{Q}^{\left(\frac{5}{2}\right)}-2 i \sqrt{2} \mathbf{U}^{\left(\frac{5}{2}\right)}+\frac{i \sqrt{2}\left(41 k^{2}+400 k+783\right)}{5(k+5)^{2}} G_{11} \mathbf{T}^{(\mathbf{1})}\right. \\
& -\frac{2 i \sqrt{2}\left(41 k^{2}+400 k+783\right)}{5(k+5)^{2}} \partial \mathbf{U}^{\left(\frac{3}{2}\right)}+\frac{2 i \sqrt{2}\left(41 k^{2}+400 k+783\right)}{5(k+5)^{2}} \mathbf{T}^{(\mathbf{1})} \mathbf{U}^{\left(\frac{3}{2}\right)} \\
& +\frac{4 \sqrt{2}}{(k+5)^{2}} A_{3} G_{11}-\frac{4 i \sqrt{2}\left(3 k^{2}+30 k+59\right)}{5(k+5)^{3}} A_{3} B_{3} F_{11}-\frac{8 \sqrt{2}}{(k+5)^{3}} A_{3} A_{3} F_{11} \\
& -\frac{4 \sqrt{2}(k+3)(5 k+34)}{3(k+5)^{3}} \partial A_{3} F_{11}-\frac{4 \sqrt{2}(k+3)(5 k+34)}{3(k+5)^{3}} A_{3} \partial F_{11} \\
& -\frac{4 i \sqrt{2}\left(3 k^{2}+30 k+59\right)}{5(k+5)^{3}} A_{3} B_{-} F_{12}-\frac{4 \sqrt{2}\left(9 k^{2}+80 k+127\right)}{5(k+5)^{4}} A_{3} F_{11} F_{12} F_{21}
\end{aligned}
$$




$$
\begin{aligned}
& +\frac{4 \sqrt{2}}{(k+5)^{2}} A_{+} G_{21}-\frac{4 i \sqrt{2}\left(3 k^{2}+30 k+59\right)}{5(k+5)^{3}} A_{+} B_{3} F_{21}+\frac{8 i \sqrt{2}}{(k+5)^{3}} A_{+} A_{-} F_{11} \\
& +\frac{4 i \sqrt{2}\left(3 k^{2}+30 k+59\right)}{5(k+5)^{3}} A_{+} B_{-} F_{22}-\frac{4 \sqrt{2}\left(9 k^{2}+80 k+127\right)}{5(k+5)^{4}} A_{+} F_{11} F_{21} F_{22} \\
& -\frac{4 \sqrt{2}\left(5 k^{2}+49 k+96\right)}{3(k+5)^{3}} \partial A_{+} F_{21}-\frac{4 \sqrt{2}(k+3)(5 k+34)}{3(k+5)^{3}} A_{+} \partial F_{21} \\
& -\frac{6 \sqrt{2}\left(k^{2}+10 k+23\right)}{5(k+5)^{2}} B_{3} G_{11}-\frac{12 i \sqrt{2}\left(k^{2}+10 k+23\right)}{5(k+5)^{3}} B_{3} B_{3} F_{11} \\
& -\frac{4 \sqrt{2}\left(7 k^{2}+65 k+96\right)}{15(k+5)^{3}} \partial B_{3} F_{11}-\frac{4 \sqrt{2}(k+3)(5 k+34)}{3(k+5)^{3}} B_{3} \partial F_{11} \\
& -\frac{4 \sqrt{2}\left(9 k^{2}+80 k+127\right)}{5(k+5)^{4}} B_{3} F_{11} F_{12} F_{21}-\frac{6 \sqrt{2}\left(k^{2}+10 k+23\right)}{5(k+5)^{2}} B_{-} G_{12} \\
& +\frac{4 \sqrt{2}\left(9 k^{2}+80 k+127\right)}{5(k+5)^{4}} B_{-} F_{11} F_{12} F_{22}-\frac{4 \sqrt{2}\left(16 k^{2}+155 k+303\right)}{15(k+5)^{3}} \partial B_{-} F_{12} \\
& -\frac{4 \sqrt{2}(k+3)(5 k+34)}{3(k+5)^{3}} B_{-} \partial F_{12}-\frac{12 i \sqrt{2}\left(k^{2}+10 k+23\right)}{5(k+5)^{3}} B_{+} B_{-} F_{11} \\
& -\frac{4 i \sqrt{2}(k+7)}{(k+5)^{3}} F_{11} F_{12} G_{21}+\frac{8 i \sqrt{2}\left(41 k^{2}+400 k+783\right)}{15(k+5)^{4}} \partial F_{11} F_{12} F_{21} \\
& +\frac{32 i \sqrt{2}\left(8 k^{2}+85 k+189\right)}{15(k+5)^{4}} F_{11} \partial F_{12} F_{21}+\frac{16 i \sqrt{2}\left(5 k^{2}+46 k+81\right)}{3(k+5)^{4}} F_{11} F_{12} \partial F_{21} \\
& \text { - } \frac{4 i \sqrt{2}\left(3 k^{2}+25 k+44\right)}{5(k+5)^{3}} F_{11} F_{21} G_{12}-\frac{2 i \sqrt{2}\left(3 k^{2}+30 k+79\right)}{5(k+5)^{3}} F_{11} F_{22} G_{11} \\
& +\frac{2 i \sqrt{2}\left(3 k^{2}+20 k+9\right)}{5(k+5)^{3}} F_{12} F_{21} G_{11}-\frac{i \sqrt{2}\left(73 k^{2}+710 k+1329\right)}{15(k+5)^{2}} \partial G_{11} \\
& +\frac{2 i \sqrt{2}}{k+5} U G_{11}-\frac{4 \sqrt{2}(k+3)}{(k+5)^{3}} U A_{3} F_{11}-\frac{4 \sqrt{2}(k+3)}{(k+5)^{3}} U A_{+} F_{21} \\
& -\frac{4 \sqrt{2}\left(3 k^{2}+35 k+94\right)}{5(k+5)^{3}} U B_{3} F_{11}-\frac{4 \sqrt{2}\left(3 k^{2}+35 k+94\right)}{5(k+5)^{3}} U B_{-} F_{12} \\
& +\frac{4 i \sqrt{2}(k+3)(5 k+34)}{3(k+5)^{3}} \partial U F_{11}+\frac{4 i \sqrt{2}(k+3)(5 k+34)}{3(k+5)^{3}} U \partial F_{11}+\frac{4 i \sqrt{2}}{(k+5)^{2}} U U F_{11} \\
& \left.+\frac{4 i \sqrt{2}\left(9 k^{2}+80 k+127\right)}{5(k+5)^{4}} U F_{11} F_{12} F_{21}-\frac{8 i \sqrt{2}\left(3 k^{2}+20 k+9\right)}{5(k+5)^{4}} F_{11} \partial F_{11} F_{22}\right](z) \\
& +\left[-2 i \sqrt{2} \mathbf{R}^{\left(\frac{5}{2}\right)}+2 i \sqrt{2} \mathbf{V}^{\left(\frac{5}{2}\right)}-\frac{4 i \sqrt{2}\left(5 k^{2}+46 k+81\right)}{(k+5)(7 k+3)}\left(F_{22} \mathbf{P}^{(\mathbf{2})}-\mathbf{P}^{(\mathbf{2})} F_{22}\right)\right. \\
& +\frac{i \sqrt{2}\left(41 k^{2}+400 k+783\right)}{5(k+5)^{2}} G_{22} \mathbf{T}^{(\mathbf{1})}-\frac{2 i \sqrt{2}\left(1061 k^{3}+10009 k^{2}+17763 k-2673\right)}{15(k+5)^{2}(7 k+3)} \partial \mathbf{V}^{\left(\frac{3}{2}\right)}
\end{aligned}
$$




$$
\begin{aligned}
& -\frac{2 i \sqrt{2}\left(41 k^{2}+400 k+783\right)}{5(k+5)^{2}} \mathbf{T}^{(\mathbf{1})} \mathbf{V}^{\left(\frac{3}{2}\right)} \\
& +\frac{4 \sqrt{2}}{(k+5)^{2}} A_{3} G_{22}-\frac{8 i \sqrt{2}}{(k+5)^{3}} A_{3} A_{3} F_{22}+\frac{4 i \sqrt{2}\left(3 k^{2}+30 k+59\right)}{5(k+5)^{3}} A_{3} B_{3} F_{22} \\
& +\frac{4 i \sqrt{2}\left(3 k^{2}+30 k+59\right)}{5(k+5)^{3}} A_{3} B_{+} F_{21}+\frac{8 \sqrt{2}\left(29 k^{3}+91 k^{2}-1143 k-2637\right)}{5(k+5)^{3}(7 k+3)} \partial A_{3} F_{22} \\
& +\frac{4 \sqrt{2}\left(5 k^{3}+24 k^{2}-109 k-264\right)}{(k+5)^{3}(7 k+3)} A_{3} \partial F_{22}-\frac{4 \sqrt{2}\left(9 k^{2}+80 k+127\right)}{5(k+5)^{4}} A_{3} F_{22} F_{21} F_{12} \\
& +\frac{4 \sqrt{2}}{(k+5)^{2}} A_{-} G_{12}-\frac{8 i \sqrt{2}}{(k+5)^{3}} A_{-} A_{+} F_{22}+\frac{4 i \sqrt{2}\left(3 k^{2}+30 k+59\right)}{5(k+5)^{3}} A_{-} B_{3} F_{12} \\
& -\frac{4 i \sqrt{2}\left(3 k^{2}+30 k+59\right)}{5(k+5)^{3}} A_{-} B_{+} F_{11}-\frac{4 \sqrt{2}\left(9 k^{2}+80 k+127\right)}{5(k+5)^{4}} A_{-} F_{22} F_{12} F_{11} \\
& +\frac{4 \sqrt{2}\left(5 k^{3}+24 k^{2}-95 k-258\right)}{(k+5)^{3}(7 k+3)} \partial A_{-} F_{12}+\frac{4 \sqrt{2}\left(5 k^{3}+24 k^{2}-109 k-264\right)}{(k+5)^{3}(7 k+3)} A_{-} \partial F_{12} \\
& -\frac{6 \sqrt{2}\left(k^{2}+10 k+23\right)}{5(k+5)^{2}} B_{3} G_{22}+\frac{12 i \sqrt{2}\left(k^{2}+10 k+23\right)}{5(k+5)^{3}} B_{3} B_{3} F_{22} \\
& -\frac{4 \sqrt{2}\left(15 k^{3}+100 k^{2}-119 k-708\right)}{(k+5)^{3}(7 k+3)} B_{3} \partial F_{22} \\
& -\frac{4 \sqrt{2}\left(9 k^{2}+80 k+127\right)}{5(k+5)^{4}} B_{3} F_{22} F_{21} F_{12}-\frac{6 \sqrt{2}\left(k^{2}+10 k+23\right)}{5(k+5)^{2}} B_{+} G_{21} \\
& +\frac{4 \sqrt{2}\left(9 k^{2}+80 k+127\right)}{5(k+5)^{4}} B_{+} F_{22} F_{21} F_{11}+\frac{4 \sqrt{2}\left(46 k^{3}+639 k^{2}+2788 k+3747\right)}{5(k+5)^{3}(7 k+3)} \partial B_{+} F_{21} \\
& +\frac{4 \sqrt{2}(k+3)\left(5 k^{2}+69 k+236\right)}{(k+5)^{3}(7 k+3)} B_{+} \partial F_{21}+\frac{12 i \sqrt{2}\left(k^{2}+10 k+23\right)}{5(k+5)^{3}} B_{-} B_{+} F_{22} \\
& -\frac{8 i \sqrt{2}(k-9)\left(5 k^{2}+46 k+81\right)}{(k+5)^{3}(7 k+3)} \partial^{2} F_{22}+\frac{4 i \sqrt{2}(k+7)}{(k+5)^{3}} F_{22} F_{21} G_{12} \\
& +\frac{8 i \sqrt{2}\left(3 k^{2}+20 k+9\right)}{5(k+5)^{4}} \partial F_{22} F_{21} F_{12}+\frac{16 i \sqrt{2}\left(3 k^{2}+20 k+9\right)}{5(k+5)^{4}} F_{22} \partial F_{21} F_{12} \\
& +\frac{4 i \sqrt{2}\left(3 k^{2}+25 k+44\right)}{5(k+5)^{3}} F_{22} F_{12} G_{21}+\frac{2 i \sqrt{2}\left(3 k^{2}+30 k+79\right)}{5(k+5)^{3}} F_{22} F_{11} G_{22} \\
& -\frac{2 i \sqrt{2}\left(3 k^{2}+20 k+9\right)}{5(k+5)^{3}} F_{21} F_{12} G_{22}+\frac{i \sqrt{2}\left(1211 k^{3}+12529 k^{2}+31053 k+18567\right)}{15(k+5)^{2}(7 k+3)} \partial G_{22} \\
& -\frac{2 i \sqrt{2}}{k+5} U G_{22}-\frac{4 \sqrt{2}(k+3)}{(k+5)^{3}} U A_{3} F_{22}-\frac{4 \sqrt{2}(k+3)}{(k+5)^{3}} U A_{-} F_{12} \\
& -\frac{4 \sqrt{2}\left(3 k^{2}+35 k+94\right)}{5(k+5)^{3}} U B_{3} F_{22}-\frac{4 \sqrt{2}\left(3 k^{2}+35 k+94\right)}{5(k+5)^{3}} U B_{+} F_{21}
\end{aligned}
$$




$$
\begin{aligned}
& -\frac{8 i \sqrt{2}\left(4 k^{3}-179 k^{2}-1978 k-3747\right)}{5(k+5)^{3}(7 k+3)} \partial U F_{22}+\frac{4 i \sqrt{2}(k+3)\left(5 k^{2}+69 k+236\right)}{(k+5)^{3}(7 k+3)} U \partial F_{22} \\
& -\frac{4 i \sqrt{2}\left(9 k^{2}+80 k+127\right)}{5(k+5)^{4}} U F_{22} F_{21} F_{12}+\frac{4 i \sqrt{2}\left(33 k^{3}+62 k^{2}-1741 k-3954\right)}{5(k+5)^{3}(7 k+3)} F_{22} G_{11} F_{22} \\
& \left.+\frac{8 i \sqrt{2}\left(3 k^{2}+20 k+9\right)}{5(k+5)^{4}} F_{22} \partial F_{22} F_{11}-\frac{4 i \sqrt{2}}{(k+5)^{2}} U U F_{22}\right](z), \\
& V_{\frac{1}{2}}^{(2), 3}(z)=\left[2 \sqrt{2} \mathbf{P}_{+}^{\left(\frac{5}{2}\right)}+2 \sqrt{2} \mathbf{W}_{+}^{\left(\frac{5}{2}\right)}-\frac{\sqrt{2}\left(41 k^{2}+400 k+783\right)}{5(k+5)^{2}} G_{21} \mathbf{T}^{(1)}\right. \\
& -\frac{2 \sqrt{2}\left(41 k^{2}+400 k+783\right)}{5(k+5)^{2}} \mathbf{T}^{(\mathbf{1})} \mathbf{T}_{+}^{\left(\frac{3}{2}\right)}+\frac{\left(41 k^{2}+400 k+783\right)}{5 \sqrt{2}(k+5)^{2}}\left(F_{21} \mathbf{P}^{(\mathbf{2})}-\mathbf{P}^{(\mathbf{2})} F_{21}\right) \\
& -\frac{4 i \sqrt{2}}{(k+5)^{2}} A_{3} G_{21}-\frac{8 \sqrt{2}}{(k+5)^{3}} A_{3} A_{3} F_{21}-\frac{4 \sqrt{2}\left(3 k^{2}+30 k+59\right)}{5(k+5)^{3}} A_{3} B_{3} F_{21} \\
& +\frac{4 \sqrt{2}\left(3 k^{2}+30 k+59\right)}{5(k+5)^{3}} A_{3} B_{-} F_{22}+\frac{4 i \sqrt{2}\left(9 k^{2}+80 k+127\right)}{5(k+5)^{4}} A_{3} F_{11} F_{21} F_{22} \\
& +\frac{2 i \sqrt{2}\left(50 k^{3}+617 k^{2}+2150 k+2151\right)}{15(k+5)^{4}} \partial A_{3} F_{21}+\frac{4 i \sqrt{2}}{(k+5)^{2}} A_{-} G_{11} \\
& +\frac{2 i \sqrt{2}\left(50 k^{3}+617 k^{2}+2270 k+2751\right)}{15(k+5)^{4}} A_{3} \partial F_{21}+\frac{4 \sqrt{2}\left(3 k^{2}+30 k+59\right)}{5(k+5)^{3}} A_{-} B_{3} F_{11} \\
& +\frac{4 \sqrt{2}\left(3 k^{2}+30 k+59\right)}{5(k+5)^{3}} A_{-} B_{-} F_{12}-\frac{4 i \sqrt{2}\left(25 k^{3}+124 k^{2}-695 k-2298\right)}{15(k+5)^{4}} \partial A_{-} F_{11} \\
& -\frac{4 i \sqrt{2}\left(25 k^{3}+124 k^{2}-665 k-2148\right)}{15(k+5)^{4}} A_{-} \partial F_{11}-\frac{6 i \sqrt{2}\left(k^{2}+10 k+23\right)}{5(k+5)^{2}} B_{3} G_{21} \\
& -\frac{4 i \sqrt{2}\left(9 k^{2}+80 k+127\right)}{5(k+5)^{4}} A_{-} F_{11} F_{12} F_{21}-\frac{8 \sqrt{2}}{(k+5)^{3}} A_{+} A_{-} F_{21} \\
& +\frac{12 \sqrt{2}\left(k^{2}+10 k+23\right)}{5(k+5)^{3}} B_{3} B_{3} F_{21}-\frac{4 i \sqrt{2}\left(9 k^{2}+80 k+127\right)}{5(k+5)^{4}} B_{3} F_{11} F_{21} F_{22} \\
& -\frac{2 i \sqrt{2}(k+3)\left(14 k^{2}+35 k-463\right)}{15(k+5)^{4}} \partial B_{3} F_{21}-\frac{2 i \sqrt{2}\left(50 k^{3}+617 k^{2}+2270 k+2751\right)}{15(k+5)^{4}} B_{3} \partial F_{21} \\
& +\frac{6 i \sqrt{2}\left(k^{2}+10 k+23\right)}{5(k+5)^{2}} B_{-} G_{22}-\frac{4 i \sqrt{2}\left(9 k^{2}+80 k+127\right)}{5(k+5)^{4}} B_{-} F_{12} F_{21} F_{22} \\
& \text { - } \frac{2 i \sqrt{2}\left(91 k^{3}+853 k^{2}+1393 k-681\right)}{15(k+5)^{4}} \partial B_{-} F_{22} \\
& -\frac{2 i \sqrt{2}\left(73 k^{3}+583 k^{2}+79 k-2751\right)}{15(k+5)^{4}} B_{-} \partial F_{22}+\frac{12 \sqrt{2}\left(k^{2}+10 k+23\right)}{5(k+5)^{3}} B_{+} B_{-} F_{21} \\
& -\frac{4 \sqrt{2}\left(3 k^{2}+25 k+44\right)}{5(k+5)^{3}} F_{11} F_{21} G_{22}+\frac{4 \sqrt{2}\left(23 k^{2}+280 k+729\right)}{15(k+5)^{4}} \partial F_{11} F_{21} F_{22}
\end{aligned}
$$




$$
\begin{aligned}
& +\frac{4 \sqrt{2}\left(41 k^{2}+400 k+783\right)}{15(k+5)^{4}} F_{11} \partial F_{21} F_{22}+\frac{4 \sqrt{2}\left(59 k^{2}+520 k+837\right)}{15(k+5)^{4}} F_{11} F_{21} \partial F_{22} \\
& +\frac{2 \sqrt{2}\left(3 k^{2}+20 k+9\right)}{5(k+5)^{3}} F_{11} F_{22} G_{21}-\frac{2 \sqrt{2}\left(3 k^{2}+30 k+79\right)}{5(k+5)^{3}} F_{12} F_{21} G_{21} \\
& +\frac{2 \sqrt{2}(k-3)\left(41 k^{2}+400 k+783\right)}{5(k+5)^{4}} \partial^{2} F_{21}+\frac{4 \sqrt{2}(k+7)}{(k+5)^{3}} F_{21} F_{22} G_{11} \\
& -\frac{\sqrt{2}\left(50 k^{3}+617 k^{2}+2270 k+2751\right)}{15(k+5)^{3}} \partial G_{21}-\frac{2 \sqrt{2}}{k+5} U G_{21}+\frac{4 i \sqrt{2}(k+3)}{(k+5)^{3}} U A_{3} F_{21} \\
& -\frac{4 i \sqrt{2}(k+3)}{(k+5)^{3}} U A_{-} F_{11}-\frac{4 i \sqrt{2}\left(3 k^{2}+35 k+94\right)}{5(k+5)^{3}} U B_{3} F_{21} \\
& +\frac{4 i \sqrt{2}\left(3 k^{2}+35 k+94\right)}{5(k+5)^{3}} U B_{-} F_{22}-\frac{4 \sqrt{2}\left(9 k^{2}+80 k+127\right)}{5(k+5)^{4}} U F_{11} F_{21} F_{22} \\
& -\frac{2 \sqrt{2}\left(50 k^{3}+617 k^{2}+2270 k+2751\right)}{15(k+5)^{4}} \partial U F_{21}-\frac{4 \sqrt{2}}{(k+5)^{2}} U U F_{21} \\
& \left.-\frac{2 \sqrt{2}\left(50 k^{3}+617 k^{2}+2270 k+2751\right)}{15(k+5)^{4}} U \partial F_{21}+\frac{8 \sqrt{2}\left(3 k^{2}+20 k+9\right)}{5(k+5)^{4}} F_{21} \partial F_{21} F_{12}\right](z) \\
& +\left[-2 \sqrt{2} \mathbf{P}_{-}^{\left(\frac{5}{2}\right)}+2 \sqrt{2} \mathbf{W}_{-}^{\left(\frac{5}{2}\right)}-\frac{\sqrt{2}(k+3)(5 k+34)}{3(k+5)^{2}}\left(F_{12} \mathbf{P}^{(\mathbf{2})}-\mathbf{P}^{(\mathbf{2})} F_{12}\right)\right. \\
& +\frac{\sqrt{2}\left(41 k^{2}+400 k+783\right)}{5(k+5)^{2}} G_{12} \mathbf{T}^{(1)}-\frac{2 \sqrt{2}\left(173 k^{2}+1690 k+3369\right)}{15(k+5)^{2}} \partial \mathbf{T}_{-}^{\left(\frac{3}{2}\right)} \\
& -\frac{2 \sqrt{2}\left(41 k^{2}+400 k+783\right)}{5(k+5)^{2}} \mathbf{T}^{(\mathbf{1})} \mathbf{T}_{-}^{\left(\frac{3}{2}\right)} \\
& +\frac{4 i \sqrt{2}}{(k+5)^{2}} A_{3} G_{12}-\frac{8 \sqrt{2}}{(k+5)^{3}} A_{3} A_{3} F_{12}-\frac{4 \sqrt{2}\left(3 k^{2}+30 k+59\right)}{5(k+5)^{3}} A_{3} B_{3} F_{12} \\
& +\frac{4 \sqrt{2}\left(3 k^{2}+30 k+59\right)}{5(k+5)^{3}} A_{3} B_{+} F_{11}+\frac{4 i \sqrt{2}\left(9 k^{2}+80 k+127\right)}{5(k+5)^{4}} A_{3} F_{11} F_{12} F_{22} \\
& -\frac{4 i \sqrt{2}}{(k+5)^{2}} A_{+} G_{22}+\frac{4 \sqrt{2}\left(3 k^{2}+30 k+59\right)}{5(k+5)^{3}} A_{+} B_{3} F_{22}-\frac{8 \sqrt{2}}{(k+5)^{3}} A_{+} A_{-} F_{12} \\
& +\frac{4 \sqrt{2}\left(3 k^{2}+30 k+59\right)}{5(k+5)^{3}} A_{+} B_{+} F_{21}-\frac{4 i \sqrt{2}\left(9 k^{2}+80 k+127\right)}{5(k+5)^{4}} A_{+} F_{12} F_{21} F_{22} \\
& -\frac{8 i \sqrt{2}}{(k+5)^{3}} \partial A_{+} F_{22}+\frac{6 i \sqrt{2}\left(k^{2}+10 k+23\right)}{5(k+5)^{2}} B_{3} G_{12}+\frac{12 \sqrt{2}\left(k^{2}+10 k+23\right)}{5(k+5)^{3}} B_{3} B_{3} F_{12} \\
& -\frac{4 i \sqrt{2}\left(9 k^{2}+80 k+127\right)}{5(k+5)^{4}} B_{3} F_{11} F_{12} F_{22}-\frac{6 i \sqrt{2}\left(k^{2}+10 k+23\right)}{5(k+5)^{2}} B_{+} G_{11} \\
& +\frac{12 \sqrt{2}\left(k^{2}+10 k+23\right)}{5(k+5)^{3}} B_{+} B_{-} F_{12}+\frac{12 i \sqrt{2}\left(k^{2}+10 k+23\right)}{5(k+5)^{3}} \partial B_{+} F_{11}
\end{aligned}
$$




$$
\begin{aligned}
& -\frac{4 i \sqrt{2}\left(9 k^{2}+80 k+127\right)}{5(k+5)^{4}} B_{+} F_{11} F_{12} F_{21}-\frac{4 \sqrt{2}(k+7)}{(k+5)^{3}} F_{11} F_{12} G_{22} \\
& -\frac{16 \sqrt{2}\left(3 k^{2}+25 k+44\right)}{5(k+5)^{4}} \partial F_{11} F_{12} F_{22}-\frac{8 \sqrt{2}\left(3 k^{2}+30 k+79\right)}{5(k+5)^{4}} F_{11} \partial F_{12} F_{22} \\
& -\frac{16 \sqrt{2}(k+7)}{(k+5)^{4}} F_{11} F_{12} \partial F_{22}-\frac{2 \sqrt{2}\left(3 k^{2}+20 k+9\right)}{5(k+5)^{3}} F_{11} F_{22} G_{12} \\
& +\frac{2 \sqrt{2}\left(3 k^{2}+30 k+79\right)}{5(k+5)^{3}} F_{12} F_{21} G_{12}+\frac{4 \sqrt{2}\left(3 k^{2}+25 k+44\right)}{5(k+5)^{3}} F_{12} F_{22} G_{11} \\
& +\frac{\sqrt{2}\left(173 k^{2}+1690 k+3369\right)}{15(k+5)^{2}} \partial G_{12}-\frac{2 \sqrt{2}}{k+5} U G_{12}-\frac{4 i \sqrt{2}(k+3)}{(k+5)^{3}} U A_{3} F_{12} \\
& +\frac{4 i \sqrt{2}(k+3)}{(k+5)^{3} U A_{+} F_{22}+\frac{4 i \sqrt{2}\left(3 k^{2}+35 k+94\right)}{5(k+5)^{3}} U B_{3} F_{12}} \\
& -\frac{4 i \sqrt{2}\left(3 k^{2}+35 k+94\right)}{5(k+5)^{3}} U B_{+} F_{11}+\frac{4 \sqrt{2}\left(9 k^{2}+80 k+127\right)}{5(k+5)^{4}} U F_{11} F_{12} F_{22} \\
& \left.-\frac{4 \sqrt{2}}{(k+5)^{2}} U F_{12}+\frac{8 \sqrt{2}\left(3 k^{2}+20 k+9\right)}{5(k+5)^{4}} F_{12} \partial F_{12} F_{21}\right](z) .
\end{aligned}
$$

Compared to the description in section 4 (the equations (4.2), (4.4), (4.6) and (4.7)), the above expressions are rather complicated because the higher spin-2 in (6.1) has many extra terms except $\mathbf{P}^{(\mathbf{2})}(z)$. Furthermore, the higher spin currents in the nonlinear version [9] has the explicit relations with its linear version via the equations (4.22)-(4.25) of [9]. Then one can write down $\tilde{V}_{0}^{(2)}(z) \equiv V_{0}^{(2)}(z)$ and $\tilde{V}_{\frac{1}{2}}^{(2), a}(z) \equiv V_{\frac{1}{2}}^{(2), a}(z)$. Once the other higher spin currents $V_{1}^{(2), \pm i}(z), V_{\frac{3}{2}}^{(2), a}(z)$ and $V_{2}^{(2)}(z)$ are found explicitly, then the corresponding higher spin currents, $\widetilde{V}_{1}^{(2), \pm i}(z), \widetilde{V}_{\frac{3}{2}}^{(2), a}(z)$ and $\widetilde{V}_{2}^{(2)}(z)$, can be written in terms of the corresponding higher spin currents in the linear versions plus four spin- $\frac{1}{2}$ currents and the spin-1 current.

\section{Appendix F The 136 OPEs between the 16 lowest higher spin currents}

In this final Appendix, the complete OPEs between the 16 lowest higher spin currents are presented. The highest spin in the right hand side of these OPEs is given by 3. The composite fields of spin- $\frac{7}{2}$, spin- 4 , spin- $\frac{9}{2}$ and spin- 5 are not present in this paper. They can be obtained from its nonlinear version of Part II as described in Appendix $E$ (just below the equation (E.1) ) in principle. All the 16 higher spin currents are known in terms of coset fields described in section 3 and one has all the singular terms of these OPEs completely. The nontrivial things here are to write them in terms of known 16 currents and 16 higher spin currents (and their 
derivatives). In this Appendix, the final OPEs are presented after determing the unknown strucuture constants. Some simple comments on each OPE are added.

\section{Appendix F.1 The OPEs between the first $\mathcal{N}=2$ multiplet and itself (and the other three $\mathcal{N}=2$ multiplets)}

From the relation (B.1), one has the following OPE

$$
\mathbf{T}^{(\mathbf{1})}(z) \mathbf{T}^{(\mathbf{1})}(w)=\frac{1}{(z-w)^{2}}\left[\frac{6 k}{(5+k)}\right]+\cdots
$$

This is the same as the OPE in the nonlinear version because one has the relation (B.1).

From the expressions (B.1) and (B.2) with the help of Part II,

$$
\begin{aligned}
\mathbf{T}^{(\mathbf{1})}(z) \mathbf{T}_{ \pm}^{\left(\frac{3}{2}\right)}(w) & =\frac{1}{(z-w)}\left[ \pm \mathbf{T}_{ \pm}^{\left(\frac{3}{2}\right)}-\frac{1}{(5+k)} U\left(\begin{array}{c}
F_{21} \\
F_{12}
\end{array}\right) \pm \frac{4}{(5+k)^{2}}\left(\begin{array}{c}
F_{21} \\
F_{12}
\end{array}\right) F_{11} F_{22}\right. \\
& \pm \frac{i}{(5+k)}\left(\begin{array}{c}
F_{21} \\
F_{12}
\end{array}\right) A_{3} \mp \frac{i}{(5+k)}\left(\begin{array}{c}
F_{11} A_{-} \\
F_{22} A_{+}
\end{array}\right) \pm \frac{i}{(5+k)}\left(\begin{array}{c}
F_{22} B_{-} \\
F_{11} B_{+}
\end{array}\right) \\
& \left.\mp \frac{i}{(5+k)}\left(\begin{array}{c}
F_{21} \\
F_{12}
\end{array}\right) B_{3}\right](w)+\cdots
\end{aligned}
$$

Due to the presence of spin- $\frac{1}{2}$ currents, there are many terms containing them in the above.

Together with (B.7), one obtains the following OPE

$$
\begin{aligned}
\mathbf{T}^{(\mathbf{1})}(z) \mathbf{T}^{(\mathbf{2})}(w) & =\frac{1}{(z-w)^{2}}\left[-\frac{6 i}{(5+k)} A_{3}-\frac{2 i k}{(5+k)} B_{3}+\frac{2(-3+k)}{(5+k)^{2}} F_{11} F_{22}-\frac{2(3+k)}{(5+k)^{2}} F_{12} F_{21}\right](w) \\
& +\frac{1}{(z-w)}\left[\frac{2 i}{(5+k)^{2}} F_{11} F_{12} A_{-}-\frac{2 i}{(5+k)^{2}} F_{21} F_{22} A_{+}-\frac{2 i}{(5+k)^{2}} F_{12} F_{22} B_{-}\right. \\
& +\frac{2 i}{(5+k)^{2}} F_{11} F_{21} B_{+}-\frac{1}{(5+k)} F_{11} G_{22} \\
& \left.+\frac{1}{(5+k)} F_{22} G_{11}-\frac{4}{(5+k)^{2}} F_{11} F_{22} U\right](w)+\cdots
\end{aligned}
$$

Compared to the OPE in the nonlinear version, the above OPE has nontrivial first order pole.

The second order pole does not have the higher spin-1 current.

With (B.4), one calculates the following OPEs

$$
\begin{aligned}
\mathbf{T}^{(\mathbf{1})}(z)\left(\begin{array}{c}
\mathbf{U}^{\left(\frac{3}{2}\right)} \\
\mathbf{V}^{\left(\frac{3}{2}\right)}
\end{array}\right)(w) & =\frac{1}{(z-w)}\left[ \pm\left(\begin{array}{c}
\mathbf{U}^{\left(\frac{3}{2}\right)} \\
\mathbf{V}^{\left(\frac{3}{2}\right)}
\end{array}\right) \mp \frac{4}{(5+k)^{2}} F_{12} F_{21}\left(\begin{array}{c}
F_{11} \\
F_{22}
\end{array}\right)\right. \\
& \mp \frac{i}{(5+k)}\left(\begin{array}{c}
F_{11} \\
F_{22}
\end{array}\right) A_{3} \mp \frac{i}{(5+k)}\left(\begin{array}{c}
F_{21} \\
F_{12}
\end{array}\right) A_{ \pm}-\frac{1}{(5+k)} U\left(\begin{array}{c}
F_{21} \\
F_{12}
\end{array}\right) \\
& \left.\mp \frac{i}{(5+k)}\left(\begin{array}{c}
F_{12} \\
F_{21}
\end{array}\right) B_{\mp} \mp \frac{i}{(5+k)}\left(\begin{array}{c}
F_{11} \\
F_{22}
\end{array}\right) B_{3}\right](w)+\cdots
\end{aligned}
$$


If one ignores the spin- $\frac{1}{2}$ current terms, then this OPE has the same form as the one in the nonlinear version.

From the previous expression for higher spin-1 current and the expression (B.5), one can describe the following OPEs

$$
\begin{aligned}
\mathbf{T}^{(\mathbf{1})}(z)\left(\begin{array}{c}
\mathbf{U}_{+}^{(2)} \\
\mathbf{V}_{-}^{(2)}
\end{array}\right)(w) & =\frac{1}{(z-w)^{2}}\left[ \pm \frac{2 k}{(5+k)} i B_{\mp} \mp \frac{4 k}{(5+k)^{2}}\left(\begin{array}{c}
F_{11} F_{21} \\
F_{12} F_{22}
\end{array}\right)\right](w) \\
& +\frac{1}{(z-w)}\left[-\frac{2 i}{(5+k)^{2}} F_{11} F_{22} B_{\mp}+\frac{2 i}{(5+k)^{2}} F_{12} F_{21} B_{\mp}\right. \\
& \pm \frac{4 i}{(5+k)^{2}}\left(\begin{array}{c}
F_{11} F_{21} \\
F_{22} F_{12}
\end{array}\right) B_{3}+\frac{4}{(5+k)^{2}}\left(\begin{array}{c}
F_{11} F_{21} \\
F_{22} F_{12}
\end{array}\right) U \\
& \left.+\frac{1}{(5+k)}\left(\begin{array}{c}
F_{11} G_{21} \\
F_{22} G_{12}
\end{array}\right)-\frac{1}{(5+k)}\left(\begin{array}{c}
F_{21} G_{11} \\
F_{12} G_{22}
\end{array}\right)\right](w)+\cdots
\end{aligned}
$$

Again, there are many spin- $\frac{1}{2}$ current terms which are new in the linear version.

Similarly, from the equation (B.6), one calculates the following OPEs

$$
\begin{aligned}
\mathbf{T}^{(\mathbf{1})}(z)\left(\begin{array}{c}
\mathbf{U}_{-}^{(2)} \\
\mathbf{V}_{+}^{(2)}
\end{array}\right)(w) & =\frac{1}{(z-w)^{2}}\left[\mp \frac{6}{(5+k)} i A_{ \pm}-\frac{12}{(5+k)^{2}}\left(\begin{array}{c}
F_{11} F_{12} \\
F_{22} F_{21}
\end{array}\right)\right](w) \\
& +\frac{1}{(z-w)}\left[\mp \frac{4 i}{(5+k)^{2}}\left(\begin{array}{c}
F_{11} F_{12} \\
F_{22} F_{21}
\end{array}\right) A_{3}+\frac{2 i}{(5+k)^{2}} F_{11} F_{22} A_{ \pm}\right. \\
& +\frac{2 i}{(5+k)^{2}} F_{12} F_{21} A_{ \pm}-\frac{1}{(5+k)}\left(\begin{array}{c}
F_{11} G_{12} \\
F_{22} G_{21}
\end{array}\right) \\
& \left.+\frac{1}{(5+k)}\left(\begin{array}{c}
F_{12} G_{11} \\
F_{21} G_{22}
\end{array}\right)-\frac{4}{(5+k)^{2}}\left(\begin{array}{c}
F_{11} F_{12} \\
F_{22} F_{21}
\end{array}\right) U\right](w)+\cdots
\end{aligned}
$$

Due to the spin- $\frac{1}{2}$ currents, this OPE has many new terms in the above.

From the relations (B.9) and (B.10), one obtains the following OPEs

$$
\begin{aligned}
\mathbf{T}^{(\mathbf{1})}(z)\left(\begin{array}{c}
\mathbf{U}^{\left(\frac{5}{2}\right)} \\
\mathbf{V}^{\left(\frac{5}{2}\right)}
\end{array}\right)(w) & =\mp \frac{1}{(z-w)^{3}} \frac{12 k}{(5+k)^{2}}\left(\begin{array}{c}
F_{11} \\
F_{22}
\end{array}\right)(w) \\
& +\frac{1}{(z-w)^{2}}\left[-\partial(\text { pole- } 3)+\frac{4 i(6+k)}{3(5+k)^{2}}\left(\begin{array}{c}
F_{11} \\
F_{22}
\end{array}\right) B_{3}\right. \\
& \mp \frac{4(-3+k)}{3(5+k)}\left(\begin{array}{c}
G_{11} \\
G_{22}
\end{array}\right) \mp \frac{8(-3+k)}{3(5+k)^{2}} U\left(\begin{array}{c}
F_{11} \\
F_{22}
\end{array}\right) \\
& +\frac{4(-3+k)}{3(5+k)^{3}} F_{12} F_{21}\left(\begin{array}{c}
F_{11} \\
F_{22}
\end{array}\right)-\frac{4 i(3+2 k)}{3(5+k)^{2}}\left(\begin{array}{c}
F_{11} \\
F_{22}
\end{array}\right) A_{3} \\
& \left.-\frac{4 i(3+2 k)}{3(5+k)^{2}}\left(\begin{array}{c}
F_{21} \\
F_{12}
\end{array}\right) A_{ \pm}+\frac{4 i(6+k)}{3(5+k)^{2}}\left(\begin{array}{c}
F_{12} \\
F_{21}
\end{array}\right) B_{\mp}\right](w) \\
& +\frac{1}{(z-w)}\left[ \pm\left(\begin{array}{c}
\mathbf{U}^{\left(\frac{5}{2}\right)} \\
\mathbf{V}^{\left(\frac{5}{2}\right)}
\end{array}\right)+\left(\begin{array}{c}
\mathbf{Q}^{\left(\frac{5}{2}\right)} \\
\mathbf{R}^{\left(\frac{5}{2}\right)}
\end{array}\right)(w)\right](w)+\cdots .
\end{aligned}
$$


Compared to the OPE in the nonlinear version, the above OPE does not contain the higher spin- $\frac{3}{2}$ currents.

Together with (B.1) and (B.8), the following OPE can be obtained

$$
\begin{aligned}
\mathbf{T}^{(\mathbf{1})}(z) \mathbf{W}^{(\mathbf{2})}(w) & =\frac{1}{(z-w)^{2}}\left[\frac{6 i}{(5+k)} A_{3}-\frac{2 i k}{(5+k)} B_{3}+\frac{2(3+k)}{(5+k)^{2}} F_{11} F_{22}-\frac{2(-3+k)}{(5+k)^{2}} F_{12} F_{21}\right](w) \\
& +\frac{1}{(z-w)}\left[-\frac{2 i}{(5+k)^{2}} F_{11} F_{12} A_{-}+\frac{2 i}{(5+k)^{2}} F_{21} F_{22} A_{+}-\frac{2 i}{(5+k)^{2}} F_{12} F_{22} B_{-}\right. \\
& +\frac{2 i}{(5+k)^{2}} F_{11} F_{21} B_{+}+\frac{1}{(5+k)} F_{12} G_{21} \\
& \left.-\frac{1}{(5+k)} F_{21} G_{12}+\frac{4}{(5+k)^{2}} F_{12} F_{21} U\right](w)+\cdots
\end{aligned}
$$

Note that in this OPE there exists nontrivial first order pole and the second order pole does not contain the higher spin-1 current.

Furthermore, the relations (B.11) and (B.12) determine the following OPEs

$$
\begin{aligned}
\mathbf{T}^{(\mathbf{1})}(z) \mathbf{W}_{ \pm}^{\left(\frac{5}{2}\right)}(w) & =\mp \frac{1}{(z-w)^{3}} \frac{12 k}{(5+k)^{2}}\left(\begin{array}{c}
F_{21} \\
F_{12}
\end{array}\right)(w) \\
& +\frac{1}{(z-w)^{2}}\left[-\partial\left(\text { pole-3) }+\frac{4 i(6+k)}{3(5+k)^{2}}\left(\begin{array}{c}
F_{21} \\
F_{12}
\end{array}\right) B_{3}\right.\right. \\
& \mp \frac{4(-3+k)}{3(5+k)}\left(\begin{array}{c}
G_{21} \\
G_{12}
\end{array}\right) \mp \frac{8(-3+k)}{3(5+k)^{2}} U\left(\begin{array}{c}
F_{21} \\
F_{12}
\end{array}\right) \\
& -\frac{4(-3+k)}{3(5+k)^{3}}\left(\begin{array}{c}
F_{21} \\
F_{12}
\end{array}\right) F_{11} F_{22}+\frac{4 i(3+2 k)}{3(5+k)^{2}}\left(\begin{array}{c}
F_{21} \\
F_{12}
\end{array}\right) A_{3} \\
& \left.-\frac{4 i(3+2 k)}{3(5+k)^{2}}\left(\begin{array}{c}
F_{11} \\
F_{22}
\end{array}\right) A_{\mp}-\frac{4 i(6+k)}{3(5+k)^{2}}\left(\begin{array}{c}
F_{22} \\
F_{11}
\end{array}\right) B_{\mp}\right](w) \\
& +\frac{1}{(z-w)}\left[ \pm \mathbf{W}_{ \pm}^{\left(\frac{5}{2}\right)}+\mathbf{P}_{ \pm}^{\left(\frac{5}{2}\right)}\right](w)+\cdots
\end{aligned}
$$

Note that there are no higher spin- $\frac{3}{2}$ currents which were present in the nonlinear version.

For the higher spin-3 current, one can use the relation (B.13) and obtain the following OPE

$$
\begin{aligned}
\mathbf{T}^{(\mathbf{1})}(z) \mathbf{W}^{(\mathbf{3})}(w) & =\frac{1}{(z-w)^{3}} \frac{24 k}{(5+k)^{2}} U(w) \\
& +\frac{1}{(z-w)^{2}}\left[-\mathbf{P}^{(\mathbf{2})}+4 \mathbf{W}^{(\mathbf{2})}+\frac{4(-3+k)}{3(5+k)} \mathbf{T}^{(\mathbf{2})}-\frac{4(-\mathbf{3}+\mathbf{k})}{(17+13 k)} \mathbf{T}^{(\mathbf{1})} \mathbf{T}^{(\mathbf{1})}\right. \\
& +\frac{8(-3+k)(17+4 k)}{3(5+k)(17+13 k)} T+\frac{4(3+2 k)}{3(5+k)^{2}} A_{3} A_{3}-\frac{4(-3+k)}{3(5+k)^{2}} A_{3} B_{3}+\frac{4 i(3+2 k)}{3(5+k)^{2}} \partial A_{3}
\end{aligned}
$$




$$
\begin{aligned}
& +\frac{4(3+2 k)}{3(5+k)^{2}} A_{+} A_{-}-\frac{4(6+k)}{3(5+k)^{2}} B_{3} B_{3}-\frac{4 i(6+k)}{3(5+k)^{2}} \partial B_{3}-\frac{4(6+k)}{3(5+k)^{2}} B_{+} B_{-} \\
& +\frac{4 i(-3+k)}{3(5+k)^{3}} A_{3} F_{11} F_{22}-\frac{8 i(15+k)}{3(5+k)^{3}} A_{-} F_{11} F_{12}+\frac{4 i(9+k)}{(5+k)^{3}} A_{+} F_{21} F_{22} \\
& -\frac{4 i(-3+k)}{3(5+k)^{3}} B_{3} F_{11} F_{22}-\frac{4 i(15+7 k)}{3(5+k)^{3}} B_{-} F_{12} F_{22}+\frac{8 i(3+k)}{(5+k)^{3}} B_{+} F_{11} F_{21} \\
& -\frac{(15+7 k)}{3(5+k)^{2}} F_{11} G_{22}+\frac{4\left(-63-9 k+2 k^{2}\right)}{3(5+k)^{3}} \partial F_{11} F_{22}-\frac{4\left(27+9 k+2 k^{2}\right)}{3(5+k)^{3}} F_{11} \partial F_{22} \\
& +\frac{(9+k)}{(5+k)^{2}} F_{12} G_{21}+\frac{8(-3+k)(6+k)}{3(5+k)^{3}} \partial F_{12} F_{21}-\frac{8(-3+k)(2+k)}{3(5+k)^{3}} F_{12} \partial F_{21} \\
& -\frac{3}{(5+k)} F_{21} G_{12}-\frac{(21+5 k)}{3(5+k)^{2}} F_{22} G_{11}+\frac{12 i}{(5+k)^{2}} U A_{3}+\frac{4 i k}{(5+k)^{2}} U B_{3} \\
& \left.-\frac{12 k}{(5+k)^{2}} \partial U+\frac{8(-3+k)}{3(5+k)^{2}} U U-\frac{16(-3+k)}{3(5+k)^{2}} U F_{11} F_{22}+\frac{12}{(5+k)^{2}} U F_{12} F_{21}\right](w) \\
& +\frac{1}{(z-w)}\left[\frac{4 i}{(k+5)^{2}} A_{3} \partial\left(F_{11} F_{22}\right)+\frac{4 i}{(k+5)^{2}} A_{3} \partial\left(F_{12} F_{21}\right)-\frac{4 i}{(k+5)^{2}} \partial A_{-} F_{11} F_{12}\right. \\
& +\frac{4 i}{(k+5)^{2}} A_{-} \partial\left(F_{11} F_{12}\right)-\frac{4 i}{(k+5)^{2}} \partial A_{+} F_{21} F_{22}+\frac{4 i}{(k+5)^{2}} A_{+} \partial\left(F_{21} F_{22}\right) \\
& \left.-\frac{4 i}{(k+5)^{2}} F_{11} G_{22} F_{11} F_{22}+\frac{4}{(k+5)^{2}} F_{21} G_{12} F_{21} F_{12}\right](w)+\cdots \\
& +
\end{aligned}
$$

The fields appearing in the second and third lines of the first order pole can be seen in the corresponding OPE in the nonlinear version. Compared to the corresponding OPE in the nonlinear version, the above OPE does not contain any composite fields between the higher spin currents and the currents from the large $\mathcal{N}=4$ linear superconformal algebra 5 . Also

${ }^{5}$ There are no composite fields written in terms of the field with a boldface and the field without a boldface. There are only composite fields with a boldface and only composite fields without a boldface. In this Appendix, what we mean by 'the nonlinear terms in the nonlinear version' are the composite fields between any higher 
note the presence of the quadratic term (nonlinear term) of higher spin-1 current. Using the following relations, some of the terms in the OPE can be written in terms of other terms

$$
\begin{aligned}
F_{22} G_{11} F_{22} F_{11} & =\left(i \partial A_{3}+i \partial B_{3}+\partial U\right) F_{22} F_{11}, \\
F_{11} G_{22} F_{11} F_{22} & =\left(-i \partial A_{3}-i \partial B_{3}+\partial U\right) F_{11} F_{22}, \\
F_{21} G_{12} F_{21} F_{12} & =\left(i \partial A_{3}-i \partial B_{3}+\partial U\right) F_{21} F_{12} .
\end{aligned}
$$

Let us move to the OPEs between the next higher spin- $\frac{3}{2}$ current and other higher spin currents (except the previous higher spin-1 current). From the description of (3.1), the following OPEs can be obtained

$$
\begin{aligned}
\mathbf{T}_{ \pm}^{\left(\frac{3}{2}\right)}(z) \mathbf{T}_{ \pm}^{\left(\frac{3}{2}\right)}(w) & =\frac{1}{(z-w)}\left[-\frac{1}{(k+5)} A_{\mp} B_{\mp} \mp \frac{2 i}{(k+5)^{2}} A_{\mp}\left(\begin{array}{c}
F_{11} F_{21} \\
F_{22} F_{12}
\end{array}\right)\right. \\
& \left. \pm \frac{2 i}{(k+5)^{2}} B_{\mp}\left(\begin{array}{c}
F_{21} F_{22} \\
F_{12} F_{11}
\end{array}\right)-\frac{2}{(k+5)^{2}}\left(\begin{array}{c}
F_{21} \partial F_{21} \\
F_{12} \partial F_{12}
\end{array}\right)\right](w)+\cdots
\end{aligned}
$$

These are new nontrivial OPEs. In the nonlinear version, the corresponding OPEs are not present. Even the terms which did not appear in the corresponding OPEs in the nonlinear version appear in the above OPEs.

Again the relations (3.1) determine the following OPE

$$
\begin{aligned}
\mathbf{T}_{+}^{\left(\frac{3}{2}\right)}(z) \mathbf{T}_{-}^{\left(\frac{3}{2}\right)}(w) & =-\frac{1}{(z-w)^{3}}\left[\frac{(4+7 k)}{(5+k)}\right] \\
& +\frac{1}{(z-w)^{2}}\left[-\mathbf{T}^{(\mathbf{1})}+\frac{7 i}{(5+k)} A_{3}+\frac{i(1+2 k)}{(5+k)} B_{3}-\frac{(k-3)}{(k+5)^{2}} F_{11} F_{22}\right. \\
& \left.+\frac{(k+3)}{(k+5)^{2}} F_{12} F_{21}\right](w) \\
& +\frac{1}{(z-w)}\left[-\mathbf{T}^{(\mathbf{2})}-\frac{1}{2} \partial \mathbf{T}^{(\mathbf{1})}\right. \\
& -\frac{1}{2(5+k)} A_{3} A_{3}+\frac{1}{(5+k)} A_{3} B_{3}+\frac{3 i}{(5+k)} \partial A_{3} \\
& -\frac{1}{2(5+k)} A_{+} A_{-}-\frac{1}{2(5+k)} B_{3} B_{3}+\frac{i k}{(5+k)} \partial B_{3}-\frac{1}{2(5+k)} B_{+} B_{-} \\
& +\frac{2 i}{(5+k)^{2}} A_{3} F_{11} F_{22}+\frac{i}{(5+k)^{2}} A_{-} F_{11} F_{12}+\frac{i}{(5+k)^{2}} A_{+} F_{21} F_{22} \\
& -\frac{2 i}{(5+k)^{2}} B_{3} F_{11} F_{22}-\frac{i}{(5+k)^{2}} B_{-} F_{12} F_{22}-\frac{i}{(5+k)^{2}} B_{+} F_{11} F_{21} \\
& -\frac{(3+k)}{(5+k)^{2}} \partial F_{11} F_{22}+\frac{6}{(5+k)^{2}} F_{11} \partial F_{22}-\frac{2}{(5+k)^{2}} \partial F_{12} F_{21}
\end{aligned}
$$

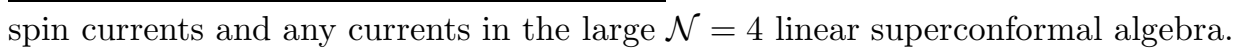




$$
\left.+\frac{1}{(5+k)} F_{12} \partial F_{21}-\frac{1}{2(5+k)} U U\right](w)+\cdots .
$$

This OPE is rather complicated due to the spin- $\frac{1}{2}$ currents and spin- 1 current which are present in the linear version.

Together with the previous relations and (3.3), the following OPEs can be obtained

$$
\begin{aligned}
& \mathbf{T}_{ \pm}^{\left(\frac{3}{2}\right)}(z) \mathbf{T}^{(\mathbf{2})}(w)=\frac{1}{(z-w)^{2}}\left[\frac{3}{2} \mathbf{T}_{ \pm}^{\left(\frac{3}{2}\right)} \mp \frac{(3 k+11)}{2(k+5)^{2}} U\left(\begin{array}{c}
F_{21} \\
F_{12}
\end{array}\right)+\frac{2}{(k+5)^{2}}\left(\begin{array}{c}
F_{21} \\
F_{12}
\end{array}\right) F_{11} F_{22}\right](w) \\
& +\frac{i(3 k+11)}{2(k+5)^{2}}\left(\begin{array}{c}
F_{21} \\
F_{12}
\end{array}\right) A_{3}-\frac{i(3 k-1)}{2(k+5)^{2}}\left(\begin{array}{c}
F_{11} \\
F_{22}
\end{array}\right) A_{\mp}-\frac{i(k-11)}{2(k+5)^{2}}\left(\begin{array}{c}
F_{22} \\
F_{11}
\end{array}\right) B_{\mp} \\
& \left.-\frac{i(3 k+11)}{2(k+5)^{2}}\left(\begin{array}{c}
F_{21} \\
F_{12}
\end{array}\right) B_{3} \pm \frac{1}{(k+5)}\left(\begin{array}{l}
G_{21} \\
G_{12}
\end{array}\right)\right](w) \\
& +\frac{1}{(z-w)}\left[\frac{1}{2} \partial \mathbf{T}_{ \pm}^{\left(\frac{3}{2}\right)} \mp \frac{1}{(k+5)^{2}} A_{3} B_{\mp}\left(\begin{array}{c}
F_{22} \\
F_{11}
\end{array}\right)+\frac{i(k+1)}{2(k+5)^{2}} \partial\left(\begin{array}{c}
A_{3} F_{21} \\
A_{3} F_{12}
\end{array}\right)\right. \\
& +\frac{i}{2(k+5)} A_{\mp}\left(\begin{array}{c}
G_{11} \\
G_{22}
\end{array}\right) \mp \frac{1}{(k+5)^{2}} A_{\mp} A_{3}\left(\begin{array}{c}
F_{11} \\
F_{22}
\end{array}\right) \pm \frac{1}{(k+5)^{2}} A_{\mp} B_{3}\left(\begin{array}{c}
F_{11} \\
F_{22}
\end{array}\right) \\
& -\frac{i(k-1)}{2(k+5)^{2}} \partial A_{\mp}\left(\begin{array}{c}
F_{11} \\
F_{22}
\end{array}\right)-\frac{i(k+7)}{2(k+5)^{2}} A_{\mp} \partial\left(\begin{array}{c}
F_{11} \\
F_{22}
\end{array}\right) \mp \frac{1}{(k+5)^{2}} A_{ \pm} A_{\mp}\left(\begin{array}{c}
F_{21} \\
F_{12}
\end{array}\right) \\
& -\frac{i(k+1)}{2(k+5)^{2}} \partial\left(\begin{array}{c}
B_{3} F_{21} \\
B_{3} F_{12}
\end{array}\right)+\frac{i}{2(k+5)} B_{\mp}\left(\begin{array}{c}
G_{22} \\
G_{11}
\end{array}\right) \pm \frac{1}{(k+5)^{2}} B_{\mp} B_{3}\left(\begin{array}{c}
F_{22} \\
F_{11}
\end{array}\right) \\
& -\frac{i(k-5)}{2(k+5)^{2}} \partial B_{\mp}\left(\begin{array}{c}
F_{22} \\
F_{11}
\end{array}\right)+\frac{i(k+7)}{2(k+5)^{2}} B_{\mp} \partial\left(\begin{array}{c}
F_{22} \\
F_{11}
\end{array}\right) \pm \frac{1}{(k+5)^{2}} B_{ \pm} B_{\mp}\left(\begin{array}{c}
F_{21} \\
F_{12}
\end{array}\right) \\
& \mp \frac{1}{(k+5)^{2}}\left(\begin{array}{c}
F_{11} F_{21} G_{22} \\
F_{22} F_{12} G_{11}
\end{array}\right) \mp \frac{8}{(k+5)^{3}}\left(\begin{array}{c}
\partial F_{11} F_{21} F_{22} \\
\partial F_{22} F_{12} F_{11}
\end{array}\right) \\
& \pm \frac{(k-3)}{2(k+5)^{2}} \partial^{2}\left(\begin{array}{c}
F_{21} \\
F_{12}
\end{array}\right) \pm \frac{1}{(k+5)^{2}}\left(\begin{array}{c}
F_{21} F_{22} G_{11} \\
F_{12} F_{11} G_{22}
\end{array}\right) \\
& +\frac{i}{(k+5)^{2}} U A_{\mp}\left(\begin{array}{c}
F_{11} \\
F_{22}
\end{array}\right)+\frac{i}{(k+5)^{2}} U B_{\mp}\left(\begin{array}{c}
F_{22} \\
F_{11}
\end{array}\right) \\
& \left.\mp \frac{1}{2(k+5)} \partial\left(\begin{array}{c}
U F_{21} \\
U F_{12}
\end{array}\right) \mp \frac{2(k+1)}{(k+5)^{3}}\left(\begin{array}{l}
F_{11} F_{21} \partial F_{22} \\
F_{22} F_{12} \partial F_{11}
\end{array}\right)\right](w)+\cdots \text {. }
\end{aligned}
$$

In this OPE, the second order pole has spin- $\frac{3}{2}$ currents living in the large $\mathcal{N}=4$ linear superconformal algebra. There are many spin- $\frac{1}{2}$ current dependent terms. Furthermore, some terms containing the above spin- $\frac{3}{2}$ currents in the first order pole do not appear in the corresponding OPEs in the nonlinear version.

From the result of (3.2), one can compute the following OPEs

$\mathbf{T}_{ \pm}^{\left(\frac{3}{2}\right)}(z)\left(\begin{array}{c}\mathbf{U}^{\left(\frac{3}{2}\right)} \\ \mathbf{V}^{\left(\frac{3}{2}\right)}\end{array}\right)(w)=\frac{1}{(z-w)^{2}}\left[ \pm \frac{i}{(5+k)} B_{\mp}+\frac{2 k}{(k+5)^{2}}\left(\begin{array}{c}F_{11} F_{21} \\ F_{22} F_{12}\end{array}\right)\right](w)$ 


$$
\begin{aligned}
& +\frac{1}{(z-w)}\left[-\frac{1}{(k+5)} A_{3} B_{\mp} \mp \frac{2 i}{(k+5)^{2}} A_{3}\left(\begin{array}{c}
F_{11} F_{21} \\
F_{22} F_{12}
\end{array}\right) \pm \frac{i}{2(k+5)} \partial B_{\mp}\right. \\
& +\frac{i}{(k+5)^{2}} B_{\mp} F_{11} F_{22}+\frac{i}{(k+5)^{2}} B_{\mp} F_{12} F_{21}+\frac{(k+1)}{(k+5)^{2}}\left(\begin{array}{c}
\partial F_{11} F_{21} \\
\partial F_{22} F_{12}
\end{array}\right) \\
& \left.+\frac{(k-1)}{(k+5)^{2}}\left(\begin{array}{c}
F_{11} \partial F_{21} \\
F_{22} \partial F_{12}
\end{array}\right)\right](w)+\cdots
\end{aligned}
$$

These OPEs are new and the corresponding OPEs in the nonlinear version are trivial.

Similarly, the following OPEs can be calculated

$$
\begin{aligned}
\mathbf{T}_{ \pm}^{\left(\frac{3}{2}\right)}(z)\left(\begin{array}{c}
\mathbf{V}^{\left(\frac{3}{2}\right)} \\
\mathbf{U}^{\left(\frac{3}{2}\right)}
\end{array}\right)(w) & =\frac{1}{(z-w)^{2}}\left[\mp \frac{7 i}{(k+5)} A_{\mp} \mp \frac{6}{(k+5)^{2}}\left(\begin{array}{c}
F_{21} F_{22} \\
F_{11} F_{12}
\end{array}\right)\right](w) \\
& +\frac{1}{(z-w)}\left[\mp\left(\begin{array}{c}
\mathbf{V}_{+}^{(2)} \\
\mathbf{U}_{-}^{(2)}
\end{array}\right)-\frac{1}{(k+5)^{2}} A_{\mp} B_{3} \mp \frac{7 i}{2(k+5)} \partial A_{\mp}\right. \\
& -\frac{i}{(k+5)^{2}} A_{\mp} F_{11} F_{22}+\frac{i}{(k+5)^{2}} A_{\mp} F_{12} F_{21} \mp \frac{2 i}{(k+5)^{2}} B_{3}\left(\begin{array}{c}
F_{22} F_{21} \\
F_{11} F_{12}
\end{array}\right) \\
& \left.+\frac{4}{(k+5)^{2}}\left(\begin{array}{c}
\partial F_{22} F_{21} \\
\partial F_{11} F_{12}
\end{array}\right)+\frac{2}{(k+5)^{2}}\left(\begin{array}{c}
F_{22} \partial F_{21} \\
F_{11} \partial F_{12}
\end{array}\right)\right](w)+\cdots .
\end{aligned}
$$

Compared to the corresponding OPEs in the nonlinear version, the above OPEs have spin- $\frac{1}{2}$ current dependent terms. The fields in the first line of the first order pole except the higher spin current do not appear in the corresponding OPEs in the nonlinear version.

The equations (3.5) determine the following OPEs

$$
\begin{aligned}
\mathbf{T}_{ \pm}^{\left(\frac{3}{2}\right)}(z)\left(\begin{array}{c}
\mathbf{U}_{+}^{(2)} \\
\mathbf{V}_{-}^{(2)}
\end{array}\right)(w) & =\frac{1}{(z-w)^{2}}\left[ \pm \frac{2 i k}{(k+5)^{2}}\left(\begin{array}{c}
F_{21} \\
F_{12}
\end{array}\right) B_{\mp}\right](w) \\
& +\frac{1}{(z-w)}\left[\frac{1}{(k+5)^{2}} A_{3} B_{\mp}\left(\begin{array}{c}
F_{21} \\
F_{12}
\end{array}\right)-\frac{1}{(k+5)^{2}} A_{\mp} B_{\mp}\left(\begin{array}{c}
F_{11} \\
F_{22}
\end{array}\right)\right. \\
& \mp \frac{i}{2(k+5)} B_{\mp}\left(\begin{array}{c}
G_{21} \\
G_{12}
\end{array}\right)+\frac{1}{(k+5)^{2}} B_{\mp} B_{3}\left(\begin{array}{c}
F_{21} \\
F_{12}
\end{array}\right) \\
& -\frac{1}{(k+5)^{2}} B_{\mp} B_{\mp}\left(\begin{array}{c}
F_{22} \\
F_{11}
\end{array}\right) \pm \frac{i k}{(k+5)^{2}} \partial B_{\mp}\left(\begin{array}{c}
F_{21} \\
F_{12}
\end{array}\right) \\
& \mp \frac{3 i}{(k+5)^{2}} B_{\mp} \partial\left(\begin{array}{c}
F_{21} \\
F_{12}
\end{array}\right)+\frac{1}{(k+5)^{2}}\left(\begin{array}{c}
F_{11} F_{21} G_{21} \\
F_{22} F_{12} G_{12}
\end{array}\right) \\
& \left.\mp \frac{i}{(k+5)^{2}} U B_{\mp}\left(\begin{array}{c}
F_{21} \\
F_{12}
\end{array}\right)+\frac{2(k+1)}{(k+5)^{3}}\left(\begin{array}{c}
F_{11} F_{21} \partial F_{21} \\
F_{22} F_{12} \partial F_{12}
\end{array}\right)\right](w)+\cdots .
\end{aligned}
$$

Note that the nonlinear term appearing in the nonlinear version disappears in these OPEs. In other words, there are no composite fields with a boldface and without a boldface. The 
field containing the spin- $\frac{3}{2}$ current in the first order pole does not appear in the corresponding OPEs in the nonlinear version.

Furthermore, the relations (3.4) allow us to calculate the following OPEs

$$
\begin{aligned}
& \mathbf{T}_{ \pm}^{\left(\frac{3}{2}\right)}(z)\left(\begin{array}{c}
\mathbf{U}_{-}^{(\mathbf{2})} \\
\mathbf{V}_{+}^{(\mathbf{2})}
\end{array}\right)(w)=\frac{1}{(z-w)^{3}}\left[\frac{6 k}{(k+5)^{2}}\left(\begin{array}{l}
F_{11} \\
F_{22}
\end{array}\right)\right](w) \\
& +\frac{1}{(z-w)^{2}}\left[\mp \frac{2(k+3)}{(k+5)}\left(\begin{array}{c}
\mathbf{U}^{\left(\frac{3}{2}\right)} \\
\mathbf{V}^{\left(\frac{3}{2}\right)}
\end{array}\right)-\frac{1}{(k+5)}\left(\begin{array}{l}
G_{11} \\
G_{22}
\end{array}\right)\right. \\
& \pm \frac{2 i(k-1)}{(k+5)^{2}}\left(\begin{array}{l}
F_{11} \\
F_{22}
\end{array}\right) A_{3} \pm \frac{4 i}{(k+5)^{2}}\left(\begin{array}{c}
F_{11} \\
F_{22}
\end{array}\right) B_{3}+\frac{2(k+2)}{(k+5)^{2}} U\left(\begin{array}{c}
F_{11} \\
F_{22}
\end{array}\right) \\
& \pm \frac{2}{(k+5)^{2}} F_{12} F_{21}\left(\begin{array}{c}
F_{11} \\
F_{22}
\end{array}\right) \pm \frac{4 i}{(k+5)^{2}}\left(\begin{array}{c}
F_{12} \\
F_{21}
\end{array}\right) B_{\mp} \\
& \left. \pm \frac{2 i(k+2)}{(k+5)^{2}}\left(\begin{array}{c}
F_{21} \\
F_{12}
\end{array}\right) A_{ \pm}\right](w) \\
& +\frac{1}{(z-w)}\left[\mp \frac{1}{2}\left(\begin{array}{c}
\mathbf{Q}^{\left(\frac{5}{2}\right)} \\
\mathbf{R}^{\left(\frac{5}{2}\right)}
\end{array}\right)-\left(\begin{array}{c}
\mathbf{U}^{\left(\frac{5}{2}\right)} \\
\mathbf{V}^{\left(\frac{5}{2}\right)}
\end{array}\right) \mp \frac{2(k+3)}{3(k+5)} \partial\left(\begin{array}{l}
\mathbf{U}^{\left(\frac{3}{2}\right)} \\
\mathbf{V}^{\left(\frac{3}{2}\right)}
\end{array}\right)\right. \\
& \mp \frac{i}{2(k+5)} A_{3}\left(\begin{array}{l}
G_{11} \\
G_{22}
\end{array}\right) \\
& +\frac{2}{(k+5)^{2}} A_{3} A_{3}\left(\begin{array}{c}
F_{11} \\
F_{22}
\end{array}\right)-\frac{2}{(k+5)^{2}} A_{3} B_{3}\left(\begin{array}{c}
F_{11} \\
F_{22}
\end{array}\right) \\
& -\frac{2}{(k+5)^{2}} A_{3} B_{\mp}\left(\begin{array}{c}
F_{12} \\
F_{21}
\end{array}\right) \pm \frac{2 i(2 k+3)}{3(k+5)^{2}} \partial A_{3}\left(\begin{array}{c}
F_{11} \\
F_{22}
\end{array}\right) \\
& \pm \frac{2 i(k+9)}{3(k+5)^{2}} A_{3} \partial\left(\begin{array}{c}
F_{11} \\
F_{22}
\end{array}\right)+\frac{1}{(k+5)^{2}} A_{ \pm} A_{3}\left(\begin{array}{c}
F_{21} \\
F_{12}
\end{array}\right) \\
& +\frac{1}{(k+5)^{2}} A_{ \pm} A_{\mp}\left(\begin{array}{c}
F_{11} \\
F_{22}
\end{array}\right)-\frac{1}{(k+5)^{2}} A_{ \pm} B_{3}\left(\begin{array}{c}
F_{21} \\
F_{12}
\end{array}\right) \\
& +\frac{1}{(k+5)^{2}} A_{ \pm} B_{\mp}\left(\begin{array}{c}
F_{22} \\
F_{11}
\end{array}\right) \pm \frac{i(2 k+3)}{3(k+5)^{2}} \partial A_{ \pm}\left(\begin{array}{c}
F_{21} \\
F_{12}
\end{array}\right) \\
& \pm \frac{i(2 k+9)}{3(k+5)^{2}} A_{ \pm} \partial\left(\begin{array}{c}
F_{21} \\
F_{12}
\end{array}\right) \pm \frac{i}{2(k+5)} B_{3}\left(\begin{array}{c}
G_{11} \\
G_{22}
\end{array}\right) \\
& \pm \frac{i(k+9)}{3(k+5)^{2}} \partial B_{3}\left(\begin{array}{c}
F_{11} \\
F_{22}
\end{array}\right) \pm \frac{2 i k}{3(k+5)^{2}} B_{3} \partial\left(\begin{array}{c}
F_{11} \\
F_{22}
\end{array}\right) \\
& \pm \frac{i}{2(k+5)} B_{\mp}\left(\begin{array}{c}
G_{12} \\
G_{21}
\end{array}\right) \mp \frac{i(k-3)}{3(k+5)^{2}} \partial B_{\mp}\left(\begin{array}{c}
F_{12} \\
F_{21}
\end{array}\right) \\
& \pm \frac{2 i k}{3(k+5)^{2}} B_{\mp} \partial\left(\begin{array}{c}
F_{12} \\
F_{21}
\end{array}\right)-\frac{3(k+3)}{2(k+5)^{2}} \partial^{2}\left(\begin{array}{l}
F_{11} \\
F_{22}
\end{array}\right) \\
& -\frac{1}{(k+5)^{2}}\left(\begin{array}{c}
F_{11} F_{12} G_{21} \\
F_{22} F_{21} G_{12}
\end{array}\right) \pm \frac{2(k+9)}{3(k+5)^{3}} \partial\left(\begin{array}{c}
F_{11} \\
F_{22}
\end{array}\right) F_{12} F_{21}
\end{aligned}
$$




$$
\begin{aligned}
& +\frac{8(k+3)}{3(k+5)^{3}}\left(\begin{array}{c}
F_{11} \partial F_{12} F_{21} \\
F_{22} \partial F_{21} F_{12}
\end{array}\right)-\frac{4(k+3)}{3(k+5)^{3}}\left(\begin{array}{c}
F_{11} F_{12} \partial F_{21} \\
F_{22} F_{21} \partial F_{12}
\end{array}\right) \\
& \mp \frac{1}{(k+5)^{2}} F_{11} F_{22}\left(\begin{array}{c}
G_{11} \\
G_{22}
\end{array}\right) \mp \frac{1}{(k+5)^{2}} F_{12} F_{21}\left(\begin{array}{c}
G_{11} \\
G_{22}
\end{array}\right) \\
& +\frac{1}{(k+5)} T\left(\begin{array}{c}
F_{11} \\
F_{22}
\end{array}\right)+\frac{1}{2(k+5)} U\left(\begin{array}{c}
G_{11} \\
G_{22}
\end{array}\right) \pm \frac{i}{(k+5)^{2}} U A_{ \pm}\left(\begin{array}{c}
F_{21} \\
F_{12}
\end{array}\right) \\
& \pm \frac{2 i}{(k+5)^{2}} U B_{3}\left(\begin{array}{c}
F_{11} \\
F_{22}
\end{array}\right) \pm \frac{2 i}{(k+5)^{2}} U B_{\mp}\left(\begin{array}{c}
F_{12} \\
F_{21}
\end{array}\right) \\
& +\frac{2(k+3)}{3(k+5)^{2}} U \partial\left(\begin{array}{c}
F_{11} \\
F_{22}
\end{array}\right)+\frac{2}{(k+5)^{2}} U U\left(\begin{array}{c}
F_{11} \\
F_{22}
\end{array}\right) \\
& \left.+\frac{2(k+3)}{3(k+5)^{2}}\left(\begin{array}{c}
F_{11} G_{22} F_{11} \\
F_{22} G_{11} F_{22}
\end{array}\right) \mp \frac{2(k+1)}{(k+5)^{3}} F_{11} F_{22} \partial\left(\begin{array}{c}
F_{11} \\
F_{22}
\end{array}\right)\right](w)+\cdots
\end{aligned}
$$

The nonlinear terms (between the higher spin currents and the currents of large $\mathcal{N}=4$ linear superconformal algebra) appearing in the nonlinear version disappear in this OPE. The structure constants in the above two OPEs are related to each other in very simple form. However, the corresponding OPEs in the nonlinear version cannot be written in this way because there are no simple relations between the structure constants. Some terms containing the spin- $\frac{3}{2}$ current in the above OPEs do not appear in the corresponding OPEs in the nonlinear version.

Similarly, the following OPEs can be described

$$
\begin{aligned}
\mathbf{T}_{ \pm}^{\left(\frac{3}{2}\right)}(z)\left(\begin{array}{c}
\mathbf{V}_{+}^{(\mathbf{2})} \\
\mathbf{U}_{-}^{(\mathbf{2})}
\end{array}\right)(w) & =\frac{1}{(z-w)^{2}}\left[\mp \frac{6 i}{(k+5)^{2}} A_{\mp}\left(\begin{array}{c}
F_{21} \\
F_{12}
\end{array}\right)\right](w) \\
& +\frac{1}{(z-w)}\left[\mp \frac{i}{2(k+5)} A_{\mp}\left(\begin{array}{c}
G_{21} \\
G_{12}
\end{array}\right)-\frac{1}{(k+5)^{2}} A_{\mp} A_{3}\left(\begin{array}{c}
F_{21} \\
F_{12}
\end{array}\right)\right. \\
& +\frac{1}{(k+5)^{2}} A_{\mp} A_{\mp}\left(\begin{array}{c}
F_{11} \\
F_{22}
\end{array}\right)-\frac{1}{(k+5)^{2}} A_{\mp} B_{3}\left(\begin{array}{c}
F_{21} \\
F_{12}
\end{array}\right) \\
& +\frac{1}{(k+5)^{2}} A_{\mp} B_{\mp}\left(\begin{array}{c}
F_{22} \\
F_{11}
\end{array}\right) \mp \frac{3 i}{(k+5)^{2}} \partial A_{\mp}\left(\begin{array}{c}
F_{21} \\
F_{12}
\end{array}\right) \\
& \pm \frac{3 i}{(k+5)^{2}} A_{\mp} \partial\left(\begin{array}{c}
F_{21} \\
F_{12}
\end{array}\right)-\frac{1}{(k+5)^{2}}\left(\begin{array}{c}
F_{21} F_{22} G_{21} \\
F_{12} F_{11} G_{12}
\end{array}\right) \\
& \left.\mp \frac{i}{(k+5)^{2}} U A_{\mp}\left(\begin{array}{c}
F_{21} \\
F_{12}
\end{array}\right)-\frac{8}{(k+5)^{3}}\left(\begin{array}{c}
F_{22} F_{21} \partial F_{21} \\
F_{11} F_{12} \partial F_{12}
\end{array}\right)\right](w)+\cdots
\end{aligned}
$$

These OPEs are new in the sense that the corresponding OPEs in the nonlinear version are trivial. 
Furthermore, one has the following OPEs

$$
\begin{aligned}
& \mathbf{T}_{ \pm}^{\left(\frac{3}{2}\right)}(z)\left(\begin{array}{l}
\mathbf{V}_{-}^{(2)} \\
\mathbf{U}_{+}^{(2)}
\end{array}\right)(w)=\frac{1}{(z-w)^{3}}\left[-\frac{6 k}{(k+5)^{2}}\left(\begin{array}{c}
F_{22} \\
F_{11}
\end{array}\right)\right](w) \\
& +\frac{1}{(z-w)^{2}}\left[\mp \frac{(k+9)}{(k+5)}\left(\begin{array}{c}
\mathbf{V}^{\left(\frac{3}{2}\right)} \\
\mathbf{U}^{\left(\frac{3}{2}\right)}
\end{array}\right)+\frac{(k+8)}{(k+5)}\left(\begin{array}{c}
G_{22} \\
G_{11}
\end{array}\right)\right. \\
& +\frac{(k+7)}{(k+5)^{2}} U\left(\begin{array}{c}
F_{22} \\
F_{11}
\end{array}\right) \mp \frac{2}{(k+5)^{2}} F_{12} F_{21}\left(\begin{array}{c}
F_{22} \\
F_{11}
\end{array}\right) \\
& \mp \frac{i(k+1)}{(k+5)^{2}}\left(\begin{array}{c}
F_{22} \\
F_{11}
\end{array}\right) A_{3} \mp \frac{i(k+1)}{(k+5)^{2}}\left(\begin{array}{c}
F_{12} \\
F_{21}
\end{array}\right) A_{\mp} \\
& \left.\mp \frac{i(k+7)}{(k+5)^{2}}\left(\begin{array}{c}
F_{21} \\
F_{12}
\end{array}\right) B_{ \pm} \pm \frac{i(k-7)}{(k+5)^{2}}\left(\begin{array}{c}
F_{22} \\
F_{11}
\end{array}\right) B_{3}\right](w) \\
& +\frac{1}{(z-w)}\left[\mp \frac{1}{2}\left(\begin{array}{c}
\mathbf{R}^{\left(\frac{5}{2}\right)} \\
\mathbf{Q}^{\left(\frac{5}{2}\right)}
\end{array}\right) \mp \frac{(k+9)}{3(k+5)} \partial\left(\begin{array}{l}
\mathbf{V}^{\left(\frac{3}{2}\right)} \\
\mathbf{U}^{\left(\frac{3}{2}\right)}
\end{array}\right)\right. \\
& \pm \frac{i}{2(k+5)} A_{3}\left(\begin{array}{c}
G_{22} \\
G_{11}
\end{array}\right)+\frac{2}{(k+5)^{2}} A_{3} B_{3}\left(\begin{array}{c}
F_{22} \\
F_{11}
\end{array}\right) \\
& +\frac{1}{(k+5)^{2}} A_{3} B_{ \pm}\left(\begin{array}{c}
F_{21} \\
F_{12}
\end{array}\right) \mp \frac{2 i(k+3)}{3(k+5)^{2}} \partial A_{3}\left(\begin{array}{c}
F_{22} \\
F_{11}
\end{array}\right) \\
& \mp \frac{i(k+3)}{3(k+5)^{2}} A_{3} \partial\left(\begin{array}{c}
F_{22} \\
F_{11}
\end{array}\right) \pm \frac{i}{2(k+5)} A_{\mp}\left(\begin{array}{l}
G_{12} \\
G_{21}
\end{array}\right) \\
& +\frac{2}{(k+5)^{2}} A_{\mp} B_{3}\left(\begin{array}{c}
F_{12} \\
F_{21}
\end{array}\right)-\frac{1}{(k+5)^{2}} A_{\mp} B_{ \pm}\left(\begin{array}{c}
F_{11} \\
F_{22}
\end{array}\right) \\
& \mp \frac{i(k-3)}{3(k+5)^{2}} \partial A_{\mp}\left(\begin{array}{c}
F_{12} \\
F_{21}
\end{array}\right) \mp \frac{i(k+3)}{3(k+5)^{2}} A_{\mp} \partial\left(\begin{array}{c}
F_{12} \\
F_{21}
\end{array}\right) \\
& \mp \frac{i}{2(k+5)} B_{3}\left(\begin{array}{c}
G_{22} \\
G_{11}
\end{array}\right)-\frac{2}{(k+5)^{2}} B_{3} B_{3}\left(\begin{array}{c}
F_{22} \\
F_{11}
\end{array}\right) \\
& \pm \frac{i(k-15)}{3(k+5)^{2}} \partial B_{3}\left(\begin{array}{c}
F_{22} \\
F_{11}
\end{array}\right) \mp \frac{i(k+21)}{3(k+5)^{2}} B_{3} \partial\left(\begin{array}{c}
F_{22} \\
F_{11}
\end{array}\right) \\
& -\frac{1}{(k+5)^{2}} B_{ \pm} B_{3}\left(\begin{array}{c}
F_{21} \\
F_{12}
\end{array}\right) \mp \frac{i(k+6)}{3(k+5)^{2}} \partial B_{ \pm}\left(\begin{array}{c}
F_{21} \\
F_{12}
\end{array}\right) \\
& \mp \frac{i(k+12)}{3(k+5)^{2}} B_{ \pm} \partial\left(\begin{array}{c}
F_{21} \\
F_{12}
\end{array}\right)-\frac{1}{(k+5)^{2}} B_{\mp} B_{ \pm}\left(\begin{array}{c}
F_{22} \\
F_{11}
\end{array}\right) \\
& +\frac{(k+15)}{2(k+5)^{2}} \partial^{2}\left(\begin{array}{c}
F_{22} \\
F_{11}
\end{array}\right) \mp \frac{4(k+3)}{3(k+5)^{3}} F_{12} F_{21} \partial\left(\begin{array}{c}
F_{22} \\
F_{11}
\end{array}\right) \\
& -\frac{2(k+9)}{3(k+5)^{3}}\left(\begin{array}{l}
F_{22} \partial F_{21} F_{12} \\
F_{11} \partial F_{12} F_{21}
\end{array}\right)+\frac{4(k+9)}{3(k+5)^{3}}\left(\begin{array}{l}
F_{22} F_{21} \partial F_{12} \\
F_{11} F_{12} \partial F_{21}
\end{array}\right) \\
& -\frac{1}{(k+5)^{2}}\left(\begin{array}{c}
F_{22} F_{12} G_{21} \\
F_{11} F_{21} G_{12}
\end{array}\right) \pm \frac{1}{(k+5)^{2}} F_{11} F_{22}\left(\begin{array}{c}
G_{22} \\
G_{11}
\end{array}\right)
\end{aligned}
$$




$$
\begin{aligned}
& \mp \frac{1}{(k+5)^{2}} F_{12} F_{21}\left(\begin{array}{c}
G_{22} \\
G_{11}
\end{array}\right)+\frac{(k+9)}{3(k+5)} \partial\left(\begin{array}{c}
G_{22} \\
G_{11}
\end{array}\right) \\
& -\frac{1}{(k+5)} T\left(\begin{array}{c}
F_{22} \\
F_{11}
\end{array}\right)-\frac{1}{2(k+5)} U\left(\begin{array}{l}
G_{22} \\
G_{11}
\end{array}\right) \pm \frac{2 i}{(k+5)^{2}} U A_{3}\left(\begin{array}{c}
F_{22} \\
F_{11}
\end{array}\right) \\
& \pm \frac{2 i}{(k+5)^{2}} U A_{\mp}\left(\begin{array}{c}
F_{12} \\
F_{21}
\end{array}\right) \pm \frac{i}{(k+5)^{2}} U B_{ \pm}\left(\begin{array}{c}
F_{21} \\
F_{12}
\end{array}\right) \\
& +\frac{(k+9)}{3(k+5)^{2}} U \partial\left(\begin{array}{c}
F_{22} \\
F_{11}
\end{array}\right)-\frac{2}{(k+5)^{2}} U U\left(\begin{array}{c}
F_{22} \\
F_{11}
\end{array}\right) \\
& \left.+\frac{(k+9)}{3(k+5)^{2}}\left(\begin{array}{c}
F_{22} G_{11} F_{22} \\
F_{11} G_{22} F_{11}
\end{array}\right)-\frac{16 i}{(k+5)^{3}}\left(\begin{array}{c}
F_{22} \\
F_{11}
\end{array}\right) A_{3} F_{11} F_{22}\right](w) \\
& +\cdots
\end{aligned}
$$

Due to the spin- $\frac{1}{2}$ currents, these OPEs are rather complicated than the ones in the nonlinear version. Some terms containing the spin- $\frac{3}{2}$ currents in the first order pole in the above OPEs do not appear in the corresponding OPEs in the nonlinear version.

From the previous relation (3.7), the following OPEs can be obtained

$$
\begin{aligned}
& \mathbf{T}_{ \pm}^{\left(\frac{3}{2}\right)}(z)\left(\begin{array}{l}
\mathbf{U}^{\left(\frac{5}{2}\right)} \\
\mathbf{V}^{\left(\frac{5}{2}\right)}
\end{array}\right)(w)=\frac{1}{(z-w)^{3}}\left[\frac{2 i k(4 k+21)}{3(k+5)^{2}} B_{\mp}-\frac{16 k(k+3)}{3(k+5)^{3}}\left(\begin{array}{c}
F_{11} F_{21} \\
F_{12} F_{22}
\end{array}\right)\right](w) \\
& +\frac{1}{(z-w)^{2}}\left[\frac{8(k+3)}{3(k+5)}\left(\begin{array}{c}
\mathbf{U}_{+}^{(2)} \\
\mathbf{V}_{-}^{(2)}
\end{array}\right) \mp \frac{2(k-3)}{3(k+5)^{2}} A_{3} B_{\mp}\right. \\
& +\frac{2 i(k-3)}{3(k+5)^{3}} A_{3}\left(\begin{array}{c}
F_{11} F_{21} \\
F_{22} F_{12}
\end{array}\right)+\frac{2 i}{(k+5)^{2}} B_{3}\left(\begin{array}{c}
F_{11} F_{21} \\
F_{22} F_{12}
\end{array}\right) \\
& \pm \frac{2 i(k+9)}{3(k+5)^{3}} B_{\mp} F_{12} F_{21} \pm \frac{(k+1)}{(k+5)^{2}}\left(\begin{array}{c}
F_{11} G_{21} \\
F_{22} G_{12}
\end{array}\right) \\
& \pm \frac{2(k+21)}{3(k+5)^{3}}\left(\begin{array}{l}
\partial F_{11} F_{21} \\
\partial F_{22} F_{12}
\end{array}\right) \pm \frac{2(5 k+9)}{3(k+5)^{3}}\left(\begin{array}{c}
F_{11} \partial F_{21} \\
F_{22} \partial F_{12}
\end{array}\right) \\
& \mp \frac{2(k+2)}{(k+5)^{2}}\left(\begin{array}{c}
F_{21} G_{11} \\
F_{12} G_{22}
\end{array}\right)+\frac{2 i k}{(k+5)^{2}} U B_{\mp} \\
& \left. \pm \frac{2}{(k+5)^{2}} U\left(\begin{array}{c}
F_{11} F_{21} \\
F_{22} F_{12}
\end{array}\right) \mp \frac{4 i(k+3)}{3(k+5)^{3}} B_{\mp} F_{11} F_{22}\right](w) \\
& +\frac{1}{(z-w)}\left[\frac{1}{2}\left(\begin{array}{c}
\mathbf{Q}_{+}^{(3)} \\
\mathbf{R}_{-}^{(3)}
\end{array}\right)+\frac{1}{4} \partial(\text { pole- } 2)\right. \\
& +\frac{i k(4 k+21)}{(k+5)(13 k+17)}\left(T B_{\mp}-\frac{1}{2} \partial^{2} B_{\mp}\right) \\
& \left.\mp \frac{8 k(k+3)}{(k+5)^{2}(13 k+17)}\left(T\left(\begin{array}{c}
F_{11} F_{21} \\
F_{22} F_{12}
\end{array}\right)-\frac{1}{2} \partial^{2}\left(\begin{array}{c}
F_{11} F_{21} \\
F_{22} F_{12}
\end{array}\right)\right)\right](w)+\cdots \text {. }
\end{aligned}
$$

One can easily see that the nonlinear terms (between the higher spin currents and the 16 
currents from the large $\mathcal{N}=4$ linear superconformal algebra or in the spirit of the footnote 5) appearing in the corresponding OPEs in the nonlinear version disappear in the above OPEs. Even the above OPEs have simple relations between the structure constants and so one can write down them together in one single equation as above. The last two expressions in the first order pole contain the quasi primary fields. The first order pole of the above OPEs is rather simple because the many composite nonderivative terms which were present in the corresponding OPEs in the nonlinear version disappear.

One also has the following OPEs

$$
\begin{aligned}
& \mathbf{T}_{ \pm}^{\left(\frac{3}{2}\right)}(z)\left(\begin{array}{l}
\mathbf{V}^{\left(\frac{5}{2}\right)} \\
\mathbf{U}^{\left(\frac{5}{2}\right)}
\end{array}\right)(w)=\frac{1}{(z-w)^{3}}\left[\frac{2 i(5 k+18)}{(k+5)^{2}} A_{\mp}+\frac{8(k+9)}{(k+5)^{3}}\left(\begin{array}{c}
F_{21} F_{22} \\
F_{11} F_{12}
\end{array}\right)\right](w) \\
& +\frac{1}{(z-w)^{2}}\left[\frac{4(k+9)}{3(k+5)}\left(\begin{array}{c}
\mathbf{V}_{+}^{(2)} \\
\mathbf{U}_{-}^{(2)}
\end{array}\right)+\frac{2 i}{(k+5)^{2}} A_{3}\left(\begin{array}{c}
F_{22} F_{21} \\
F_{11} F_{12}
\end{array}\right)\right. \\
& \pm \frac{2(k-3)}{3(k+5)^{2}} A_{\mp} B_{3} \pm \frac{2 i(k+9)}{3(k+5)^{3}} A_{\mp} F_{11} F_{22} \pm \frac{4 i(k+3)}{3(k+5)^{3}} A_{\mp} F_{12} F_{21} \\
& -\frac{2 i(k-3)}{3(k+5)^{3}} B_{3}\left(\begin{array}{c}
F_{22} F_{21} \\
F_{11} F_{12}
\end{array}\right) \mp \frac{4}{(k+5)^{2}}\left(\begin{array}{c}
F_{22} G_{21} \\
F_{11} G_{12}
\end{array}\right) \\
& \pm \frac{2(5 k+9)}{3(k+5)^{3}}\left(\begin{array}{c}
\partial F_{22} F_{21} \\
\partial F_{11} F_{12}
\end{array}\right) \pm \frac{(k+7)}{(k+5)^{2}}\left(\begin{array}{c}
F_{21} G_{22} \\
F_{12} G_{11}
\end{array}\right) \\
& \left.-\frac{6 i}{(k+5)^{2}} U A_{\mp} \mp \frac{2}{(k+5)^{2}} U\left(\begin{array}{c}
F_{22} F_{21} \\
F_{11} F_{12}
\end{array}\right) \pm \frac{2(k+21)}{3(k+5)^{3}}\left(\begin{array}{c}
F_{22} \partial F_{21} \\
F_{11} \partial F_{12}
\end{array}\right)\right](w) \\
& +\frac{1}{(z-w)}\left[\frac{1}{2}\left(\begin{array}{c}
\mathbf{R}_{+}^{(3)} \\
\mathbf{Q}_{-}^{(3)}
\end{array}\right)+\frac{1}{4} \partial(\text { pole- } 2)\right. \\
& +\frac{3 i(5 k+18)}{(k+5)(13 k+17)}\left(T A_{\mp}-\frac{1}{2} \partial^{2} A_{\mp}\right) \\
& \left. \pm \frac{12(k+9)}{(k+5)^{2}(13 k+17)}\left(T\left(\begin{array}{l}
F_{21} F_{22} \\
F_{12} F_{11}
\end{array}\right)-\frac{1}{2} \partial^{2}\left(\begin{array}{l}
F_{21} F_{22} \\
F_{12} F_{11}
\end{array}\right)\right)\right](w)+\cdots .
\end{aligned}
$$

There are no nonlinear terms (footnote 5) which appeared in the nonlinear version. The structure constants of above OPEs behave simply and so one can write them together. The last two expressions in the first order pole contain the quasi primary fields. Note that for the spin- $\frac{1}{2}$ currents dependent terms, the numerical factor in the derivative term is given by the same value $\frac{1}{2}$.

The relation (3.6) gives the higher spin-2 current and one obtains the following OPEs

$$
\begin{aligned}
\mathbf{T}_{ \pm}^{\left(\frac{3}{2}\right)}(z) \mathbf{W}^{(2)}(w) & =\frac{1}{(z-w)^{3}}\left[ \pm \frac{6 k}{(k+5)^{2}}\left(\begin{array}{c}
F_{21} \\
F_{12}
\end{array}\right)\right](w) \\
& +\frac{1}{(z-w)^{2}}\left[-\frac{(k-3)}{2(k+5)} \mathbf{T}_{ \pm}^{\left(\frac{3}{2}\right)} \pm \frac{(k-3)}{2(k+5)^{2}} U\left(\begin{array}{c}
F_{21} \\
F_{12}
\end{array}\right)\right.
\end{aligned}
$$




$$
\begin{aligned}
& -\frac{i(k+9)}{2(k+5)^{2}}\left(\begin{array}{c}
F_{21} \\
F_{12}
\end{array}\right) A_{3}+\frac{i(k-3)}{2(k+5)^{2}}\left(\begin{array}{c}
F_{11} \\
F_{22}
\end{array}\right) A_{\mp} \\
& \left.-\frac{i(k-3)}{2(k+5)^{2}}\left(\begin{array}{c}
F_{22} \\
F_{11}
\end{array}\right) B_{\mp}-\frac{3 i(k+1)}{2(k+5)^{2}}\left(\begin{array}{c}
F_{21} \\
F_{12}
\end{array}\right) B_{3}\right](w) \\
& +\frac{1}{(z-w)}\left[-\frac{1}{2} \mathbf{P}_{ \pm}^{\left(\frac{5}{2}\right)} \mp \mathbf{W}_{ \pm}^{\left(\frac{5}{2}\right)}-\frac{(k-3)}{6(k+5)} \partial \mathbf{T}_{ \pm}^{\left(\frac{3}{2}\right)}\right. \\
& -\frac{i}{2(k+5)} A_{3}\left(\begin{array}{c}
G_{21} \\
G_{12}
\end{array}\right) \mp \frac{1}{(k+5)^{2}} A_{3} B_{\mp}\left(\begin{array}{c}
F_{22} \\
F_{11}
\end{array}\right)-\frac{i(k+9)}{6(k+5)^{2}} \partial A_{3}\left(\begin{array}{c}
F_{21} \\
F_{12}
\end{array}\right) \\
& -\frac{i(k-3)}{6(k+5)^{2}} A_{3} \partial\left(\begin{array}{c}
F_{21} \\
F_{12}
\end{array}\right) \pm \frac{1}{(k+5)^{2}} A_{\mp} A_{3}\left(\begin{array}{c}
F_{11} \\
F_{22}
\end{array}\right) \\
& \mp \frac{1}{(k+5)^{2}} A_{\mp} B_{3}\left(\begin{array}{c}
F_{11} \\
F_{22}
\end{array}\right) \mp \frac{2}{(k+5)^{2}} A_{\mp} B_{\mp}\left(\begin{array}{c}
F_{12} \\
F_{21}
\end{array}\right) \\
& +\frac{i(k+3)}{6(k+5)^{2}} \partial A_{\mp}\left(\begin{array}{c}
F_{11} \\
F_{22}
\end{array}\right)+\frac{i(k+15)}{6(k+5)^{2}} A_{\mp} \partial\left(\begin{array}{c}
F_{11} \\
F_{22}
\end{array}\right) \\
& \pm \frac{1}{(k+5)^{2}} A_{ \pm} A_{\mp}\left(\begin{array}{c}
F_{21} \\
F_{12}
\end{array}\right)+\frac{i}{2(k+5)} B_{3}\left(\begin{array}{c}
G_{21} \\
G_{12}
\end{array}\right) \\
& -\frac{i(5 k-3)}{6(k+5)^{2}} \partial B_{3}\left(\begin{array}{c}
F_{21} \\
F_{12}
\end{array}\right)+\frac{i(k-3)}{6(k+5)^{2}} B_{3} \partial\left(\begin{array}{c}
F_{21} \\
F_{12}
\end{array}\right) \\
& \pm \frac{1}{(k+5)^{2}} B_{\mp} B_{3}\left(\begin{array}{c}
F_{22} \\
F_{11}
\end{array}\right)-\frac{i(k-9)}{6(k+5)^{2}} \partial B_{\mp}\left(\begin{array}{c}
F_{22} \\
F_{11}
\end{array}\right) \\
& -\frac{i(k-21)}{6(k+5)^{2}} B_{\mp} \partial\left(\begin{array}{c}
F_{22} \\
F_{11}
\end{array}\right) \pm \frac{1}{(k+5)^{2}} B_{ \pm} B_{\mp}\left(\begin{array}{c}
F_{21} \\
F_{12}
\end{array}\right) \\
& \mp \frac{1}{(k+5)^{2}}\left(\begin{array}{c}
F_{11} F_{21} G_{22} \\
F_{22} F_{12} G_{11}
\end{array}\right) \mp \frac{4(k+3)}{3(k+5)^{3}}\left(\begin{array}{c}
\partial F_{11} F_{21} F_{22} \\
\partial F_{22} F_{12} F_{11}
\end{array}\right) \\
& -\frac{2(k-3)}{3(k+5)^{3}} \partial\left(\begin{array}{c}
F_{21} \\
F_{12}
\end{array}\right) F_{11} F_{22} \pm \frac{2(k+9)}{3(k+5)^{3}}\left(\begin{array}{c}
F_{11} F_{21} \partial F_{22} \\
F_{22} F_{12} \partial F_{11}
\end{array}\right) \\
& \mp \frac{(k+6)}{(k+5)^{2}} \partial^{2}\left(\begin{array}{c}
F_{21} \\
F_{12}
\end{array}\right) \mp \frac{1}{(k+5)^{2}}\left(\begin{array}{c}
F_{21} F_{22} G_{11} \\
F_{12} F_{11} G_{22}
\end{array}\right) \\
& \pm \frac{1}{(k+5)} T\left(\begin{array}{c}
F_{21} \\
F_{12}
\end{array}\right) \pm \frac{1}{2(k+5)} U\left(\begin{array}{c}
G_{21} \\
G_{12}
\end{array}\right)-\frac{2 i}{(k+5)^{2}} U A_{3}\left(\begin{array}{c}
F_{21} \\
F_{12}
\end{array}\right) \\
& +\frac{i}{(k+5)^{2}} U A_{\mp}\left(\begin{array}{c}
F_{11} \\
F_{22}
\end{array}\right)+\frac{2 i}{(k+5)^{2}} U B_{3}\left(\begin{array}{c}
F_{21} \\
F_{12}
\end{array}\right) \\
& -\frac{i}{(k+5)^{2}} U B_{\mp}\left(\begin{array}{c}
F_{22} \\
F_{11}
\end{array}\right) \pm \frac{(k-3)}{6(k+5)^{2}} \partial\left(\begin{array}{c}
U F_{21} \\
U F_{12}
\end{array}\right) \\
& \left. \pm \frac{2}{(k+5)^{2}} U U\left(\begin{array}{c}
F_{21} \\
F_{12}
\end{array}\right)+\frac{2}{(k+5)^{2}} \partial\left(\begin{array}{c}
F_{21} \\
F_{12}
\end{array}\right) F_{12} F_{21}\right](w)+\cdots
\end{aligned}
$$

These OPEs are rather complicated due to the presence of spin- $\frac{1}{2}$ currents. Some terms 
containing the spin- $\frac{3}{2}$ currents in the above OPEs do not appear in the nonlinear version.

From the relation (3.8), the following OPEs can be obtained

$$
\begin{aligned}
& \mathbf{T}_{ \pm}^{\left(\frac{3}{2}\right)}(z) \mathbf{W}_{ \pm}^{\left(\frac{5}{2}\right)}(w)=\frac{1}{(z-w)^{2}}\left[\mp \frac{2(k-3)}{3(k+5)^{2}} A_{\mp} B_{\mp}+\frac{2 i(k-3)}{3(k+5)^{3}} A_{\mp}\left(\begin{array}{c}
F_{11} F_{21} \\
F_{22} F_{12}
\end{array}\right)\right. \\
& -\frac{2 i(k-3)}{3(k+5)^{3}} B_{\mp}\left(\begin{array}{c}
F_{21} F_{22} \\
F_{12} F_{11}
\end{array}\right) \mp \frac{(k+3)}{(k+5)^{2}}\left(\begin{array}{c}
F_{21} G_{21} \\
F_{12} G_{12}
\end{array}\right) \\
& \left. \pm \frac{8(k-3)}{3(k+5)^{3}}\left(\begin{array}{c}
F_{21} \partial F_{21} \\
F_{12} \partial F_{12}
\end{array}\right)\right](w) \\
& +\frac{1}{(z-w)}\left[ \pm \frac{(4 k+21)}{3(k+5)^{2}} \partial A_{\mp} B_{\mp} \mp \frac{(5 k+18)}{3(k+5)^{2}} A_{\mp} \partial B_{\mp}\right. \\
& -\frac{i}{(k+5)^{2}} A_{\mp}\left(\begin{array}{c}
F_{11} G_{21} \\
F_{22} G_{12}
\end{array}\right)+\frac{8 i(k+3)}{3(k+5)^{3}} \partial A_{\mp}\left(\begin{array}{c}
F_{11} F_{21} \\
F_{22} F_{12}
\end{array}\right) \\
& -\frac{2 i(5 k+21)}{3(k+5)^{3}} A_{\mp}\left(\begin{array}{c}
\partial F_{11} F_{21} \\
\partial F_{22} F_{12}
\end{array}\right)-\frac{4 i(k+3)}{3(k+5)^{3}} A_{\mp}\left(\begin{array}{c}
F_{11} \partial F_{21} \\
F_{22} \partial F_{12}
\end{array}\right) \\
& +\frac{i}{(k+5)^{2}} A_{\mp}\left(\begin{array}{c}
F_{21} G_{11} \\
F_{12} G_{22}
\end{array}\right)+\frac{i}{(k+5)^{2}} B_{\mp}\left(\begin{array}{c}
F_{21} G_{22} \\
F_{12} G_{11}
\end{array}\right) \\
& +\frac{4 i(k+9)}{3(k+5)^{3}} \partial B_{\mp}\left(\begin{array}{c}
F_{21} F_{22} \\
F_{12} F_{11}
\end{array}\right)-\frac{2 i(k+9)}{3(k+5)^{3}} B_{\mp}\left(\begin{array}{c}
\partial F_{21} F_{22} \\
\partial F_{12} F_{11}
\end{array}\right) \\
& -\frac{8 i(k+6)}{3(k+5)^{3}} B_{\mp}\left(\begin{array}{c}
F_{21} \partial F_{22} \\
F_{12} \partial F_{11}
\end{array}\right)-\frac{i}{(k+5)^{2}} B_{\mp}\left(\begin{array}{c}
F_{22} G_{21} \\
F_{11} G_{12}
\end{array}\right) \\
& \mp \frac{(k+3)}{(k+5)^{2}}\left(\begin{array}{l}
\partial F_{21} G_{21} \\
\partial F_{12} G_{12}
\end{array}\right) \pm \frac{2}{(k+5)^{2}} U A_{\mp} B_{\mp} \pm \frac{2(k-3)}{3(k+5)^{3}}\left(\begin{array}{c}
F_{21} \partial F_{21} \\
F_{12} \partial F_{12}
\end{array}\right) \\
& \pm \frac{2}{(k+5)^{2}}\left(\begin{array}{c}
F_{21} \partial F_{21} \\
F_{12} \partial F_{12}
\end{array}\right) U-\frac{2 i}{(k+5)^{2}}\left(\begin{array}{c}
F_{21} \partial F_{21} \\
F_{12} \partial F_{12}
\end{array}\right) A_{3} \\
& \left.+\frac{2 i}{(k+5)^{2}}\left(\begin{array}{c}
F_{21} \partial F_{21} \\
F_{12} \partial F_{12}
\end{array}\right) B_{3}\right](w)+\cdots \text {. }
\end{aligned}
$$

The nonlinear terms which appeared in the nonlinear version disappear in these OPEs.

Moreover, the following OPE can be obtained

$$
\begin{aligned}
\mathbf{T}_{+}^{\left(\frac{3}{2}\right)}(z) \mathbf{W}_{-}^{\left(\frac{5}{2}\right)}(w) & =\frac{1}{(z-w)^{3}}\left[-\frac{4(\mathbf{k}-\mathbf{3})}{3(k+5)} \mathbf{T}^{(\mathbf{1})}-\frac{2 i(5 k+18)}{(k+5)^{2}} A_{3}+\frac{2 i k(4 k+21)}{3(k+5)^{2}} B_{3}\right. \\
& \left.-\frac{6 k}{(k+5)^{2}} U-\frac{4\left(2 k^{2}+9 k+27\right)}{3(k+5)^{3}} F_{11} F_{22}+\frac{4(k-3)(2 k+9)}{3(k+5)^{3}} F_{12} F_{21}\right](w) \\
& +\frac{1}{(z-w)^{2}}\left[-\frac{4(k-3)}{3(k+5)} \mathbf{T}^{(\mathbf{2})}-4 \mathbf{W}^{(\mathbf{2})}+\frac{1}{2} \mathbf{P}^{(\mathbf{2})}+\frac{8 i(k+9)}{3(k+5)^{3}} A_{-} F_{11} F_{12}\right. \\
& -\frac{8 i(k+9)}{3(k+5)^{3}} A_{+} F_{21} F_{22}+\frac{16 i(k+3)}{3(k+5)^{3}} B_{-} F_{12} F_{22}-\frac{16 i(k+3)}{3(k+5)^{3}} B_{+} F_{11} F_{21}
\end{aligned}
$$




$$
\begin{aligned}
& +\frac{2(k-3)}{3(k+5)^{2}} F_{11} G_{22}+\frac{8}{(k+5)^{2}} \partial\left(F_{11} F_{22}\right)-\frac{2}{(k+5)} F_{12} G_{21} \\
& -\frac{8(k-3)}{3(k+5)^{3}} \partial\left(F_{12} F_{21}\right)+\frac{2}{(k+5)} F_{21} G_{12}-\frac{2(k-3)}{3(k+5)^{2}} F_{22} G_{11} \\
& \left.+\frac{8(k-3)}{3(k+5)^{3}} U F_{11} F_{22}-\frac{8}{(k+5)^{2}} U F_{12} F_{21}\right](w) \\
& +\frac{1}{(z-w)}\left[\frac{1}{2} \mathbf{P}^{(\mathbf{3})}+\frac{1}{8} \partial \mathbf{P}^{(\mathbf{2})}-\partial \mathbf{W}^{(\mathbf{2})}-\mathbf{W}^{(\mathbf{3})}-\frac{(k-3)}{3(k+5)} \partial \mathbf{T}^{(\mathbf{2})}\right. \\
& -\frac{2(\mathbf{k}-\mathbf{3})}{(13 k+17)}\left(T \mathbf{T}^{(\mathbf{1})}-\frac{1}{2} \partial^{2} \mathbf{T}^{(\mathbf{1})}\right) \\
& +\frac{3 i(5 k+18)}{2(k+5)(13 k+17)} \partial^{2} A_{3}-\frac{2 i}{(k+5)^{2}} A_{3} \partial\left(F_{11} F_{22}\right) \\
& -\frac{2 i}{(k+5)^{2}} A_{3} \partial\left(F_{12} F_{21}\right)+\frac{8 i(k+6)}{3(k+5)^{3}} \partial A_{-} F_{11} F_{12}-\frac{4 i(k+3)}{3(k+5)^{3}} A_{-} \partial\left(F_{11} F_{12}\right) \\
& +\frac{4 i(k+3)}{3(k+5)^{3}} \partial A_{+} F_{21} F_{22}-\frac{8 i(k+6)}{3(k+5)^{3}} A_{+} \partial\left(F_{21} F_{22}\right)-\frac{i k(4 k+21)}{2(k+5)(13 k+17)} \partial^{2} B_{3} \\
& -\frac{2 i}{(k+5)^{2}} B_{3} \partial\left(F_{11} F_{22}\right)+\frac{2 i}{(k+5)^{2}} B_{3} \partial\left(F_{12} F_{21}\right)+\frac{2 i(5 k+21)}{3(k+5)^{3}} \partial B_{-} F_{12} F_{22} \\
& -\frac{2 i(k+9)}{3(k+5)^{3}} B_{-} \partial\left(F_{12} F_{22}\right)+\frac{2 i(k+9)}{3(k+5)^{3}} \partial B_{+} F_{11} F_{21}-\frac{2 i(5 k+21)}{3(k+5)^{3}} B_{+} \partial\left(F_{11} F_{21}\right) \\
& +\frac{\left(2 k^{2}+35 k+61\right)}{(k+5)^{2}(13 k+17)} \partial^{2}\left(F_{11} F_{22}\right)+\frac{(5 k+21)}{3(k+5)^{2}} \partial F_{11} G_{22}-\frac{(k+9)}{3(k+5)^{2}} F_{11} \partial G_{22} \\
& -\frac{(k-3)\left(6 k^{2}+83 k+169\right)}{3(k+5)^{3}(13 k+17)} \partial^{2}\left(F_{12} F_{21}\right)+\frac{1}{(k+5)} \partial F_{12} G_{21}-\frac{1}{(k+5)} F_{12} \partial G_{21} \\
& +\frac{2}{(k+5)} \partial F_{21} G_{12}+\frac{4(k+6)}{3(k+5)^{2}} \partial F_{22} G_{11}-\frac{2(k+3)}{3(k+5)^{2}} F_{22} \partial G_{11} \\
& -\frac{3 i(5 k+18)}{(k+5)(13 k+17)} T A_{3}+\frac{i k(4 k+21)}{(k+5)(13 k+17)} T B_{3}-\frac{9 k}{(k+5)(13 k+17)} T U \\
& -\frac{2\left(2 k^{2}+9 k+27\right)}{(k+5)^{2}(13 k+17)} T F_{11} F_{22}+\frac{2(k-3)(2 k+9)}{(k+5)^{2}(13 k+17)} T F_{12} F_{21} \\
& +\frac{9 k}{2(k+5)(13 k+17)} \partial^{2} U+\frac{8(k+3)}{3(k+5)^{3}} \partial U F_{11} F_{22}+\frac{2(7 k+27)}{3(k+5)^{3}} U \partial F_{11} F_{22} \\
& -\frac{2(5 k+33)}{3(k+5)^{3}} U F_{11} \partial F_{22}-\frac{4}{(k+5)^{2}} \partial U F_{12} F_{21}+\frac{2}{(k+5)^{2}} U \partial F_{12} F_{21} \\
& \left.-\frac{6}{(k+5)^{2}} U F_{12} \partial F_{21}-\frac{2}{(k+5)^{2}} F_{11} G_{22} F_{11} F_{22}-\frac{2}{(k+5)^{2}} F_{21} G_{12} F_{21} F_{12}\right](w) \\
& +\cdots \text {. }
\end{aligned}
$$

Some nonlinear terms in the second order pole in the nonlinear version disappear in the above 
OPE. Note that there exist the nonlinear terms with coefficient $(k-3)$ factor containing the higher spin-1 current in the first order pole. This is the only nonlinear term between the higher spin currents and the currents from the large $\mathcal{N}=4$ linear superconformal algebra. We will see this feature in other OPEs in this Appendix.

Similarly, one has

$$
\begin{aligned}
& \mathbf{T}_{-}^{\left(\frac{3}{2}\right)}(z) \mathbf{W}_{+}^{\left(\frac{5}{2}\right)}(w)=\frac{1}{(z-w)^{3}}\left[-\frac{4(\mathbf{k}-\mathbf{3})}{3(k+5)} \mathbf{T}^{(\mathbf{1})}-\frac{2 i(5 k+18)}{(k+5)^{2}} A_{3}+\frac{2 i k(4 k+21)}{3(k+5)^{2}} B_{3}\right. \\
& \left.+\frac{6 k}{(k+5)^{2}} U-\frac{4\left(2 k^{2}+9 k+27\right)}{3(k+5)^{3}} F_{11} F_{22}+\frac{4(k-3)(2 k+9)}{3(k+5)^{3}} F_{12} F_{21}\right](w) \\
& +\frac{1}{(z-w)^{2}}\left[\frac{4(k-3)}{3(k+5)} \mathbf{T}^{(\mathbf{2})}+4 \mathbf{W}^{(\mathbf{2})}-\frac{1}{2} \mathbf{P}^{(\mathbf{2})}-\frac{2 i(k+21)}{3(k+5)^{3}} A_{-} F_{11} F_{12}\right. \\
& +\frac{2 i(k+21)}{3(k+5)^{3}} A_{+} F_{21} F_{22}-\frac{2 i(5 k+9)}{3(k+5)^{3}} B_{-} F_{12} F_{22}+\frac{2 i(5 k+9)}{3(k+5)^{3}} B_{+} F_{11} F_{21} \\
& +\frac{(k-3)}{3(k+5)^{2}} F_{11} G_{22}-\frac{4}{(k+5)^{2}} \partial\left(F_{11} F_{22}\right)+\frac{2}{(k+5)^{2}} F_{12} G_{21} \\
& +\frac{8(k-3)}{3(k+5)^{3}} \partial\left(F_{12} F_{21}\right)-\frac{2}{(k+5)^{2}} F_{21} G_{12}-\frac{(k-3)}{3(k+5)^{2}} F_{22} G_{11} \\
& \left.-\frac{8(k-3)}{3(k+5)^{3}} U F_{11} F_{22}+\frac{4}{(k+5)^{2}} U F_{12} F_{21}+\frac{12 i}{(k+5)^{2}} U A_{3}+\frac{4 i k}{(k+5)^{2}} U B_{3}\right](w) \\
& +\frac{1}{(z-w)}\left[\frac{1}{2} \mathbf{P}^{(\mathbf{3})}-\frac{1}{8} \partial \mathbf{P}^{(\mathbf{2})}+\partial \mathbf{W}^{(\mathbf{2})}-\mathbf{W}^{(\mathbf{3})}+\frac{(k-3)}{3(k+5)} \partial \mathbf{T}^{(\mathbf{2})}\right. \\
& -\frac{2(\mathbf{k}-\mathbf{3})}{(13 k+17)}\left(T \mathbf{T}^{(\mathbf{1})}-\frac{1}{2} \partial^{2} \mathbf{T}^{(\mathbf{1})}\right)-\frac{(3 k+13)}{(k+5)^{2}} \partial A_{3} B_{3}+\frac{(3 k+13)}{(k+5)^{2}} A_{3} \partial B_{3} \\
& +\frac{3 i(5 k+18)}{2(k+5)(13 k+17)} \partial^{2} A_{3}+\frac{2 i}{(k+5)^{2}} A_{3} F_{11} G_{22}-\frac{2 i}{(k+5)^{2}} A_{3} F_{22} G_{11} \\
& -\frac{2 i(k-3)}{(k+5)^{3}} \partial A_{3} F_{11} F_{22}+\frac{2 i(k+1)}{(k+5)^{3}} A_{3} \partial\left(F_{11} F_{22}\right)+\frac{i}{(k+5)^{2}} A_{-} F_{11} G_{12} \\
& -\frac{3 i}{(k+5)^{2}} A_{3} \partial\left(F_{12} F_{21}\right)+\frac{i(k+9)}{3(k+5)^{3}} \partial A_{-} F_{11} F_{12}-\frac{5 i(k+9)}{3(k+5)^{3}} A_{-} \partial F_{11} F_{12} \\
& +\frac{i(k-15)}{3(k+5)^{3}} A_{-} F_{11} \partial F_{12}-\frac{i}{(k+5)^{2}} A_{-} F_{12} G_{11}+\frac{i}{(k+5)^{2}} A_{+} F_{21} G_{22} \\
& +\frac{2 i(k+15)}{3(k+5)^{3}} \partial A_{+} F_{21} F_{22}+\frac{2 i(k+3)}{3(k+5)^{3}} A_{+} \partial F_{21} F_{22} \\
& -\frac{4 i(k+6)}{3(k+5)^{3}} A_{+} F_{21} \partial F_{22}-\frac{i}{(k+5)^{2}} A_{+} F_{22} G_{21}-\frac{i k(4 k+21)}{2(k+5)(13 k+17)} \partial^{2} B_{3} \\
& -\frac{2 i}{(k+5)^{2}} B_{3} F_{11} G_{22}+\frac{2 i}{(k+5)^{2}} B_{3} F_{22} G_{11}-\frac{i}{(k+5)^{2}} B_{-} F_{12} G_{22}
\end{aligned}
$$




$$
\begin{aligned}
& +\frac{8 i}{(k+5)^{2}} B_{3} \partial\left(F_{11} F_{22}\right)+\frac{3 i}{(k+5)^{2}} B_{3} \partial\left(F_{12} F_{21}\right)+\frac{2 i(k+3)}{3(k+5)^{3}} \partial B_{-} F_{12} F_{22} \\
& -\frac{4 i k}{3(k+5)^{3}} B_{-} \partial F_{12} F_{22}-\frac{10 i(k+3)}{3(k+5)^{3}} B_{-} F_{12} \partial F_{22}+\frac{i}{(k+5)^{2}} B_{-} F_{22} G_{12} \\
& -\frac{i}{(k+5)^{2}} B_{+} F_{11} G_{21}+\frac{i(7 k+15)}{3(k+5)^{3}} \partial B_{+} F_{11} F_{21} \\
& -\frac{i(5 k+21)}{3(k+5)^{3}} B_{+} \partial F_{11} F_{21}+\frac{i(k+9)}{3(k+5)^{3}} B_{+} F_{11} \partial F_{21}+\frac{i}{(k+5)^{2}} B_{+} F_{21} G_{11} \\
& +\frac{2\left(k^{2}-2 k+5\right)}{(k+5)^{2}(13 k+17)} \partial^{2}\left(F_{11} F_{22}\right)+\frac{(5 k+3)}{6(k+5)^{2}} \partial F_{11} G_{22}-\frac{(k+3)}{6(k+5)^{2}} F_{11} \partial G_{22} \\
& -\frac{(k-3)\left(6 k^{2}+31 k+101\right)}{3(k+5)^{3}(13 k+17)} \partial^{2}\left(F_{12} F_{21}\right)-\frac{1}{(k+5)^{2}} \partial F_{12} G_{21}+\frac{1}{(k+5)^{2}} F_{12} \partial G_{21} \\
& -\frac{2}{(k+5)^{2}} \partial F_{21} G_{12}+\frac{(2 k+3)}{3(k+5)^{2}} \partial F_{22} G_{11}-\frac{k}{3(k+5)^{2}} F_{22} \partial G_{11} \\
& -\frac{3 i(5 k+18)}{(k+5)(13 k+17)} T A_{3}+\frac{i k(4 k+21)}{(k+5)(13 k+17)} T B_{3}+\frac{17(k+2)}{(k+5)(13 k+17)} T U \\
& -\frac{2\left(2 k^{2}+9 k+27\right)}{(k+5)^{2}(13 k+17)} T F_{11} F_{22}+\frac{2(k-3)(2 k+9)}{(k+5)^{2}(13 k+17)} T F_{12} F_{21} \\
& -\frac{2}{(k+5)^{2}} U A_{3} A_{3}-\frac{4}{(k+5)^{2}} U A_{3} B_{3}+\frac{3 i}{(k+5)^{2}} \partial U A_{3}+\frac{5 i}{(k+5)^{2}} U \partial A_{3} \\
& \left.+\frac{2}{(k+5)^{2}} U F_{12} \partial F_{21}+\frac{2}{(k+5)^{2}} U U U+\frac{(k+9)}{3(k+5)^{3}} F_{22} G_{11} F_{22} F_{11} F_{21}\right](w)+\cdots \\
& +
\end{aligned}
$$

One cannot combine this OPE and previous OPE because some terms of this OPE do not appear in the previous OPE. There exist the nonlinear terms with coefficient $(k-3)$ factor containing the higher spin-1 current in the first order pole. Some nonlinear terms in the second order pole in the nonlinear version disappear in the above OPE. 
From the relation (3.9), one calculates the following OPEs

$$
\begin{aligned}
& \mathbf{T}_{ \pm}^{\left(\frac{3}{2}\right)}(z) \mathbf{W}^{(\mathbf{3})}(w)=\frac{1}{(z-w)^{4}}\left[-\frac{18 k}{(k+5)^{2}}\left(\begin{array}{l}
F_{21} \\
F_{12}
\end{array}\right)\right](w) \\
& +\frac{1}{(z-w)^{3}}\left[ \pm \frac{2(k-3)(61 k+113)}{3(k+5)(13 k+17)} \mathbf{T}_{ \pm}^{\left(\frac{3}{2}\right)}-\frac{2(k-3)(61 k+113)}{3(k+5)^{2}(13 k+17)} U\left(\begin{array}{c}
F_{21} \\
F_{12}
\end{array}\right)\right. \\
& \pm \frac{4(k-3)(5 k+73)}{3(k+5)^{3}(13 k+17)}\left(\begin{array}{c}
F_{21} \\
F_{12}
\end{array}\right) F_{11} F_{22} \pm \frac{2 i\left(61 k^{2}+164 k-33\right)}{3(k+5)^{2}(13 k+17)}\left(\begin{array}{c}
F_{21} \\
F_{12}
\end{array}\right) A_{3} \\
& \mp \frac{2 i\left(61 k^{2}+164 k-33\right)}{3(k+5)^{2}(13 k+17)}\left(\begin{array}{c}
F_{11} \\
F_{22}
\end{array}\right) A_{\mp} \mp \frac{2 i\left(17 k^{2}+172 k+339\right)}{3(k+5)^{2}(13 k+17)}\left(\begin{array}{c}
F_{22} \\
F_{11}
\end{array}\right) B_{\mp} \\
& \left. \pm \frac{2 i\left(17 k^{2}+172 k+339\right)}{3(k+5)^{2}(13 k+17)}\left(\begin{array}{c}
F_{21} \\
F_{12}
\end{array}\right) B_{3}+\frac{18 k}{(k+5)^{2}} \partial\left(\begin{array}{c}
F_{21} \\
F_{12}
\end{array}\right)\right](w) \\
& +\frac{1}{(z-w)^{2}}\left[ \pm \frac{5}{2} \mathbf{P}_{ \pm}^{\left(\frac{5}{2}\right)}+5 \mathbf{W}_{ \pm}^{\left(\frac{5}{2}\right)} \pm \frac{2(k-3)}{(13 k+17)} \partial \mathbf{T}_{ \pm}^{\left(\frac{3}{2}\right)}-\frac{6(\mathbf{k}-\mathbf{3})}{(13 k+17)} \mathbf{T}^{(\mathbf{1})} \mathbf{T}_{ \pm}^{\left(\frac{3}{2}\right)}\right. \\
& \mp \frac{10}{(k+5)^{2}} \partial\left(\begin{array}{l}
F_{21} \\
F_{12}
\end{array}\right) F_{12} F_{21} \pm \frac{3 i}{2(k+5)} A_{3}\left(\begin{array}{c}
G_{21} \\
G_{12}
\end{array}\right) \\
& +\frac{2}{(k+5)^{2}} A_{3} A_{3}\left(\begin{array}{c}
F_{21} \\
F_{12}
\end{array}\right)-\frac{4}{(k+5)^{2}} A_{3} B_{3}\left(\begin{array}{c}
F_{21} \\
F_{12}
\end{array}\right) \\
& +\frac{4}{(k+5)^{2}} A_{3} B_{\mp}\left(\begin{array}{c}
F_{22} \\
F_{11}
\end{array}\right) \pm \frac{2 i\left(k^{2}+93 k+104\right)}{(k+5)^{2}(13 k+17)} \partial A_{3}\left(\begin{array}{c}
F_{21} \\
F_{12}
\end{array}\right) \\
& \pm \frac{2 i\left(k^{2}-128 k-185\right)}{(k+5)^{2}(13 k+17)} A_{3} \partial\left(\begin{array}{c}
F_{21} \\
F_{12}
\end{array}\right) \mp \frac{3 i}{2(k+5)} A_{\mp}\left(\begin{array}{c}
G_{11} \\
G_{22}
\end{array}\right) \\
& +\frac{4}{(k+5)^{2}} A_{\mp} B_{3}\left(\begin{array}{c}
F_{11} \\
F_{22}
\end{array}\right)+\frac{4}{(k+5)^{2}} A_{\mp} B_{\mp}\left(\begin{array}{c}
F_{12} \\
F_{21}
\end{array}\right) \\
& \mp \frac{2 i\left(k^{2}+80 k+87\right)}{(k+5)^{2}(13 k+17)} \partial A_{\mp}\left(\begin{array}{c}
F_{11} \\
F_{22}
\end{array}\right) \mp \frac{2 i\left(k^{2}-128 k-185\right)}{(k+5)^{2}(13 k+17)} A_{\mp} \partial\left(\begin{array}{c}
F_{11} \\
F_{22}
\end{array}\right) \\
& +\frac{2}{(k+5)^{2}} A_{ \pm} A_{\mp}\left(\begin{array}{c}
F_{21} \\
F_{12}
\end{array}\right) \mp \frac{3 i}{2(k+5)} B_{3}\left(\begin{array}{c}
G_{21} \\
G_{12}
\end{array}\right) \\
& +\frac{2}{(k+5)^{2}} B_{3} B_{3}\left(\begin{array}{c}
F_{21} \\
F_{12}
\end{array}\right) \pm \frac{i\left(37 k^{2}+112 k+115\right)}{(k+5)^{2}(13 k+17)} \partial B_{3}\left(\begin{array}{c}
F_{21} \\
F_{12}
\end{array}\right) \\
& \mp \frac{4 i\left(20 k^{2}+33 k+1\right)}{(k+5)^{2}(13 k+17)} B_{3} \partial\left(\begin{array}{c}
F_{21} \\
F_{12}
\end{array}\right) \pm \frac{3 i}{2(k+5)} B_{\mp}\left(\begin{array}{c}
G_{22} \\
G_{11}
\end{array}\right) \\
& \mp \frac{i\left(37 k^{2}+86 k+81\right)}{(k+5)^{2}(13 k+17)} \partial B_{\mp}\left(\begin{array}{c}
F_{22} \\
F_{11}
\end{array}\right) \pm \frac{4 i\left(20 k^{2}+33 k+1\right)}{(k+5)^{2}(13 k+17)} B_{\mp} \partial\left(\begin{array}{c}
F_{22} \\
F_{11}
\end{array}\right) \\
& +\frac{2}{(k+5)^{2}} B_{ \pm} B_{\mp}\left(\begin{array}{c}
F_{21} \\
F_{12}
\end{array}\right)+\frac{2}{(k+5)^{2}}\left(\begin{array}{c}
F_{11} F_{21} G_{22} \\
F_{22} F_{12} G_{11}
\end{array}\right) \\
& +\frac{2(61 k+97)}{(k+5)^{2}(13 k+17)}\left(\begin{array}{c}
\partial F_{11} F_{21} F_{22} \\
\partial F_{22} F_{12} F_{11}
\end{array}\right) \pm \frac{8(k-3)}{(k+5)^{2}(13 k+17)}\left(\begin{array}{c}
\partial F_{21} \\
\partial F_{12}
\end{array}\right) F_{11} F_{22}
\end{aligned}
$$




$$
\begin{aligned}
& -\frac{2(69 k+73)}{(k+5)^{2}(13 k+17)}\left(\begin{array}{c}
F_{11} F_{21} \partial F_{22} \\
F_{22} F_{12} \partial F_{11}
\end{array}\right) \mp \frac{2}{(k+5)^{2}} F_{11} F_{22}\left(\begin{array}{c}
G_{21} \\
G_{12}
\end{array}\right) \\
& -\frac{3(k+3)}{2(k+5)^{2}} \partial^{2}\left(\begin{array}{c}
F_{21} \\
F_{12}
\end{array}\right)+\frac{2}{(k+5)^{2}}\left(\begin{array}{c}
F_{21} F_{22} G_{11} \\
F_{12} F_{11} G_{22}
\end{array}\right) \\
& -\frac{1}{(k+5)} T\left(\begin{array}{c}
F_{21} \\
F_{12}
\end{array}\right)-\frac{3}{2(k+5)} U\left(\begin{array}{c}
G_{21} \\
G_{12}
\end{array}\right) \pm \frac{6 i}{(k+5)^{2}} U A_{3}\left(\begin{array}{c}
F_{21} \\
F_{12}
\end{array}\right) \\
& \mp \frac{6 i}{(k+5)^{2}} U A_{\mp}\left(\begin{array}{c}
F_{11} \\
F_{22}
\end{array}\right) \mp \frac{6 i}{(k+5)^{2}} U B_{3}\left(\begin{array}{c}
F_{21} \\
F_{12}
\end{array}\right) \\
& \pm \frac{6 i}{(k+5)^{2}} U B_{\mp}\left(\begin{array}{c}
F_{22} \\
F_{11}
\end{array}\right)-\frac{2(k-3)}{(k+5)(13 k+17)} \partial\left(\begin{array}{c}
U F_{21} \\
U F_{12}
\end{array}\right) \\
& \left.-\frac{4}{(k+5)^{2}} U U\left(\begin{array}{c}
F_{21} \\
F_{12}
\end{array}\right)\right](w)+\frac{1}{(z-w)}[+\cdots](w)+\cdots .
\end{aligned}
$$

In the third order pole there is no spin- $\frac{3}{2}$ current. One can write the two OPEs together due to the simple property of the structure constants. The nonlinear terms in the spirit of the footnote 5 are not present but the nonlinear term between the higher spin currents is present. One can also remove this term by adding the $T \mathbf{T}^{(\mathbf{1})}(w)$ into the left hand side in the higher spin-3 current because the OPE $\mathbf{T}_{ \pm}^{\left(\frac{3}{2}\right)}(z) T \mathbf{T}^{(\mathbf{1})}(w)$ contributes to the above $\mathbf{T}^{(\mathbf{1})} \mathbf{T}^{\left(\frac{3}{2}\right)}(w)$ term where the factor $(k-3)$ exists. In this paper, one considers the spin-3 fields in the right hand side of the OPE. In the first order pole of above OPE, there are many nontrivial terms which can be obtained from the previous results for the corresponding OPE in the nonlinear version.

Now one can consider the OPEs between the higher spin-2 current and the 13 higher spin currents

$$
\begin{aligned}
\mathbf{T}^{(\mathbf{2})}(z) \mathbf{T}^{(\mathbf{2})}(w) & =\frac{1}{(z-w)^{4}}\left[\frac{9 k}{(5+k)}\right] \\
& +\frac{1}{(z-w)^{2}}\left[\frac{2(k+4)}{(k+5)} T+\frac{2(k+4)}{(k+5)^{2}} A_{3} A_{3}-\frac{4(k+4)}{(k+5)^{2}} A_{3} B_{3}\right. \\
& +\frac{2 i(k+1)}{(k+5)^{2}} \partial A_{3}-\frac{4 i}{(k+5)^{2}} A_{3} F_{11} F_{22}-\frac{2 i}{(k+5)^{2}} A_{-} F_{11} F_{12} \\
& +\frac{2(k+1)}{(k+5)^{2}} A_{+} A_{-}-\frac{2 i}{(k+5)^{2}} A_{+} F_{21} F_{22}+\frac{2(k+4)}{(k+5)^{2}} B_{3} B_{3} \\
& +\frac{8 i}{(k+5)^{2}} \partial B_{3}+\frac{4 i}{(k+5)^{2}} B_{3} F_{11} F_{22}+\frac{2 i}{(k+5)^{2}} B_{-} F_{12} F_{22}+\frac{8}{(k+5)^{2}} B_{+} B_{-} \\
& +\frac{2 i}{(k+5)^{2}} B_{+} F_{11} F_{21}-\frac{(k-3)}{(k+5)^{2}} F_{11} G_{22}+\frac{2\left(k^{2}+4 k+27\right)}{(k+5)^{3}} \partial F_{11} F_{22} \\
& -\frac{2\left(k^{2}+4 k+27\right)}{(k+5)^{3}} F_{11} \partial F_{22}+\frac{2}{(k+5)} \partial F_{12} F_{21}-\frac{2}{(k+5)} F_{12} \partial F_{21}
\end{aligned}
$$




$$
\left.-\frac{(k-3)}{(k+5)^{2}} F_{22} G_{11}+\frac{2(k+4)}{(k+5)^{2}} U U\right](w)+\frac{1}{(z-w)} \frac{1}{2} \partial(\text { pole- } 2)(w)+\cdots .
$$

There is no higher spin-2 current in the above which appears in the corresponding OPE in the nonlinear version. Also one sees many other terms which do not appear in the corresponding OPE in the nonlinear version.

From the relation (3.2), the following OPEs can be obtained

$$
\begin{aligned}
& \mathbf{T}^{(\mathbf{2})}(z)\left(\begin{array}{c}
\mathbf{U}^{\left(\frac{3}{2}\right)} \\
\mathbf{V}^{\left(\frac{3}{2}\right)}
\end{array}\right)(w)=\frac{1}{(z-w)^{3}}\left[\mp \frac{6 k}{(k+5)^{2}}\left(\begin{array}{c}
F_{11} \\
F_{22}
\end{array}\right)\right](w) \\
& +\frac{1}{(z-w)^{2}}\left[-\frac{(k-3)}{2(k+5)}\left(\begin{array}{c}
\mathbf{U}^{\left(\frac{3}{2}\right)} \\
\mathbf{V}^{\left(\frac{3}{2}\right)}
\end{array}\right) \pm \frac{(k-3)}{2(k+5)^{2}} U\left(\begin{array}{c}
F_{11} \\
F_{22}
\end{array}\right)\right. \\
& +\frac{i(k+9)}{2(k+5)^{2}}\left(\begin{array}{c}
F_{11} \\
F_{22}
\end{array}\right) A_{3}+\frac{i(k-3)}{2(k+5)^{2}}\left(\begin{array}{c}
F_{21} \\
F_{12}
\end{array}\right) A_{ \pm} \\
& \left.+\frac{i(k-3)}{2(k+5)^{2}}\left(\begin{array}{c}
F_{12} \\
F_{21}
\end{array}\right) B_{\mp}-\frac{3 i(k+1)}{2(k+5)^{2}}\left(\begin{array}{c}
F_{11} \\
F_{22}
\end{array}\right) B_{3} \mp \frac{6 k}{(k+5)^{2}} \partial\left(\begin{array}{c}
F_{11} \\
F_{22}
\end{array}\right)\right](w) \\
& +\frac{1}{(z-w)}\left[\frac{1}{2}\left(\begin{array}{c}
\mathbf{Q}^{\left(\frac{5}{2}\right)} \\
\mathbf{R}^{\left(\frac{5}{2}\right)}
\end{array}\right) \pm\left(\begin{array}{c}
\mathbf{U}^{\left(\frac{5}{2}\right)} \\
\mathbf{V}^{\left(\frac{5}{2}\right)}
\end{array}\right)-\frac{(k-3)}{3(k+5)} \partial\left(\begin{array}{c}
\mathbf{U}^{\left(\frac{3}{2}\right)} \\
\mathbf{V}^{\left(\frac{3}{2}\right)}
\end{array}\right)\right. \\
& -\frac{i}{2(k+5)} A_{3}\left(\begin{array}{c}
G_{11} \\
G_{22}
\end{array}\right) \pm \frac{1}{(k+5)^{2}} A_{3} B_{\mp}\left(\begin{array}{c}
F_{12} \\
F_{21}
\end{array}\right) \\
& +\frac{i\left(5 k^{2}+66 k-27\right)}{3(k+5)^{2}(2 k+9)} \partial A_{3}\left(\begin{array}{c}
F_{11} \\
F_{22}
\end{array}\right)+\frac{i(k+15)}{3(k+5)^{2}} A_{3} \partial\left(\begin{array}{c}
F_{11} \\
F_{22}
\end{array}\right) \\
& \pm \frac{1}{(k+5)^{2}} A_{ \pm} A_{3}\left(\begin{array}{c}
F_{21} \\
F_{12}
\end{array}\right) \mp \frac{1}{(k+5)^{2}} A_{ \pm} A_{\mp}\left(\begin{array}{c}
F_{11} \\
F_{22}
\end{array}\right) \\
& \pm \frac{1}{(k+5)^{2}} A_{ \pm} B_{3}\left(\begin{array}{c}
F_{21} \\
F_{12}
\end{array}\right) \mp \frac{2}{(k+5)^{2}} A_{ \pm} B_{\mp}\left(\begin{array}{c}
F_{22} \\
F_{11}
\end{array}\right) \\
& +\frac{i(k-6)}{3(k+5)^{2}} \partial A_{ \pm}\left(\begin{array}{c}
F_{21} \\
F_{12}
\end{array}\right)+\frac{i(k-12)}{3(k+5)^{2}} A_{ \pm} \partial\left(\begin{array}{c}
F_{21} \\
F_{12}
\end{array}\right) \\
& -\frac{i}{2(k+5)} B_{3}\left(\begin{array}{c}
G_{11} \\
G_{22}
\end{array}\right)-\frac{i(5 k+3)}{3(k+5)^{2}} B_{3} \partial\left(\begin{array}{c}
F_{11} \\
F_{22}
\end{array}\right) \\
& -\frac{i(k-12)(k-9)}{3(k+5)^{2}(2 k+9)} \partial B_{3}\left(\begin{array}{c}
F_{11} \\
F_{22}
\end{array}\right) \pm \frac{1}{(k+5)^{2}} B_{\mp} B_{3}\left(\begin{array}{c}
F_{12} \\
F_{21}
\end{array}\right) \\
& +\frac{i k}{3(k+5)^{2}} \partial B_{\mp}\left(\begin{array}{c}
F_{12} \\
F_{21}
\end{array}\right)+\frac{i(k+6)}{3(k+5)^{2}} B_{\mp} \partial\left(\begin{array}{c}
F_{12} \\
F_{21}
\end{array}\right) \\
& \mp \frac{1}{(k+5)^{2}} B_{ \pm} B_{\mp}\left(\begin{array}{c}
F_{11} \\
F_{22}
\end{array}\right) \mp \frac{3(k-1)}{(k+5)^{2}} \partial^{2}\left(\begin{array}{c}
F_{11} \\
F_{22}
\end{array}\right) \\
& \pm \frac{1}{(k+5)^{2}}\left(\begin{array}{c}
F_{11} F_{12} G_{21} \\
F_{22} F_{21} G_{12}
\end{array}\right)-\frac{2(k-3)}{3(k+5)^{3}} \partial\left(\begin{array}{c}
F_{11} \\
F_{22}
\end{array}\right) F_{12} F_{21}
\end{aligned}
$$




$$
\begin{aligned}
& \mp \frac{2(k+9)}{3(k+5)^{3}}\left(\begin{array}{c}
F_{11} \partial F_{12} F_{21} \\
F_{22} \partial F_{21} F_{12}
\end{array}\right) \pm \frac{4(k+3)}{3(k+5)^{3}}\left(\begin{array}{c}
F_{11} F_{12} \partial F_{21} \\
F_{22} F_{21} \partial F_{12}
\end{array}\right) \\
& \mp \frac{1}{(k+5)^{2}}\left(\begin{array}{c}
F_{11} F_{21} G_{12} \\
F_{22} F_{12} G_{21}
\end{array}\right) \mp \frac{1}{(k+5)} T\left(\begin{array}{c}
F_{11} \\
F_{22}
\end{array}\right) \mp \frac{1}{2(k+5)} U\left(\begin{array}{c}
G_{11} \\
G_{22}
\end{array}\right) \\
& -\frac{2 i}{(k+5)^{2}} U A_{3}\left(\begin{array}{c}
F_{11} \\
F_{22}
\end{array}\right)-\frac{i}{(k+5)^{2}} U A_{ \pm}\left(\begin{array}{c}
F_{21} \\
F_{12}
\end{array}\right) \\
& -\frac{2 i}{(k+5)^{2}} U B_{3}\left(\begin{array}{c}
F_{11} \\
F_{22}
\end{array}\right)-\frac{i}{(k+5)^{2}} U B_{\mp}\left(\begin{array}{c}
F_{12} \\
F_{21}
\end{array}\right) \\
& \mp \frac{(k+48 k-27)}{3(k+5)^{2}(2 k+9)} \partial U\left(\begin{array}{c}
F_{11} \\
F_{22}
\end{array}\right) \pm \frac{(k-3)}{3(k+5)^{2}} U \partial\left(\begin{array}{c}
F_{11} \\
F_{22}
\end{array}\right) \\
& \mp \frac{2}{(k+5)^{2}} U U\left(\begin{array}{c}
F_{11} \\
F_{22}
\end{array}\right) \pm \frac{(k-1)(k+18)}{(k+5)^{2}(2 k+9)}\left(\begin{array}{c}
F_{11} G_{22} F_{11} \\
F_{22} G_{11} F_{22}
\end{array}\right) \\
& \left.+\frac{2}{(k+5)^{2}} \partial\left(\begin{array}{c}
F_{11} \\
F_{22}
\end{array}\right) F_{11} F_{22}\right](w)+\cdots .
\end{aligned}
$$

There are no nonlinear terms as in the footnote 5. Also the OPE is rather complicated due to the presence of spin- $\frac{1}{2}$ currents as before. One has combined OPEs due to the symmetry of structure constants. Some terms containing the spin- $\frac{3}{2}$ currents in the above OPEs were not present in the corresponding OPEs in the nonlinear version.

One uses the relation (3.5) to obtain the following OPEs

$$
\begin{aligned}
& \mathbf{T}^{(\mathbf{2})}(z)\left(\begin{array}{c}
\mathbf{U}_{+}^{(\mathbf{2})} \\
\mathbf{V}_{-}^{(\mathbf{2})}
\end{array}\right)(w)=\frac{1}{(z-w)^{3}}\left[\frac{2 i k(k+8)}{(k+5)^{2}} B_{\mp} \mp \frac{4 k}{(k+5)^{2}}\left(\begin{array}{l}
F_{11} F_{21} \\
F_{22} F_{12}
\end{array}\right)\right](w) \\
& +\frac{1}{(z-w)^{2}}\left[ \pm \frac{2(k+4)}{(k+5)^{2}} A_{3} B_{\mp}+\frac{2 i}{(k+5)^{2}} A_{3}\left(\begin{array}{c}
F_{11} F_{21} \\
F_{22} F_{12}
\end{array}\right)\right. \\
& \mp \frac{2 k}{(k+5)^{2}} B_{\mp} B_{3}+\frac{i k(k+7)}{(k+5)^{2}} \partial B_{\mp} \mp \frac{i}{(k+5)^{2}} B_{\mp} F_{11} F_{22} \\
& \mp \frac{i}{(k+5)^{2}} B_{\mp} F_{12} F_{21} \pm \frac{(k-3)}{2(k+5)^{2}}\left(\begin{array}{c}
F_{11} G_{21} \\
F_{22} G_{12}
\end{array}\right) \mp \frac{2(k+1)^{2}}{(k+5)^{3}}\left(\begin{array}{c}
\partial F_{11} F_{21} \\
\partial F_{22} F_{12}
\end{array}\right) \\
& \left.\mp \frac{2\left(k^{2}+8 k-1\right)}{(k+5)^{3}}\left(\begin{array}{c}
F_{11} \partial F_{21} \\
F_{22} \partial F_{12}
\end{array}\right) \pm \frac{(k-3)}{2(k+5)^{2}}\left(\begin{array}{c}
F_{21} G_{11} \\
F_{12} G_{22}
\end{array}\right)\right](w) \\
& +\frac{1}{(z-w)}\left[\frac{1}{2}\left(\begin{array}{c}
\mathbf{Q}_{+}^{(3)} \\
\mathbf{R}_{-}^{(3)}
\end{array}\right)-\frac{i}{(k+5)^{2}} A_{3} A_{3} B_{\mp} \pm \frac{(k+7)}{2(k+5)^{2}} \partial A_{3} B_{\mp}\right. \\
& \pm \frac{(3 k+11)}{2(k+5)^{2}} A_{3} \partial B_{\mp}+\frac{i}{(k+5)^{2}} A_{3}\left(\begin{array}{c}
F_{11} G_{21} \\
F_{22} G_{12}
\end{array}\right) \\
& -\frac{i(k-3)}{2(k+5)^{3}} \partial A_{3}\left(\begin{array}{c}
F_{11} F_{21} \\
F_{22} F_{12}
\end{array}\right)+\frac{i(3 k+7)}{2(k+5)^{3}} A_{3}\left(\begin{array}{c}
\partial F_{11} F_{21} \\
\partial F_{22} F_{12}
\end{array}\right) \\
& +\frac{i(7 k+27)}{2(k+5)^{3}} A_{3}\left(\begin{array}{c}
F_{11} \partial F_{21} \\
F_{22} \partial F_{12}
\end{array}\right)-\frac{i}{(k+5)^{2}} A_{3}\left(\begin{array}{c}
F_{21} G_{11} \\
F_{12} G_{22}
\end{array}\right)
\end{aligned}
$$




$$
\begin{aligned}
& +\frac{i}{(k+5)^{2}} A_{\mp}\left(\begin{array}{c}
F_{11} G_{11} \\
F_{22} G_{22}
\end{array}\right)-\frac{i}{(k+5)^{2}} A_{ \pm} A_{\mp} B_{\mp} \\
& +\frac{i}{(k+5)^{2}} A_{ \pm}\left(\begin{array}{c}
F_{21} G_{21} \\
F_{12} G_{12}
\end{array}\right)-\frac{i}{(k+5)^{2}} B_{3}\left(\begin{array}{c}
F_{11} G_{21} \\
F_{22} G_{12}
\end{array}\right) \\
& +\frac{i(k-11)}{2(k+5)^{3}} B_{3}\left(\begin{array}{c}
\partial F_{11} F_{21} \\
\partial F_{22} F_{12}
\end{array}\right)-\frac{i(3 k-1)}{2(k+5)^{3}} B_{3}\left(\begin{array}{c}
F_{11} \partial F_{21} \\
F_{22} \partial F_{12}
\end{array}\right) \\
& +\frac{i}{2(k+5)^{2}} \partial B_{3}\left(\begin{array}{c}
F_{11} F_{21} \\
F_{22} F_{12}
\end{array}\right)-\frac{i}{(k+5)^{2}} B_{3}\left(\begin{array}{c}
F_{21} G_{11} \\
F_{12} G_{22}
\end{array}\right) \\
& +\frac{i}{(k+5)^{2}} B_{\mp} B_{3} B_{3} \mp \frac{(k+1)}{(k+5)^{2}} \partial B_{\mp} B_{3} \mp \frac{(k+2)}{(k+5)^{2}} B_{\mp} \partial B_{3} \\
& +\frac{i\left(14 k^{3}+67 k^{2}-345 k-306\right)}{6(k+5)^{2}(13 k+17)} \partial^{2} B_{\mp}+\frac{i}{(k+5)^{2}} B_{\mp}\left(\begin{array}{c}
F_{11} G_{22} \\
F_{22} G_{11}
\end{array}\right) \\
& -\frac{i(k-9)}{(k+5)^{3}} B_{\mp}\left(\begin{array}{c}
\partial F_{11} F_{22} \\
\partial F_{22} F_{11}
\end{array}\right) \mp \frac{i(k+7)}{(k+5)^{3}} \partial B_{\mp} F_{11} F_{22} \\
& +\frac{i(k-5)}{(k+5)^{3}} B_{\mp}\left(\begin{array}{c}
F_{11} \partial F_{22} \\
F_{22} \partial F_{11}
\end{array}\right)-\frac{i(k+9)}{2(k+5)^{3}} B_{\mp}\left(\begin{array}{c}
F_{12} \partial F_{21} \\
F_{21} \partial F_{12}
\end{array}\right) \\
& -\frac{i(k-7)}{2(k+5)^{3}} B_{\mp}\left(\begin{array}{c}
\partial F_{12} F_{21} \\
\partial F_{21} F_{12}
\end{array}\right) \mp \frac{i(k+9)}{2(k+5)^{3}} \partial B_{\mp} F_{12} F_{21} \\
& +\frac{i}{(k+5)^{2}} B_{\mp}\left(\begin{array}{c}
F_{21} G_{12} \\
F_{12} G_{21}
\end{array}\right)+\frac{2 i}{(k+5)^{2}} B_{\mp}\left(\begin{array}{c}
F_{22} G_{11} \\
F_{11} G_{22}
\end{array}\right) \\
& +\frac{i}{(k+5)^{2}} B_{ \pm} B_{\mp} B_{\mp} \pm \frac{8(k+1)}{(k+5)^{3}}\left(\begin{array}{c}
\partial^{2} F_{11} F_{21} \\
\partial^{2} F_{22} F_{12}
\end{array}\right) \mp \frac{2}{(k+5)^{2}}\left(\begin{array}{c}
\partial F_{11} \partial F_{21} \\
\partial F_{22} \partial F_{12}
\end{array}\right) \\
& \mp \frac{\left(28 k^{3}+227 k^{2}-1110 k-1581\right)}{6(k+5)^{3}(13 k+17)}\left(\begin{array}{c}
F_{11} \partial^{2} F_{21} \\
F_{22} \partial^{2} F_{12}
\end{array}\right) \pm \frac{1}{2(k+5)}\left(\begin{array}{c}
G_{11} G_{21} \\
G_{22} G_{12}
\end{array}\right) \\
& +\frac{i\left(4 k^{2}+34 k+17\right)}{(k+5)(13 k+17)} T B_{\mp} \mp \frac{2\left(4 k^{2}+25 k+17\right)}{(k+5)^{2}(13 k+17)} T\left(\begin{array}{c}
F_{11} F_{21} \\
F_{22} F_{12}
\end{array}\right) \\
& \mp \frac{2}{(k+5)^{2}} U A_{3} B_{\mp}+\frac{i(k-2)}{2(k+5)^{2}} \partial U B_{\mp}-\frac{i(k-2)}{2(k+5)^{2}} U \partial B_{\mp} \\
& \pm \frac{1}{(k+5)^{2}} U\left(\begin{array}{c}
F_{11} G_{21} \\
F_{22} G_{12}
\end{array}\right) \pm \frac{(k-11)}{2(k+5)^{3}} U\left(\begin{array}{l}
\partial F_{11} F_{21} \\
\partial F_{22} F_{12}
\end{array}\right) \\
& \mp \frac{(3 k-1)}{2(k+5)^{3}} \partial U\left(\begin{array}{c}
F_{11} F_{21} \\
F_{22} F_{12}
\end{array}\right) \pm \frac{(5 k+9)}{2(k+5)^{3}} U\left(\begin{array}{l}
F_{11} \partial F_{21} \\
F_{22} \partial F_{12}
\end{array}\right) \\
& \mp \frac{1}{(k+5)^{2}} U\left(\begin{array}{c}
F_{21} G_{11} \\
F_{12} G_{22}
\end{array}\right)+\frac{i}{(k+5)^{2}} U U B_{\mp}-\frac{8 i}{(k+5)^{3}}\left(\begin{array}{c}
F_{11} A_{-} \partial F_{11} \\
F_{22} A_{+} \partial F_{22}
\end{array}\right) \\
& +\frac{i\left(28 k^{3}+227 k^{2}+138 k+51\right)}{3(k+5)^{3}(13 k+17)}\left(\begin{array}{l}
\partial F_{21} A_{+} F_{21} \\
\partial F_{12} A_{-} F_{12}
\end{array}\right) \\
& +\frac{i\left(28 k^{3}+305 k^{2}+318 k+153\right)}{3(k+5)^{3}(13 k+17)}\left(\begin{array}{l}
F_{21} A_{+} \partial F_{21} \\
F_{12} A_{-} \partial F_{12}
\end{array}\right)
\end{aligned}
$$




$$
\begin{aligned}
& \pm \frac{(k-19)}{4(k+5)^{2}}\left(\begin{array}{l}
\partial F_{11} G_{21} \\
\partial F_{22} G_{12}
\end{array}\right) \pm \frac{(k-3)}{4(k+5)^{2}}\left(\begin{array}{c}
F_{11} \partial G_{21} \\
F_{22} \partial G_{12}
\end{array}\right) \\
& \left.\mp \frac{(k+8)}{2(k+5)^{2}}\left(\begin{array}{l}
\partial F_{21} G_{11} \\
\partial F_{12} G_{22}
\end{array}\right) \pm \frac{(k-2)}{2(k+5)^{2}}\left(\begin{array}{c}
F_{21} \partial G_{11} \\
F_{12} \partial G_{22}
\end{array}\right)\right](w)+\cdots
\end{aligned}
$$

The OPE does not contain any nonlinear terms between the higher spin currents and the currents of large $\mathcal{N}=4$ linear superconformal algebra. In the nonlinear version one cannot combine the above two OPEs. There is no higher spin-2 current in the second order pole of above OPEs.

By using the relation (3.4), the following OPEs can be obtained

$$
\begin{aligned}
& \mathbf{T}^{(\mathbf{2})}(z)\left(\begin{array}{l}
\mathbf{U}_{-}^{(\mathbf{2})} \\
\mathbf{V}_{+}^{(\mathbf{2})}
\end{array}\right)(w)=\frac{1}{(z-w)^{3}}\left[\frac{6 i(2 k+5)}{(k+5)^{2}} A_{ \pm} \pm \frac{12}{(k+5)^{2}}\left(\begin{array}{l}
F_{11} F_{12} \\
F_{22} F_{21}
\end{array}\right)\right](w) \\
& +\frac{1}{(z-w)^{2}}\left[ \pm \frac{6}{(k+5)^{2}} A_{ \pm} A_{3} \mp \frac{2(k+4)}{(k+5)^{2}} A_{ \pm} B_{3}+\frac{6 i(k+2)}{(k+5)^{2}} \partial A_{ \pm}\right. \\
& \mp \frac{i}{(k+5)^{2}} A_{ \pm} F_{11} F_{22} \pm \frac{i}{(k+5)^{2}} A_{ \pm} F_{12} F_{21}+\frac{2 i}{(k+5)^{2}} B_{3}\left(\begin{array}{c}
F_{11} F_{12} \\
F_{22} F_{21}
\end{array}\right) \\
& \mp \frac{(k-3)}{2(k+5)^{2}}\left(\begin{array}{c}
F_{11} G_{12} \\
F_{22} G_{21}
\end{array}\right) \pm \frac{32}{(k+5)^{3}}\left(\begin{array}{c}
\partial F_{11} F_{12} \\
\partial F_{22} F_{21}
\end{array}\right) \\
& \left. \pm \frac{4(3 k+7)}{(k+5)^{3}}\left(\begin{array}{c}
F_{11} \partial F_{12} \\
F_{22} \partial F_{21}
\end{array}\right) \mp \frac{(k-3)}{2(k+5)^{2}}\left(\begin{array}{c}
F_{12} G_{11} \\
F_{21} G_{22}
\end{array}\right)\right](w) \\
& +\frac{1}{(z-w)}\left[\frac{1}{2}\left(\begin{array}{c}
\mathbf{Q}_{-}^{(3)} \\
\mathbf{R}_{+}^{(3)}
\end{array}\right)+\frac{i}{(k+5)^{2}} A_{3}\left(\begin{array}{c}
F_{11} G_{12} \\
F_{22} G_{21}
\end{array}\right)\right. \\
& +\frac{i}{2(k+5)^{2}} \partial A_{3}\left(\begin{array}{c}
F_{11} F_{12} \\
F_{22} F_{21}
\end{array}\right)-\frac{i(3 k-1)}{2(k+5)^{3}} A_{3}\left(\begin{array}{c}
\partial F_{11} F_{12} \\
\partial F_{22} F_{21}
\end{array}\right) \\
& +\frac{i(k-11)}{2(k+5)^{3}} A_{3}\left(\begin{array}{c}
F_{11} \partial F_{12} \\
F_{22} \partial F_{21}
\end{array}\right)+\frac{i}{(k+5)^{2}} A_{3}\left(\begin{array}{c}
F_{12} G_{11} \\
F_{21} G_{22}
\end{array}\right) \\
& +\frac{i}{(k+5)^{2}} A_{ \pm} A_{3} A_{3} \pm \frac{4}{(k+5)^{2}} \partial A_{ \pm} A_{3} \pm \frac{1}{(k+5)^{2}} A_{ \pm} \partial A_{3} \\
& +\frac{i}{(k+5)^{2}} A_{ \pm} A_{ \pm} A_{\mp}-\frac{i}{(k+5)^{2}} A_{ \pm} B_{3} B_{3} \mp \frac{(3 k+11)}{2(k+5)^{2}} \partial A_{ \pm} B_{3} \\
& \mp \frac{(k+3)}{2(k+5)^{2}} A_{ \pm} \partial B_{3}-\frac{i}{(k+5)^{2}} A_{ \pm} B_{ \pm} B_{\mp}+\frac{i\left(24 k^{2}-13 k-185\right)}{2(k+5)^{2}(13 k+17)} \partial^{2} A_{ \pm} \\
& -\frac{i}{(k+5)^{2}} A_{ \pm}\left(\begin{array}{c}
F_{11} G_{22} \\
F_{22} G_{11}
\end{array}\right) \mp \frac{i(3 k+11)}{2(k+5)^{3}} \partial A_{ \pm} F_{11} F_{22} \\
& +\frac{i(5 k-3)}{2(k+5)^{3}} A_{ \pm}\left(\begin{array}{c}
\partial F_{11} F_{22} \\
\partial F_{22} F_{11}
\end{array}\right)-\frac{i(3 k-5)}{2(k+5)^{3}} A_{ \pm}\left(\begin{array}{c}
F_{11} \partial F_{22} \\
F_{22} \partial F_{11}
\end{array}\right) \\
& -\frac{i}{(k+5)^{2}} A_{ \pm}\left(\begin{array}{c}
F_{12} G_{21} \\
F_{21} G_{12}
\end{array}\right) \pm \frac{i(k+3)}{(k+5)^{3}} \partial A_{ \pm} F_{12} F_{21}
\end{aligned}
$$




$$
\begin{aligned}
& +\frac{i(k+3)}{(k+5)^{3}} A_{ \pm}\left(\begin{array}{c}
\partial F_{12} F_{21} \\
\partial F_{21} F_{12}
\end{array}\right)-\frac{i(k-1)}{(k+5)^{3}} A_{ \pm}\left(\begin{array}{c}
F_{12} \partial F_{21} \\
F_{21} \partial F_{12}
\end{array}\right) \\
& -\frac{2 i}{(k+5)^{2}} A_{ \pm}\left(\begin{array}{c}
F_{22} G_{11} \\
F_{11} G_{22}
\end{array}\right)-\frac{i}{(k+5)^{2}} B_{3}\left(\begin{array}{c}
F_{11} G_{12} \\
F_{22} G_{21}
\end{array}\right) \\
& +\frac{i(k-3)}{2(k+5)^{3}} \partial B_{3}\left(\begin{array}{c}
F_{11} F_{12} \\
F_{22} F_{21}
\end{array}\right)+\frac{i(k+13)}{2(k+5)^{3}} B_{3}\left(\begin{array}{c}
\partial F_{11} F_{12} \\
\partial F_{22} F_{21}
\end{array}\right) \\
& +\frac{i(5 k+33)}{2(k+5)^{3}} B_{3}\left(\begin{array}{c}
F_{11} \partial F_{12} \\
F_{22} \partial F_{21}
\end{array}\right)+\frac{i}{(k+5)^{2}} B_{3}\left(\begin{array}{c}
F_{12} G_{11} \\
F_{21} G_{22}
\end{array}\right) \\
& -\frac{i}{(k+5)^{2}} B_{\mp}\left(\begin{array}{c}
F_{12} G_{12} \\
F_{21} G_{21}
\end{array}\right)-\frac{i}{(k+5)^{2}} B_{ \pm}\left(\begin{array}{c}
F_{11} G_{11} \\
F_{22} G_{22}
\end{array}\right) \\
& \mp \frac{\left(103 k^{2}+326 k+591\right)}{2(k+5)^{3}(13 k+17)}\left(\begin{array}{l}
\partial^{2} F_{11} F_{12} \\
\partial^{2} F_{22} F_{21}
\end{array}\right) \\
& \pm \frac{\left(131 k^{2}+318 k-149\right)}{(k+5)^{3}(13 k+17)}\left(\begin{array}{l}
\partial F_{11} \partial F_{12} \\
\partial F_{22} \partial F_{21}
\end{array}\right) \\
& \pm \frac{(k-91)}{2(k+5)^{2}(13 k+17)}\left(\begin{array}{c}
F_{11} \partial^{2} F_{12} \\
F_{22} \partial^{2} F_{21}
\end{array}\right) \mp \frac{(k+1)}{(k+5)^{2}}\left(\begin{array}{l}
\partial F_{11} G_{12} \\
\partial F_{22} G_{21}
\end{array}\right) \\
& \mp \frac{(k+19)}{4(k+5)^{2}}\left(\begin{array}{c}
\partial F_{12} G_{11} \\
\partial F_{21} G_{22}
\end{array}\right) \mp \frac{(k-5)}{4(k+5)^{2}}\left(\begin{array}{c}
F_{12} \partial G_{11} \\
F_{21} \partial G_{22}
\end{array}\right) \\
& \mp \frac{1}{2(k+5)}\left(\begin{array}{c}
G_{11} G_{12} \\
G_{22} G_{21}
\end{array}\right)+\frac{i(28 k+71)}{(k+5)(13 k+17)} T A_{ \pm} \\
& \pm \frac{2(19 k+71)}{(k+5)^{2}(13 k+17)} T\left(\begin{array}{c}
F_{11} F_{12} \\
F_{22} F_{21}
\end{array}\right) \mp \frac{2}{(k+5)^{2}} U A_{ \pm} B_{3} \\
& -\frac{i}{2(k+5)^{2}} \partial U A_{ \pm}+\frac{i}{2(k+5)^{2}} U \partial A_{ \pm} \mp \frac{1}{(k+5)^{2}} U\left(\begin{array}{c}
F_{11} G_{12} \\
F_{22} G_{21}
\end{array}\right) \\
& \pm \frac{(k-11)}{2(k+5)^{3}} \partial U\left(\begin{array}{c}
F_{11} F_{12} \\
F_{22} F_{21}
\end{array}\right) \mp \frac{(3 k-1)}{2(k+5)^{3}} U\left(\begin{array}{c}
\partial F_{11} F_{12} \\
\partial F_{22} F_{21}
\end{array}\right) \\
& \pm \frac{(k+21)}{2(k+5)^{3}} U\left(\begin{array}{c}
F_{11} \partial F_{12} \\
F_{22} \partial F_{21}
\end{array}\right) \pm \frac{1}{(k+5)^{2}} U\left(\begin{array}{c}
F_{12} G_{11} \\
F_{21} G_{22}
\end{array}\right) \\
& +\frac{i}{(k+5)^{2}} U U A_{ \pm}-\frac{2 i(k+1)}{(k+5)^{3}}\left(\begin{array}{c}
F_{11} B_{+} \partial F_{11} \\
F_{22} B_{-} \partial F_{22}
\end{array}\right) \\
& \left.+\frac{8 i}{(k+5)^{3}}\left(\begin{array}{c}
F_{12} B_{-} \partial F_{12} \\
F_{21} B_{+} \partial F_{21}
\end{array}\right)\right](w)+\cdots \text {. }
\end{aligned}
$$

In this case, one sees the symmetry between the structure constants in the two OPEs. The nonlinear terms appearing in the corresponding OPEs in the nonlinear version do not appear in the above OPEs. There is no higher spin-2 current in the second order pole of above OPEs. One sees that there are many (nonderivative) terms in the first order pole which do not appear in the corresponding OPEs in the nonlinear version. 
With the help of (3.7), one calculates the following OPEs

$$
\begin{aligned}
& \mathbf{T}^{(2)}(z)\left(\begin{array}{l}
\mathbf{U}^{\left(\frac{5}{2}\right)} \\
\mathbf{V}^{\left(\frac{5}{2}\right)}
\end{array}\right)(w)=\frac{1}{(z-w)^{4}}\left[\frac{6(k-3) k}{(k+5)^{3}}\left(\begin{array}{l}
F_{11} \\
F_{22}
\end{array}\right)\right](w) \\
& +\frac{1}{(z-w)^{3}}\left[-\frac{2\left(4 k^{2}+39 k+66\right)}{3(k+5)^{2}}\left(\begin{array}{l}
G_{11} \\
G_{22}
\end{array}\right)\right. \\
& \text { - } \frac{8\left(2 k^{2}+15 k+33\right)}{3(k+5)^{3}} U\left(\begin{array}{c}
F_{11} \\
F_{22}
\end{array}\right) \mp \frac{4}{(k+5)^{2}}\left(\begin{array}{c}
F_{11} \\
F_{22}
\end{array}\right) F_{12} F_{21} \\
& \mp \frac{4 i\left(4 k^{2}+15 k-33\right)}{3(k+5)^{3}}\left(\begin{array}{l}
F_{11} \\
F_{22}
\end{array}\right) A_{3} \mp \frac{4 i\left(4 k^{2}+21 k+21\right)}{3(k+5)^{3}}\left(\begin{array}{c}
F_{21} \\
F_{12}
\end{array}\right) A_{ \pm} \\
& \left.\mp \frac{4 i\left(k^{2}+15 k+66\right)}{3(k+5)^{3}}\left(\begin{array}{c}
F_{12} \\
F_{21}
\end{array}\right) B_{\mp} \pm \frac{4 i\left(k^{2}-k-22\right)}{(k+5)^{3}}\left(\begin{array}{c}
F_{11} \\
F_{22}
\end{array}\right) B_{3}\right](w) \\
& +\frac{1}{(z-w)^{2}}\left[\mp \frac{(k-3)}{6(k+5)}\left(\begin{array}{c}
\mathbf{Q}^{\left(\frac{5}{2}\right)} \\
\mathbf{R}^{\left(\frac{5}{2}\right)}
\end{array}\right)-\frac{(k-3)}{6(k+5)}\left(\begin{array}{c}
\mathbf{U}^{\left(\frac{5}{2}\right)} \\
\mathbf{V}^{\left(\frac{5}{2}\right)}
\end{array}\right)\right. \\
& \pm \frac{i(5 k+27)}{3(k+5)^{2}} A_{3}\left(\begin{array}{c}
G_{11} \\
G_{22}
\end{array}\right)-\frac{2}{(k+5)^{2}} A_{3} A_{3}\left(\begin{array}{c}
F_{11} \\
F_{22}
\end{array}\right) \\
& +\frac{2(k+9)}{3(k+5)^{3}} A_{3}\left(\begin{array}{c}
B_{-} F_{12} \\
B_{+} F_{21}
\end{array}\right) \mp \frac{2 i\left(8 k^{2}+27 k-81\right)}{9(k+5)^{3}} \partial A_{3}\left(\begin{array}{c}
F_{11} \\
F_{22}
\end{array}\right) \\
& \mp \frac{8 i(k+3)(2 k+9)}{9(k+5)^{3}} A_{3} \partial\left(\begin{array}{c}
F_{11} \\
F_{22}
\end{array}\right) \pm \frac{i(k+1)}{(k+5)^{2}} A_{ \pm}\left(\begin{array}{c}
G_{21} \\
G_{12}
\end{array}\right) \\
& -\frac{4(k+3)}{3(k+5)^{3}} A_{ \pm} A_{3}\left(\begin{array}{c}
F_{21} \\
F_{12}
\end{array}\right)-\frac{2(k+9)}{3(k+5)^{3}} A_{ \pm} A_{\mp}\left(\begin{array}{c}
F_{11} \\
F_{22}
\end{array}\right) \\
& -\frac{4(k+3)}{3(k+5)^{3}} A_{ \pm} B_{3}\left(\begin{array}{c}
F_{21} \\
F_{12}
\end{array}\right)+\frac{2(k-3)}{3(k+5)^{3}} A_{ \pm} B_{\mp}\left(\begin{array}{c}
F_{22} \\
F_{11}
\end{array}\right) \\
& \mp \frac{4 i(k+3)(4 k-3)}{9(k+5)^{3}} \partial A_{ \pm}\left(\begin{array}{c}
F_{21} \\
F_{12}
\end{array}\right) \mp \frac{4 i(k+3)(4 k+27)}{9(k+5)^{3}} A_{ \pm} \partial\left(\begin{array}{c}
F_{21} \\
F_{12}
\end{array}\right) \\
& \mp \frac{7 i(k+3)}{3(k+5)^{2}} B_{3}\left(\begin{array}{c}
G_{11} \\
G_{22}
\end{array}\right)+\frac{2}{(k+5)^{2}} B_{3} B_{3}\left(\begin{array}{c}
F_{11} \\
F_{22}
\end{array}\right) \\
& \pm \frac{2 i(k-3)(10 k+39)}{9(k+5)^{3}} \partial B_{3}\left(\begin{array}{c}
F_{11} \\
F_{22}
\end{array}\right) \mp \frac{4 i(k+9)(k+12)}{9(k+5)^{3}} B_{3} \partial\left(\begin{array}{c}
F_{11} \\
F_{22}
\end{array}\right) \\
& \mp \frac{4 i}{(k+5)^{2}} B_{\mp}\left(\begin{array}{c}
G_{12} \\
G_{21}
\end{array}\right)+\frac{2(k+9)}{3(k+5)^{3}} B_{\mp} B_{3}\left(\begin{array}{c}
F_{12} \\
F_{21}
\end{array}\right) \\
& \pm \frac{2 i(k-12)(k+9)}{9(k+5)^{3}} \partial B_{\mp}\left(\begin{array}{c}
F_{12} \\
F_{21}
\end{array}\right) \mp \frac{2 i(k+9)(8 k+15)}{9(k+5)^{3}} B_{\mp} \partial\left(\begin{array}{c}
F_{12} \\
F_{21}
\end{array}\right) \\
& +\frac{4(k+3)}{3(k+5)^{3}} B_{ \pm} B_{\mp}\left(\begin{array}{c}
F_{11} \\
F_{22}
\end{array}\right)+\frac{2(k-3)(2 k+9)}{3(k+5)^{3}} \partial^{2}\left(\begin{array}{c}
F_{11} \\
F_{22}
\end{array}\right) \\
& +\frac{2(k+9)}{3(k+5)^{3}}\left(\begin{array}{c}
F_{11} F_{12} G_{21} \\
F_{22} F_{21} G_{12}
\end{array}\right) \mp \frac{16(k+3)(k+9)}{9(k+5)^{4}} \partial\left(\begin{array}{c}
F_{11} \\
F_{22}
\end{array}\right) F_{12} F_{21}
\end{aligned}
$$




$$
\begin{aligned}
& -\frac{16(k+3)^{2}}{9(k+5)^{4}}\left(\begin{array}{c}
F_{11} \partial F_{12} F_{21} \\
F_{22} \partial F_{21} F_{12}
\end{array}\right)-\frac{4(k+9)^{2}}{9(k+5)^{4}}\left(\begin{array}{c}
F_{11} F_{12} \partial F_{21} \\
F_{22} F_{21} \partial F_{12}
\end{array}\right) \\
& +\frac{4(k+3)}{3(k+5)^{3}}\left(\begin{array}{c}
F_{11} F_{21} G_{12} \\
F_{22} F_{12} G_{21}
\end{array}\right) \pm \frac{2}{(k+5)^{2}} F_{11} F_{22}\left(\begin{array}{c}
G_{11} \\
G_{22}
\end{array}\right) \\
& -\frac{\left(8 k^{2}+87 k+171\right)}{9(k+5)^{2}} \partial\left(\begin{array}{c}
G_{11} \\
G_{22}
\end{array}\right)+\frac{4(k-3)}{3(k+5)^{2}} T\left(\begin{array}{c}
F_{11} \\
F_{22}
\end{array}\right) \\
& -\frac{(k-3)}{3(k+5)^{2}} U\left(\begin{array}{c}
G_{11} \\
G_{22}
\end{array}\right) \pm \frac{8 i(k+3)}{3(k+5)^{3}} U A_{3}\left(\begin{array}{c}
F_{11} \\
F_{22}
\end{array}\right) \\
& \pm \frac{4 i(k+3)}{3(k+5)^{3}} U A_{ \pm}\left(\begin{array}{c}
F_{21} \\
F_{12}
\end{array}\right) \mp \frac{4 i(k+9)}{3(k+5)^{3}} U B_{3}\left(\begin{array}{c}
F_{11} \\
F_{22}
\end{array}\right) \\
& \mp \frac{2 i(k+9)}{3(k+5)^{3}} U B_{\mp}\left(\begin{array}{c}
F_{12} \\
F_{21}
\end{array}\right)-\frac{2\left(8 k^{2}+87 k+171\right)}{9(k+5)^{3}} \partial U\left(\begin{array}{c}
F_{11} \\
F_{22}
\end{array}\right) \\
& -\frac{16\left(k^{2}+3 k+18\right)}{9(k+5)^{3}} U \partial\left(\begin{array}{c}
F_{11} \\
F_{22}
\end{array}\right)+\frac{2(k-3)}{3(k+5)^{3}} U U\left(\begin{array}{c}
F_{11} \\
F_{22}
\end{array}\right) \\
& \left.-\frac{4(k-3)}{3(k+5)^{3}}\left(\begin{array}{l}
F_{11} \partial F_{11} F_{22} \\
F_{22} \partial F_{22} F_{11}
\end{array}\right)\right](w) \\
& +\frac{1}{(z-w)}[+\cdots](w)+\cdots
\end{aligned}
$$

There is no higher spin- $\frac{3}{2}$ current in the third order pole. Again, one can combine two OPEs in one expression as above due to the symmetry of structure constants. It is an open problem to fill out the first order pole of the above OPE and see whether there are nonlinear terms in the context of the footnote 5 from the corresponding OPEs in the nonlinear results.

One uses the relation (3.6) to obtain the following OPE

$$
\begin{aligned}
\mathbf{T}^{(\mathbf{2})}(z) \mathbf{W}^{(\mathbf{2})}(w) & =\frac{1}{(z-w)^{4}}\left[-\frac{3(k-3) k}{(k+5)^{2}}\right] \\
& +\frac{1}{(z-w)^{2}}\left[\frac{2(k-3)}{3(k+5)} \mathbf{T}^{(\mathbf{2})}+2 \mathbf{W}^{(\mathbf{2})}-\frac{1}{2} \mathbf{P}^{(\mathbf{2})}\right. \\
& -\frac{2(k-3)}{3(k+5)} T-\frac{2(k+6)}{3(k+5)^{2}} A_{3} A_{3}-\frac{2(k-3)}{3(k+5)^{2}} A_{3} B_{3}-\frac{2 i(k-3)}{3(k+5)^{2}} \partial A_{3} \\
& +\frac{2 i(k-3)}{3(k+5)^{3}} A_{3} F_{11} F_{22}-\frac{4 i(k+15)}{3(k+5)^{3}} A_{-} F_{11} F_{12}-\frac{2(k-3)}{3(k+5)^{2}} A_{+} A_{-} \\
& +\frac{2 i(k+9)}{(k+5)^{3}} A_{+} F_{21} F_{22}+\frac{2(2 k+3)}{3(k+5)^{2}} B_{3} B_{3}-\frac{2 i(k-3)}{3(k+5)^{2}} \partial B_{3} \\
& -\frac{2 i(k-3)}{3(k+5)^{3}} B_{3} F_{11} F_{22}-\frac{2 i(7 k+15)}{3(k+5)^{3}} B_{-} F_{12} F_{22}-\frac{2(k-3)}{3(k+5)^{2}} B_{+} B_{-} \\
& +\frac{4 i(k+3)}{(k+5)^{3}} B_{+} F_{11} F_{21}-\frac{(k-3)}{6(k+5)^{2}} F_{11} G_{22}-\frac{2(k+3)(k+9)}{3(k+5)^{3}} \partial F_{11} F_{22}
\end{aligned}
$$




$$
\begin{aligned}
& +\frac{2\left(k^{2}-6 k-63\right)}{3(k+5)^{3}} F_{11} \partial F_{22}+\frac{3}{2(k+5)} F_{12} G_{21}-\frac{2(k-3) k}{3(k+5)^{3}} \partial F_{12} F_{21} \\
& +\frac{2(k-3)(k+8)}{3(k+5)^{3}} F_{12} \partial F_{21}-\frac{(k+9)}{2(k+5)^{2}} F_{21} G_{12}+\frac{(k-3)}{6(k+5)^{2}} F_{22} G_{11} \\
& -\frac{8(k-3)}{3(k+5)^{3}} U F_{11} F_{22}+\frac{6}{(k+5)^{2}} U F_{12} F_{21}+\frac{6 i}{(k+5)^{2}} U A_{3}+\frac{2 i k}{(k+5)^{2}} U B_{3} \\
& \left.-\frac{2(k-3)}{3(k+5)^{2}} U U\right](w) \\
& +\frac{1}{(z-w)}\left[-\frac{1}{4} \partial \mathbf{P}^{(\mathbf{2})}+\partial \mathbf{W}^{(\mathbf{2})}+\frac{(k-3)}{3(k+5)} \partial \mathbf{T}^{(\mathbf{2})}-\frac{2(k+6)}{3(k+5)^{2}} \partial A_{3} A_{3}\right. \\
& -\frac{(7 k+27)}{3(k+5)^{2}} \partial A_{3} B_{3}+\frac{(5 k+33)}{3(k+5)^{2}} A_{3} \partial B_{3}+\frac{2 i(k-3)}{3(k+5)^{3}} \partial A_{3} F_{11} F_{22} \\
& +\frac{4 i(k+3)}{3(k+5)^{3}} A_{3} \partial\left(F_{11} F_{22}\right)+\frac{i}{(k+5)^{2}} \partial A_{3} F_{12} F_{21}-\frac{i}{(k+5)^{2}} A_{3} \partial\left(F_{12} F_{21}\right) \\
& +\frac{i}{(k+5)^{2}} A_{-} F_{11} G_{12}-\frac{2 i(k+15)}{3(k+5)^{3}} \partial A_{-} F_{11} F_{12}-\frac{4 i(2 k+9)}{3(k+5)^{3}} A_{-} \partial F_{11} F_{12} \\
& +\frac{4 i(k-6)}{3(k+5)^{3}} A_{-} F_{11} \partial F_{12}+\frac{i}{(k+5)^{2}} A_{-} F_{12} G_{11}-\frac{(k-3)}{3(k+5)^{2}} \partial\left(A_{+} A_{-}\right) \\
& -\frac{i}{(k+5)^{2}} A_{+} F_{21} G_{22}+\frac{i(k+9)}{(k+5)^{3}} \partial A_{+} F_{21} F_{22}+\frac{i(3 k+11)}{(k+5)^{3}} A_{+} \partial F_{21} F_{22} \\
& -\frac{i(k-7)}{(k+5)^{3}} A_{+} F_{21} \partial F_{22}-\frac{i}{(k+5)^{2}} A_{+} F_{22} G_{21}-\frac{i(k-3)}{3(k+5)^{2}} \partial^{2} B_{3} \\
& +\frac{2(2 k+3)}{3(k+5)^{2}} \partial B_{3} B_{3}+\frac{2 i(k+9)}{3(k+5)^{3}} B_{3} \partial\left(F_{11} F_{22}\right)+\frac{i}{(k+5)^{2}} B_{-} F_{12} G_{22} \\
& -\frac{i}{(k+5)^{2}} \partial B_{3} F_{12} F_{21}+\frac{i}{(k+5)^{2}} B_{3} \partial\left(F_{12} F_{21}\right)-\frac{i(7 k+15)}{3(k+5)^{3}} \partial B_{-} F_{12} F_{22} \\
& -\frac{i(7 k-9)}{3(k+5)^{3}} B_{-} \partial F_{12} F_{22}-\frac{i(7 k+39)}{3(k+5)^{3}} B_{-} F_{12} \partial F_{22}+\frac{i}{(k+5)^{2}} B_{-} F_{22} G_{12} \\
& -\frac{(k-3)}{3(k+5)^{2}} \partial\left(B_{+} B_{-}\right)-\frac{i}{(k+5)^{2}} B_{+} F_{11} G_{21}+\frac{2 i(k+3)}{(k+5)^{3}} \partial B_{+} F_{11} F_{21} \\
& +\frac{2 i(k-1)}{(k+5)^{3}} B_{+} \partial F_{11} F_{21}+\frac{2 i(k+7)}{(k+5)^{3}} B_{+} F_{11} \partial F_{21}-\frac{i}{(k+5)^{2}} B_{+} F_{21} G_{11} \\
& +\frac{(2 k+15)}{3(k+5)^{2}} \partial F_{11} G_{22}-\frac{k}{3(k+5)^{2}} F_{11} \partial G_{22}-\frac{1}{(k+5)^{2}} \partial F_{12} G_{21} \\
& +\frac{(k+4)}{(k+5)^{2}} F_{12} \partial G_{21}-\frac{(k+7)}{(k+5)^{2}} \partial F_{21} G_{12}-\frac{2}{(k+5)^{2}} F_{21} \partial G_{12} \\
& +\frac{(5 k+27)}{6(k+5)^{2}} \partial F_{22} G_{11}-\frac{(k+3)}{6(k+5)^{2}} F_{22} \partial G_{11}-\frac{(k-3)}{3(k+5)} \partial T
\end{aligned}
$$




$$
\begin{aligned}
& +\frac{3 i}{(k+5)^{2}} \partial\left(U A_{3}\right)+\frac{i k}{(k+5)^{2}} \partial\left(U B_{3}\right)-\frac{8 k}{3(k+5)^{3}} \partial U F_{11} F_{22} \\
& +\frac{2(k+21)}{3(k+5)^{3}} U \partial F_{11} F_{22}-\frac{2(5 k+9)}{3(k+5)^{3}} U F_{11} \partial F_{22}+\frac{3}{(k+5)^{2}} \partial U F_{12} F_{21} \\
& +\frac{1}{(k+5)^{2}} U \partial F_{12} F_{21}+\frac{5}{(k+5)^{2}} U F_{12} \partial F_{21}-\frac{2(k-3)}{3(k+5)^{2}} \partial U U \\
& +\frac{4(k+3)}{3(k+5)^{3}} F_{11} G_{22} F_{11} F_{22}-\frac{i(k-3)}{3(k+5)^{2}} \partial^{2} A_{3}-\frac{\left(k^{2}+15 k+18\right)}{3(k+5)^{3}} \partial^{2} F_{11} F_{22} \\
& -\frac{6}{(k+5)^{2}} \partial F_{11} \partial F_{22}+\frac{\left(k^{2}-3 k-72\right)}{3(k+5)^{3}} F_{11} \partial^{2} F_{22}-\frac{(k-3)^{2}}{3(k+5)^{3}} \partial^{2} F_{12} F_{21} \\
& \left.+\frac{8(k-3)}{3(k+5)^{3}} \partial F_{12} \partial F_{21}+\frac{(k-3)}{3(k+5)^{2}} F_{12} \partial^{2} F_{21}\right](w)+\cdots
\end{aligned}
$$

The central term in the above OPE is present. The nonlinear terms appearing in the corresponding OPE in the nonlinear version do not appear in this OPE.

The following OPEs can be obtained from (3.8)

$$
\begin{aligned}
\mathbf{T}^{(2)}(z) \mathbf{W}_{ \pm}^{\left(\frac{5}{2}\right)}(w) & =\frac{1}{(z-w)^{4}}\left[-\frac{18 k}{(k+5)^{2}}\left(\begin{array}{c}
F_{21} \\
F_{12}
\end{array}\right)\right](w) \\
& +\frac{1}{(z-w)^{3}}\left[-\frac{2(k-3)}{3(k+5)^{2}}\left(\begin{array}{c}
G_{21} \\
G_{12}
\end{array}\right)-\frac{4(k-3)}{3(k+5)^{3}} U\left(\begin{array}{c}
F_{21} \\
F_{12}
\end{array}\right)\right. \\
& \mp \frac{4(k-3)}{3(k+5)^{3}}\left(\begin{array}{c}
F_{21} \\
F_{12}
\end{array}\right) F_{11} F_{22} \pm \frac{8 i(5 k+21)}{3(k+5)^{3}}\left(\begin{array}{c}
F_{21} \\
F_{12}
\end{array}\right) A_{3} \\
& \mp \frac{16 i(k-3)}{3(k+5)^{3}}\left(\begin{array}{c}
F_{11} \\
F_{22}
\end{array}\right) A_{\mp} \pm \frac{4 i(k-3)(k+1)}{3(k+5)^{3}}\left(\begin{array}{c}
F_{22} \\
F_{11}
\end{array}\right) B_{\mp} \\
& \left. \pm \frac{4 i\left(3 k^{2}+14 k+3\right)}{3(k+5)^{3}}\left(\begin{array}{c}
F_{21} \\
F_{12}
\end{array}\right) B_{3}\right](w) \\
& +\frac{1}{(z-w)^{2}}\left[ \pm \frac{5}{2} \mathbf{P}_{ \pm}^{\left(\frac{5}{2}\right)}+\frac{5}{2} \mathbf{W}_{ \pm}^{\left(\frac{5}{2}\right)} \mp \frac{12}{(k+5)^{2}} \partial\left(\begin{array}{c}
F_{21} \\
F_{12}
\end{array}\right) F_{12} F_{21}\right. \\
& \pm \frac{i(3 k+13)}{(k+5)^{2}} A_{3}\left(\begin{array}{c}
G_{21} \\
G_{12}
\end{array}\right)+\frac{2}{(k+5)^{2}} A_{3} A_{3}\left(\begin{array}{c}
F_{21} \\
F_{12}
\end{array}\right) \\
& -\frac{4}{(k+5)^{2}} A_{3} B_{3}\left(\begin{array}{c}
F_{21} \\
F_{12}
\end{array}\right)+\frac{2(7 k+39)}{3(k+5)^{3}} A_{3} B_{\mp}\left(\begin{array}{c}
F_{22} \\
F_{11}
\end{array}\right) \\
& \pm \frac{2 i(25 k+117)}{3(k+5)^{3}} \partial A_{3}\left(\begin{array}{c}
F_{21} \\
F_{12}
\end{array}\right) \mp \frac{2 i(23 k+99)}{3(k+5)^{3}} A_{3} \partial\left(\begin{array}{l}
F_{21} \\
F_{12}
\end{array}\right) \\
& \mp \frac{i(7 k+15)}{3(k+5)^{2}} A_{\mp}\left(\begin{array}{c}
G_{11} \\
G_{22}
\end{array}\right)-\frac{4(k+3)}{3(k+5)^{3}} A_{\mp} A_{3}\left(\begin{array}{c}
F_{11} \\
F_{22}
\end{array}\right) \\
& +\frac{8(2 k+9)}{3(k+5)^{3}} A_{\mp} B_{3}\left(\begin{array}{c}
F_{11} \\
F_{22}
\end{array}\right)+\frac{6}{(k+5)^{2}} A_{\mp} B_{\mp}\left(\begin{array}{l}
F_{12} \\
F_{21}
\end{array}\right)
\end{aligned}
$$




$$
\begin{aligned}
& \mp \frac{52 i(k+3)}{3(k+5)^{3}} \partial A_{\mp}\left(\begin{array}{c}
F_{11} \\
F_{22}
\end{array}\right) \pm \frac{2 i(29 k+117)}{3(k+5)^{3}} A_{\mp} \partial\left(\begin{array}{c}
F_{11} \\
F_{22}
\end{array}\right) \\
& +\frac{2(k+9)}{3(k+5)^{3}} A_{ \pm} A_{\mp}\left(\begin{array}{c}
F_{21} \\
F_{12}
\end{array}\right) \mp \frac{i(3 k+13)}{(k+5)^{2}} B_{3}\left(\begin{array}{c}
G_{21} \\
G_{12}
\end{array}\right) \\
& +\frac{2}{(k+5)^{2}} B_{3} B_{3}\left(\begin{array}{c}
F_{21} \\
F_{12}
\end{array}\right) \pm \frac{2 i\left(6 k^{2}+35 k+33\right)}{3(k+5)^{3}} \partial B_{3}\left(\begin{array}{c}
F_{21} \\
F_{12}
\end{array}\right) \\
& \mp \frac{2 i\left(6 k^{2}+31 k+21\right)}{3(k+5)^{3}} B_{3} \partial\left(\begin{array}{c}
F_{21} \\
F_{12}
\end{array}\right) \pm \frac{2 i(k+15)}{3(k+5)^{2}} B_{\mp}\left(\begin{array}{c}
G_{22} \\
G_{11}
\end{array}\right) \\
& -\frac{2(k+9)}{3(k+5)^{3}} B_{\mp} B_{3}\left(\begin{array}{c}
F_{22} \\
F_{11}
\end{array}\right) \mp \frac{2 i(k+9)(3 k+4)}{3(k+5)^{3}} \partial B_{\mp}\left(\begin{array}{c}
F_{22} \\
F_{11}
\end{array}\right) \\
& \pm \frac{4 i\left(4 k^{2}+23 k-3\right)}{3(k+5)^{3}} B_{\mp} \partial\left(\begin{array}{c}
F_{22} \\
F_{11}
\end{array}\right)+\frac{4(k+3)}{3(k+5)^{3}} B_{ \pm} B_{\mp}\left(\begin{array}{c}
F_{21} \\
F_{12}
\end{array}\right) \\
& +\frac{8(k+6)}{3(k+5)^{3}}\left(\begin{array}{c}
F_{11} F_{21} G_{22} \\
F_{22} F_{12} G_{11}
\end{array}\right)+\frac{4\left(9 k^{2}+82 k+201\right)}{3(k+5)^{4}}\left(\begin{array}{l}
\partial F_{11} F_{21} F_{22} \\
\partial F_{22} F_{12} F_{11}
\end{array}\right) \\
& -\frac{32\left(k^{2}+10 k+27\right)}{3(k+5)^{4}}\left(\begin{array}{c}
F_{11} F_{21} \partial F_{22} \\
F_{22} F_{12} \partial F_{11}
\end{array}\right) \mp \frac{2}{(k+5)^{2}} F_{11} F_{22}\left(\begin{array}{c}
G_{21} \\
G_{12}
\end{array}\right) \\
& +\frac{4(k+3)(k+9)}{3(k+5)^{3}} \partial^{2}\left(\begin{array}{c}
F_{21} \\
F_{12}
\end{array}\right)+\frac{2(5 k+21)}{3(k+5)^{3}}\left(\begin{array}{c}
F_{21} F_{22} G_{11} \\
F_{12} F_{11} G_{22}
\end{array}\right) \\
& +\frac{(k-3)}{3(k+5)^{2}} \partial\left(\begin{array}{c}
G_{21} \\
G_{12}
\end{array}\right)-\frac{4(k+4)}{(k+5)^{2}} T\left(\begin{array}{c}
F_{21} \\
F_{12}
\end{array}\right)-\frac{(k+7)}{(k+5)^{2}} U\left(\begin{array}{c}
G_{21} \\
G_{12}
\end{array}\right) \\
& \pm \frac{8 i}{(k+5)^{2}} U A_{3}\left(\begin{array}{c}
F_{21} \\
F_{12}
\end{array}\right) \mp \frac{4 i(5 k+27)}{3(k+5)^{3}} U A_{\mp}\left(\begin{array}{c}
F_{11} \\
F_{22}
\end{array}\right) \\
& \mp \frac{8 i}{(k+5)^{2}} U B_{3}\left(\begin{array}{c}
F_{21} \\
F_{12}
\end{array}\right) \pm \frac{2 i(11 k+51)}{3(k+5)^{3}} U B_{\mp}\left(\begin{array}{c}
F_{22} \\
F_{11}
\end{array}\right) \\
& +\frac{2(k-3)}{3(k+5)^{3}} \partial U\left(\begin{array}{c}
F_{21} \\
F_{12}
\end{array}\right)-\frac{10(k-3)}{3(k+5)^{3}} U \partial\left(\begin{array}{c}
F_{21} \\
F_{12}
\end{array}\right) \\
& \left.-\frac{6}{(k+5)^{2}} U U\left(\begin{array}{c}
F_{21} \\
F_{12}
\end{array}\right)\right](w) \\
& +\frac{1}{(z-w)}[+\cdots](w)+\cdots \text {. }
\end{aligned}
$$

The OPEs are described up to the second order pole. One combines two OPEs in one single OPE using the symmetry between the structure constants. One sees that there is no higher spin- $\frac{3}{2}$ current in the third order pole of the above OPEs.

The equation (3.9) allows us to calculate the following OPE

$$
\mathbf{T}^{(\mathbf{2})}(z) \mathbf{W}^{(\mathbf{3})}(w)=\frac{1}{(z-w)^{4}}\left[\frac{36 i\left(18 k^{2}+63 k+41\right)}{(k+5)^{2}(13 k+17)} A_{3}-\frac{12 i k\left(8 k^{2}+75 k+95\right)}{(k+5)^{2}(13 k+17)} B_{3}\right.
$$




$$
\begin{aligned}
& \left.+\frac{12\left(8 k^{3}+51 k^{2}+182 k+123\right)}{(k+5)^{3}(13 k+17)} F_{11} F_{22}-\frac{12(k-3)\left(8 k^{2}+45 k+41\right)}{(k+5)^{3}(13 k+17)} F_{12} F_{21}\right](w) \\
& +\frac{1}{(z-w)^{3}}\left[-\frac{8 i\left(7 k^{2}+44 k+141\right)}{3(k+5)^{3}(13 k+17)} A_{-} F_{11} F_{12}+\frac{8 i\left(7 k^{2}+44 k+141\right)}{3(k+5)^{3}(13 k+17)} A_{+} F_{21} F_{22}\right. \\
& -\frac{4 i\left(25 k^{2}+158 k-27\right)}{3(k+5)^{3}(13 k+17)} B_{-} F_{12} F_{22}+\frac{4 i\left(25 k^{2}+158 k-27\right)}{3(k+5)^{3}(13 k+17)} B_{+} F_{11} F_{21} \\
& -\frac{(k-3)(89 k+205)}{3(k+5)^{2}(13 k+17)} F_{11} G_{22}-\frac{4}{(k+5)^{2}} \partial\left(F_{11} F_{22}\right) \\
& +\frac{(3 k+11)}{(k+5)^{2}} F_{12} G_{21}+\frac{4(k-3)(11 k+103)}{3(k+5)^{3}(13 k+17)} \partial\left(F_{12} F_{21}\right) \\
& -\frac{(3 k+11)}{(k+5)^{2}} F_{21} G_{12}+\frac{(k-3)(89 k+205)}{3(k+5)^{2}(13 k+17)} F_{22} G_{11} \\
& -\frac{4(k-3)(11 k+103)}{3(k+5)^{3}(13 k+17)} U F_{11} F_{22}+\frac{4}{(k+5)^{2}} U F_{12} F_{21}-\frac{24 i}{(k+5)^{2}} U A_{3} \\
& \left.-\frac{8 i k}{(k+5)^{2}} U B_{3}\right](w) \\
& +\frac{1}{(z-w)^{2}}\left[-3 \mathbf{P}^{(\mathbf{3})}+3 \mathbf{W}^{(\mathbf{3})}-\frac{8(\mathbf{k}-\mathbf{3})}{(13 k+17)} \mathbf{T}^{(\mathbf{1})} \mathbf{T}^{(\mathbf{2})}\right. \\
& -\frac{2 i}{(k+5)^{2}} A_{3} A_{3} A_{3}++\frac{6 i}{(k+5)^{2}} A_{3} A_{3} B_{3}+\frac{2}{(k+5)^{2}} \partial A_{3} A_{3} \\
& \text { - } \frac{6 i}{(k+5)^{2}} A_{3} B_{3} B_{3}+\frac{3(3 k+11)}{(k+5)^{2}} \partial A_{3} B_{3}-\frac{3(3 k+11)}{(k+5)^{2}} A_{3} \partial B_{3} \\
& -\frac{6 i}{(k+5)^{2}} A_{3} B_{+} B_{-}-\frac{8 i}{(k+5)^{2}} A_{3} F_{11} G_{22}+\frac{2 i(5 k+29)}{(k+5)^{3}} A_{3} \partial F_{11} F_{22} \\
& -\frac{10 i(k+1)}{(k+5)^{3}} A_{3} F_{11} \partial F_{22}+\frac{4 i}{(k+5)^{2}} A_{3} F_{22} G_{11}-\frac{3 i}{(k+5)^{2}} A_{-} F_{11} G_{12} \\
& -\frac{i\left(431 k^{2}+3082 k+3675\right)}{3(k+5)^{3}(13 k+17)} \partial A_{-} F_{11} F_{12}+\frac{i\left(739 k^{2}+4610 k+4383\right)}{3(k+5)^{3}(13 k+17)} A_{-} \partial F_{11} F_{12} \\
& +\frac{i\left(115 k^{2}+1298 k+1119\right)}{3(k+5)^{3}(13 k+17)} A_{-} F_{11} \partial F_{12}+\frac{i}{(k+5)^{2}} A_{-} F_{12} G_{11}-\frac{2 i}{(k+5)^{2}} A_{+} A_{-} A_{3} \\
& +\frac{6 i}{(k+5)^{2}} A_{+} A_{-} B_{3}-\frac{14}{(k+5)^{2}} \partial A_{+} A_{-}+\frac{14}{(k+5)^{2}} A_{+} \partial A_{-} \\
& -\frac{5 i}{(k+5)^{2}} A_{+} F_{21} G_{22}-\frac{2 i\left(77 k^{2}+772 k+687\right)}{3(k+5)^{3}(13 k+17)} \partial A_{+} F_{21} F_{22} \\
& +\frac{4 i\left(59 k^{2}+463 k+600\right)}{3(k+5)^{3}(13 k+17)} A_{+} \partial F_{21} F_{22}+\frac{4 i\left(20 k^{2}+373 k+549\right)}{3(k+5)^{3}(13 k+17)} A_{+} F_{21} \partial F_{22} \\
& +\frac{3 i}{(k+5)^{2}} A_{+} F_{22} G_{21}+\frac{2 i}{(k+5)^{2}} B_{3} B_{3} B_{3}-\frac{2}{(k+5)^{2}} \partial B_{3} B_{3}
\end{aligned}
$$




$$
\begin{aligned}
& +\frac{i\left(8 k^{3}+63 k^{2}+203 k-136\right)}{(k+5)^{2}(13 k+17)} \partial^{2} B_{3}+\frac{4 i}{(k+5)^{2}} B_{3} F_{11} G_{22} \\
& -\frac{6 i(k-3)}{(k+5)^{3}} \partial B_{3} F_{11} F_{22}-\frac{40 i}{(k+5)^{3}} B_{3} \partial F_{11} F_{22}+\frac{4 i(3 k+13)}{(k+5)^{3}} B_{3} F_{11} \partial F_{22} \\
& -\frac{8 i}{(k+5)^{2}} B_{3} F_{22} G_{11}+\frac{i}{(k+5)^{2}} B_{-} F_{12} G_{22}-\frac{4 i\left(136 k^{2}+767 k+675\right)}{3(k+5)^{3}(13 k+17)} \partial B_{-} F_{12} F_{22} \\
& +\frac{4 i\left(59 k^{2}+229 k+294\right)}{3(k+5)^{3}(13 k+17)} B_{-} \partial F_{12} F_{22}+\frac{4 i\left(176 k^{2}+1123 k+1263\right)}{3(k+5)^{3}(13 k+17)} B_{-} F_{12} \partial F_{22} \\
& -\frac{3 i}{(k+5)^{2}} B_{-} F_{22} G_{12}+\frac{2 i}{(k+5)^{2}} B_{+} B_{-} B_{3}+\frac{(3 k+5)}{(k+5)^{2}} \partial B_{+} B_{-} \\
& -\frac{(3 k+5)}{(k+5)^{2}} B_{+} \partial B_{-}+\frac{3 i}{(k+5)^{2}} B_{+} F_{11} G_{21}-\frac{i\left(275 k^{2}+1162 k+1431\right)}{3(k+5)^{3}(13 k+17)} \partial B_{+} F_{11} F_{21} \\
& +\frac{i\left(349 k^{2}+1214 k+609\right)}{3(k+5)^{3}(13 k+17)} B_{+} \partial F_{11} F_{21}+\frac{i\left(349 k^{2}+1838 k+1425\right)}{3(k+5)^{3}(13 k+17)} B_{+} F_{11} \partial F_{21} \\
& -\frac{5 i}{(k+5)^{2}} B_{+} F_{21} G_{11}-\frac{\left(8 k^{3}+521 k^{2}+1810 k+1633\right)}{(k+5)^{3}(13 k+17)} \partial^{2} F_{11} F_{22} \\
& -\frac{2\left(8 k^{3}-649 k^{2}-1826 k-1121\right)}{(k+5)^{3}(13 k+17)} \partial F_{11} \partial F_{22} \\
& -\frac{\left(8 k^{3}+521 k^{2}+1810 k+1633\right)}{(k+5)^{3}(13 k+17)} F_{11} \partial^{2} F_{22}-\frac{\left(467 k^{2}+1009 k+330\right)}{3(k+5)^{2}(13 k+17)} \partial F_{11} G_{22} \\
& +\frac{\left(118 k^{2}+341 k+435\right)}{3(k+5)^{2}(13 k+17)} F_{11} \partial G_{22}+\frac{8(k-3)\left(3 k^{2}+40 k+113\right)}{3(k+5)^{3}(13 k+17)} \partial^{2} F_{12} F_{21} \\
& +\frac{4(k-3)\left(12 k^{2}+121 k+401\right)}{3(k+5)^{3}(13 k+17)} \partial F_{12} \partial F_{21} \\
& +\frac{8(k-3)\left(3 k^{2}+40 k+113\right)}{3(k+5)^{3}(13 k+17)} F_{12} \partial^{2} F_{21}-\frac{3(3 k+13)}{2(k+5)^{2}} \partial F_{12} G_{21} \\
& +\frac{(5 k+23)}{2(k+5)^{2}} F_{12} \partial G_{21}-\frac{3(3 k+11)}{2(k+5)^{2}} \partial F_{21} G_{12}+\frac{(k+1)}{2(k+5)^{2}} F_{21} \partial G_{12} \\
& -\frac{7\left(17 k^{2}+460 k+627\right)}{6(k+5)^{2}(13 k+17)} \partial F_{22} G_{11}+\frac{\left(115 k^{2}+1064 k+813\right)}{6(k+5)^{2}(13 k+17)} F_{22} \partial G_{11} \\
& -\frac{3}{(k+5)} G_{11} G_{22}+\frac{2 i(44 k+109)}{(k+5)(13 k+17)} T A_{3}-\frac{2 i\left(8 k^{2}+62 k-17\right)}{(k+5)(13 k+17)} T B_{3} \\
& +\frac{3}{(k+5)} \partial T-\frac{6(4 k+17)}{(k+5)(13 k+17)} T U+\frac{16\left(k^{2}+13 k+20\right)}{(k+5)^{2}(13 k+17)} T F_{11} F_{22} \\
& -\frac{4(k-3)(4 k+21)}{(k+5)^{2}(13 k+17)} T F_{12} F_{21}-\frac{6}{(k+5)^{2}} U A_{3} A_{3}+\frac{12}{(k+5)^{2}} U A_{3} B_{3} \\
& +\frac{9 i}{(k+5)^{2}} \partial U A_{3}-\frac{27 i}{(k+5)^{2}} U \partial A_{3}-\frac{6}{(k+5)^{2}} U A_{+} A_{-}-\frac{6}{(k+5)^{2}} U B_{3} B_{3}
\end{aligned}
$$




$$
\begin{aligned}
& +\frac{i(k+6)}{(k+5)^{2}} \partial U B_{3}-\frac{i(5 k+12)}{(k+5)^{2}} U \partial B_{3}-\frac{6}{(k+5)^{2}} U B_{+} B_{-} \\
& +\frac{3(4 k+17)}{(k+5)(13 k+17)} \partial^{2} U-\frac{4}{(k+5)^{2}} U F_{11} G_{22}+\frac{2\left(41 k^{2}+310 k+717\right)}{3(k+5)^{3}(13 k+17)} \partial U F_{11} F_{22} \\
& -\frac{64\left(17 k^{2}+91 k+78\right)}{3(k+5)^{3}(13 k+17)} U \partial F_{11} F_{22}+\frac{16\left(49 k^{2}+374 k+453\right)}{3(k+5)^{3}(13 k+17)} U F_{11} \partial F_{22} \\
& +\frac{5}{(k+5)^{2}} \partial U F_{12} F_{21}-\frac{21}{(k+5)^{2}} U \partial F_{12} F_{21}+\frac{15}{(k+5)^{2}} U F_{12} \partial F_{21} \\
& +\frac{4}{(k+5)^{2}} U F_{22} G_{11}-\frac{2 i}{(k+5)^{2}} U U A_{3}+\frac{2 i}{(k+5)^{2}} U U B_{3} \\
& -\frac{6}{(k+5)^{2}} U U U+\frac{24}{(k+5)^{3}} F_{22} G_{11} F_{22} F_{11}-\frac{i\left(44 k^{2}+173 k+341\right)}{(k+5)^{2}(13 k+17)} \partial^{2} A_{3} \\
& -\frac{15 i}{(k+5)^{2}} \partial A_{3} F_{12} F_{21}+\frac{15 i}{(k+5)^{2}} A_{3} \partial\left(F_{12} F_{21}\right) \\
& \left.+\frac{15 i}{(k+5)^{2}} \partial B_{3} F_{12} F_{21}-\frac{15 i}{(k+5)^{2}} B_{3} \partial\left(F_{12} F_{21}\right)\right](w) \\
& +\frac{1}{(z-w)}[+\cdots](w)+\cdots
\end{aligned}
$$

There is no higher spin-1 current in the fourth order pole in the above OPE. Even there is no higher spin-2 current in the third order pole which was present in the nonlinear version. One can also remove the term between the higher spin currents by adding the $\operatorname{TT}^{(\mathbf{1})}(w)$ into the left hand side in the higher spin-3 current because the $\operatorname{OPE} \mathbf{T}^{(\mathbf{2})}(z) T \mathbf{T}^{(\mathbf{1})}(w)$ contributes to the above $\mathbf{T}^{(\mathbf{1})} \mathbf{T}^{(\mathbf{2})}(w)$ term where the factor $(k-3)$ exists.

\section{Appendix F.2 The OPEs containing the second or third $\mathcal{N}=2$ multiplets}

From the explicit result in (3.2) for the higher spin- $\frac{3}{2}$ current one can obtain the following OPEs

$$
\begin{aligned}
\left(\begin{array}{c}
\mathbf{U}^{\left(\frac{3}{2}\right)} \\
\mathbf{V}^{\left(\frac{3}{2}\right)}
\end{array}\right)(z)\left(\begin{array}{c}
\mathbf{U}^{\left(\frac{3}{2}\right)} \\
\mathbf{V}^{\left(\frac{3}{2}\right)}
\end{array}\right)(w) & =\frac{1}{(z-w)}\left[\frac{1}{(k+5)} A_{ \pm} B_{\mp} \pm \frac{2 i}{(k+5)^{2}} A_{ \pm}\left(\begin{array}{c}
F_{11} F_{21} \\
F_{22} F_{12}
\end{array}\right)\right. \\
& \left.\mp \frac{2 i}{(k+5)^{2}} B_{\mp}\left(\begin{array}{c}
F_{11} F_{12} \\
F_{22} F_{21}
\end{array}\right)-\frac{2}{(k+5)^{2}}\left(\begin{array}{c}
F_{11} \partial F_{11} \\
F_{22} \partial F_{22}
\end{array}\right)\right](w)+\cdots
\end{aligned}
$$

These are new OPEs and the corresponding OPEs in the nonlinear version are trivial.

Furthermore the result of (3.5) implies the following OPEs

$$
\left(\begin{array}{c}
\mathbf{U}^{\left(\frac{3}{2}\right)} \\
\mathbf{V}^{\left(\frac{3}{2}\right)}
\end{array}\right)(z)\left(\begin{array}{c}
\mathbf{U}_{+}^{(2)} \\
\mathbf{V}_{-}^{(2)}
\end{array}\right)(w)=\frac{1}{(z-w)^{2}}\left[ \pm \frac{2 i k}{(k+5)^{2}}\left(\begin{array}{c}
F_{11} \\
F_{22}
\end{array}\right) B_{\mp}\right](w)
$$




$$
\begin{aligned}
& +\frac{1}{(z-w)}\left[-\frac{1}{(k+5)^{2}} A_{3} B_{\mp}\left(\begin{array}{c}
F_{11} \\
F_{22}
\end{array}\right)\right. \\
& -\frac{1}{(k+5)^{2}} A_{ \pm} B_{\mp}\left(\begin{array}{c}
F_{21} \\
F_{12}
\end{array}\right) \mp \frac{i}{2(k+5)} B_{\mp}\left(\begin{array}{c}
G_{11} \\
G_{22}
\end{array}\right) \\
& +\frac{1}{(k+5)^{2}} B_{\mp} B_{3}\left(\begin{array}{c}
F_{11} \\
F_{22}
\end{array}\right)+\frac{1}{(k+5)^{2}} B_{\mp} B_{\mp}\left(\begin{array}{c}
F_{12} \\
F_{21}
\end{array}\right) \\
& \mp \frac{3 i}{(k+5)^{2}} B_{\mp} \partial\left(\begin{array}{c}
F_{11} \\
F_{22}
\end{array}\right)+\frac{1}{(k+5)^{2}}\left(\begin{array}{c}
F_{11} F_{21} G_{11} \\
F_{22} F_{12} G_{22}
\end{array}\right) \\
& \mp \frac{i}{(k+5)^{2}} U B_{\mp}\left(\begin{array}{c}
F_{11} \\
F_{22}
\end{array}\right) \pm \frac{i k}{(k+5)^{2}}\left(\begin{array}{c}
F_{11} \\
F_{22}
\end{array}\right) \partial B_{\mp} \\
& \left.+\frac{2(k+1)}{(k+5)^{3}}\left(\begin{array}{c}
F_{11} F_{21} \partial F_{11} \\
F_{22} F_{12} \partial F_{22}
\end{array}\right)\right](w)+\cdots .
\end{aligned}
$$

These are also new OPEs and the corresponding OPEs in the nonlinear version are trivial.

Similarly, the following OPEs can be obtained from the previous result in (3.4)

$$
\begin{aligned}
\left(\begin{array}{c}
\mathbf{U}^{\left(\frac{3}{2}\right)} \\
\mathbf{V}^{\left(\frac{3}{2}\right)}
\end{array}\right)(z)\left(\begin{array}{c}
\mathbf{U}_{-(\mathbf{2})}^{(2)} \\
\mathbf{V}_{+}^{(2)}
\end{array}\right)(w) & =\frac{1}{(z-w)^{2}}\left[ \pm \frac{6 i}{(k+5)^{2}}\left(\begin{array}{c}
F_{11} \\
F_{22}
\end{array}\right) A_{ \pm}\right](w) \\
& +\frac{1}{(z-w)}\left[ \pm \frac{i}{2(k+5)} A_{ \pm}\left(\begin{array}{c}
G_{11} \\
G_{22}
\end{array}\right)\right. \\
& -\frac{1}{(k+5)^{2}} A_{ \pm} A_{3}\left(\begin{array}{c}
F_{11} \\
F_{22}
\end{array}\right)-\frac{1}{(k+5)^{2}} A_{ \pm} A_{ \pm}\left(\begin{array}{c}
F_{21} \\
F_{12}
\end{array}\right) \\
& +\frac{1}{(k+5)^{2}} A_{ \pm} B_{3}\left(\begin{array}{c}
F_{11} \\
F_{22}
\end{array}\right)+\frac{1}{(k+5)^{2}} A_{ \pm} B_{\mp}\left(\begin{array}{c}
F_{12} \\
F_{21}
\end{array}\right) \\
& +\frac{1}{(k+5)^{2}}\left(\begin{array}{c}
F_{11} F_{12} G_{11} \\
F_{22} F_{21} G_{22}
\end{array}\right) \pm \frac{i}{(k+5)^{2}} U A_{ \pm}\left(\begin{array}{c}
F_{11} \\
F_{22}
\end{array}\right) \\
& \mp \frac{3 i}{(k+5)^{2}} A_{ \pm} \partial\left(\begin{array}{c}
F_{11} \\
F_{22}
\end{array}\right) \pm \frac{3 i}{(k+5)^{2}}\left(\begin{array}{l}
F_{11} \\
F_{22}
\end{array}\right) \partial A_{ \pm} \\
& \left.-\frac{8}{(k+5)^{3}}\left(\begin{array}{c}
F_{11} F_{12} \partial F_{11} \\
F_{22} F_{21} \partial F_{22}
\end{array}\right)\right](w)+\cdots
\end{aligned}
$$

These are new OPEs and the corresponding OPEs in the nonlinear version are trivial.

From the information of the higher spin- $\frac{5}{2}$ currents in (3.7), the following OPEs can be obtained

$$
\begin{aligned}
\left(\begin{array}{c}
\mathbf{U}^{\left(\frac{3}{2}\right)} \\
\mathbf{V}^{\left(\frac{3}{2}\right)}
\end{array}\right)(z)\left(\begin{array}{c}
\mathbf{U}^{\left(\frac{5}{2}\right)} \\
\mathbf{V}^{\left(\frac{5}{2}\right)}
\end{array}\right)(w) & =\frac{1}{(z-w)^{2}}\left[ \pm \frac{2(k-3)}{3(k+5)^{2}} A_{ \pm} B_{\mp}-\frac{2 i(k-3)}{3(k+5)^{3}} A_{ \pm}\left(\begin{array}{c}
F_{11} F_{21} \\
F_{22} F_{12}
\end{array}\right)\right. \\
& +\frac{2 i(k-3)}{3(k+5)^{3}} B_{\mp}\left(\begin{array}{c}
F_{11} F_{12} \\
F_{22} F_{21}
\end{array}\right) \mp \frac{(k+3)}{(k+5)^{2}}\left(\begin{array}{c}
F_{11} G_{11} \\
F_{22} G_{22}
\end{array}\right) \\
& \left. \pm \frac{8(k-3)}{3(k+5)^{3}}\left(\begin{array}{c}
F_{11} \partial F_{11} \\
F_{22} \partial F_{22}
\end{array}\right)\right](w)
\end{aligned}
$$




$$
\begin{aligned}
& +\frac{1}{(z-w)}\left[\mp \frac{(4 k+21)}{3(k+5)^{2}} \partial A_{ \pm} B_{\mp} \pm \frac{(5 k+18)}{3(k+5)^{2}} A_{ \pm} \partial B_{\mp}\right. \\
& +\frac{i}{(k+5)^{2}} A_{ \pm}\left(\begin{array}{c}
F_{11} G_{21} \\
F_{22} G_{12}
\end{array}\right)-\frac{8 i(k+3)}{3(k+5)^{3}} \partial A_{ \pm}\left(\begin{array}{c}
F_{11} F_{21} \\
F_{22} F_{12}
\end{array}\right) \\
& +\frac{4 i(k+3)}{3(k+5)^{3}} A_{ \pm}\left(\begin{array}{c}
\partial F_{11} F_{21} \\
\partial F_{22} F_{12}
\end{array}\right)+\frac{2 i(5 k+21)}{3(k+5)^{3}} A_{ \pm}\left(\begin{array}{c}
F_{11} \partial F_{21} \\
F_{22} \partial F_{12}
\end{array}\right) \\
& -\frac{i}{(k+5)^{2}} A_{ \pm}\left(\begin{array}{c}
F_{21} G_{11} \\
F_{12} G_{22}
\end{array}\right)-\frac{i}{(k+5)^{2}} B_{\mp}\left(\begin{array}{c}
F_{11} G_{12} \\
F_{22} G_{21}
\end{array}\right) \\
& -\frac{4 i(k+9)}{3(k+5)^{3}} \partial B_{\mp}\left(\begin{array}{c}
F_{11} F_{12} \\
F_{22} F_{21}
\end{array}\right)+\frac{2 i(k+9)}{3(k+5)^{3}} B_{\mp}\left(\begin{array}{l}
\partial F_{11} F_{12} \\
\partial F_{22} F_{21}
\end{array}\right) \\
& +\frac{8 i(k+6)}{3(k+5)^{3}} B_{\mp}\left(\begin{array}{c}
F_{11} \partial F_{12} \\
F_{22} \partial F_{21}
\end{array}\right)+\frac{i}{(k+5)^{2}} B_{\mp}\left(\begin{array}{c}
F_{12} G_{11} \\
F_{21} G_{22}
\end{array}\right) \\
& \mp \frac{(k+3)}{(k+5)^{2}}\left(\begin{array}{l}
\partial F_{11} G_{11} \\
\partial F_{22} G_{22}
\end{array}\right) \mp \frac{2}{(k+5)^{2}} U A_{ \pm} B_{\mp} \\
& +\frac{2 i}{(k+5)^{2}}\left(\begin{array}{l}
F_{11} \partial F_{11} \\
F_{22} \partial F_{22}
\end{array}\right) A_{3}+\frac{2 i}{(k+5)^{2}}\left(\begin{array}{c}
F_{11} \partial F_{11} \\
F_{22} \partial F_{22}
\end{array}\right) B_{3} \\
& \left. \pm \frac{2}{(k+5)^{2}}\left(\begin{array}{l}
F_{11} \partial F_{11} \\
F_{22} \partial F_{22}
\end{array}\right) U \pm \frac{2(k-3)}{3(k+5)^{3}}\left(\begin{array}{c}
F_{11} \partial^{2} F_{11} \\
F_{22} \partial^{2} F_{22}
\end{array}\right)\right](w)+\cdots
\end{aligned}
$$

As before, one has the above combined OPEs due to the symmetry of structure constants of two OPEs and there are no nonlinear terms in the spirit of footnote 5 .

Now one can construct the following OPE

$$
\begin{aligned}
\mathbf{U}^{\left(\frac{3}{2}\right)}(z) \mathbf{V}^{\left(\frac{3}{2}\right)}(w) & =\frac{1}{(z-w)^{3}}\left[-\frac{(7 k+4)}{(k+5)}\right] \\
& +\frac{1}{(z-w)^{2}}\left[-\frac{7 i}{(k+5)} A_{3}+\frac{i(2 k+1)}{(k+5)} B_{3}-\mathbf{T}^{(\mathbf{1})}\right. \\
& \left.-\frac{(k+3)}{(k+5)^{2}} F_{11} F_{22}+\frac{(k-3)}{(k+5)^{2}} F_{12} F_{21}\right](w) \\
& +\frac{1}{(z-w)}\left[-\frac{1}{2} \partial \mathbf{T}^{(\mathbf{1})}-\mathbf{W}^{(\mathbf{2})}-T\right. \\
& -\frac{1}{2(k+5)} A_{3} A_{3}-\frac{1}{(k+5)} A_{3} B_{3}-\frac{4 i}{(k+5)} \partial A_{3}+\frac{2 i}{(k+5)^{2}} A_{3} F_{12} F_{21} \\
& +\frac{i}{(k+5)^{2}} A_{-} F_{11} F_{12}-\frac{1}{2(k+5)} A_{+} A_{-}+\frac{i}{(k+5)^{2}} A_{+} F_{21} F_{22} \\
& -\frac{1}{2(k+5)} B_{3} B_{3}+\frac{i k}{(k+5)} \partial B_{3}+\frac{2 i}{(k+5)^{2}} B_{3} F_{12} F_{21} \\
& -\frac{i}{(k+5)^{2}} B_{-} F_{12} F_{22}-\frac{1}{2(k+5)} B_{+} B_{-}-\frac{i}{(k+5)^{2}} B_{+} F_{11} F_{21}
\end{aligned}
$$




$$
\begin{aligned}
& -\frac{1}{(k+5)} \partial F_{11} F_{22}+\frac{2}{(k+5)^{2}} F_{11} \partial F_{22}-\frac{6}{(k+5)^{2}} \partial F_{12} F_{21} \\
& \left.+\frac{(k+3)}{(k+5)^{2}} F_{12} \partial F_{21}-\frac{1}{2(k+5)} U U\right](w)+\cdots
\end{aligned}
$$

Compared to the nonlinear version of this OPE, the above OPE is rather complicated.

From the results in section 3, one obtains the following OPE

$$
\begin{aligned}
& \mathbf{U}^{\left(\frac{\mathbf{3}}{\mathbf{2}}\right)}(z) \mathbf{V}_{+}^{(\mathbf{2})}(w)=\frac{1}{(z-w)^{3}}\left[-\frac{6 k}{(k+5)^{2}} F_{21}\right](w) \\
& +\frac{1}{(z-w)^{2}}\left[\frac{2(k+3)}{(k+5)} \mathbf{T}_{+}^{\left(\frac{3}{2}\right)}+\frac{1}{(k+5)} G_{21}-\frac{2(k+2)}{(k+5)^{2}} U F_{21}\right. \\
& +\frac{2}{(k+5)^{2}} F_{21} F_{11} F_{22}+\frac{2 i(k-1)}{(k+5)^{2}} F_{21} A_{3}-\frac{2 i(k+2)}{(k+5)^{2}} F_{11} A_{-} \\
& \left.+\frac{4 i}{(k+5)^{2}} F_{22} B_{-}-\frac{4 i}{(k+5)^{2}} F_{21} B_{3}\right](w) \\
& +\frac{1}{(z-w)}\left[\frac{1}{2} \mathbf{P}_{+}^{\left(\frac{5}{2}\right)}+\mathbf{W}_{+}^{\left(\frac{5}{2}\right)}+\frac{2(k+3)}{3(k+5)} \partial \mathbf{T}_{+}^{\left(\frac{3}{2}\right)}\right. \\
& -\frac{i}{2(k+5)} A_{3} G_{21}-\frac{2}{(k+5)^{2}} A_{3} A_{3} F_{21}-\frac{2}{(k+5)^{2}} A_{3} B_{3} F_{21} \\
& +\frac{2}{(k+5)^{2}} A_{3} B_{-} F_{22}+\frac{2 i(k-3)}{3(k+5)^{2}} \partial A_{3} F_{21}+\frac{2 i(k+9)}{3(k+5)^{2}} A_{3} \partial F_{21} \\
& +\frac{1}{(k+5)^{2}} A_{-} A_{3} F_{11}+\frac{1}{(k+5)^{2}} A_{-} B_{3} F_{11}+\frac{1}{(k+5)^{2}} A_{-} B_{-} F_{12} \\
& -\frac{i(2 k+3)}{3(k+5)^{2}} \partial A_{-} F_{11}-\frac{i(2 k+9)}{3(k+5)^{2}} A_{-} \partial F_{11}-\frac{1}{(k+5)^{2}} A_{+} A_{-} F_{21} \\
& -\frac{i}{2(k+5)} B_{3} G_{21}+\frac{i(k-3)}{3(k+5)^{2}} \partial B_{3} F_{21}-\frac{2 i k}{3(k+5)^{2}} B_{3} \partial F_{21} \\
& +\frac{i}{2(k+5)} B_{-} G_{22}-\frac{i(k-3)}{3(k+5)^{2}} \partial B_{-} \partial F_{22}+\frac{2 i k}{3(k+5)^{2}} B_{-} \partial F_{22} \\
& +\frac{4(k+3)}{3(k+5)^{3}} \partial F_{11} F_{21} F_{22}-\frac{2(k+9)}{3(k+5)^{3}} F_{11} \partial F_{21} F_{22} \\
& -\frac{8(k+3)}{3(k+5)^{3}} F_{11} F_{21} \partial F_{22}-\frac{1}{(k+5)^{2}} F_{11} F_{22} G_{21} \\
& -\frac{1}{(k+5)^{2}} F_{12} F_{21} G_{21}+\frac{3(k+3)}{2(k+5)^{2}} \partial^{2} F_{21}+\frac{1}{(k+5)^{2}} F_{21} F_{22} G_{11} \\
& -\frac{1}{(k+5)} T F_{21}-\frac{1}{2(k+5)} U G_{21}-\frac{i}{(k+5)^{2}} U A_{-} F_{11} \\
& -\frac{2 i}{(k+5)^{2}} U B_{3} F_{21}+\frac{2 i}{(k+5)^{2}} U B_{-} F_{22}-\frac{2(k+3)}{3(k+5)^{2}} \partial U F_{21}
\end{aligned}
$$




$$
\left.-\frac{2(k+3)}{3(k+5)^{2}} U \partial F_{21}-\frac{2}{(k+5)^{2}} U U F_{21}-\frac{2(k+1)}{(k+5)^{3}} F_{21} \partial F_{21} F_{12}\right](w)+\cdots
$$

Note the spin- $\frac{3}{2}$ current dependent terms in the above OPE which were not present in the corresponding OPE in the nonlinear version.

Similarly one has the following OPE

$$
\begin{aligned}
& \mathbf{U}^{\left(\frac{3}{2}\right)}(z) \mathbf{V}_{-}^{(2)}(w)=\frac{1}{(z-w)^{3}}\left[\frac{6 k}{(k+5)^{2}} F_{12}\right](w) \\
& +\frac{1}{(z-w)^{2}}\left[\frac{(k+9)}{(k+5)} \mathbf{T}_{-}^{\left(\frac{3}{2}\right)}-\frac{(k+7)}{(k+5)^{2}} U F_{12}-\frac{2}{(k+5)^{2}} F_{12} F_{11} F_{22}\right. \\
& -\frac{i(k+1)}{(k+5)^{2}} F_{12} A_{3}+\frac{i(k+1)}{(k+5)^{2}} F_{22} A_{+}-\frac{i(k+7)}{(k+5)^{2}} F_{11} B_{+} \\
& \left.-\frac{i(k-7)}{(k+5)^{2}} F_{12} B_{3}-\frac{(k+8)}{(k+5)} G_{12}\right](w) \\
& +\frac{1}{(z-w)}\left[\frac{1}{2} \mathbf{P}_{-}^{\left(\frac{5}{2}\right)}+\frac{(k+9)}{3(k+5)} \partial \mathbf{T}_{-}^{\left(\frac{3}{2}\right)}+\frac{i}{2(k+5)} A_{3} G_{12}\right. \\
& +\frac{2}{(k+5)^{2}} A_{3} B_{3} F_{12}-\frac{1}{(k+5)^{2}} A_{3} B_{+} F_{11}-\frac{i(k-3)}{3(k+5)^{2}} \partial A_{3} F_{12} \\
& -\frac{i(k+3)}{3(k+5)^{2}} A_{3} \partial F_{12}-\frac{i}{2(k+5)} A_{+} G_{22}-\frac{2}{(k+5)^{2}} A_{+} B_{3} F_{22} \\
& -\frac{1}{(k+5)^{2}} A_{+} B_{+} F_{21}+\frac{i(k-3)}{3(k+5)^{2}} \partial A_{+} F_{22}+\frac{i(k+3)}{3(k+5)^{2}} A_{+} \partial F_{22} \\
& +\frac{i}{2(k+5)} B_{3} G_{12}+\frac{2}{(k+5)^{2}} B_{3} B_{3} F_{12}-\frac{2 i(k-6)}{3(k+5)^{2}} \partial B_{3} F_{12} \\
& +\frac{i(k+21)}{3(k+5)^{2}} B_{3} \partial F_{12}-\frac{1}{(k+5)^{2}} B_{+} B_{3} F_{11}+\frac{1}{(k+5)^{2}} B_{+} B_{-} F_{12} \\
& -\frac{i(k+6)}{3(k+5)^{2}} \partial B_{+} F_{11}-\frac{i(k+12)}{3(k+5)^{2}} B_{+} \partial F_{11}-\frac{2(k+9)}{3(k+5)^{3}} \partial F_{11} F_{12} F_{22} \\
& +\frac{4(k+3)}{3(k+5)^{3}} F_{11} \partial F_{12} F_{22}+\frac{4(k+9)}{3(k+5)^{3}} F_{11} F_{12} \partial F_{22}-\frac{1}{(k+5)^{2}} F_{11} F_{22} G_{12} \\
& -\frac{(k+15)}{2(k+5)^{2}} \partial^{2} F_{12}-\frac{(k+9)}{3(k+5)} \partial G_{12}-\frac{(k+9)}{3(k+5)^{2}} \partial U F_{12} \\
& -\frac{(k+9)}{3(k+5)^{2}} U \partial F_{12}+\frac{1}{(k+5)^{2}} F_{12} F_{21} G_{12}+\frac{1}{(k+5)^{2}} F_{12} F_{22} G_{11} \\
& +\frac{1}{(k+5)} T F_{12}+\frac{1}{2(k+5)} U G_{12}+\frac{2 i}{(k+5)^{2}} U A_{3} F_{12} \\
& -\frac{2 i}{(k+5)^{2}} U A_{+} F_{22}+\frac{i}{(k+5)^{2}} U B_{+} F_{11}+\frac{2}{(k+5)^{2}} U U F_{12}
\end{aligned}
$$




$$
\left.+\frac{16 i}{(k+5)^{3}} F_{12} B_{3} F_{12} F_{21}\right](w)+\cdots .
$$

In this case also the nonlinear terms appeared in the corresponding OPE in the nonlinear version disappear.

One can also construct the following OPE from the previous results

$$
\begin{aligned}
& \mathbf{U}^{\left(\frac{3}{2}\right)}(z) \mathbf{V}^{\left(\frac{5}{2}\right)}(w)=\frac{1}{(z-w)^{3}}\left[\frac{2 i(5 k+18)}{(k+5)^{2}} A_{3}+\frac{2 i k(4 k+21)}{3(k+5)^{2}} B_{3}-\frac{4(k-3)}{3(k+5)} \mathbf{T}^{(\mathbf{1})}\right. \\
& \left.-\frac{6 k}{(k+5)^{2}} U-\frac{4(k-3)(2 k+9)}{3(k+5)^{3}} F_{11} F_{22}+\frac{4\left(2 k^{2}+9 k+27\right)}{3(k+5)^{3}} F_{12} F_{21}\right](w) \\
& +\frac{1}{(z-w)^{2}}\left[\frac{1}{2} \mathbf{P}^{(\mathbf{2})}-\frac{8(k+3)}{3(k+5)}\left(\mathbf{T}^{(\mathbf{2})}+\mathbf{W}^{(\mathbf{2})}\right)\right. \\
& +\frac{4(k-3)}{3(k+5)^{2}} A_{3} B_{3}-\frac{2 i(k-3)}{3(k+5)^{3}} A_{3} F_{11} F_{22}+\frac{2 i(k-3)}{3(k+5)^{3}} A_{3} F_{12} F_{21} \\
& +\frac{2 i(k+21)}{3(k+5)^{3}} A_{-} F_{11} F_{12}-\frac{2 i(k+21)}{3(k+5)^{3}} A_{+} F_{21} F_{22}+\frac{2 i(k-3)}{3(k+5)^{3}} B_{3} F_{11} F_{22} \\
& +\frac{2 i(k-3)}{3(k+5)^{3}} B_{3} F_{12} F_{21}+\frac{16 i(k+3)}{3(k+5)^{3}} B_{-} F_{12} F_{22}-\frac{16 i(k+3)}{3(k+5)^{3}} B_{+} F_{11} F_{21} \\
& +\frac{5(k+3)}{3(k+5)^{2}} F_{11} G_{22}+\frac{2(11 k+39)}{3(k+5)^{3}} \partial F_{11} F_{22}+\frac{2(7 k+51)}{3(k+5)^{3}} F_{11} \partial F_{22} \\
& -\frac{2(k+3)}{(k+5)^{2}} F_{12} G_{21}-\frac{2(3 k-1)}{(k+5)^{3}} \partial F_{12} F_{21}-\frac{2(5 k+9)}{3(k+5)^{3}} F_{12} \partial F_{21} \\
& +\frac{(k+3)}{(k+5)^{2}} F_{21} G_{12}-\frac{2(k+3)}{3(k+5)^{2}} F_{22} G_{11}-\frac{12 i}{(k+5)^{2}} U A_{3} \\
& \left.+\frac{2(7 k+3)}{3(k+5)^{3}} U F_{11} F_{22}-\frac{6}{(k+5)^{2}} U F_{12} F_{21}\right](w) \\
& +\frac{1}{(z-w)}\left[\frac{1}{2} \mathbf{S}^{(\mathbf{3})}+\frac{1}{8} \partial \mathbf{P}^{(\mathbf{2})}-\mathbf{W}^{(\mathbf{3})}-\frac{2(k+3)}{3(k+5)} \partial\left(\mathbf{T}^{(\mathbf{2})}+\mathbf{W}^{(\mathbf{2})}\right)\right. \\
& -\frac{2(\mathbf{k}-\mathbf{3})}{(13 k+17)}\left(T \mathbf{T}^{(\mathbf{1})}-\frac{1}{2} \partial^{2} \mathbf{T}^{(\mathbf{1})}\right) \\
& -\frac{2(4 k+21)}{3(k+5)^{2}} \partial A_{3} B_{3}+\frac{2(5 k+18)}{3(k+5)^{2}} A_{3} \partial B_{3}-\frac{i(19 k+87)}{6(k+5)^{3}} \partial A_{3} F_{11} F_{22} \\
& +\frac{i(17 k+93)}{6(k+5)^{3}} A_{3} \partial\left(F_{11} F_{22}\right)-\frac{2 i}{(k+5)^{2}} A_{3} F_{12} G_{21} \\
& +\frac{2 i}{(k+5)^{2}} A_{3} F_{21} G_{12}-\frac{i}{(k+5)^{2}} A_{-} F_{11} G_{12}-\frac{i(k+9)}{3(k+5)^{3}} \partial A_{-} F_{11} F_{12} \\
& -\frac{i(k-15)}{3(k+5)^{3}} A_{-} \partial F_{11} F_{12}+\frac{5 i(k+9)}{3(k+5)^{3}} A_{-} F_{11} \partial F_{12}+\frac{i}{(k+5)^{2}} A_{-} F_{12} G_{11}
\end{aligned}
$$




$$
\begin{aligned}
& -\frac{i}{(k+5)^{2}} A_{+} F_{21} G_{22}-\frac{2 i(k+15)}{3(k+5)^{3}} \partial A_{+} F_{21} F_{22}+\frac{4 i(k+6)}{3(k+5)^{3}} A_{+} \partial F_{21} F_{22} \\
& -\frac{2 i(k+3)}{3(k+5)^{3}} A_{+} F_{21} \partial F_{22}+\frac{i}{(k+5)^{2}} A_{+} F_{22} G_{21}-\frac{i k(4 k+21)}{2(k+5)(13 k+17)} \partial^{2} B_{3} \\
& -\frac{i(17 k+93)}{6(k+5)^{3}} \partial B_{3} F_{11} F_{22}+\frac{i(19 k+87)}{6(k+5)^{3}} B_{3} \partial\left(F_{11} F_{22}\right) \\
& -\frac{2 i}{(k+5)^{2}} B_{3} F_{12} G_{21}+\frac{i(k-51)}{6(k+5)^{3}} \partial B_{3} F_{12} F_{21}+\frac{i(k+45)}{6(k+5)^{3}} B_{3} \partial\left(F_{12} F_{21}\right) \\
& +\frac{2 i}{(k+5)^{2}} B_{3} F_{21} G_{12}+\frac{i}{(k+5)^{2}} B_{-} F_{12} G_{22}-\frac{i(k-3)}{6(k+5)^{3}} \partial B_{-} F_{12} F_{22} \\
& +\frac{i(23 k+75)}{6(k+5)^{3}} B_{-} \partial F_{12} F_{22}+\frac{i(11 k+15)}{6(k+5)^{3}} B_{-} F_{12} \partial F_{22}-\frac{i}{(k+5)^{2}} B_{-} F_{22} G_{12} \\
& +\frac{i}{(k+5)^{2}} B_{+} F_{11} G_{21}-\frac{i(17 k+45)}{6(k+5)^{3}} \partial B_{+} F_{11} F_{21}-\frac{i(5 k+33)}{6(k+5)^{3}} B_{+} \partial F_{11} F_{21} \\
& +\frac{i(7 k+27)}{6(k+5)^{3}} B_{+} F_{11} \partial F_{21}-\frac{i}{(k+5)^{2}} B_{+} F_{21} G_{11} \\
& +\frac{\left(12 k^{3}+221 k^{2}+622 k-147\right)}{6(k+5)^{3}(13 k+17)} \partial^{2} F_{11} F_{22}+\frac{\left(4 k^{2}+45 k-3\right)}{(k+5)^{2}(13 k+17)} \partial F_{11} \partial F_{22} \\
& +\frac{\left(12 k^{3}+169 k^{2}+710 k+57\right)}{6(k+5)^{3}(13 k+17)} F_{11} \partial^{2} F_{22}+\frac{(5 k+33)}{12(k+5)^{2}} \partial F_{11} G_{22} \\
& +\frac{(5 k+9)}{12(k+5)^{2}} F_{11} \partial G_{22}-\frac{\left(4 k^{3}+77 k^{2}+182 k+253\right)}{2(k+5)^{3}(13 k+17)} \partial^{2} F_{12} F_{21} \\
& -\frac{\left(12 k^{3}+205 k^{2}+590 k+861\right)}{3(k+5)^{3}(13 k+17)} \partial F_{12} \partial F_{21}-\frac{(5 k+9)}{4(k+5)^{2}} \partial F_{12} G_{21} \\
& -\frac{\left(12 k^{3}+179 k^{2}+634 k+963\right)}{6(k+5)^{3}(13 k+17)} F_{12} \partial^{2} F_{21}-\frac{1}{4(k+5)} F_{12} \partial G_{21} \\
& -\frac{k}{2(k+5)^{2}} \partial F_{21} G_{12}+\frac{(k+2)}{2(k+5)^{2}} F_{21} \partial G_{12}-\frac{(k-6)}{6(k+5)^{2}} \partial F_{22} G_{11} \\
& -\frac{(k+6)}{6(k+5)^{2}} F_{22} \partial G_{11}+\frac{3 i(5 k+18)}{(k+5)(13 k+17)} T A_{3}+\frac{i k(4 k+21)}{(k+5)(13 k+17)} T B_{3} \\
& -\frac{17(k+2)}{(k+5)(13 k+17)} T U-\frac{2(k-3)(2 k+9)}{(k+5)^{2}(13 k+17)} T F_{11} F_{22} \\
& +\frac{2\left(2 k^{2}+9 k+27\right)}{(k+5)^{2}(13 k+17)} T F_{12} F_{21}-\frac{2}{(k+5)^{2}} U A_{3} A_{3}-\frac{4}{(k+5)^{2}} U A_{3} B_{3} \\
& -\frac{3 i}{(k+5)^{2}} \partial U A_{3}-\frac{5 i}{(k+5)^{2}} U \partial A_{3}-\frac{2}{(k+5)^{2}} U A_{+} A_{-} \\
& -\frac{2}{(k+5)^{2}} U B_{3} B_{3}-\frac{2 i}{(k+5)^{2}} U \partial B_{3}-\frac{2}{(k+5)^{2}} U B_{+} B_{-}
\end{aligned}
$$




$$
\begin{aligned}
& +\frac{17(k+2)}{2(k+5)(13 k+17)} \partial^{2} U+\frac{(7 k+3)}{6(k+5)^{3}} \partial U F_{11} F_{22}-\frac{(5 k+57)}{6(k+5)^{3}} U \partial F_{11} F_{22} \\
& +\frac{(19 k+63)}{6(k+5)^{3}} U F_{11} \partial F_{22}-\frac{3}{2(k+5)^{2}} \partial U F_{12} F_{21}-\frac{11}{2(k+5)^{2}} U \partial F_{12} F_{21} \\
& +\frac{5}{2(k+5)^{2}} U F_{12} \partial F_{21}-\frac{2}{(k+5)^{2}} U U U-\frac{3 i(5 k+18)}{2(k+5)(13 k+17)} \partial^{2} A_{3} \\
& \left.+\frac{i(13 k+9)}{6(k+5)^{3}} \partial A_{3} F_{12} F_{21}-\frac{i(11 k+15)}{6(k+5)^{3}} A_{3} \partial\left(F_{12} F_{21}\right)\right](w)+\cdots
\end{aligned}
$$

Note that there exists the nonlinear term containing the higher spin-1 current in the first order pole with $(k-3)$ factor.

With the help of (3.6), one obtains

$$
\begin{aligned}
& \left(\begin{array}{c}
\mathbf{U}^{\left(\frac{3}{2}\right)} \\
\mathbf{V}^{\left(\frac{3}{2}\right)}
\end{array}\right)(z) \mathbf{W}^{(\mathbf{2})}(w)=\frac{1}{(z-w)^{2}}\left[\frac{3}{2}\left(\begin{array}{c}
\mathbf{U}^{\left(\frac{3}{2}\right)} \\
\mathbf{V}^{\left(\frac{3}{2}\right)}
\end{array}\right) \pm \frac{1}{(k+5)}\left(\begin{array}{c}
G_{11} \\
G_{22}
\end{array}\right)\right. \\
& \mp \frac{(3 k+11)}{2(k+5)^{2}} U\left(\begin{array}{c}
F_{11} \\
F_{22}
\end{array}\right)-\frac{2}{(k+5)^{2}} F_{12} F_{21}\left(\begin{array}{c}
F_{11} \\
F_{22}
\end{array}\right) \\
& -\frac{i(3 k+11)}{2(k+5)^{2}}\left(\begin{array}{c}
F_{11} \\
F_{22}
\end{array}\right) A_{3}-\frac{i(3 k-1)}{2(k+5)^{2}}\left(\begin{array}{c}
F_{21} \\
F_{12}
\end{array}\right) A_{ \pm} \\
& \left.+\frac{i(k-11)}{2(k+5)^{2}}\left(\begin{array}{c}
F_{12} \\
F_{21}
\end{array}\right) B_{\mp}-\frac{i(3 k+11)}{2(k+5)^{2}}\left(\begin{array}{c}
F_{11} \\
F_{22}
\end{array}\right) B_{3}\right](w) \\
& +\frac{1}{(z-w)}\left[\frac{1}{2} \partial\left(\begin{array}{c}
\mathbf{U}^{\left(\frac{3}{2}\right)} \\
\mathbf{V}^{\left(\frac{3}{2}\right)}
\end{array}\right) \mp \frac{1}{(k+5)^{2}} A_{3} B_{\mp}\left(\begin{array}{c}
F_{12} \\
F_{21}
\end{array}\right)\right. \\
& +\frac{i\left(k^{2}-10 k-99\right)}{2(k+5)^{2}(2 k+9)} \partial A_{3}\left(\begin{array}{c}
F_{11} \\
F_{22}
\end{array}\right)-\frac{i(k+1)}{2(k+5)^{2}} A_{3} \partial\left(\begin{array}{c}
F_{11} \\
F_{22}
\end{array}\right) \\
& +\frac{i}{2(k+5)} A_{ \pm}\left(\begin{array}{c}
G_{21} \\
G_{12}
\end{array}\right) \pm \frac{1}{(k+5)^{2}} A_{ \pm} A_{3}\left(\begin{array}{c}
F_{21} \\
F_{12}
\end{array}\right) \\
& \mp \frac{1}{(k+5)^{2}} A_{ \pm} A_{\mp}\left(\begin{array}{c}
F_{11} \\
F_{22}
\end{array}\right) \pm \frac{1}{(k+5)^{2}} A_{ \pm} B_{3}\left(\begin{array}{c}
F_{21} \\
F_{12}
\end{array}\right) \\
& -\frac{i(k-1)}{2(k+5)^{2}} \partial A_{ \pm}\left(\begin{array}{c}
F_{21} \\
F_{12}
\end{array}\right)-\frac{i(k+1)}{2(k+5)^{2}} B_{3} \partial\left(\begin{array}{c}
F_{11} \\
F_{22}
\end{array}\right) \\
& -\frac{i(k+7)}{2(k+5)^{2}} A_{ \pm} \partial\left(\begin{array}{c}
F_{21} \\
F_{12}
\end{array}\right)+\frac{i(k-9)(k+7)}{2(k+5)^{2}(2 k+9)} \partial B_{3}\left(\begin{array}{c}
F_{11} \\
F_{22}
\end{array}\right) \\
& -\frac{i}{2(k+5)} B_{\mp}\left(\begin{array}{c}
G_{12} \\
G_{21}
\end{array}\right) \mp \frac{1}{(k+5)^{2}} B_{\mp} B_{3}\left(\begin{array}{c}
F_{12} \\
F_{21}
\end{array}\right) \\
& +\frac{i(k-5)}{2(k+5)^{2}} \partial B_{\mp}\left(\begin{array}{c}
F_{12} \\
F_{21}
\end{array}\right)-\frac{i(k+7)}{2(k+5)^{2}} B_{\mp} \partial\left(\begin{array}{c}
F_{12} \\
F_{21}
\end{array}\right) \\
& \pm \frac{1}{(k+5)^{2}} B_{ \pm} B_{\mp}\left(\begin{array}{c}
F_{11} \\
F_{22}
\end{array}\right) \pm \frac{(k-3)}{2(k+5)^{2}} \partial^{2}\left(\begin{array}{c}
F_{11} \\
F_{22}
\end{array}\right)
\end{aligned}
$$




$$
\begin{aligned}
& \pm \frac{1}{(k+5)^{2}}\left(\begin{array}{c}
F_{11} F_{12} G_{21} \\
F_{22} F_{21} G_{12}
\end{array}\right) \mp \frac{2(k+1)}{(k+5)^{3}}\left(\begin{array}{c}
F_{11} \partial F_{12} F_{21} \\
F_{22} \partial F_{21} F_{12}
\end{array}\right) \\
& \mp \frac{8}{(k+5)^{3}}\left(\begin{array}{c}
F_{11} F_{12} \partial F_{21} \\
F_{22} F_{21} \partial F_{12}
\end{array}\right) \pm \frac{1}{(k+5)^{2}}\left(\begin{array}{c}
F_{11} F_{21} G_{12} \\
F_{22} F_{12} G_{21}
\end{array}\right) \\
& +\frac{i}{(k+5)^{2}} U A_{ \pm}\left(\begin{array}{c}
F_{21} \\
F_{12}
\end{array}\right)-\frac{i}{(k+5)^{2}} U B_{\mp}\left(\begin{array}{c}
F_{12} \\
F_{21}
\end{array}\right) \\
& \mp \frac{\left(5 k^{2}+28 k-9\right)}{2(k+5)^{2}(2 k+9)} \partial U\left(\begin{array}{c}
F_{11} \\
F_{22}
\end{array}\right) \mp \frac{1}{2(k+5)} U \partial\left(\begin{array}{c}
F_{11} \\
F_{22}
\end{array}\right) \\
& \left. \pm \frac{3(k-3)(k+6)}{2(k+5)^{2}(2 k+9)}\left(\begin{array}{c}
F_{11} G_{22} F_{11} \\
F_{22} G_{11} F_{22}
\end{array}\right)\right](w)+\cdots .
\end{aligned}
$$

Note that there is a spin- $\frac{3}{2}$ current in the second and first order poles. One also writes down the OPEs together under the symmetry of the structure constants.

Similarly one describes the following OPEs from the results in (3.8)

$$
\begin{aligned}
& \left(\begin{array}{c}
\mathbf{U}^{\left(\frac{3}{2}\right)} \\
\mathbf{V}^{\left(\frac{3}{2}\right)}
\end{array}\right)(z) \mathbf{W}_{ \pm}^{\left(\frac{5}{2}\right)}(w)=\frac{1}{(z-w)^{3}}\left[-\frac{2 i k(4 k+21)}{3(k+5)^{2}} B_{\mp} \pm \frac{16 k(k+3)}{3(k+5)^{3}}\left(\begin{array}{c}
F_{11} F_{21} \\
F_{22} F_{12}
\end{array}\right)\right](w) \\
& +\frac{1}{(z-w)^{2}}\left[-\frac{8(k+3)}{3(k+5)}\left(\begin{array}{c}
\mathbf{U}_{+}^{(2)} \\
\mathbf{V}_{-}^{(\mathbf{2})}
\end{array}\right) \mp \frac{2(k-3)}{3(k+5)^{2}} A_{3} B_{\mp}\right. \\
& +\frac{2 i(k-3)}{3(k+5)^{3}} A_{3}\left(\begin{array}{c}
F_{11} F_{21} \\
F_{22} F_{12}
\end{array}\right)-\frac{2 i}{(k+5)^{2}} B_{3}\left(\begin{array}{c}
F_{11} F_{21} \\
F_{22} F_{12}
\end{array}\right) \\
& \pm \frac{2 i(k+9)}{3(k+5)^{3}} B_{\mp} F_{11} F_{22} \mp \frac{4 i(k+3)}{3(k+5)^{3}} B_{\mp} F_{12} F_{21} \\
& \mp \frac{2(k+2)}{(k+5)^{2}}\left(\begin{array}{c}
F_{11} G_{21} \\
F_{22} G_{12}
\end{array}\right) \mp \frac{2(5 k+9)}{3(k+5)^{3}}\left(\begin{array}{l}
\partial F_{11} F_{21} \\
\partial F_{22} F_{12}
\end{array}\right) \\
& \mp \frac{2(k+21)}{3(k+5)^{3}}\left(\begin{array}{c}
F_{11} \partial F_{21} \\
F_{22} \partial F_{12}
\end{array}\right) \pm \frac{(k+1)}{(k+5)^{2}}\left(\begin{array}{c}
F_{21} G_{11} \\
F_{12} G_{22}
\end{array}\right) \\
& \left.-\frac{2 i k}{(k+5)^{2}} U B_{\mp} \mp \frac{2}{(k+5)^{2}} U\left(\begin{array}{l}
F_{11} F_{21} \\
F_{22} F_{12}
\end{array}\right)\right](w) \\
& +\frac{1}{(z-w)}\left[-\frac{1}{2}\left(\begin{array}{c}
\mathbf{Q}_{+}^{(3)} \\
\mathbf{R}_{-}^{(3)}
\end{array}\right)-\frac{2(k+3)}{3(k+5)} \partial\left(\begin{array}{c}
\mathbf{U}_{+}^{(2)} \\
\mathbf{V}_{-}^{(2)}
\end{array}\right) \pm \frac{(17 k+81)}{6(k+5)^{2}} \partial A_{3} B_{\mp}\right. \\
& \mp \frac{(19 k+75)}{6(k+5)^{2}} A_{3} \partial B_{\mp}-\frac{2 i}{(k+5)^{2}} A_{3}\left(\begin{array}{c}
F_{11} G_{21} \\
F_{22} G_{12}
\end{array}\right) \\
& +\frac{i(31 k+99)}{6(k+5)^{3}} \partial A_{3}\left(\begin{array}{c}
F_{11} F_{21} \\
F_{22} F_{12}
\end{array}\right)-\frac{i(29 k+105)}{6(k+5)^{3}} A_{3} \partial\left(\begin{array}{c}
F_{11} F_{21} \\
F_{22} F_{12}
\end{array}\right) \\
& +\frac{2 i}{(k+5)^{2}} A_{3}\left(\begin{array}{c}
F_{21} G_{11} \\
F_{12} G_{22}
\end{array}\right)-\frac{i}{2(k+5)^{2}} \partial B_{3}\left(\begin{array}{c}
F_{11} F_{21} \\
F_{22} F_{12}
\end{array}\right) \\
& -\frac{5 i}{2(k+5)^{2}} B_{3}\left(\begin{array}{c}
\partial F_{11} F_{21} \\
\partial F_{22} F_{12}
\end{array}\right)+\frac{3 i}{2(k+5)^{2}} B_{3}\left(\begin{array}{c}
F_{11} \partial F_{21} \\
F_{22} \partial F_{12}
\end{array}\right)
\end{aligned}
$$




$$
\begin{aligned}
& +\frac{i k(4 k+21)}{2(k+5)(13 k+17)} \partial^{2} B_{\mp}+\frac{i}{(k+5)^{2}} B_{\mp}\left(\begin{array}{c}
F_{11} G_{22} \\
F_{22} G_{11}
\end{array}\right) \\
& -\frac{i(k+15)}{3(k+5)^{3}} B_{\mp}\left(\begin{array}{c}
\partial F_{11} F_{22} \\
\partial F_{22} F_{11}
\end{array}\right) \pm \frac{i(5 k+39)}{3(k+5)^{3}} \partial B_{\mp} F_{11} F_{22} \\
& -\frac{i(7 k+45)}{3(k+5)^{3}} B_{\mp}\left(\begin{array}{c}
F_{11} \partial F_{22} \\
F_{22} \partial F_{11}
\end{array}\right)+\frac{i}{(k+5)^{2}} B_{\mp}\left(\begin{array}{c}
F_{12} G_{21} \\
F_{21} G_{12}
\end{array}\right) \\
& -\frac{5 i(k+9)}{6(k+5)^{3}} B_{\mp}\left(\begin{array}{c}
F_{12} \partial F_{21} \\
F_{21} \partial F_{12}
\end{array}\right) \mp \frac{(k-3)}{3(k+5)^{3}}\left(\begin{array}{c}
\partial^{2} F_{11} F_{21} \\
\partial^{2} F_{22} F_{12}
\end{array}\right) \\
& -\frac{i(17 k+105)}{6(k+5)^{3}} B_{\mp}\left(\begin{array}{c}
\partial F_{12} F_{21} \\
\partial F_{21} F_{12}
\end{array}\right) \pm \frac{7 i(k+9)}{6(k+5)^{3}} \partial B_{\mp} F_{12} F_{21} \\
& -\frac{i}{(k+5)^{2}} B_{\mp}\left(\begin{array}{c}
F_{21} G_{12} \\
F_{12} G_{21}
\end{array}\right)-\frac{i}{(k+5)^{2}} B_{\mp}\left(\begin{array}{c}
F_{22} G_{11} \\
F_{11} G_{22}
\end{array}\right) \\
& \mp \frac{\left(24 k^{3}+205 k^{2}+650 k+357\right)}{6(k+5)^{3}(13 k+17)}\left(\begin{array}{c}
F_{11} \partial^{2} F_{21} \\
F_{22} \partial^{2} F_{12}
\end{array}\right) \\
& -\frac{i k(4 k+21)}{(k+5)(13 k+17)} T B_{\mp} \pm \frac{8 k(k+3)}{(k+5)^{2}(13 k+17)} T\left(\begin{array}{c}
F_{11} F_{21} \\
F_{22} F_{12}
\end{array}\right) \\
& \pm \frac{4}{(k+5)^{2}} U A_{3} B_{\mp}-\frac{i k}{2(k+5)^{2}} \partial\left(U B_{\mp}\right) \mp \frac{5}{2(k+5)^{2}} U\left(\begin{array}{l}
\partial F_{11} F_{21} \\
\partial F_{22} F_{12}
\end{array}\right) \\
& \mp \frac{1}{2(k+5)^{2}} \partial U\left(\begin{array}{c}
F_{11} F_{21} \\
F_{22} F_{12}
\end{array}\right) \pm \frac{3}{2(k+5)^{2}} U\left(\begin{array}{c}
F_{11} \partial F_{21} \\
F_{22} \partial F_{12}
\end{array}\right) \\
& +\frac{2 i}{(k+5)^{2}}\left(\begin{array}{c}
F_{11} A_{-} \partial F_{11} \\
F_{22} A_{+} \partial F_{22}
\end{array}\right)+\frac{i\left(8 k^{2}+63 k+51\right)}{(k+5)^{2}(13 k+17)}\left(\begin{array}{c}
F_{21} A_{+} \partial F_{21} \\
F_{12} A_{-} \partial F_{12}
\end{array}\right) \\
& +\frac{i\left(8 k^{2}+37 k+17\right)}{(k+5)^{2}(13 k+17)}\left(\begin{array}{l}
\partial F_{21} A_{+} F_{21} \\
\partial F_{12} A_{-} F_{12}
\end{array}\right) \\
& \mp \frac{(5 k+13)}{4(k+5)^{2}}\left(\begin{array}{c}
\partial F_{11} G_{21} \\
\partial F_{22} G_{12}
\end{array}\right) \mp \frac{(k+1)}{4(k+5)^{2}}\left(\begin{array}{l}
F_{11} \partial G_{21} \\
F_{22} \partial G_{12}
\end{array}\right) \\
& \left.\mp \frac{(k+4)}{2(k+5)^{2}}\left(\begin{array}{c}
\partial F_{21} G_{11} \\
\partial F_{12} G_{22}
\end{array}\right) \pm \frac{(k+2)}{2(k+5)^{2}}\left(\begin{array}{c}
F_{21} \partial G_{11} \\
F_{12} \partial G_{22}
\end{array}\right)\right](w)+\cdots \text {. }
\end{aligned}
$$

Due to the spin- $\frac{1}{2}$ currents the OPEs are rather complicated and the nonlinear terms in the corresponding OPEs in the nonlinear version disappear.

Moreover, one can construct the following OPEs

$$
\begin{aligned}
\left(\begin{array}{c}
\mathbf{U}^{\left(\frac{3}{2}\right)} \\
\mathbf{V}^{\left(\frac{3}{2}\right)}
\end{array}\right)(z) \mathbf{W}_{\mp}^{\left(\frac{5}{2}\right)}(w) & =\frac{1}{(z-w)^{3}}\left[\frac{2 i(5 k+18)}{(k+5)^{2}} A_{ \pm} \pm \frac{8(k+9)}{(k+5)^{3}}\left(\begin{array}{c}
F_{11} F_{12} \\
F_{22} F_{21}
\end{array}\right)\right](w) \\
& +\frac{1}{(z-w)^{2}}\left[-\frac{4(k+9)}{3(k+5)}\left(\begin{array}{c}
\mathbf{U}_{-}^{(2)} \\
\mathbf{V}_{+}^{(2)}
\end{array}\right)+\frac{2 i}{(k+5)^{2}} A_{3}\left(\begin{array}{c}
F_{11} F_{12} \\
F_{22} F_{21}
\end{array}\right)\right. \\
& \pm \frac{2(k-3)}{3(k+5)^{2}} A_{ \pm} B_{3} \mp \frac{4 i(k+3)}{3(k+5)^{3}} A_{ \pm} F_{11} F_{22}
\end{aligned}
$$




$$
\begin{aligned}
& -\frac{2 i(k+9)}{3(k+5)^{3}} A_{ \pm} F_{12} F_{21}+\frac{2 i(k-3)}{3(k+5)^{3}} B_{3}\left(\begin{array}{c}
F_{11} F_{12} \\
F_{22} F_{21}
\end{array}\right) \\
& \pm \frac{(k+7)}{(k+5)^{2}}\left(\begin{array}{c}
F_{11} G_{12} \\
F_{22} G_{21}
\end{array}\right) \mp \frac{2(k+21)}{3(k+5)^{3}}\left(\begin{array}{c}
\partial F_{11} F_{12} \\
\partial F_{22} F_{21}
\end{array}\right) \\
& \mp \frac{2(5 k+9)}{3(k+5)^{3}}\left(\begin{array}{c}
F_{11} \partial F_{12} \\
F_{22} \partial F_{21}
\end{array}\right) \mp \frac{4}{(k+5)^{2}}\left(\begin{array}{c}
F_{12} G_{11} \\
F_{21} G_{22}
\end{array}\right) \\
& \left.-\frac{6 i}{(k+5)^{2}} U A_{ \pm} \pm \frac{2}{(k+5)^{2}} U\left(\begin{array}{c}
F_{11} F_{12} \\
F_{22} F_{21}
\end{array}\right)\right](w) \\
& +\frac{1}{(z-w)}\left[\frac{1}{2}\left(\begin{array}{c}
\mathbf{Q}_{-}^{(3)} \\
\mathbf{R}_{+}^{(3)}
\end{array}\right)-\frac{(k+9)}{3(k+5)} \partial\left(\begin{array}{c}
\mathbf{U}_{-}^{(2)} \\
\mathbf{V}_{+}^{(2)}
\end{array}\right)\right. \\
& +\frac{i}{2(k+5)^{2}} \partial A_{3}\left(\begin{array}{c}
F_{11} F_{12} \\
F_{22} F_{21}
\end{array}\right)+\frac{5 i}{2(k+5)^{2}} A_{3}\left(\begin{array}{c}
\partial F_{11} F_{12} \\
\partial F_{22} F_{21}
\end{array}\right) \\
& -\frac{3 i}{2(k+5)^{2}} A_{3}\left(\begin{array}{c}
F_{11} \partial F_{12} \\
F_{22} \partial F_{21}
\end{array}\right)-\frac{3 i(5 k+18)}{2(k+5)(13 k+17)} \partial^{2} A_{ \pm} \\
& \mp \frac{(17 k+81)}{6(k+5)^{2}} \partial A_{ \pm} B_{3} \pm \frac{(19 k+75)}{6(k+5)^{2}} A_{ \pm} \partial B_{3} \\
& +\frac{i}{(k+5)^{2}} A_{ \pm}\left(\begin{array}{c}
F_{11} G_{22} \\
F_{22} G_{11}
\end{array}\right) \mp \frac{i(17 k+57)}{6(k+5)^{3}} \partial A_{ \pm} F_{11} F_{22} \\
& +\frac{i(7 k+15)}{6(k+5)^{3}} A_{ \pm}\left(\begin{array}{c}
\partial F_{11} F_{22} \\
\partial F_{22} F_{11}
\end{array}\right)+\frac{i(19 k+75)}{6(k+5)^{3}} A_{ \pm}\left(\begin{array}{c}
F_{11} \partial F_{22} \\
F_{22} \partial F_{11}
\end{array}\right) \\
& -\frac{i}{(k+5)^{2}} A_{ \pm}\left(\begin{array}{c}
F_{12} G_{21} \\
F_{21} G_{12}
\end{array}\right) \mp \frac{7 i(k+3)}{3(k+5)^{3}} \partial A_{ \pm} F_{12} F_{21} \\
& -\frac{5 i(k+3)}{3(k+5)^{3}} A_{ \pm}\left(\begin{array}{c}
\partial F_{12} F_{21} \\
\partial F_{21} F_{12}
\end{array}\right)-\frac{i(11 k+45)}{3(k+5)^{3}} A_{ \pm}\left(\begin{array}{c}
F_{12} \partial F_{21} \\
F_{21} \partial F_{12}
\end{array}\right) \\
& +\frac{i}{(k+5)^{2}} A_{ \pm}\left(\begin{array}{c}
F_{21} G_{12} \\
F_{12} G_{21}
\end{array}\right)+\frac{2 i}{(k+5)^{2}} B_{3}\left(\begin{array}{c}
F_{12} G_{11} \\
F_{21} G_{22}
\end{array}\right) \\
& -\frac{i}{(k+5)^{2}} A_{ \pm}\left(\begin{array}{c}
F_{22} G_{11} \\
F_{11} G_{22}
\end{array}\right)-\frac{2 i}{(k+5)^{2}} B_{3}\left(\begin{array}{c}
F_{11} G_{12} \\
F_{22} G_{21}
\end{array}\right) \\
& -\frac{i(17 k+141)}{6(k+5)^{3}} \partial B_{3}\left(\begin{array}{c}
F_{11} F_{12} \\
F_{22} F_{21}
\end{array}\right)+\frac{i(19 k+135)}{6(k+5)^{3}} B_{3} \partial\left(\begin{array}{c}
F_{11} F_{12} \\
F_{22} F_{21}
\end{array}\right) \\
& \mp \frac{\left(49 k^{2}+794 k+1977\right)}{6(k+5)^{3}(13 k+17)}\left(\begin{array}{c}
\partial^{2} F_{11} F_{12} \\
\partial^{2} F_{22} F_{21}
\end{array}\right) \\
& \mp \frac{25}{(k+5)(13 k+17)}\left(\begin{array}{l}
\partial F_{11} \partial F_{12} \\
\partial F_{22} \partial F_{21}
\end{array}\right) \\
& \mp \frac{\left(101 k^{2}+706 k+1773\right)}{6(k+5)^{3}(13 k+17)}\left(\begin{array}{c}
F_{11} \partial^{2} F_{12} \\
F_{22} \partial^{2} F_{21}
\end{array}\right) \pm \frac{(k+4)}{(k+5)^{2}}\left(\begin{array}{c}
\partial F_{11} G_{12} \\
\partial F_{22} G_{21}
\end{array}\right)
\end{aligned}
$$




$$
\begin{aligned}
& \pm \frac{(3 k+5)}{4(k+5)^{2}}\left(\begin{array}{c}
\partial F_{12} G_{11} \\
\partial F_{21} G_{22}
\end{array}\right) \mp \frac{(k+7)}{4(k+5)^{2}}\left(\begin{array}{c}
F_{12} \partial G_{11} \\
F_{21} \partial G_{22}
\end{array}\right) \\
& \pm \frac{1}{(k+5)^{2}}\left(\begin{array}{c}
F_{11} \partial G_{12} \\
F_{22} \partial G_{21}
\end{array}\right)+\frac{3 i(5 k+18)}{(k+5)(13 k+17)} T A_{ \pm} \\
& \pm \frac{12(k+9)}{(k+5)^{2}(13 k+17)} T\left(\begin{array}{c}
F_{11} F_{12} \\
F_{22} F_{21}
\end{array}\right) \mp \frac{4}{(k+5)^{2}} U A_{ \pm} B_{3} \\
& -\frac{3 i}{2(k+5)^{2}} \partial\left(U A_{ \pm}\right) \mp \frac{3}{2(k+5)^{2}} U\left(\begin{array}{c}
F_{11} \partial F_{12} \\
F_{22} \partial F_{21}
\end{array}\right) \\
& \pm \frac{1}{2(k+5)^{2}} \partial U\left(\begin{array}{c}
F_{11} F_{12} \\
F_{22} F_{21}
\end{array}\right) \pm \frac{5}{2(k+5)^{2}} U\left(\begin{array}{l}
\partial F_{11} F_{12} \\
\partial F_{22} F_{21}
\end{array}\right) \\
& \left.-\frac{2 i}{(k+5)^{2}}\left(\begin{array}{l}
F_{11} B_{+} \partial F_{11} \\
F_{22} B_{-} \partial F_{22}
\end{array}\right)-\frac{2 i}{(k+5)^{2}}\left(\begin{array}{l}
F_{12} B_{-} \partial F_{12} \\
F_{21} B_{+} \partial F_{21}
\end{array}\right)\right](w)+\cdots
\end{aligned}
$$

One has these OPEs by writing two OPEs together with the symmetry of the structure constants.

From the relation in (3.9), one obtains the following OPEs

$$
\begin{aligned}
\left(\begin{array}{c}
\mathbf{U}^{\left(\frac{3}{2}\right)} \\
\mathbf{V}^{\left(\frac{3}{2}\right)}
\end{array}\right)(z) \mathbf{W}^{(\mathbf{3})}(w) & =\frac{1}{(z-w)^{4}}\left[-\frac{18 k}{(k+5)^{2}}\left(\begin{array}{c}
F_{11} \\
F_{22}
\end{array}\right)\right](w) \\
& +\frac{1}{(z-w)^{3}}\left[ \pm \frac{2(k-3)(61 k+113)}{3(k+5)(13 k+17)}\left(\begin{array}{c}
\mathbf{U}^{\left(\frac{3}{2}\right)} \\
\mathbf{V}^{\left(\frac{3}{2}\right)}
\end{array}\right)\right. \\
& -\frac{2(k-3)(61 k+113)}{3(k+5)^{2}(13 k+17)} U\left(\begin{array}{c}
F_{11} \\
F_{22}
\end{array}\right) \mp \frac{4(k-3)(5 k+73)}{3(k+5)^{3}(13 k+17)} F_{12} F_{21}\left(\begin{array}{c}
F_{11} \\
F_{22}
\end{array}\right) \\
& \mp \frac{2 i\left(61 k^{2}+164 k-33\right)}{3(k+5)^{2}(13 k+17)}\left(\begin{array}{c}
F_{11} \\
F_{22}
\end{array}\right) A_{3} \mp \frac{2 i\left(61 k^{2}+164 k-33\right)}{3(k+5)^{2}(13 k+17)}\left(\begin{array}{l}
F_{21} \\
F_{12}
\end{array}\right) A_{ \pm} \\
& \pm \frac{2 i\left(17 k^{2}+172 k+339\right)}{3(k+5)^{2}(13 k+17)}\left(\begin{array}{c}
F_{12} \\
F_{21}
\end{array}\right) B_{\mp} \\
& \left.+\frac{18 k}{(k+5)^{2}} \partial\left(\begin{array}{c}
F_{11} \\
F_{22}
\end{array}\right) \pm \frac{2 i\left(17 k^{2}+172 k+339\right)}{3(k+5)^{2}(13 k+17)}\left(\begin{array}{l}
F_{11} \\
F_{22}
\end{array}\right) B_{3}\right](w) \\
& +\frac{1}{(z-w)^{2}}\left[ \pm \frac{5}{2}\left(\begin{array}{c}
\mathbf{Q}^{\left(\frac{5}{2}\right)} \\
\mathbf{R}^{\left(\frac{5}{2}\right)}
\end{array}\right)+5\left(\begin{array}{l}
\mathbf{U}^{\left(\frac{5}{2}\right)} \\
\mathbf{V}^{\left(\frac{5}{2}\right)}
\end{array}\right) \pm \frac{2(k-3)}{(13 k+17)} \partial\left(\begin{array}{l}
\mathbf{U}^{\left(\frac{3}{2}\right)} \\
\mathbf{V}^{\left(\frac{3}{2}\right)}
\end{array}\right)\right. \\
& -\frac{6(\mathbf{k}-\mathbf{3})}{(13 k+17)} \mathbf{T}^{(\mathbf{1})}\left(\begin{array}{l}
\mathbf{U}^{\left(\frac{3}{2}\right)} \\
\mathbf{V}^{\left(\frac{3}{2}\right)}
\end{array}\right)+\frac{2}{(k+5)^{2}} A_{3} A_{3}\left(\begin{array}{l}
F_{11} \\
F_{22}
\end{array}\right) \\
& +\frac{4}{(k+5)^{2}} A_{3} B_{3}\left(\begin{array}{l}
F_{11} \\
F_{22}
\end{array}\right) \mp \frac{3 i}{2(k+5)} B_{\mp}\left(\begin{array}{l}
G_{12} \\
G_{21}
\end{array}\right) \\
& \mp \frac{3 i}{2(k+5)^{2}} A_{3}\left(\begin{array}{l}
G_{11} \\
G_{22}
\end{array}\right)+\frac{4}{(k+5)^{2}} A_{3} B_{\mp}\left(\begin{array}{l}
F_{12} \\
F_{21}
\end{array}\right) \\
& \mp \frac{2 i\left(k^{2}+67 k+70\right)}{(k+5)^{2}(13 k+17)} \partial A_{3}\left(\begin{array}{l}
F_{11} \\
F_{22}
\end{array}\right) \mp \frac{2 i\left(k^{2}-128 k-185\right)}{(k+5)^{2}(13 k+17)} A_{3} \partial\left(\begin{array}{l}
F_{11} \\
F_{22}
\end{array}\right)
\end{aligned}
$$




$$
\begin{aligned}
& \mp \frac{3 i}{2(k+5)} A_{ \pm}\left(\begin{array}{c}
G_{21} \\
G_{12}
\end{array}\right)+\frac{2}{(k+5)^{2}} A_{ \pm} A_{\mp}\left(\begin{array}{c}
F_{11} \\
F_{22}
\end{array}\right) \\
& +\frac{4}{(k+5)^{2}} A_{ \pm} B_{3}\left(\begin{array}{c}
F_{21} \\
F_{12}
\end{array}\right)-\frac{4}{(k+5)^{2}} A_{ \pm} B_{\mp}\left(\begin{array}{c}
F_{22} \\
F_{11}
\end{array}\right) \\
& \mp \frac{2 i\left(k^{2}+80 k+87\right)}{(k+5)^{2}(13 k+17)} \partial A_{ \pm}\left(\begin{array}{c}
F_{21} \\
F_{12}
\end{array}\right) \mp \frac{2 i\left(k^{2}-128 k-185\right)}{(k+5)^{2}(13 k+17)} A_{ \pm} \partial\left(\begin{array}{c}
F_{21} \\
F_{12}
\end{array}\right) \\
& \mp \frac{3 i}{2(k+5)} B_{3}\left(\begin{array}{c}
G_{11} \\
G_{22}
\end{array}\right) \mp \frac{4 i\left(20 k^{2}+33 k+1\right)}{(k+5)^{2}(13 k+17)} B_{3} \partial\left(\begin{array}{c}
F_{11} \\
F_{22}
\end{array}\right) \\
& \pm \frac{i\left(37 k^{2}+112 k+115\right)(k-9)}{3(k+5)^{2}(13 k+17)} \partial B_{3}\left(\begin{array}{c}
F_{11} \\
F_{22}
\end{array}\right)+\frac{2}{(k+5)^{2}} B_{3} B_{3}\left(\begin{array}{c}
F_{11} \\
F_{22}
\end{array}\right) \\
& \pm \frac{i\left(37 k^{2}+86 k+81\right)}{(k+5)^{2}(13 k+17)} \partial B_{\mp}\left(\begin{array}{c}
F_{12} \\
F_{21}
\end{array}\right) \mp \frac{4 i\left(20 k^{2}+33 k+1\right)}{(k+5)^{2}(13 k+17)} B_{\mp} \partial\left(\begin{array}{c}
F_{12} \\
F_{21}
\end{array}\right) \\
& +\frac{2}{(k+5)^{2}} B_{ \pm} B_{\mp}\left(\begin{array}{c}
F_{11} \\
F_{22}
\end{array}\right)-\frac{3(k+3)}{2(k+5)^{2}} \partial^{2}\left(\begin{array}{c}
F_{11} \\
F_{22}
\end{array}\right) \\
& +\frac{2}{(k+5)^{2}}\left(\begin{array}{c}
F_{11} F_{12} G_{21} \\
F_{22} F_{21} G_{12}
\end{array}\right)-\frac{8(k-3)}{(k+5)^{2}(13 k+17)} \partial\left(\begin{array}{c}
F_{11} \\
F_{22}
\end{array}\right) F_{12} F_{21} \\
& -\frac{2}{(k+5)^{2}}\left(\begin{array}{c}
F_{11} F_{21} G_{12} \\
F_{22} F_{12} G_{21}
\end{array}\right)+\frac{2}{(k+5)^{2}}\left(\begin{array}{c}
F_{12} F_{21} G_{11} \\
F_{21} F_{12} G_{22}
\end{array}\right) \\
& -\frac{1}{(k+5)} T\left(\begin{array}{c}
F_{11} \\
F_{22}
\end{array}\right)-\frac{3}{2(k+5)} U\left(\begin{array}{l}
G_{11} \\
G_{22}
\end{array}\right) \\
& \mp \frac{6 i}{(k+5)^{2}} U A_{3}\left(\begin{array}{c}
F_{11} \\
F_{22}
\end{array}\right) \mp \frac{6 i}{(k+5)^{2}} U A_{ \pm}\left(\begin{array}{c}
F_{21} \\
F_{12}
\end{array}\right) \\
& \mp \frac{6 i}{(k+5)^{2}} U B_{3}\left(\begin{array}{c}
F_{11} \\
F_{22}
\end{array}\right) \mp \frac{6 i}{(k+5)^{2}} U B_{\mp}\left(\begin{array}{c}
F_{12} \\
F_{21}
\end{array}\right) \\
& -\frac{2(k-3)}{(k+5)(13 k+17)} \partial\left(\begin{array}{c}
U F_{11} \\
U F_{22}
\end{array}\right)+\frac{2(61 k+97)}{(k+5)^{2}(13 k+17)}\left(\begin{array}{c}
F_{11} F_{12} \partial F_{21} \\
F_{22} F_{21} \partial F_{12}
\end{array}\right) \\
& -\frac{4}{(k+5)^{2}} U U\left(\begin{array}{c}
F_{11} \\
F_{22}
\end{array}\right) \pm \frac{10}{(k+5)^{2}}\left(\begin{array}{l}
\partial F_{11} \\
\partial F_{22}
\end{array}\right) F_{11} F_{22} \\
& \left.-\frac{2(69 k+73)}{(k+5)^{2}(13 k+17)}\left(\begin{array}{c}
F_{11} \partial F_{12} F_{21} \\
F_{22} \partial F_{21} F_{12}
\end{array}\right)\right](w) \\
& +\frac{1}{(z-w)}[+\cdots](w)+\cdots \text {. }
\end{aligned}
$$

There is no spin- $\frac{3}{2}$ current in the third order pole. Again the nonlinear term containing the higher spin currents is present. As discussed before, this can be removed by adding the $T \mathbf{T}^{(\mathbf{1})}(w)$ term into the left hand side. Other nonlinear terms in the context of the footnote 5 disappear in this OPEs. It is an open problem to complete the first order pole by figuring out the possible composite spin- $\frac{7}{2}$ fields. 
The relation (3.5) determines the following OPEs

$$
\begin{aligned}
\left(\begin{array}{c}
\mathbf{U}_{+}^{(2)} \\
\mathbf{V}_{-}^{(2)}
\end{array}\right)(z)\left(\begin{array}{c}
\mathbf{U}_{+}^{(2)} \\
\mathbf{V}_{-}^{(2)}
\end{array}\right)(w) & =\frac{1}{(z-w)^{2}}\left[\frac{2 k}{(5+k)^{2}} B_{\mp} B_{\mp}\right](w) \\
& +\frac{1}{(z-w)}\left[\frac{2 k}{(5+k)^{2}} B_{\mp} \partial B_{\mp}\right](w)+\cdots
\end{aligned}
$$

These are new OPEs where the corresponding OPEs in the nonlinear version are trivial.

By using the result in (3.4), one has

$$
\begin{aligned}
\mathbf{U}_{+}^{(2)}(z) \mathbf{U}_{-}^{(2)}(w) & =\frac{1}{(z-w)^{2}}\left[\frac{2(k+4)}{(k+5)^{2}} A_{+} B_{-}+\frac{2 i}{(k+5)^{2}} A_{+} F_{11} F_{21}\right. \\
& \left.-\frac{2 i}{(k+5)^{2}} B_{-} F_{11} F_{12}-\frac{(k-3)}{(k+5)^{2}} F_{11} G_{11}+\frac{4(3 k-1)}{(k+5)^{3}} F_{11} \partial F_{11}\right](w) \\
& +\frac{1}{(z-w)}\left[\frac{2 i}{(k+5)^{2}} A_{3} F_{11} G_{11}-\frac{1}{(k+5)^{2}} \partial A_{+} B_{-}\right. \\
& +\frac{(2 k+9)}{(k+5)^{2}} A_{+} \partial B_{-}+\frac{i}{(k+5)^{2}} A_{+} F_{11} G_{21}+\frac{8 i}{(k+5)^{3}} A_{+} \partial F_{11} F_{21} \\
& +\frac{4 i(k+3)}{(k+5)^{3}} A_{+} F_{11} \partial F_{21}+\frac{i}{(k+5)^{2}} A_{+} F_{21} G_{11}-\frac{2 i}{(k+5)^{2}} B_{3} F_{11} G_{11} \\
& -\frac{i}{(k+5)^{2}} B_{-} F_{11} G_{12}-\frac{2 i}{(k+5)^{2}} \partial B_{-} F_{11} F_{12}-\frac{8 i}{(k+5)^{3}} B_{-} \partial F_{11} F_{12} \\
& +\frac{8 i}{(k+5)^{3}} B_{-} F_{11} \partial F_{12}-\frac{i}{(k+5)^{2}} B_{-} F_{12} G_{11}-\frac{2(k+4)}{(k+5)^{2}} \partial F_{11} G_{11} \\
& +\frac{2}{(k+5)^{2}} F_{11} \partial G_{11}+\frac{4 i(k+1)}{(k+5)^{3}} F_{11} A_{3} \partial F_{11}+\frac{16 i}{(k+5)^{3}} F_{11} B_{3} \partial F_{11} \\
& \left.+\frac{4}{(k+5)^{2}} F_{11} U \partial F_{11}+\frac{4(k+1)}{(k+5)^{3}} F_{11} \partial^{2} F_{11}\right](w)+\cdots
\end{aligned}
$$

As before, the nonlinear terms in the corresponding OPE in the nonlinear version disappear.

For the higher spin- $\frac{5}{2}$ currents, one has the result in (3.7) and can construct the following OPEs

$$
\begin{aligned}
\left(\begin{array}{c}
\mathbf{U}_{+}^{(2)} \\
\mathbf{V}_{-}^{(2)}
\end{array}\right)(z)\left(\begin{array}{c}
\mathbf{U}^{\left(\frac{5}{2}\right)} \\
\mathbf{V}^{\left(\frac{5}{2}\right)}
\end{array}\right)(w) & =\frac{1}{(z-w)^{3}}\left[-\frac{16 i k(k+3)}{3(k+5)^{3}} B_{\mp}\left(\begin{array}{c}
F_{11} \\
F_{22}
\end{array}\right)\right](w) \\
& +\frac{1}{(z-w)^{2}}\left[ \pm \frac{2(k+9)}{3(k+5)^{3}} A_{3} B_{\mp}\left(\begin{array}{c}
F_{11} \\
F_{22}
\end{array}\right) \pm \frac{2(k+9)}{3(k+5)^{3}} A_{ \pm} B_{\mp}\left(\begin{array}{c}
F_{21} \\
F_{12}
\end{array}\right)\right. \\
& +\frac{i(7 k+9)}{3(k+5)^{2}} B_{\mp}\left(\begin{array}{c}
G_{11} \\
G_{22}
\end{array}\right) \mp \frac{2(k+9)}{3(k+5)^{3}} B_{\mp} B_{3}\left(\begin{array}{c}
F_{11} \\
F_{22}
\end{array}\right) \\
& \mp \frac{2(k+9)}{3(k+5)^{3}} B_{\mp} B_{\mp}\left(\begin{array}{c}
F_{12} \\
F_{21}
\end{array}\right)-\frac{2 i k(k+1)}{(k+5)^{3}} \partial B_{\mp}\left(\begin{array}{c}
F_{11} \\
F_{22}
\end{array}\right)
\end{aligned}
$$




$$
\begin{aligned}
& -\frac{2 i(k+9)(2 k-3)}{3(k+5)^{3}} B_{\mp} \partial\left(\begin{array}{c}
F_{11} \\
F_{22}
\end{array}\right) \mp \frac{4(k+1)(k+9)}{3(k+5)^{4}}\left(\begin{array}{c}
F_{11} F_{21} \partial F_{11} \\
F_{22} F_{12} \partial F_{22}
\end{array}\right) \\
& \left.\mp \frac{2(k+9)}{3(k+5)^{3}}\left(\begin{array}{c}
F_{11} F_{21} G_{11} \\
F_{22} F_{12} G_{22}
\end{array}\right)+\frac{2 i(k+9)}{3(k+5)^{3}} U B_{\mp}\left(\begin{array}{c}
F_{11} \\
F_{22}
\end{array}\right)\right](w) \\
& +\frac{1}{(z-w)}[+\cdots](w)+\cdots .
\end{aligned}
$$

The nonlinear terms in the corresponding OPEs in the nonlinear version disappear.

As done before, one constructs

$$
\begin{aligned}
\mathbf{U}_{+}^{(2)}(z) \mathbf{V}^{\left(\frac{3}{2}\right)}(w) & =\frac{1}{(z-w)^{3}}\left[-\frac{6 k}{(k+5)^{2}} F_{21}\right](w) \\
& +\frac{1}{(z-w)^{2}}\left[-\frac{(k+9)}{(k+5)} \mathbf{T}_{+}^{\left(\frac{3}{2}\right)}-\frac{(k+8)}{(k+5)} G_{21}-\frac{i(k+1)}{(k+5)^{2}} F_{11} A_{-}\right. \\
& -\frac{(k+7)}{(k+5)^{2}} U F_{21}+\frac{2}{(k+5)^{2}} F_{21} F_{11} F_{22}+\frac{i(k+1)}{(k+5)^{2}} F_{21} A_{3} \\
& \left.+\frac{i(k+7)}{(k+5)^{2}} F_{22} B_{-}+\frac{i(k-7)}{(k+5)^{2}} F_{21} B_{3}-\frac{6 k}{(k+5)^{2}} \partial F_{21}\right](w) \\
& +\frac{1}{(z-w)}\left[\frac{1}{2} \mathbf{P}_{+}^{\left(\frac{5}{2}\right)}-\frac{2(k+9)}{3(k+5)} \partial \mathbf{T}_{+}^{\left(\frac{3}{2}\right)}\right. \\
& +\frac{i}{2(k+5)} A_{3} G_{21}-\frac{2}{(k+5)^{2}} A_{3} B_{3} F_{21}+\frac{1}{(k+5)^{2}} A_{3} B_{-} F_{22} \\
& +\frac{2 i(k+3)}{3(k+5)^{2}} \partial A_{3} F_{21}+\frac{2 i k}{3(k+5)^{2}} A_{3} \partial F_{21}-\frac{i}{2(k+5)} A_{-} G_{11} \\
& +\frac{2}{(k+5)^{2}} A_{-} B_{3} F_{11}+\frac{1}{(k+5)^{2}} A_{-} B_{-} F_{12}-\frac{2 i(k+3)}{3(k+5)^{2}} \partial A_{-} F_{11} \\
& -\frac{2 i k}{3(k+5)^{2}} A_{-} \partial F_{11}+\frac{i}{2(k+5)} B_{3} G_{21}-\frac{2}{(k+5)^{2}} B_{3} B_{3} F_{21} \\
& +\frac{i(k-15)}{3(k+5)^{2}} \partial B_{3} F_{21}+\frac{4 i k}{3(k+5)^{2}} B_{3} \partial F_{21}+\frac{1}{(k+5)^{2}} B_{-} B_{3} F_{22} \\
& +\frac{i(2 k+15)}{3(k+5)^{2}} \partial B_{-} F_{22}+\frac{i(2 k+9)}{3(k+5)^{2}} B_{-} \partial F_{22}-\frac{1}{(k+5)^{2}} B_{+} B_{-} F_{21} \\
& +\frac{1}{(k+5)^{2}} F_{11} F_{21} G_{22}-\frac{2(k-3)}{3(k+5)^{3}} \partial F_{11} F_{21} F_{22}-\frac{2(k+9)}{3(k+5)^{3}} F_{11} \partial F_{21} F_{22} \\
& -\frac{8(k+6)}{3(k+5)^{3}} F_{11} F_{21} \partial F_{22}-\frac{1}{(k+5)^{2}} F_{11} F_{22} G_{21}+\frac{1}{(k+5)^{2}} F_{12} F_{21} G_{21} \\
& -\frac{3(k+1)}{2(k+5)^{2}} \partial^{2} F_{21}-\frac{(2 k+15)}{3(k+5)} \partial G_{21}-\frac{1}{(k+5)} T F_{21} \\
& -\frac{1}{2(k+5)} U G_{21}+\frac{2 i}{(k+5)^{2}} U A_{3} F_{21}-\frac{2 i}{(k+5)^{2}} U A_{-} F_{11} \\
& \left.+\frac{1}{(k+1}\right)
\end{aligned}
$$




$$
\begin{aligned}
& +\frac{i}{(k+5)^{2}} U B_{-} F_{22}-\frac{2(k+6)}{3(k+5)^{2}} \partial U F_{21}-\frac{2(k+6)}{3(k+5)^{2}} U \partial F_{21} \\
& \left.-\frac{2}{(k+5)^{2}} U U F_{21}-\frac{8}{(k+5)^{3}} F_{21} \partial F_{21} F_{12}\right](w)+\cdots
\end{aligned}
$$

The nonderivative terms containing the spin- $\frac{3}{2}$ current in the first order pole do not appear in the corresponding OPE in the nonlinear version.

Similarly one can obtain the following OPE

$$
\begin{aligned}
\mathbf{U}_{+}^{(2)}(z) \mathbf{V}_{+}^{(2)}(w) & =\frac{1}{(z-w)^{2}}\left[-\frac{2(k+4)}{(k+5)^{2}} A_{-} B_{-}-\frac{2 i}{(k+5)^{2}} A_{-} F_{11} F_{21}\right. \\
& \left.+\frac{2 i}{(k+5)^{2}} B_{-} F_{21} F_{22}-\frac{(k-3)}{(k+5)^{2}} F_{21} G_{21}+\frac{4(3 k-1)}{(k+5)^{3}} F_{21} \partial F_{21}\right](w) \\
& +\frac{1}{(z-w)}\left[-\frac{2 i}{(k+5)^{2}} A_{3} F_{21} G_{21}+\frac{1}{(k+5)^{2}} \partial A_{-} B_{-}\right. \\
& -\frac{(2 k+9)}{(k+5)^{2}} A_{-} \partial B_{-}+\frac{i}{(k+5)^{2}} A_{-} F_{11} G_{21}-\frac{4 i(k+3)}{(k+5)^{3}} A_{-} \partial F_{11} F_{21} \\
& -\frac{8 i}{(k+5)^{3}} A_{-} F_{11} \partial F_{21}+\frac{i}{(k+5)^{2}} A_{-} F_{21} G_{11}-\frac{2 i}{(k+5)^{2}} B_{3} F_{21} G_{21} \\
& +\frac{i}{(k+5)^{2}} B_{-} F_{21} G_{22}+\frac{2 i}{(k+5)^{2}} \partial B_{-} F_{21} F_{22}+\frac{8 i}{(k+5)^{3}} B_{-} \partial F_{21} F_{22} \\
& -\frac{8 i}{(k+5)^{3}} B_{-} F_{21} \partial F_{22}+\frac{i}{(k+5)^{2}} B_{-} F_{22} G_{21}-\frac{2(k+4)}{(k+5)^{2}} \partial F_{21} G_{21} \\
& +\frac{2}{(k+5)^{2}} F_{21} \partial G_{21}+\frac{(5 k+1)}{(k+5)^{3}} F_{21} \partial^{2} F_{21}+\frac{4}{(k+5)^{2}} F_{21} \partial F_{21} U \\
& \left.-\frac{4 i(k+1)}{(k+5)^{3}} F_{21} \partial F_{21} A_{3}+\frac{16 i}{(k+5)^{3}} F_{21} \partial F_{21} B_{3}\right](w)+\cdots
\end{aligned}
$$

One sees that there are no nonlinear terms in the context of the footnote 5 .

One also presents the other case

$$
\begin{aligned}
\mathbf{U}_{+}^{(2)}(z) \mathbf{V}_{-}^{(2)}(w) & =\frac{1}{(z-w)^{4}}\left[-\frac{6 k(k+9)}{(k+5)^{2}}\right] \\
& +\frac{1}{(z-w)^{3}}\left[\frac{4 i k(k+8)}{(k+5)^{2}} B_{3}-\frac{4 k}{(k+5)^{2}} F_{11} F_{22}+\frac{4 k}{(k+5)^{2}} F_{12} F_{21}\right](w) \\
& +\frac{1}{(z-w)^{2}}\left[\frac{1}{2} \mathbf{P}^{(2)}-\frac{2(k-3)}{3(k+5)} \mathbf{T}^{(2)}-2 \mathbf{W}^{(2)}-\frac{2(2 k+15)}{3(k+5)} T\right. \\
& -\frac{4(k+3)}{3(k+5)^{2}} A_{3} A_{3}+\frac{2(k-3)}{3(k+5)^{2}} A_{3} B_{3}-\frac{4 i(k+3)}{3(k+5)^{2}} \partial A_{3} \\
& +\frac{4 i(k+9)}{3(k+5)^{3}} A_{3} F_{11} F_{22}+\frac{2 i}{(k+5)^{2}} A_{3} F_{12} F_{21}-\frac{4(k+3)}{3(k+5)^{2}} A_{+} A_{-}
\end{aligned}
$$




$$
\begin{aligned}
& +\frac{10 i(k+9)}{3(k+5)^{3}} A_{-} F_{11} F_{12}-\frac{8 i}{(k+5)^{3}} A_{+} F_{21} F_{22}+\frac{2(k-15)}{3(k+5)^{2}} B_{3} B_{3} \\
& +\frac{2 i\left(3 k^{2}+22 k-15\right)}{3(k+5)^{2}} \partial B_{3}-\frac{4 i(k+9)}{3(k+5)^{3}} B_{3} F_{11} F_{22}+\frac{2 i}{(k+5)^{2}} B_{3} F_{12} F_{21} \\
& +\frac{8 i k}{3(k+5)^{3}} B_{-} F_{12} F_{22}-\frac{2(2 k+15)}{3(k+5)^{2}} B_{+} B_{-}-\frac{2 i(3 k+11)}{(k+5)^{3}} B_{+} F_{11} F_{21} \\
& +\frac{2(k-3)}{3(k+5)^{2}} F_{11} G_{22}-\frac{2\left(5 k^{2}+24 k+51\right)}{3(k+5)^{3}} \partial F_{11} F_{22} \\
& -\frac{2\left(k^{2}-12 k-141\right)}{3(k+5)^{3}} F_{11} \partial F_{22}-\frac{(k+9)}{(k+5)^{2}} F_{12} G_{21}+\frac{2\left(k^{2}-9 k-78\right)}{3(k+5)^{3}} \partial F_{12} F_{21} \\
& +\frac{2\left(5 k^{2}+31 k+102\right)}{3(k+5)^{3}} F_{12} \partial F_{21}+\frac{(k+3)}{(k+5)^{2}} F_{21} G_{12}+\frac{(k-3)}{3(k+5)^{2}} F_{22} G_{11} \\
& \text { - } \frac{6 i}{(k+5)^{2}} U A_{3}-\frac{2 i k}{(k+5)^{2}} U B_{3}-\frac{2(2 k+15)}{3(k+5)^{2}} U U \\
& \left.+\frac{8(k-3)}{3(k+5)^{3}} U F_{11} F_{22}-\frac{6}{(k+5)^{2}} U F_{12} F_{21}\right](w) \\
& +\frac{1}{(z-w)}\left[\frac{1}{2} \mathbf{S}^{(\mathbf{3})}+\frac{1}{2} \mathbf{P}^{(\mathbf{3})}+\frac{1}{4} \partial \mathbf{P}^{(\mathbf{2})}-\mathbf{W}^{(\mathbf{3})}-\frac{(k-3)}{3(k+5)} \partial \mathbf{T}^{(\mathbf{2})}\right. \\
& \text { - } \partial \mathbf{W}^{(\mathbf{2})}-\frac{2 i}{(k+5)^{2}} A_{3} A_{3} B_{3}-\frac{4(k+3)}{3(k+5)^{2}} \partial A_{3} A_{3} \\
& -\frac{4(2 k+9)}{3(k+5)^{2}} \partial A_{3} B_{3}+\frac{2(5 k+18)}{3(k+5)^{2}} A_{3} \partial B_{3}-\frac{2 i(k+3)}{3(k+5)^{2}} \partial^{2} A_{3} \\
& +\frac{i}{(k+5)^{2}} A_{3} F_{11} G_{22}-\frac{i(11 k+15)}{6(k+5)^{3}} \partial A_{3} F_{11} F_{22} \\
& +\frac{i(13 k+57)}{6(k+5)^{3}} A_{3} \partial F_{11} F_{22}+\frac{i(25 k+117)}{6(k+5)^{3}} A_{3} F_{11} \partial F_{22}-\frac{i}{(k+5)^{2}} A_{3} F_{12} G_{21} \\
& +\frac{i(7 k+27)}{2(k+5)^{3}} \partial A_{3} F_{12} F_{21}-\frac{i(k-3)}{2(k+5)^{3}} A_{3} \partial F_{12} F_{21}-\frac{i(5 k+17)}{2(k+5)^{3}} A_{3} F_{12} \partial F_{21} \\
& +\frac{i}{(k+5)^{2}} A_{3} F_{21} G_{12}-\frac{i}{(k+5)^{2}} A_{3} F_{22} G_{11}+\frac{5 i(k+9)}{3(k+5)^{3}} \partial A_{-} F_{11} F_{12} \\
& -\frac{i(k-15)}{3(k+5)^{3}} A_{-} \partial F_{11} F_{12}+\frac{i(11 k+75)}{3(k+5)^{3}} A_{-} F_{11} \partial F_{12}-\frac{2 i}{(k+5)^{2}} A_{+} A_{-} B_{3} \\
& -\frac{2(k+3)}{3(k+5)^{2}} \partial\left(A_{+} A_{-}\right)-\frac{4 i}{(k+5)^{3}} \partial\left(A_{+} F_{21} F_{22}\right)+\frac{2 i}{(k+5)^{2}} B_{3} B_{3} B_{3} \\
& +\frac{2(k-18)}{3(k+5)^{2}} \partial B_{3} B_{3}+\frac{i\left(14 k^{3}+80 k^{2}-445 k-459\right)}{3(k+5)^{2}(13 k+17)} \partial^{2} B_{3} \\
& +\frac{i}{(k+5)^{2}} B_{3} F_{11} G_{22}-\frac{i(13 k+105)}{6(k+5)^{3}} \partial B_{3} F_{11} F_{22}
\end{aligned}
$$




$$
\begin{aligned}
& -\frac{i(k-99)}{6(k+5)^{3}} B_{3} \partial F_{11} F_{22}+\frac{11 i(k-3)}{6(k+5)^{3}} B_{3} F_{11} \partial F_{22} \\
& +\frac{i}{(k+5)^{2}} B_{3} F_{12} G_{21}-\frac{i(k+13)}{2(k+5)^{3}} \partial B_{3} F_{12} F_{21}+\frac{i(3 k+55)}{2(k+5)^{3}} B_{3} \partial F_{12} F_{21} \\
& +\frac{i(7 k+11)}{2(k+5)^{3}} B_{3} F_{12} \partial F_{21}-\frac{\left(28 k^{3}+149 k^{2}-354 k-459\right)}{3(k+5)^{3}(13 k+17)} \partial F_{11} \partial F_{22} \\
& +\frac{3 i}{(k+5)^{2}} B_{3} F_{21} G_{12}+\frac{3 i}{(k+5)^{2}} B_{3} F_{22} G_{11}+\frac{i(11 k+15)}{6(k+5)^{3}} \partial B_{-} F_{12} F_{22} \\
& +\frac{i(11 k+15)}{6(k+5)^{3}} B_{-} \partial F_{12} F_{22}-\frac{i(k+45)}{6(k+5)^{3}} B_{-} F_{12} \partial F_{22}+\frac{2 i}{(k+5)^{2}} B_{+} B_{-} B_{3} \\
& -\frac{2(k+6)}{3(k+5)^{2}} \partial B_{+} B_{-}-\frac{2(k+9)}{3(k+5)^{2}} B_{+} \partial B_{-}-\frac{i(7 k+27)}{2(k+5)^{3}} \partial B_{+} F_{11} F_{21} \\
& -\frac{i(7 k+27)}{2(k+5)^{3}} B_{+} \partial F_{11} F_{21}-\frac{i(3 k+7)}{2(k+5)^{3}} B_{+} F_{11} \partial F_{21} \\
& -\frac{\left(80 k^{3}+217 k^{2}+426 k+561\right)}{6(k+5)^{3}(13 k+17)} \partial^{2} F_{11} F_{22} \\
& +\frac{\left(8 k^{3}+285 k^{2}+1930 k+1989\right)}{2(k+5)^{3}(13 k+17)} F_{11} \partial^{2} F_{22}+\frac{(13 k-3)}{12(k+5)^{2}} \partial F_{11} G_{22} \\
& +\frac{(k-15)}{12(k+5)^{2}} F_{11} \partial G_{22}-\frac{\left(8 k^{2}+219 k+255\right)}{2(k+5)^{2}(13 k+17)} \partial^{2} F_{12} F_{21} \\
& +\frac{\left(28 k^{3}+279 k^{2}+1298 k+1479\right)}{3(k+5)^{3}(13 k+17)} \partial F_{12} \partial F_{21} \\
& +\frac{\left(80 k^{3}+399 k^{2}+1990 k+2295\right)}{6(k+5)^{3}(13 k+17)} F_{12} \partial^{2} F_{21}-\frac{(5 k+21)}{4(k+5)^{2}} \partial F_{12} G_{21} \\
& +\frac{(2 k+15)}{3(k+5)} \partial T-\frac{2\left(4 k^{2}+25 k+17\right)}{(k+5)^{2}(13 k+17)} T F_{11} F_{22} \\
& +\frac{(k+17)}{(k+5)^{2}} U F_{11} G_{22}-\frac{(k+21)}{6(k+5)^{3}} \partial U F_{11} F_{22}+\frac{(23 k+3)}{6(k+5)^{3}} U \partial F_{11} F_{22} \\
& +\frac{k}{(k+5)^{2}} F_{12} \partial G_{21}+\frac{1}{2(k+5)} G_{11} G_{22}-\frac{1}{2(k+5)} G_{12} G_{21} \\
& +\frac{(k-6)}{(k+5)^{2}} \partial\left(U F_{21} G_{12}+\frac{(k+4)}{2(k+5)^{2}} F_{21} \partial G_{12}+\frac{(k+6)}{6(k+5)^{2}} \partial F_{22} G_{11} \partial G_{11}+\frac{2 i\left(4 k^{2}+34 k+17\right)}{(k+5)(3 k+17)} T B_{3}\right. \\
& +
\end{aligned}
$$




$$
\begin{aligned}
& +\frac{(11 k-57)}{6(k+5)^{3}} U F_{11} \partial F_{22}-\frac{1}{(k+5)^{2}} U F_{12} G_{21}-\frac{(3 k+31)}{2(k+5)^{3}} \partial U F_{12} F_{21} \\
& -\frac{(11 k+39)}{2(k+5)^{3}} U \partial F_{12} F_{21}-\frac{(7 k+19)}{2(k+5)^{3}} U F_{12} \partial F_{21}+\frac{1}{(k+5)^{2}} U F_{21} G_{12} \\
& \left.-\frac{1}{(k+5)^{2}} U F_{22} G_{11}+\frac{2 i}{(k+5)^{2}} U U B_{3}-\frac{2(2 k+15)}{3(k+5)^{2}} \partial U U\right](w)+\cdots
\end{aligned}
$$

The nontrivial central term of this OPE appears. The nonlinear terms in the corresponding OPEs in the nonlinear version disappear in this OPE.

For the other higher spin current, one can obtain

$$
\begin{aligned}
& \mathbf{U}_{+}^{(2)}(z) \mathbf{V}^{\left(\frac{5}{2}\right)}(w)=\frac{1}{(z-w)^{4}}\left[\frac{12 k(k+9)}{(k+5)^{3}} F_{21}\right](w) \\
& +\frac{1}{(z-w)^{3}}\left[\frac{2\left(4 k^{2}+40 k+63\right)}{3(k+5)^{2}} G_{21}+\frac{4 i\left(4 k^{2}+25 k+9\right)}{3(k+5)^{3}} F_{11} A_{-}\right. \\
& +\frac{4\left(4 k^{2}+31 k+63\right)}{3(k+5)^{3}} U F_{21}-\frac{8(k+9)}{3(k+5)^{3}} F_{21} F_{11} F_{22} \\
& -\frac{4 i\left(4 k^{2}+25 k+9\right)}{3(k+5)^{3}} F_{21} A_{3}+\frac{4 i\left(2 k^{2}-k-63\right)}{3(k+5)^{3}} F_{22} B_{-} \\
& \left.+\frac{4 i\left(2 k^{2}+13 k+63\right)}{3(k+5)^{3}} F_{21} B_{3}\right](w) \\
& +\frac{1}{(z-w)^{2}}\left[-\frac{(7 k+39)}{3(k+5)}\left(\mathbf{P}_{+}^{\left(\frac{5}{2}\right)}+\mathbf{W}_{+}^{\left(\frac{5}{2}\right)}\right)\right. \\
& -\frac{4 i(k+3)}{3(k+5)^{2}} A_{3} G_{21}+\frac{16(k+6)}{3(k+5)^{3}} A_{3} B_{3} F_{21}-\frac{2(7 k+39)}{3(k+5)^{3}} A_{3} B_{-} F_{22} \\
& -\frac{16 i(k+3)(k+9)}{9(k+5)^{3}} \partial A_{3} F_{21}-\frac{2 i\left(8 k^{2}-9 k-189\right)}{9(k+5)^{3}} A_{3} \partial F_{21} \\
& -\frac{16(k+6)}{3(k+5)^{3}} A_{-} B_{3} F_{11}-\frac{2(7 k+39)}{3(k+5)^{3}} A_{-} B_{-} F_{12}+\frac{16 i(k+3)(k+9)}{9(k+5)^{3}} \partial A_{-} F_{11} \\
& +\frac{2 i\left(8 k^{2}-9 k-189\right)}{9(k+5)^{3}} A_{-} \partial F_{11}+\frac{2 i(k+21)}{3(k+5)^{2}} B_{3} G_{21}-\frac{8(k+3)}{3(k+5)^{3}} B_{3} B_{3} F_{21} \\
& -\frac{4 i\left(5 k^{2}+51 k+18\right)}{9(k+5)^{3}} \partial B_{3} F_{21}+\frac{2 i\left(32 k^{2}+225 k+117\right)}{9(k+5)^{3}} B_{3} \partial F_{21} \\
& -\frac{i(3 k+17)}{(k+5)^{2}} B_{-} G_{22}-\frac{2(k+9)}{3(k+5)^{3}} B_{-} B_{3} F_{22}+\frac{4 i(k+3)}{3(k+5)^{2}} A_{-} G_{11} \\
& +\frac{2 i\left(19 k^{2}+93 k-54\right)}{9(k+5)^{3}} \partial B_{-} F_{22}-\frac{4 i\left(13 k^{2}+90 k+99\right)}{9(k+5)^{3}} B_{-} \partial F_{22} \\
& -\frac{2(5 k+21)}{3(k+5)^{3}} B_{+} B_{-} F_{21}+\frac{4(k+9)^{2}}{9(k+5)^{4}} F_{11} \partial F_{21} F_{22}+\frac{4(k+3)}{3(k+5)^{3}} F_{12} F_{21} G_{21}
\end{aligned}
$$




$$
\begin{aligned}
& -\frac{2(k+9)}{3(k+5)^{3}} F_{11} F_{21} G_{22}-\frac{8\left(13 k^{2}+114 k+261\right)}{9(k+5)^{4}} \partial F_{11} F_{21} F_{22} \\
& +\frac{4\left(31 k^{2}+294 k+711\right)}{9(k+5)^{4}} F_{11} F_{21} \partial F_{22}+\frac{8(k+6)}{3(k+5)^{3}} F_{11} F_{22} G_{21} \\
& -\frac{2\left(4 k^{2}+27 k+27\right)}{3(k+5)^{3}} \partial^{2} F_{21}-\frac{4}{(k+5)^{2}} F_{21} F_{22} G_{11} \\
& +\frac{4(k+3)(2 k+15)}{9(k+5)^{2}} \partial G_{21}+\frac{4(2 k+15)}{3(k+5)^{2}} T F_{21} \\
& +\frac{2(2 k+9)}{3(k+5)^{2}} U G_{21}-\frac{16 i(k+6)}{3(k+5)^{3}} U A_{3} F_{21}+\frac{16 i(k+6)}{3(k+5)^{3}} U A_{-} F_{11} \\
& -\frac{2 i(13 k+69)}{3(k+5)^{3} U B_{-} F_{22}+\frac{8(k+3)(2 k+15)}{9(k+5)^{3}} \partial U F_{21}} \\
& +\frac{2\left(8 k^{2}+39 k+99\right)}{9(k+5)^{3}} U \partial F_{21}+\frac{8 i}{(k+5)^{2}} U B_{3} F_{21} \\
& \left.+\frac{16(k+6)}{3(k+5)^{3}} U U F_{21}+\frac{4\left(9 k^{2}+82 k+201\right)}{3(k+5)^{4}} F_{21} \partial F_{21} F_{12}\right](w) \\
& +\frac{1}{(z-w)}[+\cdots](w)+\cdots
\end{aligned}
$$

There is no higher spin current in the third order pole of this OPE.

The result (3.6) determines the following OPEs

$$
\begin{aligned}
\left(\begin{array}{c}
\mathbf{U}_{+}^{(\mathbf{2})} \\
\mathbf{V}_{-}^{(\mathbf{2})}
\end{array}\right)(z) \mathbf{W}^{(\mathbf{2})}(w) & =\frac{1}{(z-w)^{3}}\left[-\frac{2 i k(k+8)}{(k+5)^{2}} B_{\mp} \pm \frac{4 k}{(k+5)^{2}}\left(\begin{array}{c}
F_{11} F_{21} \\
F_{22} F_{12}
\end{array}\right)\right](w) \\
& +\frac{1}{(z-w)^{2}}\left[\mp \frac{2(k+4)}{(k+5)^{2}} A_{3} B_{\mp}-\frac{2 i}{(k+5)^{2}} A_{3}\left(\begin{array}{c}
F_{11} F_{21} \\
F_{22} F_{12}
\end{array}\right)\right. \\
& \mp \frac{2 k}{(k+5)^{2}} B_{\mp} B_{3}-\frac{i k(k+9)}{(k+5)^{2}} \partial B_{\mp} \\
& \pm \frac{i}{(k+5)^{2}} B_{\mp} F_{11} F_{22} \pm \frac{i}{(k+5)^{2}} B_{\mp} F_{12} F_{21} \\
& \mp \frac{(k-3)}{2(k+5)^{2}}\left(\begin{array}{c}
F_{11} G_{21} \\
F_{22} G_{12}
\end{array}\right) \pm \frac{2(k+1)^{2}}{(k+5)^{3}}\left(\begin{array}{c}
\partial F_{11} F_{21} \\
\partial F_{22} F_{12}
\end{array}\right) \\
& \left. \pm \frac{2(k+8 k-1)}{(k+5)^{3}}\left(\begin{array}{c}
F_{11} \partial F_{21} \\
F_{22} \partial F_{12}
\end{array}\right) \mp \frac{(k-3)}{2(k+5)^{2}}\left(\begin{array}{c}
F_{21} G_{11} \\
F_{12} G_{22}
\end{array}\right)\right](w) \\
& +\frac{1}{(z-w)}\left[-\frac{1}{2}\left(\begin{array}{c}
\mathbf{Q}_{+}^{(3)} \\
\mathbf{R}_{-}^{(3)}
\end{array}\right)+\frac{i}{(k+5)^{2}} A_{3} A_{3} B_{\mp} \pm \frac{(3 k+13)}{2(k+5)^{2}} \partial A_{3} B_{\mp}\right. \\
& \mp \frac{(7 k+31)}{2(k+5)^{2}} A_{3} \partial B_{\mp}-\frac{i}{(k+5)^{2}} A_{3}\left(\begin{array}{c}
F_{11} G_{21} \\
F_{22} G_{12}
\end{array}\right) \\
& +\frac{i(5 k+17)}{2(k+5)^{3}} \partial A_{3}\left(\begin{array}{c}
F_{11} F_{21} \\
F_{22} F_{12}
\end{array}\right)-\frac{i(7 k+27)}{2(k+5)^{3}} A_{3}\left(\begin{array}{c}
\partial F_{11} F_{21} \\
\partial F_{22} F_{12}
\end{array}\right)
\end{aligned}
$$




$$
\begin{aligned}
& -\frac{i(11 k+47)}{2(k+5)^{3}} A_{3}\left(\begin{array}{c}
F_{11} \partial F_{21} \\
F_{22} \partial F_{12}
\end{array}\right)+\frac{i}{(k+5)^{2}} A_{\mp}\left(\begin{array}{c}
F_{11} G_{11} \\
F_{22} G_{22}
\end{array}\right) \\
& +\frac{i}{(k+5)^{2}} A_{3}\left(\begin{array}{c}
F_{21} G_{11} \\
F_{12} G_{22}
\end{array}\right)+\frac{i}{(k+5)^{2}} A_{ \pm} A_{\mp} B_{\mp} \\
& +\frac{i}{(k+5)^{2}} A_{ \pm}\left(\begin{array}{c}
F_{21} G_{21} \\
F_{12} G_{12}
\end{array}\right)-\frac{i}{(k+5)^{2}} B_{3}\left(\begin{array}{c}
F_{11} G_{21} \\
F_{22} G_{12}
\end{array}\right) \\
& -\frac{i}{2(k+5)^{2}} \partial B_{3}\left(\begin{array}{c}
F_{11} F_{21} \\
F_{22} F_{12}
\end{array}\right)-\frac{i(k-15)}{2(k+5)^{3}} B_{\mp}\left(\begin{array}{c}
F_{12} \partial F_{21} \\
F_{21} \partial F_{12}
\end{array}\right) \\
& -\frac{i(k+21)}{2(k+5)^{3}} B_{3}\left(\begin{array}{c}
\partial F_{11} F_{21} \\
\partial F_{22} F_{12}
\end{array}\right)+\frac{i(3 k+31)}{2(k+5)^{3}} B_{3}\left(\begin{array}{c}
F_{11} \partial F_{21} \\
F_{22} \partial F_{12}
\end{array}\right) \\
& -\frac{i}{(k+5)^{2}} B_{3}\left(\begin{array}{c}
F_{21} G_{11} \\
F_{12} G_{22}
\end{array}\right)-\frac{i}{(k+5)^{2}} B_{\mp} B_{3} B_{3} \\
& \mp \frac{(k-1)}{(k+5)^{2}} \partial B_{\mp} B_{3} \mp \frac{(k-2)}{(k+5)^{2}} B_{\mp} \partial B_{3} \\
& -\frac{i(7 k+6)\left(2 k^{2}+19 k-51\right)}{6(k+5)^{2}(13 k+17)} \partial^{2} B_{\mp} \\
& -\frac{6 i}{(k+5)^{3}} B_{\mp}\left(\begin{array}{c}
\partial F_{11} F_{22} \\
\partial F_{22} F_{11}
\end{array}\right)+\frac{2 i(k+6)}{3(k+5)^{3}} \partial B_{\mp} F_{11} F_{22} \\
& -\frac{2 i(k+4)}{(k+5)^{3}} B_{\mp}\left(\begin{array}{c}
F_{11} \partial F_{22} \\
F_{22} \partial F_{11}
\end{array}\right)-\frac{i}{(k+5)^{2}} B_{\mp}\left(\begin{array}{c}
F_{12} G_{21} \\
F_{21} G_{12}
\end{array}\right) \\
& -\frac{i(k+33)}{2(k+5)^{3}} B_{\mp}\left(\begin{array}{c}
\partial F_{12} F_{21} \\
\partial F_{21} F_{12}
\end{array}\right) \pm \frac{i(3 k+19)}{2(k+5)^{3}} \partial B_{\mp} F_{12} F_{21} \\
& -\frac{2 i}{(k+5)^{2}} B_{\mp}\left(\begin{array}{c}
F_{21} G_{12} \\
F_{12} G_{21}
\end{array}\right)-\frac{i}{(k+5)^{2}} B_{\mp}\left(\begin{array}{c}
F_{22} G_{11} \\
F_{11} G_{22}
\end{array}\right) \\
& \mp \frac{(7 k+19)}{(k+5)^{3}}\left(\begin{array}{c}
\partial^{2} F_{11} F_{21} \\
\partial^{2} F_{22} F_{12}
\end{array}\right)-\frac{i}{(k+5)^{2}} B_{ \pm} B_{\mp} B_{\mp} \\
& \pm \frac{\left(28 k^{3}+71 k^{2}-846 k-969\right)}{6(k+5)^{3}(13 k+17)}\left(\begin{array}{l}
F_{11} \partial^{2} F_{21} \\
F_{22} \partial^{2} F_{12}
\end{array}\right) \\
& \mp \frac{(7 k+19)}{4(k+5)^{2}}\left(\begin{array}{c}
\partial F_{11} G_{21} \\
\partial F_{22} G_{12}
\end{array}\right) \pm \frac{1}{4(k+5)}\left(\begin{array}{c}
F_{11} \partial G_{21} \\
F_{22} \partial G_{12}
\end{array}\right) \\
& \mp \frac{(2 k+11)}{2(k+5)^{2}}\left(\begin{array}{c}
\partial F_{21} G_{11} \\
\partial F_{12} G_{22}
\end{array}\right) \pm \frac{3}{2(k+5)^{2}}\left(\begin{array}{c}
F_{21} \partial G_{11} \\
F_{12} \partial G_{22}
\end{array}\right) \\
& -\frac{i\left(4 k^{2}+34 k+17\right)}{(k+5)(13 k+17)} T B_{\mp} \pm \frac{2\left(4 k^{2}+25 k+17\right)}{(k+5)^{2}(13 k+17)} T\left(\begin{array}{c}
F_{11} F_{21} \\
F_{22} F_{12}
\end{array}\right) \\
& \pm \frac{2}{(k+5)^{2}} U A_{3} B_{\mp}-\frac{i(k-2)}{2(k+5)^{2}} \partial U B_{\mp}+\frac{i(k-2)}{2(k+5)^{2}} U \partial B_{\mp} \\
& \mp \frac{(9 k+29)}{2(k+5)^{3}} U\left(\begin{array}{c}
\partial F_{11} F_{21} \\
\partial F_{22} F_{12}
\end{array}\right) \mp \frac{1}{(k+5)^{2}} U\left(\begin{array}{c}
F_{11} G_{21} \\
F_{22} G_{12}
\end{array}\right)
\end{aligned}
$$




$$
\begin{aligned}
& \pm \frac{(3 k-1)}{2(k+5)^{3}} \partial U\left(\begin{array}{c}
F_{11} F_{21} \\
F_{22} F_{12}
\end{array}\right) \pm \frac{(3 k+31)}{2(k+5)^{3}} U\left(\begin{array}{c}
F_{11} \partial F_{21} \\
F_{22} \partial F_{12}
\end{array}\right) \\
& \pm \frac{1}{(k+5)^{2}} U\left(\begin{array}{c}
F_{21} G_{11} \\
F_{12} G_{22}
\end{array}\right)-\frac{i}{(k+5)^{2}} U U B_{\mp} \\
& +\frac{4 i(k+3)}{(k+5)^{3}}\left(\begin{array}{c}
F_{11} A_{-} \partial F_{11} \\
F_{22} A_{+} \partial F_{22}
\end{array}\right) \mp \frac{1}{2(k+5)}\left(\begin{array}{c}
G_{11} G_{21} \\
G_{22} G_{12}
\end{array}\right) \\
& -\frac{i\left(28 k^{3}+227 k^{2}+450 k+459\right)}{3(k+5)^{3}(13 k+17)}\left(\begin{array}{c}
F_{21} A_{+} \partial F_{21} \\
F_{12} A_{-} \partial F_{12}
\end{array}\right) \\
& \left.-\frac{i\left(28 k^{3}+305 k^{2}+630 k+561\right)}{3(k+5)^{3}(13 k+17)}\left(\begin{array}{l}
\partial F_{21} A_{+} F_{21} \\
\partial F_{12} A_{-} F_{12}
\end{array}\right)\right](w)+\cdots
\end{aligned}
$$

There is no higher spin current in the second order pole.

The result (3.8) determines the following OPEs

$$
\begin{aligned}
\left(\begin{array}{c}
\mathbf{U}_{+}^{(\mathbf{2})} \\
\mathbf{V}_{-}^{(\mathbf{2})}
\end{array}\right)(z) \mathbf{W}_{ \pm}^{\left(\frac{5}{2}\right)}(w) & =\frac{1}{(z-w)^{3}}\left[-\frac{16 i k(k+3)}{3(k+5)^{3}}\left(\begin{array}{c}
F_{21} \\
F_{12}
\end{array}\right) B_{\mp}\right](w) \\
& +\frac{1}{(z-w)^{2}}\left[\mp \frac{2(k+9)}{3(k+5)^{3}} A_{3} B_{\mp}\left(\begin{array}{c}
F_{21} \\
F_{12}
\end{array}\right)\right. \\
& \pm \frac{2(k+9)}{3(k+5)^{3}} A_{\mp} B_{\mp}\left(\begin{array}{c}
F_{11} \\
F_{22}
\end{array}\right)+\frac{i(7 k+9)}{3(k+5)^{2}} B_{\mp}\left(\begin{array}{c}
G_{21} \\
G_{12}
\end{array}\right) \\
& \mp \frac{2(k+9)}{3(k+5)^{3}} B_{\mp} B_{3}\left(\begin{array}{c}
F_{21} \\
F_{12}
\end{array}\right) \pm \frac{2(k+9)}{3(k+5)^{3}} B_{\mp} B_{\mp}\left(\begin{array}{c}
F_{22} \\
F_{11}
\end{array}\right) \\
& -\frac{2 i(k+9)(2 k-3)}{3(k+5)^{3}} B_{\mp} \partial\left(\begin{array}{c}
F_{21} \\
F_{12}
\end{array}\right) \mp \frac{2(k+9)}{3(k+5)^{3}}\left(\begin{array}{c}
F_{11} F_{21} G_{21} \\
F_{22} F_{12} G_{12}
\end{array}\right) \\
& +\frac{2 i(k+9)}{3(k+5)^{3}} U B_{\mp}\left(\begin{array}{c}
F_{21} \\
F_{12}
\end{array}\right)-\frac{2 i k(k+1)}{(k+5)^{3}} \partial B_{\mp}\left(\begin{array}{c}
F_{21} \\
F_{12}
\end{array}\right) \\
\mp & \left.\frac{4(k+1)(k+9)}{3(k+5)^{4}}\left(\begin{array}{c}
F_{11} F_{21} \partial F_{21} \\
F_{22} F_{12} \partial F_{12}
\end{array}\right)\right](w) \\
& +\frac{1}{(z-w)}\left[\begin{array}{l}
1 \\
(z)](w)+\cdots .
\end{array}\right.
\end{aligned}
$$

It is an open problem to obtain the first order pole of these OPEs. The nonlinear terms in the corresponding OPEs in the nonlinear version disappear.

Similarly the other combination provides the following OPEs

$$
\begin{aligned}
\left(\begin{array}{c}
\mathbf{U}_{+}^{(2)} \\
\mathbf{V}_{-}^{(2)}
\end{array}\right)(z) \mathbf{W}_{\mp}^{\left(\frac{5}{2}\right)}(w) & =\frac{1}{(z-w)^{4}}\left[\mp \frac{12 k(k+9)}{(k+5)^{3}}\left(\begin{array}{c}
F_{11} \\
F_{22}
\end{array}\right)\right](w) \\
& +\frac{1}{(z-w)^{3}}\left[\mp \frac{2\left(4 k^{2}+40 k+63\right)}{3(k+5)^{2}}\left(\begin{array}{c}
G_{11} \\
G_{22}
\end{array}\right)\right.
\end{aligned}
$$




$$
\begin{aligned}
& \mp \frac{4\left(4 k^{2}+31 k+63\right)}{3(k+5)^{3}} U\left(\begin{array}{c}
F_{11} \\
F_{22}
\end{array}\right)-\frac{8(k+9)}{3(k+5)^{3}} F_{12} F_{21}\left(\begin{array}{c}
F_{11} \\
F_{22}
\end{array}\right) \\
& \text { - } \frac{4 i\left(4 k^{2}+25 k+9\right)}{3(k+5)^{3}}\left(\begin{array}{c}
F_{11} \\
F_{22}
\end{array}\right) A_{3}-\frac{4 i\left(4 k^{2}+25 k+9\right)}{3(k+5)^{3}}\left(\begin{array}{c}
F_{21} \\
F_{12}
\end{array}\right) A_{ \pm} \\
& \left.+\frac{4 i\left(2 k^{2}-k-63\right)}{3(k+5)^{3}}\left(\begin{array}{c}
F_{12} \\
F_{21}
\end{array}\right) B_{\mp}-\frac{4 i\left(2 k^{2}+13 k+63\right)}{3(k+5)^{3}}\left(\begin{array}{c}
F_{11} \\
F_{22}
\end{array}\right) B_{3}\right](w) \\
& +\frac{1}{(z-w)^{2}}\left[\frac{(7 k+39)}{3(k+5)}\left(\begin{array}{l}
\mathbf{Q}^{\left(\frac{5}{2}\right)} \\
\mathbf{R}^{\left(\frac{5}{2}\right)}
\end{array}\right) \pm \frac{(7 k+39)}{3(k+5)}\left(\begin{array}{l}
\mathbf{U}^{\left(\frac{5}{2}\right)} \\
\mathbf{V}^{\left(\frac{5}{2}\right)}
\end{array}\right)\right. \\
& -\frac{4 i(k+3)}{3(k+5)^{2}} A_{3}\left(\begin{array}{c}
G_{11} \\
G_{22}
\end{array}\right) \pm \frac{16(k+6)}{3(k+5)^{3}} A_{3} B_{3}\left(\begin{array}{c}
F_{11} \\
F_{22}
\end{array}\right) \\
& \pm \frac{2(7 k+39)}{3(k+5)^{3}} A_{3} B_{\mp}\left(\begin{array}{c}
F_{12} \\
F_{21}
\end{array}\right) \mp \frac{2(7 k+39)}{3(k+5)^{3}} A_{ \pm} B_{\mp}\left(\begin{array}{c}
F_{22} \\
F_{11}
\end{array}\right) \\
& \text { - } \frac{16 i(k+3)(k+9)}{9(k+5)^{3}} \partial A_{3}\left(\begin{array}{c}
F_{11} \\
F_{22}
\end{array}\right)-\frac{2 i\left(8 k^{2}-9 k-189\right)}{9(k+5)^{3}} A_{3} \partial\left(\begin{array}{c}
F_{11} \\
F_{22}
\end{array}\right) \\
& -\frac{4 i(k+3)}{3(k+5)^{2}} A_{ \pm}\left(\begin{array}{c}
G_{21} \\
G_{12}
\end{array}\right) \pm \frac{16(k+6)}{3(k+5)^{3}} A_{ \pm} B_{3}\left(\begin{array}{c}
F_{21} \\
F_{12}
\end{array}\right) \\
& \text { - } \frac{16 i(k+3)(k+9)}{9(k+5)^{3}} \partial A_{ \pm}\left(\begin{array}{c}
F_{21} \\
F_{12}
\end{array}\right)-\frac{2 i\left(8 k^{2}-9 k-189\right)}{9(k+5)^{3}} A_{ \pm} \partial\left(\begin{array}{c}
F_{21} \\
F_{12}
\end{array}\right) \\
& -\frac{2 i(k+21)}{3(k+5)^{2}} B_{3}\left(\begin{array}{l}
G_{11} \\
G_{22}
\end{array}\right)-\frac{2 i\left(32 k^{2}+225 k+117\right)}{9(k+5)^{3}} B_{3} \partial\left(\begin{array}{c}
F_{11} \\
F_{22}
\end{array}\right) \\
& -\frac{i(3 k+17)}{(k+5)^{2}} B_{\mp}\left(\begin{array}{c}
G_{12} \\
G_{21}
\end{array}\right) \mp \frac{2(k+9)}{3(k+5)^{3}} B_{\mp} B_{3}\left(\begin{array}{c}
F_{12} \\
F_{21}
\end{array}\right) \\
& +\frac{4 i\left(5 k^{2}+51 k+18\right)}{9(k+5)^{3}} \partial B_{3}\left(\begin{array}{c}
F_{11} \\
F_{22}
\end{array}\right) \pm \frac{8(k+3)}{3(k+5)^{3}} B_{3} B_{3}\left(\begin{array}{c}
F_{11} \\
F_{22}
\end{array}\right) \\
& +\frac{2 i\left(19 k^{2}+93 k-54\right)}{9(k+5)^{3}} \partial B_{\mp}\left(\begin{array}{c}
F_{12} \\
F_{21}
\end{array}\right) \\
& \text { - } \frac{4 i\left(13 k^{2}+90 k+99\right)}{9(k+5)^{3}} B_{\mp} \partial\left(\begin{array}{c}
F_{12} \\
F_{21}
\end{array}\right) \\
& \pm \frac{2(5 k+21)}{3(k+5)^{3}} B_{ \pm} B_{\mp}\left(\begin{array}{c}
F_{11} \\
F_{22}
\end{array}\right) \pm \frac{2\left(4 k^{2}+27 k+27\right)}{3(k+5)^{3}} \partial^{2}\left(\begin{array}{c}
F_{11} \\
F_{22}
\end{array}\right) \\
& \pm \frac{4}{(k+5)^{2}}\left(\begin{array}{c}
F_{11} F_{12} G_{21} \\
F_{22} F_{21} G_{12}
\end{array}\right)-\frac{4(k+9)^{2}}{9(k+5)^{4}} \partial\left(\begin{array}{c}
F_{11} \\
F_{22}
\end{array}\right) F_{12} F_{21} \\
& \mp \frac{4\left(31 k^{2}+294 k+711\right)}{9(k+5)^{4}}\left(\begin{array}{l}
F_{11} \partial F_{12} F_{21} \\
F_{22} \partial F_{21} F_{12}
\end{array}\right) \\
& \pm \frac{8\left(13 k^{2}+114 k+261\right)}{9(k+5)^{4}}\left(\begin{array}{l}
F_{11} F_{12} \partial F_{21} \\
F_{22} F_{21} \partial F_{12}
\end{array}\right)
\end{aligned}
$$




$$
\begin{aligned}
& \mp \frac{2(k+9)}{3(k+5)^{3}}\left(\begin{array}{c}
F_{11} F_{21} G_{12} \\
F_{22} F_{12} G_{21}
\end{array}\right) \pm \frac{4(k+3)}{3(k+5)^{3}}\left(\begin{array}{c}
F_{11} F_{22} G_{11} \\
F_{22} F_{11} G_{22}
\end{array}\right) \\
& \pm \frac{8(k+6)}{3(k+5)^{3}}\left(\begin{array}{c}
F_{12} F_{21} G_{11} \\
F_{21} F_{12} G_{22}
\end{array}\right) \mp \frac{4(k+3)(2 k+15)}{9(k+5)^{2}} \partial\left(\begin{array}{l}
G_{11} \\
G_{22}
\end{array}\right) \\
& \mp \frac{4(2 k+15)}{3(k+5)^{2}} T\left(\begin{array}{c}
F_{11} \\
F_{22}
\end{array}\right) \mp \frac{2(2 k+9)}{3(k+5)^{2}} U\left(\begin{array}{c}
G_{11} \\
G_{22}
\end{array}\right) \\
& -\frac{16 i(k+6)}{3(k+5)^{3}} U A_{3}\left(\begin{array}{c}
F_{11} \\
F_{22}
\end{array}\right)-\frac{16 i(k+6)}{3(k+5)^{3}} U A_{ \pm}\left(\begin{array}{c}
F_{21} \\
F_{12}
\end{array}\right) \\
& -\frac{8 i}{(k+5)^{2}} U B_{3}\left(\begin{array}{c}
F_{11} \\
F_{22}
\end{array}\right)-\frac{2 i(13 k+69)}{3(k+5)^{3}} U B_{\mp}\left(\begin{array}{c}
F_{12} \\
F_{21}
\end{array}\right) \\
& \mp \frac{8(k+3)(2 k+15)}{9(k+5)^{3}} \partial U\left(\begin{array}{l}
F_{11} \\
F_{22}
\end{array}\right) \\
& \mp \frac{2\left(8 k^{2}+39 k+99\right)}{9(k+5)^{3}} U \partial\left(\begin{array}{l}
F_{11} \\
F_{22}
\end{array}\right) \mp \frac{16(k+6)}{3(k+5)^{3}} U U\left(\begin{array}{c}
F_{11} \\
F_{22}
\end{array}\right) \\
& \left.+\frac{4\left(9 k^{2}+82 k+201\right)}{3(k+5)^{4}}\left(\begin{array}{l}
\partial F_{11} \\
\partial F_{22}
\end{array}\right) F_{11} F_{22}\right](w) \\
& +\frac{1}{(z-w)}[+\cdots](w)+\cdots
\end{aligned}
$$

There is no higher spin current in the third order pole.

One also determines the following OPEs from the information on the higher spin-3 current in (3.9)

$$
\begin{aligned}
& \left(\begin{array}{c}
\mathbf{U}_{+}^{(2)} \\
\mathbf{V}_{-}^{(\mathbf{2})}
\end{array}\right)(z) \mathbf{W}^{(\mathbf{3})}(w)=\frac{1}{(z-w)^{4}}\left[ \pm \frac{12 i k\left(8 k^{2}+75 k+95\right)}{(k+5)^{2}(13 k+17)} B_{\mp}\right. \\
& \left.-\frac{96 k\left(2 k^{2}+9 k+11\right)}{(k+5)^{3}(13 k+17)}\left(\begin{array}{c}
F_{11} F_{21} \\
F_{22} F_{12}
\end{array}\right)\right](w) \\
& +\frac{1}{(z-w)^{3}}\left[ \pm \frac{8 i\left(25 k^{2}+158 k-27\right)}{3(k+5)^{3}(13 k+17)} B_{3}\left(\begin{array}{c}
F_{11} F_{21} \\
F_{22} F_{12}
\end{array}\right)\right. \\
& -\frac{4 i\left(25 k^{2}+158 k-27\right)}{3(k+5)^{3}(13 k+17)} B_{\mp} F_{11} F_{22}+\frac{4 i\left(25 k^{2}+158 k-27\right)}{3(k+5)^{3}(13 k+17)} B_{\mp} F_{12} F_{21} \\
& +\frac{2\left(103 k^{2}+260 k-27\right)}{3(k+5)^{2}(13 k+17)}\left(\begin{array}{c}
F_{11} G_{21} \\
F_{22} G_{12}
\end{array}\right)+\frac{8\left(25 k^{2}+158 k-27\right)}{3(k+5)^{3}(13 k+17)}\left(\begin{array}{l}
\partial F_{11} F_{21} \\
\partial F_{22} F_{12}
\end{array}\right) \\
& +\frac{8\left(25 k^{2}+158 k-27\right)}{3(k+5)^{3}(13 k+17)}\left(\begin{array}{c}
F_{11} \partial F_{21} \\
F_{22} \partial F_{12}
\end{array}\right)-\frac{2\left(103 k^{2}+260 k-27\right)}{3(k+5)^{2}(13 k+17)}\left(\begin{array}{c}
F_{21} G_{11} \\
F_{12} G_{22}
\end{array}\right) \\
& \left. \pm \frac{8 i k}{(k+5)^{2}} U B_{\mp}+\frac{8\left(25 k^{2}+158 k-27\right)}{3(k+5)^{3}(13 k+17)} U\left(\begin{array}{c}
F_{11} F_{21} \\
F_{22} F_{12}
\end{array}\right)\right](w) \\
& +\frac{1}{(z-w)^{2}}\left[ \pm 3\left(\begin{array}{c}
\mathbf{Q}_{+}^{(3)} \\
\mathbf{R}_{-}^{(3)}
\end{array}\right)-\frac{8(\mathbf{k}-\mathbf{3})}{(13 k+17)} \mathbf{T}^{(\mathbf{1})}\left(\begin{array}{c}
\mathbf{U}_{+}^{(\mathbf{2})} \\
\mathbf{V}_{-}^{(\mathbf{2})}
\end{array}\right)\right.
\end{aligned}
$$




$$
\begin{aligned}
& \mp \frac{6 i}{(k+5)^{2}} A_{3} A_{3} B_{\mp}-\frac{3(3 k+11)}{(k+5)^{2}} \partial A_{3} B_{\mp} \\
& +\frac{3(3 k+13)}{(k+5)^{2}} A_{3} \partial B_{\mp} \pm \frac{8 i}{(k+5)^{2}} A_{3}\left(\begin{array}{c}
F_{11} G_{21} \\
F_{22} G_{12}
\end{array}\right) \\
& \mp \frac{3 i(5 k+17)}{(k+5)^{3}} \partial A_{3}\left(\begin{array}{c}
F_{11} F_{21} \\
F_{22} F_{12}
\end{array}\right) \pm \frac{i(5 k+17)}{(k+5)^{3}} A_{3}\left(\begin{array}{l}
\partial F_{11} F_{21} \\
\partial F_{22} F_{12}
\end{array}\right) \\
& \pm \frac{5 i(5 k+17)}{(k+5)^{3}} A_{3}\left(\begin{array}{c}
F_{11} \partial F_{21} \\
F_{22} \partial F_{12}
\end{array}\right) \mp \frac{2 i}{(k+5)^{2}} A_{\mp}\left(\begin{array}{c}
F_{11} G_{11} \\
F_{22} G_{22}
\end{array}\right) \\
& \mp \frac{4 i}{(k+5)^{2}} A_{3}\left(\begin{array}{c}
F_{21} G_{11} \\
F_{12} G_{22}
\end{array}\right) \mp \frac{6 i}{(k+5)^{2}} A_{ \pm} A_{\mp} B_{\mp} \\
& \pm \frac{2 i}{(k+5)^{2}} A_{ \pm}\left(\begin{array}{c}
F_{21} G_{21} \\
F_{12} G_{12}
\end{array}\right) \pm \frac{2 i}{(k+5)^{2}} B_{3}\left(\begin{array}{c}
F_{11} G_{21} \\
F_{22} G_{12}
\end{array}\right) \\
& \pm \frac{i\left(269 k^{2}+1906 k+1269\right)}{3(k+5)^{3}(13 k+17)} \partial B_{3}\left(\begin{array}{c}
F_{11} F_{21} \\
F_{22} F_{12}
\end{array}\right) \\
& \pm \frac{i\left(113 k^{2}+298 k-567\right)}{3(k+5)^{3}(13 k+17)} B_{3}\left(\begin{array}{c}
\partial F_{11} F_{21} \\
\partial F_{22} F_{12}
\end{array}\right) \\
& \mp \frac{i(5 k+9)(71 k+403)}{3(k+5)^{3}(13 k+17)} B_{3}\left(\begin{array}{c}
F_{11} \partial F_{21} \\
F_{22} \partial F_{12}
\end{array}\right) \\
& \mp \frac{2 i}{(k+5)^{2}} B_{3}\left(\begin{array}{c}
F_{21} G_{11} \\
F_{12} G_{22}
\end{array}\right) \mp \frac{i}{(k+5)^{2}} B_{\mp}\left(\begin{array}{c}
F_{12} G_{21} \\
F_{21} G_{12}
\end{array}\right) \\
& -\frac{6(k+1)}{(k+5)^{2}} \partial B_{\mp} B_{3}+\frac{6(k+2)}{(k+5)^{2}} B_{\mp} \partial B_{3} \\
& \mp \frac{i\left(8 k^{3}+63 k^{2}+177 k-170\right)}{(k+5)^{2}(13 k+17)} \partial^{2} B_{\mp} \mp \frac{3 i}{(k+5)^{2}} B_{\mp}\left(\begin{array}{c}
F_{11} G_{22} \\
F_{22} G_{11}
\end{array}\right) \\
& \pm \frac{4 i\left(59 k^{2}+619 k+804\right)}{3(k+5)^{3}(13 k+17)} B_{\mp}\left(\begin{array}{c}
\partial F_{11} F_{22} \\
\partial F_{22} F_{11}
\end{array}\right) \\
& -\frac{2 i\left(155 k^{2}+1264 k+1197\right)}{3(k+5)^{3}(13 k+17)} \partial B_{\mp} F_{11} F_{22} \\
& \pm \frac{4 i\left(59 k^{2}+463 k+600\right)}{3(k+5)^{3}(13 k+17)} B_{\mp}\left(\begin{array}{c}
F_{11} \partial F_{22} \\
F_{22} \partial F_{11}
\end{array}\right) \\
& \mp \frac{i\left(119 k^{2}+802 k+1227\right)}{3(k+5)^{3}(13 k+17)} B_{\mp}\left(\begin{array}{c}
F_{12} \partial F_{21} \\
F_{21} \partial F_{12}
\end{array}\right) \\
& \pm \frac{i\left(349 k^{2}+2774 k+2649\right)}{3(k+5)^{3}(13 k+17)} B_{\mp}\left(\begin{array}{c}
\partial F_{12} F_{21} \\
\partial F_{21} F_{12}
\end{array}\right) \\
& -\frac{i\left(41 k^{2}+622 k+1125\right)}{3(k+5)^{3}(13 k+17)} \partial B_{\mp} F_{12} F_{21} \mp \frac{2 i}{(k+5)^{2}} B_{\mp} B_{3} B_{3} \\
& \pm \frac{3 i}{(k+5)^{2}} B_{\mp}\left(\begin{array}{c}
F_{21} G_{12} \\
F_{12} G_{21}
\end{array}\right) \pm \frac{5 i}{(k+5)^{2}} B_{\mp}\left(\begin{array}{c}
F_{22} G_{11} \\
F_{11} G_{22}
\end{array}\right)
\end{aligned}
$$




$$
\begin{aligned}
& +\frac{2(41 k+61)}{(k+5)^{3}}\left(\begin{array}{c}
\partial^{2} F_{11} F_{21} \\
\partial^{2} F_{22} F_{12}
\end{array}\right) \mp \frac{2 i}{(k+5)^{2}} B_{ \pm} B_{\mp} B_{\mp} \\
& +\frac{\left(48 k^{3}+1811 k^{2}+5374 k+2187\right)}{3(k+5)^{3}(13 k+17)}\left(\begin{array}{l}
F_{11} \partial^{2} F_{21} \\
F_{22} \partial^{2} F_{12}
\end{array}\right) \\
& +\frac{\left(583 k^{2}+38 k-1329\right)}{6(k+5)^{2}(13 k+17)}\left(\begin{array}{l}
\partial F_{11} G_{21} \\
\partial F_{22} G_{12}
\end{array}\right)-\frac{\left(41 k^{2}-470 k-303\right)}{6(k+5)^{2}(13 k+17)}\left(\begin{array}{c}
F_{11} \partial G_{21} \\
F_{22} \partial G_{12}
\end{array}\right) \\
& -\frac{\left(116 k^{2}-737 k-1353\right)}{3(k+5)^{2}(13 k+17)}\left(\begin{array}{l}
\partial F_{21} G_{11} \\
\partial F_{12} G_{22}
\end{array}\right)-\frac{\left(38 k^{2}+487 k+381\right)}{3(k+5)^{2}(13 k+17)}\left(\begin{array}{l}
F_{21} \partial G_{11} \\
F_{12} \partial G_{22}
\end{array}\right) \\
& +\frac{3}{(k+5)}\left(\begin{array}{l}
G_{11} G_{21} \\
G_{22} G_{12}
\end{array}\right) \mp \frac{16 i(k+4)}{(k+5)^{3}}\left(\begin{array}{l}
F_{11} A-\partial F_{11} \\
F_{22} A_{+} \partial F_{22}
\end{array}\right) \\
& \pm \frac{2 i\left(8 k^{2}+62 k-17\right)}{(k+5)(13 k+17)} T B_{\mp}-\frac{4\left(8 k^{2}+61 k+17\right)}{(k+5)^{2}(13 k+17)} T\left(\begin{array}{l}
F_{11} F_{21} \\
F_{22} F_{12}
\end{array}\right) \\
& -\frac{12}{(k+5)^{2}} U A_{3} B_{\mp} \mp \frac{i(k+6)}{(k+5)^{2}} \partial U B_{\mp} \pm \frac{i(5 k+6)}{(k+5)^{2}} U \partial B_{\mp} \\
& +\frac{\left(269 k^{2}+658 k-363\right)}{3(k+5)^{3}(13 k+17)} U\left(\begin{array}{l}
\partial F_{11} F_{21} \\
\partial F_{22} F_{12}
\end{array}\right)+\frac{4}{(k+5)^{2}} U\left(\begin{array}{l}
F_{11} G_{21} \\
F_{22} G_{12}
\end{array}\right) \\
& +\frac{\left(113 k^{2}+1546 k+1065\right)}{3(k+5)^{3}(13 k+17)} \partial U\left(\begin{array}{l}
F_{11} F_{21} \\
F_{22} F_{12}
\end{array}\right) \\
& -\frac{\left(199 k^{2}+2294 k+3423\right)}{3(k+5)^{3}(13 k+17)} U\left(\begin{array}{l}
F_{11} \partial F_{21} \\
F_{22} \partial F_{12}
\end{array}\right) \\
& -\frac{4}{(k+5)^{2}} U\left(\begin{array}{l}
F_{21} G_{11} \\
F_{12} G_{22}
\end{array}\right) \mp \frac{2 i}{(k+5)^{2}} U U B_{\mp} \\
& \mp \frac{2 i\left(48 k^{3}-1465 k^{2}-3746 k-4137\right)}{3(k+5)^{3}(13 k+17)}\left(\begin{array}{l}
F_{21} A_{+} \partial F_{21} \\
F_{12} A_{-} \partial F_{12}
\end{array}\right) \\
& \left.\mp \frac{2 i\left(48 k^{3}-1387 k^{2}-3566 k-4035\right)}{3(k+5)^{3}(13 k+17)}\left(\begin{array}{l}
\partial F_{21} A_{+} F_{21} \\
\partial F_{12} A-F_{12}
\end{array}\right)\right](w) \\
& +\frac{1}{(z-w)}[+\cdots](w)+\cdots \\
& +
\end{aligned}
$$

There is no higher spin current in the third order pole. In this case, the nonlinear term between the higher spin currents is present. This can be removed by adding the $T \mathbf{T}^{(\mathbf{1})}(w)$ into the left hand side for spin-3 current and then this will produce the above nonlinear term.

Again the previous result in (3.4) gives the following OPEs

$$
\begin{aligned}
\left(\begin{array}{c}
\mathbf{U}_{-}^{(\mathbf{2})} \\
\mathbf{V}_{+}^{(\mathbf{2})}
\end{array}\right)(z)\left(\begin{array}{c}
\mathbf{U}_{-}^{(\mathbf{2})} \\
\mathbf{V}_{+}^{(\mathbf{2})}
\end{array}\right)(w) & =\frac{1}{(z-w)^{2}}\left[\frac{6}{(5+k)^{2}} A_{ \pm} A_{ \pm}\right](w) \\
& +\frac{1}{(z-w)}\left[\frac{6}{(5+k)^{2}} A_{ \pm} \partial A_{ \pm}\right](w)+\cdots
\end{aligned}
$$

These are new nontrivial OPEs. The corresponding OPEs are trivial in the nonlinear version. 
From the result in (3.7), one obtains the following OPEs

$$
\begin{aligned}
\left(\begin{array}{c}
\mathbf{U}_{-}^{(2)} \\
\mathbf{V}_{+}^{(2)}
\end{array}\right)(z)\left(\begin{array}{c}
\mathbf{U}^{\left(\frac{5}{2}\right)} \\
\mathbf{V}^{\left(\frac{5}{2}\right)}
\end{array}\right)(w) & =\frac{1}{(z-w)^{3}}\left[\frac{8 i(k+9)}{(k+5)^{3}} A_{ \pm}\left(\begin{array}{c}
F_{11} \\
F_{22}
\end{array}\right)\right](w) \\
& +\frac{1}{(z-w)^{2}}\left[\frac{2 i(k+12)}{3(k+5)^{2}} A_{ \pm}\left(\begin{array}{c}
G_{11} \\
G_{22}
\end{array}\right) \mp \frac{4(k+3)}{3(k+5)^{3}} A_{ \pm} A_{3}\left(\begin{array}{c}
F_{11} \\
F_{22}
\end{array}\right)\right. \\
& \mp \frac{4(k+3)}{3(k+5)^{3}} A_{ \pm} A_{ \pm}\left(\begin{array}{c}
F_{21} \\
F_{12}
\end{array}\right) \pm \frac{4(k+3)}{3(k+5)^{3}} A_{ \pm} B_{3}\left(\begin{array}{c}
F_{11} \\
F_{22}
\end{array}\right) \\
& \pm \frac{4(k+3)}{3(k+5)^{3}} A_{ \pm} B_{\mp}\left(\begin{array}{c}
F_{12} \\
F_{21}
\end{array}\right) \pm \frac{4(k+3)}{3(k+5)^{3}}\left(\begin{array}{l}
F_{11} F_{12} G_{11} \\
F_{22} F_{21} G_{22}
\end{array}\right) \\
& +\frac{4 i(k+3)}{3(k+5)^{3}} U A_{ \pm}\left(\begin{array}{c}
F_{11} \\
F_{22}
\end{array}\right)+\frac{4 i(k+3)}{(k+5)^{3}} A_{ \pm} \partial\left(\begin{array}{l}
F_{11} \\
F_{22}
\end{array}\right) \\
& \left.\mp \frac{32(k+3)}{3(k+5)^{4}}\left(\begin{array}{c}
F_{11} F_{12} \partial F_{11} \\
F_{22} F_{21} \partial F_{22}
\end{array}\right) \mp \frac{24}{(k+5)^{3}}\left(\begin{array}{l}
F_{11} G_{12} F_{11} \\
F_{22} G_{21} F_{22}
\end{array}\right)\right](w) \\
& +\frac{1}{(z-w)}[+\cdots](w)+\cdots
\end{aligned}
$$

The nonlinear terms in the corresponding OPEs in the nonlinear version disappear. The first order pole is not determined in this paper.

Similarly one has the following OPE

$$
\begin{aligned}
\mathbf{U}_{-}^{(2)}(z) \mathbf{V}^{\left(\frac{3}{2}\right)}(w) & =\frac{1}{(z-w)^{3}}\left[\frac{6 k}{(k+5)^{2}} F_{12}\right](w) \\
& +\frac{1}{(z-w)^{2}}\left[-\frac{2(k+3)}{(k+5)} \mathbf{T}_{-}^{\left(\frac{3}{2}\right)}-\frac{2(k+2)}{(k+5)^{2}} U F_{12}-\frac{2}{(k+5)^{2}} F_{12} F_{11} F_{22}\right. \\
& -\frac{2 i(k-1)}{(k+5)^{2}} F_{12} A_{3}+\frac{2 i(k+2)}{(k+5)^{2}} F_{22} A_{+}-\frac{4 i}{(k+5)^{2}} F_{11} B_{+} \\
& \left.+\frac{4 i}{(k+5)^{2}} F_{12} B_{3}+\frac{1}{(k+5)} G_{12}+\frac{6 k}{(k+5)^{2}} \partial F_{12}\right](w) \\
& +\frac{1}{(z-w)}\left[\frac{1}{2} \mathbf{P}_{-}^{\left(\frac{5}{2}\right)}-\mathbf{W}_{-}^{\left(\frac{5}{2}\right)}-\frac{4(k+3)}{3(k+5)} \partial \mathbf{T}_{-}^{\left(\frac{3}{2}\right)}-\frac{i}{2(k+5)} A_{3} G_{12}\right. \\
& +\frac{2}{(k+5)^{2}} A_{3} A_{3} F_{12}-\frac{1}{(k+5)^{2}} A_{+} A_{3} F_{22} \\
& +\frac{2}{(k+5)^{2}} A_{3} B_{3} F_{12}-\frac{2}{(k+5)^{2}} A_{3} B_{+} F_{11}-\frac{i(8 k+3)}{3(k+5)^{2}} \partial A_{3} F_{12} \\
& -\frac{4 i(k-6)}{3(k+5)^{2}} A_{3} \partial F_{12}+\frac{1}{(k+5)^{2}} A_{+} A_{-} F_{12}-\frac{1}{(k+5)^{2}} A_{+} B_{3} F_{22} \\
& -\frac{1}{(k+5)^{2}} A_{+} B_{+} F_{21}+\frac{i(4 k+9)}{3(k+5)^{2}} \partial A_{+} F_{22}+\frac{i(4 k+3)}{3(k+5)^{2}} A_{+} \partial F_{22} \\
& -\frac{i}{2(k+5)} B_{3} G_{12}+\frac{i(5 k+18)}{3(k+5)^{2}} \partial B_{3} F_{12}-\frac{2 i(k-6)}{3(k+5)^{2}} B_{3} \partial F_{12}
\end{aligned}
$$




$$
\begin{aligned}
& +\frac{i}{2(k+5)} B_{+} G_{11}-\frac{i(k+9)}{3(k+5)^{2}} \partial B_{+} F_{11}+\frac{2 i(k-6)}{3(k+5)^{2}} B_{+} \partial F_{11} \\
& -\frac{2(k-3)}{3(k+5)^{3}} \partial F_{11} F_{12} F_{22}+\frac{1}{(k+5)^{2}} F_{11} F_{12} G_{22} \\
& +\frac{4(k+3)}{3(k+5)^{3}} F_{11} \partial F_{12} F_{22}+\frac{2(5 k+21)}{3(k+5)^{3}} F_{11} F_{12} \partial F_{22}-\frac{1}{(k+5)^{2}} F_{11} F_{22} G_{12} \\
& +\frac{3(3 k-5)}{2(k+5)^{2}} \partial^{2} F_{12}-\frac{1}{(k+5)^{2}} F_{12} F_{21} G_{12}+\frac{1}{(k+5)^{2}} \partial U F_{12} \\
& +\frac{1}{(k+5)} T F_{12}+\frac{1}{2(k+5)} U G_{12}-\frac{2 i}{(k+5)^{2}} U B_{3} F_{12}-\frac{2(2 k+3)}{3(k+5)^{2}} U \partial F_{12} \\
& -\frac{i}{(k+5)^{2}} U A_{+} F_{22}+\frac{2 i}{(k+5)^{2}} U B_{+} F_{11}+\frac{2}{(k+5)^{2}} U U F_{12} \\
& \left.+\frac{4 i(k+1)}{(k+5)^{3}} F_{12} B_{3} F_{12} F_{21}-\frac{(4 k+9)}{3(k+5)^{2}} F_{12} G_{21} F_{12}+\frac{1}{(k+5)} \partial G_{12}\right](w)+\cdots
\end{aligned}
$$

The spin- $\frac{3}{2}$ current in the second order pole was not present in the corresponding OPE in the nonlinear version.

The other combination of the OPE can be obtained

$$
\begin{aligned}
& \mathbf{U}_{-}^{(2)}(z) \mathbf{V}_{+}^{(2)}(w)=\frac{1}{(z-w)^{4}}\left[-\frac{12 k(k+3)}{(k+5)^{2}}\right] \\
& +\frac{1}{(z-w)^{3}}\left[-\frac{12 i k(2 k+5)}{(k+5)^{2}} A_{3}-\frac{12}{(k+5)^{2}} F_{11} F_{22}-\frac{12}{(k+5)^{2}} F_{12} F_{21}\right](w) \\
& +\frac{1}{(z-w)^{2}}\left[-\frac{1}{2} \mathbf{P}^{(\mathbf{2})}+\frac{2(k-3)}{3(k+5)} \mathbf{T}^{(\mathbf{2})}+2 \mathbf{W}^{(\mathbf{2})}-\frac{2(4 k+9)}{3(k+5)} T\right. \\
& -\frac{8 k}{3(k+5)^{2}} A_{3} A_{3}-\frac{2(k-3)}{3(k+5)^{2}} A_{3} B_{3}-\frac{4 i(11 k+27)}{3(k+5)^{2}} \partial A_{3} \\
& +\frac{8 i(k+3)}{3(k+5)^{3}} A_{3} F_{11} F_{22}+\frac{2 i}{(k+5)^{2}} A_{3} F_{12} F_{21}-\frac{2(4 k+9)}{3(k+5)^{2}} A_{+} A_{-} \\
& +\frac{2 i(k-15)}{3(k+5)^{3}} A_{-} F_{11} F_{12}+\frac{4 i(k+7)}{(k+5)^{3}} A_{+} F_{21} F_{22}-\frac{2(k+9)}{3(k+5)^{2}} B_{3} B_{3} \\
& -\frac{2 i(k+9)}{3(k+5)^{2}} \partial B_{3}-\frac{8 i(k+3)}{3(k+5)^{3}} B_{3} F_{11} F_{22}+\frac{2 i}{(k+5)^{2}} B_{3} F_{12} F_{21} \\
& -\frac{20 i(k+3)}{3(k+5)^{3}} B_{-} F_{12} F_{22}-\frac{2(k+9)}{3(k+5)^{2}} B_{+} B_{-}+\frac{2 i(k+1)}{(k+5)^{3}} B_{+} F_{11} F_{21} \\
& +\frac{(k-3)}{3(k+5)^{2}} F_{11} G_{22}-\frac{4\left(2 k^{2}+21 k+75\right)}{3(k+5)^{3}} \partial F_{11} F_{22} \\
& +\frac{4\left(2 k^{2}+3 k-15\right)}{3(k+5)^{3}} F_{11} \partial F_{22}+\frac{2(k+3)}{(k+5)^{2}} F_{12} G_{21}-\frac{2\left(4 k^{2}+27 k+123\right)}{3(k+5)^{3}} \partial F_{12} F_{21} \\
& +\frac{2\left(4 k^{2}+17 k+9\right)}{3(k+5)^{3}} F_{12} \partial F_{21}-\frac{6}{(k+5)^{2}} F_{21} G_{12}+\frac{2(k-3)}{3(k+5)^{2}} F_{22} G_{11}
\end{aligned}
$$




$$
\begin{aligned}
& +\frac{6 i}{(k+5)^{2}} U A_{3}+\frac{2 i k}{(k+5)^{2}} U B_{3}-\frac{2(4 k+9)}{3(k+5)^{2}} U U \\
& \left.-\frac{8(k-3)}{3(k+5)^{3}} U F_{11} F_{22}+\frac{6}{(k+5)^{2}} U F_{12} F_{21}\right](w) \\
& +\frac{1}{(z-w)}\left[-\frac{1}{2} \mathbf{S}^{(\mathbf{3})}+\frac{1}{2} \mathbf{P}^{(\mathbf{3})}-\frac{1}{4} \partial \mathbf{P}^{(\mathbf{2})}+\partial \mathbf{W}^{(\mathbf{2})}+\frac{(k-3)}{3(k+5)} \partial \mathbf{T}^{(\mathbf{2})}\right. \\
& -\frac{2 i}{(k+5)^{2}} A_{3} A_{3} A_{3}-\frac{2(4 k-3)}{3(k+5)^{2}} \partial A_{3} A_{3}+\frac{2 i}{(k+5)^{2}} A_{3} B_{3} B_{3} \\
& -\frac{(k-3)}{3(k+5)^{2}} \partial A_{3} B_{3}-\frac{(k+3)}{3(k+5)^{2}} A_{3} \partial B_{3}-\frac{i\left(124 k^{2}+263 k-249\right)}{3(k+5)^{2}(13 k+17)} \partial^{2} A_{3} \\
& +\frac{3 i}{(k+5)^{2}} A_{3} F_{11} G_{22}+\frac{i(23 k+123)}{6(k+5)^{3}} \partial A_{3} F_{11} F_{22}+\frac{2 i}{(k+5)^{2}} A_{3} B_{+} B_{-} \\
& -\frac{i(25 k+69)}{6(k+5)^{3}} A_{3} \partial F_{11} F_{22}+\frac{i(11 k-81)}{6(k+5)^{3}} A_{3} F_{11} \partial F_{22}+\frac{3 i}{(k+5)^{2}} A_{3} F_{12} G_{21} \\
& +\frac{i(7 k+43)}{2(k+5)^{3}} \partial A_{3} F_{12} F_{21}-\frac{3 i(3 k+7)}{2(k+5)^{3}} A_{3} \partial F_{12} F_{21}+\frac{i(3 k-25)}{2(k+5)^{3}} A_{3} F_{12} \partial F_{21} \\
& +\frac{i}{(k+5)^{2}} A_{3} F_{21} G_{12}+\frac{i}{(k+5)^{2}} A_{3} F_{22} G_{11}+\frac{i}{(k+5)^{2}} A_{-} F_{11} G_{12} \\
& +\frac{2 i(5 k+21)}{3(k+5)^{3}} \partial A_{-} F_{11} F_{12}+\frac{i}{(k+5)^{2}} A_{+} F_{21} G_{22} \\
& -\frac{8 i(k+9)}{3(k+5)^{3}} A_{-} \partial F_{11} F_{12}-\frac{8 i(k+9)}{3(k+5)^{3}} A_{-} F_{11} \partial F_{12}-\frac{i}{(k+5)^{2}} A_{-} F_{12} G_{11} \\
& -\frac{2 i}{(k+5)^{2}} A_{+} A_{-} A_{3}-\frac{4(k+3)}{3(k+5)^{2}} \partial A_{+} A_{-}-\frac{2(2 k+3)}{3(k+5)^{2}} A_{+} \partial A_{-} \\
& +\frac{4 i(k+7)}{(k+5)^{3}} \partial A_{+} F_{21} F_{22}-\frac{i}{(k+5)^{2}} A_{+} F_{22} G_{21} \\
& -\frac{i(k+9)}{3(k+5)^{2}} \partial^{2} B_{3}-\frac{i}{(k+5)^{2}} B_{3} F_{11} G_{22}+\frac{i(13 k+57)}{6(k+5)^{3}} \partial B_{3} F_{11} F_{22} \\
& -\frac{i(23 k+75)}{6(k+5)^{3}} B_{3} \partial F_{11} F_{22}-\frac{5 i(7 k+27)}{6(k+5)^{3}} B_{3} F_{11} \partial F_{22} \\
& +\frac{i}{(k+5)^{2}} B_{3} F_{12} G_{21}-\frac{i(5 k+17)}{2(k+5)^{3}} \partial B_{3} F_{12} F_{21}+\frac{i(7 k+27)}{2(k+5)^{3}} B_{3} \partial F_{12} F_{21} \\
& +\frac{i(11 k+47)}{2(k+5)^{3}} B_{3} F_{12} \partial F_{21}-\frac{i}{(k+5)^{2}} B_{-} F_{12} G_{22} \\
& -\frac{i}{(k+5)^{2}} B_{3} F_{21} G_{12}+\frac{i}{(k+5)^{2}} B_{3} F_{22} G_{11}+\frac{i(k+21)}{6(k+5)^{3}} \partial B_{-} F_{12} F_{22} \\
& -\frac{i(35 k+111)}{6(k+5)^{3}} B_{-} \partial F_{12} F_{22}-\frac{i(47 k+171)}{6(k+5)^{3}} B_{-} F_{12} \partial F_{22}+\frac{i}{(k+5)^{2}} B_{-} F_{22} G_{12}
\end{aligned}
$$




$$
\begin{aligned}
& -\frac{(k+9)}{3(k+5)^{2}} \partial B_{+} B_{-}-\frac{(k+9)}{3(k+5)^{2}} B_{+} \partial B_{-}+\frac{i(9 k+29)}{2(k+5)^{3}} \partial B_{+} F_{11} F_{21} \\
& -\frac{3 i}{2(k+5)^{2}} B_{+} \partial F_{11} F_{21}-\frac{7 i}{2(k+5)^{2}} B_{+} F_{11} \partial F_{21}-\frac{i}{(k+5)^{2}} B_{+} F_{11} G_{21} \\
& -\frac{\left(104 k^{3}+841 k^{2}+3234 k+2001\right)}{6(k+5)^{3}(13 k+17)} \partial^{2} F_{11} F_{22} \\
& +\frac{\left(104 k^{3}+679 k^{2}+1518 k+2079\right)}{6(k+5)^{3}(13 k+17)} F_{11} \partial^{2} F_{22}+\frac{(29 k+57)}{12(k+5)^{2}} \partial F_{11} G_{22} \\
& -\frac{(7 k+27)}{12(k+5)^{2}} F_{11} \partial G_{22}-\frac{\left(104 k^{3}+451 k^{2}+2022 k+1083\right)}{6(k+5)^{3}(13 k+17)} \partial^{2} F_{12} F_{21} \\
& -\frac{\left(367 k^{2}+1622 k+471\right)}{3(k+5)^{3}(13 k+17)} \partial F_{12} \partial F_{21}-\frac{\left(235 k^{2}+974 k+531\right)}{(k+5)^{3}(13 k+17)} \partial F_{11} \partial F_{22} \\
& +\frac{\left(104 k^{2}+445 k+681\right)}{6(k+5)^{2}(13 k+17)} F_{12} \partial^{2} F_{21}+\frac{(13 k+33)}{4(k+5)^{2}} \partial F_{12} G_{21} \\
& +\frac{1}{4(k+5)} F_{12} \partial G_{21}+\frac{1}{2(k+5)} G_{11} G_{22}+\frac{1}{2(k+5)} G_{12} G_{21} \\
& +\frac{3(k+4)}{2(k+5)^{2}} \partial F_{21} G_{12}-\frac{(k+12)}{2(k+5)^{2}} F_{21} \partial G_{12}+\frac{(11 k+48)}{6(k+5)^{2}} \partial F_{22} G_{11} \\
& -\frac{(k+24)}{6(k+5)^{2}} F_{22} \partial G_{11}-\frac{2 i(28 k+71)}{(k+5)(13 k+17)} T A_{3}+\frac{2(4 k+17)}{(k+5)(13 k+17)} T U \\
& -\frac{4(k+3)}{3(k+5)} \partial T-\frac{2(19 k+71)}{(k+5)^{2}(13 k+17)} T F_{11} F_{22}+\frac{2}{(k+5)^{2}} U A_{+} A- \\
& -\frac{2(19 k+71)}{(k+5)^{2}(13 k+17)} T F_{12} F_{21}+\frac{2}{(k+5)^{2}} U A_{3} A_{3}+\frac{2}{(k+5)^{2}} U B_{3} B_{3} \\
& +\frac{4 i}{(k+5)^{2}} \partial\left(U A_{3}\right)+\frac{i k}{(k+5)^{2}} \partial U B_{3}+\frac{i(k+2)}{(k+5)^{2}} U \partial B_{3} \\
& +\frac{2}{(k+5)^{2}} U B_{+} B_{-}-\frac{(4 k+17)}{(k+5)(13 k+17)} \partial^{2} U \\
& +\frac{1}{(k+5)^{2}} U F_{11} G_{22}+\frac{(11 k-57)}{6(k+5)^{3}} \partial U F_{11} F_{22}+\frac{(37 k+201)}{6(k+5)^{3}} U \partial F_{11} F_{22} \\
& +\frac{(47 k+219)}{6(k+5)^{3}} U F_{11} \partial F_{22}+\frac{1}{(k+5)^{2}} U F_{12} G_{21}+\frac{(5 k+41)}{2(k+5)^{3}} \partial U F_{12} F_{21} \\
& +
\end{aligned}
$$

The nonlinear terms (with the footnote 5) in this case do not appear in the above OPE. 
One continues to calculate the following OPE

$$
\begin{aligned}
\mathbf{U}_{-}^{(2)}(z) \mathbf{V}_{-}^{(2)}(w) & =\frac{1}{(z-w)^{2}}\left[-\frac{2(k+4)}{(k+5)^{2}} A_{+} B_{+}+\frac{2 i}{(k+5)^{2}} A_{+} F_{22} F_{12}\right. \\
& \left.-\frac{2 i}{(k+5)^{2}} B_{+} F_{12} F_{11}-\frac{(k-3)}{(k+5)^{2}} F_{12} G_{12}+\frac{4(3 k-1)}{(k+5)^{3}} F_{12} \partial F_{12}\right](w) \\
& +\frac{1}{(z-w)}\left[-\frac{2 i}{(k+5)^{2}} A_{3} F_{12} G_{12}-\frac{(2 k+9)}{(k+5)^{2}} \partial A_{+} B_{+}+\frac{2 i}{(k+5)^{2}} \partial A_{+} F_{22} F_{12}\right. \\
& +\frac{1}{(k+5)^{2}} A_{+} \partial B_{+}+\frac{i}{(k+5)^{2}} A_{+} F_{22} G_{12}-\frac{2 i(k+1)}{(k+5)^{3}} A_{+} \partial F_{22} F_{12} \\
& +\frac{2 i(k+1)}{(k+5)^{3}} A_{+} F_{22} \partial F_{12}+\frac{i}{(k+5)^{2}} A_{+} F_{12} G_{22}-\frac{2 i}{(k+5)^{2}} B_{3} F_{12} G_{12} \\
& +\frac{i}{(k+5)^{2}} B_{+} F_{12} G_{11}-\frac{2 i(k+1)}{(k+5)^{3}} B_{+} \partial F_{12} F_{11} \\
& -\frac{2 i(k+9)}{(k+5)^{3}} B_{+} F_{12} \partial F_{11}+\frac{i}{(k+5)^{2}} B_{+} F_{11} G_{12}+\frac{(k+11)}{(k+5)^{2}} \partial F_{12} G_{12} \\
& -\frac{(k-1)}{(k+5)^{2}} F_{12} \partial G_{12}+\frac{(7 k-5)}{(k+5)^{3}} F_{12} \partial^{2} F_{12}-\frac{4}{(k+5)^{2}} F_{12} \partial F_{12} U \\
& \left.-\frac{4 i(k+1)}{(k+5)^{3}} F_{12} \partial F_{12} A_{3}+\frac{16 i}{(k+5)^{3}} F_{12} \partial F_{12} B_{3}\right](w)+\cdots \\
& -\cdots
\end{aligned}
$$

It is obvious that there exist no boldface higher spin currents.

Furthermore, one has

$$
\begin{aligned}
\mathbf{U}_{-}^{(2)}(z) \mathbf{V}^{\left(\frac{5}{2}\right)}(w) & =\frac{1}{(z-w)^{4}}\left[\frac{24 k(k+3)}{(k+5)^{3}} F_{12}\right](w) \\
& +\frac{1}{(z-w)^{3}}\left[-\frac{4\left(4 k^{2}+29 k+69\right)}{3(k+5)^{3}} U F_{12}-\frac{16(k+3)}{3(k+5)^{3}} F_{12} F_{11} F_{22}\right. \\
& -\frac{4 i\left(4 k^{2}+17 k+33\right)}{3(k+5)^{3}} F_{12} A_{3}+\frac{4 i\left(4 k^{2}+11 k-21\right)}{3(k+5)^{3}} F_{22} A_{+} \\
& \left.+\frac{4 i(17 k+69)}{3(k+5)^{3}} F_{12} B_{3}-\frac{2\left(4 k^{2}+38 k+69\right)}{3(k+5)^{2}} G_{12}-\frac{4 i(17 k+69)}{3(k+5)^{3}} F_{11} B_{+}\right](w) \\
& +\frac{1}{(z-w)^{2}}\left[\frac{4(2 k+9)}{3(k+5)}\left(\mathbf{P}_{-}^{\left(\frac{5}{2}\right)}-\mathbf{W}_{-}^{\left(\frac{5}{2}\right)}\right)+\frac{2 i(5 k+9)}{3(k+5)^{2}} A_{3} G_{12}\right. \\
& -\frac{4(k+9)}{3(k+5)^{3}} A_{3} A_{3} F_{12}-\frac{2 i(2 k+7)}{(k+5)^{2}} A_{+} G_{22}-\frac{4(k+3)}{3(k+5)^{3}} A_{+} A_{3} F_{22} \\
& +\frac{4(5 k+21)}{3(k+5)^{3}} A_{3} B_{3} F_{12}-\frac{4(5 k+21)}{3(k+5)^{3}} A_{3} B_{+} F_{11}-\frac{2 i\left(4 k^{2}-93 k-297\right)}{9(k+5)^{3}} \partial A_{3} F_{12} \\
& -\frac{2 i\left(8 k^{2}+165 k+513\right)}{9(k+5)^{3}} A_{3} \partial F_{12}-\frac{8(k+6)}{3(k+5)^{3}} A_{+} A_{-} F_{12}-\frac{8(2 k+9)}{3(k+5)^{3}} A_{+} B_{3} F_{22}
\end{aligned}
$$




$$
\begin{aligned}
& -\frac{8(2 k+9)}{3(k+5)^{3}} A_{+} B_{+} F_{21}+\frac{8 i\left(2 k^{2}-12 k-81\right)}{9(k+5)^{3}} \partial A_{+} F_{22}+\frac{2 i(k+9)}{3(k+5)^{2}} B_{+} G_{11} \\
& +\frac{2 i\left(8 k^{2}+147 k+459\right)}{9(k+5)^{3}} A_{+} \partial F_{22}-\frac{2 i(k+9)}{3(k+5)^{2}} B_{3} G_{12} \\
& +\frac{2 i(k+9)(4 k+9)}{9(k+5)^{3}} \partial B_{3} F_{12}-\frac{2 i\left(16 k^{2}+51 k-153\right)}{9(k+5)^{3}} B_{3} \partial F_{12} \\
& -\frac{16 i(k+3)(k+9)}{9(k+5)^{3}} \partial B_{+} F_{11}+\frac{2 i\left(16 k^{2}+51 k-153\right)}{9(k+5)^{3}} B_{+} \partial F_{11} \\
& -\frac{16\left(5 k^{2}+54 k+153\right)}{9(k+5)^{4}} \partial F_{11} F_{12} F_{22}+\frac{4(k+3)}{3(k+5)^{3}} F_{11} F_{12} G_{22} \\
& +\frac{16(k+3)^{2}}{9(k+5)^{4}} F_{11} \partial F_{12} F_{22}+\frac{16\left(7 k^{2}+72 k+189\right)}{9(k+5)^{4}} F_{11} F_{12} \partial F_{22} \\
& -\frac{2(5 k+21)}{3(k+5)^{3}} F_{11} F_{22} G_{12}-\frac{2\left(8 k^{2}+9 k+189\right)}{9(k+5)^{3}} U \partial F_{12} \\
& -\frac{2(7 k+27)}{(k+5)^{3}} \partial^{2} F_{12}+\frac{2(k+9)}{3(k+5)^{3}} F_{12} F_{21} G_{12}-\frac{2(k+9)(4 k+11)}{3(k+5)^{3}} \partial U F_{12} \\
& +\frac{4}{(k+5)^{2}} F_{12} F_{22} G_{11}-\frac{2(k+9)(4 k+9)}{9(k+5)^{2}} \partial G_{12}+\frac{8 i}{(k+5)^{2}} U A_{3} F_{12} \\
& +\frac{4(4 k+9)}{3(k+5)^{2}} T F_{12}+\frac{2(k+12)}{3(k+5)^{2}} U G_{12}-\frac{4 i(5 k+21)}{3(k+5)^{3}} U B_{3} F_{12} \\
& -\frac{4 i(7 k+33)}{3(k+5)^{3}} U A_{+} F_{22}+\frac{4 i(5 k+21)}{3(k+5)^{3}} U B_{+} F_{11}+\frac{4(5 k+21)}{3(k+5)^{3}} U U F_{12} \\
& \left.+\frac{64 i\left(k^{2}+10 k+27\right)}{3(k+5)^{4}} F_{12} B_{3} F_{12} F_{21}+\frac{2(k+9)(4 k+15)}{9(k+5)^{3}} F_{12} G_{21} F_{12}\right](w) \\
& +\frac{1}{(z-w)}[+\cdots](w)+\cdots \text {. }
\end{aligned}
$$

There is no higher spin current in the third order pole of the above OPE. The structure of the first order pole is not determined.

The result in (3.6) can be used and one obtains the following OPEs

$$
\begin{aligned}
\left(\begin{array}{c}
\mathbf{U}_{-}^{(\mathbf{2})} \\
\mathbf{V}_{+}^{(\mathbf{2})}
\end{array}\right)(z) \mathbf{W}^{(\mathbf{2})}(w) & =\frac{1}{(z-w)^{3}}\left[\frac{6 i(2 k+5)}{(k+5)^{2}} A_{ \pm} \pm \frac{12}{(k+5)^{2}}\left(\begin{array}{c}
F_{11} F_{12} \\
F_{22} F_{21}
\end{array}\right)\right](w) \\
& +\frac{1}{(z-w)^{2}}\left[\mp \frac{6}{(k+5)^{2}} A_{ \pm} A_{3}+\frac{6 i(k+3)}{(k+5)^{2}} \partial A_{ \pm}\right. \\
& \mp \frac{2(k+4)}{(k+5)^{2}} A_{ \pm} B_{3} \mp \frac{i}{(k+5)^{2}} A_{ \pm} F_{11} F_{22} \\
& \pm \frac{i}{(k+5)^{2}} A_{ \pm} F_{12} F_{21}+\frac{2 i}{(k+5)^{2}} B_{3}\left(\begin{array}{c}
F_{11} F_{12} \\
F_{22} F_{21}
\end{array}\right)
\end{aligned}
$$




$$
\begin{aligned}
& \mp \frac{(k-3)}{2(k+5)^{2}}\left(\begin{array}{c}
F_{11} G_{12} \\
F_{22} G_{21}
\end{array}\right) \pm \frac{32}{(k+5)^{3}}\left(\begin{array}{c}
\partial F_{11} F_{12} \\
\partial F_{22} F_{21}
\end{array}\right) \\
& \left. \pm \frac{4(3 k+7)}{(k+5)^{3}}\left(\begin{array}{c}
F_{11} \partial F_{12} \\
F_{22} \partial F_{21}
\end{array}\right) \mp \frac{(k-3)}{2(k+5)^{2}}\left(\begin{array}{c}
F_{12} G_{11} \\
F_{21} G_{22}
\end{array}\right)\right](w) \\
& +\frac{1}{(z-w)}\left[\frac{1}{2}\left(\begin{array}{c}
\mathbf{Q}_{-}^{(3)} \\
\mathbf{R}_{+}^{(3)}
\end{array}\right)-\frac{i}{(k+5)^{2}} A_{3}\left(\begin{array}{c}
F_{11} G_{12} \\
F_{22} G_{21}
\end{array}\right)\right. \\
& +\frac{i}{2(k+5)^{2}} \partial A_{3}\left(\begin{array}{c}
F_{11} F_{12} \\
F_{22} F_{21}
\end{array}\right)+\frac{i(5 k+9)}{2(k+5)^{3}} A_{3}\left(\begin{array}{c}
\partial F_{11} F_{12} \\
\partial F_{22} F_{21}
\end{array}\right) \\
& -\frac{i(7 k+19)}{2(k+5)^{3}} A_{3}\left(\begin{array}{c}
F_{11} \partial F_{12} \\
F_{22} \partial F_{21}
\end{array}\right)-\frac{i}{(k+5)^{2}} A_{3}\left(\begin{array}{c}
F_{12} G_{11} \\
F_{21} G_{22}
\end{array}\right) \\
& +\frac{i}{(k+5)^{2}} A_{ \pm} A_{3} A_{3} \mp \frac{2}{(k+5)^{2}} \partial A_{ \pm} A_{3} \mp \frac{5}{(k+5)^{2}} A_{ \pm} \partial A_{3} \\
& +\frac{i\left(24 k^{2}+65 k-83\right)}{2(k+5)^{2}(13 k+17)} \partial^{2} A_{ \pm} \mp \frac{i(5 k+21)}{2(k+5)^{3}} \partial A_{ \pm} F_{11} F_{22} \\
& +\frac{i}{(k+5)^{2}} A_{ \pm} A_{ \pm} A_{\mp}-\frac{i}{(k+5)^{2}} A_{ \pm} B_{3} B_{3} \\
& \mp \frac{(7 k+31)}{2(k+5)^{2}} \partial A_{ \pm} B_{3} \pm \frac{(3 k+17)}{2(k+5)^{2}} A_{ \pm} \partial B_{3}-\frac{i}{(k+5)^{2}} A_{ \pm} B_{ \pm} B_{\mp} \\
& +\frac{3 i(k+1)}{2(k+5)^{3}} A_{ \pm}\left(\begin{array}{c}
\partial F_{11} F_{22} \\
\partial F_{22} F_{11}
\end{array}\right)+\frac{i(3 k+19)}{2(k+5)^{3}} A_{ \pm}\left(\begin{array}{c}
F_{11} \partial F_{22} \\
F_{22} \partial F_{11}
\end{array}\right) \\
& -\frac{2 i}{(k+5)^{2}} A_{ \pm}\left(\begin{array}{c}
F_{12} G_{21} \\
F_{21} G_{12}
\end{array}\right) \pm \frac{2 i(k+4)}{(k+5)^{3}} \partial A_{ \pm} F_{12} F_{21} \\
& +\frac{2 i k}{(k+5)^{3}} A_{ \pm}\left(\begin{array}{c}
\partial F_{12} F_{21} \\
\partial F_{21} F_{12}
\end{array}\right)-\frac{2 i(2 k+3)}{(k+5)^{3}} A_{ \pm}\left(\begin{array}{c}
F_{12} \partial F_{21} \\
F_{21} \partial F_{12}
\end{array}\right) \\
& -\frac{i}{(k+5)^{2}} A_{ \pm}\left(\begin{array}{c}
F_{21} G_{12} \\
F_{12} G_{21}
\end{array}\right) \mp \frac{(7 k+19)}{2(k+5)^{3}} U\left(\begin{array}{c}
F_{11} \partial F_{12} \\
F_{22} \partial F_{21}
\end{array}\right) \\
& -\frac{i}{(k+5)^{2}} A_{ \pm}\left(\begin{array}{c}
F_{22} G_{11} \\
F_{11} G_{22}
\end{array}\right)-\frac{i}{(k+5)^{2}} B_{3}\left(\begin{array}{c}
F_{11} G_{12} \\
F_{22} G_{21}
\end{array}\right) \\
& -\frac{i(3 k+23)}{2(k+5)^{3}} \partial B_{3}\left(\begin{array}{c}
F_{11} F_{12} \\
F_{22} F_{21}
\end{array}\right)+\frac{i(5 k+33)}{2(k+5)^{3}} B_{3}\left(\begin{array}{c}
\partial F_{11} F_{12} \\
\partial F_{22} F_{21}
\end{array}\right) \\
& +\frac{i(9 k+53)}{2(k+5)^{3}} B_{3}\left(\begin{array}{c}
F_{11} \partial F_{12} \\
F_{22} \partial F_{21}
\end{array}\right)+\frac{i}{(k+5)^{2}} B_{3}\left(\begin{array}{c}
F_{12} G_{11} \\
F_{21} G_{22}
\end{array}\right) \\
& +\frac{i}{(k+5)^{2}} B_{\mp}\left(\begin{array}{c}
F_{12} G_{12} \\
F_{21} G_{21}
\end{array}\right)+\frac{i}{(k+5)^{2}} B_{ \pm}\left(\begin{array}{c}
F_{11} G_{11} \\
F_{22} G_{22}
\end{array}\right) \\
& \mp \frac{\left(155 k^{2}+238 k+387\right)}{2(k+5)^{3}(13 k+17)}\left(\begin{array}{l}
\partial^{2} F_{11} F_{12} \\
\partial^{2} F_{22} F_{21}
\end{array}\right) \\
& \pm \frac{\left(131 k^{2}+318 k-149\right)}{(k+5)^{3}(13 k+17)}\left(\begin{array}{c}
\partial F_{11} \partial F_{12} \\
\partial F_{22} \partial F_{21}
\end{array}\right) \mp \frac{1}{2(k+5)}\left(\begin{array}{c}
G_{11} G_{12} \\
G_{22} G_{21}
\end{array}\right)
\end{aligned}
$$




$$
\begin{aligned}
& \pm \frac{\left(53 k^{2}-174 k-659\right)}{2(k+5)^{3}(13 k+17)}\left(\begin{array}{c}
F_{11} \partial^{2} F_{12} \\
F_{22} \partial^{2} F_{21}
\end{array}\right) \pm \frac{(k+17)}{2(k+5)^{2}}\left(\begin{array}{l}
\partial F_{11} G_{12} \\
\partial F_{22} G_{21}
\end{array}\right) \\
& \pm \frac{(5 k+19)}{4(k+5)^{2}}\left(\begin{array}{c}
\partial F_{12} G_{11} \\
\partial F_{21} G_{22}
\end{array}\right) \mp \frac{3(k-1)}{4(k+5)^{2}}\left(\begin{array}{c}
F_{12} \partial G_{11} \\
F_{21} \partial G_{22}
\end{array}\right) \\
& \mp \frac{(k+1)}{2(k+5)^{2}}\left(\begin{array}{c}
F_{11} \partial G_{12} \\
F_{22} \partial G_{21}
\end{array}\right)+\frac{i(28 k+71)}{(k+5)(13 k+17)} T A_{ \pm} \\
& \pm \frac{2(19 k+71)}{(k+5)^{2}(13 k+17)} T\left(\begin{array}{c}
F_{11} F_{12} \\
F_{22} F_{21}
\end{array}\right) \mp \frac{2}{(k+5)^{2}} U A_{ \pm} B_{3} \\
& -\frac{i}{2(k+5)^{2}} \partial U A_{ \pm}+\frac{i}{2(k+5)^{2}} U \partial A_{ \pm} \mp \frac{1}{(k+5)^{2}} U\left(\begin{array}{l}
F_{11} G_{12} \\
F_{22} G_{21}
\end{array}\right) \\
& \pm \frac{(k-11)}{2(k+5)^{3}} \partial U\left(\begin{array}{c}
F_{11} F_{12} \\
F_{22} F_{21}
\end{array}\right) \pm \frac{(5 k+41)}{2(k+5)^{3}} U\left(\begin{array}{c}
\partial F_{11} F_{12} \\
\partial F_{22} F_{21}
\end{array}\right) \\
& \pm \frac{1}{(k+5)^{2}} U\left(\begin{array}{l}
F_{12} G_{11} \\
F_{21} G_{22}
\end{array}\right)+\frac{i}{(k+5)^{2}} U U A_{ \pm} \\
& \left.-\frac{2 i(k+9)}{(k+5)^{3}}\left(\begin{array}{l}
F_{11} B_{+} \partial F_{11} \\
F_{22} B_{-} \partial F_{22}
\end{array}\right)-\frac{8 i}{(k+5)^{3}}\left(\begin{array}{l}
F_{12} B_{-} \partial F_{12} \\
F_{21} B_{+} \partial F_{21}
\end{array}\right)\right](w)+\cdots
\end{aligned}
$$

There is no higher spin current in the second order pole. One sees that the nonlinear terms appeared in the nonlinear version do not appear in these OPEs.

Again the result (3.8) can be used and the following OPEs can be obtained

$$
\begin{aligned}
\left(\begin{array}{c}
\mathbf{U}_{-(\mathbf{2})}^{(\mathbf{2})} \\
\mathbf{V}_{+}
\end{array}\right)(z) \mathbf{W}_{ \pm}^{\left(\frac{5}{2}\right)}(w) & =\frac{1}{(z-w)^{4}}\left[ \pm \frac{24 k(k+3)}{(k+5)^{3}}\left(\begin{array}{c}
F_{11} \\
F_{22}
\end{array}\right)\right](w) \\
& +\frac{1}{(z-w)^{3}}\left[\mp \frac{2\left(4 k^{2}+38 k+69\right)}{3(k+5)^{2}}\left(\begin{array}{c}
G_{11} \\
G_{22}
\end{array}\right)\right. \\
& \mp \frac{4\left(4 k^{2}+29 k+69\right)}{3(k+5)^{3}} U\left(\begin{array}{c}
F_{11} \\
F_{22}
\end{array}\right)-\frac{16(k+3)}{3(k+5)^{3}} F_{12} F_{21}\left(\begin{array}{c}
F_{11} \\
F_{22}
\end{array}\right) \\
& -\frac{4 i\left(4 k^{2}+17 k+33\right)}{3(k+5)^{3}}\left(\begin{array}{c}
F_{11} \\
F_{22}
\end{array}\right) A_{3}-\frac{4 i\left(4 k^{2}+11 k-21\right)}{3(k+5)^{3}}\left(\begin{array}{c}
F_{21} \\
F_{12}
\end{array}\right) A_{ \pm} \\
& \left.-\frac{4 i(17 k+69)}{3(k+5)^{3}}\left(\begin{array}{c}
F_{12} \\
F_{21}
\end{array}\right) B_{\mp}-\frac{4 i(17 k+69)}{3(k+5)^{3}}\left(\begin{array}{c}
F_{11} \\
F_{22}
\end{array}\right) B_{3}\right](w) \\
& +\frac{1}{(z-w)^{2}}\left[-\frac{4(2 k+9)}{3(k+5)}\left(\begin{array}{c}
\mathbf{Q}^{\left(\frac{5}{2}\right)} \\
\mathbf{R}^{\left(\frac{5}{2}\right)}
\end{array}\right) \mp \frac{4(2 k+9)}{3(k+5)}\left(\begin{array}{c}
\mathbf{U}^{\left(\frac{5}{2}\right)} \\
\mathbf{V}^{\left(\frac{5}{2}\right)}
\end{array}\right)\right. \\
& +\frac{2 i(5 k+9)}{3(k+5)^{2}} A_{3}\left(\begin{array}{c}
G_{11} \\
G_{22}
\end{array}\right) \mp \frac{4(k+9)}{3(k+5)^{3}} A_{3} A_{3}\left(\begin{array}{c}
F_{11} \\
F_{22}
\end{array}\right) \\
& \mp \frac{4(5 k+21)}{3(k+5)^{3}} A_{3} B_{\mp}\left(\begin{array}{c}
F_{12} \\
F_{21}
\end{array}\right) \mp \frac{4(5 k+21)}{3(k+5)^{3}} A_{3} B_{3}\left(\begin{array}{c}
F_{11} \\
F_{22}
\end{array}\right) \\
& -\frac{4 i\left(4 k^{2}-21 k-81\right)}{9(k+5)^{3}} \partial A_{3}\left(\begin{array}{c}
F_{11} \\
F_{22}
\end{array}\right)-\frac{2 i\left(8 k^{2}+165 k+513\right)}{9(k+5)^{3}} A_{3} \partial\left(\begin{array}{c}
F_{11} \\
F_{22}
\end{array}\right)
\end{aligned}
$$




$$
\begin{aligned}
& +\frac{2 i(2 k+7)}{(k+5)^{2}} A_{ \pm}\left(\begin{array}{c}
G_{21} \\
G_{12}
\end{array}\right) \mp \frac{8(2 k+9)}{3(k+5)^{3}} A_{ \pm} B_{3}\left(\begin{array}{c}
F_{21} \\
F_{12}
\end{array}\right) \\
& \pm \frac{4(k+3)}{3(k+5)^{3}} A_{ \pm} A_{3}\left(\begin{array}{c}
F_{21} \\
F_{12}
\end{array}\right) \mp \frac{8(k+6)}{3(k+5)^{3}} A_{ \pm} A_{\mp}\left(\begin{array}{c}
F_{11} \\
F_{22}
\end{array}\right) \\
& \pm \frac{8(2 k+9)}{3(k+5)^{3}} A_{ \pm} B_{\mp}\left(\begin{array}{c}
F_{22} \\
F_{11}
\end{array}\right)-\frac{16 i(k+3)(k+9)}{9(k+5)^{3}} \partial B_{3}\left(\begin{array}{c}
F_{11} \\
F_{22}
\end{array}\right) \\
& -\frac{8 i\left(2 k^{2}-12 k-81\right)}{9(k+5)^{3}} \partial A_{ \pm}\left(\begin{array}{c}
F_{21} \\
F_{12}
\end{array}\right)-\frac{2 i\left(8 k^{2}+147 k+459\right)}{9(k+5)^{3}} A_{ \pm} \partial\left(\begin{array}{c}
F_{21} \\
F_{12}
\end{array}\right) \\
& +\frac{2 i(k+9)}{3(k+5)^{2}} B_{3}\left(\begin{array}{l}
G_{11} \\
G_{22}
\end{array}\right)+\frac{2 i\left(16 k^{2}+51 k-153\right)}{9(k+5)^{3}} B_{3} \partial\left(\begin{array}{c}
F_{11} \\
F_{22}
\end{array}\right) \\
& +\frac{2 i(k+9)}{3(k+5)^{2}} B_{\mp}\left(\begin{array}{c}
G_{12} \\
G_{21}
\end{array}\right)-\frac{16 i(k+3)(k+9)}{9(k+5)^{3}} \partial B_{\mp}\left(\begin{array}{c}
F_{12} \\
F_{21}
\end{array}\right) \\
& +\frac{2 i\left(16 k^{2}+51 k-153\right)}{9(k+5)^{3}} B_{\mp} \partial\left(\begin{array}{c}
F_{12} \\
F_{21}
\end{array}\right) \\
& \mp \frac{2(7 k+27)}{(k+5)^{3}} \partial^{2}\left(\begin{array}{c}
F_{11} \\
F_{22}
\end{array}\right) \mp \frac{4(k+9)(4 k+9)}{9(k+5)^{3}} \partial U\left(\begin{array}{c}
F_{11} \\
F_{22}
\end{array}\right) \\
& \mp \frac{4(k+3)}{3(k+5)^{3}}\left(\begin{array}{c}
F_{11} F_{12} G_{21} \\
F_{22} F_{21} G_{12}
\end{array}\right)-\frac{16(k+3)^{2}}{9(k+5)^{4}} \partial\left(\begin{array}{c}
F_{11} \\
F_{22}
\end{array}\right) F_{12} F_{21} \\
& \pm \frac{16\left(5 k^{2}+54 k+153\right)}{9(k+5)^{4}}\left(\begin{array}{l}
F_{11} \partial F_{12} F_{21} \\
F_{22} \partial F_{21} F_{12}
\end{array}\right) \\
& \mp \frac{16\left(7 k^{2}+72 k+189\right)}{9(k+5)^{4}}\left(\begin{array}{l}
F_{11} F_{12} \partial F_{21} \\
F_{22} F_{21} \partial F_{12}
\end{array}\right) \\
& \pm \frac{4}{3(k+5)^{2}}\left(\begin{array}{c}
F_{11} F_{21} G_{12} \\
F_{22} F_{12} G_{21}
\end{array}\right) \pm \frac{2(k+9)}{3(k+5)^{3}}\left(\begin{array}{l}
F_{11} F_{22} G_{11} \\
F_{22} F_{11} G_{22}
\end{array}\right) \\
& \mp \frac{2(5 k+21)}{3(k+5)^{3}}\left(\begin{array}{l}
F_{12} F_{21} G_{11} \\
F_{21} F_{12} G_{22}
\end{array}\right) \mp \frac{2(k+9)(4 k+9)}{9(k+5)^{2}} \partial\left(\begin{array}{l}
G_{11} \\
G_{22}
\end{array}\right) \\
& \pm \frac{4(4 k+9)}{3(k+5)^{2}} T\left(\begin{array}{c}
F_{11} \\
F_{22}
\end{array}\right) \pm \frac{2(k+12)}{3(k+5)^{2}} U\left(\begin{array}{c}
G_{11} \\
G_{22}
\end{array}\right) \\
& +\frac{8 i}{(k+5)^{2}} U A_{3}\left(\begin{array}{c}
F_{11} \\
F_{22}
\end{array}\right)+\frac{4 i(7 k+33)}{3(k+5)^{3}} U A_{ \pm}\left(\begin{array}{c}
F_{21} \\
F_{12}
\end{array}\right) \\
& +\frac{4 i(5 k+21)}{3(k+5)^{3}} U B_{3}\left(\begin{array}{c}
F_{11} \\
F_{22}
\end{array}\right)+\frac{4 i(5 k+21)}{3(k+5)^{3}} U B_{\mp}\left(\begin{array}{c}
F_{12} \\
F_{21}
\end{array}\right) \\
& \mp \frac{2\left(8 k^{2}+9 k+189\right)}{9(k+5)^{3}} U \partial\left(\begin{array}{c}
F_{11} \\
F_{22}
\end{array}\right) \pm \frac{4(5 k+21)}{3(k+5)^{3}} U U\left(\begin{array}{c}
F_{11} \\
F_{22}
\end{array}\right) \\
& \left.-\frac{32\left(k^{2}+10 k+27\right)}{3(k+5)^{4}}\left(\begin{array}{c}
\partial F_{11} \\
\partial F_{22}
\end{array}\right) F_{11} F_{22}\right](w) \\
& +\frac{1}{(z-w)}[+\cdots](w)+\cdots \text {. }
\end{aligned}
$$


One does not see the higher spin currents in the third order pole of the above OPEs. In principle, the first order pole can be obtained from the description in the section 7 .

Similarly the other combination leads to the following OPEs

$$
\begin{aligned}
\left(\begin{array}{c}
\mathbf{U}_{-}^{(2)} \\
\mathbf{V}_{+}^{(2)}
\end{array}\right)(z) \mathbf{W}_{\mp}^{\left(\frac{5}{2}\right)}(w) & =\frac{1}{(z-w)^{3}}\left[-\frac{8 i(k+9)}{(k+5)^{3}} A_{ \pm}\left(\begin{array}{c}
F_{12} \\
F_{21}
\end{array}\right)\right](w) \\
& +\frac{1}{(z-w)^{2}}\left[-\frac{2 i(k+12)}{3(k+5)^{2}} A_{ \pm}\left(\begin{array}{c}
G_{12} \\
G_{21}
\end{array}\right) \pm \frac{4(k+3)}{3(k+5)^{3}} A_{ \pm} A_{3}\left(\begin{array}{c}
F_{12} \\
F_{21}
\end{array}\right)\right. \\
& \mp \frac{4(k+3)}{3(k+5)^{3}} A_{ \pm} A_{ \pm}\left(\begin{array}{c}
F_{22} \\
F_{11}
\end{array}\right) \pm \frac{4(k+3)}{3(k+5)^{3}} A_{ \pm} B_{3}\left(\begin{array}{c}
F_{12} \\
F_{21}
\end{array}\right) \\
& -\frac{24 i}{(k+5)^{3}} \partial A_{ \pm}\left(\begin{array}{c}
F_{12} \\
F_{21}
\end{array}\right)-\frac{4 i(k+3)}{(k+5)^{3}} A_{ \pm} \partial\left(\begin{array}{c}
F_{12} \\
F_{21}
\end{array}\right) \\
& \mp \frac{4(k+3)}{3(k+5)^{3}} A_{ \pm} B_{ \pm}\left(\begin{array}{c}
F_{11} \\
F_{22}
\end{array}\right) \pm \frac{4(k+3)}{3(k+5)^{3}}\left(\begin{array}{c}
F_{12} F_{11} G_{12} \\
F_{21} F_{22} G_{21}
\end{array}\right) \\
& \left.-\frac{4 i(k+3)}{3(k+5)^{3}} U A_{ \pm}\left(\begin{array}{c}
F_{12} \\
F_{21}
\end{array}\right) \pm \frac{32(k+3)}{3(k+5)^{4}}\left(\begin{array}{c}
F_{11} F_{12} \partial F_{12} \\
F_{22} F_{21} \partial F_{21}
\end{array}\right)\right](w) \\
& +\frac{1}{(z-w)}[+\cdots](w)+\cdots .
\end{aligned}
$$

It is clear that there are no boldface higher spin currents in the OPEs.

The result (3.9) can be used to calculate the following OPEs

$$
\begin{aligned}
& \left(\begin{array}{c}
\mathbf{U}_{-}^{(\mathbf{2})} \\
\mathbf{V}_{+}^{(\mathbf{2})}
\end{array}\right)(z) \mathbf{W}^{(\mathbf{3})}(w)=\frac{1}{(z-w)^{4}}\left[ \pm \frac{36 i\left(18 k^{2}+63 k+41\right)}{(k+5)^{2}(13 k+17)} A_{ \pm}\right. \\
& \left.+\frac{72(k+1)(5 k+41)}{(k+5)^{3}(13 k+17)}\left(\begin{array}{c}
F_{11} F_{12} \\
F_{22} F_{21}
\end{array}\right)\right](w) \\
& +\frac{1}{(z-w)^{3}}\left[ \pm \frac{16 i\left(7 k^{2}+44 k+141\right)}{3(k+5)^{3}(13 k+17)} A_{3}\left(\begin{array}{c}
F_{11} F_{12} \\
F_{22} F_{21}
\end{array}\right)\right. \\
& -\frac{8 i\left(7 k^{2}+44 k+141\right)}{3(k+5)^{3}(13 k+17)} A_{ \pm} F_{11} F_{22} \mp \frac{24 i}{(k+5)^{2}} U A_{ \pm} \\
& -\frac{8 i\left(7 k^{2}+44 k+141\right)}{3(k+5)^{3}(13 k+17)} A_{ \pm} F_{12} F_{21}+\frac{16\left(7 k^{2}+44 k+141\right)}{3(k+5)^{3}(13 k+17)} U\left(\begin{array}{c}
F_{11} F_{12} \\
F_{22} F_{21}
\end{array}\right) \\
& +\frac{28(k+2)(k+21)}{3(k+5)^{2}(13 k+17)}\left(\begin{array}{c}
F_{11} G_{12} \\
F_{22} G_{21}
\end{array}\right)-\frac{16\left(7 k^{2}+44 k+141\right)}{3(k+5)^{3}(13 k+17)} \partial\left(\begin{array}{c}
F_{11} F_{12} \\
F_{22} F_{21}
\end{array}\right) \\
& \left.-\frac{28(k+2)(k+21)}{3(k+5)^{2}(13 k+17)}\left(\begin{array}{c}
F_{12} G_{11} \\
F_{21} G_{22}
\end{array}\right)\right](w) \\
& +\frac{1}{(z-w)^{2}}\left[ \pm 3\left(\begin{array}{c}
\mathbf{Q}_{-}^{(\mathbf{3})} \\
\mathbf{R}_{+}^{(\mathbf{3})}
\end{array}\right)-\frac{8(\mathbf{k}-\mathbf{3})}{(13 k+17)} \mathbf{T}^{(\mathbf{1})}\left(\begin{array}{c}
\mathbf{U}_{-}^{(\mathbf{2})} \\
\mathbf{V}_{+}^{(\mathbf{2})}
\end{array}\right)\right. \\
& \mp \frac{2 i}{(k+5)^{2}} A_{3}\left(\begin{array}{c}
F_{11} G_{12} \\
F_{22} G_{21}
\end{array}\right) \pm \frac{2 i}{(k+5)^{2}} A_{3}\left(\begin{array}{c}
F_{12} G_{11} \\
F_{21} G_{22}
\end{array}\right)
\end{aligned}
$$




$$
\begin{aligned}
& \pm \frac{i\left(277 k^{2}+1538 k+2301\right)}{3(k+5)^{3}(13 k+17)} \partial A_{3}\left(\begin{array}{c}
F_{11} F_{12} \\
F_{22} F_{21}
\end{array}\right) \\
& \mp \frac{i\left(35 k^{2}-194 k-1077\right)}{3(k+5)^{3}(13 k+17)} A_{3}\left(\begin{array}{c}
\partial F_{11} F_{12} \\
\partial F_{22} F_{21}
\end{array}\right) \\
& \mp \frac{i\left(503 k^{2}+2758 k+1983\right)}{3(k+5)^{3}(13 k+17)} A_{3}\left(\begin{array}{c}
F_{11} \partial F_{12} \\
F_{22} \partial F_{21}
\end{array}\right) \\
& \mp \frac{2 i}{(k+5)^{2}} A_{ \pm} A_{3} A_{3}+\frac{24}{(k+5)^{2}} \partial A_{ \pm} A_{3}-\frac{22}{(k+5)^{2}} A_{ \pm} \partial A_{3} \\
& \mp \frac{i\left(44 k^{2}+173 k+341\right)}{(k+5)^{2}(13 k+17)} \partial^{2} A_{ \pm} \mp \frac{2 i}{(k+5)^{2}} A_{ \pm} A_{ \pm} A_{\mp} \\
& \text { - } \frac{3(3 k+13)}{(k+5)^{2}} \partial A_{ \pm} B_{3}+\frac{9}{(k+5)} A_{ \pm} \partial B_{3} \mp \frac{6 i}{(k+5)^{2}} A_{ \pm} B_{ \pm} B_{\mp} \\
& \mp \frac{i\left(431 k^{2}+2146 k+2451\right)}{3(k+5)^{3}(13 k+17)} \partial A_{ \pm} F_{11} F_{22} \pm \frac{3 i}{(k+5)^{2}} A_{ \pm}\left(\begin{array}{c}
F_{11} G_{22} \\
F_{22} G_{11}
\end{array}\right) \\
& \pm \frac{i\left(505 k^{2}+2198 k+1629\right)}{3(k+5)^{3}(13 k+17)} A_{ \pm}\left(\begin{array}{c}
\partial F_{11} F_{22} \\
\partial F_{22} F_{11}
\end{array}\right) \\
& \pm \frac{i\left(349 k^{2}+1838 k+1425\right)}{3(k+5)^{3}(13 k+17)} A_{ \pm}\left(\begin{array}{c}
F_{11} \partial F_{22} \\
F_{22} \partial F_{11}
\end{array}\right) \\
& \mp \frac{3 i}{(k+5)^{2}} A_{ \pm}\left(\begin{array}{c}
F_{12} G_{21} \\
F_{21} G_{12}
\end{array}\right)+\frac{2 i\left(77 k^{2}+304 k+75\right)}{3(k+5)^{3}(13 k+17)} \partial A_{ \pm} F_{12} F_{21} \\
& \pm \frac{2 i\left(77 k^{2}+460 k+279\right)}{3(k+5)^{3}(13 k+17)} A_{ \pm}\left(\begin{array}{c}
\partial F_{12} F_{21} \\
\partial F_{21} F_{12}
\end{array}\right) \\
& \mp \frac{2 i\left(235 k^{2}+1196 k+1353\right)}{3(k+5)^{3}(13 k+17)} A_{ \pm}\left(\begin{array}{c}
F_{12} \partial F_{21} \\
F_{21} \partial F_{12}
\end{array}\right) \\
& \pm \frac{i}{(k+5)^{2}} A_{ \pm}\left(\begin{array}{c}
F_{21} G_{12} \\
F_{12} G_{21}
\end{array}\right)-\frac{\left(197 k^{2}+592 k+819\right)}{6(k+5)^{2}(13 k+17)}\left(\begin{array}{c}
F_{12} \partial G_{11} \\
F_{21} \partial G_{22}
\end{array}\right) \\
& \mp \frac{5 i}{(k+5)^{2}} A_{ \pm}\left(\begin{array}{c}
F_{22} G_{11} \\
F_{11} G_{22}
\end{array}\right) \mp \frac{8 i}{(k+5)^{2}} B_{3}\left(\begin{array}{c}
F_{11} G_{12} \\
F_{22} G_{21}
\end{array}\right) \\
& \mp \frac{3 i(3 k+23)}{(k+5)^{3}} \partial B_{3}\left(\begin{array}{c}
F_{11} F_{12} \\
F_{22} F_{21}
\end{array}\right) \pm \frac{i(3 k+23)}{(k+5)^{3}} B_{3}\left(\begin{array}{c}
\partial F_{11} F_{12} \\
\partial F_{22} F_{21}
\end{array}\right) \\
& \pm \frac{5 i(3 k+23)}{(k+5)^{3}} B_{3}\left(\begin{array}{c}
F_{11} \partial F_{12} \\
F_{22} \partial F_{21}
\end{array}\right) \pm \frac{4 i}{(k+5)^{2}} B_{3}\left(\begin{array}{c}
F_{12} G_{11} \\
F_{21} G_{22}
\end{array}\right) \\
& \mp \frac{2 i}{(k+5)^{2}} B_{\mp}\left(\begin{array}{c}
F_{12} G_{12} \\
F_{21} G_{21}
\end{array}\right) \pm \frac{2 i}{(k+5)^{2}} B_{ \pm}\left(\begin{array}{l}
F_{11} G_{11} \\
F_{22} G_{22}
\end{array}\right) \\
& -\frac{\left(1315 k^{2}+5486 k+7611\right)}{3(k+5)^{3}(13 k+17)}\left(\begin{array}{c}
\partial^{2} F_{11} F_{12} \\
\partial^{2} F_{22} F_{21}
\end{array}\right) \\
& +\frac{2\left(1883 k^{2}+3454 k-1389\right)}{3(k+5)^{3}(13 k+17)}\left(\begin{array}{l}
\partial F_{11} \partial F_{12} \\
\partial F_{22} \partial F_{21}
\end{array}\right)
\end{aligned}
$$




$$
\begin{aligned}
& -\frac{\left(1315 k^{2}+5486 k+7611\right)}{3(k+5)^{3}(13 k+17)}\left(\begin{array}{c}
F_{11} \partial^{2} F_{12} \\
F_{22} \partial^{2} F_{21}
\end{array}\right)-\frac{3}{(k+5)}\left(\begin{array}{c}
G_{11} G_{12} \\
G_{22} G_{21}
\end{array}\right) \\
& -\frac{4\left(29 k^{2}-155 k-300\right)}{3(k+5)^{2}(13 k+17)}\left(\begin{array}{c}
\partial F_{11} G_{12} \\
\partial F_{22} G_{21}
\end{array}\right) \\
& +\frac{\left(583 k^{2}+272 k-1023\right)}{6(k+5)^{2}(13 k+17)}\left(\begin{array}{l}
\partial F_{12} G_{11} \\
\partial F_{21} G_{22}
\end{array}\right) \\
& +\frac{4\left(10 k^{2}+11 k+45\right)}{3(k+5)^{2}(13 k+17)}\left(\begin{array}{c}
F_{11} \partial G_{12} \\
F_{22} \partial G_{21}
\end{array}\right) \pm \frac{2 i(44 k+109)}{(k+5)(13 k+17)} T A_{ \pm} \\
& +\frac{4(43 k+143)}{(k+5)^{2}(13 k+17)} T\left(\begin{array}{c}
F_{11} F_{12} \\
F_{22} F_{21}
\end{array}\right)-\frac{12}{(k+5)^{2}} U A_{ \pm} B_{3} \\
& \pm \frac{9 i}{(k+5)^{2}} \partial U A_{ \pm} \mp \frac{21 i}{(k+5)^{2}} U \partial A_{ \pm}-\frac{4}{(k+5)^{2}} U\left(\begin{array}{c}
F_{11} G_{12} \\
F_{22} G_{21}
\end{array}\right) \\
& -\frac{\left(503 k^{2}+2134 k+1167\right)}{3(k+5)^{3}(13 k+17)} U\left(\begin{array}{c}
F_{11} \partial F_{12} \\
F_{22} \partial F_{21}
\end{array}\right) \mp \frac{2 i}{(k+5)^{2}} U U A_{ \pm} \\
& +\frac{\left(277 k^{2}+914 k+1485\right)}{3(k+5)^{3}(13 k+17)} \partial U\left(\begin{array}{c}
F_{11} F_{12} \\
F_{22} F_{21}
\end{array}\right) \mp \frac{6 i}{(k+5)^{2}} A_{ \pm} B_{3} B_{3} \\
& -\frac{\left(35 k^{2}-818 k-1893\right)}{3(k+5)^{3}(13 k+17)} U\left(\begin{array}{c}
\partial F_{11} F_{12} \\
\partial F_{22} F_{21}
\end{array}\right)+\frac{4}{(k+5)^{2}} U\left(\begin{array}{c}
F_{12} G_{11} \\
F_{21} G_{22}
\end{array}\right) \\
& \left.\mp \frac{4 i(3 k+19)}{(k+5)^{3}}\left(\begin{array}{l}
F_{11} B_{+} \partial F_{11} \\
F_{22} B_{-} \partial F_{22}
\end{array}\right) \pm \frac{16 i}{(k+5)^{3}}\left(\begin{array}{c}
F_{12} B_{-} \partial F_{12} \\
F_{21} B_{+} \partial F_{21}
\end{array}\right)\right](w) \\
& +\frac{1}{(z-w)}[+\cdots](w)+\cdots \text {. }
\end{aligned}
$$

There is no boldface higher spin current in the third order pole of the OPEs. As before, the nonlinear terms between the boldface higher spin currents can be removed by adding the $T \mathbf{T}^{(\mathbf{1})}(w)$ into the left hand side.

As before, the result (3.7) determines the following OPEs

$$
\begin{aligned}
\left(\begin{array}{c}
\mathbf{U}^{\left(\frac{5}{2}\right)} \\
\mathbf{V}^{\left(\frac{5}{2}\right)}
\end{array}\right)(z)\left(\begin{array}{c}
\mathbf{U}^{\left(\frac{5}{2}\right)} \\
\mathbf{V}^{\left(\frac{5}{2}\right)}
\end{array}\right)(w) & =\frac{1}{(z-w)^{3}}\left[\frac{8\left(8 k^{2}+51 k+135\right)}{9(k+5)^{3}} A_{ \pm} B_{\mp}\right. \\
& \pm \frac{32 i(k+3)(k+9)}{9(k+5)^{4}} A_{ \pm}\left(\begin{array}{c}
F_{11} F_{21} \\
F_{22} F_{12}
\end{array}\right) \\
& \mp \frac{32 i(k+3)(k+9)}{9(k+5)^{4}} B_{\mp}\left(\begin{array}{c}
F_{11} F_{12} \\
F_{22} F_{21}
\end{array}\right) \\
& +\frac{16\left(31 k^{2}+138 k+27\right)}{9(k+5)^{4}}\left(\begin{array}{c}
F_{11} \partial F_{11} \\
F_{22} \partial F_{22}
\end{array}\right) \\
& \left.-\frac{8(k-3)(2 k+9)}{3(k+5)^{3}}\left(\begin{array}{c}
F_{11} G_{11} \\
F_{22} G_{22}
\end{array}\right)\right](w)
\end{aligned}
$$




$$
+\frac{1}{(z-w)^{2}} \frac{1}{2} \partial(\text { pole- } 3)(w)+\frac{1}{(z-w)}[+\cdots](w)+\cdots .
$$

It is an open problem to obtain the first order pole of the OPEs.

Also one can construct the following OPE

$$
\begin{aligned}
& \mathbf{U}^{\left(\frac{5}{2}\right)}(z) \mathbf{V}^{\left(\frac{3}{2}\right)}(w)=\frac{1}{(z-w)^{3}}\left[-\frac{4(\mathbf{k}-\mathbf{3})}{3(k+5)} \mathbf{T}^{(\mathbf{1})}+\frac{2 i(5 k+18)}{(k+5)^{2}} A_{3}+\frac{2 i k(4 k+21)}{3(k+5)^{2}} B_{3}+\frac{6 k}{(k+5)^{2}} U\right. \\
& \left.-\frac{4(k-3)(2 k+9)}{3(k+5)^{3}} F_{11} F_{22}+\frac{4\left(2 k^{2}+9 k+27\right)}{3(k+5)^{3}} F_{12} F_{21}\right](w) \\
& +\frac{1}{(z-w)^{2}}\left[\frac{1}{2} \mathbf{P}^{(\mathbf{2})}-\frac{8(k+3)}{3(k+5)}\left(\mathbf{T}^{(\mathbf{2})}+\mathbf{W}^{(\mathbf{2})}\right)-\frac{4(\mathbf{k}-\mathbf{3})}{3(k+5)} \partial \mathbf{T}^{(\mathbf{1})}\right. \\
& +\frac{4(k-3)}{3(k+5)^{2}} A_{3} B_{3}+\frac{2 i(5 k+18)}{(k+5)^{2}} \partial A_{3}-\frac{4 i k}{(k+5)^{2}} U B_{3}+\frac{6 k}{(k+5)^{2}} \partial U \\
& -\frac{2 i(k-3)}{3(k+5)^{3}} A_{3} F_{11} F_{22}+\frac{2 i(k-3)}{3(k+5)^{3}} A_{3} F_{12} F_{21}+\frac{2 i(5 k+9)}{3(k+5)^{3}} B_{-} F_{12} F_{22} \\
& +\frac{8 i(k+9)}{3(k+5)^{3}} A_{-} F_{11} F_{12}-\frac{8 i(k+9)}{3(k+5)^{3}} A_{+} F_{21} F_{22}-\frac{2 i(5 k+9)}{3(k+5)^{3}} B_{+} F_{11} F_{21} \\
& +\frac{2 i k(4 k+21)}{3(k+5)^{2}} \partial B_{3}+\frac{2 i(k-3)}{3(k+5)^{3}} B_{3} F_{11} F_{22}+\frac{2 i(k-3)}{(k+5)^{2}} B_{3} F_{12} F_{21} \\
& -\frac{(k+9)}{3(k+5)^{2}} F_{11} G_{22}-\frac{2\left(4 k^{2}-5 k-93\right)}{3(k+5)^{3}} \partial F_{11} F_{22} \\
& -\frac{2(4 k-21)}{3(k+5)^{2}} F_{11} \partial F_{22}-\frac{(k+9)}{(k+5)^{2}} F_{12} G_{21}+\frac{2\left(4 k^{2}+15 k+87\right)}{3(k+5)^{3}} \partial F_{12} F_{21} \\
& +\frac{2\left(4 k^{2}+19 k+75\right)}{3(k+5)^{3}} F_{12} \partial F_{21}+\frac{6}{(k+5)^{2}} F_{21} G_{12}+\frac{2(2 k+9)}{3(k+5)^{2}} F_{22} G_{11} \\
& \left.+\frac{2(k-27)}{3(k+5)^{3}} U F_{11} F_{22}-\frac{6}{(k+5)^{2}} U F_{12} F_{21}\right](w) \\
& +\frac{1}{(z-w)}\left[\frac{1}{2} \mathbf{S}^{(\mathbf{3})}+\frac{3}{8} \partial \mathbf{P}^{(\mathbf{2})}-\mathbf{W}^{(\mathbf{3})}-\frac{2(k+3)}{(k+5)} \partial\left(\mathbf{T}^{(\mathbf{2})}+\mathbf{W}^{(\mathbf{2})}\right)\right. \\
& -\frac{2(\mathbf{k}-\mathbf{3})}{(13 k+17)}\left(T \mathbf{T}^{(\mathbf{1})}-\frac{1}{2} \partial^{2} \mathbf{T}^{(\mathbf{1})}\right)-\frac{2(\mathbf{k}-\mathbf{3})}{3(k+5)} \partial^{2} \mathbf{T}^{(\mathbf{1})} \\
& +\frac{(k-3)}{(k+5)^{2}} \partial\left(A_{3} B_{3}\right)+\frac{i(5 k+18)(23 k+19)}{2(k+5)^{2}(13 k+17)} \partial^{2} A_{3} \\
& -\frac{i(5 k+17)}{2(k+5)^{3}} \partial A_{3} F_{11} F_{22}+\frac{i(3 k+23)}{2(k+5)^{3}} A_{3} \partial\left(F_{11} F_{22}\right) \\
& -\frac{i(3 k+23)}{2(k+5)^{3}} \partial A_{3} F_{12} F_{21}+\frac{i(5 k+17)}{2(k+5)^{3}} A_{3} \partial\left(F_{12} F_{21}\right) \\
& +\frac{8 i}{(k+5)^{3}} \partial A_{-} F_{11} F_{12}+\frac{4 i(k+7)}{(k+5)^{3}} A_{-} \partial\left(F_{11} F_{12}\right)
\end{aligned}
$$




$$
\begin{aligned}
& -\frac{4 i(k+7)}{(k+5)^{3}} \partial A_{+} F_{21} F_{22}-\frac{8 i}{(k+5)^{3}} A_{+} \partial\left(F_{21} F_{22}\right) \\
& +\frac{i k(4 k+21)(23 k+19)}{6(k+5)^{2}(13 k+17)} \partial^{2} B_{3}-\frac{i(3 k+23)}{2(k+5)^{3}} \partial B_{3} F_{11} F_{22} \\
& +\frac{i(5 k+17)}{2(k+5)^{3}} B_{3} \partial\left(F_{11} F_{22}\right)+\frac{(9 k+13)}{2(k+5)^{3}} U F_{11} \partial F_{22} \\
& +\frac{i(5 k+17)}{2(k+5)^{3}} \partial B_{3} F_{12} F_{21}-\frac{i(3 k+23)}{2(k+5)^{3}} B_{3} \partial\left(F_{12} F_{21}\right) \\
& +\frac{i(k-11)}{2(k+5)^{3}} \partial B_{-} F_{12} F_{22}+\frac{i(9 k+29)}{2(k+5)^{3}} B_{-} \partial\left(F_{12} F_{22}\right) \\
& -\frac{i(9 k+29)}{2(k+5)^{3}} \partial B_{+} F_{11} F_{21}-\frac{i(9 k-11)}{2(k+5)^{2}} B_{+} \partial\left(F_{11} F_{21}\right) \\
& -\frac{\left(92 k^{3}-215 k^{2}-3210 k-3015\right)}{6(k+5)^{3}(13 k+17)} \partial^{2} F_{11} F_{22} \\
& -\frac{\left(92 k^{3}-137 k^{2}-3342 k-3321\right)}{3(k+5)^{3}(13 k+17)} \partial F_{11} \partial F_{22}-\frac{9}{2(k+5)^{2}} \partial U F_{12} F_{21} \\
& -\frac{\left(92 k^{3}-59 k^{2}-3474 k-3627\right)}{6(k+5)^{3}(13 k+17)} F_{11} \partial^{2} F_{22}-\frac{(7 k+39)}{4(k+5)^{2}} \partial F_{11} G_{22} \\
& +\frac{(k+1)}{4(k+5)^{2}} F_{11} \partial G_{22}+\frac{\left(92 k^{3}+373 k^{2}+2718 k+2709\right)}{6(k+5)^{3}(13 k+17)} \partial^{2} F_{12} F_{21} \\
& +\frac{\left(92 k^{3}+451 k^{2}+2586 k+2403\right)}{3(k+5)^{3}(13 k+17)} \partial F_{12} \partial F_{21} \\
& +\frac{\left(92 k^{3}+529 k^{2}+2454 k+2097\right)}{6(k+5)^{3}(13 k+17)} F_{12} \partial^{2} F_{21}-\frac{3(3 k+19)}{4(k+5)^{2}} \partial F_{12} G_{21} \\
& -\frac{(k+17)}{4(k+5)^{2}} F_{12} \partial G_{21}-\frac{3(k+2)}{2(k+5)^{2}} \partial F_{21} G_{12}+\frac{(k+14)}{2(k+5)^{2}} F_{21} \partial G_{12} \\
& -\frac{(k+6)}{2(k+5)^{2}} \partial F_{22} G_{11}+\frac{(3 k+14)}{2(k+5)^{2}} F_{22} \partial G_{11}+\frac{9 k}{(k+5)(13 k+17)} T U \\
& +\frac{3 i(5 k+18)}{(k+5)(13 k+17)} T A_{3}+\frac{i k(4 k+21)}{(k+5)(13 k+17)} T B_{3} \\
& -\frac{2(k-3)(2 k+9)}{(k+5)^{2}(13 k+17)} T F_{11} F_{22}+\frac{2\left(2 k^{2}+9 k+27\right)}{(k+5)^{2}(13 k+17)} T F_{12} F_{21} \\
& -\frac{3 i k}{(k+5)^{2}} \partial\left(U B_{3}\right)+\frac{3 k(23 k+19)}{2(k+5)^{2}(13 k+17)} \partial^{2} U \\
& +\frac{(k-27)}{2(k+5)^{3}} \partial U F_{11} F_{22}-\frac{(7 k+67)}{2(k+5)^{3}} U \partial F_{11} F_{22} \\
& \left.-\frac{17}{2(k+5)^{2}} U \partial F_{12} F_{21}-\frac{1}{2(k+5)^{2}} U F_{12} \partial F_{21}\right](w)+\cdots \text {. }
\end{aligned}
$$

Note the presence of a quasi primary field containing the higher spin-1 current in the first 
order pole.

Furthermore the following OPE can be obtained

$$
\begin{aligned}
& \mathbf{U}^{\left(\frac{5}{2}\right)}(z) \mathbf{V}_{+}^{(2)}(w)=\frac{1}{(z-w)^{4}}\left[-\frac{24 k(k+3)}{(k+5)^{3}} F_{21}\right](w) \\
& +\frac{1}{(z-w)^{3}}\left[-\frac{2\left(4 k^{2}+38 k+69\right)}{3(k+5)^{2}} G_{21}-\frac{4 i\left(4 k^{2}+11 k-21\right)}{3(k+5)^{3}} F_{11} A_{-}\right. \\
& -\frac{4\left(4 k^{2}+29 k+69\right)}{3(k+5)^{3}} U F_{21}+\frac{16(k+3)}{3(k+5)^{3}} F_{21} F_{11} F_{22}-\frac{4 i(17 k+69)}{3(k+5)^{3}} F_{21} B_{3} \\
& \left.+\frac{4 i\left(4 k^{2}+17 k+33\right)}{3(k+5)^{3}} F_{21} A_{3}-\frac{24 k(k+3)}{(k+5)^{3}} \partial F_{21}+\frac{4 i(17 k+69)}{3(k+5)^{3}} F_{22} B_{-}\right](w) \\
& +\frac{1}{(z-w)^{2}}\left[\frac{4(2 k+9)}{3(k+5)}\left(\mathbf{P}_{+}^{\left(\frac{5}{2}\right)}+\mathbf{W}_{+}^{\left(\frac{5}{2}\right)}\right)\right. \\
& +\frac{2 i(5 k+9)}{3(k+5)^{2}} A_{3} G_{21}-\frac{4(5 k+21)}{3(k+5)^{3}} A_{3} B_{3} F_{21}+\frac{4(5 k+21)}{3(k+5)^{3}} A_{3} B_{-} F_{22} \\
& +\frac{16 i\left(2 k^{2}+21 k+63\right)}{9(k+5)^{3}} \partial A_{3} F_{21}+\frac{2 i\left(16 k^{2}-63 k-315\right)}{9(k+5)^{3}} A_{3} \partial F_{21} \\
& -\frac{2 i(2 k+7)}{(k+5)^{2}} A_{-} G_{11}+\frac{4(k+9)}{3(k+5)^{3}} A_{3} A_{3} F_{21}+\frac{4(k+3)}{3(k+5)^{3}} A_{-} A_{3} F_{11} \\
& +\frac{8(2 k+9)}{3(k+5)^{3}} A_{-} B_{3} F_{11}+\frac{8(2 k+9)}{3(k+5)^{3}} A_{-} B_{-} F_{12}-\frac{4 i(k+3)(8 k+33)}{9(k+5)^{3}} \partial A_{-} F_{11} \\
& -\frac{2 i\left(16 k^{2}-81 k-585\right)}{9(k+5)^{3}} A_{-} \partial F_{11}-\frac{2 i(k+9)}{3(k+5)^{2}} B_{3} G_{21}+\frac{8(k+6)}{3(k+5)^{3}} A_{+} A_{-} F_{21} \\
& +\frac{4 i\left(4 k^{2}-3 k-99\right)}{9(k+5)^{3}} \partial B_{3} F_{21}-\frac{2 i\left(16 k^{2}+153 k+261\right)}{9(k+5)^{3}} B_{3} \partial F_{21} \\
& +\frac{2 i(k+9)}{3(k+5)^{2}} B_{-} G_{22}+\frac{2(k+9)}{3(k+5)^{3}} F_{12} F_{21} G_{21}-\frac{32(k+3)(k+6)}{9(k+5)^{4}} F_{11} \partial F_{21} F_{22} \\
& -\frac{4 i\left(4 k^{2}-3 k-99\right)}{9(k+5)^{3}} \partial B_{-} F_{22}+\frac{2 i\left(16 k^{2}+153 k+261\right)}{9(k+5)^{3}} B_{-} \partial F_{22} \\
& +\frac{4}{(k+5)^{2}} F_{11} F_{21} G_{22}+\frac{64(k+6)^{2}}{9(k+5)^{4}} \partial F_{11} F_{21} F_{22} \\
& -\frac{32\left(4 k^{2}+39 k+99\right)}{9(k+5)^{4}} F_{11} F_{21} \partial F_{22}-\frac{2(5 k+21)}{3(k+5)^{3}} F_{11} F_{22} G_{21} \\
& -\frac{2\left(2 k^{2}+15 k+45\right)}{(k+5)^{3}} \partial^{2} F_{21}+\frac{4(k+3)}{3(k+5)^{3}} F_{21} F_{22} G_{11} \\
& -\frac{2(k+6)(8 k+21)}{9(k+5)^{2}} \partial G_{21}-\frac{4(4 k+9)}{3(k+5)^{2}} T F_{21} \\
& -\frac{2(k+12)}{3(k+5)^{2}} U G_{21}+\frac{8 i}{(k+5)^{2}} U A_{3} F_{21}-\frac{4 i(7 k+33)}{3(k+5)^{3}} U A_{-} F_{11}
\end{aligned}
$$




$$
\begin{aligned}
& +\frac{4 i(5 k+21)}{3(k+5)^{3}} U B_{-} F_{22}-\frac{8\left(4 k^{2}+21 k+63\right)}{9(k+5)^{3}} \partial U F_{21} \\
& -\frac{2\left(16 k^{2}+165 k+225\right)}{9(k+5)^{3}} U \partial F_{21}-\frac{4 i(5+21)}{3(k+5)^{3}} U B_{3} F_{21} \\
& \left.-\frac{4(5+21)}{3(k+5)^{3}} U U F_{21}-\frac{32\left(k^{2}+10 k+27\right)}{3(k+5)^{4}} F_{21} \partial F_{21} F_{12}\right](w) \\
& +\frac{1}{(z-w)}[+\cdots](w)+\cdots
\end{aligned}
$$

One does not see the higher spin current in the third order pole. The corresponding OPE in the nonlinear version contains the higher spin current in that pole.

The following OPE can be obtained

$$
\begin{aligned}
& \mathbf{U}^{\left(\frac{5}{2}\right)}(z) \mathbf{V}_{-}^{(2)}(w)=\frac{1}{(z-w)^{4}}\left[-\frac{12 k(k+9)}{(k+5)^{3}} F_{12}\right](w) \\
& +\frac{1}{(z-w)^{3}}\left[\frac{4\left(4 k^{2}+31 k+63\right)}{3(k+5)^{3}} U F_{12}+\frac{8(k+9)}{3(k+5)^{3}} F_{12} F_{11} F_{22}\right. \\
& +\frac{4 i\left(4 k^{2}+25 k+9\right)}{3(k+5)^{3}} F_{12} A_{3}-\frac{4 i\left(4 k^{2}+25 k+9\right)}{3(k+5)^{3}} F_{22} A_{+}-\frac{4 i\left(2 k^{2}-k-63\right)}{3(k+5)^{3}} F_{11} B_{+} \\
& \left.-\frac{4 i\left(2 k^{2}+13 k+63\right)}{3(k+5)^{3}} F_{12} B_{3}+\frac{2\left(4 k^{2}+40 k+63\right)}{3(k+5)^{2}} G_{12}-\frac{12 k(k+9)}{(k+5)^{3}} \partial F_{12}\right](w) \\
& +\frac{1}{(z-w)^{2}}\left[-\frac{(7 k+39)}{3(k+5)}\left(\mathbf{P}_{-}^{\left(\frac{5}{2}\right)}-\mathbf{W}_{-}^{\left(\frac{5}{2}\right)}\right)-\frac{4 i(k+3)}{3(k+5)^{2}} A_{3} G_{12}\right. \\
& -\frac{16(k+6)}{3(k+5)^{3}} A_{3} B_{3} F_{12}+\frac{2(7 k+39)}{3(k+5)^{3}} A_{3} B_{+} F_{11}-\frac{4 i\left(k^{2}+16 k+75\right)}{3(k+5)^{3}} \partial A_{3} F_{12} \\
& +\frac{2 i\left(16 k^{2}+159 k+243\right)}{9(k+5)^{3}} A_{3} \partial F_{12}+\frac{4 i(k+3)}{3(k+5)^{2}} A_{+} G_{22}+\frac{16(k+6)}{3(k+5)^{3}} A_{+} B_{3} F_{22} \\
& +\frac{2(7 k+39)}{3(k+5)^{3}} A_{+} B_{+} F_{21}-\frac{4 i\left(8 k^{2}+27 k-81\right)}{9(k+5)^{3}} \partial A_{+} F_{22} \\
& -\frac{2 i\left(16 k^{2}+159 k+243\right)}{9(k+5)^{3}} A_{+} \partial F_{22}+\frac{2 i(k+21)}{3(k+5)^{2}} B_{3} G_{12} \\
& +\frac{8(k+3)}{3(k+5)^{3}} \partial B_{3} F_{12}+\frac{2 i\left(20 k^{2}+147 k-261\right)}{9(k+5)^{3}} B_{3} \partial F_{12} \\
& -\frac{i(3 k+17)}{(k+5)^{2}} B_{+} G_{11}+\frac{2(k+9)}{3(k+5)^{3}} B_{+} B_{3} F_{11}+\frac{2(5 k+21)}{3(k+5)^{3}} B_{+} B_{-} F_{12} \\
& +\frac{2 i(k+9)(7 k+36)}{9(k+5)^{3}} \partial B_{+} F_{11}-\frac{4 i\left(19 k^{2}+87 k-90\right)}{9(k+5)^{3}} B_{+} \partial F_{11} \\
& +\frac{4(5 k+21)^{2}}{9(k+5)^{4}} \partial F_{11} F_{12} F_{22}-\frac{4}{(k+5)^{2}} F_{11} F_{12} G_{22}+\frac{8(k+3)}{3(k+5)^{3}} B_{3} B_{3} F_{12}
\end{aligned}
$$




$$
\begin{aligned}
& -\frac{4(k+9)(5 k+21)}{9(k+5)^{4}} F_{11} \partial F_{12} F_{22}-\frac{32\left(4 k^{2}+39 k+99\right)}{9(k+5)^{4}} F_{11} F_{12} \partial F_{22} \\
& +\frac{8(k+6)}{3(k+5)^{3}} F_{11} F_{22} G_{12}-\frac{4(2 k+15)}{3(k+5)^{2}} T F_{12}-\frac{2(2 k+9)}{3(k+5)^{2}} U G_{12} \\
& -\frac{2\left(19 k^{2}+114 k-189\right)}{3(k+5)^{3}} \partial^{2} F_{12}+\frac{4(k+3)}{3(k+5)^{3}} F_{12} F_{21} G_{12} \\
& -\frac{2(k+9)}{3(k+5)^{3}} F_{12} F_{22} G_{11}+\frac{2(2 k+3)(4 k+33)}{9(k+5)^{2}} \partial G_{12}-\frac{16 i(k+6)}{3(k+5)^{3}} U A_{3} F_{12} \\
& +\frac{16 i(k+6)}{3(k+5)^{3}} U A_{+} F_{22}+\frac{8 i}{(k+5)^{2}} U B_{3} F_{12}+\frac{4\left(19 k^{2}+126 k+243\right)}{9(k+5)^{3}} \partial U F_{12} \\
& -\frac{2 i(13 k+69)}{3(k+5)^{3}} U B_{+} F_{11}-\frac{16(k+6)}{3(k+5)^{3}} U U F_{12}+\frac{2\left(16 k^{2}+147 k+279\right)}{9(k+5)^{3}} U \partial F_{12} \\
& \left.-\frac{8 i\left(9 k^{2}+82 k+201\right)}{3(k+5)^{4}} F_{12} B_{3} F_{12} F_{21}-\frac{4\left(11 k^{2}+75 k+144\right)}{9(k+5)^{3}} F_{12} G_{21} F_{12}\right](w) \\
& +\frac{1}{(z-w)}[+\cdots](w)+\cdots
\end{aligned}
$$

There is no boldface higher spin current in the third order pole.

The other combination of OPE can be described as

$$
\begin{aligned}
\mathbf{U}^{\left(\frac{5}{2}\right)}(z) \mathbf{V}^{\left(\frac{5}{2}\right)}(w) & =\frac{1}{(z-w)^{5}}\left[-\frac{32 k(k+3)(k+9)}{(k+5)^{3}}\right] \\
& +\frac{1}{(z-w)^{4}}\left[-\frac{8 i(k+9)(4 k+9)}{(k+5)^{3}} A_{3}+\frac{16 i k(k+3)(2 k+15)}{3(k+5)^{3}} B_{3}\right. \\
& \left.-\frac{8\left(4 k^{3}+27 k^{2}+90 k+243\right)}{3(k+5)^{4}} F_{11} F_{22}+\frac{8(k-3)\left(4 k^{2}+33 k+81\right)}{3(k+5)^{4}} F_{12} F_{21}\right](w) \\
& +\frac{1}{(z-w)^{3}}\left[\frac{2(k-3)}{3(k+5)} \mathbf{P}^{(2)}-\frac{8(k-3)^{2}}{9(k+5)^{2}} \mathbf{T}^{(2)}-\frac{8(k-3)}{3(k+5)} \mathbf{W}^{(2)}\right. \\
& -\frac{4\left(20 k^{2}+204 k+369\right)}{9(k+5)^{2}} T-\frac{4\left(2 k^{2}+87 k+369\right)}{9(k+5)^{3}} B_{3} B_{3} \\
& -\frac{4\left(20 k^{2}+123 k+99\right)}{9(k+5)^{3}} A_{3} A_{3}+\frac{8\left(k^{2}+5 k+16\right)}{(k+5)^{3}} A_{3} B_{3} \\
& -\frac{16 i\left(14 k^{2}+132 k+207\right)}{9(k+5)^{3}} \partial A_{3}-\frac{8 i\left(13 k^{2}+66 k+117\right)}{9(k+5)^{4}} B_{+} F_{11} F_{21} \\
& +\frac{64 i(k+3)(k+9)}{9(k+5)^{4}} A_{3} F_{11} F_{22}+\frac{8 i\left(5 k^{2}+42 k+117\right)}{9(k+5)^{4}} A_{3} F_{12} F_{21} \\
& -\frac{4\left(20 k^{2}+123 k+99\right)}{9(k+5)^{3}} A_{+} A_{-}+\frac{4(k-3)(10 k+51)}{9(k+5)^{3}} F_{22} G_{11} \\
& +\frac{8 i(k+1)(k+9)}{(k+5)^{4}} A_{-} F_{11} F_{12}+\frac{32 i\left(k^{2}+12 k+63\right)}{9(k+5)^{4}} A_{+} F_{21} F_{22}
\end{aligned}
$$




$$
\begin{aligned}
& +\frac{4 i\left(12 k^{3}+124 k^{2}+183 k-369\right)}{9(k+5)^{3}} \partial B_{3}-\frac{64 i(k+3)(k+9)}{9(k+5)^{4}} B_{3} F_{11} F_{22} \\
& +\frac{8 i\left(5 k^{2}+42 k+117\right)}{9(k+5)^{4}} B_{3} F_{12} F_{21}-\frac{16\left(2 k^{3}+45 k^{2}+270 k+747\right)}{9(k+5)^{4}} \partial F_{12} F_{21} \\
& -\frac{64 i(k+3)}{(k+5)^{4}} B_{-} F_{12} F_{22}-\frac{4\left(2 k^{2}+87 k+369\right)}{9(k+5)^{3}} B_{+} B_{-} \\
& +\frac{4(k-3)(11 k+48)}{9(k+5)^{3}} F_{11} G_{22}-\frac{32\left(4 k^{3}+29 k^{2}+132 k+387\right)}{9(k+5)^{4}} \partial F_{11} F_{22} \\
& +\frac{8\left(4 k^{3}+53 k^{2}+294 k+549\right)}{9(k+5)^{4}} F_{11} \partial F_{22} \\
& +\frac{8\left(16 k^{3}+145 k^{2}+534 k+693\right)}{9(k+5)^{4}} F_{12} \partial F_{21}+\frac{8(k-3)(k+6)}{3(k+5)^{3}} F_{21} G_{12} \\
& -\frac{8 i(k-3)}{(k+5)^{3}} U A_{3}-\frac{8 i(k-3) k}{3(k+5)^{3}} U B_{3}-\frac{4\left(20 k^{2}+177 k+369\right)}{9(k+5)^{3}} U U \\
& \left.+\frac{32(k-3)^{2}}{9(k+5)^{4}} U F_{11} F_{22}-\frac{8(k-3)}{(k+5)^{3}} U F_{12} F_{21}\right](w) \\
& +\frac{1}{(z-w)^{2}}\left[\frac{(k-3)}{3(k+5)} \mathbf{S}^{(\mathbf{3})}+3 \mathbf{P}^{(\mathbf{3})}+\frac{(k-3)}{3(k+5)} \partial \mathbf{P}^{(\mathbf{2})}-\frac{2(5 k+21)}{3(k+5)} \mathbf{W}^{(\mathbf{3})}\right. \\
& -\frac{4(k-3)^{2}}{9(k+5)^{2}} \partial \mathbf{T}^{(\mathbf{2})}-\frac{4(k-3)}{3(k+5)} \partial \mathbf{W}^{(\mathbf{2})} \\
& \text { - } \frac{8 i}{(k+5)^{2}} A_{3} A_{3} B_{3}-\frac{4\left(20 k^{2}+123 k+99\right)}{9(k+5)^{3}} \partial A_{3} A_{3}+\frac{8 i}{(k+5)^{2}} A_{3} B_{3} B_{3} \\
& -\frac{2\left(9 k^{2}+86 k+117\right)}{3(k+5)^{3}} \partial A_{3} B_{3}+\frac{2\left(21 k^{2}+146 k+309\right)}{3(k+5)^{3}} A_{3} \partial B_{3} \\
& +\frac{8 i}{(k+5)^{2}} A_{3} B_{+} B_{-}-\frac{2 i\left(392 k^{3}+3697 k^{2}+5646 k-5211\right)}{9(k+5)^{3}(13 k+17)} \partial^{2} A_{3} \\
& +\frac{10 i}{(k+5)^{2}} A_{3} F_{11} G_{22}+\frac{i\left(17 k^{2}+570 k+2169\right)}{9(k+5)^{4}} \partial A_{3} F_{11} F_{22} \\
& -\frac{i\left(79 k^{2}+774 k+2151\right)}{9(k+5)^{4}} A_{3} \partial F_{11} F_{22}+\frac{i\left(173 k^{2}+1170 k+1269\right)}{9(k+5)^{4}} A_{3} F_{11} \partial F_{22} \\
& +\frac{4 i(k+9)}{3(k+5)^{3}} A_{3} F_{12} G_{21}-\frac{i\left(97 k^{2}+1122 k+3249\right)}{9(k+5)^{4}} A_{3} F_{12} \partial F_{21} \\
& +\frac{i\left(155 k^{2}+1494 k+3915\right)}{9(k+5)^{4}} \partial A_{3} F_{12} F_{21}-\frac{i\left(133 k^{2}+1194 k+2709\right)}{9(k+5)^{4}} A_{3} \partial F_{12} F_{21} \\
& +\frac{8 i(k+3)}{3(k+5)^{3}} A_{3} F_{21} G_{12}-\frac{2 i}{(k+5)^{2}} A_{3} F_{22} G_{11}+\frac{8 i(k+6)}{3(k+5)^{3}} A_{-} F_{11} G_{12} \\
& +\frac{4 i\left(11 k^{2}+120 k+285\right)}{3(k+5)^{4}} \partial A_{-} F_{11} F_{12}-\frac{8 i}{(k+5)^{2}} A_{+} A_{-} B_{3}
\end{aligned}
$$




$$
\begin{aligned}
& -\frac{8 i\left(6 k^{2}+61 k+183\right)}{3(k+5)^{4}} A_{-} \partial F_{11} F_{12}+\frac{8 i\left(k^{2}+k-48\right)}{3(k+5)^{4}} A_{-} F_{11} \partial F_{12} \\
& \text { - } \frac{4\left(10 k^{2}+39 k-63\right)}{9(k+5)^{3}} \partial A_{+} A_{-}-\frac{8(k+3)(5 k+27)}{9(k+5)^{3}} A_{+} \partial A_{-} \\
& +\frac{2 i(7 k+39)}{3(k+5)^{3}} A_{+} F_{21} G_{22}+\frac{8 i\left(5 k^{2}+78 k+333\right)}{9(k+5)^{4}} \partial A_{+} F_{21} F_{22} \\
& \text { - } \frac{16 i(k+3)(2 k+21)}{9(k+5)^{4}} A_{+} \partial F_{21} F_{22}+\frac{16 i(k-6)(k+3)}{9(k+5)^{4}} A_{+} F_{21} \partial F_{22} \\
& -\frac{8 i(k+6)}{3(k+5)^{3}} A_{+} F_{22} G_{21}-\frac{4\left(2 k^{2}+87 k+369\right)}{9(k+5)^{3}} \partial B_{3} B_{3} \\
& +\frac{2 i\left(44 k^{4}+491 k^{3}-649 k^{2}-7743 k-4743\right)}{9(k+5)^{3}(13 k+17)} \partial^{2} B_{3} \\
& -\frac{2 i}{(k+5)^{2}} B_{3} F_{11} G_{22}+\frac{i\left(7 k^{2}-90 k-369\right)}{9(k+5)^{4}} \partial B_{3} F_{11} F_{22} \\
& -\frac{i\left(17 k^{2}-150 k-1431\right)}{9(k+5)^{4}} B_{3} \partial F_{11} F_{22}-\frac{i\left(125 k^{2}+1506 k+4149\right)}{9(k+5)^{4}} B_{3} F_{11} \partial F_{22} \\
& +\frac{4 i(k+9)}{3(k+5)^{3}} B_{3} F_{12} G_{21}-\frac{i\left(109 k^{2}+1194 k+2925\right)}{9(k+5)^{4}} \partial B_{3} F_{12} F_{21} \\
& +\frac{i\left(131 k^{2}+1494 k+4131\right)}{9(k+5)^{4}} B_{3} \partial F_{12} F_{21}-\frac{2 i(k+9)}{3(k+5)^{3}} A_{-} F_{12} G_{11} \\
& +\frac{i\left(167 k^{2}+1566 k+3591\right)}{9(k+5)^{4}} B_{3} F_{12} \partial F_{21}+\frac{(5 k+23)}{(k+5)^{2}} G_{11} G_{22} \\
& +\frac{8 i(k+3)}{3(k+5)^{3}} B_{3} F_{21} G_{12}+\frac{10 i}{(k+5)^{2}} B_{3} F_{22} G_{11}-\frac{2 i(k+9)}{3(k+5)^{3}} B_{-} F_{12} G_{22} \\
& +\frac{i\left(37 k^{2}+282 k+645\right)}{3(k+5)^{4}} \partial B_{-} F_{12} F_{22}-\frac{(k-3)}{(k+5)^{2}} G_{12} G_{21} \\
& -\frac{i\left(15 k^{2}+238 k+591\right)}{3(k+5)^{4}} B_{-} \partial F_{12} F_{22}-\frac{i\left(59 k^{2}+710 k+1851\right)}{3(k+5)^{4}} B_{-} F_{12} \partial F_{22} \\
& +\frac{8 i(k+6)}{3(k+5)^{3}} B_{-} F_{22} G_{12}-\frac{2(k+9)(11 k+51)}{9(k+5)^{3}} \partial B_{+} B_{-} \\
& +\frac{2\left(7 k^{2}-24 k-279\right)}{9(k+5)^{3}} B_{+} \partial B_{-}-\frac{8 i(k+6)}{3(k+5)^{3}} B_{+} F_{11} G_{21} \\
& +\frac{i\left(5 k^{2}+258 k+621\right)}{9(k+5)^{4}} \partial B_{+} F_{11} F_{21}-\frac{i\left(103 k^{2}+630 k+927\right)}{9(k+5)^{4}} B_{+} \partial F_{11} F_{21} \\
& -\frac{i\left(115 k^{2}+942 k+2187\right)}{9(k+5)^{4}} B_{+} F_{11} \partial F_{21}+\frac{2 i(7 k+39)}{3(k+5)^{3}} B_{+} F_{21} G_{11} \\
& -\frac{\left(608 k^{4}+657 k^{3}-10193 k^{2}+219 k+1053\right)}{9(k+5)^{4}(13 k+17)} \partial^{2} F_{11} F_{22}
\end{aligned}
$$




$$
\begin{aligned}
& -\frac{2\left(88 k^{4}+7049 k^{3}+56775 k^{2}+137475 k+81837\right)}{9(k+5)^{4}(13 k+17)} \partial F_{11} \partial F_{22} \\
& +\frac{\left(432 k^{4}+9491 k^{3}+64429 k^{2}+164721 k+141543\right)}{9(k+5)^{4}(13 k+17)} F_{11} \partial^{2} F_{22} \\
& +\frac{\left(251 k^{2}+1266 k+423\right)}{18(k+5)^{3}} \partial F_{11} G_{22}-\frac{\left(25 k^{2}+342 k+909\right)}{18(k+5)^{3}} F_{11} \partial G_{22} \\
& -\frac{\left(48 k^{4}+803 k^{3}+4117 k^{2}+10577 k+5999\right)}{(k+5)^{4}(13 k+17)} \partial^{2} F_{12} F_{21} \\
& +\frac{2(k-3)\left(88 k^{2}+89 k-1419\right)}{9(k+5)^{3}(13 k+17)} \partial F_{12} \partial F_{21}-\frac{6}{(k+5)^{2}} U F_{22} G_{11} \\
& +\frac{\left(608 k^{4}+6353 k^{3}+34775 k^{2}+91563 k+94725\right)}{9(k+5)^{4}(13 k+17)} F_{12} \partial^{2} F_{21} \\
& +\frac{\left(9 k^{2}+114 k+233\right)}{2(k+5)^{3}} \partial F_{12} G_{21}-\frac{\left(9 k^{2}+122 k+273\right)}{6(k+5)^{3}} F_{12} \partial G_{21} \\
& +\frac{\left(13 k^{2}+81 k+168\right)}{3(k+5)^{3}} \partial F_{21} G_{12}+\frac{\left(k^{2}-7 k-132\right)}{3(k+5)^{3}} F_{21} \partial G_{12} \\
& +\frac{\left(29 k^{2}+483 k+1530\right)}{9(k+5)^{3}} \partial F_{22} G_{11}+\frac{\left(17 k^{2}-105 k-918\right)}{9(k+5)^{3}} F_{22} \partial G_{11} \\
& -\frac{4 i\left(20 k^{2}+231 k+483\right)}{(k+5)^{2}(13 k+17)} T A_{3}+\frac{16 i k\left(5 k^{2}+57 k+123\right)}{3(k+5)^{2}(13 k+17)} T B_{3} \\
& -\frac{4(k+3)(10 k+81)}{9(k+5)^{2}} \partial T+\frac{16(k+6)(4 k+17)}{3(k+5)^{2}(13 k+17)} T U \\
& -\frac{8\left(10 k^{3}+123 k^{2}+552 k+903\right)}{3(k+5)^{3}(13 k+17)} T F_{11} F_{22}+\frac{16(k+6)}{3(k+5)^{3}} U A_{+} A_{-} \\
& +\frac{16(k-3)\left(5 k^{2}+45 k+108\right)}{3(k+5)^{3}(13 k+17)} T F_{12} F_{21}+\frac{16(k+6)}{3(k+5)^{3}} U A_{3} A_{3} \\
& -\frac{8(5 k+21)}{3(k+5)^{3}} U A_{3} B_{3}-\frac{8 i(3 k+8)}{(k+5)^{3}} \partial U A_{3}+\frac{8 i(8 k+45)}{3(k+5)^{3}} U \partial A_{3} \\
& -\frac{2 i\left(5 k^{2}+33 k+60\right)}{3(k+5)^{3}} \partial U B_{3}+\frac{2 i\left(k^{2}+53 k+108\right)}{3(k+5)^{3}} U \partial B_{3} \\
& +\frac{16(k+6)}{3(k+5)^{3}} U B_{+} B_{-}-\frac{8(k+6)(4 k+17)}{3(k+5)^{2}(13 k+17)} \partial^{2} U \\
& +\frac{6}{(k+5)^{2}} U F_{11} G_{22}-\frac{(k-3)(29 k+273)}{9(k+5)^{4}} \partial U F_{11} F_{22} \\
& +\frac{\left(259 k^{2}+2118 k+5139\right)}{9(k+5)^{4}} U \partial F_{11} F_{22}+\frac{16(k+6)}{3(k+5)^{3}} U B_{3} B_{3} \\
& -\frac{\left(137 k^{2}+2130 k+6201\right)}{9(k+5)^{4}} U F_{11} \partial F_{22}-\frac{(7 k+3)}{(k+5)^{3}} \partial U F_{12} F_{21}
\end{aligned}
$$




$$
\begin{aligned}
& +\frac{(43 k+375)}{3(k+5)^{3}} U \partial F_{12} F_{21}-\frac{(49 k+213)}{3(k+5)^{3}} U F_{12} \partial F_{21} \\
& \left.+\frac{16(k+6)}{3(k+5)^{3}} U U U-\frac{4\left(20 k^{2}+177 k+369\right)}{9(k+5)^{3}} \partial U U\right](w) \\
& +\frac{1}{(z-w)}[+\cdots](w)+\cdots .
\end{aligned}
$$

There is no boldface higher spin current in the fourth order pole.

One can see the following OPEs from (3.6)

$$
\begin{aligned}
& \left(\begin{array}{c}
\mathbf{U}^{\left(\frac{5}{2}\right)} \\
\mathbf{V}^{\left(\frac{5}{2}\right)}
\end{array}\right)(z) \mathbf{W}^{(2)}(w)=\frac{1}{(z-w)^{4}}\left[-\frac{18 k}{(k+5)^{2}}\left(\begin{array}{c}
F_{11} \\
F_{22}
\end{array}\right)\right](w) \\
& +\frac{1}{(z-w)^{3}}\left[\frac{2(k-3)}{3(k+5)^{2}}\left(\begin{array}{c}
G_{11} \\
G_{22}
\end{array}\right) \mp \frac{4 i\left(3 k^{2}+14 k+3\right)}{3(k+5)^{3}}\left(\begin{array}{c}
F_{11} \\
F_{22}
\end{array}\right) B_{3}\right. \\
& +\frac{4(k-3)}{3(k+5)^{3}} U\left(\begin{array}{c}
F_{11} \\
F_{22}
\end{array}\right) \mp \frac{4(k-3)}{3(k+5)^{3}} F_{12} F_{21}\left(\begin{array}{c}
F_{11} \\
F_{22}
\end{array}\right) \\
& \pm \frac{8 i(5 k+21)}{3(k+5)^{3}}\left(\begin{array}{c}
F_{11} \\
F_{22}
\end{array}\right) A_{3} \pm \frac{16 i(k-3)}{3(k+5)^{3}}\left(\begin{array}{c}
F_{21} \\
F_{12}
\end{array}\right) A_{ \pm} \\
& \left. \pm \frac{4 i(k-3)(k+1)}{3(k+5)^{3}}\left(\begin{array}{c}
F_{12} \\
F_{21}
\end{array}\right) B_{\mp}-\frac{18 k}{(k+5)^{2}} \partial\left(\begin{array}{c}
F_{11} \\
F_{22}
\end{array}\right)\right](w) \\
& +\frac{1}{(z-w)^{2}}\left[ \pm \frac{5}{2}\left(\begin{array}{l}
\mathbf{Q}^{\left(\frac{5}{2}\right)} \\
\mathbf{R}^{\left(\frac{5}{2}\right)}
\end{array}\right)+\frac{5}{2}\left(\begin{array}{l}
\mathbf{U}^{\left(\frac{5}{2}\right)} \\
\mathbf{V}^{\left(\frac{5}{2}\right)}
\end{array}\right)\right. \\
& \mp \frac{i(3 k+13)}{(k+5)^{2}} A_{3}\left(\begin{array}{c}
G_{11} \\
G_{22}
\end{array}\right)+\frac{2}{(k+5)^{2}} A_{3} A_{3}\left(\begin{array}{c}
F_{11} \\
F_{22}
\end{array}\right) \\
& +\frac{2(7 k+39)}{3(k+5)^{3}} A_{3} B_{\mp}\left(\begin{array}{c}
F_{12} \\
F_{21}
\end{array}\right)+\frac{4}{(k+5)^{2}} A_{3} B_{3}\left(\begin{array}{c}
F_{11} \\
F_{22}
\end{array}\right) \\
& \mp \frac{2 i}{(k+5)^{2}} \partial A_{3}\left(\begin{array}{c}
F_{11} \\
F_{22}
\end{array}\right) \pm \frac{2 i(43 k+183)}{3(k+5)^{3}} A_{3} \partial\left(\begin{array}{c}
F_{11} \\
F_{22}
\end{array}\right) \\
& \mp \frac{i(7 k+15)}{3(k+5)^{2}} A_{ \pm}\left(\begin{array}{c}
G_{21} \\
G_{12}
\end{array}\right)+\frac{8(2 k+9)}{3(k+5)^{3}} A_{ \pm} B_{3}\left(\begin{array}{c}
F_{21} \\
F_{12}
\end{array}\right) \\
& +\frac{4(k+3)}{3(k+5)^{3}} A_{ \pm} A_{3}\left(\begin{array}{c}
F_{21} \\
F_{12}
\end{array}\right)+\frac{2(k+9)}{3(k+5)^{3}} A_{ \pm} A_{\mp}\left(\begin{array}{c}
F_{11} \\
F_{22}
\end{array}\right) \\
& -\frac{6}{(k+5)^{2}} A_{ \pm} B_{\mp}\left(\begin{array}{c}
F_{22} \\
F_{11}
\end{array}\right) \pm \frac{2 i(7 k+27)}{3(k+5)^{3}} \partial B_{3}\left(\begin{array}{c}
F_{11} \\
F_{22}
\end{array}\right) \\
& \mp \frac{4 i(3 k+17)}{(k+5)^{3}} \partial A_{ \pm}\left(\begin{array}{c}
F_{21} \\
F_{12}
\end{array}\right) \pm \frac{2 i(37 k+93)}{3(k+5)^{3}} A_{ \pm} \partial\left(\begin{array}{c}
F_{21} \\
F_{12}
\end{array}\right) \\
& \mp \frac{i(3 k+13)}{(k+5)^{2}} B_{3}\left(\begin{array}{c}
G_{11} \\
G_{22}
\end{array}\right) \mp \frac{2 i\left(12 k^{2}+59 k+27\right)}{3(k+5)^{3}} B_{3} \partial\left(\begin{array}{c}
F_{11} \\
F_{22}
\end{array}\right)
\end{aligned}
$$




$$
\begin{aligned}
& +\frac{2}{(k+5)^{2}} B_{3} B_{3}\left(\begin{array}{c}
F_{11} \\
F_{22}
\end{array}\right)-\frac{8(k+6)}{3(k+5)^{3}}\left(\begin{array}{l}
F_{11} F_{21} G_{12} \\
F_{22} F_{12} G_{21}
\end{array}\right) \\
& \mp \frac{2 i(k+15)}{3(k+5)^{2}} B_{\mp}\left(\begin{array}{c}
G_{12} \\
G_{21}
\end{array}\right) \pm \frac{2 i\left(5 k^{2}+27 k+30\right)}{3(k+5)^{3}} \partial B_{\mp}\left(\begin{array}{l}
F_{12} \\
F_{21}
\end{array}\right) \\
& \mp \frac{4 i k(3 k+25)}{3(k+5)^{3}} B_{\mp} \partial\left(\begin{array}{c}
F_{12} \\
F_{21}
\end{array}\right)+\frac{2(k+9)}{3(k+5)^{3}} B_{\mp} B_{3}\left(\begin{array}{l}
F_{12} \\
F_{21}
\end{array}\right) \\
& -\frac{\left(25 k^{2}+159 k-54\right)}{3(k+5)^{3}} \partial^{2}\left(\begin{array}{l}
F_{11} \\
F_{22}
\end{array}\right)+\frac{4(k+3)}{3(k+5)^{3}} B_{ \pm} B_{\mp}\left(\begin{array}{l}
F_{11} \\
F_{22}
\end{array}\right) \\
& +\frac{2(5 k+21)}{3(k+5)^{3}}\left(\begin{array}{l}
F_{11} F_{12} G_{21} \\
F_{22} F_{21} G_{12}
\end{array}\right) \mp \frac{4(k-3)}{3(k+5)^{3}} \partial\left(\begin{array}{l}
F_{11} \\
F_{22}
\end{array}\right) F_{12} F_{21} \\
& -\frac{4\left(9 k^{2}+82 k+201\right)}{3(k+5)^{4}}\left(\begin{array}{l}
F_{11} \partial F_{12} F_{21} \\
F_{22} \partial F_{21} F_{12}
\end{array}\right) \\
& +\frac{32(k+10 k+27)}{3(k+5)^{4}}\left(\begin{array}{l}
F_{11} F_{12} \partial F_{21} \\
F_{22} F_{21} \partial F_{12}
\end{array}\right) \\
& +\frac{2}{(k+5)^{2}}\left(\begin{array}{l}
F_{12} F_{21} G_{11} \\
F_{21} F_{12} G_{22}
\end{array}\right)+\frac{(k-3)}{(k+5)^{2}} \partial\left(\begin{array}{l}
G_{11} \\
G_{22}
\end{array}\right) \\
& -\frac{4(k+4)}{(k+5)^{2}} T\left(\begin{array}{l}
F_{11} \\
F_{22}
\end{array}\right)-\frac{(k+7)}{(k+5)^{2}} U\left(\begin{array}{l}
G_{11} \\
G_{22}
\end{array}\right) \\
& \mp \frac{8 i}{(k+5)^{2}} U A_{3}\left(\begin{array}{l}
F_{11} \\
F_{22}
\end{array}\right) \mp \frac{4 i(5 k+27)}{3(k+5)^{3}} U A_{ \pm}\left(\begin{array}{l}
F_{21} \\
F_{12}
\end{array}\right) \\
& \mp \frac{8 i}{(k+5)^{2}} U B_{3}\left(\begin{array}{l}
F_{11} \\
F_{22}
\end{array}\right) \mp \frac{2 i(11 k+51)}{3(k+5)^{3}} U B_{\mp}\left(\begin{array}{l}
F_{12} \\
F_{21}
\end{array}\right) \\
& +\frac{2(k-3)}{(k+5)^{3}} \partial U\left(\begin{array}{l}
F_{11} \\
F_{22}
\end{array}\right)-\frac{2(k-3)}{(k+5)^{3}} U \partial\left(\begin{array}{l}
F_{11} \\
F_{22}
\end{array}\right) \\
& \left.+\frac{6}{(k+5)^{2}} U U\left(\begin{array}{l}
F_{11} \\
F_{22}
\end{array}\right) \pm \frac{12}{(k+5)^{2}}\left(\begin{array}{l}
\partial F_{11} \\
\partial F_{22}
\end{array}\right) F_{11} F_{22}\right](w) \\
& +\frac{1}{(z-w)}[+\cdots](w)+\cdots
\end{aligned}
$$

There is no boldface higher spin current in the third order pole.

Again the relation (3.8) determines the following OPEs

$$
\begin{aligned}
\left(\begin{array}{c}
\mathbf{U}^{\left(\frac{5}{2}\right)} \\
\mathbf{V}^{\left(\frac{5}{2}\right)}
\end{array}\right)(z) \mathbf{W}_{ \pm}^{\left(\frac{5}{2}\right)}(w) & =\frac{1}{(z-w)^{4}}\left[\mp \frac{16 i k(k+3)(2 k+15)}{3(k+5)^{3}} B_{\mp}+\frac{64 k(k+3)^{2}}{3(k+5)^{4}}\left(\begin{array}{c}
F_{11} F_{21} \\
F_{22} F_{12}
\end{array}\right)\right](w) \\
& +\frac{1}{(z-w)^{3}}\left[-\frac{8\left(8 k^{2}+51 k+135\right)}{9(k+5)^{3}} A_{3} B_{\mp}\right. \\
& \mp \frac{32 i(k+3)(k+9)}{9(k+5)^{4}} A_{3}\left(\begin{array}{c}
F_{11} F_{21} \\
F_{22} F_{12}
\end{array}\right) \mp \frac{8 i k(k+3)(2 k+15)}{3(k+5)^{3}} \partial B_{\mp}
\end{aligned}
$$




$$
\begin{aligned}
& +\frac{16 i(k+3)(k+9)}{9(k+5)^{4}} B_{\mp} F_{11} F_{22}+\frac{16 i(k+3)(k+9)}{9(k+5)^{4}} B_{\mp} F_{12} F_{21} \\
& -\frac{4(k-3)(2 k+9)}{3(k+5)^{3}}\left(\begin{array}{l}
F_{11} G_{21} \\
F_{22} G_{12}
\end{array}\right)+\frac{8\left(12 k^{3}+41 k^{2}-30 k-27\right)}{9(k+5)^{4}}\left(\begin{array}{c}
\partial F_{11} F_{21} \\
\partial F_{22} F_{12}
\end{array}\right) \\
& +\frac{8\left(12 k^{3}+103 k^{2}+246 k+27\right)}{9(k+5)^{4}}\left(\begin{array}{c}
F_{11} \partial F_{21} \\
F_{22} \partial F_{12}
\end{array}\right) \\
& \left.-\frac{4(k-3)(2 k+9)}{3(k+5)^{3}}\left(\begin{array}{c}
F_{21} G_{11} \\
F_{12} G_{22}
\end{array}\right)\right](w) \\
& +\frac{1}{(z-w)^{2}}\left[\mp \frac{2(5 k+21)}{3(k+5)}\left(\begin{array}{c}
\mathbf{Q}_{+}^{(3)} \\
\mathbf{R}_{-}^{(3)}
\end{array}\right)\right. \\
& \pm \frac{8 i}{(k+5)^{2}} A_{3} A_{3} B_{\mp}+\frac{2\left(29 k^{2}+246 k+369\right)}{9(k+5)^{3}} \partial A_{3} B_{\mp} \\
& -\frac{2\left(61 k^{2}+486 k+1089\right)}{9(k+5)^{3}} A_{3} \partial B_{\mp} \mp \frac{2 i(13 k+57)}{3(k+5)^{3}} A_{3}\left(\begin{array}{c}
F_{11} G_{21} \\
F_{22} G_{12}
\end{array}\right) \\
& \pm \frac{2 i\left(67 k^{2}+474 k+855\right)}{9(k+5)^{4}} \partial A_{3}\left(\begin{array}{c}
F_{11} F_{21} \\
F_{22} F_{12}
\end{array}\right) \\
& \mp \frac{2 i\left(29 k^{2}+198 k+297\right)}{9(k+5)^{4}} A_{3}\left(\begin{array}{l}
\partial F_{11} F_{21} \\
\partial F_{22} F_{12}
\end{array}\right) \\
& \mp \frac{2 i\left(137 k^{2}+1134 k+2277\right)}{9(k+5)^{4}} A_{3}\left(\begin{array}{c}
F_{11} \partial F_{21} \\
F_{22} \partial F_{12}
\end{array}\right) \pm \frac{2 i}{(k+5)^{2}} A_{\mp}\left(\begin{array}{c}
F_{11} G_{11} \\
F_{22} G_{22}
\end{array}\right) \\
& \pm \frac{2 i(7 k+27)}{3(k+5)^{3}} A_{3}\left(\begin{array}{c}
F_{21} G_{11} \\
F_{12} G_{22}
\end{array}\right) \pm \frac{8 i}{(k+5)^{2}} A_{ \pm} A_{\mp} B_{\mp} \\
& \mp \frac{2 i}{(k+5)^{2}} A_{ \pm}\left(\begin{array}{c}
F_{21} G_{21} \\
F_{12} G_{12}
\end{array}\right) \mp \frac{2 i}{(k+5)^{2}} B_{3}\left(\begin{array}{c}
F_{11} G_{21} \\
F_{22} G_{12}
\end{array}\right) \\
& \mp \frac{2 i(3 k+19)}{(k+5)^{3}} \partial B_{3}\left(\begin{array}{c}
F_{11} F_{21} \\
F_{22} F_{12}
\end{array}\right) \mp \frac{2 i(k-15)}{3(k+5)^{3}} B_{3}\left(\begin{array}{c}
\partial F_{11} F_{21} \\
\partial F_{22} F_{12}
\end{array}\right) \\
& \pm \frac{2 i(19 k+99)}{3(k+5)^{3}} B_{3}\left(\begin{array}{c}
F_{11} \partial F_{21} \\
F_{22} \partial F_{12}
\end{array}\right) \pm \frac{2 i}{(k+5)^{2}} B_{3}\left(\begin{array}{c}
F_{21} G_{11} \\
F_{12} G_{22}
\end{array}\right) \\
& +\frac{4(k+2)}{(k+5)^{2}} \partial B_{\mp} B_{3}-\frac{4(k+2)}{(k+5)^{2}} B_{\mp} \partial B_{3} \\
& \mp \frac{2 i\left(44 k^{4}+517 k^{3}+516 k^{2}-1467 k+1530\right)}{9(k+5)^{3}(13 k+17)} \partial^{2} B_{\mp} \\
& \pm \frac{4 i(k+3)}{3(k+5)^{3}} B_{\mp}\left(\begin{array}{c}
F_{11} G_{22} \\
F_{22} G_{11}
\end{array}\right)+\frac{2 i(k+9)(13 k+45)}{9(k+5)^{4}} \partial B_{\mp} F_{12} F_{21} \\
& \mp \frac{4 i\left(13 k^{2}+216 k+747\right)}{9(k+5)^{4}} B_{\mp}\left(\begin{array}{l}
\partial F_{11} F_{22} \\
\partial F_{22} F_{11}
\end{array}\right) \\
& \pm \frac{8 i(2 k+15)(5 k+21)}{9(k+5)^{4}} \partial B_{\mp} F_{11} F_{22}
\end{aligned}
$$




$$
\begin{aligned}
& \mp \frac{4 i(k+3)(19 k+99)}{9(k+5)^{4}} B_{\mp}\left(\begin{array}{c}
F_{11} \partial F_{22} \\
F_{22} \partial F_{11}
\end{array}\right) \mp \frac{2 i(k+9)}{3(k+5)^{3}} B_{\mp}\left(\begin{array}{c}
F_{12} G_{21} \\
F_{21} G_{12}
\end{array}\right) \\
& -\frac{2 i(k+9)(19 k+99)}{9(k+5)^{4}} B_{\mp}\left(\begin{array}{c}
F_{12} \partial F_{21} \\
F_{21} \partial F_{12}
\end{array}\right) \\
& \mp \frac{2 i(k+9)(29 k+141)}{9(k+5)^{4}} B_{\mp}\left(\begin{array}{c}
\partial F_{12} F_{21} \\
\partial F_{21} F_{12}
\end{array}\right) \\
& \mp \frac{8 i(2 k+9)}{3(k+5)^{3}} B_{\mp}\left(\begin{array}{c}
F_{21} G_{12} \\
F_{12} G_{21}
\end{array}\right) \mp \frac{2 i(11 k+51)}{3(k+5)^{3}} B_{\mp}\left(\begin{array}{c}
F_{22} G_{11} \\
F_{11} G_{22}
\end{array}\right) \\
& +\frac{16(7 k+27)(17 k+33)}{9(k+5)^{4}}\left(\begin{array}{l}
\partial F_{11} \partial F_{21} \\
\partial F_{22} \partial F_{12}
\end{array}\right) \\
& +\frac{2\left(88 k^{4}-1283 k^{3}-14297 k^{2}-39417 k-27387\right)}{9(k+5)^{4}(13 k+17)}\left(\begin{array}{c}
F_{11} \partial^{2} F_{21} \\
F_{22} \partial^{2} F_{12}
\end{array}\right) \\
& -\frac{\left(25 k^{2}+36 k-237\right)}{3(k+5)^{3}}\left(\begin{array}{c}
\partial F_{11} G_{21} \\
\partial F_{22} G_{12}
\end{array}\right)+\frac{(k-3)(k+3)}{(k+5)^{3}}\left(\begin{array}{c}
F_{11} \partial G_{21} \\
F_{22} \partial G_{12}
\end{array}\right) \\
& +\frac{2\left(k^{2}-42 k-159\right)}{3(k+5)^{3}}\left(\begin{array}{c}
\partial F_{21} G_{11} \\
\partial F_{12} G_{22}
\end{array}\right)-\frac{2\left(k^{2}-4 k-33\right)}{(k+5)^{3}}\left(\begin{array}{c}
F_{21} \partial G_{11} \\
F_{12} \partial G_{22}
\end{array}\right) \\
& -\frac{2(3 k+10)}{(k+5)^{2}}\left(\begin{array}{l}
G_{11} G_{21} \\
G_{22} G_{12}
\end{array}\right) \mp \frac{16 i k\left(5 k^{2}+57 k+123\right)}{3(k+5)^{2}(13 k+17)} T B_{\mp} \\
& +\frac{8\left(20 k^{3}+183 k^{2}+498 k+255\right)}{3(k+5)^{3}(13 k+17)} T\left(\begin{array}{l}
F_{11} F_{21} \\
F_{22} F_{12}
\end{array}\right) \\
& +\frac{8(5 k+21)}{3(k+5)^{3}} U A_{3} B_{\mp} \pm \frac{2 i\left(k^{2}+13 k+20\right)}{(k+5)^{3}} \partial U B_{\mp} \\
& \mp \frac{2 i\left(k^{2}+13 k+20\right)}{(k+5)^{3}} U \partial B_{\mp} \\
& -\frac{2(13 k-3)}{3(k+5)^{3}} U\left(\begin{array}{c}
\partial F_{11} F_{21} \\
\partial F_{22} F_{12}
\end{array}\right)-\frac{6}{(k+5)^{2}} U\left(\begin{array}{c}
F_{11} G_{21} \\
F_{22} G_{12}
\end{array}\right) \\
& +\frac{2(k-15)}{(k+5)^{3}} \partial U\left(\begin{array}{c}
F_{11} F_{21} \\
F_{22} F_{12}
\end{array}\right)+\frac{2(7 k+87)}{3(k+5)^{3}} U\left(\begin{array}{c}
F_{11} \partial F_{21} \\
F_{22} \partial F_{12}
\end{array}\right) \\
& +\frac{6}{(k+5)^{2}} U\left(\begin{array}{c}
F_{21} G_{11} \\
F_{12} G_{22}
\end{array}\right) \pm \frac{8 i(7 k+27)}{3(k+5)^{3}}\left(\begin{array}{c}
F_{11} A_{-} \partial F_{11} \\
F_{22} A_{+} \partial F_{22}
\end{array}\right) \\
& \mp \frac{4 i\left(88 k^{4}-2739 k^{3}-22129 k^{2}-46233 k-26163\right)}{9(k+5)^{4}(13 k+17)}\left(\begin{array}{c}
F_{21} A_{+} \partial F_{21} \\
F_{12} A_{-} \partial F_{12}
\end{array}\right) \\
& \left.\mp \frac{4 i\left(88 k^{4}-2895 k^{3}-23581 k^{2}-50205 k-29223\right)}{9(k+5)^{4}(13 k+17)}\left(\begin{array}{c}
\partial F_{21} A_{+} F_{21} \\
\partial F_{12} A_{-} F_{12}
\end{array}\right)\right](w) \\
& +\frac{1}{(z-w)}[+\cdots](w)+\cdots \text {. }
\end{aligned}
$$

There is no boldface higher spin current in the third order pole. 
The above same relation gives the other combination of the OPEs

$$
\begin{aligned}
& \left(\begin{array}{c}
\mathbf{U}^{\left(\frac{5}{2}\right)} \\
\mathbf{V}^{\left(\frac{5}{2}\right)}
\end{array}\right)(z) \mathbf{W}_{\mp}^{\left(\frac{5}{2}\right)}(w)=\frac{1}{(z-w)^{4}}\left[\mp \frac{8 i(k+9)(4 k+9)}{(k+5)^{3}} A_{ \pm}-\frac{16(k+9)^{2}}{(k+5)^{4}}\left(\begin{array}{l}
F_{11} F_{12} \\
F_{22} F_{21}
\end{array}\right)\right](w) \\
& +\frac{1}{(z-w)^{3}}\left[\frac{8\left(8 k^{2}+51 k+135\right)}{9(k+5)^{3}} A_{ \pm} B_{3} \mp \frac{4 i(k+9)(4 k+9)}{(k+5)^{3}} \partial A_{ \pm}\right. \\
& +\frac{16 i(k+3)(k+9)}{9(k+5)^{4}} A_{ \pm} F_{11} F_{22}+\frac{4(k-3)(2 k+9)}{3(k+5)^{3}}\left(\begin{array}{c}
F_{12} G_{11} \\
F_{21} G_{22}
\end{array}\right) \\
& \text { - } \frac{16 i(k+3)(k+9)}{9(k+5)^{4}} A_{ \pm} F_{12} F_{21} \mp \frac{32 i(k+3)(k+9)}{9(k+5)^{4}} B_{3}\left(\begin{array}{c}
F_{11} F_{12} \\
F_{22} F_{21}
\end{array}\right) \\
& +\frac{4(k-3)(2 k+9)}{3(k+5)^{3}}\left(\begin{array}{c}
F_{11} G_{12} \\
F_{22} G_{21}
\end{array}\right)+\frac{16\left(11 k^{2}-12 k-351\right)}{9(k+5)^{4}}\left(\begin{array}{c}
\partial F_{11} F_{12} \\
\partial F_{22} F_{21}
\end{array}\right) \\
& \left.-\frac{32\left(10 k^{2}+75 k+189\right)}{9(k+5)^{4}}\left(\begin{array}{l}
F_{11} \partial F_{12} \\
F_{22} \partial F_{21}
\end{array}\right)\right](w) \\
& +\frac{1}{(z-w)^{2}}\left[\mp \frac{8(k+6)}{3(k+5)}\left(\begin{array}{c}
\mathbf{Q}_{-}^{(3)} \\
\mathbf{R}_{+}^{(3)}
\end{array}\right)\right. \\
& \pm \frac{2 i}{(k+5)^{2}} A_{3}\left(\begin{array}{c}
F_{11} G_{12} \\
F_{22} G_{21}
\end{array}\right) \mp \frac{8 i(k+4)}{(k+5)^{3}} \partial A_{3}\left(\begin{array}{c}
F_{11} F_{12} \\
F_{22} F_{21}
\end{array}\right) \\
& \pm \frac{8 i k}{3(k+5)^{3}} A_{3}\left(\begin{array}{c}
\partial F_{11} F_{12} \\
\partial F_{22} F_{21}
\end{array}\right) \pm \frac{8 i}{(k+5)^{2}} A_{ \pm} B_{ \pm} B_{\mp} \\
& \pm \frac{8 i(5 k+24)}{3(k+5)^{3}} A_{3}\left(\begin{array}{c}
F_{11} \partial F_{12} \\
F_{22} \partial F_{21}
\end{array}\right) \mp \frac{2 i}{(k+5)^{2}} A_{3}\left(\begin{array}{c}
F_{12} G_{11} \\
F_{21} G_{22}
\end{array}\right) \\
& \mp \frac{4 i\left(22 k^{3}+293 k^{2}+378 k-1149\right)}{3(k+5)^{3}(13 k+17)} \partial^{2} A_{ \pm} \pm \frac{8 i}{(k+5)^{2}} A_{ \pm} B_{3} B_{3} \\
& +\frac{4\left(26 k^{2}+237 k+603\right)}{9(k+5)^{3}} \partial A_{ \pm} B_{3}-\frac{4\left(10 k^{2}+153 k+423\right)}{9(k+5)^{3}} A_{ \pm} \partial B_{3} \\
& \pm \frac{8 i(k+6)(13 k+45)}{9(k+5)^{4}} \partial A_{ \pm} F_{11} F_{22} \mp \frac{2 i(k+9)}{3(k+5)^{3}} A_{ \pm}\left(\begin{array}{c}
F_{11} G_{22} \\
F_{22} G_{11}
\end{array}\right) \\
& \mp \frac{8 i\left(17 k^{2}+129 k+216\right)}{9(k+5)^{4}} A_{ \pm}\left(\begin{array}{c}
\partial F_{11} F_{22} \\
\partial F_{22} F_{11}
\end{array}\right)-\frac{20}{(k+5)^{2}} \partial A_{ \pm} A_{3} \\
& \mp \frac{8 i(k+9)(5 k+24)}{9(k+5)^{4}} A_{ \pm}\left(\begin{array}{c}
F_{11} \partial F_{22} \\
F_{22} \partial F_{11}
\end{array}\right)+\frac{20}{(k+5)^{2}} A_{ \pm} \partial A_{3} \\
& \pm \frac{2 i(7 k+39)}{3(k+5)^{3}} A_{ \pm}\left(\begin{array}{c}
F_{12} G_{21} \\
F_{21} G_{12}
\end{array}\right) \mp \frac{16 i(k+3)(2 k+15)}{9(k+5)^{4}} \partial A_{ \pm} F_{12} F_{21} \\
& \mp \frac{16 i(k+3)(5 k+24)}{9(k+5)^{4}} A_{ \pm}\left(\begin{array}{c}
\partial F_{12} F_{21} \\
\partial F_{21} F_{12}
\end{array}\right) \\
& \pm \frac{16 i(k+3)(7 k+36)}{9(k+5)^{4}} A_{ \pm}\left(\begin{array}{c}
F_{12} \partial F_{21} \\
F_{21} \partial F_{12}
\end{array}\right)
\end{aligned}
$$




$$
\begin{aligned}
& \pm \frac{4 i(k+3)}{3(k+5)^{3}} A_{ \pm}\left(\begin{array}{c}
F_{21} G_{12} \\
F_{12} G_{21}
\end{array}\right) \mp \frac{2 i(5 k+33)}{3(k+5)^{3}} B_{3}\left(\begin{array}{c}
F_{12} G_{11} \\
F_{21} G_{22}
\end{array}\right) \\
& \pm \frac{4 i(5 k+27)}{3(k+5)^{3}} A_{ \pm}\left(\begin{array}{c}
F_{22} G_{11} \\
F_{11} G_{22}
\end{array}\right) \pm \frac{2 i(11 k+63)}{3(k+5)^{3}} B_{3}\left(\begin{array}{c}
F_{11} G_{12} \\
F_{22} G_{21}
\end{array}\right) \\
& \pm \frac{8 i\left(7 k^{2}+99 k+360\right)}{9(k+5)^{4}} \partial B_{3}\left(\begin{array}{c}
F_{11} F_{12} \\
F_{22} F_{21}
\end{array}\right) \\
& \mp \frac{8 i\left(2 k^{2}+39 k+153\right)}{9(k+5)^{4}} B_{3}\left(\begin{array}{l}
\partial F_{11} F_{12} \\
\partial F_{22} F_{21}
\end{array}\right) \\
& \mp \frac{8 i\left(20 k^{2}+255 k+783\right)}{9(k+5)^{4}} B_{3}\left(\begin{array}{c}
F_{11} \partial F_{12} \\
F_{22} \partial F_{21}
\end{array}\right) \\
& \pm \frac{2 i}{(k+5)^{2}} B_{\mp}\left(\begin{array}{c}
F_{12} G_{12} \\
F_{21} G_{21}
\end{array}\right) \mp \frac{2 i}{(k+5)^{2}} B_{ \pm}\left(\begin{array}{c}
F_{11} G_{11} \\
F_{22} G_{22}
\end{array}\right) \\
& +\frac{4\left(1333 k^{3}+10733 k^{2}+24315 k+26019\right)}{9(k+5)^{4}(13 k+17)}\left(\begin{array}{c}
\partial^{2} F_{11} F_{12} \\
\partial^{2} F_{22} F_{21}
\end{array}\right) \\
& -\frac{8\left(509 k^{3}+4117 k^{2}+7347 k+507\right)}{3(k+5)^{4}(13 k+17)}\left(\begin{array}{l}
\partial F_{11} \partial F_{12} \\
\partial F_{22} \partial F_{21}
\end{array}\right) \\
& +\frac{4\left(527 k^{3}+6091 k^{2}+18921 k+25101\right)}{9(k+5)^{4}(13 k+17)}\left(\begin{array}{c}
F_{11} \partial^{2} F_{12} \\
F_{22} \partial^{2} F_{21}
\end{array}\right) \\
& +\frac{2(2 k+13)}{(k+5)^{2}}\left(\begin{array}{l}
G_{11} G_{12} \\
G_{22} G_{21}
\end{array}\right)+\frac{2\left(8 k^{2}+15 k-165\right)}{3(k+5)^{3}}\left(\begin{array}{l}
\partial F_{11} G_{12} \\
\partial F_{22} G_{21}
\end{array}\right) \\
& -\frac{2\left(10 k^{2}+63 k-3\right)}{3(k+5)^{3}}\left(\begin{array}{c}
\partial F_{12} G_{11} \\
\partial F_{21} G_{22}
\end{array}\right)+\frac{2\left(2 k^{2}+9 k-9\right)}{(k+5)^{3}}\left(\begin{array}{c}
F_{12} \partial G_{11} \\
F_{21} \partial G_{22}
\end{array}\right) \\
& -\frac{2(k-3)}{(k+5)^{3}}\left(\begin{array}{c}
F_{11} \partial G_{12} \\
F_{22} \partial G_{21}
\end{array}\right) \mp \frac{4 i\left(20 k^{2}+231 k+483\right)}{(k+5)^{2}(13 k+17)} T A_{ \pm} \\
& -\frac{8\left(21 k^{2}+202 k+517\right)}{(k+5)^{3}(13 k+17)} T\left(\begin{array}{c}
F_{11} F_{12} \\
F_{22} F_{21}
\end{array}\right)+\frac{32(k+6)}{3(k+5)^{3}} U A_{ \pm} B_{3} \\
& \mp \frac{4 i(5 k+19)}{(k+5)^{3}} \partial U A_{ \pm} \pm \frac{4 i(5 k+19)}{(k+5)^{3}} U \partial A_{ \pm}+\frac{6}{(k+5)^{2}} U\left(\begin{array}{c}
F_{11} G_{12} \\
F_{22} G_{21}
\end{array}\right) \\
& +\frac{8(5 k+12)}{3(k+5)^{3}} U\left(\begin{array}{c}
F_{11} \partial F_{12} \\
F_{22} \partial F_{21}
\end{array}\right)-\frac{6}{(k+5)^{2}} U\left(\begin{array}{c}
F_{12} G_{11} \\
F_{21} G_{22}
\end{array}\right) \\
& -\frac{8 k}{(k+5)^{3}} \partial U\left(\begin{array}{c}
F_{11} F_{12} \\
F_{22} F_{21}
\end{array}\right)+\frac{8(k-12)}{3(k+5)^{3}} U\left(\begin{array}{c}
\partial F_{11} F_{12} \\
\partial F_{22} F_{21}
\end{array}\right) \\
& \left. \pm \frac{8 i(5 k+33)}{3(k+5)^{3}}\left(\begin{array}{c}
F_{11} B_{+} \partial F_{11} \\
F_{22} B_{-} \partial F_{22}
\end{array}\right) \mp \frac{8 i(k+9)}{3(k+5)^{3}}\left(\begin{array}{c}
F_{12} B_{-} \partial F_{12} \\
F_{21} B_{+} \partial F_{21}
\end{array}\right)\right](w) \\
& +\frac{1}{(z-w)}[+\cdots](w)+\cdots \text {. }
\end{aligned}
$$

There is no boldface higher spin current in the third order pole. 
Similarly the following OPEs can be obtained from (3.9)

$$
\begin{aligned}
& \left(\begin{array}{c}
\mathbf{U}^{\left(\frac{5}{2}\right)} \\
\mathbf{V}^{\left(\frac{5}{2}\right)}
\end{array}\right)(z) \mathbf{W}^{(\mathbf{3})}(w)=\frac{1}{(z-w)^{5}}\left[ \pm \frac{24(k-3) k}{(k+5)^{2}(13 k+17)}\left(\begin{array}{c}
F_{11} \\
F_{22}
\end{array}\right)\right](w) \\
& +\frac{1}{(z-w)^{4}}\left[\mp \frac{2\left(248 k^{3}+2770 k^{2}+7275 k+4509\right)}{3(k+5)^{2}(13 k+17)}\left(\begin{array}{c}
G_{11} \\
G_{22}
\end{array}\right)\right. \\
& \mp \frac{4\left(248 k^{3}+1717 k^{2}+5898 k+4509\right)}{3(k+5)^{3}(13 k+17)} U\left(\begin{array}{l}
F_{11} \\
F_{22}
\end{array}\right) \\
& -\frac{8(5 k+9)\left(11 k^{2}+118 k+219\right)}{3(k+5)^{4}(13 k+17)} F_{12} F_{21}\left(\begin{array}{c}
F_{11} \\
F_{22}
\end{array}\right) \\
& -\frac{4 i\left(248 k^{3}+1195 k^{2}+966 k-189\right)}{3(k+5)^{3}(13 k+17)}\left(\begin{array}{c}
F_{11} \\
F_{22}
\end{array}\right) A_{3} \\
& -\frac{4 i\left(248 k^{3}+1195 k^{2}+966 k-189\right)}{3(k+5)^{3}(13 k+17)}\left(\begin{array}{c}
F_{21} \\
F_{12}
\end{array}\right) A_{ \pm} \\
& +\frac{4 i\left(46 k^{3}-409 k^{2}-4404 k-4509\right)}{3(k+5)^{3}(13 k+17)}\left(\begin{array}{c}
F_{12} \\
F_{21}
\end{array}\right) B_{\mp} \\
& \left.+\frac{4 i\left(46 k^{3}-409 k^{2}-4404 k-4509\right)}{3(k+5)^{3}(13 k+17)}\left(\begin{array}{c}
F_{11} \\
F_{22}
\end{array}\right) B_{3}\right](w) \\
& +\frac{1}{(z-w)^{3}}\left[\frac{2(k-3)(41 k+109)}{3(k+5)(13 k+17)}\left(\begin{array}{c}
\mathbf{Q}^{\left(\frac{5}{2}\right)} \\
\mathbf{R}^{\left(\frac{5}{2}\right)}
\end{array}\right)\right. \\
& \pm \frac{2(k-3)(41 k+109)}{3(k+5)(13 k+17)}\left(\begin{array}{c}
\mathbf{U}^{\left(\frac{5}{2}\right)} \\
\mathbf{V}^{\left(\frac{5}{2}\right)}
\end{array}\right) \\
& +\frac{4 i(k+4)}{(k+5)^{2}} A_{3}\left(\begin{array}{c}
G_{11} \\
G_{22}
\end{array}\right) \mp \frac{8(k+9)}{3(k+5)^{3}} A_{3} A_{3}\left(\begin{array}{c}
F_{11} \\
F_{22}
\end{array}\right) \\
& \pm \frac{8(k-3)}{3(k+5)^{3}} A_{3} B_{\mp}\left(\begin{array}{c}
F_{12} \\
F_{21}
\end{array}\right) \pm \frac{8(k-3)}{3(k+5)^{3}} A_{3} B_{3}\left(\begin{array}{c}
F_{11} \\
F_{22}
\end{array}\right) \\
& -\frac{4 i\left(248 k^{3}+1039 k^{2}-642 k-2025\right)}{9(k+5)^{3}(13 k+17)} \partial A_{3}\left(\begin{array}{c}
F_{11} \\
F_{22}
\end{array}\right) \\
& \text { - } \frac{8 i\left(124 k^{3}+929 k^{2}+3198 k+2889\right)}{9(k+5)^{3}(13 k+17)} A_{3} \partial\left(\begin{array}{c}
F_{11} \\
F_{22}
\end{array}\right) \\
& +\frac{4 i(k+4)}{(k+5)^{2}} A_{ \pm}\left(\begin{array}{c}
G_{21} \\
G_{12}
\end{array}\right) \pm \frac{8(k-3)}{3(k+5)^{3}} A_{ \pm} B_{3}\left(\begin{array}{c}
F_{21} \\
F_{12}
\end{array}\right) \\
& \mp \frac{8(k+9)}{3(k+5)^{3}} A_{ \pm} A_{\mp}\left(\begin{array}{c}
F_{11} \\
F_{22}
\end{array}\right) \mp \frac{8(k-3)}{3(k+5)^{3}} A_{ \pm} B_{\mp}\left(\begin{array}{c}
F_{22} \\
F_{11}
\end{array}\right) \\
& \text { - } \frac{4 i\left(248 k^{3}+961 k^{2}-1446 k-2943\right)}{9(k+5)^{3}(13 k+17)} \partial A_{ \pm}\left(\begin{array}{c}
F_{21} \\
F_{12}
\end{array}\right) \\
& -\frac{8 i\left(124 k^{3}+929 k^{2}+3198 k+2889\right)}{9(k+5)^{3}(13 k+17)} A_{ \pm} \partial\left(\begin{array}{c}
F_{21} \\
F_{12}
\end{array}\right)
\end{aligned}
$$




$$
\begin{aligned}
& -\frac{4 i(k+4)}{(k+5)^{2}} B_{3}\left(\begin{array}{l}
G_{11} \\
G_{22}
\end{array}\right) \pm \frac{16(k+3)}{3(k+5)^{3}} B_{3} B_{3}\left(\begin{array}{c}
F_{11} \\
F_{22}
\end{array}\right) \\
& +\frac{4 i\left(202 k^{3}+419 k^{2}-3120 k-3897\right)}{9(k+5)^{3}(13 k+17)} \partial B_{3}\left(\begin{array}{c}
F_{11} \\
F_{22}
\end{array}\right) \\
& -\frac{8 i\left(133 k^{3}+896 k^{2}+3249 k+2790\right)}{9(k+5)^{3}(13 k+17)} B_{3} \partial\left(\begin{array}{c}
F_{11} \\
F_{22}
\end{array}\right) \\
& +\frac{4 i\left(202 k^{3}+263 k^{2}-3792 k-4509\right)}{9(k+5)^{3}(13 k+17)} \partial B_{\mp}\left(\begin{array}{c}
F_{12} \\
F_{21}
\end{array}\right) \\
& -\frac{8 i\left(133 k^{3}+896 k^{2}+3249 k+2790\right)}{9(k+5)^{3}(13 k+17)} B_{\mp} \partial\left(\begin{array}{c}
F_{12} \\
F_{21}
\end{array}\right) \\
& \pm \frac{16(k+3)}{3(k+5)^{3}} B_{ \pm} B_{\mp}\left(\begin{array}{c}
F_{11} \\
F_{22}
\end{array}\right)-\frac{4 i(k+4)}{(k+5)^{2}} B_{\mp}\left(\begin{array}{c}
G_{12} \\
G_{21}
\end{array}\right) \\
& \pm \frac{16(k-3)\left(8 k^{2}+43 k+27\right)}{(k+5)^{3}(13 k+17)} \partial^{2}\left(\begin{array}{c}
F_{11} \\
F_{22}
\end{array}\right) \\
& \pm \frac{16(k+3)}{3(k+5)^{3}}\left(\begin{array}{l}
F_{11} F_{12} G_{21} \\
F_{22} F_{21} G_{12}
\end{array}\right) \pm \frac{4(k-3)}{3(k+5)^{3}}\left(\begin{array}{l}
F_{12} F_{21} G_{11} \\
F_{21} F_{12} G_{22}
\end{array}\right) \\
& \mp \frac{8(5 k+9)\left(11 k^{2}+118 k+219\right)}{9(k+5)^{4}(13 k+17)} \partial\left(\begin{array}{c}
F_{11} \\
F_{22}
\end{array}\right) F_{12} F_{21} \\
& \mp \frac{8\left(211 k^{3}+1205 k^{2}+225 k-1089\right)}{9(k+5)^{4}(13 k+17)}\left(\begin{array}{l}
F_{11} \partial F_{12} F_{21} \\
F_{22} \partial F_{21} F_{12}
\end{array}\right) \\
& \pm \frac{8\left(101 k^{3}-173 k^{2}-4089 k-5031\right)}{9(k+5)^{4}(13 k+17)}\left(\begin{array}{l}
F_{11} F_{12} \partial F_{21} \\
F_{22} F_{21} \partial F_{12}
\end{array}\right) \\
& \pm \frac{8(k+9)}{3(k+5)^{3}}\left(\begin{array}{l}
F_{11} F_{21} G_{12} \\
F_{22} F_{12} G_{21}
\end{array}\right) \pm \frac{4}{(k+5)^{2}}\left(\begin{array}{l}
F_{11} F_{22} G_{11} \\
F_{22} F_{11} G_{22}
\end{array}\right) \\
& \mp \frac{2\left(248 k^{3}+3004 k^{2}+8517 k+5733\right)}{9(k+5)^{2}(13 k+17)} \partial\left(\begin{array}{l}
G_{11} \\
G_{22}
\end{array}\right) \\
& \pm \frac{16(k-3)(4 k+17)}{3(k+5)^{2}(13 k+17)} T\left(\begin{array}{c}
F_{11} \\
F_{22}
\end{array}\right) \mp \frac{4(k-3)}{(k+5)^{2}} U\left(\begin{array}{c}
G_{11} \\
G_{22}
\end{array}\right) \\
& +\frac{32 i}{(k+5)^{3}} U A_{3}\left(\begin{array}{c}
F_{11} \\
F_{22}
\end{array}\right)+\frac{32 i}{(k+5)^{3}} U A_{ \pm}\left(\begin{array}{c}
F_{21} \\
F_{12}
\end{array}\right) \\
& -\frac{8 i(k+1)}{(k+5)^{3}} U B_{3}\left(\begin{array}{c}
F_{11} \\
F_{22}
\end{array}\right)-\frac{8 i(k+1)}{(k+5)^{3}} U B_{\mp}\left(\begin{array}{c}
F_{12} \\
F_{21}
\end{array}\right) \\
& \mp \frac{4\left(248 k^{3}+2653 k^{2}+8058 k+5733\right)}{9(k+5)^{3}(13 k+17)} \partial U\left(\begin{array}{c}
F_{11} \\
F_{22}
\end{array}\right) \\
& \mp \frac{16\left(62 k^{3}+49 k^{2}+948 k+1089\right)}{9(k+5)^{3}(13 k+17)} U \partial\left(\begin{array}{c}
F_{11} \\
F_{22}
\end{array}\right)
\end{aligned}
$$




$$
\begin{aligned}
& \left.\mp \frac{8(k-3)}{3(k+5)^{3}} U U\left(\begin{array}{c}
F_{11} \\
F_{22}
\end{array}\right)+\frac{32(k-3)}{3(k+5)^{3}}\left(\begin{array}{c}
\partial F_{11} \\
\partial F_{22}
\end{array}\right) F_{11} F_{22}\right](w) \\
& +\frac{1}{(z-w)^{2}}[+\cdots](w)+\frac{1}{(z-w)}[+\cdots](w)+\cdots .
\end{aligned}
$$

There is no boldface higher spin current in the fourth order pole.

Now one can calculate the following OPE

$$
\begin{aligned}
\mathbf{V}_{+}^{(\mathbf{2})}(z) \mathbf{V}_{-}^{(\mathbf{2})}(w) & =\frac{1}{(z-w)^{2}}\left[\frac{2(k+4)}{(k+5)^{2}} A_{-} B_{+}-\frac{2 i}{(k+5)^{2}} A_{-} F_{22} F_{12}\right. \\
& \left.+\frac{2 i}{(k+5)^{2}} B_{+} F_{22} F_{21}-\frac{(k-3)}{(k+5)^{2}} F_{22} G_{22}+\frac{4(3 k-1)}{(k+5)^{3}} F_{22} \partial F_{22}\right](w) \\
& +\frac{1}{(z-w)}\left[\frac{2 i}{(k+5)^{2}} A_{3} F_{22} G_{22}+\frac{(2 k+9)}{(k+5)^{2}} \partial A_{-} B_{+}\right. \\
& -\frac{1}{(k+5)^{2}} A_{-} \partial B_{+}+\frac{i}{(k+5)^{2}} A_{-} F_{22} G_{12}-\frac{2 i(k+1)}{(k+5)^{3}} A_{-} \partial F_{22} F_{12} \\
& +\frac{2 i(k+1)}{(k+5)^{3}} A_{-} F_{22} \partial F_{12}+\frac{i}{(k+5)^{2}} A_{-} F_{12} G_{22}-\frac{2 i}{(k+5)^{2}} B_{3} F_{22} G_{22} \\
& -\frac{i}{(k+5)^{2}} B_{+} F_{22} G_{21}-\frac{2 i}{(k+5)^{2}} \partial A_{-} F_{22} F_{12}+\frac{2 i(k+1)}{(k+5)^{3}} B_{+} \partial F_{22} F_{21} \\
& +\frac{2 i(k+9)}{(k+5)^{3}} B_{+} F_{22} \partial F_{21}-\frac{i}{(k+5)^{2}} B_{+} F_{21} G_{22}+\frac{(k+11)}{(k+5)^{2}} \partial F_{22} G_{22} \\
& -\frac{(k-1)}{(k+5)^{2}} F_{22} \partial G_{22}+\frac{4 i(k+1)}{(k+5)^{3}} F_{22} A_{3} \partial F_{22}+\frac{16 i}{(k+5)^{3}} F_{22} B_{3} \partial F_{22} \\
& \left.-\frac{4}{(k+5)^{2}} F_{22} U \partial F_{22}+\frac{8(k-1)}{(k+5)^{3}} F_{22} \partial^{2} F_{22}\right](w)+\cdots \\
& -\cdots
\end{aligned}
$$

The nonlinear terms disappear in the OPE. The corresponding OPE in the nonlinear version contains those nonlinear terms.

\section{Appendix F.3 The OPEs between the last $\mathcal{N}=2$ multiplet and itself}

The result (3.6) in section 3 determines the following OPE

$$
\begin{aligned}
\mathbf{W}^{(2)}(z) \mathbf{W}^{(2)}(w) & =\frac{1}{(z-w)^{4}}\left[\frac{9 k}{(k+5)}\right] \\
& +\frac{1}{(z-w)^{2}}\left[\frac{2(k+4)}{(k+5)} T+\frac{2(k+4)}{(k+5)^{2}} A_{3} A_{3}+\frac{4(k+4)}{(k+5)^{2}} A_{3} B_{3}+\frac{2 i(k+1)}{(k+5)^{2}} \partial A_{3}\right. \\
& -\frac{4 i}{(k+5)^{2}} A_{3} F_{12} F_{21}-\frac{2 i}{(k+5)^{2}} A_{-} F_{11} F_{12}+\frac{2(k+1)}{(k+5)^{2}} A_{+} A_{-}
\end{aligned}
$$




$$
\begin{aligned}
& -\frac{2 i}{(k+5)^{2}} A_{+} F_{21} F_{22}+\frac{2(k+4)}{(k+5)^{2}} B_{3} B_{3}+\frac{8 i}{(k+5)^{2}} \partial B_{3} \\
& -\frac{4 i}{(k+5)^{2}} B_{3} F_{12} F_{21}+\frac{2 i}{(k+5)^{2}} B_{-} F_{12} F_{22}+\frac{8}{(k+5)^{2}} B_{+} B_{-} \\
& +\frac{2}{(k+5)} \partial F_{11} F_{22}-\frac{2}{(k+5)} F_{11} \partial F_{22}+\frac{2\left(k^{2}+4 k+27\right)}{(k+5)^{3}} \partial F_{12} F_{21} \\
& -\frac{2\left(k^{2}+4 k+27\right)}{(k+5)^{3}} F_{12} \partial F_{21}-\frac{(k-3)}{(k+5)^{2}} F_{21} G_{12}-\frac{(k-3)}{(k+5)^{2}} F_{12} G_{21} \\
& \left.+\frac{2(k+4)}{(k+5)^{2}} U U+\frac{2 i}{(k+5)^{2}} B_{+} F_{11} F_{21}\right](w)+\frac{1}{(z-w)} \frac{1}{2} \partial(\text { pole- } 2)(w)+\cdots
\end{aligned}
$$

There are no boldface higher spin currents in the OPE.

With the results in (3.6) and (3.8), the following OPEs can be obtained

$$
\begin{aligned}
& \mathbf{W}^{(\mathbf{2})}(z) \mathbf{W}_{ \pm}^{\left(\frac{5}{2}\right)}(w)=\frac{1}{(z-w)^{4}}\left[\frac{6 k(k-3)}{(k+5)^{3}}\left(\begin{array}{c}
F_{21} \\
F_{12}
\end{array}\right)\right](w) \\
& +\frac{1}{(z-w)^{3}}\left[-\frac{2\left(4 k^{2}+39 k+66\right)}{3(k+5)^{2}}\left(\begin{array}{l}
G_{21} \\
G_{12}
\end{array}\right)\right. \\
& \pm \frac{4}{(k+5)^{2}}\left(\begin{array}{c}
F_{21} \\
F_{12}
\end{array}\right) F_{11} F_{22} \pm \frac{4 i\left(4 k^{2}+15 k-33\right)}{3(k+5)^{3}}\left(\begin{array}{c}
F_{21} \\
F_{12}
\end{array}\right) A_{3} \\
& \mp \frac{4 i\left(4 k^{2}+21 k+21\right)}{3(k+5)^{3}}\left(\begin{array}{c}
F_{11} \\
F_{22}
\end{array}\right) A_{\mp} \pm \frac{4 i\left(k^{2}+15 k+66\right)}{3(k+5)^{3}}\left(\begin{array}{c}
F_{22} \\
F_{11}
\end{array}\right) B_{\mp} \\
& \left. \pm \frac{4 i\left(k^{2}-k-22\right)}{(k+5)^{3}}\left(\begin{array}{c}
F_{21} \\
F_{12}
\end{array}\right) B_{3}-\frac{8\left(2 k^{2}+15 k+33\right)}{3(k+5)^{3}} U\left(\begin{array}{c}
F_{21} \\
F_{12}
\end{array}\right)\right](w) \\
& +\frac{1}{(z-w)^{2}}\left[\mp \frac{(k-3)}{6(k+5)} \mathbf{P}_{ \pm}^{\left(\frac{5}{2}\right)}-\frac{(k-3)}{6(k+5)} \mathbf{W}_{ \pm}^{\left(\frac{5}{2}\right)}\right. \\
& \mp \frac{i(5 k+27)}{3(k+5)^{2}} A_{3}\left(\begin{array}{c}
G_{21} \\
G_{12}
\end{array}\right)-\frac{2}{(k+5)^{2}} A_{3} A_{3}\left(\begin{array}{c}
F_{21} \\
F_{12}
\end{array}\right) \\
& +\frac{2(k+9)}{3(k+5)^{3}} A_{3} B_{\mp}\left(\begin{array}{c}
F_{22} \\
F_{11}
\end{array}\right)-\frac{(k-3)}{3(k+5)^{2}} U\left(\begin{array}{c}
G_{21} \\
G_{12}
\end{array}\right) \\
& \pm \frac{2 i(k-3)(8 k+45)}{9(k+5)^{3}} \partial A_{3}\left(\begin{array}{c}
F_{21} \\
F_{12}
\end{array}\right) \pm \frac{8 i(k+3)(2 k+9)}{9(k+5)^{3}} A_{3} \partial\left(\begin{array}{c}
F_{21} \\
F_{12}
\end{array}\right) \\
& \pm \frac{i(k+1)}{(k+5)^{2}} A_{\mp}\left(\begin{array}{c}
G_{11} \\
G_{22}
\end{array}\right)+\frac{4(k+3)}{3(k+5)^{3}} A_{\mp} A_{3}\left(\begin{array}{c}
F_{11} \\
F_{22}
\end{array}\right) \\
& -\frac{4(k+3)}{3(k+5)^{3}} A_{\mp} B_{3}\left(\begin{array}{c}
F_{11} \\
F_{22}
\end{array}\right)-\frac{2(k-3)}{3(k+5)^{3}} A_{\mp} B_{\mp}\left(\begin{array}{c}
F_{12} \\
F_{21}
\end{array}\right) \\
& \mp \frac{4 i(k+3)(4 k-3)}{9(k+5)^{3}} \partial A_{\mp}\left(\begin{array}{c}
F_{11} \\
F_{22}
\end{array}\right) \mp \frac{4 i(k+3)(4 k+27)}{9(k+5)^{3}} A_{\mp} \partial\left(\begin{array}{c}
F_{11} \\
F_{22}
\end{array}\right) \\
& -\frac{2(k+9)}{3(k+5)^{3}} A_{ \pm} A_{\mp}\left(\begin{array}{c}
F_{21} \\
F_{12}
\end{array}\right) \mp \frac{7 i(k+3)}{3(k+5)^{2}} B_{3}\left(\begin{array}{c}
G_{21} \\
G_{12}
\end{array}\right)
\end{aligned}
$$




$$
\begin{aligned}
& +\frac{2}{(k+5)^{2}} B_{3} B_{3}\left(\begin{array}{c}
F_{21} \\
F_{12}
\end{array}\right) \pm \frac{2 i(k-3)(10 k+39)}{9(k+5)^{3}} \partial B_{3}\left(\begin{array}{c}
F_{21} \\
F_{12}
\end{array}\right) \\
& \mp \frac{4 i(k+9)(k+12)}{9(k+5)^{3}} B_{3} \partial\left(\begin{array}{c}
F_{21} \\
F_{12}
\end{array}\right) \pm \frac{4 i}{(k+5)^{2}} B_{\mp}\left(\begin{array}{c}
G_{22} \\
G_{11}
\end{array}\right) \\
& -\frac{2(k+9)}{3(k+5)^{3}} B_{\mp} B_{3}\left(\begin{array}{c}
F_{22} \\
F_{11}
\end{array}\right) \mp \frac{2 i(k+9)(k-12)}{9(k+5)^{3}} \partial B_{\mp}\left(\begin{array}{c}
F_{22} \\
F_{11}
\end{array}\right) \\
& \pm \frac{2 i(k+9)(8 k+15)}{9(k+5)^{3}} B_{\mp} \partial\left(\begin{array}{c}
F_{22} \\
F_{11}
\end{array}\right)+\frac{4(k+3)}{3(k+5)^{3}} B_{ \pm} B_{\mp}\left(\begin{array}{c}
F_{21} \\
F_{12}
\end{array}\right) \\
& -\frac{4(k+3)}{3(k+5)^{3}}\left(\begin{array}{c}
F_{11} F_{21} G_{22} \\
F_{22} F_{12} G_{11}
\end{array}\right)-\frac{4(k+9)^{2}}{9(k+5)^{4}}\left(\begin{array}{c}
\partial F_{11} F_{21} F_{22} \\
\partial F_{22} F_{12} F_{11}
\end{array}\right) \\
& -\frac{16(k+3)(k+9)}{9(k+5)^{4}}\left(\begin{array}{l}
F_{11} \partial F_{21} F_{22} \\
F_{22} \partial F_{12} F_{11}
\end{array}\right) \\
& -\frac{16(k+3)^{2}}{9(k+5)^{4}}\left(\begin{array}{c}
F_{11} F_{21} \partial F_{22} \\
F_{22} F_{12} \partial F_{11}
\end{array}\right) \mp \frac{2}{(k+5)^{2}} F_{12} F_{21}\left(\begin{array}{c}
G_{21} \\
G_{12}
\end{array}\right) \\
& +\frac{2(k-3)(2 k+9)}{3(k+5)^{3}} \partial^{2}\left(\begin{array}{c}
F_{21} \\
F_{12}
\end{array}\right)+\frac{2(k+9)}{3(k+5)^{3}}\left(\begin{array}{l}
F_{21} F_{22} G_{11} \\
F_{12} F_{11} G_{22}
\end{array}\right) \\
& -\frac{\left(8 k^{2}+87 k+171\right)}{9(k+5)^{2}} \partial\left(\begin{array}{l}
G_{21} \\
G_{12}
\end{array}\right)+\frac{4(k-3)}{3(k+5)^{2}} T\left(\begin{array}{c}
F_{21} \\
F_{12}
\end{array}\right) \\
& \mp \frac{8 i(k+3)}{3(k+5)^{3}} U A_{3}\left(\begin{array}{c}
F_{21} \\
F_{12}
\end{array}\right) \pm \frac{4 i(k+3)}{3(k+5)^{3}} U A_{\mp}\left(\begin{array}{c}
F_{11} \\
F_{22}
\end{array}\right) \\
& \mp \frac{4 i(k+9)}{3(k+5)^{3}} U B_{3}\left(\begin{array}{c}
F_{21} \\
F_{12}
\end{array}\right) \pm \frac{2 i(k+9)}{3(k+5)^{3}} U B_{\mp}\left(\begin{array}{c}
F_{22} \\
F_{11}
\end{array}\right) \\
& -\frac{2\left(8 k^{2}+87 k+171\right)}{9(k+5)^{3}} \partial U\left(\begin{array}{c}
F_{21} \\
F_{12}
\end{array}\right)-\frac{16\left(k^{2}+3 k+18\right)}{9(k+5)^{3}} U \partial\left(\begin{array}{c}
F_{21} \\
F_{12}
\end{array}\right) \\
& \left.+\frac{2(k-3)}{3(k+5)^{3}} U U\left(\begin{array}{c}
F_{21} \\
F_{12}
\end{array}\right) \mp \frac{4(k-3)}{3(k+5)^{3}} \partial\left(\begin{array}{c}
F_{21} \\
F_{12}
\end{array}\right) F_{12} F_{21}\right](w) \\
& +\frac{1}{(z-w)}[+\cdots](w)+\cdots \text {. }
\end{aligned}
$$

There is no boldface higher spin current in the third order pole of the OPEs.

With the results in (3.6) and (3.9), one obtains the following OPE

$$
\begin{aligned}
\mathbf{W}^{(2)}(z) \mathbf{W}^{(\mathbf{3})}(w) & =\frac{1}{(z-w)^{4}}\left[-\frac{36 i\left(18 k^{2}+63 k+41\right)}{(k+5)^{2}(13 k+17)} A_{3}-\frac{12 i k\left(8 k^{2}+75 k+95\right)}{(k+5)^{2}(13 k+17)} B_{3}\right. \\
& \left.+\frac{12(k-3)\left(8 k^{2}+45 k+41\right)}{(k+5)^{3}(13 k+17)} F_{11} F_{22}-\frac{12\left(8 k^{3}+51 k^{2}+182 k+123\right)}{(k+5)^{3}(13 k+17)} F_{12} F_{21}\right](w) \\
& +\frac{1}{(z-w)^{3}}\left[\frac{8 i\left(7 k^{2}+44 k+141\right)}{3(k+5)^{3}(13 k+17)} A_{-} F_{11} F_{12}\right.
\end{aligned}
$$




$$
\begin{aligned}
& -\frac{4 i\left(25 k^{2}+158 k-27\right)}{3(k+5)^{3}(13 k+17)} B_{-} F_{12} F_{22}+\frac{4 i\left(25 k^{2}+158 k-27\right)}{3(k+5)^{3}(13 k+17)} B_{+} F_{11} F_{21} \\
& -\frac{(3 k+11)}{(k+5)^{2}} F_{11} G_{22}-\frac{4(k-3)(11 k+103)}{3(k+5)^{3}(13 k+17)} \partial\left(F_{11} F_{22}\right) \\
& +\frac{4}{(k+5)^{2}} \partial\left(F_{12} F_{21}\right)-\frac{8 i\left(7 k^{2}+44 k+141\right)}{3(k+5)^{3}(13 k+17)} A_{+} F_{21} F_{22} \\
& +\frac{(k-3)(89 k+205)}{3(k+5)^{2}(13 k+17)} F_{12} G_{21}-\frac{(k-3)(89 k+205)}{3(k+5)^{2}(13 k+17)} F_{21} G_{12} \\
& +\frac{24 i}{(k+5)^{2}} U A_{3}-\frac{8 i k}{(k+5)^{2}} U B_{3}+\frac{(3 k+11)}{(k+5)^{2}} F_{22} G_{11} \\
& \text { - } \left.\frac{4}{(k+5)^{2}} U F_{11} F_{22}+\frac{4(k-3)(11 k+103)}{3(k+5)^{3}(13 k+17)} U F_{12} F_{21}\right](w) \\
& +\frac{1}{(z-w)^{2}}\left[-3 \mathbf{S}^{(\mathbf{3})}+3 \mathbf{W}^{(\mathbf{3})}-\frac{8(\mathbf{k}-\mathbf{3})}{(13 k+17)} \mathbf{T}^{(\mathbf{1})} \mathbf{W}^{(\mathbf{2})}+\frac{2 i}{(k+5)^{2}} A_{3} A_{3} A_{3}\right. \\
& +\frac{6 i}{(k+5)^{2}} A_{3} A_{3} B_{3}-\frac{2}{(k+5)^{2}} \partial A_{3} A_{3}+\frac{6 i}{(k+5)^{2}} A_{3} B_{3} B_{3} \\
& +\frac{3(3 k+11)}{(k+5)^{2}} \partial A_{3} B_{3}-\frac{9}{(k+5)} A_{3} \partial B_{3}+\frac{8 i}{(k+5)^{2}} A_{3} F_{12} G_{21} \\
& +\frac{6 i}{(k+5)^{2}} A_{3} B_{+} B_{-}+\frac{i\left(44 k^{2}+173 k+341\right)}{(k+5)^{2}(13 k+17)} \partial^{2} A_{3} \\
& +\frac{15 i}{(k+5)^{2}} \partial A_{3} F_{11} F_{22}-\frac{15 i}{(k+5)^{2}} A_{3} \partial\left(F_{11} F_{22}\right) \\
& +\frac{24 i}{(k+5)^{3}} \partial A_{3} F_{12} F_{21}-\frac{2 i(5 k+29)}{(k+5)^{3}} A_{3} \partial F_{12} F_{21}+\frac{10 i(k+1)}{(k+5)^{3}} A_{3} F_{12} \partial F_{21} \\
& \text { - } \frac{4 i}{(k+5)^{2}} A_{3} F_{21} G_{12}+\frac{i}{(k+5)^{2}} A_{-} F_{11} G_{12}+\frac{14}{(k+5)^{2}} \partial A_{+} A_{-} \\
& +\frac{i\left(431 k^{2}+3082 k+3675\right)}{3(k+5)^{3}(13 k+17)} \partial A_{-} F_{11} F_{12}-\frac{14}{(k+5)^{2}} A_{+} \partial A_{-} \\
& -\frac{i\left(115 k^{2}+1298 k+1119\right)}{3(k+5)^{3}(13 k+17)} A_{-} \partial F_{11} F_{12}-\frac{i\left(739 k^{2}+4610 k+4383\right)}{3(k+5)^{3}(13 k+17)} A_{-} F_{11} \partial F_{12} \\
& -\frac{3 i}{(k+5)^{2}} A_{-} F_{12} G_{11}+\frac{2 i}{(k+5)^{2}} A_{+} A_{-} A_{3}+\frac{6 i}{(k+5)^{2}} A_{+} A_{-} B_{3} \\
& +\frac{3 i}{(k+5)^{2}} A_{+} F_{21} G_{22}+\frac{2 i\left(77 k^{2}+772 k+687\right)}{3(k+5)^{3}(13 k+17)} \partial A_{+} F_{21} F_{22} \\
& -\frac{4 i\left(59 k^{2}+463 k+600\right)}{3(k+5)^{3}(13 k+17)} A_{+} F_{21} \partial F_{22}-\frac{5 i}{(k+5)^{2}} A_{+} F_{22} G_{21} \\
& -\frac{2}{(k+5)^{2}} \partial B_{3} B_{3}+\frac{i\left(8 k^{3}+63 k^{2}+203 k-136\right)}{(k+5)^{2}(13 k+17)} \partial^{2} B_{3}
\end{aligned}
$$




$$
\begin{aligned}
& +\frac{2 i}{(k+5)^{2}} B_{3} B_{3} B_{3}+\frac{15 i}{(k+5)^{2}} \partial B_{3} F_{11} F_{22}-\frac{15 i}{(k+5)^{2}} B_{3} \partial\left(F_{11} F_{22}\right) \\
& +\frac{4 i}{(k+5)^{2}} B_{3} F_{12} G_{21}-\frac{6 i(k+1)}{(k+5)^{3}} \partial B_{3} F_{12} F_{21}-\frac{40 i}{(k+5)^{3}} B_{3} \partial F_{12} F_{21} \\
& +\frac{4 i(3 k+13)}{(k+5)^{3}} B_{3} F_{12} \partial F_{21}-\frac{8 i}{(k+5)^{2}} B_{3} F_{21} G_{12}-\frac{3 i}{(k+5)^{2}} B_{-} F_{12} G_{22} \\
& +\frac{i\left(275 k^{2}+1162 k+1431\right)}{3(k+5)^{3}(13 k+17)} \partial B_{-} F_{12} F_{22}-\frac{(3 k+5)}{(k+5)^{2}} B_{+} \partial B_{-} \\
& -\frac{i\left(349 k^{2}+1214 k+609\right)}{3(k+5)^{3}(13 k+17)} B_{-} \partial F_{12} F_{22}-\frac{i}{(k+5)^{2}} B_{+} F_{11} G_{21} \\
& +\frac{5 i}{(k+5)^{2}} B_{-} F_{22} G_{12}+\frac{2 i}{(k+5)^{2}} B_{+} B_{-} B_{3}+\frac{(3 k+5)}{(k+5)^{2}} \partial B_{+} B_{-} \\
& +\frac{4 i\left(136 k^{2}+767 k+675\right)}{3(k+5)^{3}(13 k+17)} \partial B_{+} F_{11} F_{21}-\frac{4 i\left(59 k^{2}+229 k+294\right)}{3(k+5)^{3}(13 k+17)} B_{+} \partial F_{11} F_{21} \\
& +\frac{3 i}{(k+5)^{2}} B_{+} F_{21} G_{11}-\frac{i\left(349 k^{2}+1838 k+1425\right)}{3(k+5)^{3}(13 k+17)} B_{-} F_{12} \partial F_{22} \\
& -\frac{2\left(4 k^{3}+48 k^{2}+115 k-269\right)}{(k+5)^{3}(13 k+17)} \partial^{2} F_{11} F_{22}+\frac{3(3 k+13)}{2(k+5)^{2}} \partial F_{11} G_{22} \\
& -\frac{2\left(4 k^{3}+100 k^{2}+365 k-31\right)}{(k+5)^{3}(13 k+17)} F_{11} \partial^{2} F_{22}-\frac{(5 k+23)}{2(k+5)^{2}} F_{11} \partial G_{22} \\
& +\frac{\left(8 k^{3}+521 k^{2}+1810 k+1633\right)}{(k+5)^{3}(13 k+17)} \partial^{2} F_{12} F_{21}+\frac{3(3 k+11)}{2(k+5)^{2}} \partial F_{22} G_{11} \\
& +\frac{2(k+1)}{(k+5)^{2}(13 k+17)} F_{22} \partial G_{11} \\
& +\frac{2\left(8 k^{3}-545 k^{2}-1326 k-645\right)}{(k+5)^{3}(13 k+17)} \partial F_{12} \partial F_{21}-\frac{(k+1)}{2(k+5)^{2}} \frac{6}{(k+5)^{2}} U A_{3} A_{3} \\
& +\frac{\left(8 k^{3}+521 k^{2}+1810 k+1633\right)}{(k+5)^{3}(13 k+17)} F_{12} \partial^{2} F_{21} \\
& +\frac{\left(467 k^{2}+1009 k+330\right)}{3(k+5)^{2}(13 k+17)} \partial F_{12} G_{21}-\frac{\left(118 k^{2}+341 k+435\right)}{3(k+5)^{2}(13 k+17)} F_{12} \partial G_{21} \\
& +G_{12} G_{21}+\frac{32\left(49 k^{2}+374 k+45\right)^{2}(13 k+17)}{3(k+5)^{4}(13 k+17)} F_{11} F_{12} \partial F_{21} F_{22} \\
& +
\end{aligned}
$$




$$
\begin{aligned}
& +\frac{12}{(k+5)^{2}} U A_{3} B_{3}-\frac{9 i}{(k+5)^{2}} \partial U A_{3}+\frac{27 i}{(k+5)^{2}} U \partial A_{3} \\
& +\frac{6}{(k+5)^{2}} U A_{+} A_{-}+\frac{6}{(k+5)^{2}} U B_{3} B_{3}+\frac{i(k+6)}{(k+5)^{2}} \partial U B_{3} \\
& -\frac{5 i k}{(k+5)^{2}} U \partial B_{3}+\frac{6}{(k+5)^{2}} U B_{+} B_{-}-\frac{3(4 k+17)}{(k+5)(13 k+17)} \partial^{2} U \\
& -\frac{5}{(k+5)^{2}} \partial U F_{11} F_{22}+\frac{21}{(k+5)^{2}} U \partial F_{11} F_{22}+\frac{6}{(k+5)^{2}} U U U \\
& -\frac{15}{(k+5)^{2}} U F_{11} \partial F_{22}+\frac{4}{(k+5)^{2}} U F_{12} G_{21}-\frac{2(k-3)(41 k-35)}{3(k+5)^{3}(13 k+17)} \partial U F_{12} F_{21} \\
& +\frac{64\left(17 k^{2}+91 k+78\right)}{3(k+5)^{3}(13 k+17)} U \partial F_{12} F_{21}-\frac{16\left(49 k^{2}+374 k+453\right)}{3(k+5)^{3}(13 k+17)} U F_{12} \partial F_{21} \\
& \left.-\frac{4}{(k+5)^{2}} U F_{21} G_{12}+\frac{2 i}{(k+5)^{2}} U U A_{3}+\frac{2 i}{(k+5)^{2}} U U B_{3}\right](w) \\
& +\frac{1}{(z-w)}[+\cdots](w)+\cdots
\end{aligned}
$$

There are no boldface higher spin currents in the fourth and third order poles in the OPE. The nonlinear term containing the boldface higher spin currents in the second order pole can be removed by redefining the higher spin-3 current as a quasi primary field.

Moreover, the following OPEs can be obtained as before

$$
\begin{aligned}
\mathbf{W}_{ \pm}^{\left(\frac{5}{2}\right)}(z) \mathbf{W}_{ \pm}^{\left(\frac{5}{2}\right)}(w) & =\frac{1}{(z-w)^{3}}\left[-\frac{8\left(8 k^{2}+51 k+135\right)}{9(k+5)^{3}} A_{\mp} B_{\mp} \mp \frac{32 i(k+3)(k+9)}{9(k+5)^{4}} A_{\mp}\left(\begin{array}{c}
F_{11} F_{21} \\
F_{22} F_{12}
\end{array}\right)\right. \\
& \pm \frac{32 i(k+3)(k+9)}{9(k+5)^{4}} B_{\mp}\left(\begin{array}{c}
F_{21} F_{22} \\
F_{12} F_{11}
\end{array}\right)+\frac{16\left(31 k^{2}+138 k+27\right)}{9(k+5)^{4}}\left(\begin{array}{c}
F_{21} \partial F_{21} \\
F_{12} \partial F_{12}
\end{array}\right) \\
& \left.-\frac{8(k-3)(2 k+9)}{3(k+5)^{3}}\left(\begin{array}{c}
F_{21} G_{21} \\
F_{12} G_{12}
\end{array}\right)\right](w) \\
& +\frac{1}{(z-w)^{2}} \frac{1}{2} \partial(\operatorname{pole}-3)(w)+\frac{1}{(z-w)}[+\cdots](w)+\cdots
\end{aligned}
$$

The first order pole can be determined by using the general procedure in section 7 .

Again the different combination of OPE can be obtained

$$
\begin{aligned}
\mathbf{W}_{+}^{\left(\frac{5}{2}\right)}(z) \mathbf{W}_{-}^{\left(\frac{5}{2}\right)}(w) & =\frac{1}{(z-w)^{5}}\left[-\frac{32 k(k+3)(k+9)}{(k+5)^{3}}\right] \\
& +\frac{1}{(z-w)^{4}}\left[\frac{8 i(k+9)(4 k+9)}{(k+5)^{3}} A_{3}+\frac{16 i k(k+3)(2 k+15)}{3(k+5)^{3}} B_{3}\right. \\
& \left.-\frac{8(k-3)\left(4 k^{2}+33 k+81\right)}{3(k+5)^{4}} F_{11} F_{22}+\frac{8\left(4 k^{3}+27 k^{2}+90 k+243\right)}{3(k+5)^{4}} F_{12} F_{21}\right](w) \\
& +\frac{1}{(z-w)^{3}}\left[\frac{2(k-3)}{3(k+5)} \mathbf{P}^{(2)}-\frac{8(k-3)^{2}}{9(k+5)^{2}} \mathbf{T}^{(\mathbf{2})}-\frac{8(k-3)}{3(k+5)} \mathbf{W}^{(\mathbf{2})}\right.
\end{aligned}
$$




$$
\begin{aligned}
& -\frac{4\left(20 k^{2}+204 k+369\right)}{9(k+5)^{2}} T-\frac{4\left(20 k^{2}+123 k+99\right)}{9(k+5)^{3}} A_{+} A_{-} \\
& -\frac{4\left(20 k^{2}+123 k+99\right)}{9(k+5)^{3}} A_{3} A_{3}-\frac{8\left(7 k^{2}+57 k+126\right)}{9(k+5)^{3}} A_{3} B_{3} \\
& +\frac{8 i(k+15)(8 k+21)}{9(k+5)^{3}} \partial A_{3}+\frac{16(k-3)(k+6)}{9(k+5)^{3}} F_{22} G_{11} \\
& +\frac{32 i(k+3)(k+9)}{9(k+5)^{4}} A_{3} F_{11} F_{22}+\frac{8 i}{(k+5)^{2}} A_{3} F_{12} F_{21} \\
& +\frac{8 i(k+1)(k+9)}{(k+5)^{4}} A_{-} F_{11} F_{12}+\frac{32 i\left(k^{2}+12 k+63\right)}{9(k+5)^{4}} A_{+} F_{21} F_{22} \\
& -\frac{4\left(2 k^{2}+87 k+369\right)}{9(k+5)^{3}} B_{3} B_{3}+\frac{8 i}{(k+5)^{2}} B_{3} F_{12} F_{21} \\
& +\frac{4 i\left(12 k^{3}+124 k^{2}+183 k-369\right)}{9(k+5)^{3}} \partial B_{3}-\frac{32 i(k+3)(k+9)}{9(k+5)^{4}} B_{3} F_{11} F_{22} \\
& -\frac{64 i(k+3)}{(k+5)^{4}} B_{-} F_{12} F_{22}-\frac{4\left(2 k^{2}+87 k+369\right)}{9(k+5)^{3}} B_{+} B_{-} \\
& -\frac{8 i\left(13 k^{2}+66 k+117\right)}{9(k+5)^{4}} B_{+} F_{11} F_{21} \\
& +\frac{4(k-3)(5 k+21)}{9(k+5)^{3}} F_{11} G_{22}-\frac{16\left(8 k^{3}+69 k^{2}+252 k+423\right)}{9(k+5)^{4}} \partial F_{11} F_{22} \\
& +\frac{8\left(4 k^{3}+93 k^{2}+594 k+1305\right)}{9(k+5)^{4}} F_{11} \partial F_{22}+\frac{4(k-3)(2 k+9)}{3(k+5)^{3}} F_{12} G_{21} \\
& -\frac{16\left(2 k^{3}+25 k^{2}+120 k+369\right)}{9(k+5)^{4}} \partial F_{12} F_{21} \\
& +\frac{8\left(16 k^{3}+123 k^{2}+558 k+1395\right)}{9(k+5)^{4}} F_{12} \partial F_{21}+\frac{4(k-3)(4 k+21)}{3(k+5)^{3}} F_{21} G_{12} \\
& -\frac{8 i(k-3)}{(k+5)^{3}} U A_{3}-\frac{8 i(k-3) k}{3(k+5)^{3}} U B_{3}-\frac{4\left(20 k^{2}+177 k+369\right)}{9(k+5)^{3}} U U \\
& \left.+\frac{32(k-3)^{2}}{9(k+5)^{4}} U F_{11} F_{22}-\frac{8(k-3)}{(k+5)^{3}} U F_{12} F_{21}\right](w) \\
& +\frac{1}{(z-w)^{2}}\left[3 \mathbf{S}^{(\mathbf{3})}+\frac{(k-3)}{3(k+5)} \mathbf{P}^{(\mathbf{3})}+\frac{(k-3)}{3(k+5)} \partial \mathbf{P}^{(\mathbf{2})}-\frac{2(5 k+21)}{3(k+5)} \mathbf{W}^{(\mathbf{3})}\right. \\
& -\frac{4(k-3)^{2}}{9(k+5)^{2}} \partial \mathbf{T}^{(\mathbf{2})}-\frac{4(k-3)}{3(k+5)} \partial \mathbf{W}^{(\mathbf{2})} \\
& -\frac{8 i}{(k+5)^{2}} A_{3} A_{3} B_{3}-\frac{4\left(20 k^{2}+123 k+99\right)}{9(k+5)^{3}} \partial A_{3} A_{3}-\frac{8 i}{(k+5)^{2}} A_{3} B_{3} B_{3} \\
& -\frac{2\left(59 k^{2}+462 k+891\right)}{9(k+5)^{3}} \partial A_{3} B_{3}+\frac{2\left(31 k^{2}+306 k+747\right)}{9(k+5)^{3}} A_{3} \partial B_{3}
\end{aligned}
$$




$$
\begin{aligned}
& -\frac{8 i}{(k+5)^{2}} A_{3} B_{+} B_{-}-\frac{2 i\left(128 k^{3}+181 k^{2}+1110 k+8577\right)}{9(k+5)^{3}(13 k+17)} \partial^{2} A_{3} \\
& -\frac{4 i(k+9)}{3(k+5)^{3}} A_{3} F_{11} G_{22}-\frac{i\left(119 k^{2}+1134 k+3015\right)}{9(k+5)^{4}} \partial A_{3} F_{11} F_{22} \\
& +\frac{i\left(169 k^{2}+1554 k+3609\right)}{9(k+5)^{4}} A_{3} \partial F_{11} F_{22} \\
& -\frac{10 i}{(k+5)^{2}} A_{3} F_{12} G_{21}+\frac{i\left(133 k^{2}+1482 k+4149\right)}{9(k+5)^{4}} A_{3} F_{11} \partial F_{22} \\
& +\frac{i(17 k-27)}{3(k+5)^{3}} \partial A_{3} F_{12} F_{21}+\frac{i(49 k+261)}{3(k+5)^{3}} A_{3} \partial F_{12} F_{21} \\
& -\frac{i(35 k-33)}{3(k+5)^{3}} A_{3} F_{12} \partial F_{21}-\frac{4 i\left(5 k^{2}+60 k+231\right)}{3(k+5)^{4}} \partial A_{-} F_{11} F_{12} \\
& +\frac{2 i}{(k+5)^{2}} A_{3} F_{21} G_{12}-\frac{8 i(k+3)}{3(k+5)^{3}} A_{3} F_{22} G_{11}-\frac{2 i(k+9)}{3(k+5)^{3}} A_{-} F_{11} G_{12} \\
& +\frac{8 i\left(2 k^{2}+29 k+75\right)}{3(k+5)^{4}} A_{-} \partial F_{11} F_{12}+\frac{8 i\left(9 k^{2}+91 k+210\right)}{3(k+5)^{4}} A_{-} F_{11} \partial F_{12} \\
& +\frac{8 i(k+6)}{3(k+5)^{3}} A_{-} F_{12} G_{11}-\frac{8 i}{(k+5)^{2}} A_{+} A_{-} B_{3} \\
& -\frac{8(k+3)(5 k+27)}{9(k+5)^{3}} \partial A_{+} A_{-}-\frac{4\left(10 k^{2}+39 k-63\right)}{9(k+5)^{3}} A_{+} \partial A_{-} \\
& -\frac{8 i(k+6)}{3(k+5)^{3}} A_{+} F_{21} G_{22}-\frac{8 i(k+3)(k+27)}{9(k+5)^{4}} \partial A_{+} F_{21} F_{22} \\
& +\frac{16 i\left(k^{2}+27 k+144\right)}{9(k+5)^{4}} A_{+} \partial F_{21} F_{22}+\frac{16 i\left(4 k^{2}+51 k+189\right)}{9(k+5)^{4}} A_{+} F_{21} \partial F_{22} \\
& +\frac{2 i(7 k+39)}{3(k+5)^{3}} A_{+} F_{22} G_{21}-\frac{4\left(2 k^{2}+87 k+369\right)}{9(k+5)^{3}} \partial B_{3} B_{3} \\
& +\frac{2 i\left(44 k^{4}+491 k^{3}-649 k^{2}-7743 k-4743\right)}{9(k+5)^{3}(13 k+17)} \partial^{2} B_{3} \\
& +\frac{4 i(k+9)}{3(k+5)^{3}} B_{3} F_{11} G_{22}-\frac{i\left(145 k^{2}+1554 k+3825\right)}{9(k+5)^{4}} \partial B_{3} F_{11} F_{22} \\
& +\frac{i\left(95 k^{2}+1134 k+3231\right)}{9(k+5)^{4}} B_{3} \partial F_{11} F_{22}+\frac{i\left(131 k^{2}+1206 k+2691\right)}{9(k+5)^{4}} B_{3} F_{11} \partial F_{22} \\
& -\frac{2 i}{(k+5)^{2}} B_{3} F_{12} G_{21}+\frac{i(25 k+93)}{3(k+5)^{3}} \partial B_{3} F_{12} F_{21} \\
& +\frac{i(17 k+213)}{3(k+5)^{3}} B_{3} \partial F_{12} F_{21}-\frac{i(19 k+159)}{3(k+5)^{3}} B_{3} F_{12} \partial F_{21} \\
& +\frac{10 i}{(k+5)^{2}} B_{3} F_{21} G_{12}+\frac{8 i(k+3)}{3(k+5)^{3}} B_{3} F_{22} G_{11}+\frac{8 i(k+6)}{3(k+5)^{3}} B_{-} F_{12} G_{22}
\end{aligned}
$$




$$
\begin{aligned}
& -\frac{i\left(19 k^{2}+270 k+651\right)}{3(k+5)^{4}} \partial B_{-} F_{12} F_{22} \\
& +\frac{i\left(17 k^{2}+26 k-135\right)}{3(k+5)^{4}} B_{-} \partial F_{12} F_{22}+\frac{i\left(21 k^{2}+130 k+285\right)}{3(k+5)^{4}} B_{-} F_{12} \partial F_{22} \\
& -\frac{2 i(7 k+39)}{3(k+5)^{3}} B_{-} F_{22} G_{12}-\frac{2(k+9)(11 k+51)}{9(k+5)^{3}} \partial B_{+} B_{-} \\
& +\frac{2\left(7 k^{2}-24 k-279\right)}{9(k+5)^{3}} B_{+} \partial B_{-}+\frac{2 i(k+9)}{3(k+5)^{3}} B_{+} F_{11} G_{21} \\
& -\frac{i\left(163 k^{2}+1398 k+3267\right)}{9(k+5)^{4}} \partial B_{+} F_{11} F_{21}-\frac{i\left(7 k^{2}-162 k-441\right)}{9(k+5)^{4}} B_{+} \partial F_{11} F_{21} \\
& +\frac{i\left(125 k^{2}+1578 k+4221\right)}{9(k+5)^{4}} B_{+} F_{11} \partial F_{21}-\frac{8 i(k+6)}{3(k+5)^{3}} B_{+} F_{21} G_{11} \\
& -\frac{\left(608 k^{4}+5989 k^{3}+32739 k^{2}+97479 k+105129\right)}{9(k+5)^{4}(13 k+17)} \partial^{2} F_{11} F_{22} \\
& -\frac{2(k-3)\left(88 k^{3}+269 k^{2}-4278 k-10971\right)}{9(k+5)^{4}(13 k+17)} \partial F_{11} \partial F_{22} \\
& +\frac{\left(144 k^{4}+2461 k^{3}+13355 k^{2}+29679 k+13713\right)}{3(k+5)^{4}(13 k+17)} F_{11} \partial^{2} F_{22} \\
& -\frac{\left(61 k^{2}+1002 k+2349\right)}{18(k+5)^{3}} \partial F_{11} G_{22}+\frac{\left(47 k^{2}+390 k+567\right)}{18(k+5)^{3}} F_{11} \partial G_{22} \\
& -\frac{\left(432 k^{4}+9335 k^{3}+61417 k^{2}+170877 k+154395\right)}{9(k+5)^{4}(13 k+17)} \partial^{2} F_{12} F_{21} \\
& +\frac{2\left(88 k^{4}+7309 k^{3}+59299 k^{2}+131439 k+70209\right)}{9(k+5)^{4}(13 k+17)} \partial F_{12} \partial F_{21} \\
& +\frac{\left(608 k^{4}+1021 k^{3}-8157 k^{2}-5697 k-9351\right)}{9(k+5)^{4}(13 k+17)} F_{12} \partial^{2} F_{21} \\
& -\frac{\left(61 k^{2}+390 k+441\right)}{6(k+5)^{3}} \partial F_{12} G_{21}+\frac{\left(31 k^{2}+146 k+3\right)}{6(k+5)^{3}} F_{12} \partial G_{21} \\
& +\frac{\left(5 k^{2}-129 k-738\right)}{3(k+5)^{3}} \partial F_{21} G_{12}+\frac{(k+6)(9 k+13)}{3(k+5)^{3}} F_{21} \partial G_{12} \\
& -\frac{\left(19 k^{2}+183 k+864\right)}{9(k+5)^{3}} \partial F_{22} G_{11}+\frac{\left(17 k^{2}+81 k+36\right)}{9(k+5)^{3}} F_{22} \partial G_{11} \\
& +\frac{4 i\left(20 k^{2}+231 k+483\right)}{(k+5)^{2}(13 k+17)} T A_{3}+\frac{16 i k\left(5 k^{2}+57 k+123\right)}{3(k+5)^{2}(13 k+17)} T B_{3} \\
& -\frac{4\left(10 k^{2}+93 k+126\right)}{9(k+5)^{2}} \partial T-\frac{16(k+6)(4 k+17)}{3(k+5)^{2}(13 k+17)} T U \\
& -\frac{16(k-3)\left(5 k^{2}+45 k+108\right)}{3(k+5)^{3}(13 k+17)} T F_{11} F_{22}-\frac{(5 k+23)}{(k+5)^{2}} G_{12} G_{21}
\end{aligned}
$$




$$
\left.\begin{array}{l}
+\frac{8\left(10 k^{3}+123 k^{2}+552 k+903\right)}{3(k+5)^{3}(13 k+17)} T F_{12} F_{21}-\frac{16(k+6)}{3(k+5)^{3}} U A_{3} A_{3} \\
-\frac{8(5 k+21)}{3(k+5)^{3}} U A_{3} B_{3}+\frac{8 i(2 k+11)}{(k+5)^{3}} \partial U A_{3}-\frac{8 i(11 k+36)}{3(k+5)^{3}} U \partial A_{3} \\
-\frac{16(k+6)}{3(k+5)^{3}} U A_{+} A_{-}-\frac{16(k+6)}{3(k+5)^{3}} U B_{3} B_{3}+\frac{(k-3)}{(k+5)^{2}} G_{11} G_{22} \\
-\frac{2 i\left(5 k^{2}+33 k+60\right)}{3(k+5)^{3}} \partial U B_{3}+\frac{2 i\left(k^{2}+37 k+12\right)}{3(k+5)^{3}} U \partial B_{3} \\
-\frac{16(k+6)}{3(k+5)^{3}} U B_{+} B_{-}+\frac{8(k+6)(4 k+17)}{3(k+5)^{2}(13 k+17)} \partial^{2} U-\frac{6}{(k+5)^{2}} U F_{12} G_{21} \\
+\frac{\left(43 k^{2}+174 k+819\right)}{9(k+5)^{4}} \partial U F_{11} F_{22}-\frac{\left(149 k^{2}+1938 k+4941\right)}{9(k+5)^{4}} U \partial F_{11} F_{22} \\
+\frac{\left(127 k^{2}+1206 k+3879\right)}{9(k+5)^{4}} U F_{11} \partial F_{22}+\frac{(k-3)}{(k+5)^{3}} \partial U F_{12} F_{21} \\
-\frac{(31 k+99)}{(k+5)^{3}} U \partial F_{12} F_{21}+\frac{(13 k+153)}{(k+5)^{3}} U F_{12} \partial F_{21} \\
\left.+\frac{6}{(k+5)^{2}} U F_{21} G_{12}-\frac{16(k+6)}{3(k+5)^{3}} U U U-\frac{4\left(20 k^{2}+177 k+369\right)}{9(k+5)^{3}} \partial U U\right](w) \\
+\frac{1}{(z-w)}[+\cdots](w)+\cdots
\end{array}\right]
$$

One does not see any higher spin current in the fourth order pole.

One describes the following OPEs

$$
\begin{aligned}
\mathbf{W}_{ \pm}^{\left(\frac{5}{2}\right)}(z) \mathbf{W}^{(3)}(w) & =\frac{1}{(z-w)^{5}}\left[ \pm \frac{24(k-3) k}{(k+5)^{2}(13 k+17)}\left(\begin{array}{c}
F_{21} \\
F_{12}
\end{array}\right)\right](w) \\
& +\frac{1}{(z-w)^{4}}\left[\mp \frac{2\left(248 k^{3}+2770 k^{2}+7275 k+4509\right)}{3(k+5)^{2}(13 k+17)}\left(\begin{array}{c}
G_{21} \\
G_{12}
\end{array}\right)\right. \\
& \mp \frac{4\left(248 k^{3}+1717 k^{2}+5898 k+4509\right)}{3(k+5)^{3}(13 k+17)} U\left(\begin{array}{c}
F_{21} \\
F_{12}
\end{array}\right) \\
& +\frac{8(5 k+9)\left(11 k^{2}+118 k+219\right)}{3(k+5)^{4}(13 k+17)}\left(\begin{array}{c}
F_{21} \\
F_{12}
\end{array}\right) F_{11} F_{22} \\
& +\frac{4 i\left(248 k^{3}+1195 k^{2}+966 k-189\right)}{3(k+5)^{3}(13 k+17)}\left(\begin{array}{c}
F_{21} \\
F_{12}
\end{array}\right) A_{3} \\
& -\frac{4 i\left(248 k^{3}+1195 k^{2}+966 k-189\right)}{3(k+5)^{3}(13 k+17)}\left(\begin{array}{c}
F_{11} \\
F_{22}
\end{array}\right) A_{\mp} \\
& -\frac{4 i\left(46 k^{3}-409 k^{2}-4404 k-4509\right)}{3(k+5)^{3}(13 k+17)}\left(\begin{array}{c}
F_{22} \\
F_{11}
\end{array}\right) B_{\mp} \\
& \left.+\frac{4 i\left(46 k^{3}-409 k^{2}-4404 k-4509\right)}{3(k+5)^{3}(13 k+17)}\left(\begin{array}{c}
F_{21} \\
F_{12}
\end{array}\right) B_{3}\right](w)
\end{aligned}
$$




$$
\begin{aligned}
& +\frac{1}{(z-w)^{3}}\left[\frac{2(k-3)(41 k+109)}{3(k+5)(13 k+17)}\left(\mathbf{P}_{ \pm}^{\left(\frac{5}{2}\right)} \pm \mathbf{W}_{ \pm}^{\left(\frac{5}{2}\right)}\right)\right. \\
& -\frac{4 i(k+4)}{(k+5)^{2}} A_{3}\left(\begin{array}{c}
G_{21} \\
G_{12}
\end{array}\right) \mp \frac{8(k+9)}{3(k+5)^{3}} A_{3} A_{3}\left(\begin{array}{c}
F_{21} \\
F_{12}
\end{array}\right) \\
& \pm \frac{8(k-3)}{3(k+5)^{3}} A_{3} B_{\mp}\left(\begin{array}{c}
F_{22} \\
F_{11}
\end{array}\right) \mp \frac{8(k-3)}{3(k+5)^{3}} A_{3} B_{3}\left(\begin{array}{c}
F_{21} \\
F_{12}
\end{array}\right) \\
& +\frac{4 i\left(248 k^{3}+883 k^{2}-2250 k-3861\right)}{9(k+5)^{3}(13 k+17)} \partial A_{3}\left(\begin{array}{c}
F_{21} \\
F_{12}
\end{array}\right) \\
& +\frac{8 i\left(124 k^{3}+929 k^{2}+3198 k+2889\right)}{9(k+5)^{3}(13 k+17)} A_{3} \partial\left(\begin{array}{c}
F_{21} \\
F_{12}
\end{array}\right) \\
& +\frac{4 i(k+4)}{(k+5)^{2}} A_{\mp}\left(\begin{array}{l}
G_{11} \\
G_{22}
\end{array}\right) \pm \frac{8(k-3)}{3(k+5)^{3}} A_{\mp} B_{3}\left(\begin{array}{l}
F_{11} \\
F_{22}
\end{array}\right) \\
& \pm \frac{8(k-3)}{3(k+5)^{3}} A_{\mp} B_{\mp}\left(\begin{array}{c}
F_{12} \\
F_{21}
\end{array}\right) \pm \frac{16(k+3)}{3(k+5)^{3}} B_{3} B_{3}\left(\begin{array}{c}
F_{21} \\
F_{12}
\end{array}\right) \\
& -\frac{4 i\left(248 k^{3}+961 k^{2}-1446 k-2943\right)}{9(k+5)^{3}(13 k+17)} \partial A_{\mp}\left(\begin{array}{c}
F_{11} \\
F_{22}
\end{array}\right) \\
& \text { - } \frac{8 i\left(124 k^{3}+929 k^{2}+3198 k+2889\right)}{9(k+5)^{3}(13 k+17)} A_{\mp} \partial\left(\begin{array}{c}
F_{11} \\
F_{22}
\end{array}\right) \\
& \mp \frac{8(k+9)}{3(k+5)^{3}} A_{ \pm} A_{\mp}\left(\begin{array}{c}
F_{21} \\
F_{12}
\end{array}\right)-\frac{4 i(k+4)}{(k+5)^{2}} B_{3}\left(\begin{array}{c}
G_{21} \\
G_{12}
\end{array}\right) \\
& +\frac{4 i\left(202 k^{3}+419 k^{2}-3120 k-3897\right)}{9(k+5)^{3}(13 k+17)} \partial B_{3}\left(\begin{array}{c}
F_{21} \\
F_{12}
\end{array}\right) \\
& -\frac{8 i\left(133 k^{3}+896 k^{2}+3249 k+2790\right)}{9(k+5)^{3}(13 k+17)} B_{3} \partial\left(\begin{array}{c}
F_{21} \\
F_{12}
\end{array}\right)+\frac{4 i(k+4)}{(k+5)^{2}} B_{\mp}\left(\begin{array}{c}
G_{22} \\
G_{11}
\end{array}\right) \\
& \text { - } \frac{4 i\left(202 k^{3}+263 k^{2}-3792 k-4509\right)}{9(k+5)^{3}(13 k+17)} \partial B_{\mp}\left(\begin{array}{c}
F_{22} \\
F_{11}
\end{array}\right) \\
& +\frac{8 i\left(133 k^{3}+896 k^{2}+3249 k+2790\right)}{9(k+5)^{3}(13 k+17)} B_{\mp} \partial\left(\begin{array}{c}
F_{22} \\
F_{11}
\end{array}\right) \\
& \pm \frac{16(k+3)}{3(k+5)^{3}} B_{ \pm} B_{\mp}\left(\begin{array}{c}
F_{21} \\
F_{12}
\end{array}\right) \mp \frac{8(k+9)}{3(k+5)^{3}}\left(\begin{array}{c}
F_{11} F_{21} G_{22} \\
F_{22} F_{12} G_{11}
\end{array}\right) \\
& \pm \frac{8\left(101 k^{3}-173 k^{2}-4089 k-5031\right)}{9(k+5)^{4}(13 k+17)}\left(\begin{array}{c}
\partial F_{11} F_{21} F_{22} \\
\partial F_{22} F_{12} F_{11}
\end{array}\right) \\
& \mp \frac{8(5 k+9)\left(11 k^{2}+118 k+219\right)}{9(k+5)^{4}(13 k+17)}\left(\begin{array}{l}
F_{11} \partial F_{21} F_{22} \\
F_{22} \partial F_{12} F_{11}
\end{array}\right) \\
& \mp \frac{8\left(211 k^{3}+1205 k^{2}+225 k-1089\right)}{9(k+5)^{4}(13 k+17)}\left(\begin{array}{l}
F_{11} F_{21} \partial F_{22} \\
F_{22} F_{12} \partial F_{11}
\end{array}\right)
\end{aligned}
$$




$$
\begin{aligned}
& -\frac{4}{(k+5)^{2}} F_{12} F_{21}\left(\begin{array}{c}
G_{21} \\
G_{12}
\end{array}\right)-\frac{4(k-3)}{3(k+5)^{3}} F_{11} F_{22}\left(\begin{array}{l}
G_{21} \\
G_{12}
\end{array}\right) \\
& \pm \frac{16(k-3)\left(8 k^{2}+43 k+27\right)}{(k+5)^{3}(13 k+17)} \partial^{2}\left(\begin{array}{c}
F_{21} \\
F_{12}
\end{array}\right) \pm \frac{16(k+3)}{3(k+5)^{3}}\left(\begin{array}{c}
F_{21} F_{22} G_{11} \\
F_{12} F_{11} G_{22}
\end{array}\right) \\
& \mp \frac{2\left(248 k^{3}+3004 k^{2}+8517 k+5733\right)}{9(k+5)^{2}(13 k+17)} \partial\left(\begin{array}{c}
G_{21} \\
G_{12}
\end{array}\right) \\
& \pm \frac{16(k-3)(4 k+17)}{3(k+5)^{2}(13 k+17)} T\left(\begin{array}{c}
F_{21} \\
F_{12}
\end{array}\right) \mp \frac{4(k-3)}{(k+5)^{2}} U\left(\begin{array}{l}
G_{21} \\
G_{12}
\end{array}\right) \\
& -\frac{32 i}{(k+5)^{3}} U A_{3}\left(\begin{array}{c}
F_{21} \\
F_{12}
\end{array}\right)+\frac{32 i}{(k+5)^{3}} U A_{\mp}\left(\begin{array}{l}
F_{11} \\
F_{22}
\end{array}\right) \\
& -\frac{8 i(k+1)}{(k+5)^{3}} U B_{3}\left(\begin{array}{c}
F_{21} \\
F_{12}
\end{array}\right)+\frac{8 i(k+1)}{(k+5)^{3}} U B_{\mp}\left(\begin{array}{l}
F_{22} \\
F_{11}
\end{array}\right) \\
& \mp \frac{4\left(248 k^{3}+2653 k^{2}+8058 k+5733\right)}{9(k+5)^{3}(13 k+17)} \partial U\left(\begin{array}{l}
F_{21} \\
F_{12}
\end{array}\right) \\
& \mp \frac{16\left(62 k^{3}+49 k^{2}+948 k+1089\right)}{9(k+5)^{3}(13 k+17)} U \partial\left(\begin{array}{l}
F_{21} \\
F_{12}
\end{array}\right) \\
& \left.\mp \frac{8(k-3)}{3(k+5)^{3}} U U\left(\begin{array}{l}
F_{21} \\
F_{12}
\end{array}\right)-\frac{32(k-3)}{3(k+5)^{3}} \partial\left(\begin{array}{l}
F_{21} \\
F_{12}
\end{array}\right) F_{12} F_{21}\right](w) \\
& +\frac{1}{(z-w)^{2}}[+\cdots](w)+\frac{1}{(z-w)}[+\cdots](w)+\cdots
\end{aligned}
$$

One does not see any higher spin current in the fourth order pole.

Finally, the final OPE can be described as

$$
\begin{aligned}
\mathbf{W}^{(\mathbf{3})}(z) \mathbf{W}^{(\mathbf{3})}(w) & =\frac{1}{(z-w)^{6}}\left[\frac{64 k\left(31 k^{3}+434 k^{2}+1419 k+1080\right)}{(k+5)^{3}(13 k+17)}\right] \\
& +\frac{1}{(z-w)^{4}}\left[-\frac{2(k-3)(5 k-23)}{(k+5)(13 k+17)} \mathbf{P}^{(\mathbf{2})}+\frac{8(k-3)^{2}(5 k-23)}{3(k+5)^{2}(13 k+17)} \mathbf{T}^{(\mathbf{2})}\right. \\
& +\frac{8(k-3)(5 k-23)}{(k+5)(13 k+17)} \mathbf{W}^{(\mathbf{2})}-\frac{16(\mathbf{k}-\mathbf{3})^{\mathbf{2}}(11 k+7)}{(k+5)(13 k+17)^{2}} \mathbf{T}^{(\mathbf{1})} \mathbf{T}^{(\mathbf{1})} \\
& +\frac{8\left(3244 k^{4}+41659 k^{3}+151375 k^{2}+203133 k+83997\right)}{3(k+5)^{2}(13 k+17)^{2}} T \\
& +\frac{32\left(61 k^{3}+383 k^{2}+573 k+243\right)}{3(k+5)^{3}(13 k+17)} A_{3} A_{3}-\frac{8(k-3)^{2}(5 k-23)}{3(k+5)^{3}(13 k+17)} A_{3} B_{3} \\
& +\frac{32 i\left(61 k^{3}+383 k^{2}+573 k+243\right)}{3(k+5)^{3}(13 k+17)} \partial A_{3} \\
& -\frac{128 i\left(7 k^{3}+86 k^{2}+267 k+252\right)}{3(k+5)^{4}(13 k+17)} A_{3} F_{11} F_{22}-\frac{24 i}{(k+5)^{2}} A_{3} F_{12} F_{21} \\
& +\frac{32\left(61 k^{3}+383 k^{2}+573 k+243\right)}{3(k+5)^{3}(13 k+17)} A_{+} A_{-}
\end{aligned}
$$




$$
\begin{aligned}
& -\frac{8 i\left(127 k^{3}+1397 k^{2}+3453 k+5895\right)}{3(k+5)^{4}(13 k+17)} A_{-} F_{11} F_{12} \\
& -\frac{16 i\left(17 k^{3}+217 k^{2}+879 k+327\right)}{(k+5)^{4}(13 k+17)} A_{+} F_{21} F_{22} \\
& -\frac{8\left(5 k^{3}-827 k^{2}-5325 k-4941\right)}{3(k+5)^{3}(13 k+17)} B_{3} B_{3}-\frac{24 i}{(k+5)^{2}} B_{3} F_{12} F_{21} \\
& -\frac{8 i\left(5 k^{3}-827 k^{2}-5325 k-4941\right)}{3(k+5)^{3}(13 k+17)} \partial B_{3} \\
& +\frac{128 i\left(7 k^{3}+86 k^{2}+267 k+252\right)}{3(k+5)^{4}(13 k+17)} B_{3} F_{11} F_{22} \\
& +\frac{16 i\left(41 k^{3}+757 k^{2}+2271 k+1395\right)}{3(k+5)^{4}(13 k+17)} B_{-} F_{12} F_{22} \\
& -\frac{8\left(5 k^{3}-827 k^{2}-5325 k-4941\right)}{3(k+5)^{3}(13 k+17)} B_{+} B_{-} \\
& +\frac{8 i\left(49 k^{3}+395 k^{2}+1395 k+1689\right)}{(k+5)^{4}(13 k+17)} B_{+} F_{11} F_{21} \\
& -\frac{4(k-3)\left(193 k^{2}+1064 k+975\right)}{3(k+5)^{3}(13 k+17)} F_{11} G_{22} \\
& +\frac{8\left(244 k^{4}+2291 k^{3}+12045 k^{2}+35937 k+24723\right)}{3(k+5)^{4}(13 k+17)} \partial F_{11} F_{22} \\
& -\frac{8\left(244 k^{4}+2381 k^{3}+11811 k^{2}+33759 k+30933\right)}{3(k+5)^{4}(13 k+17)} F_{11} \partial F_{22} \\
& -\frac{8(k-3)\left(28 k^{2}+179 k+243\right)}{(k+5)^{3}(13 k+17)} F_{12} G_{21} \\
& +\frac{16\left(122 k^{4}+1183 k^{3}+5805 k^{2}+17973 k+13293\right)}{3(k+5)^{4}(13 k+17)} \partial F_{12} F_{21} \\
& -\frac{16\left(122 k^{4}+1163 k^{3}+6017 k^{2}+17241 k+14121\right)}{3(k+5)^{4}(13 k+17)} F_{12} \partial F_{21} \\
& -\frac{24(k-3)(11 k+7)}{(k+5)^{2}(13 k+17)} F_{21} G_{12}-\frac{8(k-3)\left(94 k^{2}+551 k+453\right)}{3(k+5)^{3}(13 k+17)} F_{22} G_{11} \\
& +\frac{24 i(k-3)(5 k-23)}{(k+5)^{3}(13 k+17)} U A_{3}+\frac{8 i(k-3) k(5 k-23)}{(k+5)^{3}(13 k+17)} U B_{3} \\
& +\frac{8\left(244 k^{3}+2189 k^{2}+7062 k+4941\right)}{3(k+5)^{3}(13 k+17)} U U \\
& \left.-\frac{32(k-3)^{2}(5 k-23)}{3(k+5)^{4}(13 k+17)} U F_{11} F_{22}+\frac{24(k-3)(5 k-23)}{(k+5)^{3}(13 k+17)} U F_{12} F_{21}\right](w) \\
& +\frac{1}{(z-w)^{3}} \frac{1}{2} \partial(\text { pole }-4)(w)+\frac{1}{(z-w)^{2}}[+\cdots](w)+\frac{1}{(z-w)}[+\cdots](w)+\cdots .
\end{aligned}
$$

Note the presence of the nonlinear term containing the higher spin-1 current in the fourth 
order pole. In the basis of (4.7), one realizes that this term disappears eventually.

It would be interesting to write the above 136 OPEs in the simplified notations as in Appendix $A$ or Appendix $D$.

\section{References}

[1] M. R. Gaberdiel and R. Gopakumar, "Higher Spins \& Strings," JHEP 1411, 044 (2014) arXiv:1406.6103 [hep-th]].

[2] M. R. Gaberdiel and R. Gopakumar, "Stringy Symmetries and the Higher Spin Square," arXiv:1501.07236 [hep-th].

[3] A. Sevrin, W. Troost, A. Van Proeyen and P. Spindel, "EXTENDED SUPERSYMMETRIC sigma MODELS ON GROUP MANIFOLDS. 2. CURRENT ALGEBRAS," Nucl. Phys. B 311, 465 (1988).

[4] M. R. Gaberdiel and R. Gopakumar, "Large $\mathcal{N}=4$ Holography," JHEP 1309, 036 (2013) arXiv:1305.4181 [hep-th]].

[5] C. Ahn, "Higher Spin Currents in Wolf Space. Part I," JHEP 1403 (2014) 091 arXiv:1311.6205 [hep-th]].

[6] A. Sevrin and G. Theodoridis, "N=4 Superconformal Coset Theories," Nucl. Phys. B 332, 380 (1990).

[7] N. Saulina, "Geometric interpretation of the large N=4 index," Nucl. Phys. B 706, 491 (2005) hep-th/0409175.

[8] C. Ahn, "Higher Spin Currents in Wolf Space: Part II," Class. Quant. Grav. 32, no. 1, 015023 (2015) arXiv:1408.0655 [hep-th]].

[9] M. Beccaria, C. Candu and M. R. Gaberdiel, "The large N $=4$ superconformal $W_{\infty}$ algebra," JHEP 1406 (2014) 117 [arXiv:1404.1694 [hep-th]].

[10] K. Thielemans, "A Mathematica package for computing operator product expansions," Int. J. Mod. Phys. C 2, 787 (1991).

[11] C. Ahn and H. Kim, "Higher Spin Currents in Wolf Space for Generic N," JHEP 1412 (2014) 109 [arXiv:1411.0356 [hep-th]]. 
[12] C. Ahn and J. Paeng, "Higher Spin Currents in Orthogonal Wolf Space," Class. Quant. Grav. 32, no. 4, 045011 (2015) arXiv:1410.0080 [hep-th]]. 